LIFE-SPAN EFFECTS

\title{
OF IONIZING RADIATION
}

\section{IN THE}

\author{
BEAGLE DOG
}

\begin{abstract}
A SUMMARY ACCOUNT OF FOUR DECADES OF RESEARCH FUNDED BY THE U.S. DEPARTMENT OF ENERGY AND ITS PREDECESSOR AGENCIES
\end{abstract}

BY

ROY C. THOMPSON

Senior Staff Scientist Pacific Northwest Laboratory

Richland Washington 


\title{
DISCLAIMER
}

This report was prepared as an account of work sponsored by an agency of the United States Government. Neither the United States Government nor any agency thereof, nor Battelle Memorial institute, nor any or their employees, makes any warranty, expressed or implied, or assumes any legal liability or responsibility for the accuracy, completeness, or usefulness of any information, apparatus, product or process disclosed, or represents that its use would not infringe privately owned or process disclosed, or represents that its use would not intringe privately owned
rights. Reference herein to any specific commercial product, process, or service by trade name, trademark, manufacturer, or otherwise does not necessarily const $\mathrm{i}$. tute or imply its endorsement, recommendation, or favoring by the United States Government or any agency thereof, or Battelle Memorial Institute. The views and opinions of authors expressed herein do not necessarily state or reflect those of the United States Government or any agency thereof.

\author{
PACIFIC NORTHWEST LABORATORY \\ operated by \\ BATTELLE MEMORIAL INSTITUTE \\ for the \\ UNITED STATES DEPARTMENT OF ENERGY \\ under Contract DE-AC06-76RLO 1830
}

GCDY NO. 2 
The idea for a report or book reviewing the total effort on life-span studies of radiation effects, conducted with beagles and sponsored by the Department of Energy (DOE) and its predecessor agencies, was not one that originated with the author of this book. The book was designed to fill a need that has been felt by many during the nearly 40 years that such studies have been in progress. This need was perhaps most keenly felt in DOE Headquarters, where expenditures for these studies had to be justified for annual budget requests. The need became more acute in the mid-1980s when all Federal science budgets became tighter, and the competition for funds within the DOE Office of Health and Environmental Research (OHER) became more critical. Hard questions were asked as to what scientific purposes were served by continuation of the life-span beagle studies. While the completion of studies in progress was generally supported, there were widely differing opinions as to exactly what "completion" meant in the context of these studies. To some it meant funding until the "last dog dies," while to others the death of the last dog was only a preliminary to the really important analysis and interpretation of the voluminous specimens and data accumulated over decades of observation.

The direct antecedent of the present volume was the Twenty-Second Hanford Life Sciences Symposium held at Richland, Washington, September 27-29, 1983, which was titled, Life-Span Radiation Effects Studies in Animals: What Can

They Tell Us? The Proceedings of that symposium, which Judy Mahaffey and I edited, contained over 600 pages and dealt, not entirely, but largely with the lifespan beagle studies. These Proceedings were not the general survey of the total beagle effort that was needed, but they provided a starting point from which to construct such a survey. Completion of the task of editing these Proceedings happened to coincide with my formal retirement. I was willing and available to do a job that might have little appeal to one immersed midstream in a scientific career, but which had considerable appeal to one in a somewhat nostalgic "tapering-off" mode.

A further qualification for my involvement in this project was the fact that, although I had firsthand familiarity with the people and experiments involved, over a forty-year time span, I had never been personally engaged in the beagle studies. This helped me to retain an unbiased view of the input from the various laboratories involved, both in actual fact and in the perception of the scientists in these laboratories, whose cooperation was essential to my effort. 
Although my name appears as the sole author of this book, and errors of omission or commission are my responsibility, the total effort could not have been conducted without the encouragement and cooperation of a great many other people. I would particularly like to acknowledge the early encouragement of Bill Bair and Jim Park, and their willingness to provide the support services of the Pacific Northwest Laboratory over a somewhat leisurely extended period. Bob Thomas, at DOE-OHER headquarters, has had oversight responsibility for the beagle studies since 1984 and has been a constant supporter of this book, as has Martin Minthorn, who retired in 1988 as Director of the Health Effects Research Division of OHER.

From each of the laboratories involved in life-span beagle studies, I have received continuing cooperation in terms of information and materials and comments on draft manuscripts. I am particularly indebted to Scott Miller, Ray Lloyd, and Chuck Mays, at the University of Utah; to Leon Rosenblatt and Marvin Goldman, at the University of California, Davis; to Tom Fritz at Argonne National Laboratory; to Jim Park and Chuck Watson at Pacific Northwest Laboratory; and to Bruce Boecker and Roger McClellan at the Inhalation Toxicology Research Institute.

I must confess (with some pride, actually) that the publishing effort, to the point of camera-ready copy, was done by me, on my personal computer, without secretarial or typesetting assistance. However, in the considerable correspondence accompanying the project, I am happy to acknowledge the secretarial assistance of Patty Bresina and Kris Jacobs.

Finally, I am indebted for careful and critical editing to Dev Felton, who proofed the final draft, and to Ray Baalman who proofed the final manuscript. Ray was also responsible for dealing with the printers, DOE's Office of Scientific and Technical Information.

Roy C. Thompson

January, 1989 


\section{CONTENTS}

1. INTRODUCTION

2.1 The Decade of the 1950s

2.2 The Decade of the 1960 s

2.3 The Decade of the 1970 s

2.4 The Decade of the $1980 \mathrm{~s}$

3.1 Objectives 14

3.2 Choice of Experimental Animal

3.3 Experimental Protocols 18

3.4 Exposure Modes 21

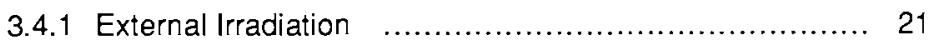

3.4 .2 Internal Irradiation ....................................... 22

3.5 Life-Span Observation ............................................ 26

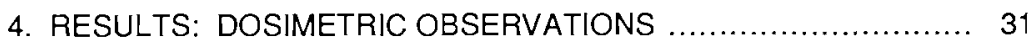

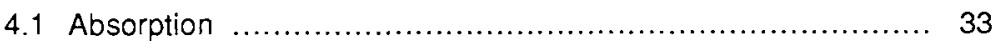

4.1.1 Absorption of Ingested Radionuclides .................. 34

4.1.2 Deposition and Absorption of Inhaled Radionuclides _.. 34

4.2 Distribution and Retention .................................... 36

4.2.1 Gross Distribution and Retention (by element) ......... 37

4.2.1.1 Strontium ..................................... 37

4.2.1.2 Yttrium ....................................... 38

4.2.1.3 Cesium ....................................... 38

4.2.1.4 Cerium .......................................... 39

4.2.1.5 Fused Aluminosilicate Particles .............. 39

4.2.1.6 Radium ...................................... 41

4.2.1.7 Thorium ......................................... 43

4.2.1.8 Plutonium ..................................... 44

4.2.1.9 Americium .................................... 47

4.2.1.10 Berkelium ....................................... 48

4.2.1.11 Californium …................................ 48

4.2.1.12 Einsteinium ................................... 49

4.2.2 Localized Distribution and Retention (by organ) .......... 49

4.2.2.1 Blood .......................................... 49

4.2.2.2 Lung ......................................... 50

4.2.2.3 Pulmonary Lymph Nodes ..................... 50 


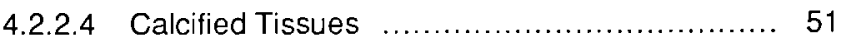

4.2.2.5 Liver ......................................... 55

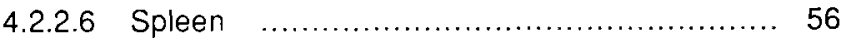

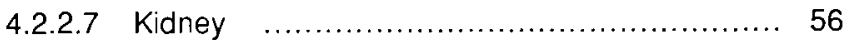

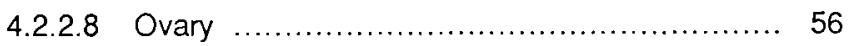

4.2.2.9 Testes ..................................... 56

4.2.2.10 Eye ......................................... 56

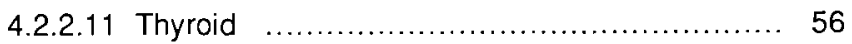

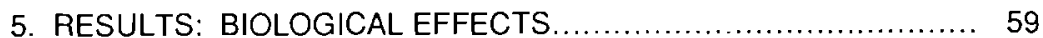

5.1 Acute Effects .............................................. 60

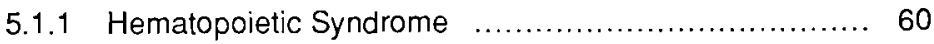

5.1 .2 Pulmonary Injury .................................. 61

5.1 .3 Gastrointestinal Injury ............................ 63

5.1 .4 Hepatic Injury ..................................... 63

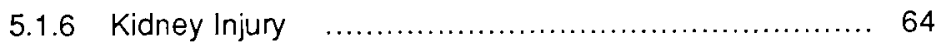

5.2 Longer-Term Nonneoplastic Effects $\quad \ldots \ldots \ldots \ldots \ldots \ldots \ldots \ldots \ldots .64$

5.2.1 Hematologic Effects ................................... 64

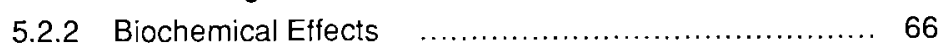

5.2.3 Nonneoplastic Effects in Calcified Tissues ............. 68

5.2.4 Nonneoplastic Effects in Lymph Nodes .................. 70

5.2.5 Nonneoplastic Effects in Thyroid ...................... 71

5.2.6 Nonneoplastic Effects in Ovaries ...................... 71

5.2.7 Nonneoplastic Effects in Eyes .......................... 71

5.3 Neoplastic Effects .......................................... 71

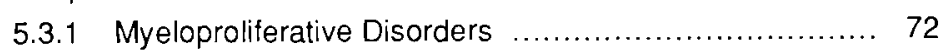

5.3 .2 Bone Tumors ................................... 73

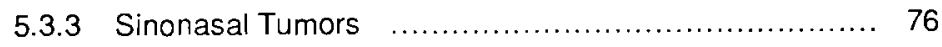

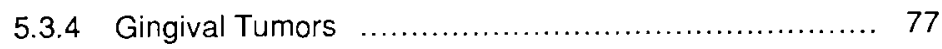

5.3 .5 Lung Tumors ..................................... 77

5.3.6 Pulmonary Lymph Node Tumors ..................... 79

5.3.7 Liver Tumors f....................................... 79

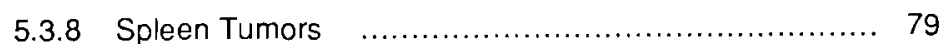

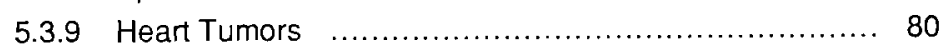

5.3.10 Eye Tumors ........................................... 80

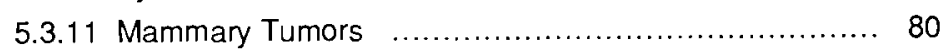

5.3.12 Urinary Tract Tumors .................................. 81

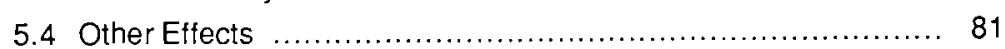

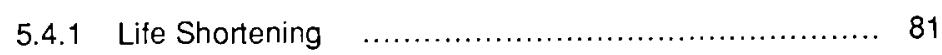

5.4 .2 Effects on Reproduction ............................. 83 
6.1 Studies Relating to Therapeutic Removal of Internally Deposited Radionuclides 86

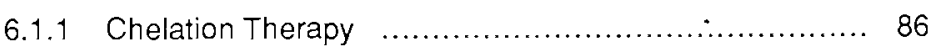

6.1 .2 Lavage Therapy ........................................ 89

6.2 Studies Relating to Pulmonary Function and

Clearance of Inhaled Particles

6.3 Studies Relating to Clearance of Wound-Deposited

Plutonium to Regional Lymph Nodes ..................... 92

6.4 Studies Relating to the Immune System ....................... 93

6.5 Studies Relating to Leukemogenesis ......................... 94

6.6 Studies Relating to Cytogenetic Effects ........................ 96

6.7 Miscellaneous Non-Life-Span Internal Exposure

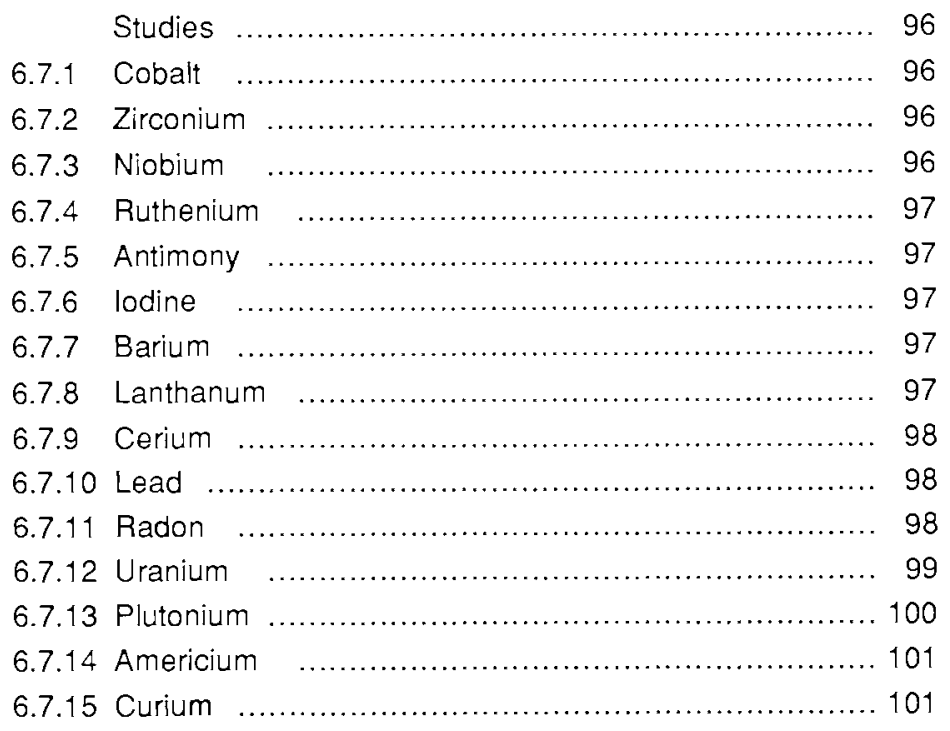

6.8 Studies of Non-Radiation-Related Toxicological and Disease Processes ............................. 102

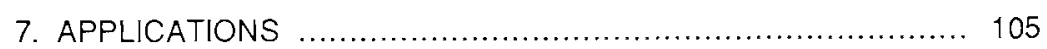

7.1 Evaluation of "Fallout" Consequences ...................... 107

7.2 Evaluation of the "Hot Particle" Hypothesis ................. 107

7.3 Development of Bioassay Models ........................... 108

7.4 Estimation of Relative Biological Effectiveness ............ 109 


\section{CONTENTS (Continued)}

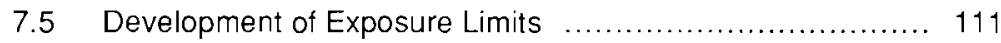

7.6 Clinical Applications to Man ...................................... 112

7.7 Estimation of Dose and Risk in Man ….................... 113

7.8 Contributions to Theories of Carcinogenesis …........... 118

7.9 Contributions to the Basic Biology of Bone ….............. 121

7.10 Applications to be Expected in the Future .................... 121

8. APPENDIX: EXPERIMENT SUMMARIES ........................ 123

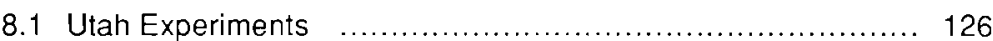

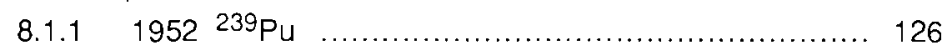

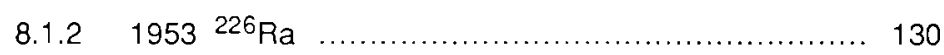

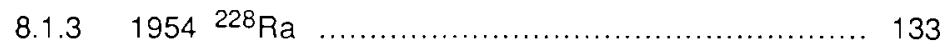

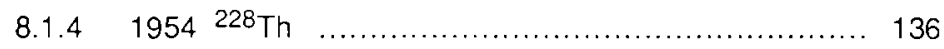

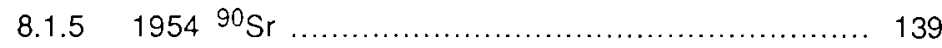

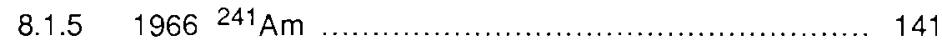

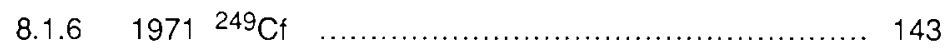

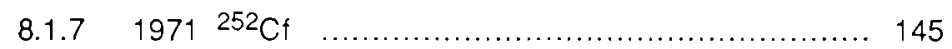

8.1.8 $1972{ }^{239} \mathrm{Pu}$ (juvenile) .............................. 147

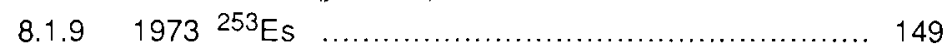

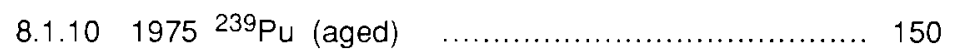

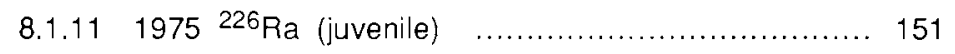

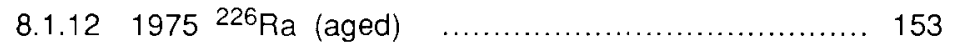

$8.1 .131977{ }^{224} \mathrm{Ra}$...................................... 154

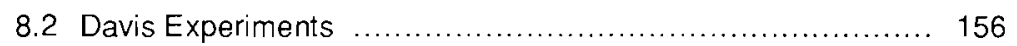

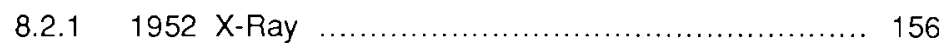

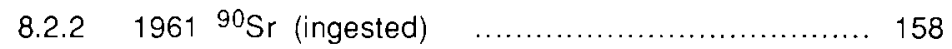

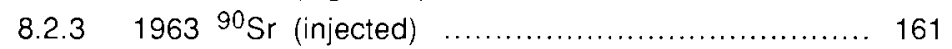

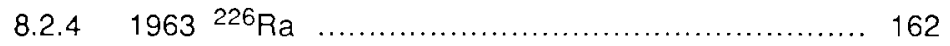

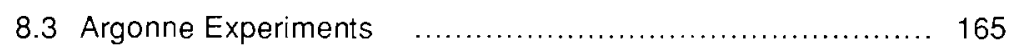

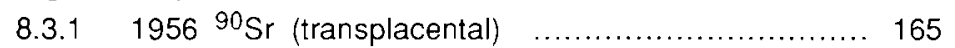

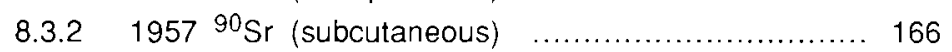

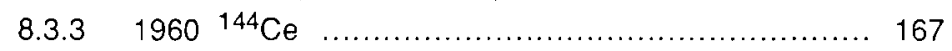

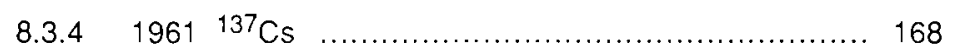

8.3.5 $1968{ }^{60} \mathrm{Co}$ (external gamma, continuous) $\ldots \ldots \ldots \ldots . . .170$

8.3.6 $1968{ }^{60} \mathrm{Co}$ (external gamma, terminated) $\ldots \ldots \ldots \ldots . . .172$

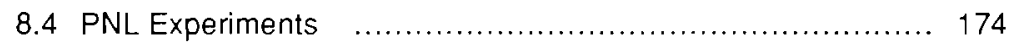

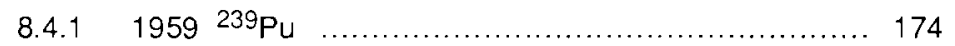

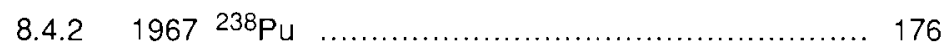

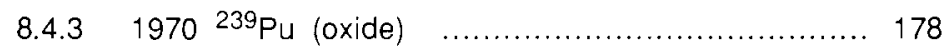

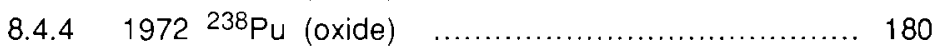




\section{CONTENTS (Continued)}

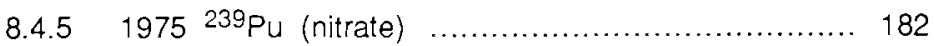

8.5 ITRI Experiments ............................................ 184

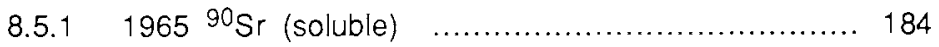

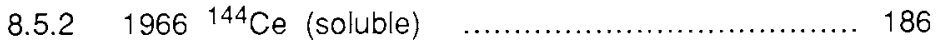

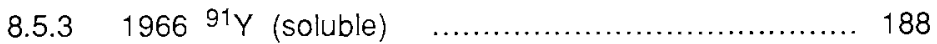

$8.5 .4 \quad 1967{ }^{144} \mathrm{Ce}$ (insoluble) ............................ 190

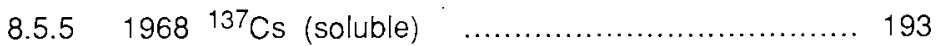

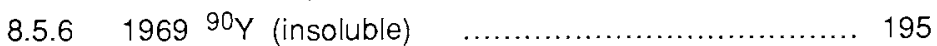

8.5.7 $197091 Y$ (insoluble) $\quad$................................. 197

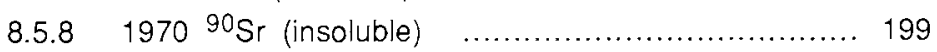

$8.5 .91972{ }^{144} \mathrm{Ce}$ (juvenile) ............................... 201

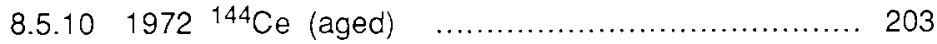

$8.5 .111972{ }^{144} \mathrm{Ce}$ (multiple exposures) ................. 205

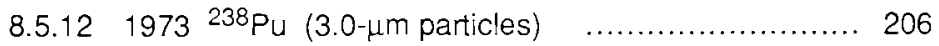

$8.5 .131974{ }^{238} \mathrm{Pu}(1.5-\mu \mathrm{m}$ particles $) \quad$.................... 208

$8.5 .141977{ }^{239} \mathrm{Pu}(0.75-\mu \mathrm{m}$ particles $) \quad \ldots \ldots \ldots \ldots \ldots \ldots \ldots . . \ldots . \ldots . . .211$

$8.5 .151977{ }^{239} \mathrm{Pu}(1.5-\mu \mathrm{m}$ particles $) \quad \ldots \ldots \ldots \ldots \ldots \ldots \ldots \ldots . . . . . . . .213$

8.5.16 $1977{ }^{239} \mathrm{Pu}(3.0-\mu \mathrm{m}$ particles) .................... 215

$8.5 .171977239 \mathrm{Pu}$ (multiple exposures) ................... 217

$8.5 .181979{ }^{239} \mathrm{Pu}$ (juvenile) ............................. 218

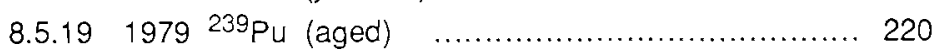

9. BIBLIOGRAPHY AND SENIOR-AUTHOR CITATION INDEX $\ldots \ldots \ldots 223$

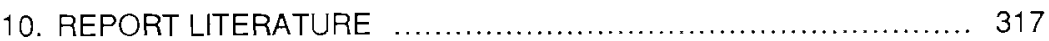





\section{INTRODUCTION}

Nearly 40 years ago, the U.S. Atomic Energy Commission made a far-reaching commitment to the support of life-span radiation-effects studies in a relatively long-lived animal, the beagle dog. As a consequence of this commitment, something in the range of 200 million dollars has already been spent on a group of closely related experiments, many of which are only now coming to fruition. Responsible fiscal management of these studies, directed toward securing an optimum return from past investments, and toward creative planning of future directions, requires a comprehensive view of this total effort. This report is designed to provide that comprehensive view.

This is primarily intended as a research management document. It is not a technical evaluation of research results. It would be neither possible nor appropriate to evaluate an ongoing effort of this magnitude in a singly authored document of this sort. Evaluation and interpretation are tasks for those directly involved in conducting these experiments. The limited objective of the present document is to describe what has been done, to give some of the background for why it was done, to describe results already realized and applications that have been made of these results-all in a manner designed to display the total effort rather than piecemeal details. While proposing no specific answers to the question "Where do we go from here?" it is hoped that the document will provide a basis for approaching that question in an informed manner. The maintenance of a continuity of scientific understanding and direction in these experiments, which often continue beyond the initiating investigators' working life, is no small part of the problem involved in conducting these experiments. It is hoped that this report will serve a useful function in support of that continuity.

Emphasis is placed on the major life-span experiments conducted under AEC/ERDA/DOE sponsorship at the University of Utah; at the University of California, Davis; at Argonne National Laboratory; at Pacific Northwest Laboratory; and at the Inhalation Toxicology Research Institute. These experiments are listed in Table 1.1, with an indication of the time period over which exposures were conducted, the general nature of those exposures, and the number of dogs held for life-span observation-total animals and those still alive. Many more animals were employed in preliminary and ancillary studies, and in the maintenance of breeding colonies. In some of the earlier experiments all of the animals are already dead; however, in none of the experiments have interpretations been completed, nor can they be considered finally completed until cross comparisons are made among all experiments. 
Table 1.1 MAJOR DOE/ERDA/AEC LIFE-SPAN BEAGLE EXPERIMENTS

\begin{tabular}{|c|c|c|c|c|c|}
\hline \multirow{2}{*}{$\begin{array}{l}\text { LABORA- } \\
\text { TORY }\end{array}$} & \multirow{2}{*}{$\begin{array}{l}\text { DATE OF } \\
\text { EXPOSURES }\end{array}$} & \multirow{2}{*}{$\begin{array}{l}\text { TYPE OF } \\
\text { EXPOSURE }\end{array}$} & \multirow{2}{*}{$\begin{array}{l}\text { EXPOSURE } \\
\text { MODE }\end{array}$} & \multicolumn{2}{|c|}{ LIFE-SPAN DOGS } \\
\hline & & & & TOTAL & ALIVE* \\
\hline \multirow[t]{14}{*}{ Utah*** } & '52-'74 & ${ }^{239} \mathrm{Pu}$ & IV inj. & 285 & 10 \\
\hline & $53-70$ & ${ }^{226} \mathrm{Ra}$ & IV inj. & 164 & 0 \\
\hline & '54-'63 & ${ }^{228} \mathrm{Ra}$ & IV inj. & 89 & 0 \\
\hline & '54-'63 & ${ }^{228} \mathrm{Th}$ & IV inj. & 94 & 0 \\
\hline & '55-'66 & ${ }^{90} \mathrm{Sr}$ & IV inj. & 99 & 0 \\
\hline & $66-75$ & ${ }^{241} \mathrm{Am}$ & IV inj. & 117 & 10 \\
\hline & $71 \cdot 74$ & ${ }^{249} \mathrm{Cf}$ & IV inj. & 36 & 5 \\
\hline & $71-73$ & ${ }^{252} \mathrm{Cf}$ & IV inj. & 35 & 3 \\
\hline & $72-78$ & ${ }^{239} \mathrm{Pu}$ & IV inj. (juvenile) & 75 & 26 \\
\hline & '73 & ${ }^{253}$ Es & IV inj. & 5 & 0 \\
\hline & $75 \cdot 78$ & ${ }^{239} \mathrm{Pu}$ & IV inj. (aged) & 34 & 3 \\
\hline & $75-78$ & ${ }^{226} \mathrm{Ra}$ & IV inj. (juvenile) & 53 & 23 \\
\hline & $75-80$ & ${ }^{226} \mathrm{Ra}$ & IV inj. (aged) & 33 & 1 \\
\hline & '77-'79 & ${ }^{224} \mathrm{Ra}$ & IV inj. (multiple) & 128 & 78 \\
\hline \multirow[t]{5}{*}{ Davis } & '52-'58 & $x$ ray & WB (fractionated) & 360 & 0 \\
\hline & $61-67$ & ${ }^{90} \mathrm{Sr}$ & Ingested (in-utero to & & \\
\hline & & & 540 days of age) & 484 & 0 \\
\hline & $63-67$ & ${ }^{90} \mathrm{Sr}$ & IV inj. & 46 & 0 \\
\hline & '63-67 & ${ }^{226} \mathrm{Ra}$ & IV inj. (multiple) & 341 & 0 \\
\hline \multirow[t]{8}{*}{ Argonne } & 56 & ${ }^{90} \mathrm{Sr}$ & Transplacental & 53 & 0 \\
\hline & 57 & ${ }^{90} \mathrm{Sr}$ & SC inj. (multiple, & & \\
\hline & & & various ages) & 98 & 0 \\
\hline & $60-' 64$ & ${ }^{144} \mathrm{Ce}$ & IV inj. & 49 & 0 \\
\hline & $61-63$ & ${ }^{137} \mathrm{Cs}$ & IV inj. & 65 & 0 \\
\hline & '68-78 & gamma ray & WB (continuous to death) & 311 & 115 \\
\hline & '68-77 & gamma ray & WB (continuous to pre- & & \\
\hline & & & determined doses) & 343 & 70 \\
\hline \multirow[t]{5}{*}{ PNL } & '59-62 & ${ }^{239} \mathrm{PuO}_{2}$ & Inhal'n & 35 & 0 \\
\hline & $' 67$ & ${ }^{238} \mathrm{PuO}_{2}$ & Inhal'n & 22 & 0 \\
\hline & $' 70-72$ & ${ }^{239} \mathrm{PuO}_{2}$ & Inhal'n & 136 & 1 \\
\hline & $' 72-' 75$ & ${ }^{238} \mathrm{PuO}_{2}$ & Inhal'n & 136 & 22 \\
\hline & '75-'77 & ${ }^{239} \mathrm{Pu}\left(\mathrm{NO}_{3}\right)_{4}$ & Inhal'n & 148 & 77 \\
\hline
\end{tabular}


Table 1.1 MAJOR DOE/ERDA/AEC LIFE-SPAN BEAGLE EXPERIMENTS (Cont.)

\begin{tabular}{|c|c|c|c|c|c|}
\hline \multirow{2}{*}{$\begin{array}{l}\text { LABORA- } \\
\text { IORY }\end{array}$} & \multirow{2}{*}{$\begin{array}{l}\text { DATE OF } \\
\text { EXPOSURES }\end{array}$} & \multirow{2}{*}{$\begin{array}{l}\text { TYPE OF } \\
\text { EXPOSURE }\end{array}$} & \multirow{2}{*}{$\begin{array}{l}\text { EXPOSURE } \\
\text { MODE }\end{array}$} & \multicolumn{2}{|c|}{ LIFE-SPAN DOGS } \\
\hline & & & & TOTAL & ALIVE* \\
\hline \multirow[t]{19}{*}{ ITRI } & '65-'67 & ${ }^{90} \mathrm{SrCl}_{2}$ & Inhal'n & 63 & 0 \\
\hline & '66-'67 & ${ }^{144} \mathrm{CeCl}_{3}$ & Inhal'n & 70 & 0 \\
\hline & '66-'67 & ${ }^{91} \mathrm{YCl}_{3}$ & Inhal'n & 54 & 0 \\
\hline & $' 67-71$ & ${ }^{144} \mathrm{Ce}$ (FAP) & Inhal'n & 126 & 1 \\
\hline & '68-'69 & ${ }^{137} \mathrm{CsCl}$ & IV inj. & 66 & 0 \\
\hline & $69-71$ & ${ }^{90} Y(F A P)$ & Inhal'n & 101 & 0 \\
\hline & $' 70-71$ & ${ }^{91}$ (FAP) & Inhal'n & 108 & 0 \\
\hline & $70-74$ & ${ }^{90} \mathrm{Sr}$ (FAP) & Inhal'n & 124 & 5 \\
\hline & $' 72-76$ & ${ }^{144} \mathrm{Ce}$ (FAP) & Inhal'n (juvenile) & 54 & 11 \\
\hline & '72-'75 & ${ }^{144} \mathrm{Ce}(\mathrm{FAP})$ & Inhal'n (aged) & 54 & 0 \\
\hline & $' 72-75$ & ${ }^{144} \mathrm{Ce}$ (FAP) & Inhal'n (multiple) & 36 & 2 \\
\hline & '73-'76 & ${ }^{238} \mathrm{PuO}_{2}$ & Inhal'n $(3.0 \mu \mathrm{m})$ & 84 & 7 \\
\hline & '74-'76 & ${ }^{238} \mathrm{PuO}_{2}$ & Inhal'n $(1.5 \mu \mathrm{m})$ & 84 & 12 \\
\hline & $' 77-79$ & ${ }^{239} \mathrm{PuO}_{2}$ & Inhal'n $(0.75 \mu \mathrm{m})$ & 60 & 22 \\
\hline & $' 77-79$ & ${ }^{239} \mathrm{PuO}_{2}$ & Inhal'n $(1.5 \mu \mathrm{m})$ & 108 & 45 \\
\hline & '77-'79 & ${ }^{239} \mathrm{PuO}_{2}$ & Inhal'n $(3.0 \mu \mathrm{m})$ & 83 & 23 \\
\hline & $' 77-78$ & ${ }^{239} \mathrm{PuO}_{2}$ & Inhal'n (mulfiple, $0.75 \mu \mathrm{m}$ ) & 72 & 34 \\
\hline & '79-'83 & ${ }^{239} \mathrm{PuO}_{2}$ & Inhal'n (juvenile, $1.5 \mu \mathrm{m}$ ) & 108 & 83 \\
\hline & '79-'82 & ${ }^{239} \mathrm{PuO}_{2}$ & Inhal'n (aged, $1.5 \mu \mathrm{m}$ ) & 60 & 3 \\
\hline Total & '52-'83 & & & 5389 & 692 \\
\hline
\end{tabular}

* As of final quarter of 1987.

** Surviving Utah dogs were moved to ITRl in September, 1987.

Abbreviations: in $=$ injection; IV = intravenous; $S C=$ subcutaneous; $W B=$ whole-body; $F A P=$ fused aluminosilicate particle; Inhal' $n$ = inhalation; $\mu \mathrm{m}$ = activity median aerodynamic diameter of particles (micrometers).

Other radiation-effects studies with beagle dogs, of shorter than life-span duration, conducted in these and other laboratories, will be considered in this report only to the extent required to place the major life-span studies of Table 1.1 in proper perspective. Thus, we will consider only briefly the important early experiments with beagle dogs conducted at the University of Rochester (Casarett, 1970; Stannard, 1988); the experiments with plutonium-contaminated wounds (Watters, 1972; Lebel, 1976) and the non-DOE-sponsored experiments at Colorado State University (Benjamin, 1986); the experiments investigating the effects of uranium-mine atmospheres conducted at Pacific Northwest Laboratory 
(Cross, 1982; Stuart, 1971); and the many ancillary short-term experiments suggested by, and often conducted in parallel with, the life-span experiments.

The general approach taken in reviewing these experiments will be apparent from the Table of Contents. It was considered important to maintain a continuing broad viewpoint on the total effort rather than to divide the report into separate sections dealing with individual experiments in individual laboratories. Such separate study-by-study consideration would have allowed a more straightforward presentation of experimental design and results, but it would have tended to obscure the fact that these experiments were planned as a total program with important interactions (Book, 1980; Bruner, 1969; Richmond, 1970b; Totter, 1972). A concession to the study-by-study approach is made in Chapter 8 , an appendix in which each experiment is outlined on an individual basis.

While many compromises were obviously required in holding this document to a reasonable size, a special effort was made to provide a complete bibliography, in Chapter 9. While knowledgeable readers will inevitably be disappointed by the omission from the text of certain details that they feel should have been included, it is hoped that the bibliography will provide them with a useful key to the total published literature on these studies. To increase the usefulness of the bibliography, entries cited in the text are indexed to the pages on which they are cited, thus providing a senior-author citation index. Another unusual feature of Chapter 9 is the inclusion of brief biographical sketches of the principal contributors to the life-span radiation effects studies in beagles.

For some detailed information, only the year-by-year progress reports from the various laboratories provide the total picture-or the nearest thing available to a total picture. These progress reports have usually not been referenced in the text, nor are they listed in the bibliography of Chapter 9. They are, however, tabulated in Chapter 10, and referenced to individual experiments in Chapter 8.

The senior-author citation index of Chapter 9 is the only index is provided. The entire report may be thought of as an index to the life-span beagle studies, and it was not clear how a useful index to this index could be constructed. Because of the limited scope of subject matter and the considerable redundancy in presentation, the Table of Contents provides the necessary and sufficient features of a subject index. 
Contents

2.1 The Decade of the $1950 \mathrm{~s}$ 6

2.2 The Decade of the $1960 \mathrm{~s}$

8

2.3 The Decade of the $1970 \mathrm{~s}$

Studies of the effects of radiation on health and longevity have been principally stimulated by the need to control to safe levels the exposure of those who must work with radiation. Data from the early decades of the twentieth century on effects in humans from external exposure to medical x-irradiation, and data from internal occupational and medical exposure to radium, provided a basis for the establishment of exposure limits in the 1920s and 1930s that seemed adequate for control of the infrequent exposures then encountered.

With the advent of the Nuclear Age, and its vastly increased potential for exposure to a bewildering array of external radiation sources and internally deposited radionuclides, the need for more extensive and more sophisticated information on radiation effects became urgently apparent. With little opportunity for the study of effects in humans-certainly no studies with controlled exposures-attention turned to studies in experimental animals. During, and in the years immediately following World War II, many studies were conducted, mostly in rats and mice, whose life spans were short enough to provide results within a few years. Based on these early studies, data on radiation effects were accumulated that led to the refinement of external exposure limits, and to the establishment of internal deposition limits for a large number of radionuclides. These exposure limits were adopted by the various laboratories and industries employing radiation workers, and codified by such groups as the National Council on Radiation Protection and Measurements (as it is now titled), and the International Commission on Radiological Protection.

Basing human exposure limits on data from animal experiments posed serious problems of extrapolation. To the extent possible, the limits were anchored to the

* The reader is directed to Dr. J. N. Stannard's comprehensive new book, Radioactivity and Health: A History (Stannard, 1988), which served as a valuable source for this chapter, and which can be profitably consulted for further details. 
human experience with $\mathrm{x}$-irradiation and radium deposition, and extended from this base by inferences drawn from the animal data. While experimental rats and mice offered the advantage of speedy and economical acquisition of life-span data, their small size and short life span were serious disadvantages from other viewpoints. Could one expect to see in the two or three years of rodent life the full array of late developing effects that were known to be characteristic of low-level radiation exposures in humans? Could one evaluate in these small animals the subtle clinical effects that could be important in extrapolation to man? It was early recognized that life-span data from a larger and longer-lived animal was essential for confident extrapolation (Stannard, 1988).

Dogs were employed in a number of experiments conducted at the University of Chicago, at the University of California (Berkeley), and at the University of Rochester, during and shortly following World War II. These, however, were experiments involving a relatively few animals, usually for short time periods, for studies of radionuclide distribution or acute radiation effects (Stannard, 1988). The initiation of life-span studies in larger animals awaited the completion of the more immediately urgent rodent studies, and perhaps most importantly, the development of confidence in the longevity of support required for consideration of these unprecedented studies.

\subsection{THE DECADE OF THE 1950s}

It would be inaccurate to conclude that the major life-span experiments in beagle dogs initiated by the AEC, as outlined in Table 1.1 of the Introduction, were conceived as a single multifaceted program. The different experiments evolved in a serial fashion, but always with the knowledge of what had gone before, and with an effort to link them together into a coherent whole.

Dr. Stannard, in his historical review, concludes that the first proposal for use of the beagle dog in life-span radiation effects experiments may have come from Dr. George Hart, Dean of the Veterinary School at the University of California's Davis campus. A contract was let by the AEC, to Davis, in January of 1951, to study effects of graded and fractionated exposure of beagles to $\mathrm{x}$ rays. Effects on reproductive performance were of primary initial interest, but life-span effects were also to be followed. Justification for this project was based, in part, on the need for information to evaluate the radiation hazard to crews of nuclear powered aircraft, which were then considered a likely future development. This first Davis experiment was directed by Dr. A. C. Andersen; the first animals were exposed in 1952 (Andersen, 1969; Stannard, 1988). 
Coincident with developments at Davis, other life-span radiation effects experiments with beagle dogs were organized at the University of Utah. The origins of the Utah program have been well documented (Dougherty, T., 1962; Stover, 1972f). The earliest planning for the Utah program may, in fact, have preceded the Davis planning. The initial Utah experiments were very specifically aimed at providing data to support an exposure limit for plutonium. As then established, the plutonium limit was derived from the radium limit, which, in turn, was based on experience with exposed humans. The link between plutonium and radium was provided by comparative toxicity studies in mice. The applicability of the mouse data was highly suspect, considering that the critical effects observed were long-delayed bone tumors, and considering the major differences in life span, and skeletal size and growth characteristics, between man and the mouse (Stover, 1972f; Mays, 1986b).

The Utah experiments were therefore designed to compare the long-term toxicity of ${ }^{239} \mathrm{Pu}$ and ${ }^{226} \mathrm{Ra}$, administered as single intravenous doses to young-adult beagles (to correspond to the usually brief exposure of the young-adult radium dial painters). Because ${ }^{228} \mathrm{Ra}$ was implicated in the exposure of some of the dial painters, it and its alpha-emitting second daughter, ${ }^{228} \mathrm{Th}$, were also included in the experimental design (Evans, 1972; Van Dilla, 1956). The contract initiating the Utah project became effective July 1, 1950. The project was initially directed by Dr. John Z. Bowers, Dean of the College of Medicine of the University of Utah, who was succeeded as project director in 1954 by Dr. Thomas Dougherty. The first life-span dogs were injected in December of 1952.

While the original Davis and Utah dog experiments seem to have arisen rather independently, their subsequent evolution, and the spawning of life-span dog experiments in other laboratories, have followed a carefully orchestrated pattern of interrelationships. The widespread public concern for ${ }^{90} \mathrm{Sr}$ in the fallout from weapons tests conducted in the atmosphere prompted extensions of both the Utah and Davis programs. To maintain comparability with the other Utah experiments, ${ }^{90} \mathrm{Sr}$, at Utah, was administered by single intravenous injection; the first animals were injected in 1954 (Dougherty, T., 1962). Because humans are more apt to ingest the fallout ${ }^{90} \mathrm{Sr}$, experiments were later initiated at Davis with the radionuclide fed daily (Book, 1980; Goldman, 1967, 1969). Dogs received ${ }^{90} \mathrm{Sr}$ in this manner from midgestation to young adulthood. For comparison, other dogs received repeated injections of ${ }^{226}$ Ra during young adulthood (another link to the dial painters). Thus, within-laboratory comparisons would evaluate the relative toxicity of ${ }^{90} \mathrm{Sr}$ and ${ }^{226} \mathrm{Ra}$, while between-laboratory comparisons would evaluate possible effects of route of entry and exposure timing. Smaller supporting studies were initiated by Dr. Miriam Finkel at Argonne National Laboratory, 
in 1956 and 1957 , with beagle dogs receiving ${ }^{90} \mathrm{Sr}$ transplacentally and by daily subcutaneous injection (Finkel, 1960, 1972).

From the outset the need had been recognized for experiments with inhaled radionuclides, since inhalation is the usual route of entry in most types of occupational exposure. Exposure of an animal as large as a beagle dog to precisely measured quantities of very toxic materials, via inhalation, poses severe technical problems. The University of Rochester was the principal source of expertise in this area, and from the late 1950 s to the early 1970 s researchers there conducted a number of usually short-term experiments in which dogs were exposed to aerosols of polonium, uranium or plutonium oxides, or radon daughters. Ultimately, the large-scale inhalation experiments were conducted in other laboratories, but usually by graduates of the University of Rochester, employing equipment originally developed at the University of Rochester (Stannard, 1988).

One such graduate, Dr. William J. Bair, led the development of inhalation exposure capabilities at Pacific Northwest Laboratory (PNL), where concern for plutonium exposure was high-plutonium then being the principal product of the Hanford Project where PNL is located. Beagles to be held for life-span observation were exposed to ${ }^{239} \mathrm{PuO}_{2}$ as early as 1959 (Park, $J ., 1964$ ); smaller groups were later exposed to ${ }^{238} \mathrm{PuO}_{2}$ (Park, J., 1976). The earliest PNL experiments with inhaled plutonium were sponsored by the U. S. Air Force, which was concerned, with some justification as later events were to prove, about the potential for plutonium exposure following crashes of aircraft carrying nuclear weapons. The appearance of lung tumors in some of these Air Force dogs prompted the extension of these experiments under AEC sponsorship.

\subsection{THE DECADE OF THE 1960s}

The early years of the 1960 s saw the development of major new beagle dog programs to study the life-span effects of fission-product radionuclides. These programs were stimulated by concern for exposure of the world's population to these radionuclides in the fallout from nuclear weapons tests, and to the potential for exposure from nuclear reactors, employed as domestic power sources, in nuclear-powered rockets or in other devices under consideration at the time. Experiments directed by Drs. Thomas E. Fritz and William P. Norris were initiated at Argonne National Laboratory, in 1960, with ${ }^{144} \mathrm{Ce}$ (Fritz, 1970), and in 1961 with ${ }^{137} \mathrm{Cs}(N C R P, 1977)$, both administered to beagles as a single intravenous injection.

The major concern, however, was for the inhalation route. It was concluded that neither Rochester nor PNL could expand their inhalation exposure 
commitments to handle such a program, and a contract was awarded in June of 1960 to the Lovelace Foundation for Medical Education and Research of Albuquerque, New Mexico, for development of a new laboratory, later to be known as the Inhalation Toxicology Research Institute (ITRI). Under the direction of Dr. Clayton S. White, with Rochester graduates in most of the key staff positions, exposure techniques and facilities were developed and a program of studies planned, which would look at selected fission products chosen for their range of radioactive half-lives and chemical behavior (McClellan, 1970). The first dogs were exposed in 1964. In 1966, direction of the ITRI program was assumed by Dr. Roger O. McClellan, who had served his apprenticeship at PNL, and who remained at ITRI until 1988.

A new radionuclide, ${ }^{241} \mathrm{Am}$, was added to the list being studied at Utah, with exposures commencing in 1966 (Stover, 1972f). Americium is only slightly less abundant than plutonium (on a radioactivity basis) in the fallout from weapons tests, and, because of its greater environmental mobility, it may constitute as great a hazard as plutonium.

At PNL, exposures were initiated in 1969 to determine the effects of daily exposure of beagle dogs to radon daughters and other components of uranium mine atmospheres (Cross, 1981, 1982; Stuart, 1970b, 1971). This experiment was designed to provide another link between effects in the dog and effects in the human; i.e., the humans that developed lung cancer from exposures in uranium mines. Because few of these animals were actually retained for life-span observation, this experiment is not given primary consideration in this report, but is briefly considered in the chapter on ancillary studies.

Argonne, in the $1960 \mathrm{~s}$, acquired ${ }^{60} \mathrm{Co}$ gamma-ray exposure facilities that were first employed in non-life-span studies of acute radiation effects in beagles (Norris, 1968). In 1967, a series of continuous, whole-body, gamma-ray exposure experiments were initiated (Norris, 1972). Dogs were exposed, 22 hours per day, 7 days per week, until they died or until a predetermined total dose had been delivered. These experiments have been extended to lower doses, and some are still in progress. In contrast to most of the other life-span dog experiments, these were designed primarily to provide insight into mechanisms of radiation effects rather than to simulate practical hazard situations (Fritz, 1986). These experiments are now cosponsored by the National Cancer Institute.

\subsection{THE DECADE OF THE 1970s}

By this time, preliminary results were accumulating from some of the earlier initiated experiments. These results, in many cases, pointed up the need for 
data from additional animals, exposed to explore effects not anticipated in the initial experimental design. At Utah, where Dr. Webster S. S. Jee had assumed direction of the project in 1974 following the death of Tom Dougherty, the high incidence of effects in the lowest ${ }^{239} \mathrm{Pu}$ and ${ }^{226} \mathrm{Ra}$ exposure levels prompted addition of groups exposed at lower levels (Stover, 1972f). Small experiments with californium isotopes (Lloyd, R., 1976) and with einsteinium (Lloyd, R., 1975) were also initiated to permit comparisons between a larger number of actinide elements, and to evaluate the biological effectiveness of fission fragments emitted in the decay of ${ }^{252} \mathrm{Cf}$.

A major experiment with ${ }^{224} \mathrm{Ra}$, initiated at Utah in 1977 (Lloyd, R., 1982; Wrenn, 1986), was designed to provide still another link between the beagle dog and the human; this time to the humans that had been treated with ${ }^{224} \mathrm{Ra}$ for relief of ankylosing spondylitis, and who were, and still are, being followed in epidemiologic studies in Germany (Mays, 1978, 1986c; Spiess, 1978). Direction of the beagle project at the University of Utah passed, in 1979, from "Web" Jee to Dr. McDonald E. Wrenn

At PNL, the early plutonium inhalation experiments had produced a nearly 100 percent incidence of lung tumors, and new experiments were initiated, extending exposures to levels equivalent in the dog to those permitted in humans by occupational exposure standards (Totter, 1972; Park, J., 1972, 1986). Parallel experiments compared the effects of inhaled ${ }^{239} \mathrm{PuO}_{2},{ }^{238} \mathrm{PuO}_{2}$, and ${ }^{239} \mathrm{Pu}\left(\mathrm{NO}_{3}\right)_{4}$.

At ITRI, techniques had been developed to produce monodisperse aerosols, i.e., aerosols with a very narrow particle-size range (Raabe, 1975). These techniques were employed in ${ }^{239} \mathrm{Pu}$ - and ${ }^{238} \mathrm{Pu}$-oxide exposure experiments with particles of various sizes (McClellan, 1972). Results from these experiments would compare with those obtained at PNL where polydisperse aerosols were employed. Experiments involving multiple inhalation exposures were also initiated during this period at ITRI (Boecker, 1977; Diel, 1982). This emphasis on plutonium was stimulated by the "hot particle controversy" instigated by allegations that plutonium aggregated in the form of highly radioactive particles possessed a carcinogenic potential much greater than the same quantity of plutonium more uniformly dispersed (Tamplin, 1974).

At Davis, Dr. Marvin Goldman assumed direction of the project in 1973, from Dr. Leo K. Bustad who had served in that capacity since 1965 . New experiments initiated at Davis during the 1970 s involved chronic whole-body exposure of beagle dogs to gamma rays from ${ }^{60} \mathrm{Co}$ (Goldman, 1986; Klein, 1984). These experiments were, in certain respects, a follow-on to the earlier chronic ${ }^{90} \mathrm{Sr}$ feeding experiment, and like that earlier experiment involved exposures starting at 
different ages, including in-utero. Designed primarily to elucidate mechanisms of leukemogenesis, they usually did not involve life-span observation; they are therefore not given primary consideration in this document, but are briefly considered in the chapter on ancillary studies.

\subsection{THE DECADE OF THE 1980s}

The 1980s saw the initiation of no new life-span dog studies. At Davis, the last life-span dog (who achieved the remarkable age of 18.5 years) died in 1986 . Much remains to be done, of course, in completing analyses and interpretation of the Davis experiments. This effort is continuing at Davis under the direction of Dr. Leon Rosenblatt.

At Utah, by September of 1987, the number of dogs remaining on life-span observation had decreased to 157 beagles and $9 \mathrm{St}$. Bernards. Two factors were mainly responsible for a decision to transfer these dogs from Utah: 1) the unfavorable economics of maintaining the small and dwindling colony, and 2) the desire of the University of Utah to build new research laboratories on the site of the existing kennels. In response to these factors, the remaining 157 beagles were transferred to the ITRI colony, and the $9 \mathrm{St}$. Bernards were transferred to PNL. Responsibility for managing completion of the Utah life-span studies was assigned to ITRI, with input from a small team of investigators at the University of Utah as well as investigators at ITRI.

Thus, by 1988, dogs remained on study at only Argonne, PNL, and ITRI. Final interpretation of experimental results must await the death of these animals, including controls, and the completion of radiochemical, clinical, and histological analyses on which interpretations must be based. The interrelationships between the results of the various studies can, of course, not be finally evaluated until all studies are completed, which will extend the period of final evaluation well into the $1990 \mathrm{~s}$.

It should also be recognized that materials derived from these life-span studies may provide the basis for experiments that could continue even further into the future. These life-span studies provide not only numerical data, but a wealth of biological material for basic studies of radiation carcinogenesis. For example, frozen cancerous and normal tissues and cell cultures from dogs of various ages and with various radiation exposure histories have been saved; they afford exciting opportunities for future molecular biological studies. Such potential spin-offs are currently receiving attention at all of the beagle laboratories (Frazier, 1987; Kelly, in press). 
Finally, it should not be assumed that the era of life-span dog studies is concluded with the animals now on experiment. Although one must acknowledge the present hiatus in newly initiated experiments, and one can appreciate the budgetary reasons for this situation, few would question that the decision to initiate this program, nearly 40 years ago, was a far-sighted decision that has paid, and will continue to pay, rich dividends. Should we assume that future planners will be less far-sighted? 


\section{THE EXPERIMENTS}

Contents

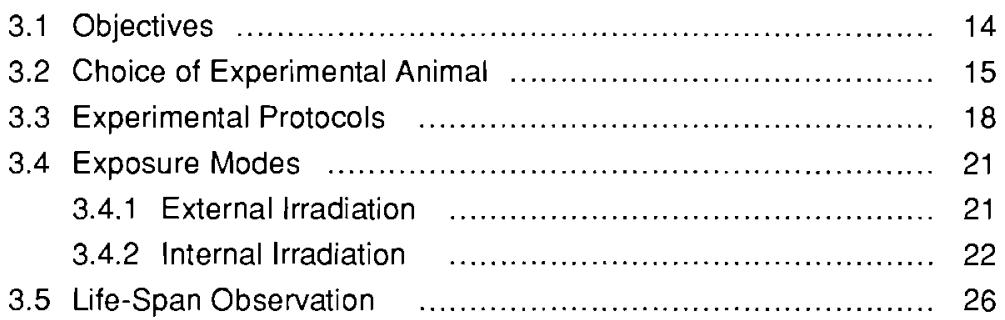

This chapter will describe the major life-span beagle experiments, which are the subject of this report. It will consider the principal objectives of these experiments. It will consider the experimental animal chosen to explore these objectives. Why the beagle dog? And what special constraints are imposed by this choice? It will consider the experimental protocols developed to accomplish these objectives. It will consider the routes of exposure employed, and the special problems encountered in the development of exposure techniques. Finally, it will consider in a general way the variety of observations required during the subsequent life span of the exposed animal. Consideration of specific experimental results will be reserved for subsequent chapters.

Several sources of information on the subject matter of this chapter require special mention. In addition to Stannard's history (Stannard, 1988), the Proceedings of the Hanford Life Sciences Symposium on Life-Span Radiation Effects Studies in Animals (Thompson, 1986) provides recent surveys of these experiments. Utah has published a lengthy review of their early studies (Dougherty, T., 1962) and several Proceedings volumes, chronicling not only their own studies, but including input from the other life-span studies (Dougherty, T., 1962d; Jee, 1976; Mays, 1969b; Stover, 1972; Wrenn, 1981). Informative Davis monographs relate specifically to the beagle as an experimental animal (Andersen, 1970; Shifrine, 1980), and to studies with ${ }^{90} \mathrm{Sr}$ (Goldman, 1972d). A recent monograph of the Commission of the European Communities includes a number of papers on the status of these experiments (Priest, 1985). Several papers relate to AEC planning in this area (Bruner, 1969; Durbin, 1978; Richmond, 1970b; Stover, 1972f; Totter, 1972). A paper by Rosenblatt is particularly helpful in succinctly pointing up the problems and potentials of long-term experiments with beagles (Rosenblatt, 1970). Finally, it must be acknowledged that details of these experiments are not always 
completely reported in the open literature, and recourse must sometimes be had to laboratory Progress Reports. These reports are listed in Chapter 10.

\subsection{OBJECTIVES}

As noted in the previous chapter, rather specific justifications were associated with each of the major life-span beagle experiments. However, certain characteristic objectives were shared by all. All were designed in response to the virtual absence of human data, and in response to the distrust of data available from more easily performed experiments in rats or mice. All should be considered as primarily "demonstration" experiments. They were experiments that ethically and politically could not be avoided.

If workers in the nuclear industry, or the general public, were to be exposed to increased levels of radiation of various types and in various manners, it was necessary to demonstrate that such exposures would cause no detectable effect in an animal that was as close a stand-in for man as could reasonably be studied. In the simplest terms, the primary objective of these experiments was to demonstrate the essential safety of a certain type of radiation exposure, at some tested level of exposure. The statistical limitations of any such demonstration were, of course, recognized, and a more scientific statement of the primary objective might be the establishment of a dose-response relationship, from which the safety of any specific radiation exposure could be estimated with some stated degree of confidence (Rosenblatt, 1970). When viewed as a doseeffect relationship, this primary objective defines two corollary objectives-the quantification of radiation dose and the identification and quantification of biological effect. It is in terms of these corollary objectives that the necessity for life-span studies in a relatively long-lived and large-sized animal becomes most apparent. Only in such an animal is it possible to make the kinds of measurements and observations that one would make in a human being suspected of suffering an effect from radiation exposure.

It is important to note that the "effect" aspect of the dose-effect relationship involves not only quantification, but, even more basically, identification. There was, and is, a concern that the difficulties of observation in the human, and other limitations in small-animal studies, may result in our overlooking certain effects of radiation exposure. Thus, while a particular type of cancer may be the most obvious finding in animals surviving the acute effects of irradiation, lifespan studies in a large, long-lived animal afford the opportunity to explore the possibility that other less dramatic effects may also result from the radiation exposure. 
Finally, in experiments such as these, serendipity must be recognized as an important objective. These are unprecedented experiments in terms of the effort expended in careful observation over extended periods of time. It would be unusual, indeed, if something unexpected were not observed. Considering only a single example, the existence of large aging populations of thoroughly investigated animals offers exciting possibilities for geriatric studies. Indeed, the impossibility of pursuing even a fraction of such potential spin-off studies was, and is, a frustrating aspect of the whole endeavor.

\subsection{CHOICE OF EXPERIMENTAL ANIMAL}

Of the animal species that might have been chosen for use in these experiments, several were rather easily eliminated. Non-human primates were too costly, were too difficult to handle and house, and had too long a life span. Pigs, burros, and horses were too large; rodents were too small and had too short a life span. Dogs were commonly used in research before the inception of the experiments considered in this report. However, the extensive use of the beagle, specifically bred for research purposes, is a development that has occurred coincident with these studies, and largely stimulated by them. Thus, the many arguments that can now be advanced for the use of this animal were unknown, or at least unsupported, when the decision to employ beagles in these experiments was made in 1950. Once that initial decision was made, the continued use of the beagle in subsequent experiments was dictated by the need for intercomparing data in the same animal model.

The American Foxhound was actually the first choice in considerations of an appropriate experimental animal for these studies, but it lost out to the beagle on grounds of availability (Stover, 1972f). The choice of the beagle seems, in retrospect, to be as good a choice as could have been made. Arguments in support of that choice would include the following (Book, 1980; Bustad, 1972b; Rehfeld, 1972):

- ready availability

- easy management-reflecting selection over centuries for field stamina, tractable disposition, and intelligent cooperation as a "pack dog"

- optimum size (about $10 \mathrm{~kg}$ )—small enough to be easily handled, while large enough to permit detailed clinical studies

- optimum life span (12 to 15 years) -an appropriate compromise between the desire to mimic humans and the need for timely results

- physiologic and anatomic similarities to humans-particularly as regards the critical hematopoietic, skeletal, and pulmonary systems. 
An extensive literature on the beagle dog as an experimental animal has developed from the AEC/ERDA/DOE-sponsored life-span studies. A full appreciation of this contribution could be conveyed only by a comprehensive review of this literature, which is beyond the scope of this report. This literature is included, however, in the Bibliography (Chapter 9). Of particular note is the treatise entitled The Beagle as an Experimental Dog, edited by A. C. Andersen, and published in 1970 (Andersen, 1970). This book summarizes much of the experience with beagles gained in the early years of the life-span studies. A later comprehensive report is The Canine as a Biomedical Research Model: Immunological, Hematological and Oncological Aspects, edited by Shifrine and Wilson (Shifrine, 1980). Particularly important have been data on skeletal structure (Gillett, in press; Jee, 1970; Park, J., 1986), mass (Garsd, 1981; Martin, 1981 ) and mineralization (Goldman, 1970); on the bone-lining cells and vasculature of the marrow (Miller, S, 1980, 1980b); on blood chemistry values (Goldthorpe, 1962; Kaspar, 1977; Pickrell, 1974); on pulmonary function parameters (Mauderly, 1972, 1973b, 1974, 1980b, 1983; Pickrell, 1971, 1973); on gastrointestinal function parameters (Miyabayashi, 1984, 1986); and on the spontaneous incidence of mammary (Chrisp, 1980; Moulton, 1986; Taylor, G., 1976b), pulmonary (Taylor, G., 1979), and bone and cartilage tumors (Pool, 1978), as these are affected by age, sex, and family line. Extensive data have also been reported on the extent of viral transmission among colony beagles, their handlers, local mongrels and other native animals (Lundgren, 1968, 1968b, 1969, 1969b, 1970, 1971). Such accumulating experience has been shared, not only through publication of scientific papers and monographs, but also through direct communication between the laboratories conducting major life-span experiments.

Of interest is the fact that dogs employed as controls in specific life-span experiments have been utilized as controls for other essentially unrelated studies (Deeg, 1980, 1983). The pooling of control-dog data from some, or all, of these experiments has been suggested as a potentially useful method of improving the statistics of specific experimental results (Crump, 1986).

The provision of optimal animal care was an essential concern in all the beagle colonies (Dougherty, T., 1962; Goldman, 1969b; McKelvie, 1970; Redman, 1970; Wolf, 1966). Ideally, all experiments would employ identical procedures, but what was optimum for one experiment might not be for another; what was optimum in 1955 might be superseded by improved procedures in 1975 . The persons responsible for the choice and application of these procedures changed over passing decades, and a continuing effort was required to maintain some semblance of uniformity and comparability. 
Animal housing facilities were different at each laboratory . Davis dogs were kept outdoors, usually 2 dogs per $377-\mathrm{ft}^{2}$ pen, with a crushed rock ground surface, and with an elevated barrel offering protection from the elements (Andersen, 1970c). With a colder winter climate, Utah dogs were maintained in outdoor runs connecting with inside housing at the dogs' choice (Dougherty, T., 1962). Floors were concrete with imbedded heating cables. Usually 2 dogs (3 maximum) shared a $50-\mathrm{ft}^{2}$ run; one-fifth of each run was indoors. Similar indoor-outdoor runs have been employed for most studies at PNL (Park, J., 1976) and ITRI (Redman, 1970). Indoor-outdoor runs have also been employed at Argonne (Norris, 1968); however, during periods of continuous exposure, the animals, including controls, are of necessity confined to cages (Fritz, 1986). The disposal of radionuclide-contaminated wastes from these large dog colonies poses a special problem (Goldman, 1963).

Disease problems unrelated to radiation exposure have complicated the interpretation of results in a number of experiments (Andersen, 1970k). An epidemic of canine distemper, and a vitamin deficiency problem influenced early deaths in the Davis x-ray study (Andersen, 1969). An epizootic of gastroenteric disease at Argonne, involving half the colony and killing $10 \mathrm{dogs}$, was shown to be associated with a parvovirus infection (Binn, 1981; Fritz, 1979). A similar parvoviral outbreak at ITRI killed 8 puppies and 3 adult dogs (Merickel, 1980), and resulted in a continuing clinical, pathological and epidemiological study of canine parvoviral enteritis (Mason, 1987). The incidence of epilepsy in certain colonies has been a complicating and incompletely understood factor (Atherton, 1986; Bielfelt, 1971; Redman, 1969, 1972b). The diagnosis of hypothyroidism in a stud dog that contributed substantially to the genetic pool of the ITRI breeding colony led to investigations involving 75 dogs, relative to the possible heritability of the thyroid condition; no evidence was found for a problem in the colony (Pickrell, 1970).

The question of genetic uniformity of the beagles employed in these experiments has been of some concern (Rosenblatt, 1986). Unlike the highly inbred strains of small rodents usually employed in laboratory studies, the beagles employed in these life-span studies possess no such genetic uniformity. Each of the major laboratories established its own breeding colony. The Davis and Utah colonies were established independently of each other (Dougherty, T., 1962; Rehfeld, 1972). Subsequently established colonies at Argonne, PNL and ITRI each obtained a part of their original breeding stock from one or both of these original colonies, but also employed animals from other sources (Rehfeld, 1970; Redman, 1970). The laboratories have followed accepted breeding practices, have maintained detailed pedigree records, and have made some effort to evaluate genetic variability (Bielfelt, 1969, 1971; Fritz, 1970b, 1976; McKelvie, 1966; Pickrell, 1970; Redman, 1970; Rehfeld, 1967, 1970, 1970b; Tyler, 1968). The comparability of 
results between experiments conducted in different laboratories, or in the same laboratory at different times, is compromised by this genetic uncertainty, to a degree that would not be involved in studies with standard strains of experimental rats or mice. On the other hand, the use of outbred beagles allows for greater confidence in extrapolating to the dog, in general, than if a single strain of inbred beagle had been employed.

\subsection{EXPERIMENTAL PROTOCOLS}

The primary objectives and basic design of most of the life-span beagle experiments are quite straightforward. Thus, for purposes of definition and superficial comparison, it is possible to provide reasonably complete experimental protocols, in simple tables, as has been done in Chapter 8. The experiments typically involve several exposure groups, exposed at different dosage levels or in different temporal patterns, with observation of the exposed animals continuing for duration of life. This observation is directed toward certain preconceived end points, such as survival time and incidence of anticipated radiation effects, but is also designed to detect unanticipated effects if they occur.

A problem associated with all of the life-span beagle studies has been that of providing appropriate control animals in statistically adequate numbers. Small control groups were sometimes justified on the basis that effects would likely not be seen in the lower exposure groups, which would effectively serve as additional controls. The combining of controls from different studies has also been considered (Atherton, 1986; Crump, 1986); and the need for controls in many applications has been questioned (Crump, 1986).

In addition to the animals retained for life-span observation, most experiments included other exposed and unexposed animals, available for sacrifice at intervals throughout the experiment. These "sacrifice" animals might be required as comparative pathology controls, or for purposes of defining radionuclide retention patterns necessary for the estimation of radiation dose. These animals are not included in the abbreviated protocols of Chapter 8 , but in some experiments may have exceeded the number of "life-span" animals. "Sacrifice" animals that were, in fact, never sacrificed but retained for life-span observation, will, in some experiments, supplement the designated "life-span" animals.

While simple in concept, the long duration, sometimes complex exposure requirements, and broad range of potential effects-all translating to high costadd complicating dimensions to the experiments. Whereas scientists normally expect to learn from a first experiment how to perform a better second experiment, the pressure in these experiments was to "do it right the first time," 
because there was apt to be neither time nor money for a second experiment. The long duration of the experiments posed extraordinary requirements for the widest acquisition and the safest retention of data, the need for which might not be fully apparent until the conclusion of the experiment, and the user of which would more than likely not be the original data collector (McKelvie, 1964).

Because of the often complex nature of the exposure processes, and the small number of beagles of appropriate age available at any given time, exposures were, of necessity, performed over an extended period of time, not infrequently amounting to several years. Under these circumstances, strict adherence to standardized and statistically randomized procedures was of critical importance (McKelvie, 1966; Rosenblatt, 1970).

Overriding all of these concerns and complications was the reality of the budget. Although, from the present historical vantage point, one may marvel at the farsighted generosity of the early funding of these experiments, there were always budgetary constraints, and compromises were inevitable.

Some specific comments on the experimental protocols, as summarized in Chapter 8, seem essential, as does some discussion of a few experiments not included among these protocols. Selection of the life-span beagle experiments to be considered in this report has involved unavoidably arbitrary decisions. One might, with some justification, have included the experiment conducted at the University of Rochester during the 1950 s and 1960s, which involved 60 dogs (including controls), exposed for their life span to daily ( 5 per week) x-ray exposures of 0.06, 0.12, or 0.60 R (Casarett, 1970; Stannard, 1988). The principal objective of this experiment, which included additional dogs exposed to higher doses and observed for shorter periods, was the effect of radiation on spermatogenesis.

Another excluded experiment is that initiated at PNL in 1969 involving chronic exposure for nearly 5 years of 69 beagles (including controls) to combinations of uranium mine air contaminants, including radon and daughters, uranium ore dust, and the smoke from individually smoked cigarettes (Cross, 1982; Stuart, 1971). Most of these animals died relatively early, or were sacrificed short of their life span, or were diverted to other studies, making the experiment difficult to evaluate as a life-span experiment.

Experiments initiated at Davis in 1976 , involving continuous ${ }^{60} \mathrm{Co}$ gamma-ray exposure of beagles, starting in-utero and at various ages after birth, have also been excluded (Goldman, 1986). These experiments are relevant to this report as 
an important application and extension of the findings from earlier Davis lifespan experiments, but are specific studies of the leukemogenic process rather than life-span studies in the sense of this report.

Excluded for administrative reasons, is the major experiment currently in progress at Colorado State University that involves the life-span observation of some 1700 beagle dogs given single ${ }^{60} \mathrm{Co}$ gamma-ray exposures of 20 or $100 \mathrm{R}$ during gestation at 8,28 , or 55 days postconception, and following birth at 2,70 , or 365 days of age (Angleton, 1977, 1988; Benjamin, 1986, 1986b). This experiment was initiated by the U.S. Public Health Service and is currently funded by the Bureau of Radiological Health, Food and Drug Administration, and the Low Level Radiation Effects Branch of the National Cancer Institute. From every point of view except source of funding, this experiment could be considered a part of the total life-span radiation-effect-in-beagles effort, and, though not considered in detail, will be referred to from time to time in this report.

Also excluded from major consideration are the Colorado State University experiments with simulated plutonium-contaminated wounds in beagle dogs, which were funded by AEC/ERDA/DOE, but in which most of the dogs were sacrificed at short intervals following exposure, and in no case were kept for life-span observation (Bistline, 1972, 1976; Dagle, 1975, 1975b, 1984b; Gomez, 1972, 1974; Lebel, 1970, 1976; Schallberger, 1976).

The Utah and ITRI protocols, as summarized in Chapter 8, are derived from their most recent Progress Reports, in which they annually publish information on the status of every dog enrolled in their experiments. A similar data tabulation has appeared during recent years for PNL and Davis experiments. Equivalent information on individual dogs for the early PNL and Davis experiments has not been published, but the protocols have been reconstructed, to the extent possible, from referenced publications of results. Published, individual animal data are available for none of the Argonne experiments. For all but the current external gamma-irradiation experiments (Fritz, 1986), published protocols are sketchy at best. The earlier Argonne experiments pose a serious documentation problem, and prompt measures are required to avoid irretrievable loss of important data.

It is important to note that the "Initial Burden" indicated in the protocols for PNL and ITRI inhalation experiments is a median level for the exposure group. Because of the technical difficulties of controlling inhalation exposures, deposition in individual animals may differ substantially from the group median, in some cases overlapping that of animals in adjacent groups. For this reason, eventual analysis of results will be based on the dosimetry for individual dogs, 
and the nominal exposure groups will have limited relevance.

\subsection{EXPOSURE MODES}

Although not purposefully planned to provide a balanced study of all possible modes of radiation delivery, the life-span beagle studies, as they have evolved, come close to providing such an all-encompassing picture. A few experiments have employed external, whole-body, $x$ - and gamma irradiation, delivered in fractionated and continuous exposures, started at various ages. Most experiments have involved internal exposure from radionuclides delivered by ingestion, injection, or inhalation routes, in various temporal patterns, at various ages. Alpha-, beta-, and gamma-emitting radionuclides of widely varying half-life and chemical form have been employed.

\subsubsection{EXTERNAL IRRADIATION}

External, whole-body exposure to $\mathrm{x}$ or gamma rays has been employed in 3 major life-span experiments. The 1952 Davis experiment involved a total of 360 life-span, female dogs, exposed at 10 to 12 months of age to total x-ray doses of 100 $\mathrm{R}$ or $300 \mathrm{R}$, delivered as a single dose or in 2 or 4 dose fractions separated by 1,2 , or 4 weeks (Andersen, 1969). Exposures were produced by an x-ray machine operated at $250 \mathrm{kVp}, 30 \mathrm{~mA}$, filtration HVL equivalent to $2.65 \mathrm{~mm}$ copper; target to midline distance was $140 \mathrm{~cm}$; midline air-dose rate averaged $8.5 \mathrm{R} / \mathrm{min}$. Dogs were suspended by slings in a standing position and irradiated bilaterally. They were randomly assigned to exposure subgroups; controls were sham-exposed. Following exposure they were randomly assigned to outdoor pens.

At Argonne, beagles were exposed to ${ }^{60} \mathrm{Co}$ gamma radiation, continuously until death, or, in a companion experiment, until the accumulation of a predetermined total dose (Fritz, 1986; Norris, 1972). Young-adult beagles of both sexes were caged singly in three-tiered fiberglass cages at appropriate distances from the ${ }^{60} \mathrm{Co}$ source, which was maintained at a reasonably constant strength by periodic removal of cylindrical steel attenuators to compensate for ${ }^{60} \mathrm{Co}$ decay (Fritz, 1982). Cages were rotated $90^{\circ}$ each day, and dogs migrated through all cages of a given dose group. In experiments initiated between 1968 and 1978, dose rates have ranged from 0.3 to $54.0 \mathrm{rad} /$ day; terminated exposures have ranged from total doses of 450 to $3000 \mathrm{rad}$.

Experiments at Davis have also employed a ${ }^{60} \mathrm{Co}$ irradiator, designed to provide both an outdoor and an indoor field for continuous exposure of beagles or other experimental animals (Momeni, 1974). This facility was designed to provide a more flexible continuous exposure regimen for follow-up of observations made in 
the Davis life-span beagle studies with internally deposited ${ }^{90} \mathrm{Sr}$, and were used subsequent to 1976 in non-life-span studies of leukemogenesis in beagles. Other external exposure studies with beagles, not considered in detail in this report, are the non-DOE-sponsored experiments at Colorado State University, which involve single, bilateral, whole-body exposure to ${ }^{60} \mathrm{Co}$ gamma radiation at doses of either 20 or $100 \mathrm{R}$, at 8,28 , or 55 days postconception, and 2,70 , or 365 days postpartum (Angleton, 1977, 1988; Benjamin, 1986, 1986b).

\subsubsection{INTERNAL IRRADIATION}

Most of the life-span beagle experiments have involved exposure to internally deposited radionuclides because this type of exposure was of particular occupational concern and, during the peak "fallout" era, of concern also for general populations. Later concerns were associated with the potential for radionuclide dispersal resulting from accidents in power-reactor, weapons-related, and space-related applications of nuclear energy. The biokinetic and dosimetric complexities of internally deposited radionuclides pose greater difficulties in estimating radiation dose and effect in man, as compared to the more straightforward situation encountered with whole-body external exposure.

Inhalation and intravenous injection have been the experimental routes of administration most commonly employed in internal exposure studies-inhalation because it is the most likely route of accidental exposure of man, and intravenous injection because it affords a greater degree of control over the chemical and physical form and precise quantity of radionuclide administered. Two early, small experiments with ${ }^{90} \mathrm{Sr}$ at Argonne employed the unusual routes of transplacental administration and subcutaneous injection (Finkel, 1960, 1972).

The ingestion route of exposure was employed in a single, major experiment with ${ }^{90} \mathrm{Sr}$, at Davis (Book, 1980; Goldman, 1969). Ingestion levels were defined in terms of the ${ }^{90} \mathrm{Sr} / \mathrm{Ca}$ ratios of the diets, in an effort to ensure uniform labeling of the growing bone; stable strontium concentration in all diets was about $1.4 \mathrm{mg} / \mathrm{g}$ calcium (Della Rosa, 1972). Actual ${ }^{90} \mathrm{Sr}$ intake within a group varied from animal to animal depending on food intake. Attained radionuclide levels were measured in each animal by periodic whole-body bremsstrahlung counting (Goldman, 1972b).

The ${ }^{90} \mathrm{Sr}$ diets, in equilibrium with ${ }^{90} \mathrm{Y}$, were fed to the dams from midgestation through the lactation period. The offspring were weaned to the same dietary ${ }^{90} \mathrm{Sr} / \mathrm{Ca}$ levels consumed by their mothers, and feeding was continued until 540 days of age. For comparative purposes, a few animals were maintained on the ${ }^{90} \mathrm{Sr}$ diets for the duration of their life $(B o o k, 1982)$. The diets employed for 7 
dosage groups ranged in ${ }^{90} \mathrm{Sr}$ content from 0.007 to $10.0 \mu \mathrm{Ci} / \mathrm{g}$ calcium; attained burdens at 540 days of age ranged from about 0.2 to $400 \mu \mathrm{Ci}$ (Momeni, 1976). The dietary regimen employed in this study was designed to mimic the "fallout" exposure pattern of the most sensitive members of the general population, i.e., the child and young adult. To provide a direct link with other studies involving acute exposure, smaller groups of dogs were given single intravenous injections of ${ }^{90} \mathrm{Sr}$ at 540 days of age (Momeni, 1976).

The intravenous injection route of exposure affords precise control of the quantity of radionuclide administered. The disadvantage is that this is not a commonly encountered route of accidental occupational exposure and is never apt to be involved in the exposure of general populations. If intravenous injection is to qualify as a dependable surrogate for more commonly encountered modes of exposure, such as ingestion or inhalation, steps must be taken to ensure that the distribution and subsequent retention of the injected radionuclide bears some resemblance to that of inhaled or ingested radionuclides and is not merely an artifact of the injection process. This problem is of particular concern with polyvalent metal ions, which are prone to hydrolyze in injectable solutions, forming colloids that may distribute very differently from the very low concentrations of these ions that would be absorbed from the lung, from the gastrointestinal tract, or through the skin (Stover, 1962).

Most of the life-span beagle experiments employing the intravenous injection route have been conducted at the University of Utah. In all of these experiments, the radionuclide under study was administered in a freshly prepared citric acidsodium citrate buffer solution, $0.08 \mathrm{M}$ in total citrate, with a pH of 3.5 (Stover, $1959 b$ ). Particular pains were taken in the preparation of plutonium solutions to ensure the presence of only monomeric Pu(IV) (Stover, 1959b). Comparative studies with polymeric ${ }^{239} \mathrm{Pu}$ (IV) in beagles have shown that it is, indeed, distributed and retained in a manner quite different from that of the monomeric form administered in citrate buffer (Bruenger, 1981; Stevens, W., 1975). Other studies showed that ${ }^{237} \mathrm{Pu}(\mathrm{IV})$ citrate, injected at mass levels $10^{5}$ times lower than those typically employed with ${ }^{239} \mathrm{Pu}(\mathrm{IV})$ citrate, was retained in blood and distributed among tissues in a manner very similar to that observed with ${ }^{239} \mathrm{Pu}(\mathrm{IV})$ citrate (Lloyd, R., 1976d). An in-vitro preparation of the ${ }^{239} \mathrm{Pu}(\mathrm{IV})$ transferrin complex, injected intravenously, was also shown to behave very similarly to injected ${ }^{239} \mathrm{Pu}(\mathrm{IV})$ citrate (Stevens, W., 1975). There is, therefore, every indication that the injection solutions employed in these studies are reasonable surrogates for the materials encountered in more realistic modes of human exposure. 
The Utah, life-span, single intravenous injection studies included experiments with ${ }^{90} \mathrm{Sr},{ }^{224} \mathrm{Ra},{ }^{226} \mathrm{Ra},{ }^{228} \mathrm{Ra},{ }^{228} \mathrm{Th},{ }^{239} \mathrm{Pu},{ }^{241} \mathrm{Am},{ }^{249} \mathrm{Cf},{ }^{252} \mathrm{Cf}$, and ${ }^{253} \mathrm{Es}$. Single intravenous injection experiments were performed at Argonne, employing ${ }^{144} \mathrm{Ce}$ (Fritz, 1970), and ${ }^{137} \mathrm{Cs}(N C R P, 1977)$. A life-span, single intravenous injection experiment with ${ }^{137} \mathrm{Cs}$ was conducted at ITRI (Boecker, 1972; Redman, 1972), after preliminary studies indicated that the metabolic behavior of inhaled ${ }^{137} \mathrm{Cs}$ chloride was indistinguishable from that of injected ${ }^{137} \mathrm{Cs}$ (Boecker, 1969b).

A major multiple intravenous injection experiment with ${ }^{226} \mathrm{Ra}$ was conducted at Davis (Book, 1980; Goldman, 1969b). Eight injections were given at 15-day intervals, the last occurring at 540 days of age, the same age at which the ${ }^{90} \mathrm{Sr}$-fed beagles, at Davis, were removed from feeding, and the approximate age at which the Utah beagles received their single intravenous injections of ${ }^{226} \mathrm{Ra}$ and other radionuclides.

Early studies of the pulmonary route of exposure to radionuclides employed techniques of intratracheal administration. It became apparent, however, that such techniques could not adequately mimic the pattern of deposition and subsequent distribution of inhaled insoluble particulate materials. Inhalation exposure to highly toxic materials poses severe problems in protecting the experimenter as well as in controlling quantitatively the exposure of the dog. From the standpoint of control of exposure, the dog is a particularly good choice of experimental animal because it can be trained to cooperate in the exposure process, and because it is large enough to be exposed in complex apparatus, employing exposure masks molded to fit the individual animal.

Experimental inhalation exposure of dogs was first employed extensively in studies with uranium compounds at the University of Rochester (Stannard, 1988). A large experiment, involving more that 100 dogs (mostly beagles) inhaling $\mathrm{UO}_{2}$ dust, chronically, for a period of 5 years, was initiated at Rochester in 1955 (Leach, 1970). This experiment is not considered in detail in this report because the dogs were not kept for their life span; however, the published exposure techniques (Leach, 1959) served as a basis for further development in subsequent life-span dog studies. These exposure techniques constitute a critical aspect of the experiments; their refinement over the past 30 years has, unfortunately, not been as extensively documented in the open literature as one might wish.

Notable early developments at ITRI included the Lovelace nebulizer (Mercer, 1968), the Mercer cascade impactor (Mercer, 1970), and the Lovelace dog plethysmograph exposure system (Boecker, 1964). Control over the administered dose 
has improved over the years. First estimated from aerosol volume and concentration parameters, it was later directly measured by external chest monitoring (Cuddihy, 1973b), employing, where necessary, a gamma-emitting tracer, such as ${ }^{169} \mathrm{Yb}$-labeled plutonium (Diel, 1982, Raabe, 1975). Studies employing ${ }^{140} \mathrm{La}$ labeled, insoluble, polydisperse aerosols, of various size ranges, demonstrated deposition patterns in the dog similar to those of man (Cuddihy, 1973).

All of the life-span PNL experiments considered in detail in this report involved single inhalation exposures of young-adult beagles to compounds of plutonium. All employed polydispersed aerosols, i.e., aerosols including a range of particle sizes. The early PNL life-span experiment with ${ }^{239} \mathrm{PuO}_{2}$ employed material obtained by calcination of plutonium oxalate in air at $300^{\circ} \mathrm{C}$, aerosolized from a suspension in $0.1 \%$ polypropyleneglycolethylene oxide polymer, with a resulting particle size mostly in the range of 0.1-1.0 $\mu \mathrm{m}$ (Park, J., 1964). Dogs were attached to the aerosol reservoir via a closely fitted, valved mask, and were trained to inhale the aerosol without anesthesia or tranquilizers (Bair, 1963). The early $\mathrm{PNL}{ }^{238} \mathrm{PuO}_{2}$ exposures were similarly performed, employing either $350^{\circ}$ calcined oxalate material, or crushed ${ }^{238} \mathrm{PuO}_{2}$ microspheres, with count median diameters of about $0.1 \mu \mathrm{m}$ (Park, J., 1976).

The later PNL life-span experiments employed similar but more sophisticated exposure techniques, affording more precise control of the quantity and quality of the material inhaled (Craig, 1972, 1973). The ${ }^{239} \mathrm{PuO}_{2}$ and ${ }^{238} \mathrm{PuO}_{2}$ were prepared from the oxalate, calcined at $750^{\circ}$ and $700^{\circ} \mathrm{C}$, respectively, with inhaled particle sizes of $2.3 \mu \mathrm{m}$ and $1.8 \mu \mathrm{m}$ activity median aerodynamic diameter (AMAD), respectively, both with a mean geometric standard deviation (GSD) of 1.9. The ${ }^{239} \mathrm{Pu}\left(\mathrm{NO}_{3}\right)_{4}$ aerosol was generated by nebulizing a nitric acid solution, producing particle sizes of $0.81 \mu \mathrm{m}$ AMAD with a GSD of 1.7 (Dagle, 1979; Park, J., 1986).

The initial life-span dog program at ITRI was designed to evaluate the effects of exposures, by inhalation of fission-product radionuclides, such as might be encountered in a catastrophic accident (McClellan, 1970, 1986). Five beta emitters ${ }^{90} \mathrm{Y},{ }^{91} \mathrm{Y},{ }^{137} \mathrm{Cs},{ }^{144} \mathrm{Ce}$, and $\left.{ }^{90} \mathrm{Sr}\right)$, with a wide range of physical half-lives $(64 \mathrm{~h}$, $58.8 \mathrm{~d}, 30 \mathrm{y}, 284 \mathrm{~d}$, and $28 \mathrm{y}$, respectively) were employed, initially in soluble forms prepared by nebulization from a chloride solution using a $\mathrm{CsCl}$ vector aerosol. Later experiments employed insoluble aluminosilicate particles formed by high temperature fusion of droplets nebulized from a suspension of montmorillonite clay in which the radionuclide was entrapped (Raabe, 1971). These fused aluminosilicate particles (FAP) showed a common kinetic behavior in the respiratory tract, and differing effects could be attributed to time and dose-rate factors imposed by the particular radionuclide involved (McClellan, 1970, 1970b). 
Most experiments involved a single inhalation exposure of young-adult beagles, but experiments with ${ }^{144} \mathrm{Ce}$ FAP were also conducted with juvenile and aged beagles, and with multiply exposed young adults.

Later ITRI life-span experiments with ${ }^{239} \mathrm{PuO}_{2}$ and ${ }^{238} \mathrm{PuO}_{2}$ employed monodispersed aerosols, i.e., aerosols of a very narrow size range. These monodispersed aerosols were produced by a tedious process of aerosol centrifugation (Kotrappa, 1971, 1972), adapted at ITRI for mass production (Raabe, 1975; Talley, 1979). While monodispersed aerosols are not normally encountered in accidental occupational exposures, they offer the only direct approach to the evaluation of the "hot particle" issue (Tamplin, 1974), i.e., the evaluation of the effect on lung cancer induction, of radiation dose distribution at the cellular level. Experiments with ${ }^{238} \mathrm{PuO}_{2}$ employed 3.0- and 1.5- $\mu \mathrm{m}$ particles administered as a single inhalation exposure to young-adult beagles. Experiments with ${ }^{239} \mathrm{PuO}_{2}$ employed 3.0-, 1.5-, and $0.75-\mu \mathrm{m}$ particles in singly exposed young adults; $1.5-\mu \mathrm{m}$ particles in juvenile and aged beagles; and, $0.75-\mu \mathrm{m}$ particles in an experiment involving a series of 20 inhalations at 6 -month intervals.

In addition to the exposure of animals to be retained for life-span study, many ancillary inhalation studies were conducted to develop the techniques (Craig, 1971, 1973), and to evaluate pulmonary function following exposure (Dubin, 1969, 1970, 1971). A challenging inhalation exposure problem was that of simulating in dogs the process by which cigarettes are smoked by humans (Bair, 1969b). These techniques were employed to determine the effect of smoking on basic clearance mechanisms (Bair, 1967), in the simulation of working conditions in uranium mines (Cross, 1982; Park, J., 1971; Stuart, 1970b, 1971), and even in studies of marijuana smoking (Sullivan, 1978).

\subsection{LIFE-SPAN OBSERVATION}

It is not our purpose in this section to consider the results of observations, but only the kinds of observations required, and special problems that these may pose (Andersen, 1962d, 1963d; Rosenblatt, 1970).

An obviously unusual feature of these experiments is the time span of observation, which poses a problem of safe retention of the data in an understandable and easily retrievable form. The more than 35 years of life-span beagle studies have seen remarkable developments in the application of computers to data handling (Doyle, 1981; McKelvie, 1964, 1967; Rosenblatt, 1970; Weller, 1986; Wittmier, 1983). The data from all current experiments are preserved in computer files, which should expedite analysis and interpretation; however, much of the primary data from earlier experiments remains in handwritten 
records. The preservation of all these data, in a format easily accessible to persons not involved in their original collection, is a concern that becomes more urgent as the original collectors die or retire. A concerted effort to preserve these observations would benefit greatly from standardization of archiving techniques and procedures.

In addition to maintenance and documentation of the general health of the animals, special measurements were required in all radionuclide experiments for assessment of radiation dose to individual animals. Special measurements were also aimed at early detection of anticipated radiation effects. Extensive postmortem studies were required, for dosimetric purposes in radionuclide experiments, and to identify and quantify effects that may have been caused or influenced by radiation, or that may have interfered in the recognition of radiation effects.

Reference was made earlier to the general animal care and housing procedures employed, and the extent to which these varied among laboratories. A particular problem is that relating to the amount and kind of supportive care given to ill and aging beagles. Such care, given or withheld, may influence the observed pattern of radiation effects. The aging beagle bitch is susceptible to mammary tumors. In some experiments, to avoid influencing survival patterns, these tumors were not treated; in other experiments they were surgically removed; in still other experiments, mammary glands were surgically removed before the animals were placed on experiment (Rosenblatt, 1986). In some experiments animals were treated surgically for bone tumors (Morgan, J., 1982). More uniformity of treatment in such situations would have simplified inter-experiment comparisons, but allowance can be made for these factors if they are recognized. Such recognition is difficult to assure when experiments extend over a score or more years, and involve a score or more investigators in different laboratories.

The radiation dose to externally irradiated animals can be estimated from measurements made on the radiation source and on suitable dosimetric phantoms (e.g. Norris, 1968; Sinclair, 1963). Estimation of the radiation dose from internally deposited radionuclides requires that measurements be made on the animal; one must know the initial deposition and the subsequent retention of the radionuclide at the point of interest. While a reasonably accurate estimate of an injected quantity can be made from measurements on the solution injected, a quantity deposited by inhalation can be estimated with reasonable accuracy only from measurements on the animal following inhalation. These measurements of deposition and retention may be made by external counting of the exposed animals if sufficiently energetic photon emitters are involved. For example, the 
radium animals at Utah and Davis were routinely counted externally (Lloyd, $R$., 1976g; Momeni, 1976c), as were the animals in several ITRI beta-emitter studies (Boecker, 1972).

If external counting is not practical, deposition and retention in the life-span animal may be inferred from analysis of tissues taken from animals periodically sacrificed during the course of the experiment. Measurement of the radionuclide in the excreta of life-span animals also gives a measure of whole-body radionuclide retention. The addition of measured life-span excretion to measured postmortem retention is a direct measure of initial deposition. This is a cumbersome approach to the estimation of initial deposition, but the only direct method for inhaled, non-photon-emitting radionuclides such as ${ }^{239} \mathrm{Pu}$ and ${ }^{238} \mathrm{Pu}$. To circumvent this problem, ITRI, in some experiments, has tagged $\mathrm{PuO}_{2}$ with ${ }^{169} \mathrm{Yb}$, which serves as a photon-emitting tracer for the inhaled $\mathrm{PuO}_{2}$ (Diel, 1982; Raabe, 1975).

Where dose to specific organs or tissues is required, measurements must be made on samples from sacrifice animals. Where it is necessary to estimate dose on a microscopic scale, quantitative autoradiographic measurements of radionuclide localization are required. Such studies have played a particularly important role in the explanation of results from experiments with bone-seeking radionuclides, where microdistribution is critical to carcinogenic effect (e.g., Jee, 1961, 1972c).

Any departure from the normal state of health may be of subsequent interest in the evaluation of dose-effect relationships; detailed clinical records are therefore maintained for all animals. A thorough physical examination is scheduled at least annually, with more frequent measurements of parameters that might reflect radiation effects. Hematology (e.g. Dougherty, J., 1971) and blood chemistry (e.g. Stevens, W., 1967) data are obtained on all animals, and particular attention is paid to any indication of tumor development. In most of the radionuclide experiments, total-body diagnostic radiography has been employed, at least during the later high-risk period, for early identification of lung and bone tumors (Christensen, 1962); radioisotopic bone scans have also proved useful for this purpose (Wolff, 1980), and liver tumors have been detected using single photon emission computed tomography (Wolff, 1988). Animals are closely observed for signs of impending death and are euthanized to avoid pain and suffering when recovery seems unlikely.

At death, a gross necropsy is performed to include all major organ systems. Gross findings are documented thoroughly, with written descriptions, photographs, radiographs, and samples taken for pathologic, dosimetric, and other 
purposes (e.g. Dougherty, T., 1962). Histologic sections of a variety of types are usually prepared from an extensive list of tissues and from any other tissues with lesions of interest (Redman, 1972). In radionuclide studies, samples of all organs and tissues, or the entire organs and tissues, are directly counted or analyzed radiochemically to provide an estimate of the radionuclide organ burdens and total-body burden. Autoradiographic studies may be made to determine radionuclide distribution within organs or tissues. Generous samples are also usually retained for unforeseen future needs. Thus, a wide variety of samples of cancerous and noncancerous tissues, and cell cultures from exposed and control animals, preserved for many years at liquid nitrogen temperatures, are proving invaluable in current studies of oncogene involvement in radiation carcinogenesis (Frazier, 1987; Kelly, in press).

The special problems of interpretation of morbidity and mortality data from the life-span beagle experiments are confounded by multiple, collinear, time-dependent variables (age, dose, survival, etc.) (Momeni, 1975), and also by the frequent desire to attempt preliminary interpretation before all the data have been collected. A variety of statistical procedures have been recommended and employed in these attempts (Crump, 1986, Gilbert, in press). Probit analysis was early recommended in the interpretation of hematological data (Rosenblatt, 1967). Linear model analysis techniques have been employed to show a lack of significant effects of radiation on mean life span in the ongoing Colorado State University ${ }^{60}$ Co gamma-ray exposure study (Angleton, 1986); "prevalence rate" methods (Peto, 1980), have been employed in another analysis of this data (Benjamin, 1986b). A proportional hazard approach has been employed in other studies, to relate elements of time, dose, and effect (Mewhinney, 1986; Peterson, 1982). Further consideration of these problems will be found in Chapter 7 on Applications. 



\section{RESULTS: DOSIMETRIC OBSERVATIONS}

\begin{tabular}{|c|c|c|c|c|}
\hline \multicolumn{5}{|c|}{ Contents } \\
\hline 4.1 & \multicolumn{3}{|c|}{ Absorption } & 33 \\
\hline & 4.1.1 & \multicolumn{2}{|c|}{ Absorption of Ingested Radionuclides } & 34 \\
\hline & 4.1 .2 & \multicolumn{2}{|c|}{ Deposition and Absorption of Inhaled Radionuclides } & 34 \\
\hline \multirow[t]{26}{*}{4.2} & \multicolumn{3}{|c|}{ Distribution and Retention $\quad \ldots \ldots \ldots \ldots \ldots \ldots \ldots$} & 36 \\
\hline & \multirow[t]{13}{*}{4.2 .1} & \multicolumn{2}{|c|}{ Gross Distribution and Retention (by element) } & 37 \\
\hline & & 4.2.1.1 & Strontium & 37 \\
\hline & & 4.2.1.2 & Yttrium $\quad \ldots$ & 38 \\
\hline & & 4.2.1.3 & Cesium & 38 \\
\hline & & 4.2.1.4 & Cerium & 39 \\
\hline & & 4.2.1.5 & Fused Aluminosilicate Particles & 39 \\
\hline & & 4.2 .1 .6 & Radium $\ldots .$. & 41 \\
\hline & & 4.2.1.7 & Thorium & 43 \\
\hline & & 4.2.1.8 & Plutonium & 44 \\
\hline & & 4.2.1.9 & Americium & 47 \\
\hline & & 4.2.1.10 & Berkelium & 48 \\
\hline & & 4.2 .1 .11 & Californium $\quad \ldots \ldots \ldots \ldots . . .$. & 48 \\
\hline & & 4.2 .1 .12 & Einsteinium & 49 \\
\hline & \multirow[t]{12}{*}{4.2 .2} & \multicolumn{2}{|c|}{ Localized Distribution and Retention (by organ) } & 49 \\
\hline & & 4.2 .2 .1 & Blood . & 49 \\
\hline & & 4.2 .2 .2 & Lung & 50 \\
\hline & & 4.2 .2 .3 & Pulmonary Lymph Nodes & 50 \\
\hline & & 4.2 .2 .4 & Calcified Tissues ... & 51 \\
\hline & & 4.2 .2 .5 & Liver & 55 \\
\hline & & 4.2 .2 .6 & Spleen & 56 \\
\hline & & 4.2 .2 .7 & Kidney & 56 \\
\hline & & 4.2 .2 .8 & Ovary & 56 \\
\hline & & 4.2 .2 .9 & Testes.. & 56 \\
\hline & & 4.2.2.10 & Eye & 56 \\
\hline & & 4.2.2.11 & Thyroid & 56 \\
\hline
\end{tabular}

This chapter, and the two chapters that follow, will place in a general perspective the results that have been realized from the life-span dog studies. This will not be a tabulation of detailed results, but will direct attention to the kinds of findings that have been described, and reference these descriptions. It will not seek to derive new understanding from raw data, but will attempt to integrate previously published interpretations and expectations. 
A problem inherent in the review of published results from long continuing and still incomplete experiments is the possibility that earlier reported findings or conclusions may need to be altered in the light of later observations. An effort has been made to direct attention to such changing interpretations when they are known to have occurred, but the reader should be alert to the possibility that such changes may not have been recognized by the author, or may not yet have been published by the experimenter.

As discussed in the previous chapter, the primary objective of these experiments may be viewed as the establishment of dose-effect relationships. It will be convenient to consider in this chapter the findings relating to the description of radiation dose, and to consider in the following chapter those relating to the identification and quantification of biological effect. A third chapter will consider a number of results that, while subordinate to the primary objective of the life-span experiments and perhaps not considered in the initial planning and justification of these experiments, are nevertheless an important legacy of the total program.

The concern of this chapter is not just with the specific evaluation of radiation dose but also with the more general body of observations necessary to that evaluation of dose. In the case of exposure to radionuclides, this will involve the distribution and retention of the radionuclide on both a macro- and microscale. These broadly defined dosimetric observations are of interest, not solely as they relate to the interpretation of effects observed in these experiments, but are of importance to the theoretical understanding and interpretation of radiation effects generally. Thus, if observation of biological effects had been excluded from these experiments, the dosimetric information derived would still have been useful in many applications.

Of the 48 life-span experiments listed in Table 1.1 of the Introduction, only 3 involved an external source of radiation exposure. Dosimetry in these $\mathrm{x}$ - and gamma-ray-exposure experiments was relatively straightforward. This is not to say that challenging problems were not encountered in the provision of reproducible exposures and in the precise estimation of relevant doses (Andersen, 1969; Barnes, 1972; Grahn, 1986; Hubbard, 1969, 1971; Momeni, 1974); however, the experiments themselves were not designed to provide new knowledge of the methods and procedures required for this dosimetry. Our concern in this chapter is therefore largely with the experiments that employed various means of internal exposure, and in which the estimation of dose to the organs and tissues of interest was an integral part of the experiment. 
Estimation of dose from internally deposited radionuclides requires information relative to the initial absorption of the radionuclide and its subsequent distribution and retention within the body. The problems of absorption will be different, depending upon the route of exposure. The evaluation of distribution and retention may be pursued at the gross organ or tissue level, or at more closely defined levels extending to cellular or molecular levels if this seems appropriate. Microscopic distribution of the radionuclide is of little concern in the case of long-range gamma emitters, but may be of critical importance in the case of short-range alpha emitters or beta emitters.

\subsection{ABSORPTION}

It is useful to give separate consideration to this initial step in the distribution of an administered radionuclide because absorption is the step that is unique to the route of entry; subsequent distribution and retention behavior may be relatively insensitive to route of entry. The route of entry in most of the life-span experiments was either inhalation or intravenous injection. Inhalation was employed because it was considered the most likely route of occupational exposure. Intravenous injection was employed because it avoided the complications of absorption; the results of injection experiments, when suitably corrected for the absorption step, would presumably be applicable to any route of entry. Ingestion was employed in one major experiment with ${ }^{90} \mathrm{Sr}$ because ingestion was considered the critical route of exposure of the general population to fallout ${ }^{90} \mathrm{Sr}$.

If distribution data obtained following intravenous injection are to be accepted as a valid indicator of postabsorption behavior following other routes of entry, this validity must be established in suitable comparison experiments. The life-span dog experiments provide several opportunities for such validation. One may compare the results of a single intravenous injection with those from chronic ingestion of ${ }^{90} \mathrm{Sr}$ in parallel Davis experiments; despite differences in age and duration of exposure, the components of long-term retention of ${ }^{90} \mathrm{Sr}$ were similar in the two experiments (Momeni, 1976). Studies at ITRI with inhaled and injected ${ }^{137} \mathrm{CsCl}$ indicated that the subsequent disribution and retention behavior of such a soluble substance was essentially similar, whether inhaled or injected (Boecker, 1969). The distribution and retention of ${ }^{239} \mathrm{Pu}$ intravenously injected as the citrate in Utah experiments (Stover, 1962b) approximated the postabsorption behavior of inhaled ${ }^{239} \mathrm{Pu}\left(\mathrm{NO}_{3}\right)_{4}$, as observed in PNL experiments, but was very different from the postabsorption behavior of inhaled ${ }^{239} \mathrm{PuO}_{2}$ (Park, J., 1986). The retention of ${ }^{90} \mathrm{Sr}$ following inhalation as the chloride in an ITRI experiment was very similar to that observed following single intravenous injection in a Utah experiment (McClellan, 1972c), and indistinguishable from that following single intravenous injection in a Davis experiment (Momeni, 1976). 


\subsubsection{ABSORPTION OF INGESTED RADIONUCLIDES}

The Davis ${ }^{90} \mathrm{Sr}$ ingestion experiment provided extensive data on ${ }^{90} \mathrm{Sr}$ absorption as a function of age, and under conditions of controlled calcium intake (Momeni, 1976); studies preliminary to the main experiment explored the effect of dietary calcium level on ${ }^{90} \mathrm{Sr}$ absorption (Della Rosa, 1965; Stover, 1961). Whether absorbed as a consequence of chronic ingestion or single intravenous injection, the long-term retention of ${ }^{90} \mathrm{Sr}$ was observed to be similar; this despite differences in age and duration of exposure (Momeni, 1976).

\subsubsection{DEPOSITION AND ABSORPTION OF INHALED RADIONUCLIDES}

Life-span experiments with inhaled radionuclides at PNL and at ITRI have provided a wealth of data on factors affecting initial deposition of inhaled materials in the lung and subsequent absorption of these materials from the lung. Solubility is, of course, a major factor. Such soluble materials as $\mathrm{CsCl}$ (Boecker, 1969 ) and $\mathrm{SrCl}_{2}$ (McClellan, 1972c) are very rapidly lost from the lung. To compare the biological responses of radionuclides inhaled as "insoluble" forms in contrast to "soluble" forms, a number of ITRI gamma-emitter experiments employed a common, very insoluble, fused aluminosilicate vector particle (FAP) of controlled size characteristics (Raabe, 1971). With less soluble materials, retention in the lung is prolonged, and factors other than solubility become important. FAP labeled with ${ }^{137} \mathrm{Cs}$ and ${ }^{144} \mathrm{Ce}$ were employed in ancillary experiments comparing their retention in the lungs of mice, rats, and beagles (Thomas, 1971). Later experiments employed FAP labeled with ${ }^{134} \mathrm{Cs}$, comparing the retention of monodisperse or polydisperse particles of various sizes in dogs, rats, and mice (Snipes, 1983, 1983c). Retention was prolonged in the dog, depending largely on solubility factors rather than on mechanical clearance, which was more important in rats and mice. Within the range studied $(0.7$ to $2.8 \mu \mathrm{m}$ AMAD), size had little influence on clearance rates. Clearance to lung-associated lymph nodes was more prominent in dogs than in the rodents. As compared to young-adult beagles, a threefold larger fraction of the initial pulmonary burden of ${ }^{144} \mathrm{Ce}$ inhaled as FAP by immature dogs (90 days of age) was cleared from the lung soon after exposure (Guilmette 1987b).

Very early distribution of inhaled ${ }^{144} \mathrm{Ce}$ or ${ }^{90} \mathrm{Y}$ FAP, as determined autoradiographically, shows an accumulation just inside the nares, on the turbinates and at the sharp ventral bend in the nasopharynx; this activity is largely cleared via the esophagus within 30 minutes (Barnes, 1971). Autoradiographs of the costal surface of the lung show a striated pattern, with deposition occurring most heavily beneath the ribs; this pattern was observed with a variety of radionuclides and persisted for at least several hundred days postexposure; a similar 
pattern has been observed in gross lesions following inhalation of ${ }^{90} \mathrm{Y}$ and ${ }^{241} \mathrm{Am}$ (Barnes, 1971).

Ancillary experiments at PNL investigated the deposition in, and absorption from, the lung of various chemical and physical forms of inhaled ${ }^{239} \mathrm{Pu}$ (Bair, 1961, 1961b, 1962b, 1963b, 1964, 1968; Stuart, 1968; West, 1964). These experiments demonstrated the tenacious retention of ${ }^{239} \mathrm{PuO}_{2}$; its relatively uniform distribution among the lobes of the lung (Bair, 1961b; West, 1964); and its translocation, principally to pulmonary lymph nodes (Bair, 1961, 1961b). During the first week or two postexposure, about half the initial lung deposit was excreted via the feces; less than 1\% was excreted in the urine (Bair, 1961, 1961b; Stuart, 1964; West, 1964). The more chemically soluble forms of plutonium, and the smaller, lower-temperature-calcined oxide particles, were more rapidly absorbed and excreted (Bair, 1962b, 1963, 1963b, 1964, 1968).

Experiments at ITRI also demonstrated effects of varying physical and chemical characteristics on pulmonary retention of ${ }^{239} \mathrm{PuO}_{2}$. Different production temperatures for ${ }^{239} \mathrm{PuO}_{2}$ aerosols resulted in lung retention half-times that increased as the production temperature increased; the proportion of plutonium excreted in the feces was greater at lower production temperatures; in-vitro solubility was inversely correlated with production temperature (Mewhinney, 1976; Miglio, 1977). Preliminary data from the life-span experiment involving repeated exposure to ${ }^{239} \mathrm{PuO}_{2}$ via inhalation indicate that, under the conditions employed, retention in the lung following each exposure was not influenced by previous exposure history (Diel, 1982).

The half-time for clearance of the major long-term component of ${ }^{239} \mathrm{PuO}_{2}$ deposited as a polydisperse aerosol in the lungs of beagles has been estimated to lie in the range of 500 to 1500 days (Bair, 1968, 1973; Fisher, D., 1986; Mahaffey, 1981; Park, J., 1972, in press; Stuart, 1970). Experiments with monodisperse aerosols of ${ }^{239} \mathrm{PuO}_{2}$ indicated half-times for the major component of retention in the lung of 680 days for $0.72 \mu \mathrm{m}$ AMAD particles, 1400 days for $1.4 \mu \mathrm{m}$ AMAD particles, and 1800 days for $2.8 \mu \mathrm{m}$ AMAD particles; the cumulative radiation dose to the lung of the 2.8- $\mu \mathrm{m}$-AMAD animals was projected to be twice that of the $0.72-\mu \mathrm{m}$ AMAD animals at 10 years postexposure (Guilmette, 1984). A more recent evaluation of the above monodisperse ${ }^{239} \mathrm{PuO}_{2}$ data, supplemented by longerterm data from life-span dogs, shows no significant difference in the lung retention of the two larger particle sizes, but significant differences in their translocation to bone and certain lymph nodes (Guilmette, 1987).

The clearance from the lung of inhaled ${ }^{238} \mathrm{PuO}_{2}$ is more rapid than that of inhaled ${ }^{239} \mathrm{PuO}_{2}$ (Bair, 1973; Dagle, 1979; Diel, 1980; Hahn, 1981; Mewhinney, 1983; 
Park, J., 1969, 1974, 1976, 1986). This more rapid clearance is attributed to radiolytic fragmentation occasioned by the high specific activity of the ${ }^{238} \mathrm{PuO}_{2}$ particles (Park, J., 1974). This fragmentation and solubilization process has been studied autoradiographically (Diel, 1983), and has been incorporated in a detailed biokinetic model (Mewhinney, 1983).

For inhaled ${ }^{239} \mathrm{Pu}\left(\mathrm{NO}_{3}\right)_{4}$, data from 15 dogs, sacrificed at intervals to 5 years postexposure, indicated a retention half-time of 100 days for the major fraction, but $18 \%$ of the initial lung deposit was retained with a half-time of about 400 days (Stevens, D., 1986); similar lung retention of ${ }^{239} \mathrm{Pu}\left(\mathrm{NO}_{3}\right)_{4}$ was indicated in earlier studies involving fewer animals (Bair, 1973). The early clearance of ${ }^{238} \mathrm{Pu}\left(\mathrm{NO}_{3}\right)_{4}$ was more rapid than that of ${ }^{239} \mathrm{Pu}\left(\mathrm{NO}_{3}\right)_{4}$, but the two rates became similar after 1 month postinhalation (Dagle, 1983).

No age-related differences have been detected in the retention of inhaled ${ }^{239} \mathrm{PuO}_{2}$ in the lungs of immature, young-adult, or aged dogs, although differences are observed in the relative fractions translocated to different organs (Guilmette, 1987b).

Concern for the practical long-term consequences of differences in absorption, and subsequent distribution of various forms of inhaled plutonium provided the primary motivation for a number of the life-span experiments currently under way at PNL (Park, J., 1986) and ITRI (McClellan, 1986).

\subsection{DISTRIBUTION AND RETENTION}

The radiation dose to various organs and tissues resulting from the internal deposition of a radionuclide will depend upon how this radionuclide is initially distributed among these organs and tissues, and the time pattern of its retention and redistribution with time. It is usually impractical to measure these radiation doses directly in a life-span study; instead they are calculated from the known physical characteristics of the radionuclide and its measured distribution as a function of time. Measurements of radionuclide distribution are obtained directly from the life-span animals at death, inferred from external counting of penetrating radiation over the life span of the animals, and/or measured in separate groups of animals exposed for this purpose and sacrificed at intervals during the course of the experiment. Distribution and retention may be determined on a gross organ or tissue basis, or microdistribution may be important in assessing dose to particular cell types or molecular species. 


\subsubsection{GROSS DISTRIBUTION AND RETENTION (by element)}

Gross distribution and retention will be considered on an element-by-element basis, with elements ordered according to atomic number.

4.2.1.1 STRONTIUM: The gross distribution and retention of strontium has been studied in life-span experiments and supporting sacrifice studies involving injected, ingested, or inhaled ${ }^{90} \mathrm{Sr}$.

The Davis chronic ingestion experiment, in which exposure commenced inutero, presented a number of special dosimetric problems. A preliminary experiment studied the comparative metabolism of ${ }^{85} \mathrm{Sr}$ and ${ }^{47} \mathrm{Ca}$ as a function of age, and provided data necessary to the establishment of dietary ${ }^{90} \mathrm{Sr} / \mathrm{Ca}$ ratios in the main experiment (Della Rosa, 1965). Another preliminary experiment provided data on transport of ${ }^{90} \mathrm{Sr}$ via the placenta and lactation (Della Rosa, 1968b). Placental discrimination resulted in a ${ }^{90} \mathrm{Sr} / \mathrm{Ca}$ ratio in fetal bone of 0.1 that in the maternal diet; this ratio increased to 0.2 during nursing and to 0.4 when the diet was fed directly. Supporting sacrifice studies provided data on the kinetics of ${ }^{9} \mathrm{Sr}$ in the growing beagle, both during ${ }^{90} \mathrm{Sr}$ feeding and subsequent to cessation of ${ }^{90} \mathrm{Sr}$ feeding at 540 days of age (Della Rosa, 1969; Goldman, 1967, 1969).

Except at very early times following exposure, ${ }^{90} \mathrm{Sr}$ is present almost entirely in the skeleton, including teeth (Jee, 1960). At long intervals following injection, less than 0.01 percent of total-body ${ }^{90} \mathrm{Sr}$ is retained in liver, spleen and kidney, and that present in these organs is apparently maintained from skeletal reservoirs (Atherton, 1966). The rate of loss of injected ${ }^{90} \mathrm{Sr}$ from plasma is similar, but not identical, to that of radium; renal clearance is less efficient than that of radium; and fecal excretion relatively less important (Stover, 1958, 1962). The lesser retention of strontium, relative to radium, was verified in a double-tracer experiment with ${ }^{85} \mathrm{Sr}$ and ${ }^{226} \mathrm{Ra}$ (Glad, 1960).

Total-body retention of ${ }^{90} \mathrm{Sr}$ in beagles of the Utah injection experiment was followed by bremsstrahlung counting over the life span of the animals (Lloyd, $R$, 1963, 1976g; Mays, 1958c). Retention data from 96 beagles were expressed as a five-component exponential function, with $36 \%$ of the injected dose lost with a half-time of less than a day, and the longest component (13\%) retained with a half-time of about 13 years (Lloyd, R., 1976h). The longest component of ${ }^{90} \mathrm{Sr}$ retention was shown to be not significantly different from the longest component of radium retention (Lloyd, $R$., 1976h). Supporting ${ }^{85} \mathrm{Sr}$ tracer experiments indicated a decrease in strontium retention with increased age at injection, for ages from 6 months to 10 years (Decker, 1964; Glad, 1960); retention also decreased at ages younger than 6 months (Decker, 1964). 
The more complex pattern of ${ }^{90} \mathrm{Sr}$ retention in the animals of the Davis chronic feeding experiment was also followed by bremsstrahlung counting (Goldman, 1972b). Results are consistent with the kinetic findings in the Davis and Utah injection experiments (Goldman, 1972c; Momeni, 1976). A small number of dogs in the Davis chronic feeding experiment were continued on ${ }^{90} \mathrm{Sr}$ intake for their life span; these dogs showed retention data that differed little from animals whose feeding of ${ }^{90} \mathrm{Sr}$ stopped at 540 days of age, which reflects the low rate of bone turnover in the mature dog (Book, 1982). Periodic external counting data have been employed to calculate individual average skeletal doses for each dog in the Davis experiment (Raabe, 1981, 1981b).

The whole-body retention of ${ }^{90} \mathrm{Sr}$ inhaled as $\mathrm{SrCl}_{2}$ has been studied in detail and, allowing for early loss of unabsorbed ${ }^{90} \mathrm{Sr}$, is very similar to the retention data from intravenous injection experiments; the longer-term retention was described by three approximately equal components with half-times of about 6 days, 130 days, and 8 years, all reflecting loss from the skeleton (McClellan, 1972c, 1983). The early distribution of inhaled and injected soluble ${ }^{90} \mathrm{Sr}$ was also shown to be virtually identical (McClellan, 1972c).

4.2.1.2 YTTRIUM: The gross distribution and retention of yttrium was followed in a single life-span experiment with inhaled ${ }^{91} \mathrm{YCl}_{3}$. Studies with ${ }^{90} \mathrm{Y}$ - and ${ }^{91}$ Y-labeled, fused aluminosilicate particles (FAP) will be considered below with other FAP. Radionuclide impurities in the ${ }^{91} \mathrm{Y}$ employed in the ${ }^{91} \mathrm{YCl}_{3}$ inhalation experiment posed dosimetric difficulties; only brief accounts of distribution and retention results have appeared in the published literature (McClellan, 1976, 1979). For long-survival dogs, inhaled ${ }^{91} \mathrm{YCl}_{3}$ delivers approximately equivalent average radiation doses to lung and skeleton, about one-third this dose to liver, about four times this dose to nasal turbinates (Boecker, 1986).

4.2.1.3 CESIUM: The distribution and retention of cesium has been studied in two life-span, single injection experiments with beagles, and in a supporting experiment comparing the behavior of inhaled and injected cesium. Experiments with cesium-labeled FAP will be considered below with other FAP.

External counting of dogs injected from 61 to 588 days of age showed a rapidly excreted component (half-time about 1 day) amounting to about $20 \%$ of the injected dose; the retention half-time of the remaining $80 \%$ increased progressively with age, achieving a value of about 30 days at an age of about 300 days; the relative concentrations of ${ }^{137} \mathrm{Cs}$ in various tissues of a dam and pups were also reported (Tyler, 1969). An incidental observation was the tenacious retention of ${ }^{137} \mathrm{Cs}$ in urinary calculi composed of the mineral struvite (Kaspar, 1969). 
Distribution and retention of systemic ${ }^{137} \mathrm{Cs}$ were similar following either inhalation or injection of ${ }^{137} \mathrm{CsCl}$ (Boecker, $1969,1969 b$ ). About $77 \%$ of ${ }^{137} \mathrm{Cs}$, inhaled as $\mathrm{CsCl}$, was retained in the body with a biological half-time of 43 days; urinary excretion was about 10 -fold higher than fecal excretion, and both decreased at a rate similar to that of total body retention (Boecker, 1968). Lifespan dogs in the ITRI injection experiment retained about $85 \%$ of injected ${ }^{137} \mathrm{Cs}$ with a half-time of about 32 days; concentration of ${ }^{137} \mathrm{Cs}$ in a variety of tissues fell in the range of 0.6-1.5 times the average concentration in the total body (Boecker, 1972).

4.2.1.4 CERIUM: The gross distribution and retention of cerium was followed in a single life-span experiment with inhaled ${ }^{144} \mathrm{CeCl}_{3}$ (Benjamin, 1973; Boecker, 1984), and in supporting sacrifice studies (Boecker, 1974b; Cuddihy, 1976b). Experiments with ${ }^{144} \mathrm{Ce}$-labeled FAP will be considered below with other FAP.

Data from animals sacrificed at intervals to 512 days postinhalation showed rapid initial clearance of about half of the inhaled ${ }^{144} \mathrm{CeCl}_{3}$. The remaining ${ }^{144} \mathrm{Ce}$ was retained with a half-life approaching its physical half-life and was distributed principally between liver and skeleton, with high local concentrations in the nasal cavity. Radiation doses were estimated, based on a detailed kinetic model, and the results were compared with data from other species and with the assumed behavior of cerium in man (Boecker, 1974b; Cuddihy, 1975).

A supporting experiment, involving 12 dogs sacrificed at intervals to 32 days following inhalation of ${ }^{144} \mathrm{CeCl}_{3}$, directed special attention to the distribution and retention of ${ }^{144} \mathrm{Ce}$ in the endocrine glands and reproductive organs. Cumulative radiation doses in pituitary and adrenal glands, pancreas, ovaries, testes, prostate, and uterus were less than $2 \%$ of the doses calculated for lung and liver; the thyroid dose was about $30 \%$ of the dose in liver (Cuddihy, 1976b).

For dogs in the life-span experiment surviving for longer than 5 years, cumulative dose to lung was about one-half the dose to liver; dose to bone, about one-third the dose to liver; and dose to the nasal cavity about 1.5 times the dose to liver (Boecker, 1984).

4.2.1.5 FUSED ALUMINOSILICATE PARTICLES: Several ITRI experiments with inhaled beta-emitting radionuclides have employed these radionuclides in the form of fused aluminosilicate particles (FAP) (Raabe, 1971). Life-span experiments have employed ${ }^{90} \mathrm{Sr},{ }^{90} \mathrm{Y},{ }^{91} \mathrm{Y}$, and ${ }^{144} \mathrm{Ce} \mathrm{FAP}$; the ${ }^{144} \mathrm{Ce}$ FAP were studied in aged and juvenile as well as young-adult animals and with repeated as well as single inhalations. Non-life-span supporting dosimetry studies have employed ${ }^{134} \mathrm{Cs}$ and ${ }^{137} \mathrm{Cs}$ FAP. 
Retention of FAP in the lung is determined by the behavior of the aluminosilicate vector particle; dosimetry in the lung is a function, also, of the radiation characteristics and the physical half-life of the radionuclide employed, but not of its chemistry (McClellan, 1970, 1974). Solubility of these FAP has been studied invitro, and compared with in-vivo behavior (Kanapilly, 1973). Since only a small fraction of the radionuclide inhaled in this form was dissolved and absorbed from the lung, these experiments are of interest primarily for the light they shed on dose-response relationships in the respiratory system, including pulmonary lymph nodes. The radionuclides employed in the life-span FAP experiments were chosen to provide a range of dose rates and exposure durations and are considered below in order of increasing half-life.

The effective half-time for retention in the lung of ${ }^{90} \mathrm{Y}$ inhaled as FAP is that dictated by its physical decay half-time of 2.6 days (Jones, R., 1974; McClellan, 1974). Results from a supporting study involving 12 beagles sacrificed at intervals from 1 hour to 12 days postexposure indicate infinite-time radiation doses per millicurie initial lung burden of: $1200 \mathrm{rad}$ to lung, $290 \mathrm{rad}$ to rib bone marrow (from ${ }^{90} \mathrm{Y}$ in the lung), 180 rad to the large intestine (from ${ }^{90} \mathrm{Y}$ cleared from the upper respiratory tract), $170 \mathrm{rad}$ to tracheobronchial lymph nodes (largely from ${ }^{90} \mathrm{Y}$ in the lung), $120 \mathrm{rad}$ to blood circulating through the lung, $0.54 \mathrm{rad}$ to skeleton, and $0.38 \mathrm{rad}$ to liver (Barnes, 1972b). Another supporting study employed 10 dogs that inhaled relatively large numbers of large-sized ${ }^{90} \mathrm{Y}$ FAP to maximize exposure of the gastrointestinal tract; animals were sacrificed from 8 to 29 days postexposure; gastrointestinal tract doses were measured with thermoluminescent dosimeters, and radiation effects were observed (Hahn, 1975).

The effective half-time for retention in the lung of ${ }^{91} \mathrm{Y}$ (physical decay half-time, 59 days) inhaled as FAP is about 50 days, allowing time for significant translocation to pulmonary lymph nodes, but dosimetrically insignificant absorption to bone and liver (Jones, R., 1974; McClellan, 1974).

The effective half-time for retention in the lung of ${ }^{144} \mathrm{Ce}$ (physical decay halftime, 285 days) inhaled as FAP is about 180 days (Jones, 1974; McClellan, 1974). Results from 24 serially sacrificed dogs indicate that only a small amount of ${ }^{144} \mathrm{Ce}$ inhaled as FAP is translocated from lung to other organs, $<5 \%$ to liver, $<3 \%$ to skeleton, and $<2 \%$ to pulmonary lymph nodes (Hahn, 1973b, 1976). In the life-span animals, cumulative radiation doses to pulmonary lymph nodes were about 3 times higher that those to lung ( $\mathrm{Hahn}$, in press $b$ ). Early results have been reported on the retention and distribution of ${ }^{144} \mathrm{Ce}$ inhaled as FAP during a series of repeated inhalation exposures (Boecker, 1980). Studies of the effect of age at inhalation showed more rapid early clearance from the lungs of immature dogs (90 days of age); coupled with the continued growth of the lung of the 
immature dog, this resulted in a $60 \%$ reduction in the cumulative radiation dose to lung, 1000 days after exposure, as compared with young adults (Guilmette, $1987 \mathrm{~b}$ ). As compared with young adults, the immature dogs showed an increased deposition of inhaled ${ }^{144} \mathrm{Ce}$ in the skeleton; aged dogs showed a decreased skeletal deposition.

The effective half-time for retention in the lung of ${ }^{90} \mathrm{Sr}$ inhaled as FAP is about 400 days, with significant translocation to pulmonary lymph nodes and bone (Jones, R., 1974; McClellan, 1974).

Supporting, non-life-span studies have investigated the short-term behavior of inhaled ${ }^{137} \mathrm{Cs}$-labeled FAP; about half of the initially inhaled material is rapidly excreted in feces, most of the remainder is retained with a biological half-time of 440 days; fecal excretion continued to exceed urinary excretion for at least 130 days postexposure (Boecker, 1968, 1969, 1969b). In another experiment, employing 12 dogs, accumulation and retention of ${ }^{137} \mathrm{Cs}$-labeled FAP was determined after 10,20, or 30 weekly inhalation exposures; deposition was about as would be predicted from single-exposure data, but retention was prolonged in the multiply exposed animals (Boecker, 1977).

Several ancillary studies of the retention of insoluble particles in the beagle lung have employed FAP labeled with ${ }^{134} \mathrm{Cs}$; these have included studies of the effect of particle size and have involved interspecies comparison, from which it was concluded that, for the clearance and translocation of such relatively insoluble particles, the dog is a much better model for man than the rat, mouse, or guinea pig (Snipes, 1983, 1983c, 1984b).

4.2.1.6 RADIUM: The gross distribution and retention of radium has been described in life-span experiments involving single injection of young-adult beagles with ${ }^{224} \mathrm{Ra},{ }^{226} \mathrm{Ra}$, and ${ }^{228} \mathrm{Ra}$; also in experiments involving multiple injection of young adults with ${ }^{224} \mathrm{Ra}$ and ${ }^{226} \mathrm{Ra}$, and in experiments involving injection of juvenile and aged dogs with ${ }^{226} \mathrm{Ra}$. A number of supporting non-life-span studies have also been conducted.

Preliminary to the earliest life-span experiments with radium, a number of exploratory studies were conducted. One of these compared the metabolism of ${ }^{226} \mathrm{Ra}$ and ${ }^{45} \mathrm{Ca}$ in a 5-month-old beagle; the principal gross metabolic difference was the more rapid clearance of ${ }^{226} \mathrm{Ra}$ from blood and its preferential excretion by both kidney and gut (Stover, 1957). The extent of biological or physical redistribution of the longer-lived members of the complex decay-product chains of the different radium isotopes was a matter of critical concern. Such information is important not only in the estimation of radiation dose to various 
organs, but is also essential in the interpretation of external counting data, on which estimates of retention are based (Lloyd, $R ., 1976 \mathrm{~g}$ ).

Supporting studies focusing on these problems have included detailed investigations of the substantial exhalation of the ${ }^{222} \mathrm{Rn}$ daughter following single (Mays, 1958, 1975; Van Dilla, 1958) or multiple (Parks, 1978) injection of ${ }^{226} \mathrm{Ra}$, and the effect of age (Lloyd, R., 1983d; Parks, 1978) and radiation dose (Parks, 1978) on this process. Skeletal ${ }^{222} \mathrm{Rn} /{ }^{226} \mathrm{Ra}$ ratios in neonatal and juvenile beagles could be described by the same equation, but this ratio was higher in young-adult beagles and in St. Bernards (Lloyd, R., 1983d). Less than $2 \%$ of the shorter-lived ${ }^{220} \mathrm{Rn}$, in the decay chain of ${ }^{228} \mathrm{Ra}$, is lost by exhalation (Mays, $1958 \mathrm{~b}$ ); there is essentially complete retention of the ${ }^{212} \mathrm{~Pb}$ daughter (Stover, 1959c).

The complex internal dosimetry of ${ }^{224} \mathrm{Ra}$ and its daughters has been studied in dogs sacrificed at intervals to 7 days postinjection (Lloyd, R., 1982); other studies with ${ }^{224} \mathrm{Ra}$ have focused on the state of equilibrium with its daughters, ${ }^{220} \mathrm{Rn}$ and ${ }^{212} \mathrm{~Pb}$, at the endosteal surfaces of dog bone (Schlenker, 1986).

Clearance of radium from the plasma was more rapid than that of other radionuclides in Utah injection studies, and the fecal-to-urinary excretion ratio higher (Stover, 1962; Van Dilla, 1958). Radium is deposited predominantly in bone, including teeth (Jee, 1960). About 15\% remains in soft tissues at 1 day postinjection, which is reduced to less than $3 \%$ at 60 days; liver, kidney and spleen are principal sites of soft tissue retention (Atherton, 1965). Concentration and retention of radium also occur in the eye, where its presence is correlated with biological effects (Fisher, G., 1976; Stover, 1962; Taylor, G., 1962b, 1964b).

In-vivo counting data from 125 dogs, over a period of as long as 14 years following injection with ${ }^{226} \mathrm{Ra}$, as young adults, shows evidence of greater retention in dogs receiving more than $4 \mu \mathrm{Ci} / \mathrm{kg}$; retention in dogs receiving lower injected doses could be expressed as a five-component exponential, with $25 \%$ lost with a half-time of less than 1 day, and the longest component (15\%) retained with a half-time of about 13 years (Lloyd, R., 1976h, 1986b; Wrenn, 1986b). The definitive ${ }^{226}$ Ra skeletal dosimetry for the Utah injection study, including dose from daughters injected and daughters grown in, is that of Lloyd, Mays and Atherton (Lloyd, R., 1986b). Data from 78 dogs injected, as young adults, with ${ }^{228} \mathrm{Ra}$ showed retention essentially similar to that of ${ }^{226} \mathrm{Ra}$ (Lloyd, R., 1976h).

The distribution, retention, and complex internal dosimetry of injected ${ }^{224} \mathrm{Ra}$ and its daughter products were studied in 6 young-adult beagles sacrificed at intervals to 7 days following injection of ${ }^{224} \mathrm{RaCl}_{2}$; the injection of $1 \mu \mathrm{Ci} / \mathrm{kg}$ was 
estimated to result in cumulative doses of about $40 \mathrm{rad}$ to skeleton (since revised to $30 \mathrm{rad}$, based on a larger assumed skeleton), $13 \mathrm{rad}$ to kidneys, $11 \mathrm{rad}$ to eyes, 8 rad to liver, and $0.8 \mathrm{rad}$ to testes and ovaries (Lloyd, R., 1982).

The retention of ${ }^{226} \mathrm{Ra}$ has been measured by in-vivo counting procedures over the life span of 46 multiply injected Davis beagles (Momeni, 1976c, 1976d; Parks, 1978b). The Davis and Utah data are remarkably consistent, both showing the increased long-term retention at high radiation dose levels. The Davis data also identify an effect of age-at-initiation of the multiple ${ }^{226} \mathrm{Ra}$ injections. Long-term retention of radium in the beagle is substantially greater than that in man (Momeni, 1976c). Periodic external counting data have been employed to calculate individual average skeletal doses for each dog in the Davis life-span experiment (Raabe, 1981, 1981b).

The retention and dosimetry of ${ }^{226} \mathrm{Ra}$ were studied in St. Bernards, in an experiment designed to compare the effect of radiation in a breed with a much higher spontaneous incidence of osteosarcoma (Jee, 1976b). Radium retention in the St. Bernard is slightly greater than that in beagles; radium distribution is similar, as is radon retention in bone (Lloyd, $R ., 1983 b, 1983 d$ ).

Effects of age on radium retention were observed in early Utah studies (Van Dilla, 1958). In more extensive later studies, beagles injected with ${ }^{226} \mathrm{Ra}$ at 5 years of age showed a diminished retention, but an essentially similar distribution, as compared with young adults (Lloyd, R., 1983c). Those injected at 3 months of age showed an increased retention, as compared with young adults, but a lower average radiation dose to bone per microcurie injected because of dilution due to growth (Lloyd, R., 1983); the juvenile animals retained a significantly smaller fraction of the ${ }^{222} \mathrm{Rn}$ daughter in their bones for about the first 600 days after injection. Preliminary distribution and retention data have also been reported from experiments involving the serial sacrifice, from 1 to 14 days following injection of 6 neonatal beagles, and from 7 to 360 days following injection of 7 three-month-old beagles (Bruenger, 1983).

4.2.1.7 THORIUM: The single life-span experiment employing ${ }^{228} \mathrm{Th}$ was supported by a number of sacrifice studies designed to explore the biological and physical redistribution of the longer-lived members of its complex chain of decay products (Jee, 1967; Stover, 1968c, 1981). These include experiments on the distribution and retention of the decay products ${ }^{224} \mathrm{Ra}$ (Van Dilla, 1956, 1957, Stover, $1965 b, 1965 c, 1968 c$ ) ${ }^{220} \mathrm{Rn}$ (Mays, $1958 b$; Van Dilla, 1956), and ${ }^{212} \mathrm{~Pb}$ and ${ }^{212} \mathrm{Bi}$ (Stover, 1959c, 1965b, 1965c, 1968c). A 20-dog sacrifice study has supplied detailed data on decay-chain equilibria for the period to 18 months postinjection (Lloyd R., $1984 d)$. 
Early clearance of thorium from the plasma was slower than that of radium or strontium but, after the first hour, was more rapid than that of plutonium; the early fecal-to-urinary excretion ratio was the lowest of the four elements (Stover, 1960,1962 ). At the low mass levels employed in the Utah experiment, $70 \%$ to $80 \%$ of injected thorium deposited in bone, about $4 \%$ in liver, about $1.5 \%$ in kidney, $0.3 \%$ in spleen, and the remainder was widely distributed in other soft tissues. Concentration and retention of thorium also occur in teeth (Jee, 1960) and in the eye, where its presence is correlated with biological effects (Stover, 1962; Taylor, G., 1962b).

In-vivo counting data from 40 life-span dogs, obtained over a period of nearly 8 years, and from 20 dogs sacrificed from 7 days to 18 months postinjection, show evidence of greater retention at the highest dose levels; a single biological halftime of about 10 years was estimated for ${ }^{228} \mathrm{Th}$ in bone (Lloyd, R., 1984d). Loss from soft tissues was more rapid, with a biological half-time of about 2 years (Stover, 1960).

4.2.1.8 PLUTONIUM: The gross distribution and retention of plutonium has been described in a number of life-span experiments, and supporting sacrifice studies, conducted at three laboratories, involving its administration by injection or inhalation, in various forms, to dogs of various ages.

The Utah single intravenous injection experiments, with juvenile, young-adult, and aged dogs, employed a carefully standardized ${ }^{239} \mathrm{Pu}$ citrate injection solution, which was minimally colloidal, and with at least $98 \%$ of the plutonium in the tetravalent state (Stover, 1962b). A supporting study with intravenously injected ${ }^{237} \mathrm{Pu}$ showed that this isotope, present at very much smaller mass levels, was distributed similarly to ${ }^{239} \mathrm{Pu}$ injected as the citrate (Lloyd, R., 1976b). Another study had indicated that ${ }^{237} \mathrm{Pu}$ was more readily excreted than ${ }^{239} \mathrm{Pu}$ injected as the nitrate (Bair, 1974d). It seems likely that the nitrate form would undergo polymerization, which would explain its different behavior; such polymerization would not occur in the case of the citrate. Thus, administration of ${ }^{239} \mathrm{Pu}$ citrate, at the relatively high plutonium mass concentrations required in biological studies with ${ }^{239} \mathrm{Pu}$, may be considered an appropriate stand-in for the very low mass levels of plutonium present in the environment, which is the circumstance of primary interest to radiation protection considerations (Lloyd, $R ., 1976 d)$.

The colloidal status of the injection solution has a profound effect on initial distribution, with colloidal ${ }^{239} \mathrm{Pu}(\mathrm{IV})$ being cleared more rapidly from blood and deposited, primarily by phagocytic processes, in liver, spleen, and bone marrow, rather than on bone surfaces. Phagocytically deposited colloidal plutonium was 
shown to be eventually translocated to bone surfaces and liver parenchyma, but only slowly, with half-times of approximately a year or longer (Bruenger, 1981; Stevens, W., 1975). The noncolloidal ${ }^{239} \mathrm{Pu}(\mathrm{IV})$ citrate solution, when injected, behaved very similarly to an injected ${ }^{239} \mathrm{Pu}$-transferrin complex (Stevens, W., 1975).

The distribution and retention of ${ }^{239} \mathrm{Pu}$ in the Utah, young-adult, life-span dogs has been studied exhaustively (Stover, 1959, 1959b, 1962, 1962b, 1968, 1969, 1971, $1972 c, 1974,1976,1977)$. About $85 \%$ of injected plutonium remains in the blood at 1 minute postinjection, and only about half of this is lost from blood in the next 10 hours; about $12 \%$ is excreted in the first 3 weeks, another $16 \%$ in the next 4 years; nearly $30 \%$ is deposited in liver, a few percent in spleen, kidney, and other soft tissues, and the major fraction in the skeleton, including teeth (Jee, 1960).

Because of the low energy and low abundance of penetrating radiation from ${ }^{239} \mathrm{Pu}$, data on its distribution and retention have been obtained largely from alpha-counting of tissue and excreta samples, although some success was achieved in counting gamma rays from relatively high-dose animals (Lloyd, $R$., 1962 ), and a ${ }^{169} \mathrm{Yb}$ tracer has been successfully employed in following the early retention of inhaled plutonium (Diel, 1982; Hahn, 1981).

Plutonium concentration in plasma, from 1 minute to $\mathbf{1 2 . 5}$ years postinjection, has been described by a four-term exponential function of time (Stover, 1977). Retention of plutonium in the skeleton, at dose levels below about $0.1 \mu \mathrm{Ci} / \mathrm{kg}$, has been described by a single exponential plus a constant term amounting to $21 \%$ of the injected dose; at higher dose levels, the constant retention term is greater because of radiation damage (Stover, 1976, 1977). A more elaborate kinetic model for skeletal retention, taking account of differences in bone types and the microdistribution within bone, has also been reported (Stover, 1974, 1975, 1976).

Retention of plutonium in the liver, at dose levels below about $0.1 \mu \mathrm{Ci} / \mathrm{kg}$, has been described by a single exponential expression corresponding to a retention half-time of about 10 years; at higher dose levels, the half-time is shorter because of radiation damage (Stover, 1968, 1971, 1977). Thus, radiation damage has opposite effects on skeletal and hepatic retention of ${ }^{239} \mathrm{Pu}$. There is also evidence of a radiation effect on retention in spleen and kidney (Stover, 1971). Based on the above relationships, calculations show the average cumulative radiation dose to liver, at injection levels less than $0.1 \mu \mathrm{Ci} / \mathrm{kg}$, to be about twice the average cumulative dose to the skeleton (Stover, 1977). A comprehensive biokinetic model of ${ }^{239} \mathrm{Pu}$ distribution and retention in blood, liver, and cortical and trabecular bone of the beagle has been recently described (Polig, in press). 
The retention and dosimetry of plutonium were studied in St. Bernards, which were also employed in life-span studies designed to compare the effect of radiation on induction of bone tumors in a dog with a much higher spontaneous incidence of bone tumor than the beagle (Jee, 1976b); distribution and retention were similar to what was observed in the young-adult beagle (Lloyd, R., 1984b).

The behavior of inhaled plutonium in the lung has been previously considered in Section 4.1.2. It might seem reasonable that plutonium, once translocated from the lung, would be distributed and retained within the body in a manner unaffected by the form in which it was inhaled. This is often not the case, however. There is a variable degree of plutonium translocation to, and retention in, pulmonary lymph nodes, depending on the size and chemical nature (solubility) of the plutonium particles inhaled (Bair, 1962b). Very little of the relatively soluble ${ }^{239} \mathrm{Pu}\left(\mathrm{NO}_{3}\right)_{4}$ is translocated to lymph nodes (Park, J., 1986; Stevens, D., 1986). Much more of the insoluble ${ }^{239} \mathrm{PuO}_{2}$ and ${ }^{238} \mathrm{PuO}_{2}$ is translocated to lymph nodes (Bair, 1962, 1980; Mewhinney, 1983; Park, J., 1964, 1966, 1971, 1972, 1981, 1986, in press) but retained less tenaciously in the case of ${ }^{238} \mathrm{PuO}_{2}$, probably as a consequence of radiolytic fragmentation (Diel, 1980; Mewhinney, 1983). Whatever form of plutonium is inhaled, however, the highest concentration, within 100 days postexposure, is present in pulmonary lymph nodes (Park, J., 1972). Within 3 to 4 years postinhalation, the quantity of ${ }^{239} \mathrm{PuO}_{2}$ in pulmonary lymph nodes is about equal to that retained in the lung (Clarke, 1964b, 1966b). As much as $5 \%$ of inhaled ${ }^{239} \mathrm{PuO}_{2}$ is translocated, at later times postexposure, to hepatic and splenic lymph nodes (Park, $J_{.}, 1972$ ). Recently reported ITRI data for the period extending to 1100 days following exposure to monodisperse aerosols of $0.7,1.5$, and $3.0 \mu \mathrm{m}$ AMAD show an effect of particle size on both retention half-times in the lung and on the fractions translocated to skeleton and certain lymph nodes (Guilmette, 1984, 1987).

Comprehensive biokinetic models have been derived for the distribution and retention in the beagle dog of inhaled ${ }^{239} \mathrm{PuO}_{2}$ (Mahaffey, 1981; Stuart, 1970), ${ }^{238} \mathrm{PuO}_{2}$ (Mewhinney, 1983), and ${ }^{239} \mathrm{Pu}\left(\mathrm{NO}_{3}\right)_{4}$ (Stevens, D., 1986), and for the correlation of both inhalation and intravenous injection data (Cuddihy, 1976). Systemic distribution of plutonium absorbed from the lung, when inhaled as ${ }^{239} \mathrm{Pu}\left(\mathrm{NO}_{3}\right)_{4}$ or ${ }^{238} \mathrm{PuO}_{2}$, is similar to that observed following intravenous injection of $\mathrm{Pu}(\mathrm{IV})$ citrate, with roughly equivalent deposition in liver and bone (Dagle, 1979; Mewhinney, 1983; Park, J., 1969, 1976, 1986; Stevens, D., 1986). When plutonium is inhaled as ${ }^{239} \mathrm{PuO}_{2}$, however, the fraction deposited in bone is greatly reduced (Dagle, 1985; Guilmette, 1984, 1987; Park, J., 1964, 1966, 1971, 1972), in a manner reminiscent of injected colloidal $\mathrm{Pu}(\mathrm{IV})$, suggesting that absorption from the lung occurs in particulate form (Dagle, 1979; Guilmette, 1987; Park, J., 1986). 
A supporting study, involving 24 dogs, compared distribution and retention of inhaled ${ }^{239} \mathrm{Pu}\left(\mathrm{NO}_{3}\right)_{4}$ with that of inhaled ${ }^{238} \mathrm{Pu}\left(\mathrm{NO}_{3}\right)_{4}$, in animals sacrificed at intervals to 1 year postinjection; eventual systemic distribution was similar, but was more rapidly attained following ${ }^{238} \mathrm{Pu}_{\left(\mathrm{NO}_{3}\right)}{ }_{4}$ inhalation (Dagle, 1983; Park, J., 1981).

Deposition and retention of inhaled or injected plutonium in ovary and testis have been of particular interest. Relatively high concentrations were reported in ovaries following inhalation of ${ }^{239} \mathrm{PuO}_{2}$, due perhaps to the particulate nature of the translocated material (Bair, 1963). Data on the gonadal concentration of plutonium and other actinides, from life-span dog studies and from other animal studies, have been reviewed (Richmond, 1975).

The effect of age on plutonium distribution and retention was explored in supporting studies with dogs injected at 2 days of age (Bruenger, 1978), and at about 3 months of age (Atherton, 1976; Bruenger, 1980, 1983, 1984; Lloyd, R., 1978c). As compared to young adults, the juvenile dogs cleared plutonium more rapidly from the blood, excreted less, deposited and retained less in liver, and more in bone. A supporting study with dogs injected at 4 to 7 years of age, employing ${ }^{239} \mathrm{Pu}(\mathrm{IV})$ citrate with a ${ }^{237} \mathrm{Pu}$ tracer, showed little difference in early distribution and retention as compared to dogs injected as young adults (Lloyd, R., 1978).

Following inhalation of ${ }^{239} \mathrm{PuO}_{2}$, age-related effects were seen in the translocation of plutonium to the skeleton, with the largest uptake in immature dogs and the smallest in aged dogs; significant age-related differences in retention in the lung or translocation to pulmonary lymph nodes were not seen (Guilmette, $1987 b, 1988 b$ ).

4.2.1.9 AMERICIUM: Gross distribution and retention of ${ }^{241} \mathrm{Am}$ has been followed in a single life-span experiment involving administration via intravenous injection (Lloyd, R., 1970, 1984c), and in ancillary sacrifice studies involving its inhalation (Craig, 1979; Mewhinney, 1981, 1982; Thomas, 1970, 1972b).

Retention following intravenous injection was measured by whole body counting, with liver content estimated from counts with and without shielding in the liver region (Lloyd, R., 1970, 1975d, 1976g). Clearance of ${ }^{241} \mathrm{Am}$ from blood is much faster than that of plutonium; comparable to the rate of clearance of divalent alkaline earths (Bruenger, 1969). Early excretion was largely via the urine.

About $50 \%$ of initially injected ${ }^{241} \mathrm{Am}$ was deposited in liver and about $30 \%$ in skeleton. Both liver and skeletal burdens were retained with a half-time of about 10 years, except at relatively high dose levels, where there was a sharp decrease 
in liver retention beginning about 100 days postinjection, accompanied by increased deposition in skeleton (Lloyd $R ., 1970,1972 b$ ). With nearly all animals dead in the life-span injection experiment, detailed retention and dosimetry data have been reported (Lloyd R., 1984c). Retention of the $49 \%$ of injected dose deposited in liver was characterized by a single exponential function, with retention half-time varying from 0.8 year at the highest injection levels, to 8.8 years at levels of $0.1 \mu \mathrm{Ci} / \mathrm{kg}$ or less. The $36 \%$ of injected dose initially deposited in skeleton increased with time as americium was translocated from liver.

Although the fraction deposited was low, notably high concentrations of ${ }^{241} \mathrm{Am}$ occurred in the thyroid gland, where it was selectively bound to connective tissue elements (Lloyd, R., 1970, Stevens, W., 1969). Relatively high concentrations also occurred in the kidney, with selective deposition in the glomerulus (Lloyd, R., 1970 ).

A supporting sacrifice study described the retention and distribution of ${ }^{241} \mathrm{Am}$ in neonatal beagles injected at 1 day of age and sacrificed from 1 to 5 days later (Stevens, W., 1977). As compared with young adults, the neonates deposited a larger fraction in the skeleton $(\sim 80 \%)$; only $7 \%$ was deposited in liver.

Three sacrifice studies showed somewhat different rates of removal of inhaled ${ }^{241} \mathrm{AmO}_{2}$ from the lung, and of deposition in pulmonary lymph nodes, due presumably to differences in the chemical and physical state of the oxide particles inhaled (Craig, 1979; Thomas, 1972b; Mewhinney, 1982). Systemic deposition and retention were similar to what was observed following injection. A detailed biokinetic model for inhaled ${ }^{241} \mathrm{AmO}_{2}$ has been developed from these data (Mewhinney, 1980, 1981, 1982, 1982b, 1983b).

4.2.1.10 BERKELIUM: Data on the distribution of ${ }^{249} \mathrm{Bk}$ in the beagle was obtained from studies with ${ }^{249} \mathrm{Cf}$ in which berkelium was a contaminant; the general distribution of intravenously injected ${ }^{249} \mathrm{Bk}$ was almost identical to that of ${ }^{249} \mathrm{Cf}$ (Taylor, G., 1972).

4.2.1.11 CALIFORNIUM: Gross distribution and retention of californium have been followed in life-span experiments involving the administration via intravenous injection of either ${ }^{249} \mathrm{Cf}$ or ${ }^{252} \mathrm{Cf}$ (Lloyd, R., 1976). Retention of either radionuclide was measured by whole-body counting, with liver content estimated from counts with and without shielding in the liver region (Lloyd, $R$., $1972,1975 d$ ). Both isotopes were rapidly absorbed from the blood stream, less than $1 \%$ of the injected quantity remaining after 24 hours (Stevens, W., 1972). Early excretion was largely via the urine (Lloyd, R., 1972). About $50 \%$ of the injected dose was deposited in skeleton, about $20 \%$ in liver; relatively high 
concentrations were initially present, and retained, in kidney and thyroid (Atherton, 1972; Lloyd, R., 1972, 1976). Based on measurements to about 4 years postinjection, the major fraction of either californium isotope was retained in the total body with a half-time of about 8.5 years; the major fraction of californium deposited in liver was retained with a half-time of about 4.2 years (Lloyd, R., 1976).

4.2.1.12 EINSTEINIUM: Data from the very few dogs involved in the einsteinium experiment indicate an excretory, distribution, and retention behavior very similar to that of californium. Three dogs sacrificed at intervals to 8 weeks postinjection showed an average of about $45 \%$ of the injected dose in skeleton and $12 \%$ in liver, with significant concentrations also present in thyroid and kidney (Lloyd, R., 1975).

\subsubsection{LOCALIZED DISTRIBUTION AND RETENTION (by organ or tissue)}

Correlation of radiation dose with biological effect often requires knowledge of dose to local regions of an organ or tissue, or even to individual cell types or molecular species. Materials derived from life-span dog studies have provided a wealth of information of this type. It will be convenient to consider this information in relation to the organs or tissues studied.

4.2.2.1 BLOOD: Supporting studies have described the distribution of ${ }^{226} \mathrm{Ra}$ between blood cells and plasma and have compared the results with those obtained from similar measurements in a sample of human blood (Stover, 1965). The distribution of ${ }^{228} \mathrm{Th}$ between red cells and plasma has also been studied (Lloyd, R., 1984d).

Studies of the interaction of blood constituents with $\mathrm{Pu}(\mathrm{IV})$, injected in citrate buffer, indicated extensive binding of the plutonium to proteins (Stevens, W., 1965), later identified as albumin and transferrin, with predominant binding to transferrin (Stevens, W., 1968; Stover, 1972b). In-vitro studies with human blood showed similar binding of $\mathrm{Pu}(\mathrm{IV})$ to transferrin (Stover, 1968b). In-vitro studies demonstrated exchange of ${ }^{239} \mathrm{Pu}(\mathrm{IV})$ between transferrin and ferritin in beagle blood (Bruenger, 1969b).

Americium(III) also formed a complex with transferrin, which was less stable than that formed with $\mathrm{Fe}(\mathrm{III})$ or $\mathrm{Pu}(\mathrm{IV})$; americium was also bound by albumin, and possibly by an alpha-globulin; similar results were obtained from in-vitro studies with human sera (Bruenger, 1969; Stover, 1972b). Californium exhibited behavior similar to that of americium, with binding to transferrin less stable than that shown by plutonium (Stevens, W., 1972). 
4.2.2.2 LUNG: The uniformity of distribution of inhaled FAP within the lung was studied in experiments involving 16 dogs that inhaled ${ }^{90} \mathrm{Sr}-{ }^{90} \mathrm{Y}-$ labeled FAP, and 14 dogs that inhaled FAP labeled with ${ }^{147} \mathrm{Pm}$ and ${ }^{169} \mathrm{Yb}$ (Snipes, 1975). A 3to 4 -fold ratio of observed local dose to average thorax dose was observed in the case of ${ }^{90} \mathrm{Sr}$-labeled FAP. Other experiments, with ${ }^{134} \mathrm{Cs}$-labeled FAP, investigated the translocation of particles deposited in various lobes of the lung to the various regional lymph nodes (Snipes, 1983b).

Autoradiographic studies of the very early distribution of inhaled ${ }^{144} \mathrm{Ce}$ or ${ }^{90} \mathrm{Y}$ FAP showed an accumulation just inside the nares, on the turbinates and at the sharp ventral bend in the nasopharynx; this activity was largely cleared via the esophagus within 30 minutes (Barnes, 1971). Autoradiographs of the costal surface of the lung showed a striated pattern, with deposition occurring most heavily beneath the ribs; this pattern was observed with a variety of radionuclides and persisted for at least several hundred days postexposure; a similar pattern has been observed in gross lesions following inhalation of ${ }^{90} \mathrm{Y}$ and ${ }^{241} \mathrm{Am}$ (Barnes, 1971).

The distribution of inhaled ${ }^{239} \mathrm{PuO}_{2}$ within the lung has been described in several autoradiographic studies (Clarke, 1964, 1966; McShane, 1980; Park, J., 1964) and considered in relation to the induction of neoplasia (Sanders, 1971, 1972). These studies indicate an initial uniform distribution of alpha-emitting particles that become focally concentrated in subpleural areas with the passage of time. Phagocytosis and entrapment in fibrotic areas appear to be involved in these processes. Fragmentation of ${ }^{238} \mathrm{PuO}_{2}$ particles, inhaled by dogs as monodisperse aerosols, was observed autoradiographically in lung sections (Diel, 1983).

The microscopic distribution of radiation dose around ${ }^{239} \mathrm{PuO}_{2}$ particles was calculated from autoradiographs of lung tissue sections of dogs, and the results were compared with similar calculations for rats and hamsters; the results did not explain the much lower tumor incidence in hamsters (Diel, 1984).

4.2.2.3 PULMONARY LYMPH NODES: Autoradiographic studies of tracheobronchial lymph nodes, following inhalation of ${ }^{144} \mathrm{Ce}$ as FAP, showed ${ }^{144} \mathrm{Ce}$ particles in macrophages in the paracortical zone as early as 2 days postinhalation; concentrations in the paracortical zone and medullary cords continued to increase over the next year, with concentrations exceeding that in the lung by 100 days postexposure (Hahn, 1976).

Autoradiographic studies have demonstrated an early accumulation of inhaled ${ }^{239} \mathrm{PuO}_{2}$ particles in medullary sinuses, followed by later concentration in the cortex of the nodes (Clarke, 1964, 1966; Dagle, 1976b; Park, J., 1962, 1964). The 
very nonuniform distribution of inhaled ${ }^{239} \mathrm{PuO}_{2}$ among the different lymph nodes draining the respiratory tract, and the influence of particle size on this distribution, have been demonstrated (Guilmette, 1987).

4.2.2.4 CALCIFIED TISSUES: Many of the radionuclides employed in these experiments deposit selectively in bone and teeth. The alkaline earth elements, strontium and radium, can substitute for calcium in the bone matrix; the actinide and lanthanide elements deposit initially on bone surfaces but may be buried by apposition of new bone. Localization of radionuclides within bone, together with the range and quality of the radiations emitted, will determine the cells or tissues irradiated and the kinds of effects observed (Marshall, 1969).

Extensive data have been collected on the distribution of radionuclides among the different bones of the beagle skeleton, with correlations made to the presence of cortical versus trabecular bone, and to rates of bone remodeling. Nasal turbinates accumulate higher-than-average-bone concentrations of ${ }^{90} \mathrm{Sr},{ }^{91} \mathrm{Y}$ and ${ }^{144} \mathrm{Ce}$, when these radionuclides are inhaled in soluble form (Boecker, 1986). The distribution of injected ${ }^{90} \mathrm{Sr}$ (Lloyd, R., 1976e), chronically ingested ${ }^{90} \mathrm{Sr}$ (Parks, 1985), and injected ${ }^{226} \mathrm{Ra}$ (Lloyd, R., 1976e; Parks, 1980) among individual bones of the beagle skeleton has been reported for various times postinjection; distribution is similar for the two radioelements, and similar to that of calcium. Such distribution data have also been reported for ${ }^{224} \mathrm{Ra}$ in the beagle (Lloyd, $R$., 1982 ), and for ${ }^{226} \mathrm{Ra}$ in the St. Bernard (Lloyd, R., 1983b). In young-adult beagles, cortical bone takes up ${ }^{90} \mathrm{Sr}$ less avidly than does trabecular bone, but this difference is not maintained at long times following injection. The complex redistribution of ${ }^{226} \mathrm{Ra}$ among the different bones of 12 beagles sacrificed at intervals following 8 semimonthly injections was described in terms of the fraction of cancellous and compact bone in each skeletal component (Parks, 1980). The dynamics of life-span ${ }^{90} \mathrm{Sr}$ distribution among different bones were described in a similar fashion, in terms of the proportions of cancellous and compact bone present (Parks, 1985). An effect of radiation on the distribution of ${ }^{226} \mathrm{Ra}$ among different bones is seen within a month following injection of doses as large as $10 \mu \mathrm{Ci} / \mathrm{kg}$ (Lloyd, R., 1976e).

The distribution of ${ }^{226} \mathrm{Ra}$ among the various bones of the juvenile beagle was shown to be quite different from that observed in young-adult beagles, with a greater proportion deposited in parts of the skeleton containing much cortical bone, and a lesser deposition in parts containing much trabecular bone (Lloyd, $R$., 1983). Mature beagles, injected at 5 years of age, showed a distribution of ${ }^{226} \mathrm{Ra}$ among the various bones of the skeleton that was quite similar to that of the young adult (Lloyd, R., 1983c). 
The distribution of injected ${ }^{228} \mathrm{Th}$ among individual bones of the beagle skeleton at various times postinjection, as well as distribution along the length of humerus, femur, and ulna, displayed a pattern more typical of a surface-seeking radionuclide, with highest deposition in areas with much trabecular bone and with high rates of bone remodeling (Lloyd, R., 1984d).

Detailed data have been obtained on the distribution of ${ }^{239} \mathrm{Pu}$ among different bones, and within different regions of specific bones, as a function of exposure level and time postinjection; average dose rates within bone regions varied as much as 7-fold, even between relatively large pieces of bone (Stover, 1969; 1972c). A detailed biokinetic model of the distribution of ${ }^{239} \mathrm{Pu}$ between trabecular and cortical bone has been recently described (Polig, in press).

The much greater vascularization of red marrow, as compared to yellow marrow, correlates with the greater deposition of plutonium in red marrow sites, and the greater incidence of bone tumors at these sites (Smith, J., 1984). The concentration of ${ }^{238} \mathrm{Pu}$, inhaled as ${ }^{238} \mathrm{PuO}_{2}$, was found to vary in different bones from about one-half to twice the skeletal average (Park, J, 1976); a similar distribution of concentrations in different bones was observed following inhalation of ${ }^{239} \mathrm{Pu}\left(\mathrm{NO}_{3}\right)_{4}$ (Dagle, 1985). Distribution among different bones of ${ }^{239} \mathrm{Pu}$ present at very low levels in a Utah control dog showed a somewhat similar spread of values (Singh, 1987). Data have also been presented on the partition of plutonium, inhaled as ${ }^{239} \mathrm{Pu}\left(\mathrm{NO}_{3}\right)_{4}$, between bone and marrow in different regions of the humerus, radius, and lumbar vertebra (Dagle, 1985).

The distribution of plutonium among the various bones of the neonatal (Bruenger, 1978) and juvenile (Atherton, 1976; Bruenger, 1980; Lloyd, R., 1978c, 1983) beagle was shown to be significantly different from the distribution observed in beagles injected as young adults. Compared to young-adult beagles, animals injected as juveniles showed a greater deposition in parts of the skeleton containing much cortical bone, such as paws and lower leg bones, and a lesser deposition in parts containing much trabecular bone (Lloyd, R., 1978c). Active bone growth and turnover in the very young beagle also produced a different pattern of plutonium redistribution within bone from that observed in older animals (Bruenger, 1980).

Distribution of injected americium (Lloyd, R., 1972b) and californium (Lloyd, R., 1972) among bones of the beagle skeleton was generally similar to that of plutonium. Detailed analyses of autopsy data from the ${ }^{241} \mathrm{Am}$ life-span study showed distribution of americium among bones to vary with injection level, due to radiation effects and to systematic differences in time between injection and autopsy, (Lloyd, R., 1984c). The distribution of ${ }^{241} \mathrm{Am}$ among the various bones of 
the neonatal beagle was measured in animals injected at 1 day of age; distribution was dependent on the state of development of the individual bones and differed considerably from that seen in dogs injected as young adults (Stevens, W., 1977).

Autoradiographic procedures have been widely employed in studies at the microscopic level of radionuclide distribution. Microdensitometric techniques were developed for the quantitation of localized concentrations of ${ }^{239} \mathrm{Pu}$ as depicted on autoradiographs (Twente, 1961). Techniques of neutron-induced autoradiography have proved particularly useful in studying the microdistribution of plutonium present in low concentrations in bone (Fellows, 1975; Jee, 1972b, 1972c; Smith, J., 1980; Wronski, 1980).

Autoradiographic studies of the highly nonuniform microscopic deposition and redistribution within bone of ${ }^{90} \mathrm{Sr}$ (Jee, 1971) and the alpha emitters ${ }^{226} \mathrm{Ra},{ }^{228} \mathrm{Th}$, and ${ }^{239} \mathrm{Pu}$ (Arnold, 1959; Jee, 1960b, 1962c, 1964, 1971) show similar patterns for the distribution of the volume seekers strontium and radium, and for the surface-seekers plutonium and thorium. The redistribution of plutonium within bone as a function of time postinjection shows a pronounced dose-level effect, clearly attributable to radiation damage; at the highest dose levels of the Utah experiment, remodeling of bone and redistribution of plutonium is virtually stopped (Arnold, 1959, 1962). In teeth, there is an initial concentration on the newly formed dentinal surface of the pulp chambers; at long times following injection, bone-seekers exhibit a minimal diffuse distribution in dentine formed after injection, whereas volume-seekers show a more uniform distribution, throughout, with maximum concentration on most recently formed dentine, cementum, and bone (Jee, 1960, 1962c).

Thermoluminescent dosimetry, autoradiography, and radiochemical analysis of excised humeri and lumbar vertebrae from beagles of the Davis ${ }^{90} \mathrm{Sr}$ chronicfeeding experiment (Goldman, 1972b; Momeni, 1975b, 1975d, 1976, 1976b; Spiers, 1969, 1972; Zanelli, 1971), and ${ }^{226} \mathrm{Ra}$ multiple-injection experiment (Momeni, $1976 \mathrm{~b}, 1976 \mathrm{~d}$ ), provide a detailed picture of dose distribution within these bones as a function of time; the local dose to hematopoietic tissues (marrow), and to osseous tissues, was lower than that estimated as average skeletal dose. Similar local dosimetry studies of the mandible and teeth identify this region as one of unusually high dose, because of the lack of removal of ${ }^{90} \mathrm{Sr}$ from teeth by remodeling (Parks, 1984).

A detailed picture has emerged of the kinetic behavior of low concentrations of plutonium in bone, with initial nonuniform deposition on bone surfaces, subsequent burial of these surface deposits by new bone growth, removal of the buried 
deposits by osteoclastic activity, with a resulting concentration of plutonium within osteoclasts and bone marrow macrophages; at low injected doses, plutonium is essentially removed from bone surfaces within 6 months postinjection (Arnold, 1959, 1962; Jee, 1964, 1971, 1972, 1972c). The fraction of plutonium accumulated in marrow increases with decreasing plutonium dose, but at the lowest dose levels is not retained in the marrow beyond about 4 years postinjection ( $\mathrm{Jee}, 1972,1972 \mathrm{C}$ ). A similarly detailed picture is available for the microscopic distribution, retention, and dosimetry of ${ }^{226} \mathrm{Ra}$ and ${ }^{90} \mathrm{Sr}$ on bone surfaces of beagles, with comparative data from other species, including man in the case of ${ }^{226} \mathrm{Ra}$ (Jee, 1964).

The microdosimetric pattern of plutonium and radium in the dog has been extrapolated to man using comparative morphometric techniques (Lloyd, E., 1971, 1972; Spiers, 1976, 1988). These techniques have been employed in the calculation of absorbed dose rates to the tissues of beagle and human bone, for ${ }^{90} \mathrm{Sr}$ (Spiers, 1969, 1972, 1988), for ${ }^{226} \mathrm{Ra}$ and ${ }^{239} \mathrm{Pu}$ (Spiers, 1976, 1988), and for other bone-seeking radionuclides (Spiers, 1978).

Detailed morphometric and autoradiographic studies of the ulna and second lumbar vertebral body of dogs sacrificed from 5 to 746 days following plutonium injection, showed low initial uptake and slow loss from the ulna with $50 \%$ of its surface trabecular, and high initial uptake and rapid loss from the vertebral body with $90 \%$ of its surface trabecular (Kimmel, 1976). Detailed studies have also been reported of the microdistribution of ${ }^{239} \mathrm{Pu}$ in the cortical bone regions of these same two bones over times ranging from 41 to 1950 days postinjection (Ingrao, 1985).

Thicknesses of the deposits of plutonium and radium at bone surfaces have been compared in studies measuring the energy loss of alpha particles emitted from the bone surfaces (Schlenker, 1975). Ninety days after injection of monomeric ${ }^{239} \mathrm{Pu}$, the thickness of the endosteal surface deposit was not greater than 0.1 $\mu \mathrm{m}$; for ${ }^{226} \mathrm{Ra}$, the thickness was as great as $6.5 \mu \mathrm{m}$, within 5 hours postinjection. Studies of ${ }^{224} \mathrm{Ra}$ daughter-product equilibrium at bone surfaces indicate an endosteal tissue dose of one-half to one-third that which would be calculated assuming complete equilibrium (Schlenker, 1986).

Preliminary results have been reported from a comparative study of the microscopic distribution within bone of ${ }^{226} \mathrm{Ra}$ and ${ }^{239} \mathrm{Pu}$ following injection of neonatal, juvenile, young-adult, and aged beagles (Bruenger, 1983). The local distribution of radiation dose within the humerus of growing beagles following injection with ${ }^{239} \mathrm{Pu}$ at 90 days of age has been compared with the corresponding distribution of dose in beagles injected as young adults (Bruenger, 1984). 
Autoradiographic studies reveal a somewhat more uniform distribution of americium on bone surfaces than is the case with plutonium (Lloyd, R., 1972b). Recently developed methods employing computer-controlled microphotometry (Polig, 1986, 1986c) have provided analyses of microdistribution and microdosimetry at a level of detail hitherto unavailable. For ${ }^{241} \mathrm{Am}$ on bone surfaces, the estimated dose to the $10-\mu \mathrm{m}$ marrow band adjacent to bone surfaces was 8 to 16 times the average skeletal dose, and a positive correlation was demonstrated between specific surface activity and local bone turnover rate (Polig, 1984, 1984b). This approach can be extended to the consideration of dose to individual cells or cell types (Polig, 1985, 1986b, 1986c, in press).

4.2.2.5 LIVER: Autoradiographic studies have indicated an initial uniform diffuse distribution in the liver, of $\mathrm{Pu}$ (IV) injected as the citrate complex (Cochran, 1962; Jee, 1976b; Taylor, G., 1971, 1972b, 1986). This distribution became less uniform with time; "hot spots" appeared as early as a few months postinjection in sinusoidal reticuloendothelial cells; concentration also occurred in periportal areas in association with iron-staining pigment. Regenerating nodules, which might constitute as much as $85 \%$ of the liver volume, showed no significant redeposition of plutonium. A similar behavior was noted for ${ }^{241} \mathrm{Am}$ (Taylor G., $1972 b, 1986$ ). Early concentration of injected ${ }^{249} \mathrm{Cf}$ occurred in the hepatic cells of the liver, with little if any deposition in reticuloendothelial cells (Taylor, G., 1972). Redistribution and loss of plutonium from the liver was not observed in dogs injected at 3 months of age (Bruenger, 1980).

A very different and nonuniform pattern of distribution within the liver was observed following injection of colloidal $\mathrm{Pu}(\mathrm{IV})$; plutonium was retained predominantly within reticuloendothelial cells; very little was associated with soluble proteins (Stevens, W., 1975).

Plutonium in the liver of dogs that have inhaled ${ }^{239} \mathrm{PuO}_{2}$ is heterogeneously distributed, showing on autoradiographs as large "stars," and suggesting translocation in particulate form (Guilmette, 1987). Following ${ }^{239} \mathrm{Pu}\left(\mathrm{NO}_{3}\right)_{4}$ inhalation, liver autoradiographs show a distribution of single tracks; following ${ }^{238} \mathrm{PuO}_{2}$ inhalation, both stars and single tracks are observed (Dagle, 1986). Following inhalation of ${ }^{238} \mathrm{PuO}_{2}$, clustering of liver plutonium in particulate form was increased at high exposure levels, and it increased with time following exposure; at low exposure levels, distribution of inhaled ${ }^{238} \mathrm{PuO}_{2}$ in the liver was similar to that of injected ${ }^{238} \mathrm{Pu}$ - or ${ }^{239} \mathrm{Pu}$-citrate (Gearhart, 1980 ).

Studies of the subcellular distribution of plutonium in liver showed a somewhat ubiquitous distribution, with evidence for its association with ferritin (Bruenger, 1971, 1976; Stover, 1972b); similar patterns of distribution and binding were 
observed for americium (Stover, 1970, 1972b), californium (Bruenger, 1972) and curium (Bruenger, 1976c). A review has been published of the comparative subcellular behavior of five actinides in the beagle liver (Bruenger, 1976b). Subcellular distribution of ${ }^{241} \mathrm{Am}$ in the liver of neonatal beagles differed significantly from that in the liver of young adults (Stevens, W., 1977).

The deposition patterns and toxicity of plutonium and americium in liver were reviewed by Lindenbaum and Rosenthal, comparing data from beagles and other experimental animals (Lindenbaum, 1972).

4.2.2.6 SPLEEN: Plutonium deposited in the spleen following inhalation of ${ }^{239} \mathrm{PuO}_{2}$ was present as discrete particles, suggesting transport from lung to spleen in particulate form (Guilmette, 1987).

4.2.2.7 KIDNEY: Deposition of ${ }^{241} \mathrm{Am}$ (Lloyd, $R ., 1970$ ) and ${ }^{249} \mathrm{Cf}$ (Taylor, G., 1972 . in the beagle kidney was shown to occur principally in the glomeruli and renal papillae.

4.2.2.8 OVARY: Moderate localization of ${ }^{249} \mathrm{Cf}$ was observed, adjacent to the ovum, in the region of the zona pellucida of larger and more mature Graafian follicles; selective localization in the smaller follicles did not occur (Taylor, G., 1972).

4.2.2.9 TESTES: Investigations employing light- and electron-microscopic autoradiography described the distribution and localization of ${ }^{241} \mathrm{Pu}$ in the testes of the beagle, rat, and guinea pig; in all species the plutonium was localized in the lysosomal system of macrophages of the interstitial tissue, and little was evident in seminiferous tubules (Miller, S., 1982, 1985). Together with morphometric measurements in aged, adult, and immature humans, these data indicate that the radiation dose to the spermatogonia of man is less than would be predicted assuming a uniform distribution of plutonium (Brooks, 1979).

4.2.2.10 EYE: Several papers have been published on the microscopic distribution of radium, thorium, and plutonium within the beagle eye (Fisher, G., 1976; Stover, 1962; Taylor, G., 1962b, 1964b, 1972d). Radium is concentrated in the iridiocytes of the tapetum and in the melanocytes of the ocular vascular tunic (Taylor, G., 1964b); concentrations as high as 66 times the average concentration in the eye were measured in cells of the tapetum lucidum (Fisher, G., 1976).

4.2.2.11 THYROID: Deposition of ${ }^{241} \mathrm{Am}$ in the beagle thyroid was shown to occur principally in the interfollicular areas, with lesser amounts retained in the tunica media, and in the intima of some of the arterioles; the deposition pattern did 
not change over a period of 448 days (Taylor, G., 1969c). Californium in the thyroid was also present, almost exclusively, in the interfollicular connective tissue (Taylor, G., 1972). Subcellular fractionation showed that americium is selectively bound to connective tissue elements of the thyroid (Stevens, W., 1969). 



\section{Contents}

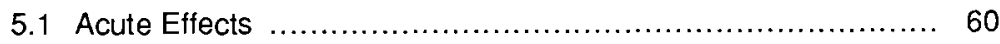

5.1.1 Hematopoietic Syndrome ............................. 60

5.1 .2 Pulmonary Injury ...................................... 61

5.1 .3 Gastrointestinal Injury ................................. 63

5.1 .4 Hepatic Injury ........................................ 63

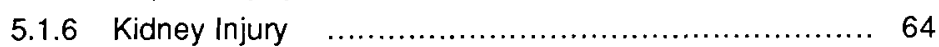

5.2 Longer-Term Nonneoplastic Effects ............................. 64

5.2.1 Hematologic Effects .................................... 64

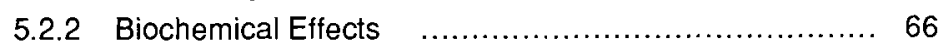

5.2.3 Nonneoplastic Effects in Calcified Tissues ............. 68

5.2.4 Nonneoplastic Effects in Lymph Nodes ................ 70

5.2.5 Nonneoplastic Effects in Thyroid ...................... 71

5.2.6 Nonneoplastic Effects in Ovaries ....................... 71

5.2.7 Nonneoplastic Effects in Eyes ........................... 71

5.3 Neoplastic Effects ......................................... 71

5.3.1 Myeloproliferative Disorders ........................... 72

5.3.2 Bone Tumors .......................................... 73

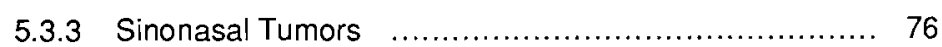

5.3.4 Gingival Tumors ...................................... 77

5.3 .5 Lung Tumors ........................................... 77

5.3.6 Pulmonary Lymph Node Tumors ....................... 79

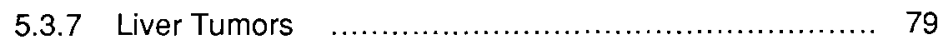

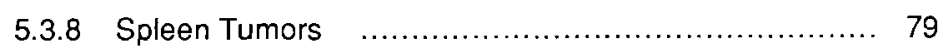

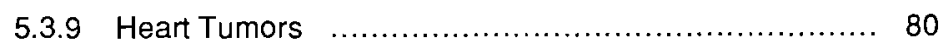

5.3.10 Eye Tumors .......................................... 80

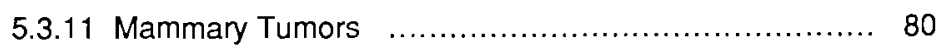

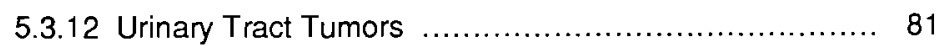

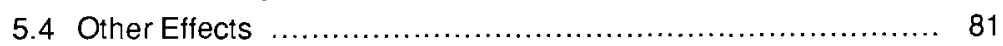

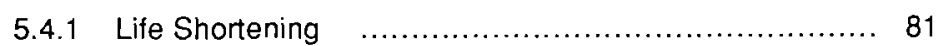

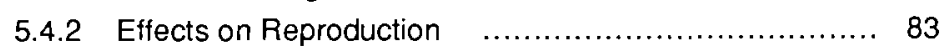

5.4.3 Psychological Evaluations $\quad \ldots \ldots \ldots \ldots \ldots \ldots \ldots \ldots \ldots . \ldots . \ldots . \ldots . \ldots$

Biological effects observed in the life-span studies will be considered in 3 major categories: those resulting in early death or debilitation; delayed, nonneoplastic effects, such as may be detected by hematologic, biochemical or morphologic studies; and long-delayed neoplastic effects, sometimes, but not always, 
resulting in shortened life span. A final group of effects will be considered that do not fit neatly into these categories. Life shortening, per se, will be briefly considered in this final category. Data on life shortening and on specific causes of death also appear in the experiment summaries of Chapter 8.

\subsection{ACUTE EFFECTS}

The description of acute effects of radiation was not a primary purpose of most of the life-span dog experiments. Information on such effects was available from other sources, including many observations on man. However, most of the life-span dog experiments included groups that received exposures calculated to produce early effects. This was due in some cases to uncertainty regarding acutely damaging levels of exposure, but more generally reflected a desire to include, in a single experiment, the complete spectrum of potential biological effects. High-exposure groups usually contained fewer animals. It will be most instructive to consider these acute effects in relation to the type of syndrome encountered, which will, of course, reflect the early distribution of radiation dose.

\subsubsection{HEMATOPOIETIC SYNDROME}

The classic hematopoietic syndrome of bone marrow irradiation has been described in dogs exposed to $\mathrm{x}$ - (Andersen, 1957) or gamma-irradiation (Fritz, 1970), and in dogs exposed to whole-body irradiation by injection of ${ }^{137} \mathrm{CsCl}$ (Redman, 1972). The Argonne continuous gamma-irradiation experiments were designed particularly to study these effects. In life-span-irradiated animals, early deaths were due to septicemia associated with granulocytopenia, at doses of 12.8 and $26.3 \mathrm{rad} /$ day; later deaths were due to anemia, at doses of 1.9 to $12.8 \mathrm{rad} /$ day; longer delayed myeloproliferative disorders, mostly myelogenous leukemias, occurred at dose rates of 0.8 to $12.8 \mathrm{rad} /$ day; only a few dogs died of delayed radiation-induced effects unrelated to the hematopoietic system (Fritz, 1973, 1978, 1986; Norris, 1972, 1976). Hematopoietic effects were less pronounced when the continuous irradiation was terminated at predetermined total doses (Fritz, 1986).

High dose levels of many bone-seeking radionuclides have also resulted in early death from irradiation of the bone marrow. The usual clinical signs include a progressive severe thrombocytopenia and neutropenia; anorexia; petechial hemorrhages of mucous membranes and skin; pyrexia; and hemorrhagic diarrhea. Pathological findings include hemorrhage in the gastrointestinal tract, epicardium, subcutaneous tissues, and lungs, and marked destruction of marrow elements (Gillett, 1987; McClellan, 1972c). Such effects have been described following inhalation of soluble forms of ${ }^{90} \mathrm{Sr}$ (Gillett, 1987; McClellan, 1972c; 
1973), ${ }^{144} \mathrm{Ce}$ (Benjamin, 1973; Boecker, 1984), ${ }^{91} \mathrm{Y}$ (Scott, 1980); and intravenous injection of ${ }^{90} \mathrm{Sr}$ (Dougherty, J., 1972b), ${ }^{137} \mathrm{Cs}$ (NCRP, 1977; Redman, 1972), and ${ }^{144} \mathrm{Ce}$ (Fritz, 1970). An $\mathrm{LD}_{50}$ of $256 \mu \mathrm{Ci} / \mathrm{kg}$ was estimated for hematopoietic death following a single inhalation of ${ }^{90} \mathrm{SrCl}_{2}$ (McClellan, 1972c; Scott, 1980), and of 150 $\mu \mathrm{Ci} / \mathrm{kg}$ for a single injection (Finkel, 1960, 1972). Acute hematopoietic death has also been reported following injection or inhalation of alpha emitters.

\subsubsection{PULMONARY INJURY}

Acute pulmonary injury, usually described as radiation pneumonitis and pulmonary fibrosis, was a commonly observed cause of early death in dogs that inhaled relatively high levels of radionuclides that were retained for appreciable periods in the lung. Such early deaths were observed with the beta emitters, ${ }^{144} \mathrm{Ce},{ }^{90} \mathrm{Y},{ }^{91} \mathrm{Y}$, and ${ }^{90} \mathrm{Sr}$ when inhaled as FAP (McClellan, 1970, 1974; Slauson, 1976, 1977), less frequently when inhaled in soluble forms (Benjamin, 1973); and were extensively studied in terms of pulmonary histopathology (Hobbs, 1972), in terms of the effects on pulmonary vasculature (Slauson, 1977), of the physiology and pathology of pulmonary lipids (Pfleger, 1975), of the effects on pulmonary connective tissue (Pickrell, 1975, 1978), effects on microbiological flora of the lung and other organs (Hobbs, 1972), effects on cardiopulmonary function and histopathology (Hobbs, 1972; Mauderly, 1973, 1980, 1980b), effects on peripheral lymphocytes and lymphocyte function (Benjamin, 1975, 1976, 1978; Jones, R., 1976), and morphologic effects in pulmonary lymph nodes (Hahn, 1976). These observations of "nonstochastic" effects in lung have been recently reviewed (Hahn, 1988).

Similar acute effects in the lung were seen in dogs inhaling ${ }^{144} \mathrm{Ce}$ FAP as juveniles or as aged dogs (Pickrell, 1978); unlike the effects seen in adults, the juvenile radiation pneumonitis deaths were sometimes complicated by congestive heart failure. Deaths from radiation pneumonitis were much less frequent, and were long delayed, when ${ }^{144} \mathrm{Ce}$ FAP was inhaled as a series of individually smaller doses (Boecker, 1980). Pulmonary deaths were also less frequent when ${ }^{144} \mathrm{Ce}$ was inhaled as the more soluble chloride (Boecker, 1984).

Acute deaths from pulmonary damage following inhalation of ${ }^{239} \mathrm{PuO}_{2}$ have been described in several reports (Bair, 1962; Clarke, 1964; Dagle, 1976; Park, J., 1962, 1986; West, 1964). Similar observations have been reported following inhalation of ${ }^{238} \mathrm{PuO}_{2}$ or ${ }^{239} \mathrm{Pu}\left(\mathrm{NO}_{3}\right)_{4}$ (Dagle, 1979, 1986; Hahn, 1981; Muggenburg, 1980; Park, J., 1969, 1972, 1986). A practical threshold for radiation pneumonitis of about $2300 \mathrm{rad}$ was estimated for dogs inhaling ${ }^{239} \mathrm{PuO}_{2}$ (Fisher, D., 1986). 
With ${ }^{239} \mathrm{PuO}_{2}$ depositions of $0.1 \mu \mathrm{Ci} / \mathrm{g}$ lung, or more, acute respiratory death was usually noted within 1 to 12 months postinhalation, with symptoms of pulmonary edema, severe vascular damage, fibrinous accumulations in the bronchioles and alveoli, and pulmonary fibrosis; with depositions in the range of $0.05 \mu \mathrm{Ci} / \mathrm{g}$ lung, symptoms occurred from 1 to 5 years postinhalation and death was more likely to result from lung cancer (Howard, 1970). A markedly increased respiratory rate was a characteristic feature of the acute effect of plutonium inhalation (West, 1964).

Preliminary data from studies comparing the effect of different sizes of inhaled ${ }^{239} \mathrm{PuO}_{2}$ particles suggest that the more homogeneous radiation dose from smaller particles results in earlier deaths from radiation pneumonitis and pulmonary fibrosis than does the more heterogeneous dose from larger particles (Diel, 1983b). As compared to dogs exposed as young adults, those exposed at about 3 months of age show a lower incidence of radiation pneumonitis, while those exposed at 8 to 10 years of age show a higher incidence, when exposed at comparable radiation doses to the lung (Guilmette, 1988b).

A detailed comparison of pulmonary function in dogs that inhaled ${ }^{238} \mathrm{PuO}_{2}$ or ${ }^{144} \mathrm{Ce}$ FAP, showed a different pattern of effects, with death at comparable dose levels being substantially later in the plutonium dogs, probably because of their much less homogeneous pattern of dose distribution within the lung (Mauderly, 1980). Detailed studies of pulmonary function in dogs surviving exposure to ${ }^{239} \mathrm{PuO}_{2}$ aerosols suggest that restrictive lung disease may occur at long times (1 to 5 years) after initial exposure; cardiac function in these same animals was not affected (Muggenburg, 1988).

The pattern of early death following inhalation of ${ }^{239} \mathrm{PuO}_{2}$ in the dog has been compared to that observed in studies with baboons; considering only early mortality, the baboon and beagle were judged to show a similar sensitivity (Bair, 1980).

Pulmonary fibrosis was also observed in dogs inhaling radon and daughters with uranium-ore dust; the effect was attributed to radiation from the radon and daughters, rather than to the ore dust, because of the correspondence of the dose-effect relationship with that seen in plutonium exposures where no ore dust was present (Cross, 1986).

Data on early death from radiation pneumonitis have been subjected to extensive dose-response modeling (Raabe, 1979; Scott, 1979, 1980, 1980b, 1980c), and applied to the prediction of potential effects in acutely exposed humans (McClellan, 1982). Beta emitters with short half-lives, and higher initial dose rates, were 
more effective, per rad, in producing early death from radiation penumonitis and pulmonary fibrosis than beta emitters producing lower initial dose rates (Hahn, 1983b).

\subsubsection{GASTROINTESTINAL INJURY}

Inhaled, insoluble materials are largely cleared through the gastrointestinal tract and may result in radiation damage in those regions. This route of exposure was specifically targeted in a supporting study that involved inhalation of ${ }^{90} \mathrm{Y}$ FAP; the short half-life of the ${ }^{90} \mathrm{Y}$, and the large-sized particles employed, maximized exposure of the GI tract relative to the lung and other organs (Hahn, 1975). Radiation doses as high as 5700 rad were measured by thermoluminescent dosimeters in the colon, and focal colonic lesions were observed, but nasal dermatitis and radiation pneumonitis were more severe and life threatening than the gastrointestinal injury.

\subsubsection{HEPATIC INJURY}

Liver degeneration was noted as a frequent cause of early death in the Argonne ${ }^{137} \mathrm{Cs}$ injection experiment (NCRP, 1977). Acute necrosis, fibrosis and atrophy were also observed at somewhat lower dose levels in the ITRI ${ }^{137} \mathrm{Cs}$ injection experiment (Muggenburg, 1986). Liver lesions consisting of alternating areas of degeneration, atrophy, and regeneration were consistent postmortem findings in dogs injected with ${ }^{144} \mathrm{Ce}$ (Fritz, 1970). Hepatic injury was noted as an early cause of death following inhalation of soluble forms of ${ }^{144} \mathrm{Ce}$ (Benjamin, 1973; Boecker, 1984). These observations of "nonstochastic" effects in liver have been recently reviewed (Hahn, 1988).

Liver lesions were a prominent finding in dogs injected with plutonium (Cochran, 1962; Taylor, G., 1971, 1972b, 1986), and to an even greater extent in the case of americium (Lloyd, R., 1970, 1972b; Taylor, G., 1972b), where liver degeneration was listed as the cause of death for several high-dose animals. Even at relatively low dose levels, extensive nodular hyperplasia was a frequent observation, occurring as early as 1 year postinjection and eventually extending to as much as $85 \%$ of the liver volume (Cochran, 1962). Liver changes were less evident in beagles injected as juveniles (Bruenger, 1980; Jee, 1976b).

Passive congestion with central lobular degeneration was observed in the livers of dogs that died early following inhalation of ${ }^{239} \mathrm{PuO}_{2}($ Park, J., 1964) and ${ }^{238} \mathrm{PuO}_{2}$ (Park, J., 1969); this was thought to be related to circulatory changes caused by severe lung pathology (Park, J., 1972). Hepatic nodular hyperplasia was observed in dogs that inhaled ${ }^{239} \mathrm{Pu}\left(\mathrm{NO}_{3}\right)_{4}$ or ${ }^{238} \mathrm{PuO}_{2}$, but was seldom 
observed following inhalation of ${ }^{239} \mathrm{PuO}_{2}$, and was not a cause of death (Dagle, 1986; Park, J., 1986).

\subsubsection{KIDNEY INJURY}

Histologically evident kidney damage was a common observation following higher levels of external gamma-irradiation (Fritz, 1970), and following internal deposition of ${ }^{144} \mathrm{Ce}$ (Fritz, 1970). In the Davis fractionated $\mathrm{x}$-ray exposure experiment, progressive intercapillary glomerulosclerosis was followed by light-, electron-, and fluorescence-microscopy in aging beagles; those exposed to as little as $100 \mathrm{R}$, in 50-R fractions, showed earlier and more severe lesions than were observed in control dogs (Guttman, 1968, 1970).

Studies in support of the Colorado State perinatal radiation program investigated effects on the developing kidney from ${ }^{60} \mathrm{Co}$ gamma radiation delivered at 4 and 30 days of age. The progressive glomerulosclerosis and renal failure associated with perinatal renal irradiation was shown to result from direct radiation damage to deep cortical nephrons and compensatory functional changes occurring in response to loss of renal mass (Peneyra, 1985). Early effects of a 1500-rad dose of $6 \mathrm{MeV}$ electrons to the externalized kidneys of 6 dogs were reported from a supporting study at Colorado State University (Hoopes, 1985).

Kidney degeneration was listed as cause of death for several high-dose ${ }^{241} \mathrm{Am}$ injected dogs (Lloyd, R., 1972b). Kidney degeneration occurring in high-dose ${ }^{228} \mathrm{Th}$-injected dogs was attributed to both ${ }^{228} \mathrm{Th}$ and its daughter ${ }^{224} \mathrm{Ra}$, and correlates with increased kidney disease in ${ }^{224}$ Ra-injected humans (Spiess, 1978).

\subsection{LONGER-TERM NONNEOPLASTIC EFFECTS}

\subsubsection{HEMATOLOGIC EFFECTS}

Changes in the peripheral blood picture are an easily measured and sensitive indicator of exposure to radiation. The normal beagle hemogram has been described in several publications (Andersen, 1970g; Dougherty, J., 1955, 1965).

Hematological responses observed in the Argonne continuous gamma-irradiation studies have been described in detail (Fritz, 1973, 1982, 1986; Norris, 1972, 1976; Seed, 1977, 1978, 1980, 1982b; Tolle, 1977, 1979, 1979b, 1982, 1983, 1983b). There is a dose-related depression in numbers of all types of circulating blood cells, effects on leukocytes and thrombocytes occur before those on erythrocytes. Significant changes in bone-marrow function and structure have been shown to parallel the peripheral-blood responses (Seed, 1977, 1978, 1982, 1982b, 1983). 
The essentially total-body exposure from injected ${ }^{137} \mathrm{Cs}$, which resulted in early hematopoietic deaths at high dose levels, caused a dose-related depression in lymphocytes, granulocytes and platelets in all exposure groups (Redman, 1972).

Female pups, exposed to ${ }^{60} \mathrm{Co}$ gamma radiation in-utero, throughout gestation, at doses as high as $5 \mathrm{R}$ per day, exhibited normal hematopoiesis and peripheral blood values within the normal range, despite their subsequent inability to bear young (Sanderson, 1978; Seed, 1977b).

The hematological response, as a function of time postexposure, has been described in a series of papers comparing effects of ${ }^{90} \mathrm{Sr},{ }^{226} \mathrm{Ra},{ }^{228} \mathrm{Ra},{ }^{228} \mathrm{Th}$, and ${ }^{239} \mathrm{Pu}$ in the Utah injection experiments (Dougherty, J., 1955, 1962, 1969, 1970, 1971, 1972, 1972b; Parkinson, 1956, 1958, 1959); as has the hematological response to chronically fed ${ }^{90} \mathrm{Sr}$ (Bustad, 1969, 1969b; Della Rosa, 1969; Dungworth, 1969; Goldman, 1972c) and multiply injected ${ }^{226} \mathrm{Ra}$ (Bustad, 1969b; Goldman, 1969b; Rosenblatt, 1967) in the Davis experiments, and for injected ${ }^{144} \mathrm{Ce}$ (Fritz, 1970) in the Argonne experiment. Detailed hematologic data have also been reported for dogs exposed to a single inhalation of ${ }^{90} \mathrm{SrCl}_{2}$ (Gillett, 1987).

Significant changes in red cell values were not seen with ${ }^{90} \mathrm{Sr}$, and for the other radionuclides in the Utah injection experiments, only at the highest exposure levels (Parkinson, 1958, 1959). The response of white blood cells was similar for all radionuclides, with minimal values of all cell types except lymphocytes observed within 2 to 4 weeks postinjection; maximal lymphocyte depression occurred after about 1 year. In the chronically fed ${ }^{90} \mathrm{Sr}$ dogs of the Davis experiment the severity of the neutropenia increased with time during exposure, but there was a tendency for recovery when ${ }^{90} \mathrm{Sr}$ feeding was terminated; recovery from neutropenia seemed to correlate with a return of the depressed myeloid/erythroid ratio in bone marrow to near normal levels (Goldman 1972c). Only in the chronically fed Davis dogs was there observed a significantly increased incidence of hematopoietic neoplasia (Bustad, 1969; Dungworth, 1968, 1969; Goldman, 1969b; Wilson, 1972).

Hematological relative biological effectiveness (RBE) values (defined as the average dose rate to bone from ${ }^{226} \mathrm{Ra}$ required to produce a $50 \%$ depression in blood cells, divided by the average dose rate from the radionuclide in question required to produce the same $50 \%$ depression) were calculated as $\left({ }^{226} \mathrm{Ra}=1\right):{ }^{228} \mathrm{Th},-4$; ${ }^{239} \mathrm{Pu}, \sim 4{ }^{228} \mathrm{Ra}, \sim 2$; and ${ }^{90} \mathrm{Sr}, \sim 0.5$ (Dougherty, J., 1970, 1971). It is of interest that these hematological RBEs are in reasonable agreement with early estimates of RBEs based on osteosarcoma as the endpoint (Mays, 1969). 
Hematologic data from juvenile dogs injected with ${ }^{239} \mathrm{Pu}$ indicated similar effects, but of a lesser magnitude, than those observed in young-adult beagles injected with similar radionuclide concentrations (Bruenger, 1980).

Hematologic effects have also been studied following inhalation of beta or alpha emitters. A dose-related lymphopenia was observed at early times following inhalation of ${ }^{144} \mathrm{Ce},{ }^{90} \mathrm{Sr},{ }^{90} \mathrm{Y}$, or ${ }^{91} \mathrm{Y}$ as FAP (Benjamin, 1975 ; Hobbs, 1972 ; Jones, R., 1976); the magnitude of lymphocyte depression was related to the initial dose rate to the lung, but recovery occurred unless radiation exposure was maintained. Anemia was a common observation during the period shortly before death of dogs dying with lung tumors following inhalation of ${ }^{144} \mathrm{Ce}$ as FAP $(H a h n, 1973 b)$. This anemia was not a direct result of irradiation but resulted from hemorrhage related to the neoplasia.

A dose-related lymphopenia was the earliest and most sensitive clinical indication of toxicity from inhaled ${ }^{239} \mathrm{PuO}_{2}$; effects on blood cells other than lymphocytes were minimal (Bair, 1961, 1962; Guilmette, 1984b; Park, J., 1962, 1964, 1966, 1971, 1972, 1975, 1986; Ragan, 1976, 1986; West, 1964). Inhalation of ${ }^{238} \mathrm{PuO}_{2}$ caused an even more pronounced effect on lymphocytes, and at high dose levels, a moderate neutropenia (Dagle, 1979; Muggenburg, 1980; Park, J., 1969, 1975, 1976, 1986; Ragan, 1986). After ${ }^{239} \mathrm{Pu}\left(\mathrm{NO}_{3}\right)_{4}$ inhalation, a significant lymphopenia was seen only at relatively high dose levels (Dagle, 1979; Park, J., 1986; Ragan, 1986). Recovery from lymphocyte depression occurred in all plutonium-exposed groups, complete recovery occurring in surviving ${ }^{239} \mathrm{Pu}\left(\mathrm{NO}_{3}\right)_{4}{ }^{-}$ exposed animals. Observations are consistent with the conclusion that the observed effects are due, primarily, to irradiation of lymphocytes during transit through pulmonary lymph nodes (Dagle, 1979; Ragan, 1986).

Lymphocytes from dogs suffering from a severe lymphopenia following inhalation of ${ }^{90} \mathrm{Sr}$ or ${ }^{144} \mathrm{Ce}$ as FAP, showed an impaired ability to respond to plant mitogens in-vitro; this effect was not observed for lymphocytes from dogs that had recovered from a severe lymphopenia following inhalation of the short-lived ${ }^{90} \mathrm{Y}$ (Benjamin, 1975, 1975c, 1976, 1978). These findings were considered to be evidence of immune suppression that might be involved in subsequent neoplasia.

\subsubsection{BIOCHEMICAL EFFECTS}

Normal blood chemistry values in the beagle dog, as influenced by such factors as age, sex, and family line, have been described in several publications (Goldthorpe, 1962; Kaspar, 1977; McKelvie, 1970b; Pickrell, 1974), as have the levels of various metal ions in the liver (Keen, 1981). 
Serum lactic acid dehydrogenase (LDH) was depressed during the early period following injection of relatively high levels of ${ }^{137} \mathrm{CsCl}$ (Redman, 1972). Elevated LDH levels were reported at longer intervals following injection of a number of radionuclides, but the effect was not considered a consistent indicator of pathologic states in irradiated dogs (Ruhmann, 1965).

Biochemical changes were studied in the blood of dogs from the Utah injection studies with ${ }^{90} \mathrm{Sr},{ }^{226} \mathrm{Ra},{ }^{228} \mathrm{Ra},{ }^{228} \mathrm{Th}$, and ${ }^{239} \mathrm{Pu}$ (Berliner, 1969; Goldthorpe, 1962; Ruhmann, 1965), and from the Davis ${ }^{90} \mathrm{Sr}$ ingestion experiment (McKelvie, 1968; Bustad, 1969). A dose-related increase in serum alkaline phosphatase was observed for all except ${ }^{90} \mathrm{Sr}$ animals, and was also shown to correlate with osteosarcoma incidence (Goldthorpe, 1962; Stevens, W., 1967). Elevated lysozyme concentrations were seen in dogs with ${ }^{90} \mathrm{Sr}$-induced myelomonocytic leukemia and also in the recipients of transplants of these tumors (Shifrine, 1973d).

Supporting studies in 36 beagle pups raised on a diet containing $10 \mu \mathrm{Ci}{ }^{90} \mathrm{Sr} / \mathrm{g} \mathrm{Ca}$ showed elevation in bilirubin, total protein, calcium, inorganic phosphorus, alkaline phosphatase, blood urea nitrogen, serum glutamic oxalacetic transaminase (SGOT) and alpha-2 globulin, and a depression in serum glutamic pyruvic transaminase (SGPT), by 4 months of age; the effects began to return to normal by 5 months of age despite continued ${ }^{90} \mathrm{Sr}$ ingestion (McKelvie, 1968).

Levels of SGPT and SGOT were markedly increased in ${ }^{239} \mathrm{Pu}$-injected animals and were considered to reflect liver damage (Stevens, W., 1964, 1967; Taylor, G., 1971). Dose-dependent increases in serum inorganic phosphorus were observed in all except the ${ }^{90} \mathrm{Sr}$-injected animals of the early Utah studies. For all of these radionuclides, increases in serum globulins were accompanied by decreases in serum albumin.

Studies employing minced adrenal tissue from dogs that had been injected with ${ }^{90} \mathrm{Sr},{ }^{226} \mathrm{Ra},{ }^{228} \mathrm{Ra},{ }^{228} \mathrm{Th}$, or ${ }^{239} \mathrm{Pu}$, showed effects on corticosteroid biosynthesis (Berliner, 1962); similar studies with testicular tissue showed significant reductions in androgen biosynthesis (Ellis, 1967). In another study, no statistically significant difference in urinary steroid levels were noted between control dogs and dogs burdened with ${ }^{90} \mathrm{Sr}$ (Mitchell, 1970).

A comparison of effects from injected ${ }^{239} \mathrm{Pu}$ and ${ }^{241} \mathrm{Am}$ showed ${ }^{241} \mathrm{Am}$ to be more effective in causing early elevation of SGPT, SGOT and serum alkaline phosphatase. These effects, attributed to liver damage from the deposited actinide, were generally more pronounced in males than in females (Nabors, 1972). Cortisol metabolism was shown to be significantly elevated in the skin of beagles bearing ${ }^{241} \mathrm{Am}$ (Nabors, 1975). 
A variety of serum chemistry abnormalities were reported in dogs dying with lung tumors following inhalation of ${ }^{144} \mathrm{Ce}$ as FAP (Hahn, 1973b). Effects on pulmonary lipids (Pfleger, 1975) and on collagen constituents (Pickrell, 1975, 1978 ) were studied following inhalation of the beta emitters ${ }^{144} \mathrm{Ce},{ }^{90} \mathrm{Sr},{ }^{90} \mathrm{Y}$, or ${ }^{91} \mathrm{Y}$. Dose-related effects on the mucopolysaccharides of cartilage were measured in beagles multiply injected with ${ }^{226} \mathrm{Ra}$ ( $T_{\text {sai, }} 1970$ ).

Blood chemistry data were reported following inhalation of ${ }^{239} \mathrm{PuO}_{2}$, with values normal as compared to controls (Park, J., 1962). Evidence of elevated SGPT and SGOT levels were reported in dogs that inhaled ${ }^{238} \mathrm{PuO}_{2}$ or ${ }^{239} \mathrm{Pu}\left(\mathrm{NO}_{3}\right)_{4}($ Park, J., 1976, 1986). A dose-related elevation of alanine aminotransferase levels, indicative of liver damage, was observed at long intervals following inhalation of ${ }^{238} \mathrm{PuO}_{2}$ (Gillett, in press).

Radioimmunoassay techniques revealed a significant decrease in primary antibody response in dogs that inhaled ${ }^{239} \mathrm{PuO}_{2}$ (Dagle, 1979).

Increased urinary taurine levels observed as early as 3 months following inhalation of ${ }^{239} \mathrm{PuO}_{2}$ were shown to correlate with the onset of lymphopenia; a similar inverse correlation between urinary taurine and circulating lymphocytes was noted in humans undergoing treatment for chronic lymphocytic leukemia by extracorporeal irradiation (Dilley, 1972).

\subsubsection{NONNEOPLASTIC EFFECTS IN CALCIFIED TISSUES}

A wide variety of nonneoplastic effects have been observed in the bones and teeth of life-span beagles with depositions of bone-seeking radionuclides.

Morphologic effects in bone were demonstrated both grossly and microradiographically in dogs from the higher dose levels of the Davis ${ }^{90} \mathrm{Sr}$ feeding experiment (Andersen, 1962; Della Rosa, 1969). The bones exhibited a characteristic "flinty" condition related to decreased endosteal resorption and remodeling (Goldman, 1969). Effects were more varied, more prominent, and observed at lower radiation dose levels in the ${ }^{226} \mathrm{Ra}$ multiple injection study, especially in animals whose injections commenced at 2 or 4 months of age (Goldman, 1969b; Momeni, 1976b, 1976d, 1976e; Morgan, J., 1982, 1983; Pool, 1983). These effects included pathological fractures and a variety of dental lesions. Preliminary studies on the effects of ${ }^{226} \mathrm{Ra}$ on the mucopolysaccharides of cartilage were also reported (Tsai, 1970).

Comparison of these effects with those observed in humans exposed to ${ }^{226} \mathrm{Ra}$ indicates that the beagle affords a useful model for understanding the human 
pathology (Morgan, $J ., 1982,1983$ ). From a comprehensive study of these effects it was concluded that a beagle surviving to 16 years of age would not show detectable skeletal change if injected at about 1 year of age with $0.053 \mu \mathrm{Ci}$, or less, of ${ }^{226} \mathrm{Ra}$ (Momeni, 1976e).

Similar pathologic effects of bone-seeking radionuclides injected in Utah experiments have been studied radiographically (Christensen, 1962; Jee, 1961, 1962b; Stover, 1963; Taylor, G., 1976), in light-(Jee, 1960b, 1961, 1962b, 1962c, 1969, 1971, 1972), in electron-microscopic studies (Park, H., 1972), and in terms of changes in gross chemical composition (Stover, 1963). Effects of radiation on bone remodeling processes have been inferred from dose-dependent differences in the redistribution of ${ }^{239} \mathrm{Pu}$ and ${ }^{226} \mathrm{Ra}$ as observed in autoradiographic studies (Arnold, 1959, 1962). From the above observations there has developed a "vascular theory of radiation injury to bone," which interprets a variety of radiation effects in terms of the efficiency of diffusion of nutrients between vessels and bone (Christensen, 1962; Jee, 1960b, 1960c, 1961, 1962b, 1969, 1971).

Pathologic fractures have been a frequently observed gross manifestation of nonneoplastic effects at relatively high dose levels of bone-seeking radionuclides (Christensen, 1962; Gillett, 1987b; Jee, 1961; Momeni, 1976e; Morgan, J., 1982, 1983; Rehfeld, 1962; Taylor, G., 1962, 1966, 1966b, 1971, 1976). Effects of daily ${ }^{90} \mathrm{Sr}$ ingestion were less severe than those from alpha emitters, being mostly evidenced by an increased hardness and a narrowing of the marrow cavity (Bustad, $1969 \mathrm{~b}$ ). The highest incidence, and slowest healing, of pathologic fractures were seen in dogs injected with ${ }^{228} \mathrm{Ra}$; fractures were particularly numerous in the rib cage, a region notably free of tumors (Taylor, G., 1966). As compared to young adults injected with the same high concentration of ${ }^{239} \mathrm{Pu}$, juvenile beagles exhibited a much lower incidence of pathologic fractures and more rapid healing of these fractures (Bruenger, 1980). Tensile stress tests on tibias from radionuclide-injected dogs from the Utah studies could not account for fractures, suggesting that these fractures were due to localized faults (Taysum, 1962).

Radiation osteodystrophy (osteodysplasia) in which the normal process of bone tissue replacement appears to be defective, characterized by peritrabecular fibrosis and considered a possible precursor of fractures and neoplasia, has been reported in dogs exposed to relatively high levels of ${ }^{238} \mathrm{PuO}_{2}$ or ${ }^{239} \mathrm{Pu}\left(\mathrm{NO}_{3}\right)_{4}$ (Dagle, 1979, 1985, 1986; Hahn, 1981; Park, J., 1986), ${ }^{241} \mathrm{AmO}_{2}$ (Gillett, 1985), ${ }^{226} \mathrm{Ra}$ (Morgan, J., 1982; Pool, 1973, 1983), or ${ }^{90} \mathrm{Sr}$ (Gillett, 1987b; Nilsson, 1985;

Pool, 1973). Material available from 42 dogs of the Davis ${ }^{226} \mathrm{Ra}$ experiment, whose limbs were amputated because of the development of painful bone lesions, have been particularly valuable in the study of this syndrome (Morgan, J., 1982). 
The very much lower incidence of radiation osteodystrophy in dogs exposed to a single inhalation of ${ }^{90} \mathrm{Sr}$ as compared to those exposed by chronic ingestion of ${ }^{90} \mathrm{Sr}$, suggests that this disturbance in bone remodeling is not a necessary precursor to osteosarcoma development (Gillett, 1987b). These observations of "nonstochastic" effects in bone have been recently reviewed (Hahn, 1988).

Turbinate osteolysis was a dose-related consequence of intravenously injected ${ }^{239} \mathrm{Pu},{ }^{226} \mathrm{Ra},{ }^{228} \mathrm{Ra}$, and ${ }^{228} \mathrm{Th}$ (Taylor, G., 1971). Atrophy of the maxilloturbinates was associated with fibrous dysplasia of the turbinate bones in dogs that inhaled ${ }^{238} \mathrm{PuO}_{2}$ (Hahn, 1981). A trend toward higher than usual incidence of spondylosis deformans is seen in dogs inhaling ${ }^{238} \mathrm{PuO}_{2}$, and hypertrophic osteoarthropathy has been observed secondary to plutonium-induced lung tumors (Dagle, 1985).

Histopathologic effects reported from the deposition of ${ }^{239} \mathrm{Pu}$ in teeth include the formation of secondary dentine, disturbance in cementum formation, resorption of cementum and dentine with metaplastic bone formation, damage to periodontal membrane, ankylosis of teeth and loss of teeth (Jee, 1960). Relatively high doses of intravenously injected ${ }^{224} \mathrm{Ra},{ }^{226} \mathrm{Ra}$, or ${ }^{239} \mathrm{Pu}$ did not have an effect on the narrowing of the dental pulp cavity as a function of age (Taylor, G., 1969b). Pathological changes in the mandible have been an early and consistent effect of alpha-emitting radionuclides (Christensen, 1962; Taylor, G., 1976). Gingival ulceration was observed as a consequence of ${ }^{226} \mathrm{Ra},{ }^{228} \mathrm{Ra}$, or ${ }^{228} \mathrm{Th}$ deposition on tooth surfaces (Taylor, G., 1964). Similar but lesser changes were induced by ${ }^{239} \mathrm{Pu}$, but gingival ulceration did not occur (Taylor G., 1976). Tooth loss was an ultimate consequence of these pathological changes (Taylor, G., 1971, 1976).

\subsubsection{NONNEOPLASTIC EFFECTS IN LYMPH NODES}

The high concentration of inhaled, insoluble radionuclides that typically accumulate in pulmonary lymph nodes, and the low incidence of effects of this exposure, have been subjects of particular interest in many life-span experiments involving inhaled radionuclides. A review of the subject, referring to results of the beagle studies, has been published (Leeds, 1976).

The effects on tracheobronchial lymph nodes of ${ }^{144} \mathrm{Ce}$ inhaled as FAP was followed in a supporting experiment involving 30 dogs sacrificed at intervals to 2 years postexposure; histologic changes were described, including atrophy of the germinal centers and lymphocytic follicles; primary neoplasms were not observed until more than 2000 days following exposure (Hahn, 1976). 
At relatively high doses of inhaled ${ }^{239} \mathrm{PuO}_{2}$, pulmonary lymph nodes were severely affected, with the germinal centers gradually replaced by dense, sclerotic connective tissue (Clarke, 1964, 1966; Dagle, 1976b; Park, J., 1962, 1971, 1986). Similar effects were seen with inhaled ${ }^{238} \mathrm{PuO}_{2}$ (Dagle, 1979; Hahn, 1981; Park, J., 1969, 1986), and similar but less severe effects with ${ }^{239} \mathrm{Pu}^{2}\left(\mathrm{NO}_{3}\right)_{4}$ (Dagle, 1979; Park, J., 1986). Hepatic lymph nodes in the ${ }^{239} \mathrm{PuO}_{2}$ - and ${ }^{239} \mathrm{Pu}\left(\mathrm{NO}_{3}\right)_{4}$-exposed dogs showed similar but less severe effects (Dagle, 1979).

Damage to popliteal lymph nodes subsequent to injection of ${ }^{239} \mathrm{PuO}_{2}$ into the hind paws of beagles was described in studies involving light- and electron-microscopy and autoradiography (Dagle, 1975, 1976b).

\subsubsection{NONNEOPLASTIC EFFECTS IN THYROID}

Effects in the thyroids of life-span dogs injected with high-levels of ${ }^{241} \mathrm{Am}$ included a marked loss of the epithelial component and the absence of welldefined follicles (Lloyd, R., 1972b).

\subsubsection{NONNEOPLASTIC EFFECTS IN OVARIES}

Light- and electron-microscopic studies revealed severe ultrastructural abnormalities in the ovarian cortex of pups exposed in-utero, throughout gestation, to ${ }^{60} \mathrm{Co}$ gamma-ray doses of $5 \mathrm{R}$ per day. Less severe effects were noted in animals exposed at a rate of $2.5 \mathrm{R}$ per day. The 5-R-per-day animals were subsequently unable to bear young (Sanderson, 1978; Seed, 1977b).

\subsubsection{NONNEOPLASTIC EFFECTS IN EYES}

Concentration of ${ }^{226} \mathrm{Ra},{ }^{228} \mathrm{Ra},{ }^{228} \mathrm{Th}$, and less frequently ${ }^{239} \mathrm{Pu}$ in the beagle eye results in degeneration and necrosis of iridiocytes and melanocytes, producing a marked depigmentation of the choroid and iris and complete loss of the tapetum (Taylor, G., 1962b, 1964b).

\subsection{NEOPLASTIC EFFECTS}

The observation and quantification of long-delayed neoplastic effects has been a principal focus of all of the life-span beagle experiments. These observations will be considered in relation to the organ or organ systems involved, except for the case of the Davis fractionated, whole-body, $x$-ray study, in which the incidence of neoplasia was not significantly greater for irradiated dogs than for controls, but the latency period decreased as dose increased. This study involved a total of 360 dogs exposed to total doses of 100 or $300 \mathrm{R}$, with varying patterns of 
fractionation. Protraction of exposure lengthened survival in dogs that received total doses of $300 \mathrm{R}$, due solely to amelioration of incidence rates of nonmammary neoplasia (Rosenblatt, 1986).

\subsubsection{MYELOPROLIFERATIVE DISORDERS}

A variety of neoplastic manifestations of bone marrow irradiation are considered under this heading.

Myeloproliferative disorders were a major cause of death in the intermediate dose ranges of the Argonne continuous gamma-irradiation experiments. The majority of these disorders were classified as myelogenous leukemias (Fritz, 1970, 1973, 1986; Norris, 1972, 1976; Seed, 1977, 1978; Tolle, 1979b, 1982). Erythroleukemias (Tolle, 1977) and acute monocytic leukemia (Tolle, 1979) were also described. There appears to be a critical period for the development of myeloproliferative disorders, none occurring before about 400 days of continuous irradiation, and none after 2000 days, irrespective of total dose or dose rate. The incidence of myeloproliferative disorders was much lower, but still significant, when exposures were terminated at predetermined accumulated doses. Data from these studies combine to provide a rather detailed picture of the probable mechanism of myelogenous leukemia induction (Frazier, 1987, Fritz, 1985, 1986; Grahn, 1986; Seed, 1977, 1978, 1984, 1985, 1987; Tolle, 1979b, 1982). These mechanistic applications are discussed at greater length in Section 7.8.

Myeloproliferative disorders, including myelogenous leukemia, were also a prominent finding in the Davis ${ }^{90} \mathrm{Sr}$ chronic feeding experiment (Andersen, 1962; Book, 1980, 1986; Bustad, 1969; Dungworth, 1968, 1969; Goldman, 1969b, 1972c; Wilson, 1972). These effects were observed at relatively high dose levels and usually occurred much earlier than bone tumors. In the acute form these disorders appear "as granulocytic leukemia and in the chronic form resemble myelofibrosis with myeloid metaplasia; anemia, poikilocytosis, anisocytosis, hypochromasia and terminal thrombocytopenia are constant features" (Dungworth, 1969). Such myeloproliferative disorders were also observed as a significant finding in the early Argonne ${ }^{90} \mathrm{Sr}$ studies, which also involved multiple exposure of very young dogs (Finkel, 1972).

In other life-span ${ }^{90} \mathrm{Sr}$ experiments, involving exposure of young-adult beagles by single inhalation (Gillett, 1987b) or injection (Taylor, G., 1976), myeloproliferative neoplasia has not been a major cause of death; two cases of myelomonocytic leukemia following inhalation of ${ }^{9} \mathrm{SrCl}_{2}$ were considered to be radiation induced (Gillett, 1987). Atypical myeloproliferation, including at least one case of myelogenous leukemia, has been reported following injection of ${ }^{144} \mathrm{Ce}$ 
to young-adult beagles (Fritz, 1970). Two cases of myelogenous leukemia were reported as relatively early causes of death in dogs that inhaled ${ }^{144} \mathrm{CeCl}_{3}$ (Benjamin, 1973; Boecker, 1984). Leukemia has not been a significant finding in life-span studies with plutonium in the beagle, despite its deposition in bone marrow; there has been speculation, however, as to the possibility of a leukemogenic risk from plutonium in humans (Vaughan, 1976).

Based on the observation that chronic exposure of the very young animal appeared to be a critical factor in the development of myeloproliferative disorders, extensive studies were undertaken at Davis to develop a reliable "preleukemic model," in which chronic external irradiation of the beagle fetus produces a leukemic response in about 2 years (Goldman, 1986). A brief consideration of these non-life-span studies will be found in Section 6.5 .

\subsubsection{BONE TUMORS}

The increased incidence of bone tumors has been a commonly observed delayed effect of exposure to bone-seeking radionuclides. The spontaneous incidence of bone tumors in the beagle is extremely low, although perhaps higher than early estimates based on veterinary hospital surveys (Mays, 1969). An incidence of no more than $1 \%$ is suggested by the 3 or 4 observed bone tumors in control beagles in all of the life-span experiments (Wrenn, 1986b).

Bone-tumor data from the Utah ${ }^{226} \mathrm{Ra}$ injection experiment, with all animals in the experiment dead, have been briefly summarized (Wrenn, 1986b). Such findings have been described in greater detail in a number of progress reports, which also describe preliminary observations of bone tumor incidence in the ${ }^{228} \mathrm{Ra},{ }^{228} \mathrm{Th},{ }^{90} \mathrm{Sr}$, and ${ }^{239} \mathrm{Pu}$ injection experiments (Blair, 1972; Dougherty, $T$., 1962, 1962c, 1969; Gridgeman, 1971; Mays, 1969, 1972, 1972c, 1980b, 1987). Bone tumors induced by injected ${ }^{226} \mathrm{Ra}$ were mostly osteosarcomas, with a variable degree of osteogenesis. Dogs were euthanized when pain became evident, or clinical management difficult, otherwise the immediate cause of death would frequently have been metastatic disease (Wrenn, 1986b). The pattern of bonetumor induction following repeated ${ }^{226} \mathrm{Ra}$ injection, in the Davis experiment, was very similar to that reported for the Utah single-injection experiment (Goldman, 1969b).

The dose-vs-response curve for bone tumor induction by intravenously injected ${ }^{90} \mathrm{Sr}$, in contrast to that for ${ }^{226} \mathrm{Ra}$, is decidedly nonlinear (Mays, 1972c). RBEs for bone tumor induction, for ${ }^{90} \mathrm{Sr}$ relative to ${ }^{226} \mathrm{Ra}$, consequently vary with dose level. Based on average dose to bone calculated to 1 year prior to death, they range from 0.2 to $0.04\left({ }^{226} \mathrm{Ra}=1\right)($ Mays, $1980 \mathrm{~b})$. Similarly estimated RBE values 
from other Utah injection studies, as most recently reported, are: for ${ }^{228} \mathrm{Ra}, 2.0 \pm$ 0.5; for ${ }^{228} \mathrm{Th}, 8.5 \pm 2.3$; for ${ }^{239} \mathrm{Pu}, 16.6 \pm 4.5$; and for ${ }^{241} \mathrm{Am}, 5.4 \pm 1.6$ (Lloyd, $R$., 1986; Mays, 1987).

The pattern of bone tumor incidence in the Davis ${ }^{90} \mathrm{Sr}$ and ${ }^{226} \mathrm{Ra}$ chronic exposure experiments was very similar to that observed in the Utah injection experiments (Andersen, 1962; Book, 1980, 1986; Goldman, 1972c; Nilsson, 1985, 1987; Pool, 1972b, 1973, 1973b; Raabe, 1981). As in the Utah experiments, the nonlinear dose response curve for ${ }^{90} \mathrm{Sr}$ bone tumors resulted in a wide range of $\mathrm{RBE}$ values (Raabe, 1981). A somewhat similar pattern of bone tumor incidence was observed in life-span experiments involving the inhalation of ${ }^{90} \mathrm{Sr}$, as ${ }^{90} \mathrm{SrCl}_{2}$, except for a relatively higher incidence of hemangiosarcomas (Benjamin, 1975b; Gillett, 1987b; McClellan, 1973). Bone tumors were a prominent finding in dogs injected intravenously with relatively high doses of ${ }^{144} \mathrm{Ce}$ (Fritz, 1970), but were not observed following inhalation of ${ }^{144} \mathrm{Ce}$ in soluble form (Boecker, 1984).

Bone tumors resulting from ${ }^{239} \mathrm{Pu}$ injection were predominantly osteogenic sarcomas. These tumors originated almost always in trabecular bone, usually in the long bones or vertebrae, this distribution being especially pronounced at lower dose levels. A longer average latent period was exhibited at lower injection doses (Jee, 1962; Taylor, G., 1971, 1976).

Relative to average skeletal dose, injected ${ }^{241}$ Am produced a substantially lower incidence of bone tumors than did ${ }^{239} \mathrm{Pu}$ (Wrenn, 1986). Bone tumors have thus far been observed only in the highest dose levels of the ${ }^{249} \mathrm{Cf}$ and ${ }^{252} \mathrm{Cf}$ life-span studies; these preliminary results suggest that the carcinogenicity of fission fragments from ${ }^{252} \mathrm{Cf}$ is much lower than that of alpha particles (Lloyd, R., 1976; Taylor, G., 1983b, Wrenn, 1986).

Bone tumors have not been observed following inhalation of ${ }^{239} \mathrm{PuO}_{2}$, but are the most common cause of delayed death in dogs inhaling ${ }^{238} \mathrm{PuO}_{2}$ (Dagle, 1985, 1986; Hahn, 1981; Muggenburg, 1980; Park, J., 1976, 1986) or ${ }^{239} \mathrm{Pu}^{2}\left(\mathrm{NO}_{3}\right)_{4}$ (Dagle, 1985, 1986; Park, J., 1986). Although there are no life-span studies involving inhaled ${ }^{241} \mathrm{Am}$, bone tumors have been reported in long-term sacrifice studies with inhaled ${ }^{241} \mathrm{AmO}_{2}$ (Gillett, 1985).

Supporting studies with a few ${ }^{239} \mathrm{Pu}$ - and ${ }^{226} \mathrm{Ra}$-injected St. Bernards have compared the incidence of bone tumors in this animal, prone to spontaneous bone tumors (Tjalma, 1966), with that in the beagle. The St. Bernard was estimated to be about 5 times as sensitive to radiation induction of osteosarcoma as the beagle, with latent periods in the St. Bernard about one-half those for comparable dose levels in the beagle (Jee, 1976b, Mays, 1986b; Taylor, G., 1981). The anatomical 
distribution of tumors in the St. Bernard was unlike that in the beagle, with only 1 axial ${ }^{239} \mathrm{PuO}_{2}$ tumor (Taylor, G., 1981). Despite the greater radiosensitivity of the St. Bernard, the ${ }^{239} \mathrm{Pu} /{ }^{226} \mathrm{Ra}$ toxicity ratio, as measured by relative times to tumor appearance, was similar to that observed in the beagle (Mays, 1986b).

A supporting study employing young-adult beagles injected with polymeric ${ }^{239} \mathrm{Pu}$ suggested a shorter latent period for bone tumor induction than that seen with monomeric plutonium; the difference was attributed to delayed translocation to bone of polymeric plutonium initially deposited in liver, and consequent longer retention on the bone surfaces of a more mature skeleton (Jee, 1976b; Stevens, W., 1976). Early results from the life-span experiments in variously aged dogs suggest that older dogs are more sensitive to induction of bone sarcoma than young adults or juveniles, presumably because of longer retention of plutonium on bone surfaces in the older animals (Wrenn, 1986).

Osteosarcomas are the predominant type of bone tumor induced by internally deposited radionuclides in the beagle, as is also the case in human radium victims; a much wider variety of bone tumors are observed spontaneously in humans (Thurman, 1973). Growth dynamics were studied for 256 radiationinduced beagle bone tumors from the Utah studies with injected ${ }^{90} \mathrm{Sr},{ }^{226} \mathrm{Ra}$, ${ }^{239} \mathrm{Pu},{ }^{228} \mathrm{Ra}$, and ${ }^{228} \mathrm{Th}$; tumors appeared to enlarge exponentially, with an average volume doubling time of $\mathbf{1 2 . 4}$ days, which was independent of radionuclide or dose administered, age, sex or tumor location (Thurman, 1971).

The distribution of radiation-induced bone tumors among various skeletal sites in the beagle has received considerable attention (Christensen, 1972; Dagle, 1985; Gillett, 1987b; Hahn, 1981; Jee, 1972; Miller, S., 1986; Nilsson, 1985, 1987; Park, J., 1976; Parks, 1980; Pool, 1972b, 1973, 1973b; Spiers, 1983; Thurman, 1973; Wrenn, 1986b). This distribution is significantly different for man and beagle (Thurman, 1973), for spontaneous and radiation-induced bone tumors in dogs (Christensen, 1972; Hahn, 1981; Thurman, 1973), for volume-seeking and surface-seeking radionuclides (Christensen, 1972; Miller, S., 1986; Wrenn, 1986b), and for dogs exposed to inhaled ${ }^{90} \mathrm{Sr}$ as opposed to chronically ingested ${ }^{90} \mathrm{Sr}$ (Gillett, 1987b). The pattern of distribution can be related to the radiation dose level (Pool, 1973, 1973b; Nilsson, 1987), the time pattern of radionuclide administration (Spiers, 1983), and, in long bones, to the trabecular surface area (Spiers, 1983, 1988). The distribution also varies strikingly among different animal species (Christensen, 1972). Comparison of estimates of average dose to bone groups and site-specific tumor occurrence revealed a 90 -fold variation of dose-response among bone groups (Parks, 1980). 
A number of investigations have attempted (with varying degrees of success) to correlate the sites of tumor occurrence with microdistribution of plutonium on bone surfaces and with rates of skeletal remodeling (Arnold, 1976; Hahn, 1981; Kimmel, 1976, 1982; Miller, S., 1986; Wronski, 1980). The degree of initial deposition on trabecular bone surfaces and the rate of trabecular bone turnover appear to be critical factors in the production of osteosarcomas by ${ }^{239} \mathrm{Pu}$. Bone-marrow microvasculature has also been implicated as influencing the tumorigenic process (Miller, S., 1980b, 1986).

Recent evaluation of the relationship between the cortical and trabecular composition of bones and the differing incidence of ${ }^{226} \mathrm{Ra}-,{ }^{239} \mathrm{Pu}-$, and ${ }^{241} \mathrm{Am}$-induced cancers in those bones shows a positive correlation between the occurrence of ${ }^{226} \mathrm{Ra}$-induced bone sarcomas and the percentage of cortical bone, but no correlation with cortical bone mass or cortical endosteal surface area, and a negative correlation with activated trabecular calcium and trabecular surface area; the frequency of occurrence of ${ }^{239} \mathrm{Pu}$-induced bone sarcomas (but not ${ }^{241} \mathrm{Am}$-induced bone sarcomas) was positively correlated with trabecular surface-to-volume ratios and trabecular turnover (Jee, 1985, 1986).

A number of statistical approaches have been employed in efforts to describe the effect of radiation in the production of bone tumors. In addition to the straightforward, but sometimes misleading (Rosenblatt, 1971), relationship between quantity of radionuclide injected, or radiation dose delivered, and tumor incidence, attempts have been made to relate incidence, dose, and time-toappearance of tumor, employing three-dimensional response surfaces. These attempts have employed logistic models (Goldman, 1972c, 1973, 1986; Rosenblatt, 1971, 1976; Stover, 1970b), log-normal models (Raabe, 1979, 1980, 1981, 1981b, 1983, 1984, 1986, 1986b), and Weibull models (DuMouchel, 1981; Whittemore, 1982). A proportional hazard model has also been employed in relating probability of survival with bone tumor, dose, and time following inhalation of ${ }^{90} \mathrm{SrCl}_{2}$ or ${ }^{238} \mathrm{PuO}_{2}$ (Mewhinney, 1986); and following injection of ${ }^{239} \mathrm{PuO}_{2}$ (Peterson, 1982). A more extended discussion of these efforts will be found in Section 7.7 .

\subsubsection{SINONASAL TUMORS}

An increased incidence of squamous cell carcinoma in the nasal cavity following inhalation of ${ }^{90} \mathrm{Sr},{ }^{144} \mathrm{Ce}$, or ${ }^{91} \mathrm{Y}$ in soluble form, or injection of ${ }^{137} \mathrm{Cs}$, appears to be associated with concentration of the radionuclide in the nasal turbinates (Benjamin, 1979; Boecker, 1984, 1986; Gillett, 1987b); lifetime risk estimates ranged from 9 tumors per $10^{6}$ rad to turbinates, for ${ }^{90} \mathrm{Sr}$, to 98 tumors per $10^{6}$ rad, for ${ }^{137} \mathrm{Cs}$. Nasal carcinomas have also resulted from the exposure of dogs to radon and daughters inhaled with uranium-ore dust (Cross, 1982, 1986). 
The absence of head-sinus carcinomas in life-span beagles injected with ${ }^{226} \mathrm{Ra}$ or ${ }^{228} \mathrm{Ra}$ contrasts with the high incidence of such tumors in the human ${ }^{226} \mathrm{Ra}$ and ${ }^{228}$ Ra cases (Jee, 1976b).

\subsubsection{GINGIVAL TUMORS}

Gingival carcinomas have been a prominent finding only in the Davis ${ }^{90} \mathrm{Sr}$ chronic feeding study, where they correlate with a high radiation dose due to deposition of ${ }^{90} \mathrm{Sr}$ on the forming tooth surface, and to the lack of remodeling of this surface (Parks, 1984). They are postulated to arise from epithelial rests, embryonic tissue trapped in the periodontal membrane. Gingival ulceration was observed following single injection of ${ }^{226} \mathrm{Ra},{ }^{228} \mathrm{Ra}$, or ${ }^{228} \mathrm{Th}$ (Taylor, G., 1964).

\subsubsection{LUNG TUMORS}

Lung tumors occur spontaneously in beagles, but at a relatively low incidence. Data from the Utah colony indicate an overall incidence of $0.1 \%$ per dog-year at risk, which rises to as high as $0.7 \%$ at ages in excess of 13 years (Taylor, G., 1979).

Preliminary evaluation of results from the Davis whole-body $x$-ray experiment indicated an increased incidence of lung tumors, with 9 tumors in irradiated animals and none in controls (Andersen, 1966); re-evaluation of these data indicated only 3 primary lung tumors, 2 of which were considered to be the cause of death, and all appearing in irradiated animals (Rosenblatt, 1986).

Lung tumors were the major radiation-induced cause of delayed death in dogs inhaling radionuclides in forms sufficiently insoluble to be retained for long periods in the lung. Such insoluble radionuclides include the beta emitters ${ }^{144} \mathrm{Ce},{ }^{90} \mathrm{Sr},{ }^{90} \mathrm{Y}$, and ${ }^{91} \mathrm{Y}$, when inhaled as FAP (McClellan, 1976, 1979, 1986).

Hemangiosarcomas were observed in the surviving dogs at higher dose levels of ${ }^{144} \mathrm{Ce}$ and ${ }^{90} \mathrm{Sr}$, from 2 to 4 years postexposure (Benjamin, 1975b; Hahn, 1973, 1973b, 1977; Jones, R., 1974); hemangiosarcomas were not observed following inhalation of the shorter-lived ${ }^{90} \mathrm{Y}$ or ${ }^{91} \mathrm{Y}$. Later-occurring lung cancers were primarily bronchioalveolar carcinomas and were observed with all inhaled beta emitters studied ( $H a h n, 1977,1983 b, 1986$, in press), including the more soluble ${ }^{144} \mathrm{CeCl}_{3}$ (Boecker, 1984).

Hemangiosarcomas are seen much less frequently with repeated inhalation of ${ }^{144} \mathrm{Ce}$ FAP and are not seen at all in aged dogs inhaling ${ }^{144} \mathrm{Ce}$ FAP; juvenile dogs inhaling ${ }^{144} \mathrm{Ce}$ FAP showed hemangiosarcomas earlier and at lower cumulative doses than did adult animals (Boecker, 1980). 
Bronchioalveolar carcinoma is the most common cause of delayed death from inhaled ${ }^{239} \mathrm{PuO}_{2}$. These tumors originate in peripheral lung areas in association with plutonium deposits, fibrosis, and bronchioalveolar metaplasia (Clarke, 1964b, 1966, 1966b; Park, 1964, 1966, 1971, 1972, 1986, in press). Less commonly observed types of plutonium-induced lung tumors include adenocarcinoma, adenosquamous carcinoma, and epidermoid carcinoma (Dagle, 1976, 1986; Howard, 1970).

Incomplete results from the ITRI life-span experiments comparing the response to inhaled 0.75-, 1.5- and 3.0- $\mu \mathrm{m} \mathrm{AMAD}{ }^{239} \mathrm{PuO}_{2}$ particles indicate no effect of particle size on the relative risk of causing lung cancer, thus appearing to refute the "hot particle hypothesis" that nonuniform alpha irradiation of the lung should be more carcinogenic than more uniform irradiation (Muggenburg, in press).

Preliminary data have been reported on the effect of age at exposure on the induction of lung tumors by inhaled ${ }^{239} \mathrm{PuO}_{2}$ (Guilmette, 1988b). Dogs exposed at 8 to 10 years of age exhibit a relatively low incidence of incidental pulmonary carcinomas, with radiation-related deaths ascribable to radiation pneumonitis. Dogs exposed at 3 months of age exhibit an early incidence of fatal pulmonary carcinomas similar to those observed in dogs exposed as young adults.

Lung tumors were also observed following inhalation of ${ }^{238} \mathrm{PuO}_{2}$ or ${ }^{239} \mathrm{Pu}\left(\mathrm{NO}_{3}\right)_{4}$, but in these dogs bone tumors were the more common causes of death, with lung tumors usually present only as incidental findings (Dagle, 1986; Hahn, 1981; Muggenburg, 1980; Park, J., 1976, 1986). Bronchioalveolar carcinomas, epidermoid carcinomas, and fibrosarcomas have also been produced in the lungs of dogs chronically inhaling radon and daughters and uranium-ore dust (Cross, 1982,1986 ).

Preliminary results from studies of high-molecular-weight DNA from plutonium-induced lung tumors indicate that dominant-acting transforming oncogene activity is associated with these tumors (Frazier, 1987; Kelly, in press).

Risk factors for lung tumors, based on average dose to lung, have been estimated as about 20 per $10^{6} \mathrm{rad}$ for ${ }^{144} \mathrm{Ce}$ and ${ }^{90} \mathrm{Sr} \mathrm{FAP}$, and about 50 per $10^{6} \mathrm{rad}$ for ${ }^{90} \mathrm{Y}$ and ${ }^{91} \mathrm{Y}$ FAP (Hahn, 1983b, 1986, in press $b$ ). A risk factor of about 600 per $10^{6}$ rad to lung was estimated for inhaled ${ }^{239} \mathrm{PuO}_{2}$ (Fisher, D., 1986). Risk factor ratios for ${ }^{91} \mathrm{Y} / 239 \mathrm{Pu}$ of 18,15 , and 10 , for $0.75,1.5$, and $3.0 \mu \mathrm{m}{ }^{239} \mathrm{PuO}_{2}$ particles, respectively, have been estimated from a completed ${ }^{91} \mathrm{Y}$ inhalation experiment and ${ }^{239} \mathrm{PuO}_{2}$ inhalation experiments still in progress (Boecker, 1988). 


\subsubsection{PULMONARY LYMPH NODE TUMORS}

An increased incidence of hemangiosarcomas, presumed to originate in pulmonary lymph nodes, has been observed following inhalation, as FAP, of the longer-lived beta emitters ${ }^{144} \mathrm{Ce}$ and ${ }^{90} \mathrm{Sr}$ (Hahn, 1980, 1986, in press, in press b). These tumors appear at relatively long times postexposure and, considering the relatively high radiation dose to lymph nodes, exhibit a lower risk factor than pulmonary tumors.

Primary pulmonary lymph node tumors were reported to have been observed in studies with inhaled plutonium (Howard, 1970; Park, 1962; Thompson, 1968); however, later re-evaluation suggested that the lesions were epithelial lungtumor metastases (Dagle, 1976b, 1986).

\subsubsection{LIVER TUMORS}

Liver tumors of a variety of types have been described in dogs exposed to betaemitting radionuclides, most notably ${ }^{144} \mathrm{Ce}$ (Benjamin, 1975b; Boecker, 1984) and ${ }^{137} \mathrm{Cs}$ (Muggenburg, 1986), leading to a risk estimate of 90 liver cancers per $10^{6}$ rad (Muggenburg, 1986). These tumors typically are observed at long times following exposure and result in little, if any, shortening of life span.

Liver tumors were also observed in dogs injected with ${ }^{239} \mathrm{Pu}$ and ${ }^{241} \mathrm{Am}$ (Taylor, G., 1969, 1972b, 1976, 1983, 1986). The most frequent tumor type was the bile duct adenoma, followed in frequency by bile duct carcinomas, and a variety of sarcomas; hepatic cell tumors were relatively uncommon. Liver tumors were usually very late effects and, compared to bone tumors, were a less common cause of death, except in a few low-dose ${ }^{241} \mathrm{Am}$ groups. Contrary to earlier belief, and supported by epidemiologic study of human Thorotrast patients, the liver appears to be a highly radiosensitive tissue (Mays, 1970, 1982; Taylor, G., 1986).

Hepatic nodular hyperplasia, but no liver tumors, were reported from the PNL plutonium inhalation experiments (Dagle, 1986). However, an increasing incidence of usually nonfatal liver tumors has been reported at long periods following exposure in the ITRI ${ }^{238} \mathrm{PuO}_{2}$ inhalation experiments (Gillett, in press).

\subsubsection{SPLEEN TUMORS}

The Argonne dogs, continuously gamma-irradiated to a predetermined dose, developed 14 spleen tumors, as compared to a single spleen tumor in controls; 10 were fibrosarcomas, probably of nerve sheath origin, and 4 were angiosarcomas 
(Fritz, 1986). Neurofibrosarcoma in the spleen was the most common radiationinduced tumor in the Argonne ${ }^{137} \mathrm{Cs}$ injection experiment (NCRP, 1977).

\subsubsection{HEART TUMORS}

An increased incidence of hemangiosarcomas of the heart has been observed in dogs that inhaled intermediate dose levels of ${ }^{90} \mathrm{Sr}$ FAP (Hahn, in press $b$; McClellan, 1979). These tumors are thought to be the result of irradiation from radionuclide-laden bronchial lymph nodes located at the base of the heart.

\subsubsection{EYE TUMORS}

An increased incidence of intraocular melanomas was observed in beagles injected with ${ }^{226} \mathrm{Ra},{ }^{228} \mathrm{Ra}$, or ${ }^{228} \mathrm{Th}$ (Taylor, G., 1969, 1972d, 1976).

\subsubsection{MAMMARY TUMORS}

In considering the rather limited amount of data on mammary tumors that have been reported from the life-span beagle studies, it must be recalled that the different experiments varied in their handling of mammary tumors. In some experiments, to increase life span and maximize information obtainable on other tumor types, mammary tumors were surgically removed; in other experiments mammary glands were surgically removed before the animals were placed on experiment (Rosenblatt, 1986).

Spontaneous mammary tumors were not observed in male beagles, but were the most common spontaneous tumor in females; they first appeared at 5 to 7 years of age, and their incidence increased with age; there was no evidence of an increased incidence in dogs injected with ${ }^{226} \mathrm{Ra},{ }^{228} \mathrm{Ra},{ }^{228} \mathrm{Th},{ }^{239} \mathrm{Pu}$, or ${ }^{90} \mathrm{Sr}$ (Taylor, G., 1976b). However, in the Argonne continuous gamma-irradiationto-predetermined-dose experiment, mean time to death with mammary tumor was significantly shorter in irradiated dogs than in controls (Fritz, 1986). In the Davis fractionated $x$-ray experiment, mammary tumors occurred somewhat earlier in animals exposed to $100 \mathrm{R}$, but this incidence was not increased at larger doses nor influenced by fractionation of the dose (Rosenblatt, 1986). In the Davis repeated ${ }^{226} \mathrm{Ra}$-injection, and chronic ${ }^{90} \mathrm{Sr}$-ingestion experiments, the cumulative incidence rates for both benign and malignant mammary tumors were not significantly different from controls (Chrisp, 1980). A comprehensive description of mammary tumor incidence in the Davis colony has been recently published (Moulton, 1986). 


\subsubsection{URINARY TRACT TUMORS}

A ureteral transitional cell carcinoma, a type previously unreported in dogs, was observed in a 12-year-old life-span beagle previously injected with ${ }^{137} \mathrm{CsCl}$ (Hanika, 1980).

\subsection{OTHER EFFECTS}

\subsubsection{LIFE SHORTENING}

Nonspecific life shortening has sometimes been considered an effect of irradiation, and the life-span beagle studies frequently have been interpreted in terms of life shortening (Crump, 1986). In most instances, however, it has been possible to explain this life shortening in terms of more specific effects.

In the continuous ${ }^{60} \mathrm{Co}$ gamma-irradiation experiments conducted at Argonne, in the dose-rate range from about 3.5 to $35 \mathrm{rad} /$ day, the mean radiation-specific death rate for the beagle increased as the square of dose, indicating a dependence on dose rate; in this range the dog was about 2.5 times as sensitive as the mouse, an appreciably lower ratio than that of their $\mathrm{LD}_{50} \mathrm{~s}$ (Norris, 1976). An earlier experiment at Argonne involving 61 dogs given single, graded, wholebody doses of ${ }^{60} \mathrm{Co}$ gamma radiation at a rate of $15 \mathrm{R} / \mathrm{min}$, led to an $\mathrm{LD}_{50}$ (30 days) of $258 \mathrm{rad}$ average absorbed dose (Norris, 1968). Detailed clinical hematological, histological, biochemical, and gross and microscopic inspection methods led to the conclusion that acute bacteremia secondary to bone marrow damage was the primary cause of death.

In the Davis experiment with fractionated $\mathrm{x}$-ray exposure it was difficult to discern differences in causes of death between control and irradiated animals, the principal effect of radiation appearing to be a dose-dependent shortening of the latency period for an essentially "normal" variety of neoplastic diseases; protraction of a total dose of $300 \mathrm{R}$ increased survival, but protraction had no effect with a total dose of $100 \mathrm{R}$ (Andersen, 1969; Bustad, 1969b; Rosenblatt, 1986).

A "steady state theory of mutation rates" has been applied in the analysis of survival data from several of the Utah life-span experiments (Stover, 1970b, $1972 d, 1972 e, 1975,1986)$. A preliminary analysis, employing linear model analysis techniques, has been made of life-shortening effects as a function of age at exposure to ${ }^{60} \mathrm{Co}$ gamma rays, employing data from the ongoing Colorado State study (Angleton, 1986; Benjamin, 1986). In the Rochester experiment involving life-span daily $\mathrm{x}$-ray doses ( 5 per week) of $0.06,0.12$, and $0.60 \mathrm{R}$, no significant life shortening was observed (Casarett, 1970). 
Publications detailing survival data in the life-span experiments involving exposure to radionuclides are referenced in Table 5.1.

The possibility of an increased life span, or suppression of cancer, at the lowest levels of alpha-particle exposure in the Utah injection experiments was examined, with no evidence found for such effects (Mays 1987).

\begin{tabular}{|c|c|c|}
\hline $\begin{array}{l}\text { Radio- } \\
\text { nuclide }\end{array}$ & $\begin{array}{l}\text { Mode of } \\
\text { Administration }\end{array}$ & References \\
\hline${ }^{90} \mathrm{Sr}$ & chronic ingestion & $\begin{array}{l}\text { Book, 1980, 1982, 1986; Goldman, } \\
\text { 1972c; Nilsson, 1987; Raabe, } 1981 b\end{array}$ \\
\hline${ }^{90} \mathrm{Sr}$ & $\begin{array}{l}\text { transplacental or } \\
\text { subcutaneous }\end{array}$ & Finkel, 1960, 1972 \\
\hline${ }^{90} \mathrm{Sr}$ & single injection & Mewhinney, 1986; Stover, $1972 d$ \\
\hline${ }^{90} \mathrm{SrCl}_{2}$ & inhalation & $\begin{array}{l}\text { McClellan, 1973, 1983, 1986; } \\
\text { Mewhinney, } 1986\end{array}$ \\
\hline${ }^{90} \mathrm{Sr}$ FAP & inhalation & Hahn, 1983, 1983b, 1986 \\
\hline $90 Y$ FAP & inhalation & Hahn, 1983, 1983b, 1986 \\
\hline${ }^{91} Y$ FAP & inhalation & Hahn, 1983, 1983b, 1986 \\
\hline${ }^{144} \mathrm{CeCl}_{3}$ & inhalation & Benjamin, 1973; McClellan, 1986 \\
\hline${ }^{144} \mathrm{Ce}$ FAP & inhalation & Hahn, 1983, 1983b, 1986, in press \\
\hline${ }^{226} \mathrm{Ra}$ & single injection & $\begin{array}{l}\text { Mays, 1969, 1987; Mewhinney, 1986; } \\
\text { Stover, 1972d }\end{array}$ \\
\hline${ }^{226} \mathrm{Ra}$ & repeated injection & Book, 1980, 1986; Raabe, $1981 b$ \\
\hline${ }^{228} \mathrm{Ra}$ & single injection & Mays, 1969, 1987; Stover, $1972 d$ \\
\hline${ }^{228} \mathrm{Th}$ & single injection & Mays, 1969, 1987; Stover, $1972 d$ \\
\hline${ }^{239} \mathrm{Pu}$ & single injection & $\begin{array}{l}\text { Mays, 1969, 1987; Mewhinney, 1986; } \\
\text { Stover, 1972d, 1972e }\end{array}$ \\
\hline${ }^{239} \mathrm{PuO}_{2}$ & inhalation & $\begin{array}{l}\text { Bair, 1970, 1974; Dagle, 1979; Diel, } \\
\text { 1983b; Fisher, D., 1986; Mahaffey, } 1981 \text {; } \\
\text { Park, J., 1972, 1975; Thompson, } 1972\end{array}$ \\
\hline${ }^{238} \mathrm{PuO}_{2}$ & inhalation & Dagle, 1979; Mewhinney, 1986 \\
\hline${ }^{239} \mathrm{Pu}\left(\mathrm{NO}_{3}\right)_{4}$ & inhalation & Dagle, 1979 \\
\hline${ }^{241} \mathrm{Am}$ & single injection & Mays, 1969, 1987 \\
\hline \multicolumn{2}{|c|}{ None (control dogs) } & Atherton, 1986 \\
\hline
\end{tabular}




\subsubsection{EFFECTS ON REPRODUCTION}

Female beagles exposed to either 100 or $300 \mathrm{R}$ at age 1 year were tested for reproductive ability by whelping two litters before the age of 4 years. Results from 297 litters, including 644 pups, showed no effects of the previous irradiation (Andersen, 1961; Bustad, 1969b). Effects of aging on reproductive ability were accelerated in the 300-R group, with fewer estrus periods as senescence approached (Andersen, 1963e, 1963 $f$ ). Effects on reproductive ability were observed at exposures of $290 \mathrm{R}$ in ancillary studies with dogs exposed at 9 months, and 3 to 87 days of age. Exposure at 9 months of age led to a $9 \%$ reduction in litter size and to an $18 \%$ reduction in number weaned. Exposure at 3 to 87 days of age led to a $17 \%$ reduction in litter size and to a $38 \%$ reduction in number weaned (Andersen, 1976). An absence of effects on puppy mortality and litter size was noted in the Davis ${ }^{90} \mathrm{Sr}$ chronic-feeding experiment at exposure levels $10^{5}$ times that received from worldwide ${ }^{90} \mathrm{Sr}$ fallout (Rosenblatt, $1972 b$ ), although a reduction in sperm motility was reported at the highest feeding level (Della Rosa, 1972). It was concluded from such observations as noted above, that the genital tract of the female beagle was relatively radioresistant as regards effects on reproduction (Andersen, 1963f).

Beagles exposed prenatally, throughout gestation, to ${ }^{60} \mathrm{Co}$ gamma radiation at a rate of $5 \mathrm{R}$ per day were subsequently unable to bear young; severe structural abnormalities were noted in the ovarian cortex of animals irradiated at $5 \mathrm{R}$ per day but not in those irradiated at $2.5 \mathrm{R}$ per day (Sanderson, 1978; Seed, 1977b). Prenatal and early neonatal mortality was observed in beagles of the Colorado State studies exposed during gestation to single $x$-ray doses of $83 \mathrm{R}$ or $16 \mathrm{R}$ (Angleton, 1988).

In the University of Rochester experiment involving life-span daily $\mathrm{x}$-ray doses (5 per week) of $0.06,0.12$, and $0.60 \mathrm{R}$, seminal sperm counts were reduced after 20 to 30 weeks of exposure at the $0.60 \mathrm{R} /$ day level; from the third to tenth year of exposure all dogs at this highest exposure level were essentially aspermic; a trend toward reduced sperm count at the $0.12 \mathrm{R} /$ day level was not statistically significant (Casarett, 1970).

\subsubsection{PSYCHOLOGICAL EVALUATIONS}

Efforts to evaluate effects of $x$-irradiation on dominance behavior and other emotional factors were unsuccessful because of the predominant influence of other uncontrolled factors (Solarz, 1964, 1964b). 



\section{Contents}

6.1 Studies Relating to Therapeutic Removal of Internally Deposited Radionuclides $\quad \ldots \ldots \ldots \ldots \ldots \ldots \ldots . . \ldots 6$

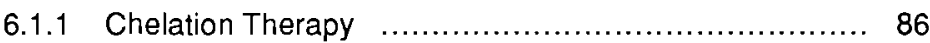

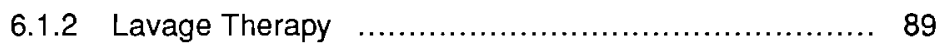

6.2 Studies Relating to Pulmonary Function and

Clearance of Inhaled Particles ............................ 91

6.3 Studies Relating to Clearance of Wound-Deposited

Plutonium to Regional Lymph Nodes $\ldots . . \ldots \ldots \ldots \ldots \ldots \ldots . . \ldots 2$

6.4 Studies Relating to the Immune System $\ldots \ldots \ldots \ldots \ldots \ldots \ldots . \ldots 3$

6.5 Studies Relating to Leukemogenesis f...................... 94

6.6 Studies Relating to Cytogenetic Effects $\ldots \ldots \ldots \ldots \ldots \ldots \ldots \ldots . \ldots 6$

6.7 Miscellaneous Non-Life-Span Internal Exposure

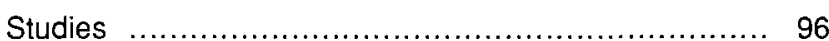

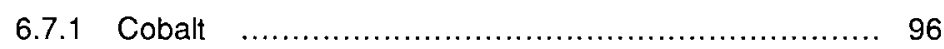

6.7 .2 Zirconium .............................................. 96

6.7.3 Niobium ................................................ 96

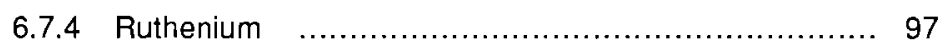

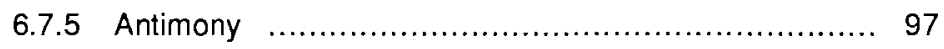

6.7 .6 lodine ............................................... 97

6.7.7 Barium .................................................. 97

6.7.8 Lanthanum ......................................... 97

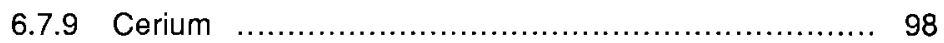

6.7.10 Lead .................................................. 98

6.7.11 Radon .................................................. 98

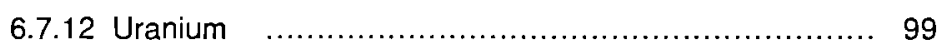

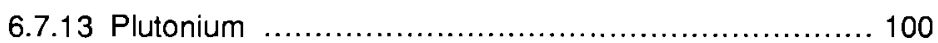

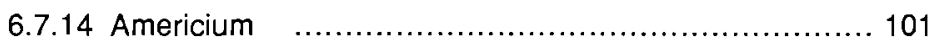

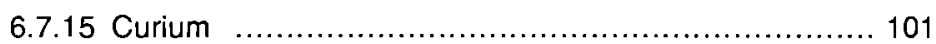

6.8 Studies of Non-Radiation-Related Toxicological

and Disease Processes ............................... 102

The selection of material for this chapter has posed problems. The distinction between studies that are "supportive of" the life-span studies, and hence considered in previous chapters, and studies that are "ancillary to" the life-span studies, and considered in this chapter, is clearly a somewhat arbitrary one. It has been difficult, also, to distinguish between studies that are truly ancillary, in 
the sense that they would probably not have been conducted were it not for the larger effort with life-span dogs, and studies whose only relation to the life-span studies is the fact that they employed beagle dogs. Such latter studies have not been included, although, again, the choice is often a somewhat arbitrary one. Thus, the work of Richmond, Furchner, and colleagues at Los Alamos, on interspecies comparisons of radionuclide metabolism (Richmond, 1970c) has been excluded, as have many other studies that employed beagles, but not as a corollary to any life-span study.

\subsection{STUDIES RELATING TO THERAPEUTIC REMOVAL OF INTERNALLY DEPOSITED RADIONUCLIDES}

A number of ancillary studies have explored methods for speeding the removal of radionuclides from the body, as an aid in the development of practical procedures for treatment of exposed workers (Bair, 1969, Mays, 1981).

\subsubsection{CHELATION THERAPY}

Most attempts at radionuclide decorporation have involved the use of chelating agents, in particular diethylenetriamine pentaacetic acid (DTPA). The retention and distribution of intravenously injected ${ }^{14} \mathrm{C}$-labeled DTPA was studied in 2 beagles; more than $99 \%$ was lost from plasma with a half-time of 0.77 hour; about $0.1 \%$ was present in liver and in kidney after 52 hours (Stevens, W., 1978).

Dogs previously injected with ${ }^{228} \mathrm{Th}$ have a significant steady-state concentration of ${ }^{212} \mathrm{~Pb}$ in their blood and have been employed as reusable test animals for assaying the effectiveness of various chelating agents and treatment regimens for temporary reduction of their ${ }^{212} \mathrm{~Pb}$ level in blood (Stover, 1966).

Demonstration of the toxicity of repeatedly administered Ca-DTPA, and the attribution of this toxicity to the depletion of zinc levels, led to studies of the therapeutic effectiveness and toxicity of Zn-DTPA (Mays, 1977). Administration of Ca-DTPA at 5-hour intervals proved fatal to beagles as early as 4 days after initiation of treatment, with the most serious lesions produced in the epithelial lining of the gastrointestinal tract; the same frequency of administration of Zn-DTPA produced no significant damage (Taylor, G., 1974). Fetal injury was produced by daily Ca-DTPA treatment of pregnant dogs at dose levels commonly employed in human therapy (30 $\mu \mathrm{mole} / \mathrm{kg})$ (Taylor, G., 1978b).

Another comparison of Ca-DTPA and Zn-DTPA therapy regimens for removal of ${ }^{239} \mathrm{Pu}$ demonstrated the superior effectiveness of an initial Ca-DTPA treatment, and the lack of improved effectiveness when treatments with Zn-DTPA were 
given more frequently than once per day; the relative distribution of plutonium among different bones of the skeleton was not significantly affected by Zn-DTPA treatment (Taylor, G., 1978).

Fourteen beagles were employed in an experiment comparing the effectiveness of Ca-DTPA administered weekly, and Zn-DTPA administered daily, in removing ${ }^{239} \mathrm{Pu}$ administered in polymeric form, tagged with ${ }^{237} \mathrm{Pu}$ for in-vivo counting. The Zn-DTPA, under these conditions, was substantially more effective than Ca-DTPA in removing plutonium from both liver and skeleton (Lloyd, $R ., 1978 b$ ).

In preliminary studies of the comparative effectiveness of Ca-DTPA or Zn-DTPA for delayed treatment ( 2 weeks after intravenous injection of ${ }^{241} \mathrm{Am}$ ), the zinc chelate was shown to be equally effective and far less toxic (Lloyd, R., 1976c). The long-term therapeutic effectiveness of chronically administered $\mathrm{Zn}$-DTPA in removing ${ }^{241} \mathrm{Am}$ from beagles was studied in 6 dogs, with treatment commencing 2 weeks after americium injection. Thirteen months of daily treatment resulted in reduction of liver americium to less than $1 \%$ of pretreatment levels, and skeletal americium to $27 \%$ of pretreatment levels; retention half-time in liver and skeleton of untreated animals was on the order of 10 years (Lloyd, R. 1975b). Delayed treatment with Zn-DTPA was as effective as treatment with Ca-DTPA, and far less toxic (Lloyd, R., 1977). A 10-week daily-treatment study with $\mathrm{Zn}$-DTPA showed similar effectiveness in preventing deposition of ${ }^{241} \mathrm{Am}$ injected into simulated wound sites; treatment 5 times per day was, in this case, somewhat more effective than once-daily treatment (Lloyd, $R$., 1975c). The experience gained in these studies was of inestimable value in determining the treatment of a worker seriously overexposed to americium (Thompson, 1983).

The feasibility of administering Zn-DTPA in the form of a slow-release implant was studied in beagle dogs, where such a procedure proved effective in promoting the removal of ${ }^{241} \mathrm{Am}$ (Calder, 1978). Continuous Zn-DTPA therapy for ${ }^{241} \mathrm{Am}$ removal has also been effectively achieved employing subcutaneously implanted osmotic pumps (Guilmette, 1988).

The dependence of chelation efficacy upon time of administration following radionuclide deposition was studied in experiments involving 14 beagles given either ${ }^{241} \mathrm{Am}$ (III) citrate or ${ }^{237,239} \mathrm{Pu}$ (IV) citrate; for Ca-DTPA treatments at 1, 6, and 30 minutes following radionuclide injection, retention of ${ }^{241} \mathrm{Am}$ was $3 \%$, $10 \%$, and $29 \%$, respectively; plutonium removal was less sensitive to the timing of treatment (Lloyd, R., 1979). An experiment involving 5 beagles injected with both ${ }^{241} \mathrm{Am}$ and ${ }^{237,239} \mathrm{Pu}$, tested the effect of magnitude of initial Ca-DTPA injection, and demonstrated that the largest tolerated doses were the most 
effective (Lloyd, R., 1979b). It was deduced from these studies that treatment of contaminated humans should begin as soon as possible, with a large dose of Ca-DTPA, to be followed at daily intervals by treatments with Zn-DTPA.

The efficacy of chelation therapy in decreasing or delaying the incidence of bone sarcoma has been demonstrated in preliminary results from experiments in which beagles were given protracted therapy with Zn-DTPA or Ca-DTPA (Jones, C., 1986).

Among extensive studies conducted at Argonne on the efficacy of various agents for therapeutic removal of monomeric and polymeric plutonium, a number were conducted in beagles (Baxter, 1973). They included studies of optimal time-dose relationships, employing DTPA for removal of monomeric plutonium (Guilmette, 1979). The polysaccharide, glucan, when employed in conjunction with DTPA, improved decorporation of polymeric plutonium (Rosenthal, 1975) but was not effective with monomeric plutonium (Baxter, 1973b). Liposome encapsulation improved the effectiveness of DTPA in removal of polymeric plutonium from liver (Rosenthal, 1975b).

Beagles injected with both ${ }^{241} \mathrm{Am}$ and ${ }^{237,239} \mathrm{Pu}$ have been employed in tests of the therapeutic effectiveness and toxicity of tetrameric catechoylamide ligands (LICAM). Retention in untreated dogs of $91 \%$ and $87 \%$, for plutonium and americium, respectively, was reduced to $12 \%$ and $70 \%$, respectively, with prompt treatment with a CAM ligand, which compares to a reduction to $30 \%$ and $20 \%$ with Ca-DTPA treatment; LICAM(S) showed evidence of toxicity; LICAM(C) did not (Lloyd, R., 1984). When treatment was delayed for two weeks, LICAM(C) was somewhat more effective than Zn-DTPA in removing plutonium from nonliver tissue, but much less effective in removing plutonium from liver and in removing americium from either liver or non-liver tissue (Mays, 1986).

DTPA was found to be relatively ineffective in influencing the movement of either ${ }^{239} \mathrm{PuO}_{2}$ or ${ }^{239} \mathrm{Pu}\left(\mathrm{NO}_{3}\right)_{4}$ from simulated puncture wounds to regional lymph nodes (Bistline, 1972), but had some effect in enhancing excretion, particularly that of the americium contaminant (Bistline, 1976; Johnson, L., 1970). Removal of lymph nodes burdened with plutonium absorbed from contaminated wound sites was studied as a potentially useful therapeutic measure but was found to result in more, rather than less, plutonium being ultimately translocated to liver and spleen (Gomez, 1972, 1974).

Substantial absorption of radiolabeled DTPA from the respiratory tract of beagle dogs was observed, whether the DTPA was instilled in nasopharyngeal, tracheobronchial or pulmonary regions (Dudley, 1980). The possibly toxic effects of 
Ca-DTPA administered by inhalation were studied in 24 dogs; effects were minor and/or of questionable significance (Smith, V., 1976).

Chelating agents have also been employed for therapeutic removal of inhaled radionuclides. In preliminary studies on a few dogs, repeated treatments with DTPA, administered as an aerosol or by subcutaneous injection, following inhalation of ${ }^{144} \mathrm{CeO}_{2}$, achieved a substantial reduction in retained ${ }^{144} \mathrm{Ce}$ (Bair, $1963 \mathrm{~b}$ ). In other studies, the effectiveness of intravenous DTPA in removing inhaled ${ }^{144} \mathrm{CeCl}_{3}$ was shown to be greatly decreased when treatment was delayed from 1 hour to 5 days following inhalation (Pfleger, 1972b). Treatment with DTPA by aerosol inhalation or subcutaneous injection was relatively ineffective in removing inhaled ${ }^{239} \mathrm{PuO}_{2}$; aerosol treatment with "Pluronics," a non-ionic wetting agent, showed some promise for plutonium removal (Bair, 1963b; Tombropoulos, 1963). Repeated intraperitoneal injection of Ca-DTPA was ineffective in removing inhaled ${ }^{239} \mathrm{PuF}_{4}(\mathrm{McD}$ onald, 1979). Early efforts at therapeutic removal of inhaled radionuclides, employing beagles, have been reviewed (Tombropoulos, 1964).

Very effective therapy for inhaled ${ }^{241} \mathrm{AmO}_{2}$ was achieved by a single injection of Ca-DTPA, one hour postexposure, followed by continuous infusion, for 64 days, of $\mathrm{Zn}$-DTPA from subcutaneously implanted osmotic pumps (Guilmette, 1988). This treatment blocked translocation of $99.5 \%$ of the americium that would otherwise have been deposited in liver, and $98.3 \%$ of the americium that would have been deposited in bone; removal of americium from the lung was also accelerated.

\subsubsection{LAVAGE THERAPY}

Pulmonary lavage has been extensively studied in beagles as a method for removing inhaled insoluble radioactive materials, and, based on these studies, was employed in the treatment of a worker that inhaled ${ }^{239} \mathrm{Pu}$ (McClellan, 1972;

Muggenburg, 1977). Lavage with isotonic saline was shown to be effective in removing inhaled ${ }^{137} \mathrm{Cs}$ FAP (Pfleger, 1969) and ${ }^{95} \mathrm{Zr}-{ }^{95} \mathrm{Nb}$-labeled $\mathrm{ZrO}_{2}$ (Pfleger, $1969 \mathrm{~b}$ ). Lavage with a DTPA solution or saline was effective in removing inhaled ${ }^{144} \mathrm{CeCl}_{3}$ (Muggenburg, 1972; Pfleger, 1972). In another study with inhaled ${ }^{144} \mathrm{Ce}$ FAP, 8 dogs that received 10 saline lavage treatments over a 56 -day period retained an average $44 \%$ less ${ }^{144} \mathrm{Ce}$ than untreated controls (Boecker, 1974; Silbaugh, 1975). In other studies with ${ }^{144} \mathrm{Ce}$ FAP, neither flow-rate nor sequence of right- or left-lung lavage was found to be a significant factor in removal of particles from the lung (Felicetti, 1975). Prompt lavage was most effective, but a sizable fraction of particles retained in the lung remained accessible to removal by lavage for periods of up to 6 months postexposure (Felicetti, 1975b). The 
distribution pattern of inhaled ${ }^{147} \mathrm{Pm}-{ }^{169} \mathrm{Yb}$-labeled FAP was studied, with and without prior lavage treatment, showing that the treatment did not preferentially remove particles from any particular area of the lung (Snipes, 1979).

A series of 19 weekly lavage treatments, limited to the right lung, was effective in removing about one-fourth of an initial lung deposit of ${ }^{239} \mathrm{PuO}_{2}$ (McDonald, 1975). Other studies of ${ }^{239} \mathrm{Pu}$ and ${ }^{238} \mathrm{Pu}$ removal by combined lavage and chelation therapy explored the effect of particle size and solubility. Lavage was generally more effective on less soluble and smaller particles, while chelation therapy was more effective with the more soluble materials (Muggenburg, 1976, $1976 b$ ). Similar results were obtained in studies of the removal of inhaled ${ }^{241} \mathrm{AmO}_{2}$ particles of different sizes; the combined treatments reduced the 64-day cumulative absorbed radiation doses to lung, liver and skeleton by about $50 \%$, $90 \%$, and $85 \%$, respectively (Muggenburg, 1981). Similar studies were also conducted with industrial mixed oxides of ${ }^{239} \mathrm{PuO}_{2}$ and $\mathrm{UO}_{2}$ (Muggenburg, 1981b).

The life-prolonging potential of pulmonary lavage and chelation therapy was demonstrated in an experiment in which 12 dogs inhaled marginally acutely lethal quantities of ${ }^{144} \mathrm{Ce}$ FAP; 8 were treated with a series of 10 bronchopulmonary lavages and 10 intravenous injections of Ca-DTPA (Muggenburg, 1975). Only 1 of the treated dogs died; 3 of the 4 untreated dogs died by 550 days postexposure. Later results from this experiment, with additional animals added, showed 21 of 24 untreated dogs dying of radiation pneumonitis in less than 750 days, and 2 of 11 treated dogs dying during the same period; the remainder of the treated dogs died from 750 to 1800 days postexposure, from lung tumors or other neoplasia (Muggenburg, 1981c).

Pulmonary lavage has also been considered as a possible bioassay tool. Measurements on lavage samples from beagles that had inhaled ${ }^{239} \mathrm{PuO}_{2}$ provided estimates of lung burden of precision comparable to those obtained from external $\mathrm{x}$-ray counting (Guilmette, 1986).

Improved procedures for bronchopulmonary lavage have been developed (Muggenburg, 1975b). Pathologic abnormalities associated with the treatment have been studied and found to be limited to a period of 24 hours postlavage; histologic alterations were found for only 48 hours (Muggenburg, 1972b, 1980b). Techniques were developed, and applied, for measuring the temporary detriment to pulmonary function caused by the lavage treatment (Dubin, 1971; Silbaugh, 1977). No alterations in physiologic parameters were found after 24 hours postlavage (Muggenburg, 1972b), nor in 6 dogs, lavaged 10 or more times, followed for 4 years after their last lung lavage (Muggenburg, 1980b). 
In connection with the investigation of pulmonary lavage as a measure for removal of inhaled radionuclides, the normal pulmonary cytology of the beagle has been described (Rebar, 1980), and a number of studies have been conducted on the alveolar lining cells removed with the lavage fluid. These cells were shown to be the site of synthesis of the lipid components of pulmonary surfactant (Pfleger, 1971). Dipalmitoyl lecithin and phosphatidyl glycerol were shown to be important components of the surfactant material (Henderson, 1972; Pfleger, $1971 b$ ). Surfactant removed by lavage was shown to be rapidly replaced and was not a contraindication for the procedure (Henderson, 1974, 1975). Serum proteins of surfactant were studied electrophoretically, and the presence of a trypsin inhibitor was demonstrated (Tuttle, 1974).

\subsection{STUDIES RELATING TO PULMONARY FUNCTION AND CLEARANCE OF INHALED PARTICLES}

A number of ancillary studies relating to the respiratory tract were conducted in preparation for, or in efforts to understand the results of, the inhalation exposure of beagles in life-span experiments. A number of these studies were concerned with morphometry of the respiratory airways (Phalen, 1978; Schreider, 1980, 1981, 1983). Fiberoptic bronchoscopy was employed in studies of regional variations in epithelial permeability to small deposited volumes of $99 \mathrm{~m}$ Tc-labeled DTPA (Wolff, in press). A technique was described for aerosol deposition restricted to the nose in beagles (Whaley, 1988).

Cardiopulmonary function of awake, sedated, and anesthetized beagles was studied, repeatedly, in 4 dogs; results identified problems with the use of chemical restraint in inhalation exposures (Muggenburg, 1974).

Employing 10 dogs repeatedly exposed to ${ }^{67} \mathrm{Ga}$-labeled $\mathrm{Ga}_{2} \mathrm{O}_{3}$ (half-life, 78 hours), a study was made of the effect of postexposure hyperpnea (induced by a $7 \% \mathrm{CO}_{2}$ atmosphere) on clearance and redistribution. Clearance was generally retarded by the hyperpnea, but the pattern of retention was not significantly affected (Valberg, 1985). Twenty dogs were employed in a study of the deposition of ultrafine $0.1 \mu \mathrm{m}$ chain aggregate aerosols of ${ }^{67} \mathrm{Ga}_{2} \mathrm{O}_{3}$ (Wolff, 1981). Comparative data on the deposition and retention of the ultrafine aggregate particles were also reported for beagles, rats, and mice (Wolff, 1984).

Methods have been developed for the measurement of tracheal mucus transport rates employing $99 \mathrm{~m} \mathrm{Tc}$-labeled macroaggregated albumin (Wolff, 1979, 1982). The effect of aging on tracheal mucociliary clearance was studied in 24 beagles; mucous velocity was significantly greater in young-adult and middle-aged animals than in immature or aged animals (Whaley, 1987). Comparative data 
from rats, guinea pigs, rabbits, dogs, and man showed mucous velocity to be proportional to the 0.4 power of body weight, making the dog a much better model for humans than the smaller animals (Felicetti, 1981). Other studies investigated the influence of particle-size of sulfuric acid aerosols on tracheal mucus clearance (Wolff, 1981b).

Experiments with radiolabeled polystyrene microspheres of 3, 7, and $13 \mu \mathrm{m}$ diameter, instilled into the lung, showed essentially no clearance of the two larger-sized particles, either to the gastrointestinal tract or to lymph nodes (Snipes, 1984). In other experiments with labeled microspheres, the ability of both macrophages (Harmsen, 1985b) and neutrophils (Harmsen, 1987) to phagocytize particles and translocate these particles from lung to lymph node was demonstrated. Mucociliary clearance and particle retention in the maxillary and ethmoid turbinate regions of beagle dogs has also been studied with radiolabeled polystyrene microspheres (Whaley, 1986). A recent review compares and models the retention patterns of chronically inhaled particles in the lungs of beagles, rats, guinea pigs, nonhuman primates and humans; the largest concentrations occur in the lungs of guinea pigs, the smallest in the lungs of rats (Snipes, in press).

Several studies have explored possible effects of cigarette smoking on the retention and toxicity of inhaled radionuclides. This was a major factor investigated in the dog studies with inhaled radon and other components of uranium mine atmospheres, which are not, for the purposes of this report considered to be life-span studies; they are appropriately considered, however, later in this chapter (Section 6.7.11), as important ancillary studies. In an early PNL experiment, 6 dogs smoked 20 cigarettes per day, 5 days per week, for over a year, without showing effects on the periodically tested clearance of ${ }^{59} \mathrm{Fe}_{2} \mathrm{O}_{3}$ or ${ }^{51} \mathrm{Cr}_{2} \mathrm{O}_{3}$ (Bair, 1967). The apparatus employed in simulating human patterns of smoking in the dog has been described (Bair, 1969b; Cross, 1982; Stuart, 1970b, 1971).

The effect of $\mathrm{SO}_{2}$-induced bronchitis on the deposition and retention of inhaled ${ }^{134} \mathrm{Cs}$-labeled FAP was studied in 4 beagles; deposition in the pulmonary region was markedly reduced by the preexisting pulmonary disease, but early and longterm clearance pathways and rates were not affected (Greene, 1987).

\subsection{STUDIES RELATING TO CLEARANCE OF WOUND-DEPOSITED PLUTONIUM TO REGIONAL LYMPH NODES}

Experiments at Colorado State University, conducted in cooperation with the Rocky Flats Plutonium Weapons Plant, investigated the movement of plutonium deposited as ${ }^{239} \mathrm{PuO}_{2}$ or ${ }^{239} \mathrm{Pu}\left(\mathrm{NO}_{3}\right)_{4}$ in simulated puncture wounds in the paw, 
to the first major downstream lymph node and to other major body organs. Early results of these experiments have been reviewed (Watters, 1972). Excision of downstream lymph nodes increased translocation to other body organs; treatment with DTPA had little effect; detailed kinetic data were obtained using invivo counting techniques (Bistline, 1972, 1976; Dagle, 1975, 1975b; Gomez, 1972, 1974; Lebel, 1976). Distribution of the translocated ${ }^{241} \mathrm{Am}$ contaminant differed from that of the ${ }^{239} \mathrm{Pu}$ and was differentially affected by administration of DTPA (Johnson, L., 1970). Uptake and transport mechanisms in afferent and efferent lymph were studied using a variety of biochemical methods (Schallberger, 1976). Effects in the lymph nodes were studied histologically and autoradiographically (Dagle, 1975, 1976b; Lebel, 1970). A lymphosarcoma associated with nodal concentration of $\mathrm{PuO}_{2}$ was probably not related to plutonium exposure, since it appeared only 4 months postinjection (Lebel, 1970).

Ten $\mathrm{PuO}_{2}$-implanted animals and $8 \mathrm{Pu}\left(\mathrm{NO}_{3}\right)_{4}$-implanted animals were sacrificed after 8 and 5 years, respectively. Of the initial quantity implanted, the $\mathrm{PuO}_{2}$ animals retained an average $21 \%$ in their paw, $7 \%$ in the regional lymph node, $1 \%$ in skeleton, and $5 \%$ in liver; the $\mathrm{Pu}\left(\mathrm{NO}_{3}\right)_{4}$ animals retained $16 \%$ in their paw, $0.3 \%$ in the regional lymph node, $7 \%$ in skeleton, and $7 \%$ in liver (Dagle, $1984 \mathrm{~b}$ ). The chemical form of implanted plutonium thus had a major effect on ultimate distribution and retention. Pathology included bone and liver tumors, probably related to the plutonium exposure, but no serious effects unique to the subcutaneous route of exposure.

\subsection{STUDIES RELATING TO THE IMMUNE SYSTEM}

Use of the beagle in studies of the immune system has been considered broadly in recent monographs (Shifrine, 1980, 1984), including specific information derived from radiation effects studies (Colgrove, 1980, 1980b; Holmberg, 1980).

Cell surface markers on beagle lymphocytes were studied and techniques described for the identification of $\mathrm{B}$ and $\mathrm{T}$ lymphocytes in peripheral blood (Miller, C., 1978, 1980).

In connection with studies of chronic ${ }^{60} \mathrm{Co}$ gamma-ray exposure at dose levels of 0.07 and $0.33 \mathrm{rad} / \mathrm{day}$, measurements were made of cell-mediated immunity, using the whole-blood lymphocyte stimulation test (Shifrine, 1978; Thilsted, $1979 \mathrm{~b}$ ). These studies showed a seasonal variation in cell-mediated immunity, with a summer peak and a winter trough, that was not dependent on radiation, photoperiod, or temperature (Shifrine, 1980b, 1980c, 1982). An examination of other environmental factors indicated that sunspot activity showed the highest correlation (Garsd, 1982). 
Regression of beagle sarcomas induced by feline sarcoma virus was studied morphologically (Slauson, 1975) and immunologically (Slauson, 1975b). The role of natural killer (NK) activity in immunosurveillance has been studied in whole-body chronically irradiated beagles. Radiation increased NK activity at lower doses and depressed it at higher doses, the effect being evident in both clinically normal and leukemic animals (Dyck, 1986).

The effect of ${ }^{90} \mathrm{Sr}$, inhaled or intravenously injected, on secondary immune response was studied in beagles, employing Leptospira canicola and infectious canine hepatitis antigens (Clapper, 1970). A depressed response was observed, directly related to initial body burden and cumulative skeletal dose; at lower radiation doses, recovery occurred after 5 months. Similar studies, employing a T-cell-dependent antigen, keyhole limpet hemocyanin, in dogs that had inhaled ${ }^{239} \mathrm{PuO}_{2}$, showed a significant decrease in primary antibody response in exposed versus unexposed dogs (Dagle, 1979). Earlier studies had shown an impaired ability to form antibodies against sheep red blood cells following inhalation of ${ }^{239} \mathrm{PuO}_{2}$ and ${ }^{238} \mathrm{PuO}_{2}$ (Park, J., 1971).

The preferential retention of many insoluble radionuclide particles in pulmonary lymph nodes, and the pronounced lymphopenia associated with this deposition, has stimulated studies, in the beagle, of immunologic response in the respiratory tract (Bice, 1985b), including effects of localized deposition of antigen in the lung (Bice, 1980, 1980b; Brownstein, 1980; Galvin, 1986; Harmsen, 1985, 1985b, 1987; Schnizlein, 1980), and effects of age (Bice, 1985).

A technique was developed in beagles for selective irradiation of tissues responsible for the homograft rejection response, employing injected ${ }^{90} \mathrm{Y}$ chelated with DTPA. At optimum dosage a severe lymphopenia developed without depression of granulocytes, platelets, or reticulocytes (Winchell, 1964). Studies have been reported on the antigenicity of several beagle kidney cell lines in tissue culture (Levy, 1969). The decline and restoration of antibody-forming capacity was studied in aging beagles (Jaroslow, 1974).

\subsection{STUDIES RELATING TO LEUKEMOGENESIS}

The myeloproliferative disorders that developed in beagles continuously exposed at early ages to external irradiation or internally deposited ${ }^{90} \mathrm{Sr}$ (Dungworth, 1969) have been employed extensively as a model system for the study of leukemogenesis (Wilson, 1978, 1980). A myelomonocytic leukemia developing in a ${ }^{90} \mathrm{Sr}$-exposed dog was successfully transplanted and carried through multiple passages in beagle fetuses (Shifrine, 1971; Wilson, 1972); a cytogenetic marker allowed determination of the host-recipient nature of the leukemic cell 
populations (Shifrine, 1973c). Hematological values and lysozyme levels of beagles with transplantable leukemias have been characterized (Shifrine, 1973d; Wilson, 1972), and the influence of host age and immune responsiveness on such transplants have been studied (Shifrine, 1976).

Extensive research at Davis has employed externally irradiated beagles in nonlife-span studies of effects on blood and bone marrow cells, particularly as these relate to leukemogenesis. This research has, in many respects, complemented that conducted in life-span studies at Argonne. Dogs were exposed chronically in an outdoor ${ }^{60} \mathrm{Co}$-gamma-radiation field (Momeni, 1974) at rates of 10,4 , or 2 $\mathrm{rad} /$ day, beginning at 50,150 , or 400 days of age, or, for the two higher rates, at 21 days of gestation (Stitzel, 1982). Dogs whose exposure began in-utero, live up to ten times longer than dogs exposed at the same rate starting after birth. Dogs exposed in-utero almost invariably develop acute nonlymphocytic leukemia or preleukemic changes, which is a rare occurrence in animals exposed after birth. This model system offers many opportunities for study of the cellular and molecular changes associated with the transformation of normal hematopoietic cells to malignant cells. Comprehensive descriptions of the overall Davis experiment have not appeared in the journal literature, although specific aspects have been described. Ancillary studies have also been conducted at Argonne, involving in-utero exposure to ${ }^{60} \mathrm{Co}$ gamma rays, and the resulting hematopathologic effects compared to those obtained with the life-span animals whose exposure began as young adults (Seed, 1987).

A bone marrow culture system was developed and employed to study basic doseresponse relationships of granulocyte-monocyte colonies and adherent fibroblast colonies from 2- to 3-month-old beagles exposed to ${ }^{60} \mathrm{Co}$ whole-body gamma radiation (Knox, 1981; Wilson, 1977, 1978c, 1980b, 1981). The effect of ${ }^{60} \mathrm{Co}$ gamma-irradiation on the appearance of a substance in serum that enhances the formation of bone-marrow colony-forming units was explored in ancillary studies with both acutely and chronically irradiated beagles (Kaspar, 1984). The serum colony stimulating activity was higher, at similar total dose levels, following acute than following chronic irradiation. These techniques have been employed in the characterization of fetal lymphohematopoiesis in the beagle (Klein, 1983).

The effect of continuous, whole-body, ${ }^{60} \mathrm{Co}$ gamma-irradiation on lymphohematopoietic progenitors, and on a possible hematopoietic regulatory population, has been reported (Klein, 1985), as have effects on bone marrow fibroblasts (Klein, 1984). 
Radiation-induced megakaryoblastic leukemia has been recently observed and studied in the chronically exposed beagle (Cain, 1985, 1986b).

\subsection{STUDIES RELATING TO CYTOGENETIC EFFECTS}

Chromosomes of the dog are acrocentric and therefore do not represent the best material for cytogenetic studies (Moore, 1970). Studies of peripheral blood lymphocytes in whole-body irradiated dogs indicate a dicentric yield not significantly different from that observed in humans; the half-time of beagle lymphocytes carrying chromosome aberrations was estimated to be less than 30 days (Leonard, 1982).

\subsection{MISCELLANEOUS NON-LIFE-SPAN INTERNAL EXPOSURE STUDIES}

Data on the internal distribution, retention, and effects of radionuclides were obtained in many experiments with beagles, unrelated to specific life-span studies. These are considered below, by element.

\subsubsection{COBALT}

Three dogs exposed by inhalation to ${ }^{60} \mathrm{Co}_{3} \mathrm{O}_{4}$, and 3 dogs exposed to ${ }^{60} \mathrm{CoO}$, were sacrificed at intervals to 128 days to provide short-term retention and distribution data. Lung and pulmonary lymph nodes were the predominantly exposed organs, with ${ }^{60} \mathrm{Co}_{3} \mathrm{O}_{4}$ more tenaciously retained in the lung and translocated in greater amount to the lymph nodes (Barnes, 1976). These data were pertinent to the interpretation of several human exposure cases.

\subsubsection{ZIRCONIUM}

The clearance from the lung of ${ }^{95} \mathrm{Nb}$-labeled $\mathrm{ZrO}_{2}$ was measured in 3 dogs and compared to retention in an accidentally exposed human (Waligora, 1971). Retention half-times in man and dogs were of the order of one year, suggesting a "Class Y" classification, rather than the ICRP assumption of "Class W."

\subsubsection{NIOBIUM}

A detailed kinetic model was derived from data on the distribution and retention of ${ }^{95} \mathrm{Nb}$ inhaled as the oxide or oxalate in studies involving 33 dogs sacrificed at intervals to 128 days postexposure (Cuddihy, 1978). The oxalate cleared more rapidly from the lung, but the lung was projected to receive the largest radiation dose in either case. For longer-lived niobium isotopes, skeleton and kidney were identified as organs of potential concern. 


\subsubsection{RUTHENIUM}

A detailed kinetic model for the distribution and retention of ${ }^{106} \mathrm{Ru}$ inhaled as ${ }^{106} \mathrm{RuO}_{4}$ was derived from an experiment with 26 dogs sacrificed at intervals to 512 days postexposure (Snipes, 1981). Ninety-nine percent of the initially deposited ${ }^{106} \mathrm{RuO}_{4}$ was lost from the body with a half-time of 1.2 days. The nasopharyngeal region received the highest radiation dose.

\subsubsection{ANTIMONY}

Data on the distribution and retention of ${ }^{124} \mathrm{Sb}$, inhaled as aerosols produced at different temperatures and with different AMADs, were obtained in studies with 9 dogs (Felicetti, 1974). Particles produced at higher temperatures were smaller and were retained more tenaciously. After lung, highest concentrations were retained in the thyroid.

\subsubsection{IODINE}

A detailed study of ${ }^{131} \mathrm{I}$ distribution and retention was conducted in $30 \mathrm{dogs}$, involving inhalation at 6,18 and 48 months of age, and ingestion and intravenous injection at 18 months of age (Foreman, 1969). Distribution and retention were followed for 8 days by whole-body counting, thyroid monitoring and periodic autopsy. Neither age- nor route-related effects were observed. The influence of age on thyroidal ${ }^{131}$ I uptake in beagle pups was described (Book, 1976), as were age-related changes in serum thyroxine and ${ }^{125}$ I-triiodo-thyronine resin sponge uptake in the young $\operatorname{dog}(B o o k, 1977)$.

\subsubsection{BARIUM}

A detailed kinetic model for the distribution and retention of inhaled barium compounds was derived from a series of experiments involving inhalation of a number of ${ }^{133} \mathrm{Ba}$ - or ${ }^{140} \mathrm{Ba}$-labeled compounds (Cuddihy, 1972, 1974, 1974b). The skeleton received the highest radiation dose from inhaled ${ }^{140} \mathrm{BaCl}_{2}$; lung would receive a substantially higher dose from inhalation of less soluble barium compounds.

\subsubsection{LANTHANUM}

A detailed kinetic model for the distribution and retention of inhaled ${ }^{140} \mathrm{LaCl}_{3}$ was derived from an experiment with 14 dogs sacrificed at intervals to 8 days postexposure; additional animals were exposed by injection and by gavage (Cuddihy, 1970). Markedly different distribution patterns were obtained 
following different routes of administration. The highest radiation dose following inhalation of ${ }^{140} \mathrm{La}$ was received by the nasal turbinates, followed in decreasing order by lung, gastrointestinal tract, liver, skeleton and bronchial lymph nodes.

\subsubsection{CERIUM}

Data on the acute and chronic effects of inhaled ${ }^{144} \mathrm{CeO}_{2}$ were reported from an experiment involving 15 animals exposed to aerosols of various particle sizes and dose levels and followed for 21 months (Stuart, 1964). Depressed lymphocyte levels were the earliest and most sensitive indicator of toxicity. Liver and skeleton were the principal sites of systemic deposition, but highest concentrations of ${ }^{144} \mathrm{Ce}$ were found in pulmonary lymph nodes.

\subsubsection{LEAD}

Studies of ${ }^{210} \mathrm{~Pb}$ distribution and retention were conducted in 10 beagles, some of whom were observed for as long as 1600 days (Lloyd, $R$., 1975e). Results of this experiment are of interest for what they tell us about the metabolism of lead, and also because ${ }^{210} \mathrm{~Pb}$ was a contaminant in the Utah ${ }^{226} \mathrm{Ra}$ and ${ }^{228} \mathrm{Th}$ life-span studies, and data on ${ }^{210} \mathrm{~Pb}$ distribution, retention, and effects, are necessary to the interpretation of these life-span experiments. Of the injected ${ }^{210} \mathrm{~Pb}, 40 \%$ remained in the blood after 1 day and 3\% after 1 month, with more than 99\% associated with red cells. Skeleton was the principal site of deposition, but concentration in liver was higher during early times following injection. About 70\% of the injected ${ }^{210} \mathrm{~Pb}$ was lost from the body with a half-life of 12 days, $17 \%$ with a half-life of about 180 days, and $13 \%$ with a half-life of about 14 years. Data are presented on the distribution of ${ }^{210} \mathrm{~Pb}$ among different bone types (Lloyd $R$., 1975e). Additional in-vivo and in-vitro studies with ${ }^{210} \mathrm{~Pb}$ in beagles, and comparative in-vitro studies with human blood, have provided information on the association of lead with constituents of erythrocytes (Bruenger, 1973).

Data from only 3 dogs, on the time of appearance of bone tumors following injection of ${ }^{210} \mathrm{~Pb}$, suggest that its toxicity is similar to that of injected ${ }^{226} \mathrm{Ra}$ (Lloyd, R., 1975e).

\subsubsection{RADON}

An extensive program of studies at PNL, jointly supported by AEC/ERDADOE and by the National Institute of Environmental Health Sciences (NIEHS), investigated the effect of various components of uranium mine atmospheres on several animal species (Cross, 1981, 1982, 1986; Filipy, 1974; Palmer, 1975; Park, 
J., 1971; Stuart 1970b, 1971, 1977). As a part of this program, 69 dogs inhaled, 5 days per week, for periods of up to 54 months, various combinations of radon and radon daughters, uranium-ore dust, and the smoke from individually smoked cigarettes. Contrary to reported behavior in uranium miners, thorium isotopes from the ore dust were more tenaciously retained in the lungs of these dogs than were uranium isotopes (Singh, 1986).

Survival time was shortened in dogs whose exposures included radon and daughters and uranium-ore dust; smoking had no effect on the survival of either exposed or control animals. Pulmonary fibrosis was produced in all animals inhaling radon and daughters with uranium ore dust. A variety of respiratory tract tumors were produced in the exposed animals, but were less prevalent in smoking than in nonsmoking animals; this protective effect of cigarette smoke was not clarified by histologic studies (Gies, 1987). Additional experiments are under way to distinguish between the effects of radon and daughters, and the effects of uranium-ore dust (Cross, 1981).

\subsubsection{URANIUM}

Experiments were conducted at the University of Rochester on the retention and biological effects of natural $\mathrm{UO}_{2}$ dust, chronically inhaled over periods of up to 5 years, by rats, monkeys, and dogs (Leach, 1970). Although the animals were not maintained for life-span observation, these experiments were of particular importance because they provided the experience drawn upon in many later life-span experiments at other laboratories. With cumulative radiation doses after 5 years of about 500 rad to lung and 10,000 rad to tracheobronchial lymph nodes, no effects were observed other than occasional fibrotic changes in lymph nodes. Doses to skeleton, kidney, liver, and spleen were on the order of 0.1 to 1.0 $\operatorname{rad}($ Leach, 1970).

Experiments involving chronic inhalation of uranium ore dust by beagles at PNL have resulted in more dramatic effects than those observed in the Rochester experiments, due perhaps to longer periods of exposure. The most notable pulmonary lesions observed, in experiments still in progress, include vesicular emphysema, peribronchiolitis and focal pneumoconiosis (Cross, 1981).

Early distribution and effects of inhaled ammonium diuranate and $\mathrm{U}_{3} \mathrm{O}_{8}$ powders have been described for periods of up to 180 days following exposure of 2 groups of 10 beagles, and the results have been applied to the interpretation of human excretion data (Eidson, in press). 
The distribution and retention of hexavalent ${ }^{233} \mathrm{U}$ was studied in 7 beagles, injected intravenously, and sacrificed at intervals from 1 to 726 days postinjection (Stevens, W. , 1980). Uranium was lost rapidly from blood, where it was present as a transferrin-albumin, or carbonate-bicarbonate complex. About $65 \%$ was excreted the first day, mostly in urine; less than $5 \%$ was retained at 2 years. Kidneys contained $22 \%$ at 1 day postinjection. The skeleton contained $7.7 \%$ at 1 day postinjection, where it was retained with a half-time of 883 days.

Studies involving the inhalation of uranium-ore dust in combination with radon are considered in Section 6.7.11; studies involving the inhalation of mixed oxides of uranium and plutonium are considered in Section 6.7.13.

\subsubsection{PLUTONIUM}

The acute toxicity of inhaled ${ }^{238} \mathrm{PuO}_{2}$ was studied in an early experiment at PNL, involving 16 beagles and supported by the Air Force Weapons Laboratory (Park, $J ., 1969$ ). Effects were similar to those observed in the high dose levels of the inhaled ${ }^{239} \mathrm{PuO}_{2}$ life-span experiments, except that lesions in the skeleton were more prominent in the ${ }^{238} \mathrm{PuO}_{2}$-exposed dogs. This study was of particular importance in first demonstrating the unanticipated dissimilarity in distribution of inhaled ${ }^{238} \mathrm{PuO}_{2}$ and ${ }^{239} \mathrm{PuO}_{2}$.

Distribution, retention and excretion of ${ }^{239} \mathrm{Pu}$, inhaled as ${ }^{239} \mathrm{PuF}_{4}$, was studied over a period of 85 days, in 6 dogs, half of them treated with repeated injections of Ca-DTPA, which was not effective in promoting excretion (McDonald, 1979).

The distribution of very low-level concentrations of ${ }^{239} \mathrm{Pu}$ in the tissues of a Utah control beagle has been reported (Singh, 1987); the source of this plutonium was unknown, and its distribution, particularly among different bones of the skeleton, was quite irregular.

The metabolism and dosimetry of inhaled mixed oxides of plutonium and uranium, of a type used in nuclear fuel, was followed in a 6.5-year study employing beagle dogs, rats and cynomolgus monkeys. Substantial differences were observed in the behavior of uranium and plutonium both within and between species. The behavior of plutonium was little influenced by the presence of the uranium oxide (Mewhinney, 1984; Stanley, 1982).

Adult beagle dogs have been employed in experiments studying the absorption from the gastrointestinal tract of ${ }^{238} \mathrm{Pu}\left(\mathrm{NO}_{3}\right)_{4}$. The fraction absorbed was on the order of $3 \times 10^{-4}$, which was similar to results obtained with adult rats and guinea pigs (Sullivan, 1980). 
Beagles have been employed in the development of techniques for the whole-body counting of inhaled plutonium and americium in humans (Swinth, 1967, 1968), including the use of intraesophageal probes for measuring plutonium in tracheobronchial lymph nodes (Swinth, 1972b, 1976). They have also been employed in the development of pulmonary lavage techniques potentially applicable to the bioassay of plutonium in the lung (Guilmette 1986).

\subsubsection{AMERICIUM}

The distribution and retention of ${ }^{241} \mathrm{Am}$ has been followed in several experiments involving inhalation of the oxide, and results have been compared with data from other species (Mewhinney, 1981). The first of these experiments involved 5 animals, sacrificed at intervals to 1022 days postexposure (Thomas, $1970,19726)$. Another involved 15 dogs, exposed at 3 dose levels, and sacrificed at 5 time intervals to 810 days postexposure (Craig, 1979). A third experiment involved $52 \mathrm{dogs}$, exposed to 3 sizes of monodisperse particles and a polydisperse aerosol, with sacrifices at 7 time intervals to 730 days postexposure (Mewhinney, 1981, 1982). The 3 experiments resulted in somewhat different rates of lung clearance, presumably due to differences in the chemical and physical state of the oxide inhaled; clearance of smaller particles was more rapid than that of larger particles. Although clearance from the lung was, in all studies, more rapid than that of plutonium oxide, a long-term retention component resulted in greater dose to lung than predicted by the "Class W" designation of the ICRP lung model (Mewhinney, 1981, 1982). Systemic deposition and retention were similar to those observed following injection of americium, and quite different from those of inhaled plutonium. Hematologic and pathologic effects were also reported for some of these dogs (Thomas, 1972b), including the production of osteosarcomas in 4 out of 15 animals sacrificed at times greater than 1000 days postexposure (Gillett, 1985).

Preliminary data from inhalation experiments with juvenile and aged beagles indicate an effect of age on ${ }^{241} \mathrm{Am}$ clearance from the lung and on relative quantities excreted and translocated to liver and skeleton (Mewhinney, in press).

\subsubsection{CURIUM}

Short-term experiments on the distribution and retention of curium have been conducted following inhalation (McClellan, 1972b; Guilmette, in press) and following injection (Lloyd, R., 1974).

The curium injection experiment involved 5 dogs studied over a period of 57 days. Early excretion was mainly via the urine. About $35 \%$ of injected ${ }^{243,244} \mathrm{Cm}$ 
was deposited in liver, and about $53 \%$ in nonliver tissues, principally skeleton; significant concentration was also noted in the thyroid and kidney (Lloyd, $R$., $1974,1975 d)$.

In an early inhalation experiment, 24 dogs inhaled aerosols of either ${ }^{244} \mathrm{CmCl}_{3}$ or ${ }^{244} \mathrm{CmO}_{1.73}$ and were sacrificed at intervals to 256 days postexposure; both forms were relatively rapidly cleared from the lung, with about $3 \%, 30 \%$, and $45 \%$ retained in lung, liver, and skeleton, respectively, at 256 days postexposure (McClellan, 1972b). A later inhalation experiment, involving 32 dogs, compared the distribution and retention of curium over time intervals to 2 years following inhalation of the oxide or the nitrate (Guilmette, in press). The nitrate was cleared from the lung with a half-time of about 0.6 day, as compared to a halftime of about 8 days for the oxide. A detailed biokinetic model has been developed for curium in the beagle based on these data and on data from inhalation of ${ }^{241} \mathrm{Am}$ (Guilmette, in press b).

\subsection{STUDIES OF NON-RADIATION-RELATED TOXICOLOGICAL AND DISEASE PROCESSES}

A number of toxicological studies and investigations of disease processes, unrelated to radiation exposure, have been undertaken because of their potential influence on the life-span radiation effect experiments, or because materials from the life-span experiments made such studies possible.

The pathology and familial incidence of lymphocytic thyroiditis was studied in more than 400 dogs of the Argonne beagle colony. The condition, resembling Hashimoto's disease in man, occurred spontaneously in nearly $20 \%$ of untreated animals over 1 year of age; the incidence was clearly genetically related (Fritz, $1970 b$ ). Experiments with ${ }^{131}$ I showed lowered and earlier uptake, and shortened retention in dogs with severe thyroiditis (Fritz, 1970c). Other experiments investigated the effect of dietary iodide on tracer ${ }^{131}$ I uptake in irradiated (Fritz, 1972 ) and nonirradiated (Norris, 1970) animals.

Spontaneous lymphocytic orchitis, culminating in infertility, exhibited a high incidence in aging dogs of the Argonne colony and was shown to be correlated with thyroiditis and genetic factors (Fritz, 1971, 1976). These and other autoimmune diseases that occur in the beagle, make it a useful animal model for study of this little-understood group of diseases (Fritz, 1980).

A case of hypothyroidism in a stud dog of the ITRI breeding colony led to extensive investigation of its possible heritability, with negative findings (Pickrell, 1970). 
The distribution of bone calcium between morphologically identifiable cortical and trabecular bone in the beagle skeleton has been studied employing techniques of neutron activation analysis (Parks, 1986), yielding information of critical importance to the interpretation of radiation dose-effect relationships in bone. Bone turnover rates varying from $20 \%$ to $200 \%$ per year were measured in double-tetracycline-labeling studies of 36 regions of trabecular bone; these rates in the young-adult beagles were two to three times those that have been measured in young-adult humans (Kimmel, 1982).

Studies of joint disease in aging beagles have been reported in several papers (Carrig, 1974, 1975b; Morgan, J., 1986b). The normal range of motion of the cervical spine of beagles was determined in a radiographic study (Morgan, J., 1986). Mechanical and morphological evaluations of age-related changes in the beagle spine may improve understanding of human intervertebral disc disease (Gillett, in press $b$ ). Bone abnormalities associated with hypergravity exposure have also been studied (Amtmann, 1976; Morgan, J., 1979).

The incidence and distribution of spirochetes in the digestive tract of beagles were investigated (Pindak, 1965). Human enteric viruses were isolated from beagles (Pindak, 1964); beagles free of the virus (Echo Type 6) were experimentally infected and showed symptoms of enteric disease (Pindak, 1966). Parvoviral outbreaks at Argonne (Binn, 1981; Fritz, 1979) and ITRI (Mason, 1987; Merickel, 1980) have resulted in clinical, pathological and epidemiological studies of canine parvoviral enteritis. Gastric (Miyabayashi, 1984) and small intestinal (Miyabayashi, 1986) emptying times have been studied in normal beagles, employing a contrast radiographic technique.

Extensive clinical, histochemical and ultrastructural studies of corneal opacities in beagles have been reported (Spangler, 1982; Waring, 1977, 1978, 1979, 1979b).

Using an electronmicroscope, the juxtaglomerular apparatus of the beagle kidney has been studied in the normal animal (Spangler, 1979), as has its pathophysiologic response in animals with restricted sodium intake (Spangler, 1979b). Light- and electronmicroscopic studies of gentamicin nephrotoxicity have also been reported (Spangler, 1980).

Studies have been reported on the pharmacokinetics of ${ }^{14} \mathrm{C}$-labeled "Paraquat," a widely employed nonselective herbicide, including associated biochemical and pathological changes following intravenous administration to beagles (Giri, 1982, 1983). 
Data have been reported on the effect of disease processes, including neoplasia, on the behavior of copper and zinc (Fisher, G., 1977, 1977b), and of vanadium (Harris, 1984), in the blood of beagles.

A case of pituitary-dependent hyperadrenocorticism (Cushing's disease), complicated with pulmonary embolism, was reported, with extensive clinical data, including pulmonary function studies (King, 1985).

The incidence of epilepsy in certain colonies has resulted in several neurological studies (Bielfelt, 1971; Redman, 1969, 1972b). 


\section{APPLICATIONS}

\begin{tabular}{|c|c|c|}
\hline \multicolumn{3}{|c|}{ Contents } \\
\hline 7.1 & Evaluation of "Fallout" Consequences & 107 \\
\hline 7.2 & Evaluation of the "Hot Particle" Hypothesis & 107 \\
\hline 7.3 & Development of Bioassay Models & 108 \\
\hline 7.4 & Estimation of Relative Biological Effectiveness & 109 \\
\hline 7.5 & Development of Exposure Limits $\ldots . . . \ldots \ldots$. & 111 \\
\hline 7.6 & Clinical Applications to Man ......... & 112 \\
\hline 7.7 & Estimation of Dose and Risk in Man & 113 \\
\hline 7.8 & Contributions to Theories of Carcinogenesis & 118 \\
\hline 7.9 & Contributions to the Basic Biology of Bone & 121 \\
\hline 7.10 & Applications to be Expected in the Future .... & 121 \\
\hline
\end{tabular}

In this chapter attention is directed to the applications that have been made of results from the life-span beagle experiments and supporting and ancillary studies, as these applications are reflected in the scientific literature. The categories employed in considering these applications are necessarily broad and somewhat arbitrary. In some cases the applications were of such a general nature that attempts at categorization seemed inappropriate. These very general applications are considered at the beginning of this chapter.

Results from these experiments have been employed in numerous attempts to review and integrate our state of knowledge in critical areas of concern for human radiation risks. Very early examples were papers by Langham, who drew upon the dog studies in reviewing the physiology and toxicology of plutonium (Langham, 1959, 1964), including sensitive considerations of the possible effects of nonnuclear incidents involving nuclear warheads (Langham, 1969). Also notable was the early report of the National Academy of Sciences Effects of Inhaled Radioactive Particles (1961), and the paper by Bair, "Inhalation of Radionuclides and Carcinogenesis," which enumerated the clear evidence that inhaled radionuclides are indeed carcinogenic (Bair 1970).

An early review by Janet Vaughan on the nonuniformity of radiation dose from deposited radionuclides in a number of tissues, and the influence of this nonuniformity on radiological protection considerations, employed extensive data from the early dog experiments (Vaughan, 1965), as did her later definitive reviews on plutonium biology (Vaughan, 1973, 1973b). Also notable were reviews by Thompson on the metabolism of radionuclides in general (Thompson, 1960) 
and plutonium in particular (Thompson, 1967). Other early examples include the review by Betsy Stover comparing the metabolism of bone-seeking radionuclides (Stover, 1959), papers by David Taylor reviewing the metabolism of the transuranic elements (Taylor, D., 1964) and their interactions with components of cells and tissues (Taylor D., 1972, 1972b), and papers by Thomas reviewing the influence of aerosol properties on transport from lung to lymph nodes (Thomas, 1968), and on other aspects of radionuclide distribution and retention (Thomas, 1964).

A paper coauthored by representatives from the major laboratories involved in life-span dog studies evaluated "long-term effects of exposure to internally deposited radionuclides" at the United Nations Conference on Peaceful Uses of Atomic Energy (Bustad, 1972). A comprehensive review of the comparative metabolism of radionuclides in a number of animal species derived extensive data from life-span dog experiments (Stara, 1971). Dolphin, in 1971, reviewed the biological problems involved in the protection of workers exposed to ${ }^{239} \mathrm{Pu}$, drawing heavily on early results from life-span dog experiments (Dolphin, 1971). Volume 36 of the Handbook of Experimental Pharmacology: Uranium, Plutonium, Transplutonic Elements includes important chapters on "Plutonium in Soft Tissues with Emphasis on the Respiratory Tract" (Bair, 1973), and "Metabolism and Biological Effects of the Transplutonium Elements" (Durbin, 1973), both of which derive extensive data from the life-span dog experiments.

A Science feature article by Bair and Thompson on Plutonium: Biomedical Research, appearing at the height of public concern over plutonium in fallout, was based largely upon results of life-span dog experiments (Bair, 1974), as were several other contemporary review articles (Bair, 1974b: Durbin, 1975; Edsall, 1976; Thompson, 1975, 1977). An influential publication by the Medical Research Council, in England, The Toxicity of Plutonium, featured data from the lifespan dog experiments (Medical Research Council, 1975). A publication of the Commission of the European Communities, Toxicity of Plutonium, Americium and Curium, drew heavily on the life-span beagle experiments (Nenot, 1979), as did a recent review of health effects from internally deposited radionuclides released in nuclear disasters (McClellan, 1982).

The United Nations Scientific Committee on the Effects of Atomic Radiation has made extensive use of the data from life-span beagle experiments in its periodic reviews of the biological effects of ionizing radiation $(1972,1977,1982)$, as has also the National Academy of Science's Committee on the Biological Effects of Ionizing Radiations (1988). 


\subsection{EVALUATION OF "FALLOUT" CONSEQUENCES}

The inclusion of ${ }^{90} \mathrm{Sr}$ among the radionuclides employed in life-span dog experiments was due largely to concern for the possible effect on populations of the presence of that radionuclide in worldwide fallout from atmospheric testing of nuclear weapons. Early beagle data on the gastrointestinal absorption, retention, and excretion of ${ }^{90} \mathrm{Sr}$, and its behavior relative to calcium, particularly as influenced by age, were employed in the evaluation of possible fallout consequences (Andersen, 1963h, Della Rosa, 1961, 1965), and in the assessment of ${ }^{90} \mathrm{Sr}$ body burdens based on measurements in hair, teeth, and excreta (Della Rosa, 1966, 1968). Useful data were also obtained on the effects of gestation and lactation on ${ }^{90} \mathrm{Sr}$ kinetics, in the dam and in the offspring (Della Rosa, 1968, 1968b, 1969), and on the behavior of inhaled ${ }^{90} \mathrm{Sr}$ (McClellan, 1972c). Data from the Davis ${ }^{90} \mathrm{Sr}$ chronic feeding experiment were reviewed, in respect to their applicability to man, with the conclusion that similar ${ }^{90} \mathrm{Sr} / \mathrm{Ca}$ ratios in the diet of dog and man would result in about a 4 -fold higher dose rate in the dog to critical cells at risk in the skeleton (Della Rosa, 1972).

Data on the effects of ${ }^{90} \mathrm{Sr}$, as observed in life-span dog experiments, were influential in allaying unreasonable fears of fallout consequences (Mays, 1959, 1972c, 1972d). Life-span studies with ${ }^{137} \mathrm{Cs}$ in beagles provided a major input to the evaluation of the potential consequences of its presence in fallout (NCRP, 1977). The topic of animal studies in relation to fallout has been reviewed (Book, 1983b).

\subsection{EVALUATION OF THE "HOT PARTICLE" HYPOTHESIS}

The issue was raised in 1974 that plutonium, when present as critically sized insoluble particles, was unusually toxic-the so-called "hot particle" hypothesis (Bair, 1977; Gillette, 1974; Gofman, 1975, 1975b, 1976; Lovins, 1975; Morgan, K., 1975; Tamplin, 1974, 1974b, 1974c). These extreme claims were effectively countered by experimental data, much of it derived from life-span dog experiments, which were presented in U.S. Atomic Energy Commission documents (Bair, 1974c, 1974e; Healy, 1974), in publications of the National Council on Radiation Protection and Measurements (1975), of the National Academy of Sciences (1976), of the Medical Research Council in England (1975), of the National Radiological Protection Board in England (Dolphin, 1974), of the German Ministry of the Interior (Bonn) (1976), and in numerous other publications (Bair, 1974, 1974b, 1974f, 1976; Cuddihy, 1982; Dagle, 1984; Diel, 1983b; McClellan, 1986; Richmond, 1976; Thompson, 1975, 1975b, 1977). These analyses demonstrated that particulate exposure was probably less hazardous than a more homogeneous exposure, and led to the denial, by the U.S. Nuclear Regulatory Commission, of a Natural Resources Defense Council petition for rule making (USNRC, 1976). 
The "hot particle" controversy also led to the initiation of a series of life-span beagle experiments at ITRI, employing monodisperse ${ }^{238} \mathrm{PuO}_{2}$ and ${ }^{239} \mathrm{PuO}_{2}$ aerosols, designed to definitively demonstrate the effect of particle size on tumor incidence. Preliminary data from the ${ }^{239} \mathrm{PuO}_{2}$ experiments indicate lung cancer risk factors for the larger particles that are no greater than those for the smaller particles, thus contradicting the "hot particle" hypothesis. (Boecker, 1988; Muggenburg, in press).

\subsection{DEVELOPMENT OF BIOASSAY MODELS}

Distribution, retention, and excretion data from life-span beagle experiments have been employed in the development of models useful for estimating the radionuclide content of the body, or the radiation doses to organs of exposed humans, based on excreta data, or other postexposure measurements. Detailed comparison of beagle and human excretion of plutonium at long time periods following injection was instrumental in detecting errors in the human data, and in the development of an improved human excretion model (Moss, in press). Early data from plutonium inhalation studies in beagles were used to test the performance of bioassay computer codes designed for application to humans (Snyder, 1964); they were also used to demonstrate the inadequacy of bioassay models based solely on data from injected plutonium (Robertson, 1964). More recently, data from a variety of sources, including life-span dog experiments, have been employed in the development of a detailed retention-excretion model for systemic plutonium (Leggett, 1984c, 1985, 1987). Data from studies with inhaled plutonium in beagles have been employed in evaluating the impact of isotopic effects on the interpretation of human bioassay data (Ryan, 1987).

Early studies with inhaled ${ }^{241} \mathrm{AmO}_{2}$ in a few beagles were conducted as an approach to solving some of the practical problems that arise in estimating the ${ }^{241} \mathrm{Am}$ content of exposed humans (Thomas, 1970). Available data from dogs, rats, cynomolgus monkeys, baboons, and human exposure cases, have been employed in developing a model relating excretion rates to body burden of ${ }^{241} \mathrm{Am}$ (Griffith, 1983). A similar biokinetic model has been derived from beagle data on curium distribution and retention and has been applied to the interpretation of human exposure data (Guilmette, in press b).

Data on the skeletal distribution and toxicity of ${ }^{239} \mathrm{Pu}$ in beagles have been employed in the selection of appropriate samples for analysis from humans coming to autopsy after known plutonium exposures (Jee, 1981). 


\subsection{ESTIMATION OF RELATIVE BIOLOGICAL EFFECTIVENESS}

Data on the comparative toxicity of different radionuclides in animals is vital to the extrapolation of the limited human toxicity data, in order to estimate effects from radionuclides for which there are no human data. The Utah life-span beagle studies with ${ }^{226} \mathrm{Ra}$ and ${ }^{239} \mathrm{Pu}$ were primarily designed to provide data on the relative biological effectiveness (RBE) of these two radionuclides, so that the human radium data could be used to estimate effects of plutonium in man. Many of the other life-span dog experiments afford similar opportunities for evaluation of RBEs for application in the estimation of human hazards.

The Utah injection experiments have been utilized in many attempts to estimate RBEs. An early attempt, based on average dose to bone, and bone cancer data to 8 years postinjection, led to $\mathrm{RBE}$ values $\left({ }^{226} \mathrm{Ra}=1\right)$ of: ${ }^{239} \mathrm{Pu}, \sim 6 ;{ }^{228} \mathrm{Th}, \sim 8 ;{ }^{228} \mathrm{Ra}$, $\sim 2.5$; and ${ }^{90} \mathrm{Sr}, 0.07$ to 0.24 (Dougherty T., 1969; Mays, 1969). Later evalulation of the Utah data, with nearly all life-span animals dead, indicated a ${ }^{90} \mathrm{Sr} /{ }^{226} \mathrm{Ra}$ toxicity ratio as low as 0.04 at low dose levels (Mays, 1980b), and values for alpha emitters of $16.6 \pm 4.5$ for ${ }^{239} \mathrm{Pu}, 8.5 \pm 2.3$ for ${ }^{228} \mathrm{Th}, 2.0 \pm 0.5$ for ${ }^{228} \mathrm{Ra}$, and $5.4 \pm 1.6$ for ${ }^{241} \mathrm{Am}$ (Lloyd, R., 1986; Mays, 1987).

Comparison of the bone tumor response data from the Davis chronic ${ }^{90} \mathrm{Sr}$ feeding experiment and from the ${ }^{226} \mathrm{Ra}$ repeated-injection experiment, employing a logistic dose-time-effect model, indicated $R B E$ values for ${ }^{90} \mathrm{Sr}\left({ }^{226} \mathrm{Ra}=1\right)$ varying from 1.5 at average dose rates to bone of $16 \mathrm{rad} /$ day, to 0.05 at dose rates of 1.6 $\mathrm{rad} /$ day (Raabe, 1981, 1983). A similar computational approach, employing data from 9 life-span beagle experiments, resulted in RBEs for bone tumor production of 3.0 for ${ }^{226} \mathrm{Ra}$; 6.4 for ${ }^{241} \mathrm{Am},{ }^{249} \mathrm{Cf}$, and ${ }^{252} \mathrm{Cf}$; 9.0 for ${ }^{239} \mathrm{Pu} ; 10.7$ for ${ }^{228} \mathrm{Th}$; and 15.5 for ${ }^{238} \mathrm{Pu}$ (Raabe, 1984, 1986, 1986b; Wrenn, 1986c).

With bone cancer as the effect of interest, and on an average-dose-to-bone basis, Dolphin calculated ${ }^{239} \mathrm{Pu}$ to be about 8 times as toxic as ${ }^{226} \mathrm{Ra}$; with appropriate assumptions regarding distribution within bone, and calculating dose to osteoprogenitor cells near bone surfaces, the relative toxicity was close to unity (Dolphin, 1978). The Medical Research Council, in England, concluded from the life-span dog data that a factor of 5 would account for differences in the effectiveness of ${ }^{239} \mathrm{Pu}$ and ${ }^{226} \mathrm{Ra}$ in humans (Medical Research Council, 1975). Several other approaches to a bone cancer RBE, based on the Utah data, have been explored (Gridgeman, 1971; Marshall, 1969). Lloyd and Marshall evaluated differences in the structure and physiology of bone in humans and beagles, and concluded that the relative toxicity of ${ }^{239} \mathrm{Pu}$ to ${ }^{226} \mathrm{Ra}$ should be higher in adult humans than in young-adult beagles (Lloyd, E., 1972; Marshall, 1973). 
Applying a proportional hazard model to the average skeletal dose data from the Utah single-injection studies, an RBE for bone cancer induction ( ${ }^{226} \mathrm{Ra}=1$ ) of 8.6 was calculated for ${ }^{239} \mathrm{Pu}$, and of 0.18 for ${ }^{90} \mathrm{Sr}$; an identical RBE was calculated for inhaled ${ }^{90} \mathrm{Sr}$ (ITRI data), and a value of 5.5 for inhaled ${ }^{238} \mathrm{PuO}_{2}$ (ITRI data) (Mewhinney, 1986).

Toxicity data from the life-span experiments with ${ }^{252} \mathrm{Cf}$, half of whose decay energy is contained in fission fragments, and with ${ }^{249} \mathrm{Cf}$, which shows no significant spontaneous fission, can be used to estimate an RBE for fission fragments relative to alpha particles. Incomplete data suggest that this $\mathrm{RBE}$ is very much smaller than 1 , and that fission fragments are, in fact, very inefficient carcinogenic agents (Lloyd, R., 1976; Taylor, G., 1983b).

In terms of radiographically detectable skeletal change by 10 years of age in the Davis experiments, a 23 -fold greater total dose of ${ }^{90} \mathrm{Sr}$ than of ${ }^{226} \mathrm{Ra}$ was required to produce a comparable effect (Momeni, 1976b). This ratio held only at the lowest effective dose levels, however, since the dose-effect relationships for both radionuclides were markedly nonlinear (Momeni, 1976d).

Hematologic effects have been used as an endpoint for estimation of RBEs. Defined as the average dose rate to bone from ${ }^{226} \mathrm{Ra}$ required to produce a $50 \%$ depression in blood cells, divided by the average dose rate to bone from the radionuclide in question required to produce the same $50 \%$ depression, values were: for ${ }^{228} \mathrm{Th}$, $\sim 4$; for ${ }^{239} \mathrm{Pu}, \sim 4$; for ${ }^{228} \mathrm{Ra}, \sim 2$; and for ${ }^{90} \mathrm{Sr}, \sim 0.5$ (Dougherty, J., 1970, 1971).

Early mortality from single inhalations of radionuclides have been analyzed by comparison of the lethal cumulative dose to lung tissue. The RBE for such early mortality, normalized to a value of 1 for inhaled ${ }^{90} \mathrm{Sr}$ FAP, was 14 for inhaled ${ }^{239} \mathrm{PuO}_{2}$ and 5 for inhaled ${ }^{238} \mathrm{PuO}_{2}$; the differing $\mathrm{RBEs}$ for the two plutonium isotopes being attributed to the less uniform irradiation by ${ }^{238} \mathrm{Pu}$ because of its much higher specific activity (Raabe, 1979). Another approach to the evaluation of early-mortality RBEs for inhaled radionuclides led to values ( ${ }^{90} \mathrm{Sr} F A P=1$ ) of: 7.0 for ${ }^{239} \mathrm{Pu}, 5.0$ for ${ }^{90} \mathrm{Y} \mathrm{FAP}, 2.5$ for ${ }^{91} \mathrm{Y} \mathrm{FAP}$, and 1.1 for ${ }^{144} \mathrm{Ce}$ FAP (Scott, 1979).

The RBE for induction of bronchioloalveolar tumors by inhaled alpha- as compared to beta-emitting particles appears to fall in the range of 4 to 5 , which contrasts with the RBE of about 10 for induction of bronchioloalveolar tumors by inhaled alpha emitters as compared to the induction of hemangiosarcomas by inhaled beta emitters ( $H a h n, 1977)$. Overall, the risk of pulmonary tumors from high-LET irradiation, for the period from 4 to 6 years postinhalation, is about 10 times that from an equal rad dose from low-LET irradiation, but this reflects only data from relatively high exposures (Hahn, 1986). A specific comparison of 
lung-cancer risk factors for inhaled ${ }^{91} \mathrm{Y}$ (experiment completed) and inhaled ${ }^{239} \mathrm{PuO}_{2}$ (experiments in progress) resulted in estimated ${ }^{91} \mathrm{Y} /{ }^{239} \mathrm{PuO}_{2}$ risk-factor ratios of 18,15 , and 10 for $0.75-, 1.5-$, and $3.0-\mu \mathrm{m}{ }^{239} \mathrm{PuO}_{2}$ particles, respectively (Boecker, 1988).

\subsection{DEVELOPMENT OF EXPOSURE LIMITS}

The RBEs discussed above are important to the derivation of "quality factors" employed in the development of exposure limits. In addition, data on the distribution and retention of radionuclides in the beagle have been used to develop kinetic models for application in estimating dose to humans. Of the models employed by the ICRP in their derivation of limits for radionuclide intake (ICRP, $1979,1980 b, 1981$ ), those for 12 elements employ critical parameter values obtained primarily from studies with beagle dogs; for another 33 elements, values for critical parameters were based on experimental animal data and/or human data that included comparative studies in dogs. Application of life-span dog data to such derivation of protection standards has been specifically considered in a number of publications, which are listed in Table 7.1.

Table 7.1 PUBLICATIONS THAT APPLY DATA FROM LIFE-SPAN DOG STUDIES TO THE DERIVATION OF PROTECTION STANDARDS

\begin{tabular}{|c|c|}
\hline Element & References \\
\hline Strontium & Blair, 1972; Hems, 1966 \\
\hline Barium & Cuddihy, 1972 \\
\hline Cesium & NCRP, 1977 \\
\hline Cerium & Boecker, 1974b; Cuddihy, $1976 b$ \\
\hline Zirconium & Waligora, 1971 \\
\hline Niobium & Cuddihy, 1978 \\
\hline Ruthenium & Snipes, 1981 \\
\hline Barium & Cuddihy, 1972 \\
\hline Lanthanum & Cuddihy, 1972 \\
\hline Radium & Wrenn, 1985 \\
\hline Uranium & Wrenn, 1985 \\
\hline Plutonium & $\begin{array}{l}\text { Bair, 1974, 1974c, 1974f, 1976; Dolphin, 1971, 1972; Durbin, } \\
\text { 1976; Gavankar, 1972, 1974; Gofman, 1975, 1976; Guilmette, } \\
\text { 1984; Harley, 1976; Healy, 1974; Hems, 1966; ICRP, 1972; Jee, } \\
\text { 1980; Langham, 1959, 1952; Leggett, 1984, 1984b, 1985; Long, } \\
\text { 1971; Mays, 1976b; Medical Research Council, 1975; Mewhinney, } \\
\text { 1983; Morgan, K., 1975; Norwood, 1959; Park, J., 1981; } \\
\text { Richmond, 1976; Robertson, 1964; Schofield, 1974; Spiers, } \\
\text { 1976b; Thomas, 1972c; Thompson, 1972, 1974, 1974b, 1975, } \\
\text { 1975b }\end{array}$ \\
\hline Americium & Leggett, 1984b; Mewhinney, 1980, 1981, 1982, 1982b, 1983b \\
\hline Curium & Guilmette, in press; Leggett, $1984 b ;$ McClellan, $1972 b$ \\
\hline Californium & Lloyd, R., 1976 \\
\hline
\end{tabular}


Data from dog studies have also been employed in the development or refinement of generic organ-system models employed by the ICRP and NCRP. Thus, the ICRP in its Review of the Radiosensitivity of the Tissues in Bone (ICRP, 1968) drew heavily on early data from life-span dog experiments. The improved dosimetric model for bone employed in the derivation of ICRP's current limits on radionuclide intake (ICRP, 1979), which distinguishes between deposition in cortical and trabecular bone, has received important validation from the life-span dosimetric studies on ${ }^{226} \mathrm{Ra}$ (Parks, 1980) and ${ }^{90} \mathrm{Sr}$ (Parks, 1985).

Data from beagles on the deposition, clearance, and observed effects of a number of inhaled radionuclides were used extensively in the ICRP's recent review of Biological Effects of Inhaled Radionuclides (ICRP, 1980); they have also been used in support of suggested modifications to models of the human respiratory tract (Cuddihy, 1972, 1979, 1981; Guilmette, 1984, 1987; Hahn, 1986; McClellan, 1979; Mewhinney, 1980, 1981, 1982, 1982b, 1983, 1983b; Park, J., 1981; Snipes, 1983, 1984b; Thomas, 1972, 1972c; Watts, 1975).

Other data from beagle studies point to problems with current dosimetric models for bone and liver (Cuddihy, 1981). Computer simulation of bone-cell ${ }^{239} \mathrm{Pu}$ disintegration interactions, based on beagle data, has been employed in one approach to the evaluation of exposure limits (Jee, 1980). Data from life-span beagle experiments were extensively employed in the ICRP's recent review of The Metabolism of Plutonium and Related Elements (ICRP, 1986; Taylor, D., 1985), as they were in an earlier publication on the same subject (ICRP, 1972).

Also of importance to the standard-setting activities of such groups as the ICRP and NCRP are the indications of previously neglected critical organs or tissues. Thus, results of life-span dog experiments have called attention to sinonasal tumors arising from radionuclide deposition in nasal turbinates (Benjamin, 1979; Boecker, 1984, 1986) and to lymph node tumors arising at relatively long time periods following inhalation of beta emitters ( $\mathrm{Hahn}, 1986)$. Extensive data on the concentration of plutonium and other actinides in gonads, derived from life-span dog studies and from studies with other animal species, have been compiled (Richmond, 1975) and used by standard-setting bodies (ICRP, 1979, 1980b, 1981, 1986).

\subsection{CLINICAL APPLICATIONS TO MAN}

Experience gained from studies on the therapeutic removal of internally deposited radionuclides from beagles (see Section 6.1) has played an important role in the selection of treatment regimens in cases of serious human overexposure to radionuclides (Norwood, 1962b, 1972). In one of these cases, involving external 
deposition in excess of $1 \mathrm{Ci}$ of ${ }^{241} \mathrm{Am}$, and initial systemic deposition of about 1 $\mathrm{mCi}$, long-continued treatment with $\mathrm{Zn}$-DTPA, based on its demonstrated effectiveness and lack of toxicity in beagles (Lloyd, R.,1975b, 1975c), was probably a life-saving measure (Thompson, 1983). In another case, bronchopulmonary lavage was employed, in conjunction with intravenous DTPA, to substantially reduce the ${ }^{239} \mathrm{Pu}$ lung burden of an exposed worker (McClellan, 1972). The factors involved in a decision to treat an exposed human with bronchopulmonary lavage have been discussed (Muggenburg, 1977).

Experience in the total-body counting of beagles has also been applied to man, specifically in the prediction of ${ }^{90} \mathrm{Sr}^{90} \mathrm{Y}$ bremsstrahlung efficiency by extrapolation of data from a number of animal species (Lloyd, R., 1976f).

\subsection{ESTIMATION OF DOSE AND RISK IN MAN}

Application of the life-span dog data to the estimation of human risks has been a specific objective of these studies. Establishing dose-response relationships in the beagle is only the first step toward estimating such a relationship for man. Methodologies for approaching this task have been the subject of a number of publications (Crump, 1986; Cuddihy, in press; Evans, 1972; Gilbert, in press; Goldman, 1973, 1986; Mays, 1986b; Rosenblatt, 1971, 1976).

The widely employed "toxicity ratio" approach is based on the assumption that the ratio of toxicities of two different radionuclides in man should be approximately the same as the ratio of toxicities of the same two radionuclides in an experimental animal (Evans, 1972). Thus, for example, there are human data on the toxicity of ${ }^{226} \mathrm{Ra}$; if data are obtained on the ratio of ${ }^{239} \mathrm{Pu}$ toxicity to ${ }^{226} \mathrm{Ra}$ toxicity in the beagle, that ratio may be used to obtain an estimate of ${ }^{239} \mathrm{Pu}$ toxicity in man. The life-span beagle experiments have provided support for this approach and also demonstrated some limitations (Mays, 1986b). Early Utah data on the relative toxicities for bone tumor induction of ${ }^{226} \mathrm{Ra},{ }^{90} \mathrm{Sr}$, and ${ }^{239} \mathrm{Pu}$ were employed in this manner, and the conclusions were used to support the then-current exposure limits for ${ }^{239} \mathrm{Pu}$ and to support a relaxation of the thencurrent exposure limits for ${ }^{90} \mathrm{Sr}$ (Hems, 1966).

Direct extrapolation of the accumulating beagle data from Utah has been employed to estimate human risk of bone and liver tumors from internally deposited ${ }^{239} \mathrm{Pu}$ (Mays, 1970). A similar approach has developed risk estimates for exposure to ${ }^{90} \mathrm{Sr}$, employing data from a variety of animal species, including the beagle (Mays, 1972c, 1972d). 
A risk estimate for ${ }^{239} \mathrm{Pu}$ of 200 bone sarcomas per $10^{6}$ person rad, as derived from epidemiologic data from ${ }^{224} \mathrm{Ra}$ exposure in humans, bears about the same relationship to the lower risk of ${ }^{226} \mathrm{Ra}$ exposure in humans as do the corresponding risks of ${ }^{239} \mathrm{Pu}$ and ${ }^{226} \mathrm{Ra}$ in beagles. These comparisons provide support for application of the human ${ }^{224} \mathrm{Ra}$ risk factor in the estimation of ${ }^{239} \mathrm{Pu}$ risks in humans. This is also supported by dosimetric considerations, since both ${ }^{224} \mathrm{Ra}$ and ${ }^{239} \mathrm{Pu}$ decay on bone surfaces (Mays, 1976, 1976b).

The "toxicity ratio" approach has been modified by limiting its application to the relatively lower dose groups, where the problem of "wasted radiation" is presumably not a factor. Employing such an approach, a risk factor of about 1200 bone cancers per $10^{6}$ rad was estimated for injected ${ }^{239} \mathrm{Pu}$; a similar value was estimated from incomplete results for inhaled ${ }^{238} \mathrm{Pu}$ (Muggenburg, 1983).

An interesting recent approach to the estimation of bone cancer risk from plutonium in the human has employed a Bayesian methodology to combine human epidemiology data for radium, human injection data for plutonium, beagle injection data for radium and plutonium, beagle inhalation data for plutonium, and rat inhalation data for plutonium, with a resulting risk estimate of about 300 bone cancer deaths per million person rad, with a $95 \%$ confidence interval of about 80 to 1100 (National Academy of Sciences, 1988).

Bone tumor sites in beagles continually fed ${ }^{90} \mathrm{Sr}$ or repeatedly injected with ${ }^{226} \mathrm{Ra}$ have been compared with radiation-induced bone tumor sites in man, and spontaneous bone tumor sites in beagle and man, as an approach to scaling risk to humans (Pool, 1973). These comparisons have been extended to the spectrum of premalignant changes observed in ${ }^{226}$ Ra-exposed beagles and humans, employing radiographic, microradiographic, light- and electronmicroscopic procedures ( $P o o l$, 1983). A similar comparison of ${ }^{226} \mathrm{Ra}$-induced lesions in beagles and humans, together with their similarity to lesions induced by ${ }^{239} \mathrm{Pu}$ in beagles, has been employed in support of the "toxicity ratio" approach to the estimation of ${ }^{239} \mathrm{Pu}$ effects in humans (Taylor, G., 1976).

Data on ${ }^{239} \mathrm{Pu}$-induced liver tumors in the beagle, together with data on Thorotrast-induced liver tumors in man, were employed to derive a risk factor for ${ }^{239} \mathrm{Pu}$ in humans of 100 liver tumors per $10^{6} \mathrm{rad}(\mathrm{Jee}, 1976 \mathrm{~b} ;$ Mays, 1976b). More recent human data suggest a risk factor of 300 liver tumors per $10^{6} \mathrm{rad}$ (Mays, 1982). Liver cancer risks from beta emitters, derived from the ITRI ${ }^{144} \mathrm{CeCl}_{3}$ inhalation study and ${ }^{137} \mathrm{CsCl}$ injection study, came to about 90 liver cancers per $10^{6} \mathrm{rad}$ of beta radiation; this was extrapolated to an estimated human risk of about 30 liver cancers per $10^{6} \mathrm{rad}$ of beta radiation, based on a comparison of dog data and human Thorotrast risk data (Muggenburg, 1986). 
Employing data from experiments still in progress at Utah and PNL, and avoiding the traditional dosimetric approach, a total tumor incidence of "a few percent" was estimated to result from an initial "permissible deposition" in the lung of $0.04 \mu \mathrm{Ci}$ of ${ }^{239} \mathrm{Pu}$. Of more significance than this numerical estimate was the conclusion that risks of lung, bone, or liver cancer were approximately equivalent (Bair, 1974f; Thompson, 1972). Extensions of this approach have been employed in the estimation of plutonium risks from the operation of breeder reactors (Gavankar, 1972, 1974), and in a number of environmental impact statements (e.g., USAEC, 1974; USERDA, 1975).

The relationship between the incidence of lung cancer and the average dose to lung from inhaled ${ }^{144} \mathrm{Ce}$ in relatively insoluble form has been compared, employing data from life-span beagle experiments and similar experiments in mice, rats, and Syrian hamsters. The animal data (excluding that from hamsters) are in reasonable agreement with the available risk data from epidemiologic studies of externally exposed humans, despite differences in the morphological type of lung cancer in the different species (Griffith, 1986).

Cuddihy has compared radiation-induced lung-cancer risk-factor data from a number of life-span dog experiments with data from other species, including man; all of these risk factors were of a like magnitude (Cuddihy, 1982, in press). A similar approach, involving the summation of annual risk factors, has been employed to compare human and beagle risk factors from high-LET and lowLET irradiation of lungs ( $\operatorname{Tahn}, 1986$ ). The same approach has been employed to derive and compare risk factors for lung, bone, liver, and nasal-cavity irradiation (McClellan, 1986b). In a more general vein, data from a variety of species on radionuclide injury to the lung have been compared, as these data relate to the prediction of human hazards (Dagle, 1984). More specific comparisons have been made of pulmonary carcinogenesis data from rats and dogs exposed to inhaled plutonium (Dagle, 1980; Mahaffey, 1981).

Several attempts have been made to include dose, time, and effect parameters in a single dose-response relationship. A nonlinear, 3-dimensional surface, relating dose, time, and effect has been proposed (Rosenblatt, 1971, 1972), employing logistic equations that may bear some relationship to biological and physical phenomena (Stover, 1970b). This approach was used to compare data on ${ }^{226} \mathrm{Ra}$ induced osteosarcoma from Utah and Davis beagle experiments with similar data from experiments with mice, and the results were employed as a basis for extrapolation of animal risk estimates for ${ }^{226} \mathrm{Ra}$ to man (Goldman, 1973). A similar approach, augmented by animal and human "toxicity ratio" data, was employed to estimate risks of ${ }^{239} \mathrm{Pu}$-induced bone tumor in man (Rosenblatt, 1976). A modification of the logistic model has been described and applied to the 
Utah ${ }^{226}$ Ra data (Sedlak, 1986). A similar approach, based on the proportional hazards model, has also been applied to the bone cancer induction data from inhaled ${ }^{90} \mathrm{SrCl}_{2}$, and ${ }^{238} \mathrm{PuO}_{2}$, and from injected ${ }^{90} \mathrm{Sr},{ }^{226} \mathrm{Ra}$, and ${ }^{239} \mathrm{Pu}$; the results have been compared to the toxicity data from human ${ }^{226} \mathrm{Ra}$ and ${ }^{224} \mathrm{Ra}$ exposures (Mewhinney, 1986).

A modification of the above approach employs log-normal rather than logistic equations in the construction of time-dose-effect surfaces, assuming that the logarithm of average dose rate to the tissue of interest is linearly related to the logarithm of time to death (Raabe, 1979, 1980, 1981, 1981b, 1983, 1984, 1986, 1986b, 1987 , in press). Application of this model to early mortality data following radionuclide inhalation provided an approach to the calculation of $\mathrm{RBE}$ values for this endpoint (Raabe, 1979). Application of the same model to ${ }^{226} \mathrm{Ra}$ osteosarcomainduction data from life-span beagle studies, from mouse studies, and from human epidemiologic studies, showed median cumulative doses (or times) for bone-tumor deaths that were 2.9 times larger for dogs than for mice and 3.6 times larger for humans than for dogs, values that correlate well with normal life expectancies for the three species (Raabe, 1980, 1981, 1983). From this model it was also possible to estimate a "practical threshold" for bone tumor production in man of about $80 \mathrm{rad}$; at lower doses, tumors were predicted to occur at times beyond the normal life expectancy. Beagle and other animal data have also been employed to question the existence of such a "practical threshold" (Mays, 1988).

The 3-dimensional log-normal response model was employed in comparisons of the carcinogenicity, as derived from life-span beagle experiments, of ${ }^{226} \mathrm{Ra}$, ${ }^{228} \mathrm{Ra},{ }^{228} \mathrm{Th},{ }^{238} \mathrm{Pu},{ }^{239} \mathrm{Pu},{ }^{241} \mathrm{Am},{ }^{249} \mathrm{Cf}$, and ${ }^{252} \mathrm{Cf}$ (Raabe, 1984, 1986, 1986b;

Wrenn, 1986c). These bone-seeking alpha emitters were shown to follow mathematically similar log-normal dose-rate/time-response relationships, displaced by RBE factors $\left({ }^{226} \mathrm{Ra}=1\right.$ ) of 3.0 for ${ }^{228} \mathrm{Ra}$; 6.4 for ${ }^{241} \mathrm{Am},{ }^{249} \mathrm{Cf}$, and ${ }^{252} \mathrm{Cf} ; 9.0$ for ${ }^{239} \mathrm{Pu}$; 10.7 for ${ }^{228} \mathrm{Th}$; and 15.5 for ${ }^{228} \mathrm{Pu}$. Dose-rate/time-response relationships for people were predicted based on a response ratio of 3.6 for ${ }^{226} \mathrm{Ra}$-induced bone tumors in beagles and man. The 3-dimensional log-normal response model has also been employed, together with computer graphics, to illustrate the timerelated competing risks of death from radiation pneumonitis/fibrosis, lung cancer, and natural aging, and applied to the scaling of risks from experimental animals to humans (Raabe, 1987, in press).

Weibull functions have also been employed in modeling dose-time-effect surfaces (DuMouchel, 1981; Scott, 1980; Whittemore, 1982). Comparisons of models employing logistic, log-normal, and Weibull functions have been made (Raabe, 1986). 
Approaches to human radiation-induced-cancer-risk estimation have also been based on dosimetric comparisons between beagle and human, without recourse to the beagle cancer incidence data. Skeletal ${ }^{212} \mathrm{~Pb}$ retention in the human following ${ }^{224} \mathrm{Ra}$ injection has been estimated by extrapolation from beagle and rodent data (Schlenker, 1988). Total tumor risk to bone, including estimates for leukemia, trabecular and cortical bone sarcoma, and sinus carcinoma, were estimated for ${ }^{239} \mathrm{Pu}$, based on human ${ }^{226} \mathrm{Ra}$ toxicity data and a strictly dosimetric comparison of the presumed distribution of radium and plutonium in human bone, the distribution of plutonium being deduced largely from the life-span dog data (Spiers, 1976b, 1988).

Extrapolation of skeletal effects data from dog to man will benefit from comparative information on the microscopic structure of bone in the two species (Lloyd, E., 1971, 1972; Rowland, 1969). Such data have been reported for man, monkey, pig, and beagle, and factors have been calculated relating average bone dose to more meaningful doses to marrow and endosteal cells for a variety of beta- and alpha-emitting radionuclides (Beddoe, 1979; Harley, 1976; Lloyd, E., 1972; Spiers, $1969,1972,1976,1978,1988$ ). In general, these factors are very much larger for beagle than for man, supporting the conclusion that, on an average-dose-to-bone basis, the beagle is more sensitive to radiation-induced bone tumor than man.

In another approach to the extrapolation of skeletal effects, the concentration of ${ }^{239} \mathrm{Pu}$ on endosteal bone surfaces, as related to concentration in the total body, was compared for beagle, rat, and rabbit, and the relationship was extrapolated to man (James, 1972). Computer modeling of the microscopic distribution and redistribution patterns of ${ }^{239} \mathrm{Pu}$ in the beagle skeleton, of the relationship of tumor incidence to bone turnover rate, and of comparative bone biology of beagles and humans, has also been employed to estimate tumor risk in man (Jee, 1980).

The incidence of early leukopenia following ${ }^{239} \mathrm{Pu},{ }^{226} \mathrm{Ra}$, or ${ }^{90} \mathrm{Sr}$ deposition has been correlated with the later appearance of osteosarcoma, and the relationship has been suggested as a means of predicting the late effect from the early observation (Goldman, 1976).

Problems of interspecies differences in pulmonary anatomy, in sensitivity to the induction of functional abnormalities, and in the measurement of these abnormalities are important to the extrapolation of animal data from inhalation studies to man; they have been given specific consideration (Mauderly, 1983).

Life-span dog data have been employed in the interpretation of the data from the human plutonium injection cases, particularly in regard to models for the deposition and retention of plutonium in skeleton and liver (Durbin, 1972, 1976). 
Knowledge of the comparative metabolism of calcium and radium in beagles, as a function of age, has been applied in estimating the age-dependence of radium retention in humans (Parks, 1984). Distribution and retention data on ${ }^{241} \mathrm{Am}$ in humans and beagles have been reviewed and employed in the estimation of human carcinogenic risks (Wrenn, 1981b).

Data on the retention and distribution of ${ }^{241} \mathrm{AmO}_{2}$ inhaled by dogs have been combined with retention and exceretion data from several human exposures to develop models that take account of physical and chemical characteristics of the inhaled aerosol. These models describe behavior in the human more adequately than does the ICRP lung model for Class W particles (Mewhinney, 1980, 1982b, $1983 b$ ). The most recent versions of this model also include revised tissue distribution parameters, derived from dog data, which seem to adequately represent the observed behavior in the human cases. A modification of this model can also be employed for bioassay purposes (Griffith, 1983).

In addition to the concern for radiation-induced cancer, data on early death from radiation pneumonitis has been subjected to extensive dose-response modeling (Raabe, 1979; Scott, 1979, 1980, 1980b, 1980c, 1982, 1986), as have data on the hematopoietic syndrome (Scott, in press); both have been applied to the prediction of potential effects in acutely exposed humans (McClellan, 1982; Scott, 1980c, in press). Beta emitters with short half-lives and higher initial dose rates were more effective, per rad, in producing early death from radiation pneumonitis and pulmonary fibrosis than beta emitters producing lower initial dose rates (Hahn, 1983b).

\subsection{CONTRIBUTIONS TO THEORIES OF CARCINOGENESIS}

Dose-response data from life-span beagle experiments have been employed in many attempts to provide a theoretical basis for tumor prediction. These include early attempts by Blair, employing data from the Utah studies, in which he assumed that two processes were involved, one requiring a relatively high dose and a short development phase, the other a lower dose, with an intervening latency period before the development phase (Blair, 1968, 1972).

The myeloproliferative disorders that developed in beagles continuously exposed at early ages to external irradiation or internally deposited ${ }^{90} \mathrm{Sr}$ have been employed extensively as a model system for the understanding of leukemogenesis (Fritz, 1985, 1986; Holmberg, 1980; Seed, 1977, 1978, 1984, 1985; Tolle, 1979b, 1982; Wilson, 1978, 1980). Dogs chronically exposed at Davis to whole-body ${ }^{60} \mathrm{Co}$-irradiation at rates of 10 or $4 \mathrm{rad}$ per day, starting in-utero at 21 days of gestation, almost invariably developed acute nonlymphocytic leukemia or 
preleukemic changes; such developments were a rare occurrence in animals similarly exposed after birth (Momeni, 1974 Stitzel, 1982). This model system offers many opportunities for study of the cellular and molecular changes associated with the transformation of normal hematopoietic cells to malignant cells.

The patterns of survival and hematologic responses in the Argonne continuous gamma-irradiation studies suggest that the hematopoietic recovery and increased radioresistance of dogs surviving the first 300 days of irradiation is in some manner related to the later development of leukemia, perhaps through the mechanism of an error-prone enhanced repair of sublethal damage (Fritz, 1985, 1986; Seed, 1977, 1978, 1984, 1985, 1987; Tolle, 1979b, 1982). These studies have included the development of advanced techniques, particularly in the area of cell culture and observation (Seed, 1979, 1979b), the application of scanning electron microscopy (Seed, 1982, 1983, 1987b; Tolle, 1983b), the investigation of bone marrow architecture and pathology (Seed, 1982, 1983), and studies on the mobilization and altered radiosensitivity of hemopoietic progenitors (Seed, 1980, 1982b). Precise control of the ${ }^{60} \mathrm{Co}$ gamma-ray exposure allows production in the beagle of a high incidence of both myelogenous leukemias and erythroleukemias that are clinically, hematologically, and histologically similar to the human disease, thus providing an attractive animal model for study of the leukemogenic process. The variety of long-term neoplastic responses to the internal deposition of ${ }^{90} \mathrm{Sr}$, as observed following different routes and patterns of administration, at different ages, and in different species, has also provided important data for the understanding of these processes (McClellan, 1969).

Data from life-span beagle experiments have been extensively employed in the derivation of hypotheses concerning the pathogenesis of radiation-induced lung tumors, which seem to involve a progression from pulmonary fibrosis to metaplasia to neoplasia, possibly influenced by immunologic factors (Clarke, 1966, 1966b; Dagle, 1976). Life-span data on lung-tumor incidence following inhalation of insoluble forms of beta emitters with widely varying physical halflife, and consequent differences in protraction of dose, have elucidated effects of radiation dose patterns on the process of tumor induction; protracted irradiation from ${ }^{90} \mathrm{Sr}$ or ${ }^{144} \mathrm{Ce}$ produced fewer lung tumors per rad (by a factor of 4 to 8 ) than less-protracted irradiation from ${ }^{90} \mathrm{Y}$ or ${ }^{91} \mathrm{Y}$ (Hahn, 1983, 1983b, 1986).

Data from life-span beagle studies have also been employed in the development of theories relating to the pathogenesis of radiation-induced osteosarcoma ( $\mathrm{Jee}$, 1971; Marshall, 1969; Miller, S., 1986; Nilsson, 1985). These theories usually consider the endosteal cells of trabecular bone as the most likely cells at risk (Hashimoto, 1976; Miller, S., 1980; Wronski, 1980), emphasize the importance of the proliferative activity at the site of tumor induction (Jee, 1972; Kimmel, 1976; 
Miller, S., 1986), the importance of the marrow microvasculature (Miller, S., 19806,1986 ), or emphasize the importance (or lack of importance) of pre-existing lesions of radiation osteodystrophy (Gillett, 1985, 1987b; Nilsson, 1985; Pool, $1973 \mathrm{~b}, 1983)$. A careful comparison has been made of the pathogenesis of ${ }^{90} \mathrm{Sr}-$ induced bone tumors in the beagle and the mouse (Nilsson, 1985). Mole was impressed by the apparent uniform radiosensitivity of the whole endosteum, considered as an organ, in the dog, rabbit, rat, and mouse (Mole, 1969).

Recent evaluation of the relationship between the cortical and trabecular composition of bones and the differing incidence of ${ }^{226} \mathrm{Ra}$-, ${ }^{239} \mathrm{Pu}$-, and ${ }^{241} \mathrm{Am}$-induced cancers in those bones raises provocative questions as to the mechanism of carcinogenesis (Jee, 1985, 1986). The frequency of occurrence of ${ }^{226} \mathrm{Ra}$-induced bone sarcomas was positively correlated with the percentage of cortical bone, but not related to cortical bone mass or cortical endosteal surface area, and was negatively correlated to activated trabecular calcium and trabecular surface area. The frequency of occurrence of ${ }^{239} \mathrm{Pu}$-induced bone sarcomas (but not ${ }^{241} \mathrm{Am}$-induced bone sarcomas) was positively correlated with trabecular surface-to-volume ratios and trabecular turnover.

The linear, or nonlinear nature of the dose-response curve for osteosarcoma induction has been a matter of basic interest, with data from the life-span beagle experiments inconclusive at low dose levels, but seeming to support a lessthan-linear relationship in the case of ${ }^{226} \mathrm{Ra}$ and ${ }^{228} \mathrm{Ra}$, and a linear relationship in the case of ${ }^{239} \mathrm{Pu}$ (Mays, 1972, 1980). In the case of ${ }^{90} \mathrm{Sr}$ the relationship is markedly nonlinear (Mays, 1972c).

Extensive data on the spontaneous incidence of benign and malignant mammary tumors in control beagles have been described and employed in efforts to model human breast cancer (Chrisp, 1980; Taylor, G., 1976b).

Autopsy material from life-span dog studies has been employed in a number of experiments designed to elucidate the molecular-biological basis of observed effects. Tumor cell preparations cultured from osteosarcomas present in dogs that inhaled ${ }^{238} \mathrm{PuO}_{2}$ were transplanted, in-utero, into beagle fetuses; this resulted in the appearance, shortly after birth, of extensive nodules characterized as fibrosarcomas (Park, J., 1976). Tissues from five beagles with radiation-induced osteosarcomas were shown to contain RNA-instructed DNA polymerase (RIDP), which was indicative of the presence of retrovirus or oncornavirus infection; RIDP was not detected in dogs without tumors, or in dogs with lung tumors (Frazier, 1976). Cell cultures from plutonium-induced bone tumors were killed without delay by circulating lymphocytes from dogs with bone tumors; similar toxicity was exhibited by circulating lymphocytes from dogs with no bone 
tumors, only in the presence of macrophages, and only after a 3- to 5-day delay (Frazier, 1976b).

In preliminary studies of high-molecular-weight DNA from both plutoniuminduced lung tumors and gamma-ray-induced myeloproliferative disorders, dominant-acting, transforming oncogene activity has been observed (Frazier, 1987; Kelly, in press).

\subsection{CONTRIBUTIONS TO THE BASIC BIOLOGY OF BONE}

Data from life-span experiments in dogs, employing bone-seeking radionuclides, have provided important new understanding of the basic nature and functioning of bone as a tissue. This includes significant advances in the understanding of the role of the vasculature in bone (Jee, 1960b, 1960c, 1961, 1962b, 1964c, 1969,1971) and marrow (Miller, S., 1980b, l986), of bone remodeling rates (Jee, 1964b; Kimmel, 1976, 1982; Polig, 1987), and of the significance of various cell types in bone and marrow (Hashimoto, 1976; Jee, 1963, 1971, 1977; Miller, S., 1980; Momeni, 1975c; Wilson, 1976). Advanced morphometric (Kimmel, 1976, 1977), autoradiographic (Fellows, 1975; Wronski, 1980), and bone-marrow culture techniques (Kaspar, 1984; Wilson, 1976, 1978b, 1978c) have been developed and employed in these studies.

The dog skeleton has been recommended for its utility in modeling problems of the human elderly, including gingivitis and periodontitis, degenerative joint disease and osteoporosis (Jee, 1981b). Mechanical and morphological evaluations of age-related changes in the aging beagle may prove useful in extending our understanding of intervertebral disc disease in humans (Gillett, in press).

\subsection{APPLICATIONS TO BE EXPECTED IN THE FUTURE}

It would be inappropriate to speculate at length, in this report, on possible future applications of the results from these life-span studies of radiation effects in the beagle. It is important to emphasize, however, that applications made to date, as reviewed in this chapter, represent only a fraction of the benefits to be expected from these studies. Many of the experiments are still incomplete, in the sense that animals remain alive, on experiment. Other experiments, in which animals are all dead, are still incomplete in the sense that many samples and much data remain to be processed and interpreted. Most of the conclusions thus far drawn, as considered in the previous chapters of this report, must be thought of as provisional findings subject to reinterpretation in the light of final results. 
The data still to be obtained from animals still alive is, in many respects, the most interesting data of all, since it is usually data from the longest-lived animals-those exposed to the lowest radiation doses, and therefore of greatest importance for establishing the incidence of long-delayed effects of low-level exposure. Because the most interesting results are the last to be obtained from an experiment, there has been an understandable tendency to delay interpretation until all the results are in. And, of course, definitive comparisons between experiments must await completion and interpretation of the individual experiments.

Aside from the tumor incidence data, which have been of primary initial interest, there is a wealth of additional observations, recorded in nearly all of the experiments, to which little or no attention has yet been given. In fact, there are undoubtedly more data than it will ever be practically possible to totally comprehend. This being the case, a critical future task will be to set priorities to explore these data. As a first step, it is to be hoped that a thorough and comprehensive description of the design, conduct, and findings, of each experiment will be published in some reasonably uniform format that will facilitate future study and comparison. Such complete descriptions cannot, as yet, be said to exist for any of the experiments.

In addition to the data recorded and awaiting interpretation, there also exist collections of biological material from which may be extracted data unanticipated in the original design of the experiments. These materials include a variety of biological specimens, ranging from gross organ and tissue samples to histological slides to frozen viable cell cultures. These materials represent an invaluable resource for future study, employing modern biological and measurement techniques undreamed of when the material was collected. The preservation and storage of this material in readily retrievable form are among the more urgent challenges of the immediate future. 


\section{Contents}

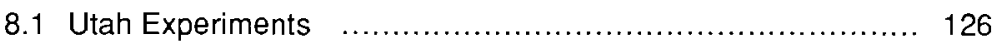

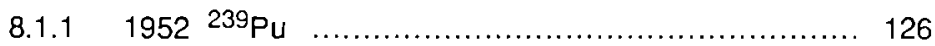

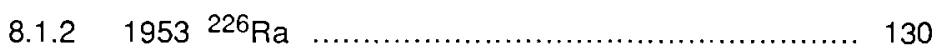

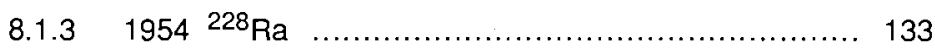

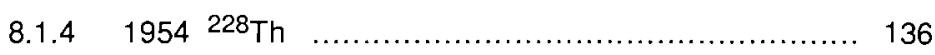

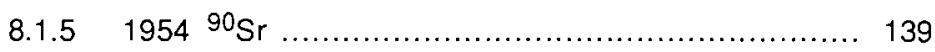

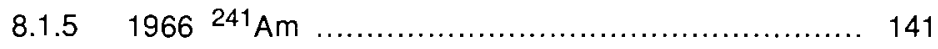

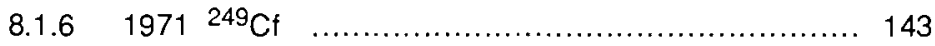

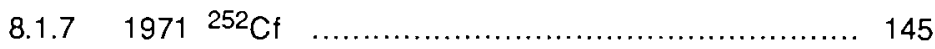

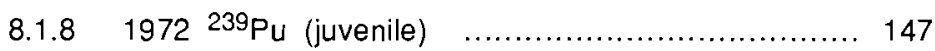

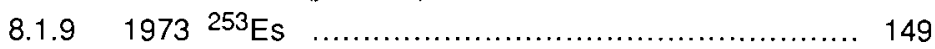

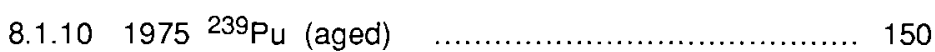

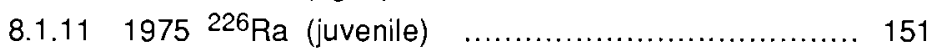

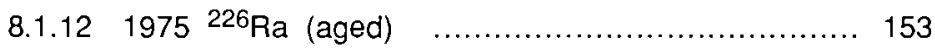

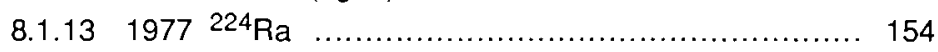

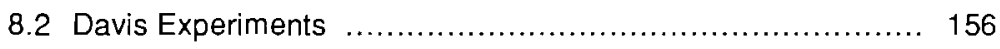

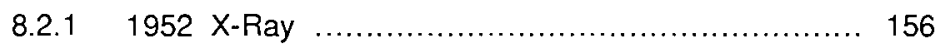

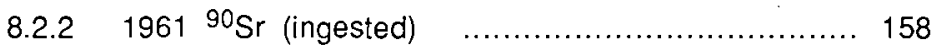

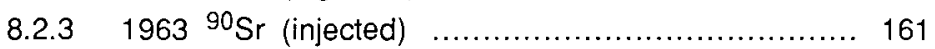

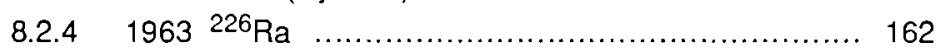

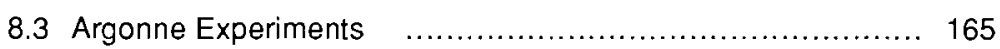

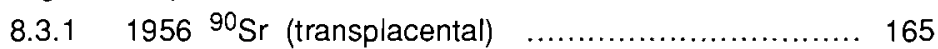

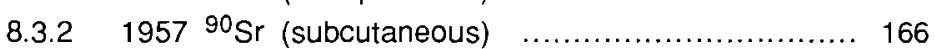

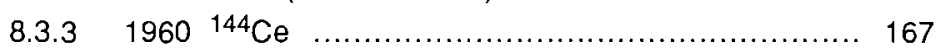

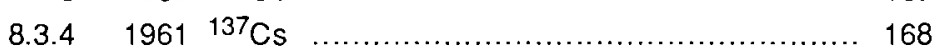

8.3.5 $1968{ }^{60} \mathrm{Co}$ (external gamma, continuous) $\ldots \ldots \ldots \ldots . . \ldots \quad 170$

8.3.6 $1968{ }^{60} \mathrm{Co}$ (external gamma, terminated) $\ldots \ldots \ldots \ldots . . . . .172$

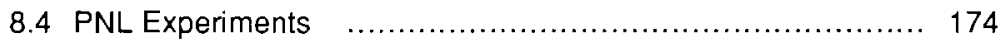

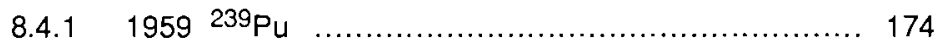

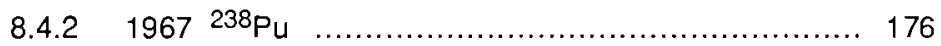

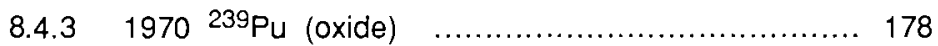

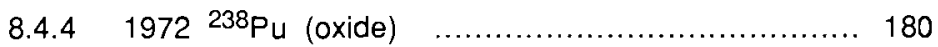

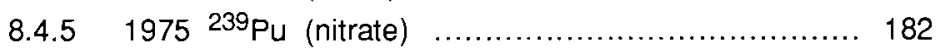

8.5 ITRI Experiments ................................................. 184

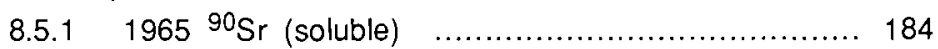

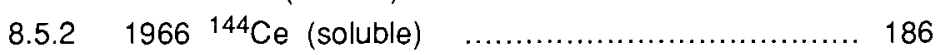

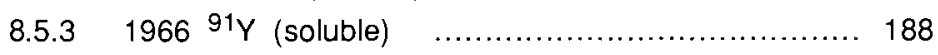




\begin{tabular}{|c|c|c|c|}
\hline 8.5 .4 & 1967 & ${ }^{144} \mathrm{Ce}$ (insoluble) & 190 \\
\hline 8.5 .5 & 1968 & ${ }^{137} \mathrm{Cs}$ (soluble) & 193 \\
\hline 8.5 .6 & 1969 & $90 Y$ (insoluble) & 195 \\
\hline 8.5 .7 & 1970 & $91 Y$ (insoluble) & 197 \\
\hline 8.5 .8 & 1970 & ${ }^{90} \mathrm{Sr}$ (insoluble) & 199 \\
\hline 8.5 .9 & 1972 & ${ }^{144} \mathrm{Ce}$ (juvenile) & 201 \\
\hline 8.5 .10 & 1972 & ${ }^{144} \mathrm{Ce}$ (aged) & 203 \\
\hline 8.5 .11 & 1972 & ${ }^{144} \mathrm{Ce}$ (multiple exposures) & 205 \\
\hline 8.5 .12 & 1973 & ${ }^{238} \mathrm{Pu}(3.0-\mu \mathrm{m}$ particles $)$ & 206 \\
\hline 8.5 .13 & 1974 & ${ }^{238} \mathrm{Pu}(1.5-\mu \mathrm{m}$ particles $)$ & 208 \\
\hline 8.5 .14 & 1977 & ${ }^{239} \mathrm{Pu}(0.75-\mu \mathrm{m}$ particles $)$ & 211 \\
\hline 8.5 .15 & 1977 & ${ }^{239} \mathrm{Pu}(1.5-\mu \mathrm{m}$ particles $)$ & 213 \\
\hline 8.5 .16 & 1977 & ${ }^{239} \mathrm{Pu} \quad(3.0-\mu \mathrm{m}$ particles $)$ & 215 \\
\hline 8.5 .17 & 1977 & ${ }^{239} \mathrm{Pu}$ (multiple exposures) & 217 \\
\hline 8.5 .18 & 1979 & ${ }^{239} \mathrm{Pu}$ (juvenile) & 218 \\
\hline 8.5 .19 & 1979 & ${ }^{239} \mathrm{Pu}$ (aged) & 220 \\
\hline
\end{tabular}

Summaries of the major life-span beagle experiments are collected here in a uniform format. Experiments are ordered by laboratory, by date of initiation, and by radiation source or radionuclide employed. The identification of each experiment also includes information on the physical and/or chemical nature of the radiation source or radionuclide employed, on the nature of the exposure, on the age and sex of dogs exposed, and on the range of years over which dogs were exposed. A principal reference is given for the tabulated data.

The tabulated data relate only to the life-span animals; they do not include animals sacrificed as pathology controls or for dosimetric purposes, nor do they include animals maintained as a breeding colony or employed for ancillary research projects not involving life-span observation.

The tables list information on experimental design, including the defining characteristics of the exposure groups and the number of animals in each group. Numbers given for "quantity injected" are mean values for the exposure group. For inhalation exposures, the "initial burden" tabulated is the burden measured after completion of initial rapid clearance from the upper respiratory tract; a quantity sometimes referred to as the "deep-lung burden" or "alveolar burden." Because inhalation exposures cannot be precisely controlled, these burdens may vary widely within exposure groups; the values listed are medians for the group. In the case of ITRI experiments, the exposure groups were redefined, following exposure, to avoid burden overlap between adjacent groups. This adjustment was not made for PNL experiments, where such overlap was minimal. 
Tabulated results are a necessarily abbreviated selection, varying in nature from experiment to experiment, depending on the objective of the experiment and the present status of available results. In all cases the results include the number of dogs that are dead in each group and some group measure of the time interval of postexposure survival. The number of dogs dead with the principal radiationrelated effect(s) is listed, together with the percentage incidence (number with effect/number dead). In a few cases, specifically noted, the number of primary tumors rather than the number of animals with tumors is listed. Not included in these tables are data related to radionuclide retention, to physiologic or pathologic observations other than gross incidence of major effects, or data on the time of appearance of effects other than death.

Derived data, such as estimates of radiation dose to organs or tissues, and inferred conclusions, such as estimates of carcinogenic risk, are intentionally excluded from the tables. Such derived data are often of a provisional nature and should be considered only in the full context of the methods employed in their derivation; they are, in any case, not essential to the purpose of these tables, i.e., to facilitate gross comparison of current status and principal findings.

It must be emphasized that the tabulated data of these appendices should never be employed as a basis for further analysis. For any such purpose. referenced sources should be consulted for a more detailed and fully qualified presentation of the data.

Following the tabulated data is a brief narrative description of the experiment and supporting studies, with references to principal published papers. At the conclusion of these remarks there is a listing of reports in the document literature, which may contain details of the experiment that have not found their way into more widely available sources. 


\subsection{UTAH EXPERIMENTS}

\subsection{1 $1952{ }^{239} \mathrm{Pu}$}

Single intravenous injection of plutonium citrate solution; dogs 13 to 25 months old, of both sexes, placed on experiment in 3 series: "A", from 1952 to 1958; " $B$ ", from 1964 to 1970; and " $C$ ", from 1973 to 1974. Results as of September, 1987 (Doc. COO-119-263).

\begin{tabular}{|c|c|c|c|c|c|c|c|c|}
\hline \multirow{2}{*}{\multicolumn{3}{|c|}{$\begin{array}{l}\text { EXPERIMENTAL } \\
\text { DESIGN }\end{array}$}} & \multicolumn{6}{|c|}{ EXPERIMENTAL RESULTS } \\
\hline & & & & \multirow{4}{*}{$\begin{array}{l}\text { Median } \\
\text { Postexp. } \\
\text { Survival } \\
\text { (years) }\end{array}$} & & & & \\
\hline \multirow{3}{*}{$\begin{array}{l}\text { Quantity } \\
\text { Injected } \\
\text { (uci/kg) }\end{array}$} & \multirow{3}{*}{\multicolumn{2}{|c|}{$\begin{array}{l}\text { Series, } \\
\text { and No. } \\
\text { Injected }\end{array}$}} & \multirow{3}{*}{$\begin{array}{l}\text { No. of } \\
\text { Animals } \\
\text { Dead } \\
\end{array}$} & & \multicolumn{2}{|c|}{ №. (\% Incidence) } & \\
\hline & & & & & Bone & & Liver & \\
\hline & & & & & Tumor & & Tumor & \\
\hline None & A & 12 & 12 & 11.2 & 0 & (0) & 0 & (0) \\
\hline (Control) & & 31 & 31 & 12.1 & 1 & (3) & 0 & (0) \\
\hline & $\mathrm{C}$ & 7 & 5 & 11.6 & 0 & (0) & 0 & (0) \\
\hline 0.001 & B & 20 & 20 & 11.7 & 0 & (0) & 0 & (0) \\
\hline & & 8 & 6 & 12.0 & 0 & (0) & 0 & $(0)$ \\
\hline 0.002 & B & 39 & 39 & 11.9 & 2 & (5) & 2 & (5) \\
\hline & $\mathrm{C}$ & 7 & 5 & 12.7 & 0 & (0) & 0 & (0) \\
\hline 0.005 & B & 23 & 23 & 11.9 & 3 & (13) & 0 & (0) \\
\hline & C & 15 & 13 & 11.1 & 1 & (8) & 0 & (0) \\
\hline 0.010 & $\mathrm{~B}$ & 11 & 11 & 10.8 & 3 & (27) & 1 & (9) \\
\hline & $\mathrm{C}$ & 27 & 25 & 11.6 & 4 & (16) & 0 & (0) \\
\hline 0.016 & A & 14 & 14 & 11.3 & 4 & (29) & 2 & (14) \\
\hline & B & 12 & 12 & 11.5 & 4 & (33) & 0 & (0) \\
\hline 0.05 & A & 14 & 14 & 8.6 & 8 & (57) & 1 & (7) \\
\hline 0.1 & A & 12 & 12 & 7.1 & 10 & (83) & 0 & (0) \\
\hline 0.3 & A & 12 & 12 & 4.4 & 12 & $(100)$ & 0 & (0) \\
\hline 1.0 & A & 12 & 12 & 3.5 & 12 & $(100)$ & 0 & (0) \\
\hline 3.0 & A & 9 & 9 & 3.6 & 7 & (78) & 0 & (0) \\
\hline
\end{tabular}

Series A and B animals are all dead, as are most of series C. Several papers provide general descriptions of the experiment (Dougherty, T., 1962; Jee, 1976b; Mays, 1969, 1986b; Stover, 1972f, 1975; Taylor, G., 1972c; Wrenn, 1986). These and other publications often present comparative data from the Utah ${ }^{239} \mathrm{Pu},{ }^{226} \mathrm{Ra}$, ${ }^{228} \mathrm{Ra},{ }^{228} \mathrm{Th}$, and ${ }^{90} \mathrm{Sr}$ experiments. Control dogs from these other contemporary Utah experiments are sometimes combined in analyses of results from the plutonium experiment (Atherton, 1986). 
The carefully standardized injection solutions were shown to be minimally colloidal and at least $98 \% \mathrm{Pu}(\mathrm{IV})$ (Stover, 1962b). Supporting studies with ${ }^{237} \mathrm{Pu}$ showed that plutonium distribution was not a sensitive function of mass injected (Lloyd, R., 1976b); injection of particulate plutonium, however, resulted in a very different pattern of distribution and retention (Bruenger, 1981).

Data on the gross distribution and retention of plutonium, from excreta and tissue measurements, were reported in a series of papers, which also derived dosimetric parameters for the experiment (Dougherty, T.,1962; Lloyd, R., 1976b; Stover, 1959, 1959b, 1962, 1962b, 1968, 1969, 1971, 1972c, 1974, 1976, 1977). Estimated median-survival average radiation dose to bone ranged from about 2 rad in the lowest injection group to about $4000 \mathrm{rad}$ in the highest (Doc. COO119-262). A detailed biokinetic model of plutonium distribution between blood, liver, and trabecular and cortical bone compartments has been recently described (Polig, in press)

A number of papers have dealt with local distribution and dosimetry of plutonium within bone (Arnold, 1959, 1962; Jee, 1964, 1971, 1972c; Kimmel, 1976; Smith, J., 1984; Spiers, 1976; Wronski, 1980), teeth (Jee, 1960), and liver (Cochran, 1962, Taylor, G., 1972b, 1986), as well as its subcellular distribution and binding in liver (Bruenger, 1971, 1976, 1976b) and blood (Stevens, W., 1968).

Hematologic effects were described and analyzed in a series of papers (Dougherty, J., 1955, 1962, 1969, 1970, 1971, 1972; Goldman, 1976). A variety of biochemical effects measured in blood have been reported (Berliner, 1969;

Nabors, 1972; Ruhmann, 1965; Stevens, W., 1964, 1967). Nonneoplastic effects described in bone have included fractures (Jee, 1961; Rehfeld, 1962; Taylor, G., 1962, 1966, 1976), radiographic changes (Christensen, 1962), chemical changes (Stover, 1963), histopathologic changes (Jee, 1961, 1962, 1962b, 1971), and effects on blood vessals (Jee, 1960b, 1962c, 1969, 1971; Park, H., 1972). Nonneoplastic effects have also been reported in teeth (Jee, 1960; Taylor, G., 1969b, 1971, 1976), in the liver (Cochran, 1962; Stover, 1968; Taylor, G., 1971, 1972b, 1986), and in the eye (Taylor, G., 1962b).

The greatly increased incidence of bone tumors is described in a number of early papers (Dougherty, T., 1962, 1962c; Jee, 1962). Later papers on this subject usually include mathematical analysis in terms of dose-response relationships (Blair, 1968, 1972; Gridgeman, 1971; Mays, 1969, 1972; Peterson, 1982; Raabe, 1984, 1986, 1986b; Stover, 1970b, 1986; Whittemore, 1982; Wrenn, 1986c), and discuss possible carcinogenic mechanisms in relation to bone structure and turnover rates (Arnold, 1962; Kimmel, 1976, 1982; Marshall, 1973; Miller, S., 
1986; Wronski, 1980), the distribution of spontaneous and radiation-induced tumors (Christensen, 1972; Miller, S., 1986; Spiers, 1983), and the dynamics of tumor growth (Thurman, 1971). Supporting studies with St. Bernard dogs have explored the influence of spontaneous osteosarcoma sensitivity on the induction of osteosarcomas by plutonium (Lloyd, R., 1984b; Taylor, G., 1981). The increased incidence of liver tumors is described (Mays, 1970; Taylor, G., 1969, $1972 b, 1986)$, as is the lack of effect of plutonium on the incidence of mammary tumors (Taylor, G., 1976b).

A number of papers have dealt with estimation of the relative biological effectiveness of the various radionuclides involved in the Utah life-span experiments (Dougherty, J., 1970; Gridgeman, 1971; Marshall, 1973; Mays, 1969, 1986b), and with the extension of these data to the estimation of risk factors for man (Mays, 1970, 1976, 1976b; Muggenburg, 1983; Rosenblatt, 1976; Spiers, 1976, 1988; Taylor, G., 1976).

Information relevant to this experiment will be found in the following University of Utah Radiobiology Laboratory periodic reports:

\begin{tabular}{|c|c|c|c|c|c|c|}
\hline Issue Date & Doc. No. & Page Refs. & \multicolumn{2}{|c|}{ Issue Date } & Doc. No. & Page Refs \\
\hline 1955 (Mar) & AECU-3418 & $7-13$ & 1960 & (Mar) & $\mathrm{COO}-220$ & $29-207$ \\
\hline & & $32-33$ & 1960 & (Sep) & $\mathrm{C} 0 \mathrm{O}-222$ & $49-52$ \\
\hline 1955 (Sep) & AECU-3109 & $44-61$ & 1961 & (Sep) & $\mathrm{COO}-224$ & $32-56$ \\
\hline 1956 (Mar) & TID-16458 & $24-53$ & 1962 & (Mar) & $C 00-225$ & $31-47$ \\
\hline 1956 (Sep) & TID-16459 & $13-15$ & & & & $49-58$ \\
\hline & & $19-44$ & & & & $62-72$ \\
\hline 1957 (Mar) & AECU-3522 & $13-148$ & & & & $76-84$ \\
\hline 1957 (Sep) & AECU-3583 & $13-61$ & & & & $109-128$ \\
\hline & & $68-73$ & 1962 & (Sep) & $\mathrm{COO}-226$ & $95-127$ \\
\hline & & $77-86$ & 1963 & (Sep) & $\mathrm{COO}-228$ & $61-68$ \\
\hline & & $94-104$ & & & & $95-108$ \\
\hline 1958 (Mar) & COO-215 & $14-126$ & 1964 & (Mar) & COO-229 & $93-132$ \\
\hline 1958 (Sep) & $\mathrm{COO}-217$ & $14-101$ & 1964 & (Sep) & COO-119-231 & 99-109 \\
\hline & & $118-125$ & 1965 & (Mar) & $\mathrm{COO}-119-232$ & $88-121$ \\
\hline & & $147-180$ & 1966 & (Mar) & COO-119-234 & $70-84$ \\
\hline 1959 (Mar) & COO-218 & $21-84$ & & & & $94-119$ \\
\hline & & $121-145$ & 1966 & (Sep) & COO-119-235 & $71-75$ \\
\hline & & $148-209$ & 1967 & (Mar) & $\mathrm{COO}-119-236$ & $97-195$ \\
\hline 1959 (Sep) & $\mathrm{COO}-219$ & $24-25$ & & & & $207-251$ \\
\hline 1959 & & $42-43$ & & & & \\
\hline
\end{tabular}




\begin{tabular}{|c|c|c|c|c|c|c|}
\hline \multicolumn{2}{|c|}{ Issue Date } & Doc. No. & Page Refs. & Issue Date & Doc. No. & Page Refs. \\
\hline \multirow[t]{3}{*}{1969} & (Mar) & COO-119-240 & $86-96$ & 1980 (Mar) & COO-119-256 & $33-41$ \\
\hline & & & $188-198$ & & & $91-101$ \\
\hline & & & $224-274$ & 1982 (Mar) & $\mathrm{COO}-119-257$ & $12-22$ \\
\hline \multirow[t]{3}{*}{1970} & (Mar) & $\mathrm{COO}-119-242$ & $131-144$ & & & $52-88$ \\
\hline & & & 176-191 & 1983 (Mar) & $\mathrm{COO}-119-258$ & $14-21$ \\
\hline & & & $385-401$ & & & $26-34$ \\
\hline 1971 & (Mar) & COO-119-244 & $175-249$ & & & $46-90$ \\
\hline 1972 & (Mar) & COO-119-246 & $106-262$ & 1984 (Dec) & COO-119-259 & $1-12$ \\
\hline \multirow[t]{3}{*}{1973} & (Mar) & COO-119-248 & $229-273$ & & & $25-50$ \\
\hline & & & $308-335$ & & & $73-76$ \\
\hline & & & $370-377$ & & & $113-127$ \\
\hline 1974 & (Mar) & COO-119-249 & $180-233$ & 1985 (Dec) & CoO-119-261 & $1-14$ \\
\hline \multirow[t]{2}{*}{1975} & (Mar) & COO-119-250 & $128-137$ & & & $27-40$ \\
\hline & & & $166-181$ & 1986 (Dec) & COO-119-262 & $1-14$ \\
\hline 1976 & (Mar) & COO-119-251 & $172-180$ & & & $20-25$ \\
\hline \multirow[t]{2}{*}{1977} & (Mar) & $\mathrm{COO}-119-252$ & $152-190$ & & & $58-66$ \\
\hline & & & $203-227$ & & & $97-102$ \\
\hline 1978 & (Mar) & COO-119-253 & $158-253$ & & & \\
\hline \multirow[t]{2}{*}{1979} & (Mar) & COO-119-254 & $9-14$ & & & \\
\hline & & & $55-73$ & & & \\
\hline
\end{tabular}




\subsection{2 $1953^{226} \mathrm{Ra}$}

Single intravenous injection of radium citrate solution; dogs 12 to 28 months old, of both sexes, placed on experiment in 2 series: "A", from 1953 to 1963; and "B", from 1964 to 1970. Results as of September, 1987 (Doc. COO-119-263).

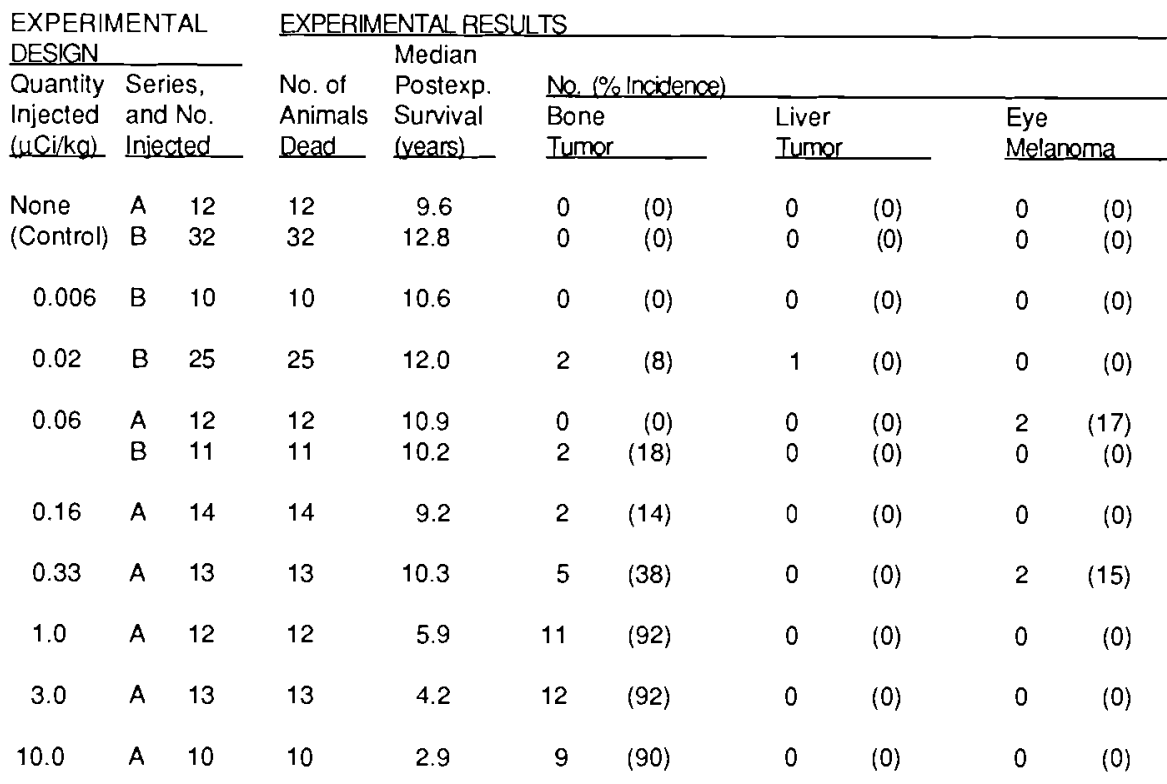

This experiment was designed to provide a link between epidemiologic studies of radium effects in humans, and studies in the $\mathrm{dog}$ with various radionuclides (but particularly plutonium) for which no effects have been observed in humans (Stover, 1972d). All dogs are dead. A brief summary of the experiment has recently appeared (Wrenn, 1986b); greater detail appeared in an earlier general status report (Dougherty, T., 1962). These and other publications often present comparative data from the Utah ${ }^{239} \mathrm{Pu},{ }^{226} \mathrm{Ra},{ }^{228} \mathrm{Ra},{ }^{228} \mathrm{Th}$, and ${ }^{90} \mathrm{Sr}$ experiments. Control dogs from these other contemporary Utah experiments are sometimes combined in analyses of results from the radium experiment (Atherton, 1986).

Gross retention of ${ }^{226} \mathrm{Ra}$ was followed by total-body counting ( $L l o y d, R$, 1976g); interpretation of these data involved detailed study of the retention of ${ }^{226} \mathrm{Ra}$ decay products (Lloyd, R., 1976h, 1983d, 1986b; Mays, 1958, 1975; Van Dilla, 1958). The general problem of radium distribution and retention was considered in a series of papers, which also derived dosimetric parameters for the study (Atherton, 
1965; Lloyd, R., 1976e, 1976h, 1986b; Stover, 1958, 1959, 1962). The most recent description of average skeletal dosimetry, including dose from daughters injected or grown in, was published in 1986 (Lloyd, R., 1986b). Estimated mediansurvival average radiation dose to bone ranged from about $30 \mathrm{rad}$ at the lowest injection level to about $16,000 \mathrm{rad}$ at the highest (Doc. COO-119-262).

A number of papers have dealt with the distribution of radium among bones (Lloyd, R., 1976e), and local distribution and dosimetry of radium within bone (Arnold, 1959; Jee, 1960b, 1962c, 1964), teeth (Jee, 1960), in the eye (Fisher, G., 1976; Stover, 1962; Taylor, G., 1962b, 1964b, 1972d) and in blood cells and plasma (Stover, 1965).

Hematologic effects were described and analyzed in a series of papers (Dougherty, J., 1955, 1962, 1969, 1970, 1971, Goldman, 1976). A variety of biochemical effects measured in blood have been reported (Berliner, 1969; Ruhman, 1965; Stevens, W., 1967). Nonneoplastic effects described in bone have included fractures (Rehfeld, 1962; Taylor, G., 1966), radiographic changes (Christensen, 1962), histopathologic changes (Jee, 1962b, 1971), and effects on blood vessals (Jee, 1960b, 1962c, 1969; Park, H., 1972). Nonneoplastic effects have also been reported in the eye (Taylor, G., 1962b, 1964b).

The increased incidence of bone tumors is described in early papers (Dougherty, T., 1962, 1962c). Later papers on this subject usually include mathematical analysis in terms of dose-response relationships (Blair, 1968, 1972; Dougherty, T., 1969; Gridgeman, 1971; Mays, 1969, 1972; Raabe, 1984, 1986, 1986b; Stover, $1970 b, 1986$; Wrenn, 1986c), and discuss possible carcinogenic mechanisms in relation to bone structure and turnover rates (Marshall, 1973; Spiers, 1983, 1988), the distribution of spontaneous and radiation-induced tumors (Christensen, 1972; Spiers, 1983), and the dynamics of tumor growth (Thurman, 1971). Supporting studies with St. Bernard dogs have explored the influence of spontaneous osteosarcoma sensitivity on the induction of osteosarcomas by ${ }^{226} \mathrm{Ra}$ (Lloyd, R., 1983b, 1983d; Wrenn, 1986). The increased incidence of intraocular melanomas is described (Taylor, G., 1969, 1972d, 1976), as is the lack of effect of ${ }^{226} \mathrm{Ra}$ on the incidence of mammary tumors (Taylor, G., 1976b).

A number of papers have employed the ${ }^{226} \mathrm{Ra}$ data in estimating the relative biological effectiveness of other radionuclides involved in the Utah studies (Dougherty, J., 1970; Gridgeman, 1971; Marshall, 1973; Mays, 1969, 1986b), and in the extension of these data to the estimation of risk factors for man (Goldman, 1973; Mays, 1976, 1976b; Mewhinney, 1986; Muggenburg, 1983; Rosenblatt, 1976; Spiers, 1976, 1988; Taylor, G., 1976). 
Information relevant to this experiment will be found in the following University of Utah Radiobiology Laboratory periodic reports:

\begin{tabular}{|c|c|c|c|c|c|c|c|}
\hline Issue & Date & Doc. No. & Page Refs. & Issue & Date & Dac. No. & Page Refs. \\
\hline 1955 & (Mar) & AECU-3418 & $7-13$ & 1964 & (Mar) & COO-229 & $59-79$ \\
\hline & & & $32-33$ & & & & $133-151$ \\
\hline & & & 55 & 1965 & (Mar) & COO-119-232 & $88-121$ \\
\hline 1955 & (Sep) & AECU-3109 & $13-23$ & 1965 & (Sep) & COO-119-233 & $68-80$ \\
\hline & & & $44-56$ & 1966 & (Mar) & COO-119-234 & $94-119$ \\
\hline 1956 & (Mar) & TID-16458 & $23-81$ & 1967 & (Mar) & $\mathrm{COO}-119-236$ & $97-163$ \\
\hline & & & $109-135$ & 1969 & (Mar) & COO-119-240 & $86-96$ \\
\hline 1956 & (Sep) & TID-16459 & $16-36$ & & & & $224-261$ \\
\hline 1957 & (Mar) & AECU-3522 & $13-148$ & 1970 & (Mar) & $\mathrm{COO}-119-242$ & $211-257$ \\
\hline 1957 & (Sep) & AECU-3583 & 13-61 & & & & $385-401$ \\
\hline & & & $77-81$ & 1973 & (Mar) & COO-119-248 & $370-377$ \\
\hline & & & $91-115$ & 1975 & (Mar) & COO-119-250 & $182-187$ \\
\hline 1958 & (Mar) & COO-215 & $14-115$ & 1977 & (Mar) & COO-119-252 & $152-161$ \\
\hline & & & $158-179$ & & & & $188-190$ \\
\hline 1958 & (Sep) & $\mathrm{COO}-217$ & $14-113$ & 1978 & (Mar) & $\mathrm{COO}-119-253$ & $158-164$ \\
\hline & & & $118-125$ & & & & $220-223$ \\
\hline 1959 & (Mar) & $\mathrm{COO}-218$ & $21-92$ & 1979 & (Mar) & COO-119-254 & $9-14$ \\
\hline & & & $121-147$ & 1982 & (Mar) & $\mathrm{COO}-119-257$ & $12-51$ \\
\hline 1959 & (Sep) & COO-219 & $24-43$ & 1983 & (Mar) & COO-119-258 & $14-21$ \\
\hline & & & 60 & & & & $29-32$ \\
\hline 1960 & (Mar) & $\mathrm{COO}-220$ & $29-143$ & & & & $43-45$ \\
\hline & & & $150-165$ & 1984 & (Dec) & COO-119-259 & $1-12$ \\
\hline 1960 & (Sep) & $\mathrm{COO}-222$ & $49-139$ & & & & $31-36$ \\
\hline 1961 & (Sep) & $\mathrm{COO}-224$ & $32-56$ & & & & $63-64$ \\
\hline 1962 & (Mar) & $\mathrm{COO}-225$ & $31-58$ & & & & $101-127$ \\
\hline & & & $109-128$ & 1985 & (Dec) & COO-119-261 & $1-14$ \\
\hline 1962 & (Sep) & $\mathrm{COO}-226$ & $34-56$ & & & & $31-32$ \\
\hline & & & $78-94$ & & & & $49-66$ \\
\hline 1963 & (Mar) & $\mathrm{COO}-227$ & $57-150$ & 1986 & (Dec) & COO-119-262 & $1-14$ \\
\hline & & & $185-203$ & & & & $22-23$ \\
\hline 1963 & (Sep) & $\mathrm{COO}-228$ & $61-68$ & & & & $52-57$ \\
\hline & & & $95-140$ & & & & \\
\hline
\end{tabular}




\subsection{3 $1954{ }^{228} \mathrm{Ra}$}

Single intravenous injection of radium citrate solution; dogs 13 to 24 months old, of both sexes, placed on experiment from 1954 to 1963. Results as of September, 1987 (Doc. COO-119-263).

\begin{tabular}{|c|c|c|c|c|c|c|c|c|c|}
\hline EXPERIN & ENTAL & EXPER $\mathbb{N}$ & ENTAL RES & LTS & & & & & \\
\hline DESIGN & & & Median & & & & & & \\
\hline Quantity & No. of & No. of & Postexp. & & \% Incidence) & & & & \\
\hline $\begin{array}{l}\text { Injected } \\
\text { (uCi/kg) }\end{array}$ & $\begin{array}{l}\text { Animals } \\
\text { Iniected }\end{array}$ & $\begin{array}{l}\text { Animals } \\
\text { Dead }\end{array}$ & $\begin{array}{l}\text { Survival } \\
\text { (years) }\end{array}$ & Bo & & $\begin{array}{l}\text { Liv } \\
\text { Tun }\end{array}$ & & $\begin{array}{l}\text { Eye } \\
\text { Mel }\end{array}$ & \\
\hline $\begin{array}{l}\text { None } \\
\text { (Control) }\end{array}$ & 13 & 13 & 12.6 & 0 & $(0)$ & 0 & $(0)$ & 0 & (0) \\
\hline 0.02 & 12 & 12 & 11.5 & 0 & $(0)$ & 0 & (0) & 6 & (50) \\
\hline 0.05 & 13 & 13 & 10.6 & 1 & (8) & 0 & $(0)$ & 6 & (46) \\
\hline 0.15 & 12 & 12 & 8.1 & 10 & (83) & 0 & $(0)$ & 3 & (25) \\
\hline 0.3 & 12 & 12 & 6.4 & 9 & (75) & 0 & $(0)$ & 0 & (0) \\
\hline 0.9 & 12 & 12 & 4.0 & 12 & $(100)$ & 0 & $(0)$ & 0 & (0) \\
\hline 2.7 & 8 & 8 & 3.0 & 6 & (75) & 0 & $(0)$ & 0 & (0) \\
\hline 9.0 & 7 & 7 & 2.1 & 1 & (14) & 0 & $(0)$ & 0 & (0) \\
\hline
\end{tabular}

This experiment supplements the ${ }^{226} \mathrm{Ra}$ experiment in providing a link between epidemiologic studies of radium effects in humans, and studies in the dog with various radionuclides (but particularly plutonium) for which no effects have been observed in humans (Evans, 1972). All dogs are dead. Brief recent summaries of results have been published (Lloyd, R., 1986; Mays, 1987). An early publication gave a detailed account of experimental design and early results (Dougherty, T., 1962); it and other publications on specific aspects of the experiment usually present comparative data from the Utah ${ }^{239} \mathrm{Pu},{ }^{226} \mathrm{Ra},{ }^{228} \mathrm{Ra},{ }^{228} \mathrm{Th}$, and ${ }^{90} \mathrm{Sr}$ experiments. Control dogs from these other contemporary Utah experiments are sometimes combined in analyses of results (Atherton, 1986).

Gross retention of ${ }^{228} \mathrm{Ra}$ was followed by total-body counting ( $L l o y d, R$., 1976g); interpretation of these data involved detailed study of the retention of ${ }^{228} \mathrm{Ra}$ decay products (Mays, 1958b; Stover, 1959c). The general problem of ${ }^{228} \mathrm{Ra}$ distribution and retention was considered in several papers, which also derived dosimetric parameters for the study (Dougherty, T., 1962; Lloyd, R., 1976h, 1986; Stover, 1962). Estimated median-survival average radiation dose to bone ranged from about $100 \mathrm{rad}$ in the lowest injection group to nearly $10,000 \mathrm{rad}$ in the highest (Doc. COO-119-262). 
Hematologic effects were described and analyzed in a series of papers (Dougherty, J., 1962, 1970, 1971). A variety of biochemical effects measured in blood have been reported (Berliner, 1969; Ruhmann, 1965; Stevens, W., 1967). Nonneoplastic effects described in bone have included fractures (Taylor, G., 1966), radiographic changes (Christensen, 1962), histopathologic changes (Jee, $1962 b, 1971$ ), and effects on blood vessals (Jee, 1969; Park, H., 1972). Nonneoplastic effects have also been reported in the eye (Taylor, 1962b).

The increased incidence of bone tumors is described in early papers (Dougherty, $T ., 1962,1962 c$ ). Later papers on this subject usually include mathematical analysis in terms of dose-response relationships (Blair, 1968; Dougherty, T., 1969; Gridgeman, 1971; Mays, 1969, 1972; Raabe, 1984, 1986, 1986b; Wrenn, 1986c) and discuss possible carcinogenic mechanisms in relation to bone structure and turnover rates (Spiers, 1983), the distribution of spontaneous and radiationinduced tumors (Spiers, 1983), and the dynamics of tumor growth (Thurman, 1971). The increased incidence of intraocular melanomas is described (Taylor, G., $1969,1972 d, 1976$ ), as is the lack of effect of ${ }^{228} \mathrm{Ra}$ on the incidence of mammary tumors (Taylor, G., 1976b).

Several papers have dealt with estimation of the biological effectiveness of ${ }^{228} \mathrm{Ra}$ relative to ${ }^{226} \mathrm{Ra}$ (Dougherty, J., 1970; Gridgeman, 197l; Lloyd, R., 1986; Mays, 1969, 1987).

Information relevant to this experiment will be found in the following University of Utah Radiobiology Laboratory periodic reports:

\begin{tabular}{|c|c|c|c|c|c|}
\hline Issue Date & Dos. No. & Page Refs. & Issue Date & Doc, No. & Page Refs \\
\hline 1955 (Mar) & AECU-3418 & $7-17$ & 1958 (Sep) & $\mathrm{COO}-217$ & $118-125$ \\
\hline & & $34-54$ & 1959 (Mar) & $\mathrm{COO}-218$ & 21-145 \\
\hline 1955 (Sep) & AECU-3109 & $24-53$ & 1959 (Sep) & COO-219 & $24-25$ \\
\hline 1956 (Mar) & TID-16458 & $23-53$ & & & $42-43$ \\
\hline & & $61-67$ & 1960 (Mar) & COO-220 & 29-165 \\
\hline & & $82-88$ & 1960 (Sep) & $\mathrm{COO}-222$ & $27-52$ \\
\hline 1956 (Sep) & TID-16459 & $19-35$ & 1961 (Sep) & $\mathrm{COO}-224$ & $32-56$ \\
\hline 1957 (Mar) & $A E C \cup-3522$ & $13-148$ & 1962 (Mar) & $\mathrm{COO}-225$ & $31-48$ \\
\hline \multirow[t]{2}{*}{1957} & AECU-3583 & $13-61$ & & & $92-106$ \\
\hline & & $68-81$ & & & $109-128$ \\
\hline & & $94-114$ & 1962 (Sep) & COO-226 & $35-49$ \\
\hline 1958 (Mar) & COO-215 & $14-115$ & 1963 (Sep) & $\mathrm{COO}-228$ & $61-66$ \\
\hline 1958 (Sep) & $\mathrm{COO}-217$ & $14-113$ & & & $95-108$ \\
\hline
\end{tabular}




\begin{tabular}{|c|c|c|c|c|c|}
\hline Issue Date & Doc. No. & Page Refs. & Issue Date & Doc. №. & Page Refs. \\
\hline 1966 (Mar) & COO-119-234 & $94-119$ & 1973 (Mar) & COO-119-248 & $370-377$ \\
\hline 1969 (Mar) & COO-119-240 & $86-96$ & 1979 (Mar) & COO-119-254 & $9-14$ \\
\hline & & $224-261$ & 1985 (Dec) & COO-119-261 & $61-66$ \\
\hline \multirow[t]{2}{*}{1970} & COO-119-242 & $211-257$ & & & \\
\hline & & $385-401$ & & & \\
\hline
\end{tabular}


8.1.4 $1954{ }^{228} \mathrm{Th}$

Single intravenous injection of thorium citrate solution; dogs 10 to

24 months old, of both sexes, placed on experiment from 1954 to 1963. Results as of September, 1987 (Doc. COO-119-263).

\begin{tabular}{|c|c|c|c|c|c|c|c|}
\hline EXPERIN & NTAL & EXPERIN & TAL_RESUL & & & & \\
\hline DESIGN & & & Median & & & & \\
\hline Quantity & No. of & No. of & Postexp. & No. $1 \%$ & ence) & & \\
\hline Injected & Animals & Animals & Survival & Bone & & Liver & \\
\hline$(\mu \mathrm{Ci} / \mathrm{kg})$ & Injected & Dead & (vears) & Tumor & & Turnor & \\
\hline $\begin{array}{l}\text { None } \\
\text { (Control) }\end{array}$ & 13 & 13 & 12.5 & 0 & $(0)$ & 0 & $(0)$ \\
\hline 0.002 & 13 & 13 & 12.5 & 0 & (0) & 2 & (15) \\
\hline 0.005 & 12 & 12 & 11.1 & 2 & (17) & 0 & (0) \\
\hline 0.015 & 12 & 12 & 9.2 & 5 & (42) & 1 & (8) \\
\hline 0.03 & 13 & 13 & 6.5 & 11 & (85) & 0 & $(0)$ \\
\hline 0.09 & 13 & 13 & 3.0 & 12 & (92) & 0 & (0) \\
\hline 0.3 & 12 & 12 & 2.4 & 12 & $(100)$ & 0 & $(0)$ \\
\hline 0.9 & 4 & 4 & 2.1 & 2 & (50) & 0 & $(0)$ \\
\hline 2.7 & 2 & 2 & 0.4 & 0 & (0) & 0 & (0) \\
\hline
\end{tabular}

This experiment was designed to supplement the ${ }^{226} \mathrm{Ra}$ experiment in providing a link between epidemiologic studies of radium effects in humans, and studies in the dog with various radionuclides (but particularly plutonium) for which no effects have been observed in humans (Evans, 1972). Perhaps more importantly, the experiment is one of the few sources of information on the toxicology of radionuclides of concern in any future implementation of a thorium fuel cycle (Stover, 1981).

All dogs are dead. The experiment has been briefly summarized (Lloyd, R., 1986; Stover, 1981), and detailed retention and dosimetry data have been published (Lloyd, 1984d). An earlier publication gave greater detail on experimental design and early results (Dougherty, T., 1962); it and other publications on specific aspect of the experiment usually present comparative data from the Utah ${ }^{239} \mathrm{Pu},{ }^{226} \mathrm{Ra},{ }^{228} \mathrm{Ra},{ }^{228} \mathrm{Th}$, and ${ }^{90} \mathrm{Sr}$ experiments. Control dogs from these other contemporary Utah experiments are sometimes combined in analyses of results (Atherton, 1986). 
Gross retention of ${ }^{228} \mathrm{Th}$ was followed by total-body counting (Lloyd, R., 1976g). Interpretation of these data involved extensive study of the retention of ${ }^{228} \mathrm{Th}$ decay products (Lloyd, 1984d; Mays, 1958b; Stover, 1959c, 1960, 1965b, 1965c, 1968c, 1981; Van Dilla, 1956, 1957). A supporting 20-dog sacrifice study provided detailed data for the period to 18 months postinjection (Lloyd, R., 1984d). The general problem of ${ }^{228} \mathrm{Th}$ distribution and retention was considered in several papers, which also derived dosimetric parameters for the study (Dougherty, T., 1962; Lloyd, R., 1984d; Stover 1960, 1962, 1965b, 1965c, 1981). Estimated mediansurvival average radiation dose to bone ranged from about $15 \mathrm{rad}$ in the lowest injection group to about 4,000 rad in the highest (Doc. COO-119-262). A number of papers have dealt with local and microscopic distribution and dosimetry in bone (Jee, 1964, 1971; Lloyd, R., 1984d) and teeth (Jee, 1960).

Hematologic effects were described and analyzed in a series of papers (Dougherty, J., 1962, 1970, 1971). A variety of biochemical effects measured in blood have been reported (Berliner, 1969; Ruhmann, 1965; Stevens, W., 1967). Nonneoplastic effects described in bone have included fractures (Taylor, G., $1966 b$ ), radiographic changes (Christensen, 1962), histopathologic changes (Jee, $1962 b, 1971$ ), and effects on blood vessals (Jee, 1960b, 1969; Park, H., 1972). Nonneoplastic effects have also been reported in the eye (Taylor, G., 1962b).

The increased incidence of bone tumors is described in early papers (Dougherty, $T ., 1962,1962 \mathrm{c}$ ). Later papers on this subject usually include mathematical analysis in terms of dose-response relationships (Blair, 1968; Dougherty, T., 1969; Gridgeman, 1971; Lloyd, R., 1986; Mays, 1969, 1972, 1987; Raabe, 1984, 1986, 1986b; Wrenn, 1986b) and discuss possible carcinogenic mechanisms in relation to bone structure and turnover rates (Spiers, 1983), the distribution of spontaneous and radiation-induced tumors (Spiers, 1983), and the dynamics of tumor growth (Thurman, 1971). The increased incidence of intraocular melanomas is described (Taylor, G., 1972d, 1976), as is the lack of effect of ${ }^{228} \mathrm{Th}$ on the incidence of mammary tumors (Taylor, G., 1976b).

Several papers have dealt with estimation of the biological effectiveness of ${ }^{228} \mathrm{Th}$ relative to ${ }^{226} \mathrm{Ra}$ (Dougherty, J., 1970; Gridgeman, 1971; Lloyd, R., 1986; Mays, 1969, 1987).

Information relevant to this experiment will be found in the following University of Utah Radiobiology Laboratory periodic reports: 


\begin{tabular}{|c|c|c|c|c|c|c|c|}
\hline \multicolumn{2}{|c|}{ Issue Date } & Doc. No. & Page Refs. & \multicolumn{2}{|c|}{ Issue Date } & Doc. No. & Page Refs. \\
\hline \multirow[t]{2}{*}{1955} & (Mar) & AECU-3418 & $7-17$ & 1962 & (Mar) & $\mathrm{COO}-225$ & $31-47$ \\
\hline & & & $34-54$ & & & & $85-106$ \\
\hline 1955 & (Sep) & AECU-3109 & $24-61$ & & & & $109-122$ \\
\hline \multirow[t]{2}{*}{1956} & (Mar) & TID-16458 & $24-53$ & 1962 & (Sep) & $\mathrm{COO}-226$ & $35-49$ \\
\hline & & & $79-88$ & & & & $78-85$ \\
\hline 1956 & (Sep) & TID-16459 & $19-35$ & 1963 & (Sep) & $\mathrm{COO}-228$ & $61-66$ \\
\hline 1957 & (Mar) & AECU-3522 & $13-148$ & & & & $95-108$ \\
\hline \multirow[t]{3}{*}{1957} & (Sep) & AECU-3583 & $13-61$ & 1964 & (Mar) & COO-229 & $59-73$ \\
\hline & & & $68-81$ & 1964 & (Sep) & COO-119-231 & 110 \\
\hline & & & $94-114$ & 1965 & (Mar) & COO-119-232 & $74-121$ \\
\hline 1958 & (Mar) & $C 00.215$ & $14-115$ & 1966 & (Mar) & COO-119-234 & $94-119$ \\
\hline 1958 & (Sep) & COO-217 & $14-101$ & 1968 & (Mar) & COO-119-237 & $110-116$ \\
\hline \multirow[t]{2}{*}{1959} & (Mar) & COO-218 & $21-84$ & 1969 & (Mar) & COO-119-240 & $86-96$ \\
\hline & & & $93-145$ & & & & $224-261$ \\
\hline \multirow[t]{2}{*}{1959} & (Sep) & CoO-219 & $24-25$ & 1970 & (Mar) & COO-119-242 & $211-257$ \\
\hline & & & $52-59$ & & & & $385-401$ \\
\hline \multirow[t]{2}{*}{1960} & (Mar) & $\mathrm{COO}-220$ & $29-167$ & 1973 & (Mar) & COO-119-248 & $370-377$ \\
\hline & & & $211-219$ & 1985 & (Dec) & COO-119-261 & $61-66$ \\
\hline 1960 & (Sep) & $\mathrm{COO}-222$ & $27-52$ & & & & \\
\hline \multirow[t]{2}{*}{1961} & (Sep) & COO-224 & $38-56$ & & & & \\
\hline & & & $91-93$ & & & & \\
\hline
\end{tabular}




\subsection{5 $1954^{90} \mathrm{Sr}$}

Single intravenous injection of strontium citrate solution; dogs 14 to 21 months old, of both sexes, placed on experiment from 1955 to 1966. Results as of September, 1987 (Doc. COO-119-263).

\begin{tabular}{|c|c|c|c|c|c|c|c|}
\hline \multirow{2}{*}{\multicolumn{2}{|c|}{$\begin{array}{l}\text { EXPERIMENTAL } \\
\text { DESIGN }\end{array}$}} & \multicolumn{6}{|c|}{ EXPERIMENTAL RESULTS } \\
\hline & & & Median & & & & \\
\hline Quantity & No. of & No. of & Postexp. & №. $1 \%$ & nee) & & \\
\hline Injected & Animals & Animals & Survival & Bone & & Liver & \\
\hline$(\mu \mathrm{Ci} / \mathrm{kg})$ & Injected & Dead & (vears) & Tumor & & Iumor & \\
\hline $\begin{array}{l}\text { None } \\
\text { (Control) }\end{array}$ & 13 & 13 & 11.4 & 0 & (0) & 0 & (0) \\
\hline 0.6 & 12 & 12 & 14.1 & 0 & $(0)$ & 0 & (0) \\
\hline 1.8 & 12 & 12 & 12.7 & 0 & (0) & 1 & (8) \\
\hline 3.6 & 12 & 12 & 10.6 & 0 & (0) & 2 & $(17)$ \\
\hline 11. & 12 & 12 & 13.0 & 1 & (8) & 0 & (0) \\
\hline 32. & 12 & 12 & 10.8 & 2 & $(17)$ & 0 & (0) \\
\hline 64. & 12 & 12 & 6.1 & 8 & $(67)$ & 0 & (0) \\
\hline 100 & 14 & 14 & 3.4 & 8 & $(57)$ & 0 & (0) \\
\hline
\end{tabular}

All animals are dead, but no comprehensive report on the experiment has been published. Progress reports on various aspects of the experiment typically present comparative data from the Utah ${ }^{239} \mathrm{Pu},{ }^{226} \mathrm{Ra},{ }^{228} \mathrm{Ra},{ }^{228} \mathrm{Th}$, and ${ }^{90} \mathrm{Sr}$ studies. Control dogs from these other contemporary Utah experiments are sometimes combined in analyses of results (Atherton, 1986).

Gross retention of ${ }^{90} \mathrm{Sr}$ was followed by total-body bremsstrahlung counting (Lloyd, R., 1963, 1976g, 1976h; Mays, 1958c). Distribution and retention of ${ }^{90} \mathrm{Sr}$ was described in a series of papers, which also derived dosimetric parameters for the study (Stover, 1958, 1962). Estimated median-survival average radiation dose to bone ranged from about $100 \mathrm{rad}$ in the lowest injection group to nearly $10,000 \mathrm{rad}$ in the highest ( $D O c$. COO-119-262). The internal dosimetry of injected ${ }^{90} \mathrm{Sr}$, as derived from this experiment, has been compared with that of inhaled (McClellan, 1972c), and ingested (Momeni, 1976) ${ }^{90} \mathrm{Sr}$. Other papers have considered the effect of age at injection, confinement, and level of radiation exposure on ${ }^{90} \mathrm{Sr}$ retention (Decker, 1964; Glad, 1960), the distribution of ${ }^{90} \mathrm{Sr}$ among bones (Lloyd, R., 1976e), and local distribution and dosimetry within bone (Jee, 1971; Spiers, 1969, 1988 ) and teeth (Jee, 1960). 
Hematologic effects were described and analyzed in a series of papers (Dougherty, J., 1962, 1970, 1971, 1972b; Goldman, 1976). Early hematopoietic deaths at high dose levels have been described (Dougherty, J., 1972b). A variety of biochemical effects have been measured in blood (Berliner, 1969; Ruhmann, 1965). Histopathologic effects have been studied in bone at the electronmicroscopic level (Park, H., 1972).

The increased incidence of bone tumors has been described and analyzed in terms of dose-response relationships (Blair, 1972; Dougherty, T., 1969; Gridgeman, 1971; Mays, 1969, 1972c). Other papers discuss possible carcinogenic mechanisms in relation to bone structure and turnover rates (Spiers, 1983, 1988), the distribution of spontaneous and radiation-induced tumors (Spiers, 1983), and the dynamics of tumor growth (Thurman, 1971). There was no effect of ${ }^{90} \mathrm{Sr}$ on the incidence of mammary tumors (Taylor G., 1976b).

Several papers have dealt with the estimation of biological effectiveness of ${ }^{90} \mathrm{Sr}$ relative to ${ }^{226} \mathrm{Ra}$ (Dougherty, J., 1970; Gridgeman, 1971; Mays, 1969, 19806) and with the estimation of risk factors for man (Blair, 1972; Mays, 1972c).

Information relevant to this experiment will be found in the following University of Utah Radiobiology Laboratory periodic reports:

\begin{tabular}{|c|c|c|c|c|c|c|c|}
\hline \multicolumn{2}{|c|}{ Issue Date } & Doc. No. & Page Refs. & \multicolumn{2}{|c|}{ Issue Date } & Doc. No. & Page Refs \\
\hline 1955 & (Mar) & AECU-3418 & $18-31$ & 1959 & (Sep) & COO-219 & $24-25$ \\
\hline \multirow[t]{2}{*}{1955} & (Sep) & AECU-3109 & $44-56$ & & & & $42-51$ \\
\hline & & & $68-70$ & 1960 & (Mar) & $\mathrm{COO}-220$ & $29-143$ \\
\hline \multirow[t]{2}{*}{1956} & (Mar) & TID-16458 & $24-53$ & 1961 & (Sep) & $\mathrm{COO}-224$ & $38-74$ \\
\hline & & & $89-108$ & & & & $83-90$ \\
\hline 1956 & (Sep) & TID-16459 & $19-35$ & 1963 & (Sep) & COO-228 & $61-68$ \\
\hline 1957 & (Mar) & AECU-3522 & $13-148$ & & & & $95-108$ \\
\hline \multirow[t]{4}{*}{1957} & (Sep) & AECU-3583 & $13-61$ & 1965 & (Mar) & $\mathrm{COO}-119-232$ & $81-96$ \\
\hline & & & $68-73$ & 1966 & (Mar) & COO-119-234 & p. 69 \\
\hline & & & $87-90$ & 1968 & (Mar) & COO-119-237 & $90-109$ \\
\hline & & & $94-104$ & 1969 & (Mar) & COO-119-240 & $86-96$ \\
\hline \multirow[t]{2}{*}{1958} & (Mar) & $\mathrm{COO}-215$ & $14-115$ & & & & $224-261$ \\
\hline & & & $133-146$ & 1970 & (Mar) & COO-119-242 & $385-401$ \\
\hline \multirow[t]{2}{*}{1958} & (Sep) & $\mathrm{COO}-217$ & $14-101$ & 1985 & (Dec) & COO-119-261 & $61-66$ \\
\hline & & & $114-141$ & & & & \\
\hline \multirow[t]{2}{*}{1959} & (Mar) & COO-218 & $21-84$ & & & & \\
\hline & & & $113-145$ & & & & \\
\hline
\end{tabular}


Single intravenous injection of americium citrate solution; dogs 15 to 19 months old, of both sexes, placed on experiment in 2 series: "A", from 1966 to 1970; and " $B$ ", from 1974 to 1975. Results as of September, 1987 (Doc. COO-119-263).

\begin{tabular}{|c|c|c|c|c|c|c|c|c|}
\hline \multirow{2}{*}{\multicolumn{3}{|c|}{$\begin{array}{l}\text { EXPERIMENTAL } \\
\text { DESIGN }\end{array}$}} & \multicolumn{6}{|c|}{ EXPERIMENTAL RESULTSS } \\
\hline & & & \multirow{4}{*}{$\begin{array}{l}\text { No. of } \\
\text { Animals } \\
\text { Dead }\end{array}$} & \multirow{4}{*}{$\begin{array}{l}\text { Median } \\
\text { Postexp. } \\
\text { Survival } \\
\text { (vears) }\end{array}$} & & & & \\
\hline Quantity & & ies, & & & \multicolumn{2}{|c|}{ №. (\% Incidence) } & \multirow{3}{*}{\multicolumn{2}{|c|}{$\begin{array}{l}\text { Liver } \\
\text { Iumor }\end{array}$}} \\
\hline Injected & & No. & & & Bone & & & \\
\hline (1) & & cted & & & Iumor & & & \\
\hline 0.002 & A & 14 & 14 & 11.5 & 0 & (0) & 0 & (0) \\
\hline 0.005 & A & 14 & 14 & 12.5 & 1 & (7) & 2 & (14) \\
\hline \multirow[t]{2}{*}{0.015} & A & 14 & 14 & 10.6 & 1 & (7) & 3 & (21) \\
\hline & B & 12 & 3 & $>11.3$ & 0 & (0) & 0 & (0) \\
\hline \multirow[t]{2}{*}{0.05} & A & 13 & 13 & 10.1 & 4 & (31) & 2 & (15) \\
\hline & B & 11 & 10 & 9.5 & 4 & (40) & 2 & (20) \\
\hline 0.1 & A & 12 & 12 & 7.8 & 9 & (75) & 2 & (17) \\
\hline 0.3 & A & 13 & 13 & 4.8 & 12 & (92) & 1 & (8) \\
\hline 0.9 & A & 12 & 12 & 3.8 & 8 & (67) & 1 & (8) \\
\hline 2.8 & A & 2 & 2 & 1.1 & 0 & (0) & 0 & (0) \\
\hline
\end{tabular}

All series A dogs are dead. No comprehensive description of the total experiment has been published, but detailed retention and dosimetry data have been reported (Lloyd $R$., 1984c). Retention of ${ }^{241} \mathrm{Am}$ was measured by whole-body counting (Lloyd, R., 1970, 1976g), with liver content estimated from counts with and without shielding in the liver region (Lloyd, $R ., 1970,1975 d$ ). Papers have described the early retention of americium in the blood (Bruenger, 1969), and its gross distribution and retention in bone and liver (Lloyd, R., 1970, 1984c; Stevens, W., 1969). Estimated median-survival average radiation dose to bone ranged from about $10 \mathrm{rad}$ in the lowest injection group to about $1500 \mathrm{rad}$ in the highest (Doc. COO-119-262).

The distribution of americium among different bones (Lloyd, R., 1972b, 1984c) and its microscopic distribution and dosimetry within bone (Lloyd, R., 1972b; Polig, 1984, 1984b) have been reported. Microscopic (Taylor, G., 1972b) and subcellular (Bruenger, 1976b) distribution in liver was investigated. Retention in liver was shown to be a function of deposition level (Lloyd, R., 1972b, 1984c). Americium concentrates in the thyroid (Stevens, W., 1969; Lloyd, R., 1970), 
where its microscopic (Taylor, G., 1969c) and subcellular (Stevens, W., 1969) distribution and damaging effects (Lloyd, $R$., 1972b) have been studied. A supporting experiment studied the retention and distribution of americium administered to neonatal beagles (Stevens, W., 1977).

Early histopathologic effects have been described for liver (Lloyd, R., 1972b; Taylor, G., 1972b), kidney (Lloyd, R., 1972b), and bone (Park, H., 1972). Serum biochemical effects have been compared with those observed in plutoniuminjected animals (Nabors, 1972). Cortisol metabolism in the skin was shown to be elevated in dogs bearing ${ }^{241} \mathrm{Am}$ (Nabors, 1975).

The increased incidence of liver tumors (Taylor, G., 1972b, 1986) and bone tumors (Mays, 1987; Raabe, 1984, 1986, 1986b; Wrenn, 1986, 1986c) has been subjected to preliminary analysis.

Information relevant to this experiment will be found in the following University of Utah Radiobiology Laboratory periodic reports:

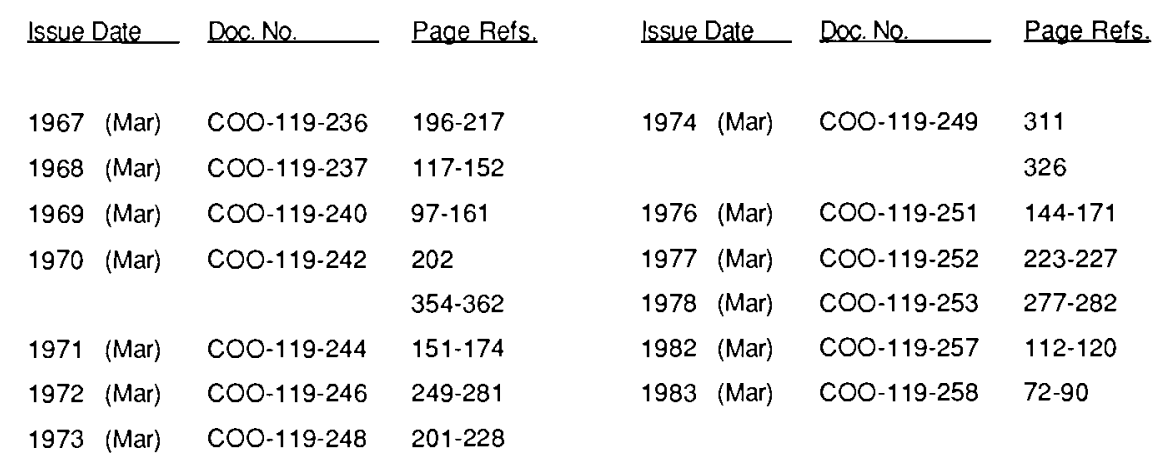




\subsection{7 $1971^{249} \mathrm{Cf}$}

Single intravenous injection of californium citrate solution; dogs 15 to 19 months old, of both sexes, placed on experiment from 1971 to 1974. Results as of September, 1987 (Doc. COO-119-263).

\begin{tabular}{|c|c|c|c|c|c|c|c|}
\hline \multirow{2}{*}{\multicolumn{2}{|c|}{$\begin{array}{l}\text { EXPERIMENTAL } \\
\text { DESIGN }\end{array}$}} & \multicolumn{6}{|c|}{ EXPERIMENTAL RESULTS } \\
\hline & & & Median & & & & \\
\hline Quantity & No. of & No. of & Postexp. & №. (\%) & ence) & & \\
\hline Injected & Animals & Animals & Survival & Bone & & Liver & \\
\hline$(\mu \mathrm{Ci} / \mathrm{kg})$ & Injected & Dead & (vears) & Tumor & & Iumor & \\
\hline $\begin{array}{l}\text { None } \\
\text { (Control) }\end{array}$ & 6 & 4 & $>12.8$ & 0 & (0) & 0 & (0) \\
\hline 0.0006 & 6 & 4 & $>13.1$ & 0 & (0) & 0 & (0) \\
\hline 0.005 & 6 & 5 & 11.0 & 0 & (0) & 0 & $(0)$ \\
\hline 0.015 & 6 & 6 & 11.2 & 0 & (0) & 0 & (0) \\
\hline 0.09 & 6 & 6 & 7.1 & 5 & (83) & 0 & $(0)$ \\
\hline 0.3 & 6 & 6 & 4.4 & 6 & $(100)$ & 0 & $(0)$ \\
\hline
\end{tabular}

Descriptions of this experiment and the rationale for comparative ${ }^{249} \mathrm{Cf}$ and ${ }^{252} \mathrm{Cf}$ studies appear in several progress reports (Lloyd, R., 1976; Mays, 1972b; Wrenn, 1986). Retention of ${ }^{249} \mathrm{Cf}$ was measured by whole-body counting (Lloyd, R., 1972), with liver content estimated from counts with and without shielding in the liver region (Lloyd, $R ., 1975 d$ ). Estimated median-survival average radiation dose to bone ranged from about 4 rad in the lowest injection group to about 800 rad in the highest (Doc. COO-119-262).

The retention and protein-binding of ${ }^{249} \mathrm{Cf}$ in the blood during the first 48 hours postinjection have been described (Stevens, W., 1972). Its gross distribution among soft tissues (Atherton, 1972), its microscopic distribution in soft tissues (Taylor G., 1972), and its subcellular distribution in liver (Bruenger, 1972, 1976b) were studied at 1 and 3 weeks postinjection. Its longer-term distribution and retention is reported to 160 days (Lloyd, $R$., 1972) and to about 4 years (Lloyd, $R$., 1976 ) postinjection.

The increased incidence of bone tumors has been subjected to preliminary analysis (Raabe, 1984, 1986, 1986b; Wrenn, 1986, 1986c).

Information relevant to this experiment will be found in the following University of Utah Radiobiology Laboratory periodic reports: 


\begin{tabular}{|c|c|c|c|c|c|}
\hline Issue Date & Doc. No. & Page Refs. & Issue Date & Dec. No. & Page Refs. \\
\hline 1971 (Mar) & COO-119-244 & $126-150$ & 1975 (Mar) & COO-119-250 & $231-237$ \\
\hline 1972 (Mar) & COO-119-246 & $287-330$ & 1978 (Mar) & COO-119-253 & $283-286$ \\
\hline 1973 (Mar) & COO-119-248 & $156-162$ & 1982 (Mar) & $\mathrm{COO}-119-257$ & $112-115$ \\
\hline & & $201-212$ & & & \\
\hline
\end{tabular}




\subsection{8 $1971^{252} \mathrm{Cf}$}

Single intravenous injection of californium citrate solution; dogs 15 to 19 months old, of both sexes, placed on experiment from 1971 to 1973. Results as of September, 1987 (Doc. COO-119-263).

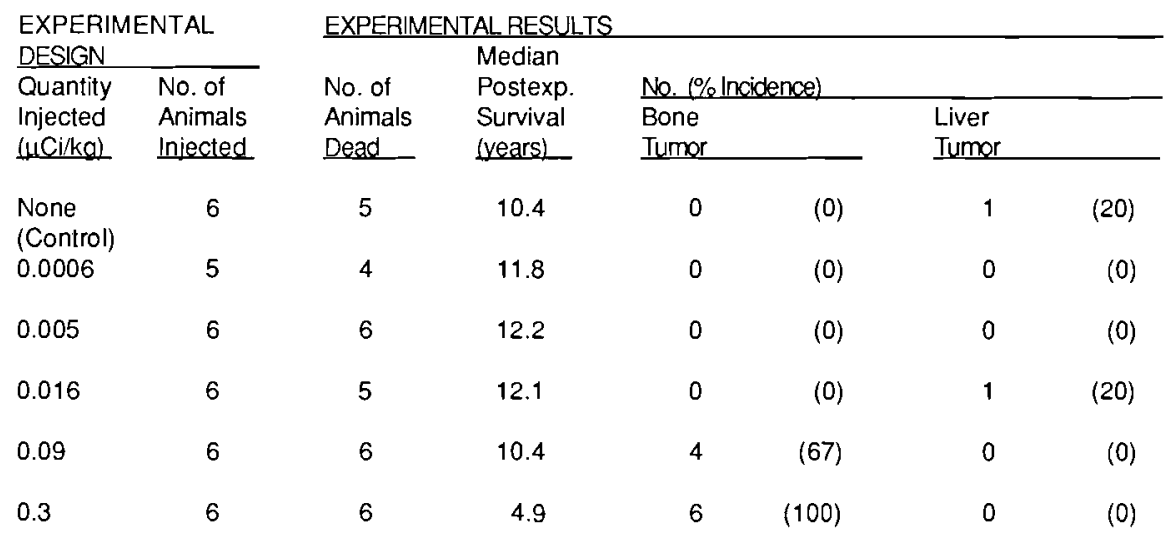

Descriptions of this experiment and the rationale for comparative ${ }^{249} \mathrm{Cf}$ and ${ }^{252} \mathrm{Cf}$ studies appear in several progress reports (Lloyd, R., 1976; Mays, 1972b; Wrenn, 1986). Retention of ${ }^{252} \mathrm{Cf}$ was measured by whole-body counting (Lloyd, R., 1972), with liver content estimated from counts with and without shielding in the liver region (Lloyd, $R$., 1975d). Estimated median-survival average radiation dose to bone ranged from about $2 \mathrm{rad}$ in the lowest injection group to nearly $1000 \mathrm{rad}$ in the highest (Doc. COO-119-262).

The retention and protein-binding of ${ }^{252} \mathrm{Cf}$ in the blood during the first 48 hours postinjection have been described (Stevens, W., 1972), as has its early subcellular distribution in liver (Bruenger, 1976b). Its longer-term distribution and retention is reported to 160 days (Lloyd, R., 1972) and to about 4 years (Lloyd, R., 1976) postinjection.

The increased incidence of bone tumors has been subjected to preliminary analysis (Raabe, 1984, 1986, 1986b; Wrenn, 1986, 1986c). From early observations, the carcinogenicity of fission fragments from ${ }^{252} \mathrm{Cf}$ appears to be much lower than that of alpha particles (Lloyd, R., 1976; Taylor, G., 1983b; Wrenn, 1986).

Information relevant to this experiment will be found in the following University of Utah Radiobiology Laboratory periodic reports: 


\begin{tabular}{|c|c|c|c|c|c|}
\hline Issue Date & Doc. №. & Page Refs. & Issue Date & Doc. No. & Page Refs. \\
\hline 1971 (Mar) & COO-119-244 & $147-148$ & 1975 (Mar) & COO-119-250 & $231-237$ \\
\hline 1972 (Mar) & COO-119-246 & $287-330$ & 1978 (Mar) & COO-119-253 & $283-286$ \\
\hline 1973 (Mar) & COO-119-248 & $156-162$ & 1982 (Mar) & $\mathrm{COO}-119-257$ & $112-115$ \\
\hline & & $201-212$ & & & \\
\hline
\end{tabular}


8.1.9 $1972{ }^{239 P u}$

Single intravenous injection of plutonium citrate solution; dogs 2.9 to 3.5 months old, of both sexes, placed on experiment from 1972 to 1978. Results as of September, 1987 (Doc. COO-119-263).

\begin{tabular}{|c|c|c|c|c|c|c|c|}
\hline \multirow{2}{*}{\multicolumn{2}{|c|}{$\begin{array}{l}\text { EXPERIMENTAL } \\
\text { DESIGN }\end{array}$}} & \multicolumn{6}{|c|}{ EXPERIMENTAL RESULTS } \\
\hline & & & Median & & & & \\
\hline Quantity & No. of & No. of & Postexp. & \multicolumn{4}{|c|}{ No. (\% Incidence) } \\
\hline Injected & Animals & Animals & Survival & Bone & & Liver & \\
\hline$(\mu \mathrm{Ci} / \mathrm{kg})$ & Injected & Dead & (years) & Tumor & & Tumor & \\
\hline $\begin{array}{l}\text { None } \\
\text { (Control) }\end{array}$ & 8 & 7 & 12.6 & 0 & (0) & 0 & (0) \\
\hline 0.005 & 11 & 4 & $>11.5$ & 0 & (0) & 0 & (0) \\
\hline 0.016 & 10 & 5 & $>10.5$ & 0 & $(0)$ & 0 & (0) \\
\hline 0.05 & 11 & 4 & $>10.9$ & 1 & (25) & 1 & (25) \\
\hline 0.1 & 11 & 5 & $>10.1$ & 1 & (33) & 1 & (33) \\
\hline 0.3 & 12 & 12 & 7.1 & 8 & (67) & 2 & (17) \\
\hline 3.0 & 12 & 12 & 3.5 & 12 & $(100)$ & 0 & (0) \\
\hline
\end{tabular}

Published details of this experiment are limited to descriptions of experimental design (Jee, 1976b; Wrenn, 1986) and an account of results from the highest injection level (Bruenger, 1980), where the 12 dogs exhibited 17 bone tumors and 33 healed fractures. Estimated median-survival average dose to bone in the highest injection level was about $2500 \mathrm{rad}$ (Doc. COO-119-262).

From supporting sacrifice studies, the early distribution and retention of plutonium has been described (Lloyd, R., 1978c; Bruenger, 1980, 1983, 1984), including data from dogs injected at 2 days of age (Bruenger, 1978). As compared to young adults, the juvenile dogs deposited and retained less plutonium in liver and more plutonium in bone. Plutonium deposited on bone surfaces was rapidly buried, however, and may be less hazardous than plutonium deposited in older animals (Wrenn, 1986).

Information relevant to this experiment will be found in the following University of Utah Radiobiology Laboratory periodic reports: 


\begin{tabular}{|c|c|c|c|c|c|}
\hline Issue Date & Doc. No. & Page Refs. & Issue Date & Doc. No. & Page Refs. \\
\hline 1973 (Mar) & COO-119-248 & $317-335$ & 1983 (Mar) & COO-119-258 & $16-19$ \\
\hline 1976 (Mar) & COO-119-251 & $172-180$ & & & 46 \\
\hline 1977 & COO-119-252 & $188-190$ & 1984 (Dec) & COO-119-259 & $3-8$ \\
\hline & & $210-222$ & & & $63-64$ \\
\hline 1978 (Mar) & CoO-119-253 & $165-185$ & & & $113-127$ \\
\hline 1979 (Mar) & $\mathrm{COO}-119-254$ & $65-73$ & 1985 (Dec) & COO-119-261 & $5-10$ \\
\hline 1982 (Mar) & COO-119-257 & $12-22$ & 1986 (Dec) & COO-119-262 & $4-8$ \\
\hline
\end{tabular}




\subsubsection{0 $1973{ }^{253} \mathrm{Es}$}

Single intravenous injection of einsteinium citrate solution; dogs 16 months old, of both sexes, placed on experiment in 1973. Results as of September, 1987 (Doc. COO-119-263).

$\begin{array}{ll}\text { EXPERIMENTAL } \\ \text { DESIGN } & \\ \text { Quantity } & \text { No. of } \\ \text { Injected } & \text { Animals } \\ (\mu \mathrm{Ci} / \mathrm{kgl} & \text { Injected }\end{array}$

0.3

3.0

\section{EXPERIMENTAL RESULTS}

Median

Animals Survival

Dead _ (years)

$2 \quad 12.9$

3
No. $1 \%$ Incidence)

0

(0)

Liver

Tumor

$\begin{array}{ll}0 & (0) \\ 0 & (0)\end{array}$

Papers have appeared on retention and distribution of einsteinium to 55 days postinjection as determined from 3 sacrifice dogs (Lloyd, R., 1975), and on its subcellular distribution in liver (Bruenger, 1976b).

Information relevant to this experiment will be found in the following University of Utah Radiobiology Laboratory periodic reports:
Issue Date Doc. No. Page Refs.
1974 (Mar) COO-119-249 234-280
1984 (Dec) COO-119-259 113-127 


\subsubsection{1 $1975{ }^{239} \mathrm{Pu}$}

Single intravenous injection of plutonium citrate solution; dogs 4.1 to 5.2 years old, of both sexes, placed on experiment from 1975 to 1978. Results as of September, 1987 (Doc. COO-119-263).

\begin{tabular}{|c|c|c|c|c|c|c|c|}
\hline \multirow{2}{*}{\multicolumn{2}{|c|}{$\begin{array}{l}\text { EXPERIMENTAL } \\
\text { DESIGN }\end{array}$}} & \multicolumn{6}{|c|}{ EXPERIMENTAL RESULTS } \\
\hline & & & Median & & & & \\
\hline Quantity & No. of & No. of & Postexp. & \multicolumn{4}{|c|}{ №. $(\%$ Incidence) } \\
\hline Injected & Animals & Animals & Survival & \multirow{2}{*}{\multicolumn{2}{|c|}{ Bone }} & \multirow{2}{*}{\multicolumn{2}{|c|}{$\begin{array}{l}\text { Liver } \\
\text { Iumor }\end{array}$}} \\
\hline$(\mu C i / k q)$ & Injected & Dead & (vears) & & & & \\
\hline 0.016 & 4 & 3 & 8.2 & 1 & (33) & 1 & (33) \\
\hline 0.05 & 10 & 8 & $>7.6$ & 2 & (25) & 0 & 10 \\
\hline 0.1 & 10 & 10 & 5.8 & 4 & $(40)$ & 1 & $(10)$ \\
\hline 0.3 & 10 & 10 & 3.9 & 8 & $(80)$ & 0 & (0) \\
\hline
\end{tabular}

Published details of this experiment are limited to descriptions of experimental design and tabulation of early effects (Jee, 1976b; Wrenn, 1986). Supporting sacrifice studies showed few differences between aged dogs and young adults in early distribution and retention of plutonium (Lloyd, $R ., 1978$ ); local distribution and retention within bone has been compared between aged, young-adult and juvenile animals (Bruenger, 1983). As compared to young adults, the aged dogs retain more plutonium on bone surfaces and thus may be more sensitive to bonetumor production despite their restricted life expectancy (Wrenn, 1986). Estimated median-survival average radiation dose to bone was about $400 \mathrm{rad}$ in the highest injection group (Doc. COO-119-262).

Information relevant to this experiment will be found in the following University of Utah Radiobiology Laboratory periodic reports:

\begin{tabular}{|c|c|c|c|c|c|c|}
\hline \multicolumn{2}{|c|}{ Issue Date } & Doc. No. & Page Refs. & Issue Date & Doc. No. & Page Refs. \\
\hline 1976 & (Mar) & COO-119-251 & $172-180$ & 1984 (Dec) & COO-119-259 & $3-8$ \\
\hline 1977 & (Mar) & COO-119-252 & $188-190$ & & & $113-127$ \\
\hline 1982 & (Mar) & COO-119-257 & $12-15$ & 1985 (Dec) & COO-119-261 & $5-10$ \\
\hline \multirow[t]{2}{*}{1983} & (Mar) & COO-119-258 & $16-19$ & 1986 (Dec) & COO-119-262 & $4-8$ \\
\hline & & & 46 & & & \\
\hline
\end{tabular}




\subsubsection{2 $1975{ }^{226} \mathrm{Ra}$}

Single intravenous injection of radium citrate solution; dogs $\underline{3}$ to 5 months old, of both sexes, placed on experiment from 1975 to 1978.

Results as of September, 1987 (Doc. COO-119-263).

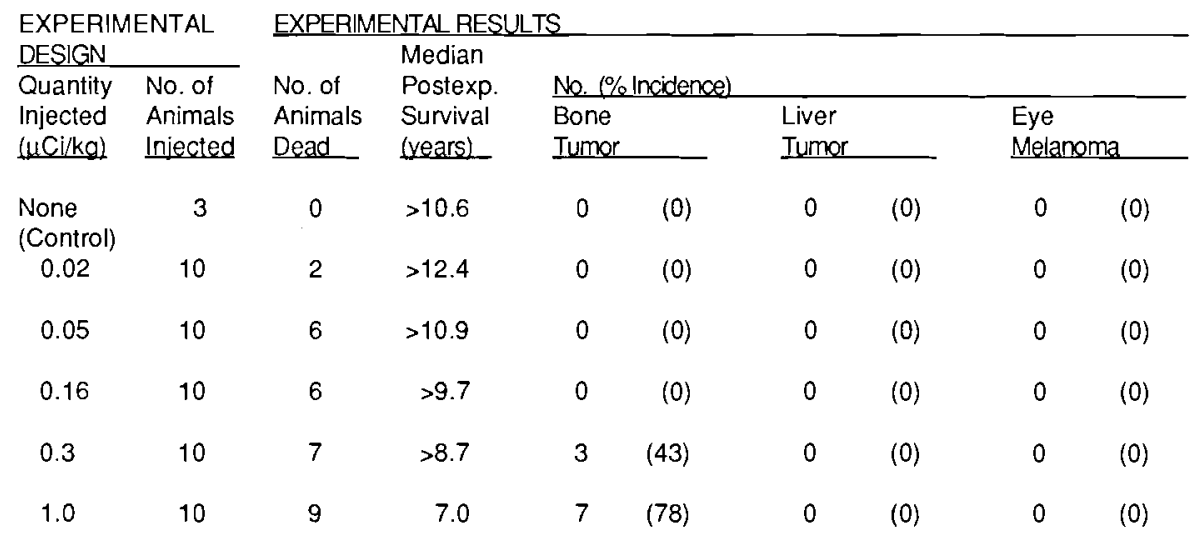

Brief descriptions of the experiment and status reports have been published (Lloyd, R., 1983; Wrenn, 1986). Retention of ${ }^{226}$ Ra by the juvenile beagle has been described in detail, based on sacrificed animals and life-span animals dying to about 6 years postexposure (Lloyd, R., 1983). Estimated median-survival average radiation dose to bone was about $3000 \mathrm{rad}$ in the highest injection group (Doc. COO-119-262).

Data on skeletal retention and distribution of ${ }^{226} \mathrm{Ra}$ (Bruenger, 1983), on skeletal radon to radium ratios (Lloyd, $R ., 1983 d$ ), and on microanatomical distribution within bone (Bruenger, 1983) have been compared for neonatal, juvenile, and older beagles. Retention of ${ }^{226} \mathrm{Ra}$ was substantially greater in juveniles than in young adults, but the ${ }^{222} \mathrm{Rn} /{ }^{226} \mathrm{Ra}$ ratio in bone is lower for about the first 600 days postinjection, and average radiation dose to bone per $\mu \mathrm{Ci}$ injected is lower for juveniles than for young adults (Lloyd, R., 1983).

Information relevant to this experiment will be found in the following University of Utah Radiobiology Laboratory periodic reports: 


\begin{tabular}{|c|c|c|c|c|c|}
\hline Issue Date & Doc. No. & Page Rets. & Issue Date & Doc. No. & Page Refs. \\
\hline 1977 (Mar) & COO-119-252 & $188-190$ & 1984 (Dec) & COO-119-259 & $3-8$ \\
\hline \multirow[t]{2}{*}{1982 (Mar) } & COO-119-257 & $12-30$ & & & $63-64$ \\
\hline & & $38-44$ & 1985 (Dec) & COO-119-261 & $5-10$ \\
\hline \multirow[t]{2}{*}{1983 (Mar) } & CoO-119-258 & $16-19$ & 1986 (Dec) & COO-119-262 & $4-8$ \\
\hline & & 46 & & & \\
\hline
\end{tabular}


$8.1 .13 \quad 1975{ }^{226} \mathrm{Ra}$

Single intravenous injection of radium citrate solution; dogs $\underline{4.9 \text { to }}$ 6.2 years old, of both sexes, placed on experiment from 1975 to 1980. Results as of September, 1987 (Doc. COO-119-263).

\begin{tabular}{ll} 
EXPERIMENTAL \\
DESIGN \\
\hline Quantity & No. of \\
Injected & Animals \\
(uCi/kg) & Injected
\end{tabular}

$\begin{array}{rr}1.0 & 9 \\ 3.0 & 20 \\ 10 . & 4\end{array}$

$\begin{array}{ll}\frac{\text { EXPERIMENTAL RESULTS }}{\text { Median }} \\ \text { No. of } & \text { Postexp. } \\ \text { Animals } & \text { Survival }\end{array}$

$$
\text { Dead }
$$

$$
8
$$

20

4 (years)

5.7
3.7

1.2

\section{№. $(\%$ Incidence) \\ Bone}

Tumor$$
5
$$

(63)

11

1
Kidney Degeneration

1

(12)

10

(50)

3

(75)

Brief descriptions of the study and status reports have been published (Lloyd, $R$., 1983c; Wrenn, 1986). Retention of ${ }^{226} \mathrm{Ra}$ by the aged beagle has been described in some detail to about 5 years postexposure (Lloyd, R., 1983c). Estimated mediansurvival average radiation dose to bone was about $1600 \mathrm{rad}$ in the lowest injection group, about $4000 \mathrm{rad}$ in the middle injection group, and somewhat lower due to shortened life-span in the highest injection group (Doc. COO-119-262).

Data on skeletal retention and distribution of ${ }^{226} \mathrm{Ra}$ (Bruenger, 1983) and on skeletal radon-to-radium ratios (Lloyd, $R$., 1983d) have been compared for aged, and younger beagles. Retention of ${ }^{226} \mathrm{Ra}$ in aged dogs was somewhat lower than that observed in young adults; distribution was essentially similar (Lloyd, R., 1983c). The high mortality at higher dose levels, attributed largely to kidney degeneration, differs from observations in animals exposed at younger ages, but no detailed analysis of effects in aged animals has been published.

Information relevant to this experiment will be found in the following University

\begin{tabular}{|c|c|c|c|c|c|c|c|}
\hline \multicolumn{2}{|c|}{ Issue Date } & Doc. No. & Page Refs. & \multicolumn{2}{|c|}{ Issue Date } & Doc. No. & Page Refs \\
\hline 1977 & (Mar) & COO-119-252 & $188-190$ & 1984 & (Dec) & COO-119-259 & $3-8$ \\
\hline \multirow[t]{2}{*}{1982} & (Mar) & $\mathrm{COO}-119-257$ & $12-15$ & & & & $63-64$ \\
\hline & & & $31-44$ & 1985 & (Dec) & $\mathrm{COO}-119-261$ & $5-10$ \\
\hline \multirow[t]{2}{*}{1983} & (Mar) & $\mathrm{COO}-119-258$ & $16-19$ & & & & $53-60$ \\
\hline & & & 46 & 1986 & (Dec) & $\mathrm{COO}-119-262$ & $4-8$ \\
\hline
\end{tabular}
of Utah Radiobiology Laboratory periodic reports: 
Intravenous injection of radium citrate solution; dogs 15 to 24 months old, of both sexes, placed on experiment from 1977 to 1979 .

Series " $A$ " received a single injection; series " $B$ ", 10 injections at 1-week intervals; and series " $C$ ", 50 injections at 1-week intervals. Results as of September, 1987 (Doc. COO-119-263).

\begin{tabular}{|c|c|c|c|c|c|c|}
\hline \multirow{2}{*}{\multicolumn{3}{|c|}{$\begin{array}{l}\text { EXPERIMENTAL } \\
\text { DESIGN }\end{array}$}} & \multicolumn{4}{|c|}{ EXPEBIMENTAL RESULTS } \\
\hline & & & \multirow{5}{*}{$\begin{array}{l}\text { No. of } \\
\text { Animals } \\
\text { Dead } \\
0\end{array}$} & \multirow{4}{*}{$\begin{array}{l}\text { Median } \\
\text { Postexp. } \\
\text { Survival } \\
\text { (years) }\end{array}$} & & \\
\hline \multirow{6}{*}{$\begin{array}{l}\text { Total } \\
\text { Injected } \\
(\mu \mathrm{Ci} / \mathrm{kgl}) \\
\text { None } \\
\text { (Control) }\end{array}$} & \multirow{3}{*}{\multicolumn{2}{|c|}{$\begin{array}{l}\text { Series, } \\
\text { and No. } \\
\text { Injected }\end{array}$}} & & & \multicolumn{2}{|c|}{ No. $(\%$ Incidence) } \\
\hline & & & & & \multicolumn{2}{|c|}{ Bone } \\
\hline & & & & & \multicolumn{2}{|c|}{ Tumor } \\
\hline & $A$ & 6 & & $>8.4$ & 0 & $(0)$ \\
\hline & $B$ & 6 & 1 & $>7.9$ & 0 & (0) \\
\hline & $c$ & 6 & 4 & $>8.7$ & 0 & (0) \\
\hline \multirow[t]{3}{*}{0.3} & $A$ & 12 & 4 & $>8.2$ & 0 & (0) \\
\hline & B & 12 & 1 & $>8.0$ & 0 & (0) \\
\hline & C & 12 & 6 & $>9.9$ & 0 & (0) \\
\hline \multirow[t]{3}{*}{1.0} & $A$ & 12 & 3 & $>8.3$ & 0 & (0) \\
\hline & $\mathrm{B}$ & 12 & 3 & $>8.0$ & 1 & (33) \\
\hline & C & 12 & 5 & $>9.9$ & 0 & (0) \\
\hline \multirow[t]{3}{*}{3.0} & $A$ & 6 & 0 & $>8.4$ & 0 & $(0)$ \\
\hline & $\mathrm{B}$ & 6 & 2 & $>8.0$ & 0 & (0) \\
\hline & $\mathrm{C}$ & 6 & 5 & $>8.9$ & 0 & (0) \\
\hline \multirow[t]{3}{*}{10.} & $A$ & 8 & 5 & $>7.0$ & 0 & (0) \\
\hline & $\mathrm{B}$ & 6 & 5 & $>7.3$ & 2 & $(40)$ \\
\hline & $\mathrm{C}$ & 6 & 6 & 5.5 & 5 & (83) \\
\hline
\end{tabular}

This experiment was designed to provide a link between epidemiologic studies of ${ }^{224} \mathrm{Ra}$ effects in humans, and studies in the dog with various radionuclides (but particularly plutonium) for which no effects had been observed in humans (Wrenn, 1986). Because of the 3.6-day half-life of ${ }^{224} \mathrm{Ra}$, virtually all of the radiation dose is received within the first month following injection. Estimated average radiation dose to bone was about $30 \mathrm{rad}$ per $\mu \mathrm{Ci} / \mathrm{kg}$ injected (Doc. COO-119-262).

The distribution, retention, and complex internal dosimetry of ${ }^{224} \mathrm{Ra}$ and its decay products have been reported from experiments on 6 dogs sacrificed at intervals to 7 days postinjection (Lloyd, R., 1982). Another supporting study of ${ }^{224} \mathrm{Ra}$ daughter-product equilibrium at bone surfaces indicates an endosteal tissue dose of one-third to one-half that which would be calculated assuming complete equilibrium (Schlenker, 1986). 
Information relevant to this experiment will be found in the following University of Utah Radiobiology Laboratory periodic reports:

\begin{tabular}{|c|c|c|c|c|c|}
\hline Issue Date & Doc. No. & Page Refs. & Issue Date & Doc. No. & Page Refs \\
\hline 1977 (Mar) & COO-119-252 & $272-287$ & 1984 (Dec) & COO-119-259 & $19-24$ \\
\hline 1978 (Mar) & COO-119-253 & $260-276$ & & & $113-127$ \\
\hline 1979 (Mar) & COO-119-254 & 81 & 1985 (Dec) & COO-119-261 & $21-26$ \\
\hline 1983 (Mar) & COO-119-258 & 25 & 1986 (Dec) & COO-119-262 & $15-19$ \\
\hline
\end{tabular}




\subsection{DAVIS EXPERIMENTS}

\subsection{1 $1952 \times$ Ray}

Bilateral, $250 \mathrm{kVp} x$-ray exposures, delivered in different numbers of fractions and different fractionation intervals. Female dogs received first exposures at 8 to 15 months of age, from 1952 to 1958. Some dogs were bred subsequent to exposure, distinguished below as parous (Par.) us nulliparous (Nul.). Survival data from Andersen, 1969; effects data from Rosenblatt, 1986.

\begin{tabular}{|c|c|c|c|c|c|c|c|c|c|c|c|}
\hline \multirow{3}{*}{\multicolumn{2}{|c|}{$\begin{array}{l}\text { EXPERIMENTAL } \\
\text { DESIGN } \\
\text { Exposure (R) }\end{array}$}} & \multicolumn{10}{|c|}{ EXPERIMENTAL RESULTS } \\
\hline & & \multirow{3}{*}{$\begin{array}{l}\text { Median Post- } \\
\text { exposure } \\
\text { Survival } \\
\text { (years) } \\
\end{array}$} & \multicolumn{9}{|c|}{ Percentage Incidence Among Parous, Nulliparous, and All Doas } \\
\hline & & & \multirow{2}{*}{\multicolumn{3}{|c|}{$\begin{array}{l}\text { Nonneoplastic } \\
\text { Diseases }\end{array}$}} & \multirow{2}{*}{\multicolumn{3}{|c|}{$\begin{array}{l}\text { Mammary } \\
\text { Tumors }\end{array}$}} & \multirow{2}{*}{\multicolumn{3}{|c|}{$\begin{array}{l}\text { Nonmammary } \\
\text { Tumors }\end{array}$}} \\
\hline $\begin{array}{l}x \text { Fractions } \\
\text { (Interval) }\end{array}$ & $\begin{array}{l}\text { No. of } \\
\text { Animals }\end{array}$ & & & & & & & & & & \\
\hline $\begin{array}{l}\text { None } \\
\text { (Control) }\end{array}$ & 57 & 11.6 & 38 & 48 & 44 & 27 & 16 & 21 & 35 & 35 & 35 \\
\hline $25 \times 4(28 d)$ & 22 & 11.0 & & & & & & & & & \\
\hline $25 \times 4(14 d)$ & 25 & 10.0 & & & & & & & & & \\
\hline $25 \times 4(7 d)$ & 20 & 11.3 & & & & & & & & & \\
\hline $50 \times 2(28 d)$ & 21 & 10.6 & 49 & 44 & 46 & 24 & 23 & 23 & 27 & 33 & 31 \\
\hline $50 \times 2(14 d)$ & 21 & 9.8 & & & & & & & & & \\
\hline $50 \times 2(7 d)$ & 20 & 10.7 & & & & & & & & & \\
\hline $100 \times 1$ & 23 & 10.8 & & & & & & & & & \\
\hline $75 \times 4(28 d)$ & 22 & 10.3 & & & & & & & & & \\
\hline $75 \times 4(14 d)$ & 23 & 9.3 & & & & & & & & & \\
\hline $75 \times 4(7 d)$ & 26 & 9.0 & & & & & & & & & \\
\hline $150 \times 2(28 d)$ & 25 & 9.2 & 62 & 49 & 54 & 18 & 16 & 17 & 20 & 35 & 29 \\
\hline $150 \times 2(14 d)$ & 21 & 8.5 & & & & & & & & & \\
\hline $150 \times 2(7 d)$ & 23 & 8.7 & & & & & & & & & \\
\hline $300 \times 1$ & 11 & 10.4 & & & & & & & & & \\
\hline
\end{tabular}

The number of dogs listed for each group are those surviving at least 90 days postirradiation. Doses are midline air doses. Two general summaries have been published, one before all animals were dead (Andersen, 1969), the other emphasizing effects on life span and tumor induction (Rosenblatt, 1986). Other 
publications described exposure techniques and early acute lethality at $300 \mathrm{R}$ (Andersen, 1957); summarized effects on fertility, which were minimal (Andersen, 1961, 1963e, 1963f, 1976); summarized progressive effects in the kidney, which increased in all treated groups except the $4 \times 25 \mathrm{R}$ group (Guttman, 1968); and noted the absence of radiation effects on emotional display (Solarz, 1964) or dominance behavior (Solarz, 1964b).

All irradiated beagles exhibited life shortening relative to controls, averaging, on a linear scale, $6.7 \%$ per $100 \mathrm{R}$. An effect of fractionation was seen only at total doses of $300 \mathrm{R}$, attributable solely to amelioration of nonmammary neoplasia. Major causes of death were similar in irradiated and control dogs. The development of malignant neoplasms at an earlier age in irradiated dogs explains, in large part, the observed life-span shortening. A number of identified variables not considered in this brief tabulation may have significantly affected results (Andersen, 1969; Rosenblatt, 1986).

Information relevant to this experiment and to ancillary external irradiation studies in beagles will be found in the following University of California, Davis (Radiobiology Laboratory or Laboratory for Energy-Related Health Research) periodic reports:

\begin{tabular}{|c|c|c|c|c|c|}
\hline Issue Date & Doc. №. & Page Refs. & Issue Date & Doc. №. & Page Refs. \\
\hline 1956 & Project 4 Report & all & 1970 (Jun) & UCD $472-116$ & $1-30$ \\
\hline 1957 (Jun) & Project 4 Report & all & 1971 (Jun) & UCD $472-117$ & $3-16$ \\
\hline 1958 (Jun) & Project 4 Report & all & & & $22-38$ \\
\hline 1959 (Jun) & Project 4 Report & all & 1972 (Jun) & UCD $472-118$ & $2-9$ \\
\hline 1960 (Jun) & Project 4 Report & all & & & $14-24$ \\
\hline 1961 (Jul) & UCD 101 & all & 1973 (Jun) & UCD $472-119$ & $2-51$ \\
\hline 1961 & UCD 102 & all & 1974 (Jun) & UCD $472-120$ & $18-33$ \\
\hline 1961 & UCD 103 & all & 1975 (Jun) & UCD 472-121 & $2-5$ \\
\hline 1962 (Jun) & UCD 105 & all & & & $8-19$ \\
\hline 1963 (Jun) & UCD 107 & all & 1976 (Jun) & UCD 472-122 & $1-12$ \\
\hline 1964 (Jun) & UCD 472-109 & all & & & $17-23$ \\
\hline 1965 (Apr) & UCD 472-111 & all & 1977 (Oct) & UCD $472-123$ & $28-49$ \\
\hline 1967 (Jun) & UCD $472-113$ & $1-16$ & & & $53-56$ \\
\hline 1968 (Jun) & UCD 472-114 & $1-20$ & 1984 (Jul) & UCD 472-129 & $113-118$ \\
\hline 1969 (Jun) & UCD $472-115$ & $1-14$ & & & \\
\hline
\end{tabular}




\subsection{2 $1961^{90} \mathrm{Sr}$}

Ingested with food, in-utero to 540 days, except "*" fed for life span; both sexes placed on experiment from 1961 to 1967. Results as of January, 1988 (Doc. UCD 472-131, modified by Rosenblatt, private communication).

\begin{tabular}{|c|c|c|c|c|c|c|c|c|c|c|c|}
\hline \multirow{2}{*}{\multicolumn{2}{|c|}{$\begin{array}{l}\text { EXPERIMENTAL } \\
\text { DESIGN }\end{array}$}} & \multicolumn{10}{|c|}{ EXPERIMENTAL RESULTS } \\
\hline & & \multirow{3}{*}{$\begin{array}{l}\text { No. of } \\
\text { Animals } \\
\text { Dead } \\
80\end{array}$} & \multirow{3}{*}{$\begin{array}{l}\text { Median } \\
\text { Age at } \\
\text { Death } \\
\text { years) } \\
14.4\end{array}$} & \multicolumn{6}{|c|}{ No ${ }^{* *}$ (\% Incidence) } & \multirow{2}{*}{\multicolumn{2}{|c|}{$\begin{array}{l}\text { Myelopro- } \\
\text { liferative } \\
\text { Disorder }\end{array}$}} \\
\hline $\begin{array}{l}\text { Total } \\
\text { Ingested** } \\
(\mu \mathrm{Ci} / \mathrm{kg})\end{array}$ & $\begin{array}{l}\text { No. of } \\
\text { Animals } \\
\text { Exposed }\end{array}$ & & & \multicolumn{2}{|c|}{$\begin{array}{l}\text { Bone } \\
\text { Tumor }\end{array}$} & \multicolumn{2}{|c|}{$\begin{array}{l}\text { Oral } \\
\text { Tumor }\end{array}$} & \multicolumn{2}{|c|}{$\begin{array}{l}\text { Nasal } \\
\text { Tumor }\end{array}$} & & \\
\hline $\begin{array}{l}\text { None } \\
\text { (Control) }\end{array}$ & 80 & & & 4 & (5) & 7 & (9) & 1 & (1) & 1 & (1) \\
\hline 1 & 77 & 77 & 14.2 & 0 & (0) & 5 & (6) & 1 & (1) & 0 & $(0)$ \\
\hline 4 & 42 & 42 & 13.5 & 0 & $(0)$ & 1 & (2) & 0 & (0) & 0 & $(0)$ \\
\hline 24 & 66 & 66 & 14.4 & 0 & $(0)$ & 5 & (8) & 1 & (2) & 1 & (2) \\
\hline 70 & 64 & 64 & 14.1 & 5 & (8) & 11 & $(17)$ & 4 & (6) & 1 & (2) \\
\hline $70^{*}$ & 7 & 7 & $12.5^{*}$ & $0^{*}$ & $(0)^{*}$ & $3^{*}$ & $(43)^{*}$ & $1^{*}$ & $(14)^{*}$ & $1^{*}$ & $(14)^{*}$ \\
\hline 226 & 61 & 61 & 12.0 & 9 & (15) & 16 & (26) & 3 & (5) & 6 & $(10)$ \\
\hline $226^{*}$ & $4^{*}$ & $4^{*}$ & $6.4^{*}$ & $0^{*}$ & $(0)^{*}$ & $1^{*}$ & $(25)^{*}$ & $0^{*}$ & $(0)^{*}$ & $1^{*}$ & $(25)^{\star}$ \\
\hline 656 & 60 & 60 & 5.2 & 20 & (33) & 13 & (22) & 1 & (2) & 23 & (38) \\
\hline $656^{*}$ & $4^{*}$ & $4^{*}$ & $5.1^{*}$ & $1^{*}$ & $(25)^{*}$ & $0^{*}$ & $(0)^{*}$ & $0^{*}$ & $(0)^{*}$ & $2^{*}$ & $(50)^{*}$ \\
\hline 1940 & 19 & 19 & 2.2 & 10 & (53) & 1 & (5) & $0^{*}$ & $(0)^{*}$ & 4 & (21) \\
\hline
\end{tabular}

This experiment, initiated in response to the concern for possible effects from ${ }^{90} \mathrm{Sr}$ in the fallout from tests of nuclear weapons, is the largest, and probably the most extensively described and interpreted, of the life-span dog studies. General summaries of the partially completed study are contained in several recent publications (Book, 1980, 1986; Raabe, 1981, 1981b). Feeding of ${ }^{90} \mathrm{SrCl}_{2}$ to pregnant dogs began at 21 days after conception and was continued until their offspring were weaned; at weaning and until 18 months of age the offspring received the same diet, in which a constant ratio of ${ }^{90} \mathrm{Sr}$ was maintained relative to wellcontrolled dietary calcium levels.

Several early papers were concerned with supporting sacrifice studies performed to explore the complex dosimetry of ${ }^{90} \mathrm{Sr}$ when administered under circumstances involving cross placental transport, lactation, and deposition in a 
rapidly growing skeletal system (Andersen, 1962; Della Rosa, 1965, 1966, 1968b, 1969; Goldman, 1967; Stover, 1961). Relatively low dose rates observed during fetal development and lactation increased sharply upon weaning, as maternal discrimination was removed and skeletal mineralization increased.

Early effects described included hematological observations (Bustad, 1969; Della Rosa, 1969; Dungworth, 1969; Goldman, 1972c), serum chemistry changes (McKelvie, 1968), the occurrence of myeloproliferative disorders at the higher dose levels (Bustad, 1969; Dungworth, 1968, 1969; Goldman, 1969b, 1972c; Wilson, 1972), and the occurrence of neoplastic (Goldman, 1972c) and nonneoplastic effects in bone (Andersen, 1962; Della Rosa, 1969; Goldman, 1969). A 1972 monograph (Goldman, 1972), which summarized the then-current status of the study, included papers on metabolic data and dosimetry, with comparison to man (Della Rosa, 1972); on theoretical and experimental aspects of local dosimetry within bone (Goldman, 1972b; Spiers, 1972); on the absence of effects on puppy mortality and litter size, at ${ }^{90} \mathrm{Sr}$ exposure levels $10^{5}$ times that from worldwide fallout (Rosenblatt, 1972b); and on the occurrence of bone tumors, which appeared in similar exposure groups but much later than myeloproliferative disorders (Pool, 1972b).

The dosimetric aspects of this study were comprehensively considered in a 1976 paper (Momeni, 1976). Other papers of this period considered specific aspects of local dosimetry in bone (Momeni, 1975b, 1976b, 1976d; Zanelli, 1971) and correlated this dosimetry with the nature and distribution of observed effects in bone (Goldman, 1972c; Momeni, 1976b, 1976d; Pool, 1973, 1973b; Spiers, 1983, 1988). Supporting studies have investigated the redistribution of ${ }^{90} \mathrm{Sr}$ among different bones following cessation of intake, and as a function of the proportion of compact and cancellous bone present (Parks, 1985). A single paper has focused on the $15 \mathrm{dogs}$ that ingested ${ }^{90} \mathrm{Sr}$ until death; dosimetry and effects in these dogs were little different from those in animals removed from feeding at 18 months of age, reflecting the low rate of calcium turnover in mature dogs (Book, 1982).

Two recent papers give detailed consideration to the occurrence and distribution of bone tumors (Nilsson, 1987), and to the pathogenesis of these tumors (Nilsson, 1985 ) in the completed experiment. Another paper has concentrated on the greatly enhanced incidence of squamous-cell carcinoma of the gingiva, as related to the concentration and retention of ${ }^{90} \mathrm{Sr}$ in teeth (Parks, 1984). A high incidence of benign and malignant mammary tumors appears to be unrelated to radionuclide exposure (Chrisp, 1980).

The representation of dose-effect-time relationships as three-dimensional surfaces has been the subject of several papers (Goldman, 1972c; Raabe, 1981, 1983), 
as have the prediction of late effects from early observations (Goldman, 1976), and the extrapolation of conclusions from the dog study to humans (Della Rosa, 1972; Pool, 1973; Raabe, 1981, 1983; Spiers, 1983, 1988). Many of the recent reports compare the results of this study with those from the companion, chronic ${ }^{226} \mathrm{Ra}-$ exposure study.

Information relevant to this experiment will be found in the following University of California, Davis (Radiobiology Laboratory or Laboratory for Energy-Related Health Research) periodic reports:

\begin{tabular}{|c|c|c|c|c|c|}
\hline Issue Date & Doc. No. & Page Rets. & Issue Date & Doc. No & Page Refs. \\
\hline 1958 & Project 6 Report & all & 1974 (Jun) & UCD $472-120$ & $56-104$ \\
\hline $1959-60$ & Project 6 Report & all & 1975 (Jun) & UCD $472-121$ & $24-30$ \\
\hline 1961 (Sep) & UCD 104 & all & & & $40-59$ \\
\hline 1962 (Jun) & UCD 106 & all & & & $66-69$ \\
\hline 1963 (Aug) & UCD 108 & all & & & $80-99$ \\
\hline 1964 (Jul) & UCD $472-110$ & all & 1976 (Jun) & UCD 472-122 & $39-76$ \\
\hline 1965 (Jun) & UCD $472-112$ & all & & & $102-108$ \\
\hline 1967 (Jun) & UCD $472-113$ & $17-47$ & & & $112-127$ \\
\hline \multirow[t]{2}{*}{1968 (Jun) } & UCD $472-114$ & $21-39$ & 1977 (Oct) & UCD $472-123$ & $89-108$ \\
\hline & & $42-67$ & 1978 (Oct) & UCD $472-124$ & $144-153$ \\
\hline \multirow[t]{4}{*}{1969 (Jun) } & UCD $472-115$ & $15-30$ & 1979 (Oct) & UCD $472-125$ & $129-164$ \\
\hline & & 39.47 & & & $200-232$ \\
\hline & & $50-70$ & 1981 & UCD $472-126$ & $230-292$ \\
\hline & & $72-74$ & 1982 (Sep) & UCD $472-127$ & $9-54$ \\
\hline \multirow[t]{2}{*}{1970 (Jun) } & UCD $472-116$ & $31-38$ & & & $71-80$ \\
\hline & & $41-113$ & 1983 (Aug) & UCD $472-128$ & $2-43$ \\
\hline 1971 (Jun) & UCD $472-117$ & $39-86$ & 1984 (Jul) & UCD $472-129$ & $2-45$ \\
\hline \multirow[t]{4}{*}{1972 (Jun) } & UCD $472-118$ & $36-46$ & & & $63-81$ \\
\hline & & $66-99$ & 1985 (Nov) & UCD $472-130$ & $2-46$ \\
\hline & & $109-114$ & & & $90-95$ \\
\hline & & $121-136$ & 1987 (Jun) & UCD 472-131 & $2-43$ \\
\hline \multirow[t]{2}{*}{1973 (Jun) } & UCD $472-119$ & $52-55$ & & & \\
\hline & & $58-127$ & & & \\
\hline
\end{tabular}




\subsection{3 $1963^{90} \mathrm{Sr}$}

Single intravenous injection of strontium solution; dogs 540 days of age, of both sexes, placed on experiment from 1963 to 1967. Results as of January, 1988 (Doc. UCD 472-131, modified by Rosenblatt, private communication).

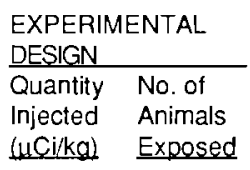

EXPERIMENTAL RESULTS

\begin{tabular}{|c|c|c|c|c|c|c|c|c|c|}
\hline & Median & \multicolumn{8}{|c|}{ No: (\% Incidence) } \\
\hline $\begin{array}{l}\text { No. of } \\
\text { Animals } \\
\text { Dead }\end{array}$ & $\begin{array}{l}\text { Age at } \\
\text { Death } \\
\text { (years) }\end{array}$ & \multicolumn{2}{|c|}{$\begin{array}{l}\text { Bone } \\
\text { Iumer }\end{array}$} & \multicolumn{2}{|c|}{$\begin{array}{l}\text { Oral } \\
\text { Iumer }\end{array}$} & \multicolumn{2}{|c|}{$\begin{array}{l}\text { Nasal } \\
\text { Tumor }\end{array}$} & \multicolumn{2}{|c|}{$\begin{array}{l}\text { Myelopro- } \\
\text { liferative } \\
\text { Disorder }\end{array}$} \\
\hline 20 & 13.5 & 1 & (5) & 1 & (5) & 0 & (0) & 1 & (5) \\
\hline 26 & 13.3 & 4 & (15) & 6 & (23) & 1 & (4) & 0 & (0) \\
\hline
\end{tabular}

33

26

- Numbers listed are numbers of primary tumors, not numbers of animals with tumors

This study supplemented the Davis ${ }^{90} \mathrm{Sr}$ chronic feeding study, providing a comparison of single and repeated administration, and also serving as a link to the more extensive single injection study at the University of Utah (Goldman, 1969, 1969b). A single paper has compared, in some detail, the dosimetry of the chronically fed and singly injected dogs, in terms of both gross retention and dose to local regions of bone (Momeni, 1976). No difference was seen in retention of a single injection of ${ }^{90} \mathrm{Sr}$ and a single inhalation, as studied at the Inhalation Toxicology Research Institute (Momeni, 1976). No detailed consideration of effects has been published; the incidence of myeloproliferative disorders in the high dose group is lower than that observed at similar dose levels from chronic feeding.

Information relevant to this experiment will be found in many of the University of California, Davis (Radiobiology Laboratory or Laboratory for Energy-Related Health Research) periodic reports, as referenced for the Davis ${ }^{90} \mathrm{Sr}$ ingestion experiment. 


\begin{tabular}{|c|c|c|c|c|c|c|c|c|c|c|c|}
\hline 8.2 .4 & \multicolumn{11}{|c|}{$\begin{array}{l}\text { Eight semimonthly intravenous injections, started at } 435 \text { days of } \\
\text { age, except "*" started at } 60 \text { or } 120 \text { days. Dogs of both sexes placed } \\
\text { on experiment from } 1963 \text { to } 1967 . \text { Results as of January, } 1988 \\
\text { (Doc. UCD } 472-131 \text {, modified by Rosenblatt, private communica- } \\
\text { tion). }\end{array}$} \\
\hline \multicolumn{2}{|c|}{$\begin{array}{l}\text { EXPERIMENTAL } \\
\text { DESIGN }\end{array}$} & \multicolumn{10}{|c|}{ EXPERIMENTAL RESULTS } \\
\hline $\begin{array}{l}\text { Total } \\
\text { Injected } \\
(\mu \mathrm{Ci} / \mathrm{kgl} \\
\end{array}$ & $\begin{array}{l}\text { No. of } \\
\text { Animals } \\
\text { Exposed }\end{array}$ & $\begin{array}{l}\text { No. of } \\
\text { Animals } \\
\text { Dead } \\
\end{array}$ & $\begin{array}{l}\text { Age at } \\
\text { Death } \\
\text { (vears) }\end{array}$ & $\begin{array}{l}\text { Bo } \\
\text { Tu }\end{array}$ & & $\begin{array}{l}\text { Ora } \\
\text { Tum }\end{array}$ & & $\begin{array}{l}\text { Nas } \\
\text { Ium }\end{array}$ & & $\begin{array}{l}\text { My } \\
\text { lifer } \\
\text { Dis }\end{array}$ & $\begin{array}{l}\text { opro- } \\
\text { tive } \\
\text { der }\end{array}$ \\
\hline $\begin{array}{l}\text { None } \\
\text { (Control) }\end{array}$ & 85 & 85 & 14.6 & 1 & (1) & 8 & (9) & 1 & (1) & 0 & $(0)$ \\
\hline 0.024 & 46 & 46 & 14.5 & 0 & (0) & 5 & (11) & 1 & (2) & 0 & $(0)$ \\
\hline 0.064 & 40 & 40 & 13.8 & 5 & (13) & 1 & (3) & 0 & (0) & 0 & $(0)$ \\
\hline 0.376 & 42 & 42 & 10.9 & 30 & (71) & 5 & (12) & 1 & (2) & 0 & $(0)$ \\
\hline 1.12 & 40 & 40 & 7.4 & 35 & (88) & 1 & (3) & 0 & (0) & 0 & $(0)$ \\
\hline 3.36 & 41 & 41 & 5.1 & 45 & $(110)$ & 0 & (0) & 0 & (0) & 0 & $(0)$ \\
\hline 10.0 & 41 & 41 & 4.3 & 25 & (61) & 0 & (0) & 0 & (0) & 0 & $(0)$ \\
\hline $10.0^{*}$ & $6^{*}$ & $6^{*}$ & $2.3^{*}$ & 4 & $(67)$ & 0 & (0) & 0 & (0) & 0 & (0) \\
\hline
\end{tabular}

- Numbers listed are numbers of primary tumors, not numbers of animals with tumors

This study was designed to simulate the exposure pattern of the human dial painters and thus to provide a link between ${ }^{226} \mathrm{Ra}$ effects in humans and the dog. The extended period of exposure also allows direct comparison with the Davis chronic ${ }^{90} \mathrm{Sr}$ ingestion study and complements the University of Utah single injection ${ }^{226}$ Ra study. General summaries of the partially completed study are contained in several recent publications (Book, 1980, 1986; Raabe, 1981, 1981b). Most published reports compare the results of this study and the companion chronic ${ }^{90} \mathrm{Sr}$ ingestion study, and many include a comparison with data on human ${ }^{226}$ Ra exposure.

Dosimetric aspects of the study have been considered in some detail, including both gross distribution and retention as a function of age and dose level (Goldman, 1973; Momeni, 1976c, 1976d; Parks, 1978, 1978b; Pool, 1973), localized dosimetry within bone (Momeni, 1976b, 1976c), concentration of radium in the tapetum lucidum of the eye (Fisher, G., 1976), and radon retention as a function of age and dose level (Parks, 1978). Supporting studies have investigated the redistribution of ${ }^{226} \mathrm{Ra}$ among different bones as a function of time, and of the 
relative proportions of compact and cancellous bone present (Park, 1980). The rate of loss of ${ }^{226} \mathrm{Ra}$ from the human skeleton appears to be about ten times as rapid as its loss from the beagle skeleton (Goldman, 1973).

The earliest noted effect was a leukocyte depression (Goldman, 1969b; Rosenblatt, 1967), which could be correlated with later appearance of osteosarcoma (Goldman, 1976). A variety of bone defects, including pathologic fractures and radiation osteodystrophy, caused early deaths at high dose levels and were detected radiologically at lower dose levels (Goldman, 1969b; Momeni, 1976b, $1976 e$ ); these effects were more evident in dogs injected at 2 or 4 months of age, and were much more prominent than in the chronic ${ }^{90} \mathrm{Sr}$ ingestion study (Momeni, 1976b). Radiation effects on the mucopolysaccharides of cartilage were also described (Tsai, 1970).

Bone tumors appeared earlier than in the ${ }^{90} \mathrm{Sr}$ ingestion study (Goldman, 1969b; Rosenblatt, 1971, 1972); myeloproliferative disorders were notably absent. The preferred sites of osteosarcoma incidence have been studied in relation to ${ }^{226} \mathrm{Ra}$ localization and the structure and function of the bone (Parks, 1980; Spiers, 1983, 1988; Wilson, 1976). A high incidence of benign and malignant mammary tumors appears to be unrelated to radionuclide exposure (Chrisp, 1980).

There has been a continuing effort to develop dose-effect-time relationships, which can be used in comparing the bone tumor data from dogs to similar data from human epidemiological studies and from studies in other animal species, and with other radionuclides (DuMouchel, 1981; Goldman, 1973; Pool, 1973; Raabe, 1980, 1983, 1984, 1986, 1986b; Rosenblatt, 1976; Wrenn, 1986c). Detailed comparisons of noncarcinogenic effects in the skeletons of $\mathrm{dog}$ and humans have also been reported (Morgan, J., 1982, 1983: Pool, 1983).

Information relevant to this experiment will be found in the following University of California, Davis (Radiobiology Laboratory or Laboratory for Energy-Related Health Research) periodic reports:

\begin{tabular}{|c|c|c|c|c|c|}
\hline Issue Date & Doc. No. & Page Refs. & Issue Date & Doc. No. & Page Refs \\
\hline 1965 (Jun) & UCD $472-112$ & $97-108$ & 1970 (Jun) & UCD $472-116$ & $102-116$ \\
\hline 1967 (Jun) & UCD $472-113$ & $18-25$ & 1971 (Jun) & UCD $472-117$ & 51 \\
\hline \multirow[t]{2}{*}{1968 (Jun) } & UCD $472-114$ & $30-48$ & & & 82.86 \\
\hline & & $60-62$ & 1972 (Jun) & UCD $472-118$ & $86-89$ \\
\hline 1969 (Jun) & UCD 472-115 & $21-71$ & & & $100-112$ \\
\hline \multirow[t]{2}{*}{1970 (Jun) } & UCD $472-116$ & $39-67$ & & & $115-117$ \\
\hline & & $78-84$ & 1973 (Jun) & UCD $472-119$ & $66-70$ \\
\hline
\end{tabular}




\begin{tabular}{|c|c|c|c|c|c|}
\hline Issue Date & Doc. No. & Page Refs. & Issue Date & Doc. No. & Page Refs. \\
\hline 1973 (Jun) & UCD $472-119$ & $79-119$ & 1979 (OCt) & UCD $472-125$ & $130-164$ \\
\hline \multirow[t]{2}{*}{1974 (Jun) } & UCD $472-120$ & $56-77$ & & & $200-232$ \\
\hline & & $96-114$ & 1981 & UCD $472-126$ & $226-229$ \\
\hline \multirow[t]{2}{*}{1975 (Jun) } & UCD 472-121 & $24-69$ & & & $252-292$ \\
\hline & & $71-99$ & 1982 (Sep) & UCD $472-127$ & $9-49$ \\
\hline \multirow[t]{2}{*}{1976 (Jun) } & UCD 472-122 & $40-101$ & & & $55-80$ \\
\hline & & $112-131$ & 1983 (Aug) & UCD $472-128$ & $2-64$ \\
\hline 1977 (Oct) & UCD $472-123$ & $90-110$ & 1984 (Jul) & UCD 472-129 & $2-43$ \\
\hline \multirow[t]{4}{*}{1978 (Oct) } & UCD 472-124 & $144-153$ & & & $46-81$ \\
\hline & & $157-162$ & 1985 (Nov) & UCD $472-130$ & $2-46$ \\
\hline & & $182-184$ & & & $90-95$ \\
\hline & & & 1987 (Jun) & UCD $472-131$ & $2-50$ \\
\hline
\end{tabular}




\subsection{ARGONNE EXPERIMENTS}

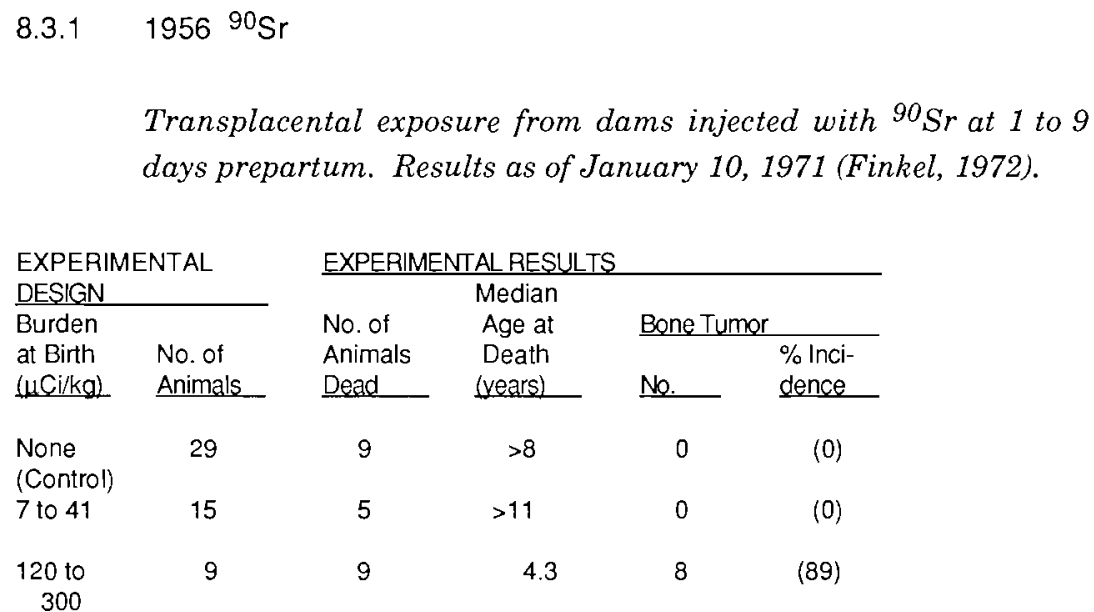

A single report describes early results of this study (Finkel, 1972). Initial burdens were determined by whole-body bremsstrahlung measurement at birth. High-level litters were from dams injected with $100 \mu \mathrm{Ci} / \mathrm{kg}{ }^{90} \mathrm{Sr}$ (in equilibrium with $\left.{ }^{90} \mathrm{Y}\right) 8$ days before delivery. Low-level litters were from dams injected with 10 to $15 \mu \mathrm{Ci} / \mathrm{kg} 1$ to 9 days before delivery. Controls are general colony controls. Final results have not been published.

Information relevant to this experiment will be found in the following Argonne National Laboratory periodic reports:

$\begin{array}{lll}\text { Issue Date } & \text { Doc. No. } & \text { Page Refs. } \\ 1959 \text { (Dec) } & \text { ANL-6093 } & 15-19 \\ 1961 \text { (Nov) } & \text { ANL-6464 } & 38-49\end{array}$




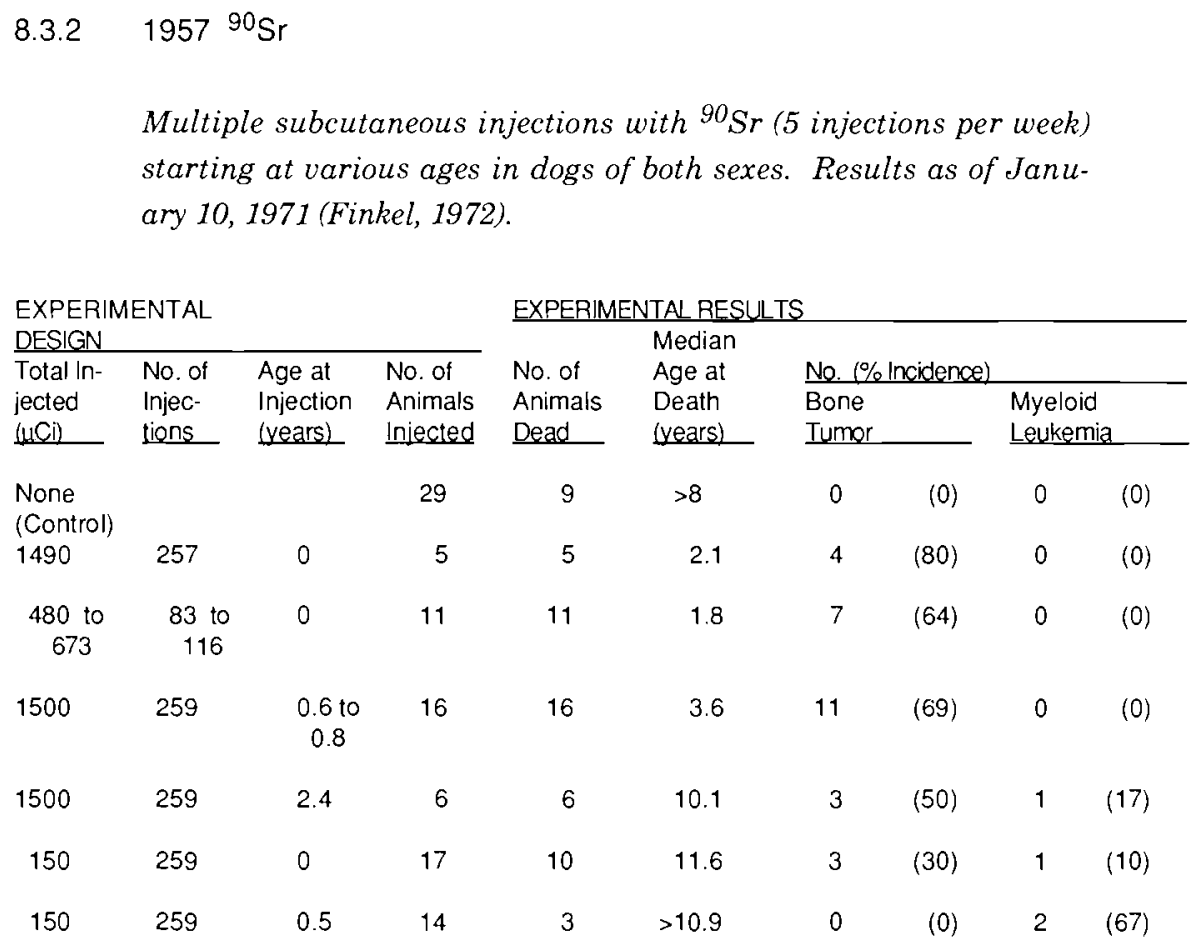

Only early results from this study have been reported (Finkel, 1960, 1972). Injections started at 4 to 8 days of age in the 0 year groups. The high-and low-dose groups received daily injections (except weekends and holidays) of 5.8 and 0.58 $\mu \mathrm{Ci}{ }^{90} \mathrm{Sr}$ (in equilibrium with ${ }^{90} \mathrm{Y}$ ), respectively. The injections of two litters at the high dose level were terminated before completion of the planned 257 doses because of excessive mortality. Seven animals dying of distemper during the second year are excluded from the newborn, $150-\mu \mathrm{Ci}$ group. Controls are general colony controls.

"The purpose of this dog experiment [including the preceding experiment] was not to establish dose-response relationships but to obtain data on certain parameters of ${ }^{90} \mathrm{Sr}$ dosage in dogs that would be useful in extrapolating mouse data, through dog data, to man." (Finkel, 1972).

Information relevant to this experiment will be found in the following Argonne National Laboratory periodic report:

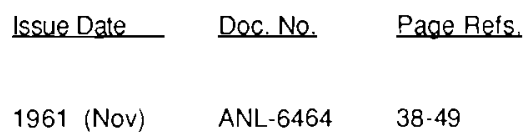


8.3.3 $1960{ }^{144} \mathrm{Ce}$

Single intravenous injection of cerium citrate solution; dogs "approximately 13 months" of age, of both sexes, placed on experiment from 1960 to 1964. Results as of 1970 (Fritz, 1970).

\begin{tabular}{ll} 
EXPERIMENTAL \\
DESIGN & \\
\hline Quantity & No. of \\
Injected & Animals \\
(uCi/kg) & Injected
\end{tabular}

23 to 253

\section{9}

EXPERIMENTAL RESULTS

Total Specific Pathology

No of

Animals Cause

of Death

39 chronic anemia

bone tumors
Time of Death Range of Injec-

(days post- ted Radionu- No. of incidence iniection)

$<100$
$>1000$
$>900$

\section{7 to 253}

28 to 73

23 to 80
Deaths $(\%)$

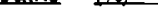

$\begin{array}{rr}17 & 44 \\ 5 & 13 \\ 16 & 41\end{array}$

A single report describes early results of this study (Fritz, 1970). Of the 16 dogs dying with bone tumors, 7 also showed mild to severe anemia. Atypical myeloproliferation was observed in bone specimens from 5 dogs with anemia at times later than 100 days postinjection. Liver lesions consisting of alternating areas of degeneration, atrophy, and regeneration were also seen in these five dogs. One of these dogs was considered to exhibit a clear case of myelogenous leukemia, with the diagnosis questionable for the other four. Final results have not been published.

Information relevant to this experiment will be found in the following Argonne National Laboratory periodic reports:

\begin{tabular}{|c|c|c|c|c|c|}
\hline Issue Date & Doc. No. & Page Refs. & Issue Date & Doc. No. & Page Refs. \\
\hline 1962 (Apr) & ANL- 6535 & $40-45$ & 1966 (Dec) & ANL-7278 & $112-114$ \\
\hline 1963 (May) & ANL- 6723 & $117-120$ & 1967 (Dec) & ANL-7409 & $260-265$ \\
\hline 1964 (Jan) & ANL-6823 & $73-74$ & $1968(\mathrm{Dec})$ & ANL-7535 & $162-163$ \\
\hline 1964 (Jun) & ANL-6906 & $41-42$ & $1970(\mathrm{Dec})$ & ANL-7770 & $143-144$ \\
\hline 1964 (Dec) & ANL-6971 & $138-142$ & 1971 (Dec) & ANL-7870 & $145-146$ \\
\hline
\end{tabular}




\begin{tabular}{|c|c|c|c|c|c|c|c|}
\hline 8.3 .4 & \multicolumn{7}{|c|}{$\begin{array}{l}\text { Single intravenous injection of cesium solution; dogs of various } \\
\text { ages, of both sexes, placed on experiment from } 1961 \text { to 1963. Re- } \\
\text { sults are as published in NCRP Report No. } 52 \text { (NCRP, 1977). }\end{array}$} \\
\hline \multirow{2}{*}{\multicolumn{3}{|c|}{$\begin{array}{l}\text { EXPERIMENTAL } \\
\text { DESIGN }\end{array}$}} & \multicolumn{5}{|c|}{ EXPERIMENTAL RESULTS } \\
\hline & & & & Median & Whole-Body & & \\
\hline $\begin{array}{l}\text { Age at } \\
\text { Injection }\end{array}$ & Quantity & No. of & $\begin{array}{l}\text { No. of } \\
\text { Animals }\end{array}$ & Postexp. & Dose to Med- & Notable Pathologic Find & $\frac{\text { tings }}{\text { Incidence }}$ \\
\hline (months) & (uCikgal & Iniected & Dead & (years) & & & \\
\hline 5 & 3.2 to 3.8 & 15 & 15 & 7.5 & 770 & $\begin{array}{l}3 \text { acute marrow deaths } \\
3 \text { liver degeneration } \\
1 \text { neurofibrosarcoma } \\
4 \text { miscellaneous tumors }\end{array}$ & $\begin{array}{r}20 \\
20 \\
7\end{array}$ \\
\hline 13 & 1.6 to 2.8 & 19 & 19 & 8.4 & 890 & $\begin{array}{l}1 \text { acute marrow death } \\
3 \text { liver degeneration } \\
3 \text { neurofibrosarcoma } \\
7 \text { miscellaneous tumors }\end{array}$ & $\begin{array}{r}5 \\
16 \\
16\end{array}$ \\
\hline 13 & 3.1 to 4.3 & 19 & 19 & 6.3 & 1260 & $\begin{array}{l}9 \text { acute marrow deaths } \\
2 \text { liver degeneration } \\
2 \text { neurofibrosarcoma } \\
5 \text { miscellaneous tumors }\end{array}$ & $\begin{array}{l}47 \\
11 \\
11\end{array}$ \\
\hline 34 to & 2.0 to 3.7 & 12 & 12 & 0.07 & $<1000$ & 12 acute marrow deaths & 100 \\
\hline
\end{tabular}

A detailed description of the design and results of this experiment has not been published; limited information is available only from an NCRP report (NCRP, 1977). Higher acute toxicity in older dogs was attributed to higher radiation doses occasioned by increased biological retention of cesium with age. Relative to nonirradiated controls (not described) a shortening in average life occurred in dogs surviving the acute effects of ${ }^{137} \mathrm{Cs}$ irradiation. Nearly all long-term survivors showed significant liver degeneration. The most frequently observed cancer was the neurofibrosarcoma, a nerve-sheath tumor that is "quite rare" in controls (NCRP, 1977).

Information relevant to this experiment will be found in the following Argonne National Laboratory periodic reports:

\begin{tabular}{|c|c|c|c|c|c|}
\hline Issue Date & Doc. No. & Page Refs. & Issue Date & Doc. No. & Page Refs. \\
\hline 1962 (Apr) & ANL-6535 & $45-48$ & 1964 (Jan) & ANL- 6823 & $44-62$ \\
\hline 1963 (May) & ANL-6723 & $107-116$ & 1964 (Jun) & ANL- 6906 & $42-46$ \\
\hline 1963 (Oct) & ANL-6790 & $31-39$ & 1964 (Dec) & ANL-6971 & $133-137$ \\
\hline
\end{tabular}




\begin{tabular}{|c|c|c|c|c|c|}
\hline Issue Date & Doc. No. & Page Refs. & Issue Date & Doc. No. & Page Refs. \\
\hline 1965 (Dec) & ANL-7136 & $110-113$ & 1970 (Dec) & ANL-7770 & $139-141$ \\
\hline 1966 (Dec) & ANL-7278 & $95-102$ & & & 143 \\
\hline 1967 (Dec) & ANL-7409 & $254-256$ & 1971 (Dec) & ANL-7870 & $146-149$ \\
\hline 1968 (Dec) & ANL-7535 & $163-165$ & 1972 (Dec) & ANL-7970 & $206-208$ \\
\hline \multirow[t]{2}{*}{1969 (Dec) } & ANL-7635 & $102-103$ & 1974 (Dec) & ANL-75-30 & $17-20$ \\
\hline & & & 1975 (Dec) & ANL-76-99 & $19-21$ \\
\hline
\end{tabular}




\subsubsection{Gamma Ray}

External ${ }^{60}$ Co gamma-ray exposure, continued until death; dogs 13 months old, of both sexes, placed on experiment in two series: "A" from 1968 to 1970, and "B" from 1976 to 1978. Results are as of October, 1987 (Fritz, private communication).

\begin{tabular}{|c|c|c|c|c|c|c|c|c|c|c|c|}
\hline EXPE & & & EXPERIM & NTAL RES & LTS & & & & & & \\
\hline DESIGN & & & & & & Cau & of $D e$ & No. 1 & incids & & \\
\hline $\begin{array}{l}\text { Dose Rate } \\
\text { (rad/day) }\end{array}$ & & $\begin{array}{l}\text { es } \\
\text { No. } \\
\text { ased }\end{array}$ & $\begin{array}{l}\text { No. of } \\
\text { Animals } \\
\text { Dead }\end{array}$ & $\begin{array}{l}\text { Mean Sun } \\
\text { of Dead } \\
\text { Animals }\end{array}$ & 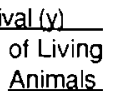 & Sep & cemia & Anen & & $\begin{array}{l}\text { Myelc } \\
\text { liferat } \\
\text { Disore }\end{array}$ & \\
\hline Controls & $\mathrm{B}$ & 46 & 12 & 8.4 & 10.0 & 0 & (0) & 0 & (0) & 0 & $(0)$ \\
\hline 0.3 & $\mathrm{~B}$ & 92 & 33 & 8.2 & 9.7 & 0 & (0) & 0 & (0) & 0 & (0) \\
\hline 0.8 & $\mathrm{~B}$ & 46 & 28 & 8.0 & 10.0 & 0 & (0) & 0 & (0) & 1 & (4) \\
\hline 1.9 & $\mathrm{~B}$ & 46 & 42 & 7.3 & 8.9 & 0 & (0) & 1 & (2) & 7 & (17) \\
\hline 3.8 & A & 24 & 24 & 5.2 & & 0 & (0) & 2 & (8) & 11 & (46) \\
\hline 7.5 & $A$ & 16 & 16 & 1.9 & & 0 & (0) & 7 & (44) & 7 & (44) \\
\hline 12.8 & A & 13 & 13 & 0.8 & & 7 & (54) & 4 & (31) & 2 & (12) \\
\hline 26.3 & A & 16 & 16 & 0.15 & & 16 & $(100)$ & 0 & (0) & 0 & (0) \\
\hline 37.5 & $A$ & 8 & 8 & 0.10 & & 8 & $(100)$ & 0 & (0) & 0 & (0) \\
\hline 54.0 & $A$ & 4 & 4 & 0.07 & & 4 & $(100)$ & 0 & (0) & 0 & (0) \\
\hline
\end{tabular}

Dogs were irradiated 22 hours per day, 7 days per week, in a specially constructed facility (Grahn, 1986; Norris, 1976). Particular attention was given to dosimetry; all factors contributing to the dose rate and total dose were normalized in the irradiation field by migrating dogs through all positions and orientations with respect to the irradiation source. Dose rates shown above are average absorbed dose; doses in air were quoted in early publications.

Several recent papers have summarized the status of the total experiment (Fritz, 1985, 1986; Grahn, 1986; Seed, 1985); earlier papers reported results from the higher-dose, Series A, animals (Norris, 1972, 1976; Seed, 1984). Published papers on specialized aspects of this study include those dealing with hematological effects (Fritz, 1973, 1982; Seed, 1978, 1980, 1987; Tolle, 1982, 1983), myelogenous leukemia (Fritz, 1973; Seed, 1977), erythroleukemia (Seed, 1977; Tolle, 1977), electron microscopic studies of bone marrow (Seed, 1982, 1983, 1987b; Tolle, 1983b), and leukemogenesis modeling (Seed, 1978, 1982b, 1985, 1987; Tolle, $1979 b)$. 
Results at higher dose rates defined three distinct radiation-induced causes of death; all involve the hematopoietic system and seem to be related more directly to dose rate than to total dose. At the highest dose rates, early death resulted from septicemia associated with granulocytopenia. At somewhat lower dose rates, anemia was the predominant cause of death, with survival times of a few hundred days. At still lower dose rates, myeloproliferative disorders (the majority of which were myelogenous leukemias) occurred between 400 and 2000 days after onset of exposure. Myeloproliferative disorders did not occur beyond 2000 days, regardless of dose rate or total dose. After 7 years of continuous irradiation there is no difference in survival rate between controls and the 0.3 $\mathrm{rad} /$ day group. Among all dogs still alive, except for testicular atrophy and aspermia, the only clinically detectable effect of irradiation is a depression of circulating blood cell levels.

Information relevant to this experiment will be found in the following Argonne National Laboratory periodic reports:

\begin{tabular}{|c|c|c|c|c|c|}
\hline Issue Date & Doc. No. & Page Refs. & Issue Date & Doc. No. & Page Refs. \\
\hline 1964 (Dec) & ANL- 5971 & $137-138$ & 1975 (Dec) & ANL-76-99 & $10-19$ \\
\hline 1965 (Dec) & ANL-7136 & $116-120$ & 1976 (Dec) & ANL-77-55 & $34-42$ \\
\hline $1966(\mathrm{Dec})$ & ANL-7278 & $102-106$ & 1977 (Dec) & ANL-78-90 & $78-86$ \\
\hline 1967 (Dec) & ANL-7409 & $258-260$ & 1978 (Dec) & ANL-79-90 & $6-12$ \\
\hline 1968 (Dec) & ANL-7535 & $151-162$ & 1980 (Dec) & ANL-80-90 & $8-13$ \\
\hline 1969 (Dec) & ANL-7635 & $94-102$ & 1981 (Aug) & ANL-81-50 & $8-13$ \\
\hline 1970 (Dec) & ANL-7770 & $130-135$ & 1982 (Jun) & ANL-82-35 & $11-19$ \\
\hline 1971 (Dec) & ANL-7870 & $138-145$ & 1983 (May) & ANL $-83-40$ & $27-36$ \\
\hline 1972 (Dec) & ANL-7970 & $192-206$ & 1984 (Aug) & ANL-84-30 & $37-46$ \\
\hline 1973 (Dec) & ANL-8070 & $31-54$ & 1985 (Aug) & ANL-85-30 & $63-72$ \\
\hline 1974 (Dec) & ANL-75-30 & $9-14$ & & & \\
\hline
\end{tabular}




\subsubsection{Gamma Ray}

Continuous external ${ }^{60}$ Co gamma-ray exposure, at various dose rates, terminated at various total doses; dogs of mean age 463 days, of both sexes, placed on experiment from 1968 to 1977. Results as of October, 1987 (Fritz, private communication).

\begin{tabular}{|c|c|c|c|c|c|c|c|c|c|}
\hline \multirow{2}{*}{\multicolumn{3}{|c|}{$\begin{array}{l}\text { EXPERIMENTAL } \\
\text { DESIGN }\end{array}$}} & \multicolumn{7}{|c|}{ EXPERIMENTAL RESULTS } \\
\hline & & & \multirow{2}{*}{$\begin{array}{l}\text { No. of } \\
\text { Animais } \\
\text { Dead }\end{array}$} & \multirow{2}{*}{\multicolumn{2}{|c|}{$\frac{\text { Mean Survival ( }(v)}{\text { of Dead of Living }}$}} & \multicolumn{4}{|c|}{ №. (\% Incidence) } \\
\hline $\begin{array}{l}\text { Dose Rate } \\
\text { (rad/day) }\end{array}$ & $\begin{array}{l}\text { Total Dose } \\
\text { (rad) }\end{array}$ & $\begin{array}{l}\text { No. of } \\
\text { Animals }\end{array}$ & & & & \multicolumn{2}{|c|}{$\begin{array}{l}\text { Fatal } \\
\text { Tumors* }\end{array}$} & \multicolumn{2}{|c|}{$\begin{array}{l}\text { Myeloproliferative } \\
\text { Disorders }\end{array}$} \\
\hline $\begin{array}{l}\text { None } \\
\text { (Control) }\end{array}$ & 0 & 86 & 66 & 11.4 & 12.4 & 28 & $(42)$ & 0 & (0) \\
\hline \multirow[t]{3}{*}{3.8} & 450 & 20 & 10 & 10.3 & 12.1 & 5 & (50) & 0 & (0) \\
\hline & 1050 & 24 & 24 & 10.7 & & 14 & (58) & 0 & (0) \\
\hline & 1500 & 20 & 19 & 10.2 & 13.6 & 13 & (68) & 1 & (5) \\
\hline \multirow[t]{4}{*}{7.5} & 450 & 20 & 10 & 10.4 & 12.4 & 8 & (80) & 1 & (10) \\
\hline & 1050 & 24 & 23 & 10.2 & 13.1 & 14 & (61) & 0 & (0) \\
\hline & 1500 & 19 & 19 & 9.0 & & 15 & (79) & 2 & (11) \\
\hline & 3000 & 20 & 20 & 7.8 & & 16 & (80) & 3 & (15) \\
\hline \multirow[t]{4}{*}{12.8} & 450 & 20 & 10 & 10.7 & 12.2 & 7 & (70) & 0 & (0) \\
\hline & 1050 & 21 & 17 & 10.0 & 13.3 & 10 & (59) & 0 & (0) \\
\hline & 1500 & 19 & 15 & 9.2 & 11.7 & 8 & (53) & 1 & (7) \\
\hline & 3000 & 10 & 10 & 10.6 & & 7 & $(70)$ & 0 & (0) \\
\hline \multirow[t]{2}{*}{26.3} & 450 & 20 & 10 & 8.0 & 11.8 & 4 & $(40)$ & 0 & (0) \\
\hline & 1050 & 20 & 20 & 10.1 & & 12 & $(60)$ & 0 & (0) \\
\hline
\end{tabular}

* Including myeloproliferative disorders

This experiment complements the preceding continuous lifetime-irradiation experiment. Dogs were irradiated 22 hours per day, 7 days per week, in a specially constructed facility (Grahn, 1986; Norris, 1976). Particular attention was given to dosimetry; all factors contributing to the dose rate and total dose were normalized in the irradiation field by migrating dogs through all positions and orientations with respect to the irradiation source. Dose rates shown above are average absorbed dose; doses in air were quoted in early publications. Dogs listed above include only those surviving 100 days following termination of exposure.

Several papers have been published describing the then-current status of this study (Fritz, 1978, 1986; Grahn, 1986; Seed, 1985). The partial results have been employed in modeling the leukemogenic process (Seed, 1985, 1987, 1987b; Tolle, $1979 b$ ). A detailed account has appeared of an acute monocytic leukemia in a dog from this study (Tolle, 1979). 
Interim conclusions drawn from this experiment include the following: 1) There is a positive trend in the proportions of dogs dying of tumors as total dose is increased at all dose rates; there is no indication of a dose-rate effect except at a total dose of $3000 \mathrm{rad}$, where the trend is negative. 2) The timing of deaths in all irradiated groups differs from the control group, except for the 450 rad total dose group where meaningful analysis is not yet possible. 3) Only total dose influences the timing of fatal tumors, but neither total dose nor dose rate influences the timing of nontumor deaths. 4) Although a significant increase in myeloproliferative disorders is observed, it is smaller than observed in dogs irradiated continuously; dogs appear to recover from hematopoietic damage after irradiation is terminated, and die at later times of soft tissue malignancies (Fritz, 1986; Seed, 1985).

Information relevant to this experiment will be found in the following Argonne National Laboratory periodic reports:

\begin{tabular}{|c|c|c|c|c|c|}
\hline Issue Date & Doc. No. & Page Refs. & Issue Date & Doc. No. & Page Refs. \\
\hline 1971 (Dec) & ANL-7870 & $138-145$ & $1978(\mathrm{Dec})$ & ANL-79-90 & 6-12 \\
\hline 1972 (Dec) & ANL-7970 & $192-203$ & 1980 (Dec) & ANL-80-90 & $8-13$ \\
\hline 1973 (Dec) & ANL-8070 & $31-54$ & 1981 (Aug) & ANL-81-50 & $8-13$ \\
\hline 1974 (Dec) & ANL-75-30 & $9-14$ & 1982 (Jun) & ANL-82-35 & $11-19$ \\
\hline 1975 (Dec) & ANL-76-99 & $10-19$ & 1983 (May) & ANL-83-40 & $27-36$ \\
\hline 1976 (Dec) & ANL-77-55 & $34-40$ & 1984 (Aug) & ANL-84-30 & $37-46$ \\
\hline 1977 (Dec) & ANL-78-90 & $78-86$ & 1985 (Aug) & ANL-85-30 & $63-72$ \\
\hline
\end{tabular}




\subsection{PNL EXPERIMENTS}

8.4.1 $1959{ }^{239} \mathrm{Pu}$

Single inhalation of plutonium oxide aerosol, CMD 0.1 to $0.5 \mu \mathrm{m}$; dogs 12 to 43 months old, of both sexes, placed on experiment between 1959 and 1962 (Park, J., 1964, 1972;ICRP, 1980)

\begin{tabular}{|c|c|c|c|c|c|c|c|}
\hline \multirow{2}{*}{\multicolumn{2}{|c|}{$\begin{array}{l}\text { EXPERIMENTAL } \\
\text { DESIGN } \\
\end{array}$}} & \multicolumn{4}{|c|}{ EXPERIMENTAL RESULTS } & & \\
\hline & & & Median & & & & \\
\hline Initial & No. of & No. of & Postexp. & №. $1 \%$ & ce) & & \\
\hline $\begin{array}{l}\text { Burden } \\
(\mu \mathrm{Ci} / \mathrm{kg})\end{array}$ & $\begin{array}{l}\text { Animals } \\
\text { Exposed }\end{array}$ & $\begin{array}{l}\text { Animals } \\
\text { Dead }\end{array}$ & $\begin{array}{l}\text { Survival } \\
\text { (years) } \\
\end{array}$ & $\begin{array}{l}\text { Lung } \\
\text { Tumor }\end{array}$ & & $\begin{array}{l}\text { Radia } \\
\text { monit }\end{array}$ & $\begin{array}{l}\text { Pneu- } \\
\text { ath }\end{array}$ \\
\hline 0.06 & 11 & 11 & 8.4 & 10 & (91) & 10 & (91) \\
\hline 0.13 & 13 & 13 & 5.5 & 11 & (84) & 13 & $(100)$ \\
\hline 0.20 & 6 & 6 & 4.4 & 4 & $(67)$ & 6 & $(100)$ \\
\hline 0.31 & 5 & 5 & 2.7 & 2 & $(40)$ & 5 & $(100)$ \\
\hline
\end{tabular}

All dogs are dead. A brief summary of results has been published (ICRP, 1980); more detailed general accounts appear in earlier progress reports (Bair, 1970b; Park, 1964, 1972).

Animals in the above tabulation include only those surviving for as long as 855 days. Acute effects have been described for an additional 31 dogs that died within a year of exposure (Bair, 1962; Clarke, 1964; Dagle, 1976; Park, J., 1962), and for another 8 dogs employed in an ancillary study to define the early radiation syndrome following plutonium inhalation (West, 1964). These data have been employed in several attempts to model acute effects of inhaled plutonium (Bair, 1980; Raabe, 1979; Scott, 1980, 1980b). Other early ancillary studies investigated the effect of physical and chemical properties of the exposure aerosol on early deposition and retention (Bair, 1961b, 1962b, 1963, 1963b, 1964, 1968; Stuart, 1968).

The oxide employed in the long-term experiment was produced by calcining the oxalate at 300 to $350^{\circ} \mathrm{C}$; the aerosol was produced by atomization and administered via a mask (Bair, 1963). Plutonium distribution and retention were determined from excreta and postmortem tissue analyses (Bair, 1970b, 1973; Park, J., 1964, 1972); these data have been employed in the development of several models of the biokinetics of inhaled plutonium (Cuddihy, 1976; Mahaffey, 1981; Stuart, 1970). Percent of terminal body burden present in lungs decreased from $71 \%$ in dogs surviving less than 3 years, to $17 \%$ in dogs surviving more than 10 years; comparable <3- and $>10$-year-survival percentages for pulmonary lymph nodes 
were $18 \%$ and $33 \%$; for liver, $7 \%$ and $33 \%$; and for skeleton, $2 \%$ and $7 \%$ (ICRP, 1980). Local and microscopic distribution of plutonium within the lung and pulmonary lymph nodes has been studied autoradiographically (Clarke, 1964, 1966; Dagle, 1976b; McShane, 1980; Park, J., 1964). Estimated average dose to lung ranged from about 1200 to 4000 rad over the four exposure groups (ICRP, 1980).

Effects in the long-term experiment were described in a series of general progress reports (Bair, 1970b, 1973, 1974, 1974b; Park, J., 1964, 1966, 1972) and in papers emphasizing histopathology (Clarke, 1966; Dagle, 1976; Howard, 1970), biochemical effects (Dilley, 1972), effects in pulmonary lymph nodes (Dagle, 1976b), and carcinogenesis (Clarke, 1964b, 1966b; Dagle, 1976, 1980). All tumorbearing dogs had pulmonary adenocarcinomas (bronchioloalveolar carcinomas), often with multiple metastases; five dogs also had peripherally located epidermoid carcinomas of the lungs, two had lymphangiosarcomas, and one a capillary hemangioma of the lungs (ICRP, 1980).

Results of this experiment have been used to estimate cancer risk factors for inhalation of plutonium (Bair, 1974f; Cuddihy, 1976, 1982; Mahaffey, 1981), including particular emphasis on the risk from so-called "hot particles" (Bair, 1974c). The data have also been employed, using 3-dimensional dose-response models, to illustrate the time-related contributions of the competing risks of death from radiation pneumonitis/fibrosis, lung cancer, and natural aging (Raabe, 1987).

Information relevant to this experiment will be found in the following Hanford Laboratory or Pacific Northwest Laboratory periodic reports:

\begin{tabular}{|c|c|c|c|c|c|}
\hline Issue Date & Doc. No. & Page Refs. & Issue Date & Doc. No. & Page Refs \\
\hline 1960 (Jan) & HW-65500 & $116-128$ & 1967 (Jul) & BNWL-480 & $57-65$ \\
\hline 1961 (Jan) & $H W-69500$ & $67-76$ & 1968 (May) & BNWL-714 & $3.3-3.10$ \\
\hline 1962 (Jan) & $\mathrm{HW}-72500$ & $61-66$ & 1970 (Jan) & BNWL-1050 & 3.3-3.5 \\
\hline 1963 (Jan) & $\mathrm{HW}-76000$ & $118-135$ & & & $3.14-3.18$ \\
\hline 1964 (Jan) & $\mathrm{HW}-80500$ & $30-43$ & 1970 (Aug) & BNWL-1306 & $44-47$ \\
\hline 1965 (Jan) & BNWL-122 & $1-15$ & 1971 (Oct) & BNWL-1550 & $66-74$ \\
\hline 1966 (Jan) & BNWL-280 & $45-49$ & & & \\
\hline
\end{tabular}




\subsection{2 $1967^{238} \mathrm{Pu}$}

Single inhalation of plutonium oxide aerosol, CMD $0.1 \mu \mathrm{m} ; \mathrm{dogs} 8$ to 42 months old, of both sexes, placed on experiment in 1967 (Park, J., 1976; ICRP, 1980).

\begin{tabular}{|c|c|c|c|c|c|c|c|}
\hline \multirow{2}{*}{\multicolumn{2}{|c|}{$\begin{array}{l}\text { EXPERIMENTAL } \\
\text { DESIGN }\end{array}$}} & \multicolumn{4}{|c|}{ RIMENTAL RESULTS } & & \\
\hline & & & Median & & & & \\
\hline Terminal & No. of & No. of & Postexp. & №. $1 \%$ & acel & & \\
\hline Burden & Animals & Animals & Survival & Bone & & Lung & \\
\hline$(\omega \mathrm{Ci} / \mathrm{kg})$ & Exposed & Dead & (years) & Tumor & & Tumor & \\
\hline $\begin{array}{c}0.02 \text { to } \\
0.8\end{array}$ & 10 & 10 & 4.9 & 6 & $(60)$ & 1 & (10) \\
\hline $\begin{array}{c}0.02 \text { to } \\
0.3\end{array}$ & 12 & 12 & 6.0 & 6 & (50) & 1 & (8) \\
\hline
\end{tabular}

All dogs are dead. No summary of results has been published but rather detailed progress reports appeared when 6 dogs remained alive (Park, J., 1975, 1976). In an earlier acute toxicity study, 10 dogs died within 6 months of exposures with terminal body burdens in excess of $2 \mu \mathrm{Ci} / \mathrm{kg}$; effects were similar to those observed with ${ }^{239} \mathrm{PuO}_{2}$ (Park, J., 1969, 1976).

The first group of 10 dogs listed above was exposed to an oxide produced by calcining the oxalate at $350^{\circ} \mathrm{C}$; the second group of 12 dogs was exposed to crushed oxide microspheres of a type developed for application in thermoelectric power generators (Park, J., 1976). The aerosol was produced by atomization and administered via a mask (Bair, 1963). Distribution and retention, as determined from excreta and postmortem tissue analyses, were very different for inhaled ${ }^{238} \mathrm{PuO}_{2}$ than for ${ }^{239} \mathrm{PuO}_{2}$, with ${ }^{238} \mathrm{Pu}$ being more rapidly translocated from the lung and depositing in much higher concentrations in bone (Bair, 1973; Park, J., 1972). This apparent greater solubility of ${ }^{238} \mathrm{PuO}_{2}$ is attributed to radiolytic effects caused by the higher specific activity of ${ }^{238} \mathrm{Pu}$ (Park, J., 1974; Vaughan, 1973). An average $19 \%$ of terminal body burden was present in lungs, $12 \%$ in pulmonary lymph nodes, $22 \%$ in liver, and $41 \%$ in skeleton (Park, J., 1976).

Hematologic and carcinogenic effects have been compared with those from inhaled ${ }^{239} \mathrm{PuO}_{2}$ (Park, J., 1975, 1976); this comparison has been of particular interest in assessing the effect of spatial distribution of radiation dose from inhaled plutonium (Bair, 1974c). The high incidence of bone, rather than lung, tumors in the ${ }^{238} \mathrm{PuO}_{2}$ dogs correlates with the greater and more rapid translocation of ${ }^{238} \mathrm{Pu}$ from lung to bone. 
Information relevant to this experiment will be found in the following Hanford Laboratory or Pacific Northwest Laboratory periodic reports:

\begin{tabular}{|c|c|c|c|c|c|}
\hline Issue Date & Doc. No. & Page Refs. & Issue Date & Doc. No. & Page Refs. \\
\hline 1968 (May) & BNWL-714 & $4.9-4.10$ & 1974 (Aug) & BNWL-1850 Pt 1 & $41-44$ \\
\hline 1970 (Jan) & BNWL-1050 & $3.6-3.16$ & 1975 (Mar) & BNWL-1950 Pt 1 & $10-16$ \\
\hline 1970 (Aug) & BNWL-1306 & $66-67$ & 1976 (Jan) & BNWL-2000 Pt 1 & $18-22$ \\
\hline 1973 (Apr) & BNWL-1750 Pt 1 & $37-38$ & & & \\
\hline
\end{tabular}


8.4.3 $1970{ }^{239} \mathrm{Pu}$

Single inhalation of plutonium oxide aerosol, mean AMAD 2.3 $\mu \mathrm{m}$, mean GSD 1.9; dogs 14 to 22 months old, of both sexes, placed on experiment from 1970 to 1972 . Results as of 30 September 1987 (Doc. PNL-6500 Pt 1).

\begin{tabular}{|c|c|c|c|c|c|c|c|c|c|}
\hline \multirow{2}{*}{\multicolumn{2}{|c|}{$\begin{array}{l}\text { EXPERIMENTAL } \\
\text { DESIGN }\end{array}$}} & \multicolumn{8}{|c|}{ EXPERIMENTAL RESULTS } \\
\hline & & \multirow[b]{2}{*}{$\begin{array}{l}\text { No. of } \\
\text { Animals } \\
\text { Dead } \\
\end{array}$} & \multirow{2}{*}{$\begin{array}{l}\text { Median } \\
\text { Postexp. } \\
\text { Survival } \\
\text { (years) }\end{array}$} & \multicolumn{4}{|c|}{ №. (\% Incidence) } & & \\
\hline $\begin{array}{l}\text { Initial } \\
\text { Burden } \\
\text { (uCi/kg) }\end{array}$ & $\begin{array}{l}\text { No. of } \\
\text { Animals } \\
\text { Exposed }\end{array}$ & & & \multicolumn{2}{|c|}{$\begin{array}{l}\text { Bone } \\
\text { Tumor }\end{array}$} & \multicolumn{2}{|c|}{$\begin{array}{l}\text { Lung } \\
\text { Tumor }\end{array}$} & \multicolumn{2}{|c|}{$\begin{array}{l}\text { Radiation } \\
\text { Pneumonitis } \\
\text { Death }\end{array}$} \\
\hline $\begin{array}{l}\text { None } \\
\text { (Control) }\end{array}$ & 20 & 20 & 12.7 & 0 & $(0)$ & 4 & (20) & 0 & $(0)$ \\
\hline 0.0003 & 24 & 24 & 12.8 & 1 & (4) & 1 & (4) & 0 & $(0)$ \\
\hline 0.0020 & 21 & 21 & 13.2 & 1 & (5) & 4 & (19) & 0 & $(0)$ \\
\hline 0.007 & 20 & 19 & 12.9 & 0 & $(0)$ & 10 & (53) & 0 & $(0)$ \\
\hline 0.026 & 22 & 22 & 10.1 & 0 & $(0)$ & 16 & (73) & 0 & $(0)$ \\
\hline 0.09 & 21 & 21 & 6.3 & 0 & $(0)$ & 20 & $(95)$ & 2 & $(10)$ \\
\hline 0.60 & 8 & 8 & 1.5 & 0 & $(0)$ & 1 & (13) & 7 & (88) \\
\hline
\end{tabular}

This experiment was initiated when it became apparent that dogs in the lowest exposure levels of the earlier ${ }^{239} \mathrm{PuO}_{2}$ experiment were nearly all developing lung tumors. The design and current status of the experiment has been described in several progress reports (Dagle, 1979; Park, J., 1975, 1981, 1986, in press).

The oxide was produced by calcining the oxalate at $750^{\circ} \mathrm{C}$ for 2 hours; improved exposure procedures and deposition results have been described in some detail (Craig, 1972, 1973). Data on the translocation and retention of the deposited plutonium have been described (Dagle, 1979; Park, J., 1981, 1986, in press) and subjected to preliminary dosimetric analysis (Fisher, D., 1986; Park, in press).

Hematologic effects have been described in some detail (Ragan, 1976, 1986); lymphopenia developed soon after exposure in all but the two lowest exposure groups, with a trend toward recovery after about 3 years in all but the highest exposure group. Radioimmunoassay techniques revealed a significant decrease in primary antibody response in exposed versus unexposed dogs (Dagle, 1979). Later effects have been described briefly (Dagle, 1986; Park, J., 1986, in press); the predominant lung tumor was bronchiolar-alveolar carcinoma, with a lesser number of adenosquamous carcinoma, adenocarcinoma, and epidermoid carcinoma. Preliminary studies have shown that dominant-acting transforming 
oncogene activity is associated with the plutonium-induced tumors (Frazier, 1987).

Preliminary dose-response relationships have been derived (Fisher, D., 1986). Three-dimensional dose-response models have been employed to illustrate the time-related contributions of the competing risks of death from radiation pneumonitis/fibrosis, lung cancer, and natural aging (Raabe, 1987).

Information relevant to this experiment will be found in the following Hanford Laboratory or Pacific Northwest Laboratory periodic reports:

\begin{tabular}{|c|c|c|c|c|c|c|}
\hline \multicolumn{2}{|c|}{ Issue Date } & Doc. No. & Page Refs. & Issue Date & Doc. No. & Page Rets. \\
\hline 1970 & (Aug) & BNWL-1306 & $55-56$ & 1981 (Feb) & PNL-3700 Pt 1 & $66-78$ \\
\hline 1972 & (Sep) & BNWL-1650 & $177-230$ & 1982 (Feb) & PNL-4100 Pt 1 & $61-71$ \\
\hline 1973 & (Apr) & BNWL-1750 Pt 1 & $21-27$ & 1983 (Feb) & PN-4600 Pt 1 & $51-60$ \\
\hline 1974 & (Aug) & BNWL-1850 Pt 1 & $35-40$ & 1984 (Feb) & PNL-5000 Pt 1 & $13-22$ \\
\hline 1975 & (Mar) & BNWL-1950 Pt 1 & $3-9$ & 1985 (Feb) & PNL-5500 Pt 1 & $13-22$ \\
\hline 1976 & (Jan) & BNWL-2000 Pt 1 & $11-17$ & 1986 (Feb) & PNL-5750 Pt 1 & $3-17$ \\
\hline 1977 & (May) & BNWL-2100 Pt 1 & $17-21$ & & & $25-26$ \\
\hline 1978 & (Feb) & PNL-2500 Pt 1 & $3.13-3.20$ & 1987 (Feb) & PNL-6100 Pt 1 & $5-19$ \\
\hline 1979 & (Feb) & PNL-2850 Pt 1 & $3.9-3.16$ & & & $27-30$ \\
\hline \multirow[t]{2}{*}{1980} & (Feb) & PNL-3300 Pt 1 & $87-98$ & 1988 (Feb) & PNL-6500 Pt 1 & $9-23$ \\
\hline & & & & & & $45-47$ \\
\hline
\end{tabular}




\subsection{4 $1972{ }^{238} \mathrm{Pu}$}

Single inhalation of plutonium oxide aerosol, mean AMAD 1.8 $\mu \mathrm{m}$, mean GSD 1.9; dogs 15 to 20 months old, of both sexes, placed on experiment from 1972 to 1975 . Results as of 30 September 1987 (Doc. PNL-6500 Pt 1)

\begin{tabular}{|c|c|c|c|c|c|c|c|c|c|}
\hline \multirow{2}{*}{\multicolumn{2}{|c|}{$\begin{array}{l}\text { EXPERIMENTAL } \\
\text { DESIGN }\end{array}$}} & \multicolumn{8}{|c|}{ EXPERIMENTAL RESULTS } \\
\hline & & \multirow{4}{*}{$\begin{array}{l}\text { No. of } \\
\text { Animals } \\
\text { Dead }\end{array}$} & \multirow{4}{*}{$\begin{array}{l}\text { Median } \\
\text { Postexp. } \\
\text { Survival } \\
\text { (years) }\end{array}$} & \multicolumn{4}{|c|}{ No. (\% Incidence) } & \multirow{2}{*}{\multicolumn{2}{|c|}{ Radiation }} \\
\hline _Initial & No. of & & & \multirow{2}{*}{\multicolumn{2}{|c|}{ Bone }} & \multirow{2}{*}{\multicolumn{2}{|c|}{ Lung }} & & \\
\hline Burden & Animals & & & & & & & \multicolumn{2}{|c|}{ Pneumonitis } \\
\hline$(\mu \mathrm{Cl} / \mathrm{kg})$ & Exposed & & & \multicolumn{2}{|c|}{ Iumor } & \multicolumn{2}{|c|}{ Tumor } & \multicolumn{2}{|c|}{ Death } \\
\hline $\begin{array}{l}\text { None } \\
\text { (Control) }\end{array}$ & 20 & 12 & 13.5 & 0 & (0) & 0 & $(0)$ & 0 & (0) \\
\hline 0.0002 & 20 & 17 & 12.6 & 1 & (6) & 0 & $(0)$ & 0 & $(0)$ \\
\hline 0.0016 & 21 & 16 & 13.0 & 0 & (0) & 2 & $(13)$ & 0 & $(0)$ \\
\hline 0.007 & 22 & 17 & 12.7 & 0 & (0) & 1 & (6) & 1 & (6) \\
\hline 0.029 & 20 & 19 & 11.7 & 7 & (37) & 3 & $(16)$ & 1 & (5) \\
\hline 0.11 & 20 & 20 & 7.4 & 16 & $(80)$ & 5 & $(25)$ & 0 & (0) \\
\hline 0.47 & 13 & 13 & 5.2 & 9 & (69) & 9 & $(69)$ & 0 & $(0)$ \\
\hline
\end{tabular}

This experiment was designed to compare the behavior and effects of inhaled ${ }^{238} \mathrm{PuO}_{2}$ with those of inhaled ${ }^{239} \mathrm{PuO}_{2}$ and ${ }^{239} \mathrm{Pu}\left(\mathrm{NO}_{3}\right)_{4}$ in concurrent experiments. The design and current status of the experiment has been described in several progress reports (Dagle, 1979; Park, J., 1975, 1981, 1986).

The oxide was produced by calcining the oxalate at $700^{\circ} \mathrm{C}$ for 2 hours; exposure procedures have been described in some detail (Craig, 1972, 1973). Data on the translocation and retention of the deposited plutonium have been described (Dagle, 1979; Park, J., 1981, 1986); methods for estimation of the initial lung deposit from subsequent retention and excretion data were studied in detail for one group of animals (Stevens, D., 1986b).

Hematologic effects have been described in some detail (Ragan, 1986); lymphopenia developed sooner and was more pronounced than was the case with ${ }^{239} \mathrm{PuO}_{2}$; it occurred in all but the two lowest exposure groups, with a trend toward recovery after 2 or 3 years in all groups. Later effects have been described briefly (Dagle, 1986; Park, J., 1986) and include, in addition to bone and lung tumors, sclerotic lymph nodes, osteodystrophy, hepatic nodular hyperplasia, and elevated serum glutamic pyruvic transaminase levels. The predominant bone tumor was osteosarcoma; the predominant lung tumor was bronchiolar- 
alveolar carcinoma, with lesser numbers of adenosquamous carcinoma, adenocarcinoma, bronchiolar-alveolar adenoma, and fibrosarcoma.

Information relevant to this experiment will be found in the following Hanford Laboratory or Pacific Northwest Laboratory periodic reports:

\begin{tabular}{|c|c|c|c|c|c|}
\hline Issue Date & Doc. No. & Page Refs. & Issue Date & Doc. No. & Page Refs. \\
\hline 1970 (Aug) & BNWL-1306 & $55-56$ & 1981 (Feb) & PNL-3700 Pt 1 & $66-78$ \\
\hline 1973 (Aug) & BNWL-1750 Pt 1 & $21-37$ & 1982 (Feb) & PNL-4100 Pt 1 & $61-71$ \\
\hline 1974 (Aug) & BNWL-1850 Pt 1 & $35-40$ & 1983 (Feb) & PNL-4600 Pt 1 & $51-60$ \\
\hline 1975 (Mar) & BNWL-1950 Pt 1 & $3-9$ & 1984 (Feb) & PNL-5000 Pt 1 & $13-22$ \\
\hline 1976 (Jan) & BNWL-2000 Pt 1 & $11-17$ & 1985 (Feb) & PNL-5500 Pt 1 & $13-22$ \\
\hline 1977 (May) & BNWL-2100 Pt 1 & $17-21$ & 1986 (Feb) & PNL-5750 Pt 1 & $3-17$ \\
\hline 1978 (Feb) & PNL-2500 Pt 1 & $3.13-3.20$ & & & $25-26$ \\
\hline 1979 (Feb) & PNL-2850 Pt 1 & $3.9-3.16$ & 1987 (Feb) & PNL-6100 Pt 1 & $5-19$ \\
\hline 1980 (Feb) & PNL-3300 Pt 1 & $87-96$ & & & $27-30$ \\
\hline & & & 1988 (Feb) & PNL-6500 Pt 1 & $9-23$ \\
\hline & & & & & $45-47$ \\
\hline
\end{tabular}




\subsection{5 $1975^{239} \mathrm{Pu}$}

Single inhalation of plutonium nitrate aerosol, mean AMAD 0.81 um, mean GSD 1.7; dogs 17 to 23 months old, of both sexes, placed on experiment from 1975 to 1977. Results as of 30 September 1987 (Doc. PNL-6500 Pt 1).

\begin{tabular}{|c|c|c|c|c|c|c|c|c|c|}
\hline \multirow{2}{*}{\multicolumn{2}{|c|}{$\begin{array}{l}\text { EXPERIMENTAL } \\
\text { DESIGN }\end{array}$}} & \multicolumn{8}{|c|}{ EXPERIMENTAL RESULTS } \\
\hline & & \multirow{3}{*}{$\begin{array}{l}\text { No. of } \\
\text { Animals } \\
\text { Dead } \\
\end{array}$} & \multirow{3}{*}{$\begin{array}{l}\text { Median } \\
\text { Postexp. } \\
\text { Survival } \\
\text { (years) }\end{array}$} & \multicolumn{4}{|c|}{ №. $(\%$ Incidence) } & \multirow{2}{*}{\multicolumn{2}{|c|}{ Radiation }} \\
\hline _Initial & No. of & & & \multirow{2}{*}{\multicolumn{2}{|c|}{ Bone }} & \multirow{2}{*}{\multicolumn{2}{|c|}{$\begin{array}{l}\text { Lung } \\
\text { Iumor }\end{array}$}} & & \\
\hline $\begin{array}{l}\text { Burden } \\
\text { ( } \mathrm{Ci} / \mathrm{kg})\end{array}$ & $\begin{array}{l}\text { Animals } \\
\text { Exposed }\end{array}$ & & & & & & & \multicolumn{2}{|c|}{$\begin{array}{l}\text { Pneumonitis } \\
\text { Death }\end{array}$} \\
\hline $\begin{array}{l}\text { None } \\
\text { (Control) }\end{array}$ & 23 & 10 & $>10.4$ & 0 & (0) & 0 & (0) & 0 & (0) \\
\hline $\begin{array}{l}\text { None } \\
\text { (Vehicle } \\
\text { control) }\end{array}$ & 20 & 2 & $>11.2$ & 0 & (0) & 0 & (0) & 0 & (0) \\
\hline 0.0002 & 20 & 6 & $>10.4$ & 0 & (0) & 0 & (0) & 0 & (0) \\
\hline 0.0007 & 20 & 4 & $>10.4$ & 0 & (0) & 0 & (0) & 0 & $(0)$ \\
\hline 0.005 & 20 & 11 & 11.3 & 0 & (0) & 2 & (18) & 0 & $(0)$ \\
\hline 0.026 & 20 & 13 & 9.9 & 5 & $(38)$ & 6 & (46) & 0 & (0) \\
\hline 0.15 & 20 & 20 & 5.4 & 17 & $(85)$ & 11 & (55) & 2 & $(10)$ \\
\hline 0.52 & 5 & 5 & 1.4 & 0 & (0) & 1 & (20) & 5 & $(100)$ \\
\hline
\end{tabular}

This experiment was designed to compare the behavior and effects of inhaled ${ }^{239} \mathrm{Pu}\left(\mathrm{NO}_{3}\right)_{4}$ with those of inhaled ${ }^{239} \mathrm{PuO}_{2}$ and ${ }^{238} \mathrm{PuO}_{2}$ in concurrent experiments. The design and current status of the experiment have been described in several progress reports (Dagle, 1979; Park, J., 1981, 1986). Earlier short-term studies with inhaled $\mathrm{Pu}\left(\mathrm{NO}_{3}\right)_{4}$ provided background data for this experiment (Bair, 1964, 1970b, 1974b; Park, J., 1972; Stuart, 1968).

The exposure aerosol was produced by nebulizing a $0.27 \mathrm{~N}$ nitric acid solution (Dagle, 1979). Data on the translocation and retention of the deposited plutonium have been described (Park, J., 1981, 1986). A supporting 1-year sacrifice study compared the early distribution and retention of inhaled ${ }^{238} \mathrm{Pu}\left(\mathrm{NO}_{3}\right)_{4}$ and ${ }^{239} \mathrm{Pu}\left(\mathrm{NO}_{3}\right)_{4}$ (Dagle, 1983 ); although ${ }^{238} \mathrm{Pu}$ was initially translocated more rapidly from the lung, the rate of translocation was similar at 1 year postexposure. In another periodic sacrifice study extending to 5 years postexposure, estimates were made of radiation doses to various tissues following ${ }^{239} \mathrm{Pu}\left(\mathrm{NO}_{3}\right)_{4}$ inhalation (Stevens, D, 1986). 
Hematologic effects have been described in some detail (Ragan, 1986); lymphopenia was less pronounced than was the case following exposure to either ${ }^{239} \mathrm{PuO}_{2}$ or ${ }^{238} \mathrm{PuO}_{2}$; it occurred only in the two highest exposure groups. Later effects have been described briefly (Dagle, 1986; Park, J., 1986) and include, in addition to bone and lung tumors, sclerotic lymph nodes, osteodystrophy, hepatic nodular hyperplasia, and elevated serum glutamic pyruvic transaminase levels. All bone tumors were osteosarcomas. Lung tumors were usually incidental findings at death and included bronchiolar-alveolar carcinoma, papillary adenocarcinoma, and epidermoid carcinoma. Preliminary studies have shown that dominant-acting transforming oncogene activity is associated with the plutonium-induced lung tumors (Frazier, 1987).

Information relevant to this experiment will be found in the following Hanford Laboratory or Pacific Northwest Laboratory periodic reports:

\begin{tabular}{|c|c|c|c|c|c|}
\hline Issue Date & Doc. No. & Page Refs. & Issue Date & Doc. №. & Page Refs. \\
\hline 1976 (Jan) & BNWL-2000 Pt 1 & 27 & 1982 (Feb) & PNL-4100 Pt 1 & $73-76$ \\
\hline 1977 (May) & BNWL-2100 Pt 1 & $25-27$ & 1983 (Feb) & PNL-4600 Pt 1 & $61-64$ \\
\hline 1978 (Feb) & PNL-2500 Pt 1 & $3.4-3.5$ & 1984 (Feb) & PNL-5000 Pt 1 & $23-26$ \\
\hline & & $3.27-3.29$ & 1985 (Feb) & PNL-5500 Pt 1 & $23-27$ \\
\hline 1979 (Feb) & PNL-2850 P1 1 & $3.19-3.26$ & 1986 (Feb) & PNL-5750 Pt 1 & $19-23$ \\
\hline 1980 (Feb) & PNL-3300 P1 1 & $99-104$ & 1987 (Feb) & PNL-6100 Pt 1 & $21-25$ \\
\hline 1981 (Feb) & PNL-3700 Pt 1 & $81-85$ & 1988 (Feb) & PNL-6500 Pt 1 & $25-29$ \\
\hline
\end{tabular}




\subsection{ITRI EXPERIMENTS}

\subsection{1 $1965^{90} \mathrm{Sr}$}

Single inhalation of strontium chloride in a cesium chloride vector aerosol; dogs 12 to 15 months old, of both sexes, placed on experiment from 1965 to 1967. Results as of 30 September 1987 (Doc. $L M F-120$ ).

\begin{tabular}{|c|c|c|c|c|c|c|c|}
\hline \multirow{2}{*}{\multicolumn{2}{|c|}{$\begin{array}{l}\text { EXPERIMENTAL } \\
\text { DESIGN }\end{array}$}} & \multicolumn{6}{|c|}{ EXPERIMENTAL RESULTS } \\
\hline & & & Median & & & & \\
\hline Initial & No. of & No. of & Postexp. & \multicolumn{4}{|c|}{ No. $(\%$ Incidence) } \\
\hline Burden & Animals & Animals & Survival & Bone & & Lung & \\
\hline$(\mu \mathrm{Ci} / \mathrm{kg})$ & Exposed & Dead & (years) & Tumor & & Tumor & \\
\hline $\begin{array}{l}\text { None } \\
\text { (Control) }\end{array}$ & 15 & 15 & 12.6 & 0 & $(0)$ & 0 & $(0)$ \\
\hline 2 & 12 & 12 & 13.0 & 0 & (0) & 1 & (8) \\
\hline 8 & 12 & 12 & 12.7 & 1 & (8) & 1 & (8) \\
\hline 43 & 12 & 12 & 5.1 & 11 & $(92)$ & 0 & (0) \\
\hline 100 & 12 & 12 & 1.8 & 5 & (42) & 0 & (0) \\
\hline
\end{tabular}

All dogs are dead, and several summaries of results have been published (Gillett, 1987, 1987b; McClellan, 1983; Mewhinney, 1986). Exposure aerosols had an AMAD of 1.4 to $2.7 \mu \mathrm{m}$, with a GSD of about 2.0. Quantity of ${ }^{90} \mathrm{Sr}$ deposited and retained was determined by whole-body counting. About $60 \%$ of the initial deposit was lost with a half-time of 0.3 day, reflecting rapid clearance from the respiratory and gastrointestinal tract. The initial burdens, as tabulated above, do not include this rapidly lost component, whose estimation was relatively uncertain. The retention of the remainder was described by 3 approximately equal components with half-times of about 6 days, 130 days, and 8 years, all reflecting loss from the skeleton (McClellan, 1983). Estimated median-survival average radiation dose to bone ranged from about $500 \mathrm{rad}$ in the lowest exposure group to about $18,000 \mathrm{rad}$ in the highest (Doc, $L M F-120$ ). A supporting study with intravenously injected dogs showed a systemic distribution pattern almost identical to that seen following inhalation (McClellan, 1972c).

In addition to the 48 life-span dogs tabulated above, 25 "sacrifice" dogs, including 7 controls, were, in fact, not sacrificed, but retained for life-span observation. Including these dogs, a total of 6 died acutely, 2 died with leukemia, 30 died with bone tumors, and 4 died with sinonasal carcinomas (Gillett, 1987b). The median 
survival time of dogs with long-retained burdens of less than $10 \mu \mathrm{Ci} / \mathrm{kg}$ was similar to that of controls (McClellan, 1983).

Early publications give details of partial results (McClellan, 1972c, 1973), including reports emphasizing the production of hemangiosarcomas (Benjamin, 1975b) and sinonasal carcinomas (Benjamin, 1979; Boecker, 1986); a study of the excretion of urinary steroids was also reported (Mitchell, 1970). Several publications have compared the preliminary results from this experiment with those from experiments with beta emitters of different retention and distribution characteristics (Boecker, 1986; McClellan, 1976, 1979, 1982, 1986). Detailed data have been recently reported, for individual animals, relating particularly to the early hematopoietic deaths and hematological effects (Gillett, 1987), and to the late neoplastic and preneoplastic effects in bone (Gillett, 1987b). Preliminary consideration was given to dose-response relationships for late-occurring neoplastic (McClellan, 1973) and early hematopoietic effects (Scott, 1980). It was noted that late effects in this experiment closely resemble those seen in the Utah experiment with intravenously injected ${ }^{90} \mathrm{Sr}$; in neither experiment was there observed the high incidence of myeloproliferative disorders seen in the Davis ${ }^{90} \mathrm{Sr}$ chronic ingestion experiment (McClellan, 1973). Bone cancer data from this experiment have been compared with those from studies with other inhaled and injected bone seekers, and efforts have been made to extrapolate findings to humans, employing a proportional hazard analysis (Mewhinney, 1986).

Information relevant to this experiment will be found in the following Inhalation Toxicology Research Institute periodic reports:

\begin{tabular}{|c|c|c|c|c|c|}
\hline Issue Date & Doc. No. & Page Refs. & Issue Date & Doc. No. & Page Refs. \\
\hline 1966 (Nov) & LF-33 & $122-136$ & 1976 (Dec) & LF-56 & $154-157$ \\
\hline & & $153-156$ & & & $200-204$ \\
\hline & & $211-219$ & 1977 (Dec) & $L F-58$ & $62-65$ \\
\hline 1967 (Nov) & $L F-38$ & $1-18$ & 1978 (Dec) & $L F-60$ & $68-71$ \\
\hline 1968 (Nov) & $L F-39$ & $1-13$ & 1979 (Dec) & $L F-69$ & $57-61$ \\
\hline 1969 (Nov) & $L F-41$ & $1-7$ & 1980 (Dec) & LMF-84 & $48-52$ \\
\hline & & $59-60$ & 1981 (Dec) & LMF-91 & $67-72$ \\
\hline 1970 (Nov) & LF-43 & $123-127$ & 1982 (Dec) & LMF-102 & $271-275$ \\
\hline 1971 (Nov) & $L F-44$ & $121-125$ & 1983 (Dec) & LMF-107 & $183-189$ \\
\hline 1972 (Nov) & $L F-45$ & $129-136$ & 1984 (Dec) & LMF-113 & 154-158 \\
\hline 1973 (Dec) & $L F-46$ & $86-90$ & 1985 (Dec) & LMF-114 & $175-180$ \\
\hline 1974 (Dec) & $L F-49$ & $89-92$ & & & $275-279$ \\
\hline 1975 (Dec) & LF-52 & $134-138$ & 1986 (Dec) & $L M F-115$ & $167-176$ \\
\hline
\end{tabular}




\subsection{2 $1966{ }^{144} \mathrm{Ce}$}

Single inhalation of cerium chloride in a cesium chloride vector aerosol; dogs 12 to 15 months old, of both sexes, placed on experiment from 1966 to 1967. Results as of 30 September 1987 (Doc. LMF-120).

\begin{tabular}{|c|c|c|c|c|c|c|c|c|c|}
\hline EXPERI & AENTAL & EXPERIN & NTAL RES & & & & & & \\
\hline DESIGN & & & Median & & & & & & \\
\hline Initial & No. of & No. of & Postexp. & №. & Incidence) & & & & \\
\hline Burden & Animals & Animals & Survival & Liv & & Lur & & $\mathrm{Na}$ & Cavity \\
\hline$(\mu \mathrm{Ci} / \mathrm{kg})$ & Exposed & Dead _ & (years) & Tun & & Tur & & $\underline{\text { Tur }}$ & \\
\hline $\begin{array}{l}\text { None } \\
\text { (Control) }\end{array}$ & 15 & 15 & 13.9 & 0 & (0) & 2 & (13) & 0 & $(0)$ \\
\hline 4.8 & 12 & 12 & 11.5 & 2 & (17) & 0 & (0) & 0 & (0) \\
\hline 14 & 12 & 12 & 11.1 & 4 & (33) & 2 & (17) & 2 & (17) \\
\hline 44 & 12 & 12 & 7.4 & 5 & (42) & 2 & (17) & 4 & (33) \\
\hline 140 & 12 & 12 & 1.2 & 2 & (17) & 0 & $(0)$ & 1 & (8) \\
\hline 210 & 7 & 7 & 0.1 & 0 & (0) & 0 & (0) & 0 & (0) \\
\hline
\end{tabular}

All dogs are dead. A brief summary of results has been published (Boecker, 1984).

Exposure aerosols had an AMAD of 1.5 to $2.4 \mu \mathrm{m}$, with a GSD of 1.6 to 2.1. Quantity of ${ }^{144} \mathrm{Ce}$ deposited and retained was determined by whole-body counting. In addition to the life-span dogs detailed above, 27 dogs were exposed for dosimetric purposes and sacrificed at intervals to 512 days postexposure. Estimated median-survival average radiation dose to liver ranged from about $8900 \mathrm{rad}$ in the lowest dose group to about 19,000 rad in the next to highest dose group (Doc. $L M F-120$ ). For long-survival dogs, cumulative lung dose was about one-half the liver dose; skeleton dose, about one-third the liver dose (Boecker, 1974b; Cuddihy, $1975,1979)$. High local concentrations of ${ }^{144} \mathrm{Ce}$ were noted in the nasal turbinates (Cuddihy, 1976b).

Early deaths, at high exposure levels, were due to bone marrow aplasia, pulmonary, and/or hepatic injury (Benjamin, 1973; Boecker, 1984; McClellan, 1976). Data on the early hematopoietic deaths have been modeled (Scott, 1980) and applied in the prediction of health effects resulting from nuclear disasters (McClellan, 1982). 
Late effects probably related to ${ }^{144} \mathrm{Ce}$ exposure include neoplasia in all tissues receiving high radiation doses (Benjamin, 1973; Boecker, 1984). The highest cancer incidence was in the liver, mostly hemangiosarcomas (Benjamin, 1975); a liver-cancer risk factor of about 90 cancers per $10^{6}$ rad has been estimated (Muggenburg, 1986). Also notable was the substantial incidence of nasal carcinomas (Benjamin, 1979); a risk factor for nasal cavity cancer of about 30 per $10^{6}$ rad has been estimated (Boecker, 1986). Several publications have compared the preliminary results from this experiment with those from experiments with beta emitters of different retention and distribution characteristics (Boecker, 1986; Mc Clellan, 1976, 1979, 1982, 1986).

Information relevant to this experiment will be found in the following Inhalation Toxicology Research Institute periodic reports:

\begin{tabular}{|c|c|c|c|c|c|}
\hline Issue Date & Doc. No. & Page Refs. & Issue Date & Doc. No. & Page Refs. \\
\hline 1966 (Nov) & $L F-33$ & $153-166$ & $1976(\mathrm{Dec})$ & LF-56 & $158-163$ \\
\hline 1967 (Nov) & $L F-38$ & $19-39$ & & & $200-204$ \\
\hline 1968 (Nov) & $L F-39$ & $14-25$ & 1977 (Dec) & LF-58 & $69-73$ \\
\hline \multirow[t]{2}{*}{1969 (Nov) } & $L F-41$ & $8-14$ & 1978 (Dec) & $L F-60$ & $76-79$ \\
\hline & & $59-60$ & 1979 (Dec) & $L F-69$ & $66-70$ \\
\hline 1970 (Nov) & $L F-43$ & $128-136$ & $1980(\mathrm{Dec})$ & LMF-84 & $57-61$ \\
\hline 1971 (Nov) & $L F-44$ & $126-135$ & 1981 (Dec) & LMF-91 & $79-83$ \\
\hline 1972 (Nov) & $L F-45$ & $137-139$ & & & $126-129$ \\
\hline \multirow[t]{2}{*}{1973 (Dec) } & $L F-46$ & $61-66$ & 1982 (Dec) & LMF-102 & $280-283$ \\
\hline & & $91-95$ & 1983 (Dec) & LMF-107 & 194-197 \\
\hline 1974 (Dec) & $L F-49$ & $93-97$ & 1984 (Dec) & LMF-113 & $163-167$ \\
\hline 1975 (Dec) & $L F-52$ & $139-142$ & 1986 (Dec) & LMF-115 & $247-250$ \\
\hline
\end{tabular}


Single inhalation of yttrium chloride in a cesium chloride vector aerosol; dogs 12 to 15 months old, of both sexes, placed on experiment from 1966 to 1967. Results as of 30 September 1987 (Doc. LMF-120).

\begin{tabular}{|c|c|c|c|c|c|c|c|c|c|}
\hline \multirow{2}{*}{\multicolumn{2}{|c|}{$\begin{array}{l}\text { EXPERIMENTAL } \\
\text { DESIGN }\end{array}$}} & \multicolumn{8}{|c|}{ EXPERIMENTAL RESULTS } \\
\hline & & \multirow{4}{*}{$\begin{array}{l}\text { No. of } \\
\text { Animals } \\
\text { Dead }\end{array}$} & \multirow{4}{*}{$\begin{array}{l}\text { Median } \\
\text { Postexp. } \\
\text { Survival } \\
\text { (years) }\end{array}$} & & & & & & \\
\hline Initial & No. of & & & \multicolumn{2}{|c|}{ No. $(\%$ Incidence) } & & & & \\
\hline Burden & Animals & & & \multicolumn{2}{|c|}{ Liver } & \multicolumn{2}{|c|}{ Lung } & \multirow{2}{*}{\multicolumn{2}{|c|}{ Nasal Cavity }} \\
\hline$(\mu \mathrm{Ci} / \mathrm{kg})$ & Exposed & & & Tur & & Tur & & & \\
\hline $\begin{array}{l}\text { None } \\
\text { (Control) }\end{array}$ & 12 & 12 & 12.6 & 1 & (8) & 0 & $(0)$ & 0 & (0) \\
\hline 44 & 12 & 12 & 12.5 & 0 & (0) & 0 & (0) & 0 & (0) \\
\hline 71 & 12 & 12 & 12.2 & 1 & (8) & 1 & (8) & 1 & (8) \\
\hline 180 & 12 & 12 & 8.6 & 0 & (0) & 0 & $(0)$ & 2 & (17) \\
\hline 270 & 6 & 6 & 0.1 & 0 & (0) & 0 & $(0)$ & 0 & (0) \\
\hline
\end{tabular}

All dogs are dead. No comprehensive summary of the total experiment has been published. In addition to the 54 life-span dogs tabulated above, 4 "sacrifice dogs" exposed at the nominal $180 \mu \mathrm{Ci} / \mathrm{kg}$ level were retained for life-span observation; one of these developed a lung tumor.

Estimated median-survival average radiation dose to lung ranged from about $600 \mathrm{rad}$ in the lowest dose group to about $2600 \mathrm{rad}$ in the highest (Doc. LMF-120). For long-survival dogs, skeletal dose was about $85 \%$ of the lung dose; liver dose, about $30 \%$ of the lung dose; dose to nasal turbinates was about 5 times the average skeletal dose (Boecker, 1986).

Several publications have compared the preliminary results from this experiment with those from experiments with beta emitters of different retention and distribution characteristics (McClellan, 1976, 1979); special attention has been given to modeling the early-occurring hematopoietic deaths (McClellan, 1982; Scott, 1980). Late deaths appear to be not markedly influenced by radiation exposure; interest has centered particularly on the incidence of nasal cavity tumors (Benjamin, 1979; Boecker, 1986).

Information relevant to this experiment will be found in the following Inhalation Toxicology Research Institute periodic reports: 


\begin{tabular}{|c|c|c|c|c|c|}
\hline Issue Date & Doc. No. & Page Refs. & Issue Date & Doc. No & Page Refs. \\
\hline 1967 (Nov) & LF-38 & $40-64$ & 1976 (Dec) & LF-56 & $164-166$ \\
\hline 1968 (Nov) & LF-39 & $26-32$ & & & $200-204$ \\
\hline \multirow[t]{2}{*}{1969 (Nov) } & LF-41 & $15-18$ & 1977 (Dec) & LF-58 & $66-68$ \\
\hline & & $59-60$ & $1978(\mathrm{Dec})$ & $L F-60$ & $72-75$ \\
\hline 1970 (Nov) & $L F-43$ & $137-139$ & 1979 (Dec) & LF-69 & $62-65$ \\
\hline 1971 (Nov) & $L F \cdot 44$ & $136-138$ & 1980 (Dec) & LMF-84 & $53-56$ \\
\hline 1972 (Nov) & $L F-45$ & $140-143$ & 1981 (Dec) & LMF-91 & $73-78$ \\
\hline 1973 (Dec) & $L F-46$ & $96-99$ & 1982 (Dec) & LMF-102 & $276-279$ \\
\hline 1974 (Dec) & LF -49 & $98-100$ & 1983 (Dec) & LMF-107 & $190-193$ \\
\hline 1975 (Dec) & LF-52 & $143-145$ & 1984 (Dec) & LMF-113 & 159-162 \\
\hline
\end{tabular}




\subsection{4 $1967{ }^{144} \mathrm{Ce}$}

Single inhalation of cerium adsorbed to an insoluble fused aluminosilicate vector aerosol (FAP); dogs 12 to 14 months old, of both sexes, placed on experiment from 1967 to 1968 (Series A), and from 1969 to 1971 (Series B). Results as of 30 September 1987 (Doc. $L M F-120)$.

\begin{tabular}{|c|c|c|c|c|c|c|c|c|c|c|}
\hline \multirow{2}{*}{\multicolumn{3}{|c|}{$\begin{array}{l}\text { EXPERIMENTAL } \\
\text { DESIGN }\end{array}$}} & \multicolumn{8}{|c|}{ EXPERIMENTAL RESULTS } \\
\hline & & & & Median & №. & $\%$ Incids & & & & \\
\hline $\begin{array}{l}\text { Initial } \\
\text { Burden } \\
\text { (uCi/kg) }\end{array}$ & $\begin{array}{l}\text { Serie } \\
\text { and } N \\
\text { Expos }\end{array}$ & & $\begin{array}{l}\text { No. of } \\
\text { Animals } \\
\text { Dead } \\
\end{array}$ & $\begin{array}{l}\text { Postexp. } \\
\text { Survival } \\
\text { (years) }\end{array}$ & $\begin{array}{l}\text { Lur } \\
\text { Tun }\end{array}$ & & & $\begin{array}{l}\text { obron- } \\
\text { ymph } \\
\text { umor }\end{array}$ & $\begin{array}{l}\text { Bo } \\
\text { Iu }\end{array}$ & \\
\hline None & A & 3 & 3 & 13.0 & 0 & (0) & 0 & $(0)$ & 0 & (0) \\
\hline (Control) & & 12 & 12 & 12.7 & 1 & (8) & 0 & $(0)$ & 0 & (0) \\
\hline 0.015 & B & 12 & 12 & 14.2 & 0 & (0) & 0 & $(0)$ & 0 & (0) \\
\hline 0.069 & B & 12 & 11 & 13.7 & 2 & (18) & 0 & $(0)$ & 0 & (0) \\
\hline 0.34 & B & 12 & 12 & 12.8 & 1 & (8) & 0 & (0) & 0 & (0) \\
\hline 1.4 & $B$ & 12 & 12 & 11.6 & 2 & (17) & 1 & (8) & 0 & (0) \\
\hline 6.1 & $B$ & 12 & 12 & 9.9 & 4 & (33) & 3 & (25) & 0 & (0) \\
\hline 15 & A & 3 & 3 & 6.8 & 0 & (0) & 1 & (33) & 1 & (33) \\
\hline 13 & B & 12 & 12 & 6.7 & 2 & (17) & 3 & (25) & 1 & (8) \\
\hline 27 & A & 3 & 3 & 3.6 & 3 & $(100)$ & 0 & (0) & 0 & (0) \\
\hline 24 & B & 12 & 12 & 4.6 & 5 & (42) & 0 & (0) & 1 & (8) \\
\hline 45 & B & 12 & 12 & 0.8 & 5 & (42) & 0 & (0) & 0 & (0) \\
\hline 66 & A & 3 & 3 & 0.7 & 0 & (0) & 0 & (0) & 0 & (0) \\
\hline 120 & A & 3 & 3 & 0.5 & 0 & (0) & 0 & $(0)$ & 0 & (0) \\
\hline 190 & A & 3 & 3 & 0.5 & 0 & (0) & 0 & (0) & 0 & (0) \\
\hline
\end{tabular}

Series A differs experimentally from Series B only in that aluminosilicate particles were fused prior to, rather than during, exposure. Only one dog survives. In addition to the life-span dogs detailed above, 77 sacrifice dogs were exposed, many of which were retained for substantial time periods, and may provide input to the final analysis of effects.

Life-span dogs were exposed to polydisperse aerosols of FAP (Raabe, 1971) with AMADs of 1.5 to $2.7 \mu \mathrm{m}$ (Cuddihy, 1973b) and GSDs of about 2. The quantity of ${ }^{144} \mathrm{Ce}$ deposited and retained was determined by whole-body counting and by analyses on 24 dogs sacrificed at intervals to 2 years postexposure; an effective 
half-life of about 200 days was observed for ${ }^{144} \mathrm{Ce}$ in lung, with less than $2 \%$ of the initial lung burden translocating to tracheobronchial lymph nodes, less than $5 \%$ to liver, and less than $3 \%$ to skeleton $(\mathrm{Hahn}, 1973 \mathrm{~b})$. Distribution data from the sacrifice dogs have been compared with similar data from immature and aged dogs (Guilmette, 1987b). Lung and tracheobronchial lymph nodes received the highest cumulative radiation doses (Hahn, 1976), the estimated median-survival average radiation dose to lung ranging from about $25 \mathrm{rad}$ in the lowest dose group to more than $100,000 \mathrm{rad}$ in the highest (Doc. LMF-120). The cumulative radiation dose to tracheobronchial lymph nodes was about 3 times that to lung (Hahn, in press b).

At the higher exposure levels dogs showed an early, dose-related lymphopenia (Jones, R., 1976), and at the highest levels died from radiation pneumonitis, with severe damage also evident in the heart and in tracheobronchial lymph nodes (McClellan, 1970). Acute and subacute nonneoplastic effects were studied in a number of supporting sacrifice experiments exploring effects on pulmonary lipid composition, physiology and pathology (Pfleger, 1975), on connective tissue alteration (Pickrell, 1975, 1978), on cardiopulmonary function and histopathology (Mauderly, 1980, 1980b), on lymphocyte function (Benjamin, 1975, 1976, 1978), and on dosimetry and morphologic effects in tracheobronchial lymph nodes (Hahn, 1976). In an ancillary study, early mortality from radiation pneumonitis was substantially reduced by pulmonary lavage, but most of the dogs subsequently died of pulmonary neoplasia (Muggenburg, 1981c). A considerable effort has been made on dose-response modeling of early effects (Raabe, 1979; Scott, $1979,1980,1980 \mathrm{~b}, 1980 \mathrm{c}$ ) and on the application of these data to the prediction of the potential effects in humans of radionuclide releases from nuclear disasters (McClellan, 1982).

Hemangiosarcomas of the lung were observed in surviving dogs at the higher dose levels from 2 to 4 years postexposure (Benjamin, 1975b; Hahn, 1973b, in press; Jones, $R$., 1974); later-occurring lung cancers were carcinomas, primarily bronchioalveolar (Hahn, 1977, 1986, in press). Cancers presumed to arise in tracheobronchial lymph nodes were hemangiosarcomas, mostly observed at 6 years or more postexposure (Hahn, 1980, in press, in press b). Risk factors based on average dose to lung have been estimated as 20 per $10^{6} \mathrm{rad}$ for lung tumors and 27 per $10^{6} \mathrm{rad}$ for lung and lymph node tumors combined (Hahn, 1986). The cancer risk factor based on radiation dose to tracheobronchial lymph nodes was about $40 \%$ of that for lung cancer (Hahn, in press $b$ ). The pattern of pulmonary neoplasia in these dogs has been compared with that observed in other species, including humans (Griffith, 1986). 
Several publications have compared the preliminary results from this experiment with those from experiments with beta emitters of different retention and distribution characteristics (Boecker, 1986; McClellan, 1976, 1979, 1982, 1986).

Information relevant to this experiment will be found in the following Inhalation Toxicology Research Institute periodic reports:

\begin{tabular}{|c|c|c|c|c|c|}
\hline Issue Date & Doc. No. & Page Refs. & Issue Date & Doc. No. & Page Refs. \\
\hline 1967 (Nov) & LF-38 & $92-100$ & 1975 (Dec) & LF-52 & $160-164$ \\
\hline 1968 (Nov) & LF-39 & $33-53$ & & & $182-190$ \\
\hline 1969 (Nov) & LF-41 & $19-35$ & & & $272-274$ \\
\hline \multirow[t]{3}{*}{1970 (Nov) } & LF-43 & $183-187$ & $1976(\mathrm{Dec})$ & LF-56 & $180-185$ \\
\hline & & $197-204$ & 1977 (Dec) & LF-58 & $87-92$ \\
\hline & & $218-223$ & 1978 (Dec) & LF-60 & $94-98$ \\
\hline \multirow[t]{2}{*}{1971 (Nov) } & $L F-44$ & $164-180$ & 1979 (Dec) & LF-69 & $83-91$ \\
\hline & & 193-195 & $1980(\mathrm{Dec})$ & LMF-84 & $76-81$ \\
\hline \multirow[t]{2}{*}{1972 (Nov) } & $L F-45$ & $157-166$ & 1981 (Dec) & LMF-91 & $101-108$ \\
\hline & & $209-221$ & 1982 (Dec) & LMF-102 & $300-305$ \\
\hline \multirow[t]{5}{*}{1973 (Dec) } & LF-46 & $112-114$ & 1983 (Dec) & LMF-107 & $213-219$ \\
\hline & & $155-162$ & 1984 (Dec) & LMF-113 & $182-187$ \\
\hline & & $169-172$ & 1985 (Dec) & LMF-114 & $196-201$ \\
\hline & & $180-186$ & 1986 (Dec) & LMF-115 & $187-192$ \\
\hline & & 219-222 & & & $247-250$ \\
\hline \multirow[t]{2}{*}{1974 (Dec) } & LF-49 & $113-117$ & 1987 (Dec) & LMF-120 & $213-216$ \\
\hline & & $189-202$ & & & \\
\hline
\end{tabular}


Single intravenous injection of cesium chloride solution; dogs 12 to 14 months old, of both sexes, placed on experiment from 1968 to 1969. Results as of 30 September 1987 (Doc. LMF-120).

\begin{tabular}{|c|c|c|c|c|c|c|c|c|c|}
\hline \multirow{2}{*}{\multicolumn{2}{|c|}{$\begin{array}{l}\text { EXPERIMENTAL } \\
\text { DESIGN }\end{array}$}} & \multicolumn{8}{|c|}{ EXPERIMENTAL RESULTS } \\
\hline & & \multirow{4}{*}{$\begin{array}{l}\text { No. of } \\
\text { Animals } \\
\text { Dead }\end{array}$} & \multirow{4}{*}{$\begin{array}{l}\text { Median } \\
\text { Postexp. } \\
\text { Survival } \\
\text { (vears) }\end{array}$} & & & & & & \\
\hline Quantity & No. of & & & \multicolumn{2}{|c|}{ No. $(\%$ Incidence) } & & & & \\
\hline Injected & Animals & & & \multicolumn{2}{|c|}{ Liver } & \multirow{2}{*}{\multicolumn{2}{|c|}{$\begin{array}{l}\text { Lung } \\
\text { Tumor }\end{array}$}} & \multirow{2}{*}{\multicolumn{2}{|c|}{$\begin{array}{l}\text { Nasal Cavity } \\
\text { Tumor }\end{array}$}} \\
\hline$(\mu \mathrm{Ci} / \mathrm{kg})$ & Injected & & & Tun & & & & & \\
\hline $\begin{array}{l}\text { None } \\
\text { (Control) }\end{array}$ & 12 & 12 & 13.7 & 0 & (0) & 1 & (8) & 0 & (0) \\
\hline 970 & 12 & 12 & 12.4 & 3 & (25) & 1 & (8) & 1 & (8) \\
\hline 1400 & 12 & 12 & 12.3 & 1 & (8) & 0 & $(0)$ & 1 & (8) \\
\hline 1900 & 12 & 12 & 11.3 & 0 & (0) & 1 & (8) & 0 & (0) \\
\hline 2800 & 12 & 12 & 6.0 & 0 & (0) & 0 & (0) & 2 & $(17)$ \\
\hline 3900 & 6 & 6 & 0.1 & 0 & (0) & 0 & (0) & 0 & (0) \\
\hline
\end{tabular}

All dogs are dead. No comprehensive summary of the total experiment has been published. Injection rather than inhalation (the usual exposure mode for ITRI studies) was employed because of the greater precision attainable, the smaller risk to laboratory personnel, and because preliminary studies showed that route of administration had little effect on distribution, retention, or cumulative radiation dose (Boecker, 1969).

Retention was measured by whole-body counting. About $15 \%$ of the injected dose was lost with a half-time of less than one day, the balance with a half-time of 32 days. No dose-related effects on retention were noted. Estimated median-survival whole-body radiation dose ranged from about $600 \mathrm{rad}$ in the lowest dose group to about $1900 \mathrm{rad}$ in the next to highest dose group (Doc. $L M F-120$ ). Data on tissue distribution from 11 dogs that died 19 to 81 days after injection indicated absorbed tissue doses that were 0.6 to 1.5 times that calculated as an average whole-body dose (Boecker, 1972).

A detailed account has been published of biological effects occurring during the first year after injection (Redman, 1972). A dose-related depression in lymphocytes, granulocytes and platelets was observed in all groups. Nine deaths occurred between 19 and 33 days after injection, 2 at 77 and 81 days, all attributable to severe bone marrow damage. Data on the early hematopoietic deaths have been modeled (Scott, 1980) and applied in the prediction of health effects that might 
result from a nuclear disaster (McClellan, 1982). Although no comprehensive analysis of late effects has been published, an increased cancer incidence in irradiated animals is apparent, notably in the nasal cavity (Boecker, 1986) and liver (Muggenburg, 1986).

Information relevant to this experiment will be found in the following Inhalation Toxicology Research Institute periodic reports:

\begin{tabular}{|c|c|c|c|c|c|}
\hline Issue Date & Doc. No. & Page Refs. & Issue Date & Dec. Ne. & Page Refs. \\
\hline 1965 (Sep) & LF-28 & $111-127$ & 1976 (Dec) & LF-56 & $167-171$ \\
\hline 1968 (Nov) & LF-39 & $54-75$ & 1977 (Dec) & LF-58 & $74-77$ \\
\hline \multirow[t]{2}{*}{1969 (Nov) } & $L F-41$ & $36-45$ & 1978 (Dec) & LF-60 & $80-83$ \\
\hline & & $59-60$ & 1979 (Dec) & LF-69 & 71.74 \\
\hline 1970 (Nov) & $L F-43$ & $140-145$ & 1980 (Dec) & LMF-84 & $62-66$ \\
\hline 1971 (Nov) & $L F-44$ & $139-144$ & 1981 (Dec) & LMF-91 & $84-89$ \\
\hline 1972 (Nov) & $L F-45$ & $144-146$ & 1982 (Dec) & LMF-102 & $284-288$ \\
\hline 1973 (Dec) & LF-46 & $100-102$ & 1983 (Dec) & LMF-107 & $198-202$ \\
\hline 1974 (Dec) & LF-49 & $101-103$ & 1984 (Dec) & LMF-113 & $168-171$ \\
\hline 1975 (Dec) & LF-52 & $146-149$ & 1985 (Dec) & LMF-114 & $181-184$ \\
\hline
\end{tabular}




\section{$8.5196990 Y$}

Single inhalation of yttrium adsorbed to an insoluble fused aluminosilicate vector aerosol (FAP); dogs 12 to 14 months old, of both sexes, placed on experiment from 1969 to 1971. Results as of 30 September 1987 (Doc. LMF-120).

\begin{tabular}{|c|c|c|c|c|c|c|c|}
\hline \multirow{2}{*}{\multicolumn{2}{|c|}{$\begin{array}{l}\text { EXPERIMENTAL } \\
\text { DESIGN }\end{array}$}} & \multicolumn{6}{|c|}{ EXPERIMENTAL RESULTS } \\
\hline & & & Median & & & & \\
\hline Initial & No. of & No. of & Postexp. & №. $\%$ & (ce) & & \\
\hline Burden & Animals & Animals & Survival & Lung & & Radi & \\
\hline$(\mu \mathrm{Ci} / \mathrm{ka})$ & Exposed & Dead & (years) & Tumor & & Pnel & is Death \\
\hline None & 12 & 12 & 13.0 & 4 & (33) & 0 & (0) \\
\hline 105 & 12 & 12 & 12.5 & 1 & (8) & 0 & (0) \\
\hline 210 & 12 & 12 & 11.7 & 1 & (8) & 0 & (0) \\
\hline 300 & 12 & 12 & 12.1 & 2 & (17) & 0 & (0) \\
\hline 390 & 12 & 12 & 10.3 & 4 & (33) & 0 & (0) \\
\hline 640 & 12 & 12 & 0.6 & 2 & (17) & 9 & (75) \\
\hline 750 & 12 & 12 & 0.3 & 1 & (8) & 12 & $(100)$ \\
\hline 1200 & 4 & 4 & 0.3 & 0 & $(0)$ & 4 & $(100)$ \\
\hline 1500 & 5 & 5 & 0.2 & 0 & (0) & 5 & $(100)$ \\
\hline 1900 & 4 & 4 & 0.2 & 0 & (0) & 4 & $(100)$ \\
\hline 3200 & 4 & 4 & 0.1 & 0 & (0) & 4 & $(100)$ \\
\hline
\end{tabular}

All dogs are dead. In addition to the life-span animals detailed above, five dogs were exposed for autoradiographic study of initial deposition in the respiratory tract (Barnes, 1971); 12 dogs were sacrificed in pairs, at intervals to 12 days postexposure, for dosimetry purposes (Barnes, 1972b); nine dogs were employed in pulmonary function, clinical, and radiographic evaluations of early pulmonary damage (Mauderly, 1973); 10 dogs were sacrificed at intervals to 29 days postexposure to study dose and effects in the gastrointestinal tract (Hahn, 1975).

Life-span dogs were exposed to polydisperse aerosols of FAP (Raabe, 1971) with AMADs of 0.8 to $1.4 \mu \mathrm{m}$ (Cuddihy, 1973b) and GSDs of about 2. Because of the short physical half-life of ${ }^{9} \mathrm{Y}$, only lung, tracheobronchial lymph nodes, and gastrointestinal tract received appreciable radiation exposure. Estimated radiation dose to lung ranged from about $1500 \mathrm{rad}$ in the lowest exposure group to $30,000 \mathrm{rad}$, or more, in the 3 highest exposure groups (Doc. LMF-120). 
Early deaths due to radiation pneumonitis and pulmonary fibrosis have been described (Hobbs, 1972; Slauson, 1976, 1977); a special effort has been made on dose-response modeling for this period (Scott, 1979, 1980, 1980b,1980c) and on the application of these data to the prediction of the potential effects in humans of radionuclide releases from nuclear disasters (McClellan, 1982). Other noted effects include changes in collagen constituents in the lung (Pickrell, 1978), effects on peripheral lymphocytes (Jones, $R ., 1976$ ), and effecsts on lymphocyte function (Benjamin, 1976).

Several publications have compared the preliminary results from this experiment with those from experiments with beta emitters of different retention and distribution characteristics (Hahn, 1983b, in press; McClellan, 1976, 1979, 1986). A preliminary estimate of the risk factor for development of primary lung tumor of about 50 per $10^{6} \mathrm{rad}$, is similar to that estimated for ${ }^{91} \mathrm{Y}$, but substantially higher than that for the longer-lived beta emitters ${ }^{144} \mathrm{Ce}$ and ${ }^{90} \mathrm{Sr}$ (Hahn, 1983b).

Information relevant to this experiment will be found in the following Inhalation Toxicology Research Institute periodic reports:

\begin{tabular}{|c|c|c|c|c|c|}
\hline Issue Date & Doc. No. & Page Refs. & Issue Date & Doc. No. & Page Refs. \\
\hline 1969 (Nov) & $L F-41$ & $46-58$ & $1976(\mathrm{Dec})$ & LF-56 & $172-175$ \\
\hline \multirow[t]{2}{*}{1970 (Nov) } & $L F \cdot 43$ & $146 \cdot 162$ & 1977 (Dec) & LF-58 & $78-82$ \\
\hline & & $197-204$ & 1978 (Dec) & $L F-60$ & $84-88$ \\
\hline \multirow[t]{3}{*}{1971 (Nov) } & $L F-44$ & $145-150$ & & & $122 \cdot 124$ \\
\hline & & $193-195$ & 1979 (Dec) & $L F-69$ & $75-78$ \\
\hline & & $227-236$ & $1980(\mathrm{Dec})$ & LMF-84 & $67-70$ \\
\hline \multirow[t]{2}{*}{1972 (Nov) } & $L F-45$ & $147-150$ & & & $107-110$ \\
\hline & & $216-221$ & 1981 (Dec) & LMF-91 & $90-95$ \\
\hline \multirow[t]{2}{*}{1973 (Dec) } & $L F-46$ & $103-107$ & 1982 (Dec) & LMF-102 & $289-294$ \\
\hline & & $169-172$ & 1983 (Dec) & LMF-107 & $203-207$ \\
\hline \multirow[t]{3}{*}{1974 (Dec) } & $L F-49$ & $104-107$ & 1984 (Dec) & LMF-113 & $172-176$ \\
\hline & & $160-164$ & 1985 (Dec) & LMF-114 & $185-190$ \\
\hline & & $192-196$ & 1986 (Dec) & LMF-115 & $177-181$ \\
\hline \multirow[t]{3}{*}{1975 (Dec) } & LF-52 & $150-153$ & 1987 (Dec) & LMF-120 & $205-208$ \\
\hline & & $187-190$ & & & \\
\hline & & $295-301$ & & & \\
\hline
\end{tabular}




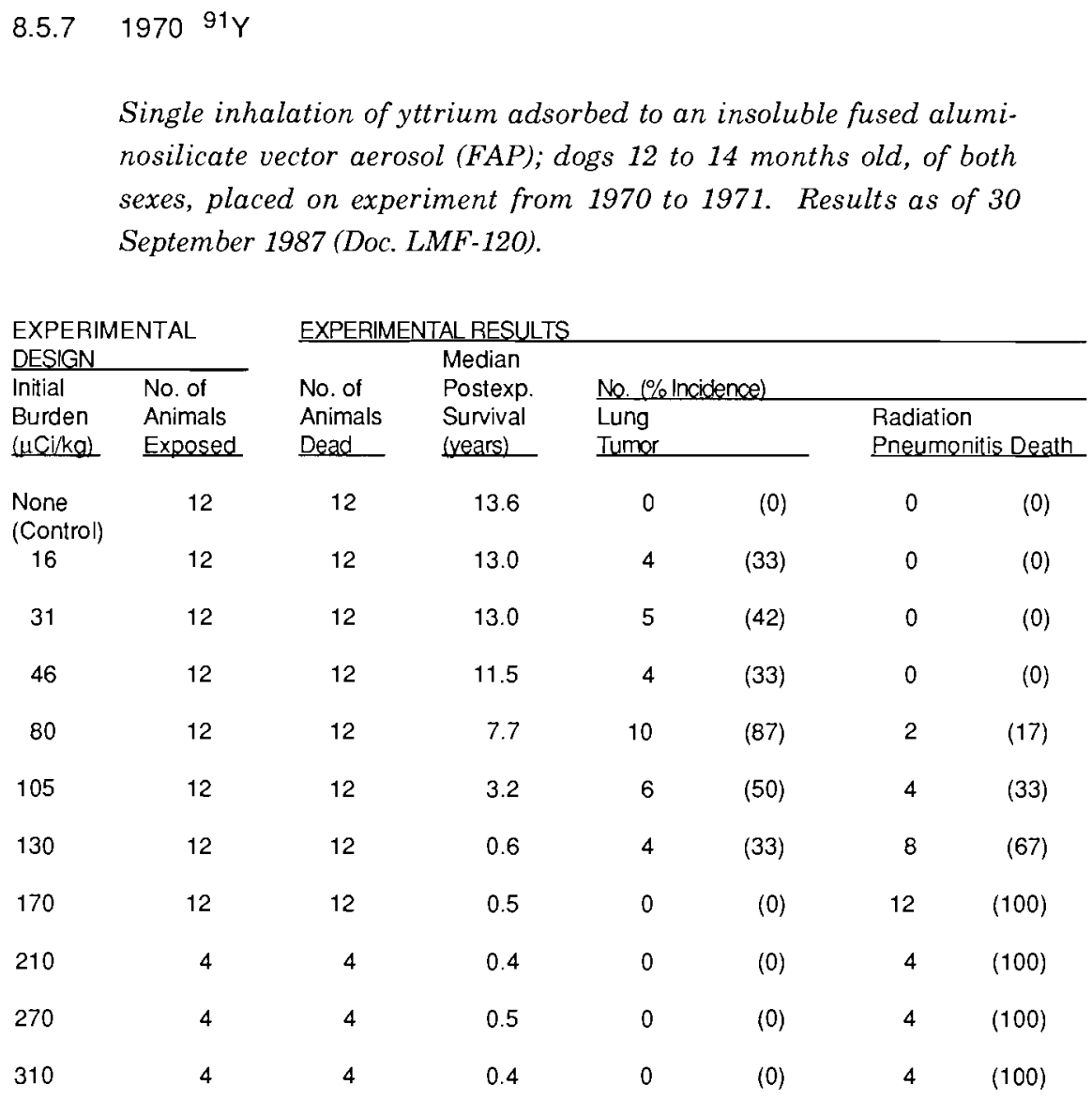

All dogs are dead. No comprehensive summary of the total experiment has been published. Dogs were exposed to polydisperse aerosols of FAP (Raabe, 1971) with AMADs of 1.2 to $2.4 \mu \mathrm{m}$ (Cuddihy, 1973b) and GSDs of about 2. Quantity of radionuclide deposited and retained was determined by whole-body counting. The effective half-time for retention of ${ }^{91} \mathrm{Y}$ in the lung was 50 days. There was substantial translocation of ${ }^{91} \mathrm{Y}$ to tracheobronchial lymph nodes, with a much smaller distribution to bone and liver (McClellan, 1974). Estimated radiation dose to lung ranged from $4000 \mathrm{rad}$ in the lowest exposure group to more than $20,000 \mathrm{rad}$ in groups with initial depositions of $100 \mu \mathrm{Ci} / \mathrm{kg}$ or more (Doc. LMF-120).

Radiation pneumonitis and pulmonary fibrosis were responsible for deaths occurring during the early period following exposure; a special effort has been made on dose-response modeling for this period (Scott, 1979, 1980, 1980b, 1980c) and on the application of these data to the prediction of the potential effects in 
humans of radionuclide releases from nuclear disasters (McClellan, 1982). Specific effects considered in other publications include changes in collagen constituents in the lung (Pickrell, 1978) and effects on peripheral lymphocytes (Jones, R., 1976).

Several publications have compared the carcinogenic effects of ${ }^{91} \mathrm{Y}$ with those from beta emitters of different retention and distribution characteristics (Hahn, 1977, 1986, in press; McClellan, 1976, 1979). No hemangiosarcomas, only pulmonary carcinomas, were seen in ${ }^{91} \mathrm{Y}$-exposed dogs. At 10 years postexposure, the risk factor for development of primary lung tumor was on the order of 50 per $10^{6} \mathrm{rad}$ to lung, which was similar to the risk factor for ${ }^{90} \mathrm{Y}$, but substantially higher than that for the longer-lived beta emitters ${ }^{144} \mathrm{Ce}$ and ${ }^{90} \mathrm{Sr}$ (Hahn, 1983b, 1986). With all animals dead, the lung cancer risk factor calculated from this experiment was compared to risk factors based on incomplete returns from the ${ }^{239} \mathrm{PuO}_{2}$ inhalation experiments; the ${ }^{239} \mathrm{Pu} /{ }^{91} \mathrm{Y}$ ratios for linear risk coefficients were 18,15 , and 10 when compared to $0.75,1.5$, and $3.0 \mu \mathrm{m}{ }^{239} \mathrm{PuO}_{2}$ particles, respectively (Boecker, 1988).

Information relevant to this experiment will be found in the following Inhalation Toxicology Research Institute periodic reports:

\begin{tabular}{|c|c|c|c|c|c|}
\hline Issue Date & Doc. No. & Page Refs. & Issue Date & DOC. No & Page Refs. \\
\hline 1967 (Nov) & LF-38 & $92-100$ & 1976 (Dec) & LF-56 & $176-179$ \\
\hline \multirow[t]{2}{*}{1970 (Nov) } & $L F-43$ & $163-182$ & 1977 (Dec) & $L F-58$ & $83-86$ \\
\hline & & $197-204$ & 1978 (Dec) & $L F-60$ & $89-93$ \\
\hline \multirow[t]{4}{*}{1971 (Nov) } & $L F-44$ & $40-50$ & & & $122-124$ \\
\hline & & $76-80$ & 1979 (Dec) & $L F-69$ & $79-82$ \\
\hline & & $151-163$ & 1980 (Dec) & LMF-84 & $71-75$ \\
\hline & & $193-195$ & 1981 (Dec) & LMF-91 & $96-100$ \\
\hline \multirow[t]{2}{*}{1972 (Nov) } & $L F-45$ & $151-156$ & 1982 (Dec) & LMF-102 & $295-299$ \\
\hline & & $216-221$ & 1983 (Dec) & LMF-107 & $208-212$ \\
\hline 1973 (Dec) & $L F-46$ & $108-111$ & 1984 (Dec) & LMF-113 & $177-181$ \\
\hline 1974 (Dec) & $L F \cdot 49$ & $108-112$ & 1985 (Dec) & LMF-114 & $191-195$ \\
\hline \multirow[t]{2}{*}{1975 (Dec) } & $L F-52$ & $154-159$ & 1986 (Dec) & LMF-115 & $182-186$ \\
\hline & & $187-190$ & 1987 (Dec) & LMF-120 & $209-212$ \\
\hline
\end{tabular}




\subsection{8 $1970^{90} \mathrm{Sr}$}

Single inhalation of strontium adsorbed to an insoluble fused aluminosilicate vector aerosol (FAP); dogs 11 to 15 months old, of both sexes, placed on experiment from 1970 to 1974. Results as of 30 September 1987 (Doc. LMF-120).

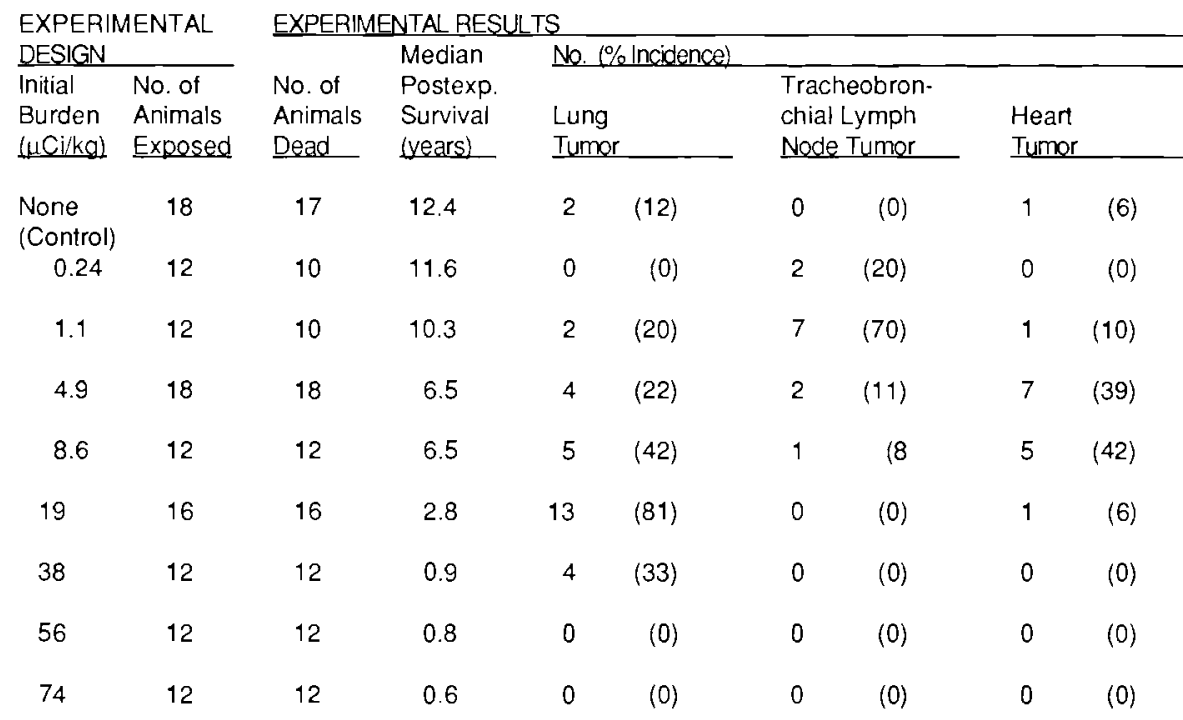

A few dogs remain alive in the control and lowest dose groups. Dogs were exposed to polydisperse aerosols of FAP (Raabe, 1971) with AMADs of 1.5 to $2.8 \mu \mathrm{m}$ (Cuddihy, 1973b) and GSDs of about 2. Quantity of radionuclide deposited and retained was determined by whole-body counting. The effective half-time for retention of ${ }^{90} \mathrm{Sr}$ in the lung was 400 days; there was substantial transfer to tracheobronchial lymph nodes and some transfer to bone (McClellan, 1974). Uniformity of distribution within the lung was examined in a supporting study (Snipes, 1975). Estimated median-survival radiation dose to lung ranged from about $1300 \mathrm{rad}$ in the lowest exposure group to $50,000 \mathrm{rad}$ or more in groups with initial burdens of $19 \mu \mathrm{Ci} / \mathrm{kg}$ or more (Doc. $L M F-120$ ).

Radiation pneumonitis and pulmonary fibrosis were responsible for deaths occurring during the early period following exposure (McClellan, 1974). Specific effects considered in ancillary studies include changes in collagen constituents in the lung (Pickrell, 1978) and effects on peripheral lymphocytes (Jones, $R$., 1976) and lymphocyte function (Benjamin, 1976). A special effort has been made on dose-response modeling for acute effects (Raabe, 1979; Scott, 1979, 1980, 1980b, 
$1980 \mathrm{c}$ ), and on the application of these data to the prediction of the potential effects in humans of radionuclide releases from nuclear disasters (McClellan, 1982; Scott, 1986).

Several publications have compared the carcinogenic effects of ${ }^{90} \mathrm{Sr}$ with those from beta emitters of different retention and distribution characteristics (Hahn, 1977, 1986; McClellan, 1976, 1979). Hemangiosarcomas of the lung were observed in surviving dogs at the higher dose levels from 2 to 4 years postexposure (Benjamin, 1975b; Jones, R., 1974); later-occurring lung cancers were carcinomas, primarily bronchioalveolar ( $H a h n, 1977,19836,1986$, in press). Cancers presumed to arise in tracheobronchial lymph nodes were hemangiosarcomas (Hahn, 1986, in press). The high incidence of heart tumors has been attributed to radiation from external sources, primarily from tracheobronchial lymph nodes ( $H a h n$, in press). Risk factors based on average dose to lung have been estimated as 19 per $10^{6}$ rad for lung tumors, and 22 per $10^{6}$ rad for lung and lymph node tumors combined, which are similar to those estimated for ${ }^{144} \mathrm{Ce}$ but substantially lower than those estimated for the shorter-lived ${ }^{90} \mathrm{Y}$ and ${ }^{91} \mathrm{Y}$ (Hahn, 1986).

Information relevant to this experiment will be found in the following Inhalation Toxicology Research Institute periodic reports:

\begin{tabular}{|c|c|c|c|c|c|}
\hline Issue Date & Dec. No. & Page Refs. & Issue Date & Dec. Ne. & Page Reis. \\
\hline 1970 (Nov) & $L F-43$ & $188-196$ & 1977 (Dec) & $L F-58$ & $102-106$ \\
\hline 1971 (Nov) & $L F-44$ & $181-195$ & 1978 (Dec) & $L F-60$ & $108-112$ \\
\hline \multirow[t]{2}{*}{1972 (Nov) } & $L F-45$ & $177-188$ & & & $122-124$ \\
\hline & & $216-221$ & 1979 (Dec) & $L F-69$ & $101-106$ \\
\hline \multirow[t]{3}{*}{$1973(\mathrm{Dec})$} & $L F-46$ & $43-49$ & $1980(\mathrm{Dec})$ & LMF-84 & $90-94$ \\
\hline & & $128-136$ & $1981(\mathrm{Dec})$ & LMF-91 & $117-121$ \\
\hline & & $169-172$ & 1982 (Dec) & LMF-102 & $314-318$ \\
\hline \multirow[t]{2}{*}{$1974(\mathrm{Dec})$} & $L F-49$ & $126-129$ & 1983 (Dec) & LMF-107 & $228-231$ \\
\hline & & $192-196$ & 1984 (Dec) & LMF-113 & $196-200$ \\
\hline \multirow[t]{2}{*}{1975 (Dec) } & LF-52 & $88-90$ & 1985 (Dec) & LMF-114 & $207-211$ \\
\hline & & $173-177$ & 1986 (Dec) & LMF-115 & $198-203$ \\
\hline 1976 (Dec) & LF-56 & 195-199 & 1987 (Dec) & LMF-120 & $222-227$ \\
\hline
\end{tabular}


Single inhalation of cerium adsorbed to an insoluble fused aluminosilicate vector aerosol (FAP); dogs 3 months old, of both sexes, placed on experiment from 1972 to 1976. Results as of 30 September 1987 (Doc. LMF-120).

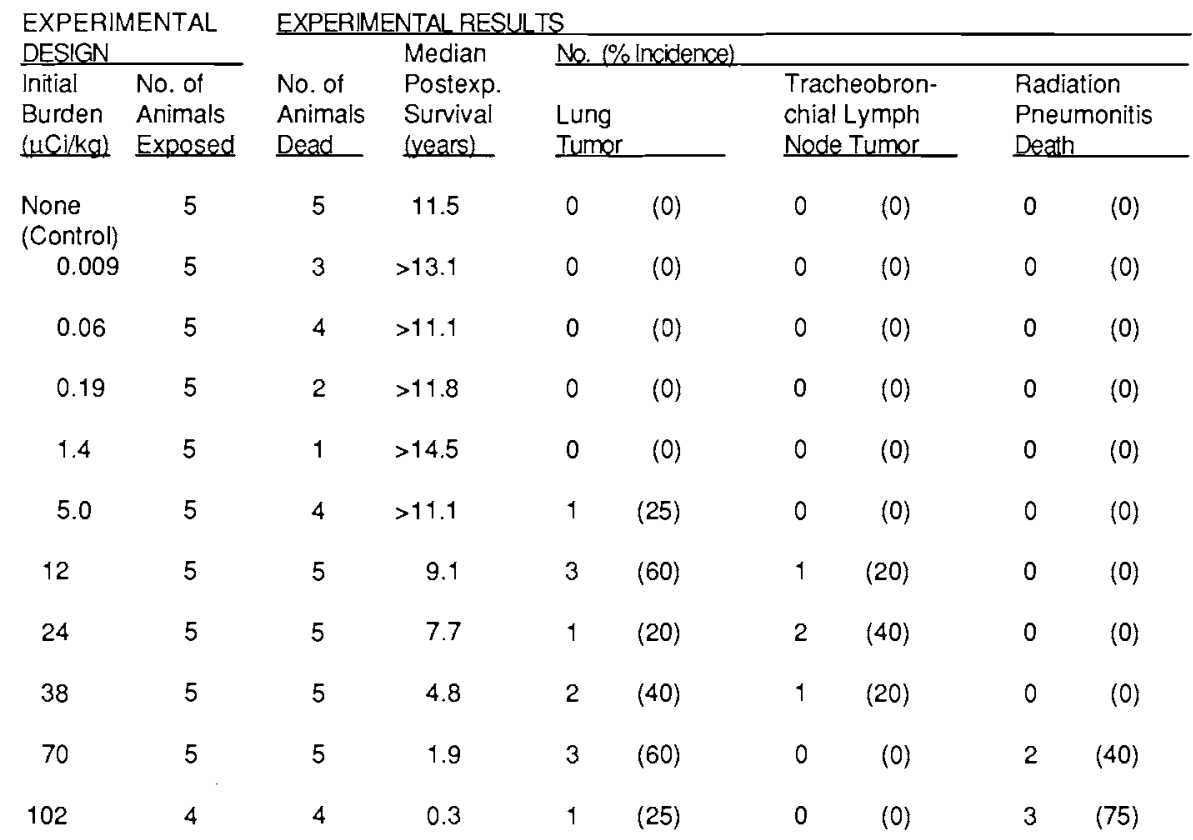

Detailed descriptions of this experiment appear only in the report literature (Doc. LMF-114). Protocols employed were similar to those of the young-adult experiment. Early distribution data from sacrifice dogs have been compared with similar data from young-adult and aged dogs, the immature animals showing a more rapid early clearance from the lung and a larger fraction deposited in the skeleton (Guilmette, 1987b).

Information relevant to this experiment will be found in the following Inhalation Toxicology Research Institute periodic reports:

\begin{tabular}{|c|c|c|c|c|c|}
\hline Issue Date & Dec. No. & Page Rets. & Issue Date & Dec. No. & Page Refs. \\
\hline \multirow[t]{2}{*}{1972 (Nov) } & LF-45 & $167-171$ & 1973 (Dec) & LF-46 & $116-121$ \\
\hline & & $195-205$ & $1974(\mathrm{Dec})$ & LF-49 & $118-121$ \\
\hline
\end{tabular}




\begin{tabular}{|c|c|c|c|c|c|}
\hline Issue Date & Doc. №. & Page Refs. & Issue Date & Doc. No. & Page Refs \\
\hline 1975 (Dec) & LF-52 & $165-168$ & $1982(\mathrm{Dec})$ & LMF-102 & $306-309$ \\
\hline 1976 (Dec) & LF-56 & $186-189$ & $1983(\mathrm{Dec})$ & LMF-107 & $220-223$ \\
\hline 1977 (Dec) & LF-58 & $93-96$ & $1984(\mathrm{Dec})$ & LMF-113 & $188-192$ \\
\hline $1978(\mathrm{Dec})$ & $L F-60$ & $99-102$ & 1985 (Dec) & LMF-114 & $202-206$ \\
\hline 1979 (Dec) & LF-69 & $92-95$ & 1986 (Dec) & LMF-115 & $193-197$ \\
\hline $1980(\mathrm{Dec})$ & LMF-84 & $82-85$ & 1987 (Dec) & LMF-120 & $154-161$ \\
\hline 1981 (Dec) & LMF-91 & $109-112$ & & & $217-221$ \\
\hline
\end{tabular}


Single inhalation of cerium adsorbed to an insoluble fused aluminosilicate vector aerosol (FAP); dogs 8 to 10 years old, of both sexes, placed on experiment from 1972 to 1975 . Results as of 30 September 1987 (Doc. LMF-120).

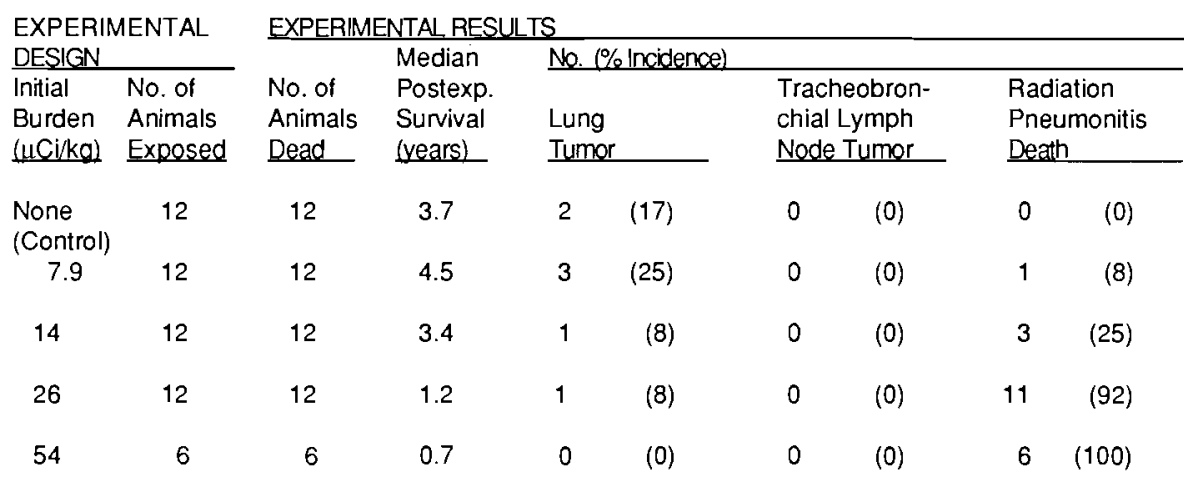

All dogs are dead. A brief summary of the experiment appears only in the report literature (Doc. LMF-113). Protocols employed were similar to those of the young-adult study. Distribution data from life-span dogs have been compared with similar data from sacrifice studies with young-adult and immature dogs; the aged dogs show a smaller fraction deposited in the skeleton (Guilmette, $1987 \mathrm{~b}$ ). Estimated median-survival radiation dose to lung ranged from about $12,000 \mathrm{rad}$ at the lowest exposure level to nearly 50,000 rad at the highest ( $D o c$. LMF-120).

Effects in aged dogs differ from those in young adults in that no pulmonary hemangiosarcomas, but only carcinomas, are seen in the aged animals. Changes in collagen constituents of dogs dying of pulmonary fibrosis in this experiment have been compared with similar effects in young-adult dogs (Pickrell, 1978).

Information relevant to this experiment will be found in the following Inhalation Toxicology Research Institute periodic reports:

\begin{tabular}{|c|c|c|c|c|c|}
\hline Issue Date & Doc. No & Page Refs. & Issue Date & Dec. No & Page Refs. \\
\hline \multirow[t]{2}{*}{1972 (Nov) } & $L F-45$ & $172-176$ & 1974 (Dec) & LF-49 & $122-125$ \\
\hline & & $195-205$ & 1975 (Dec) & $L F-52$ & $169-172$ \\
\hline 1973 (Dec) & $L F-46$ & $122-127$ & 1976 (Dec) & $L F-56$ & $190-194$ \\
\hline
\end{tabular}




\begin{tabular}{|c|c|c|c|c|c|}
\hline Issue Date & Doc No & Page Refs & Issue Date & Doc. No. & Page Refs \\
\hline 1977 (Dec) & LF-58 & $97-101$ & 1982 (Dec) & LMF-102 & $310-313$ \\
\hline 1978 (Dec) & LF-60 & 103-107 & 1983 (Dec) & LMF-107 & $224-227$ \\
\hline 1979 (Dec) & LF 69 & $96-100$ & 1984 (Dec) & LMF-113 & $154-161$ \\
\hline 1980 (Dec) & LMF-84 & $86-89$ & & & 193-195 \\
\hline 1981 (Dec) & LMF-91 & $113-116$ & & & \\
\hline
\end{tabular}


Thirteen inhalations, at 56-day intervals, of cerium adsorbed to an insoluble fused aluminosilicate vector aerosol (FAP); dogs of both sexes, 14 to 17 months old at initial exposure, placed on experiment from 1972 to 1975. Results as of 30 September 1987 (Doc. LMF-120).

\begin{tabular}{|c|c|c|c|c|c|c|c|c|c|}
\hline EXPER & NTAL & EXPERIM & NTAL RES & & & & & & \\
\hline DESIGN & & & Median & & Incidence) & & & & \\
\hline $\begin{array}{l}\text { Initial } \\
\text { Burden } \\
(\mu \mathrm{Ci} / \mathrm{kg})\end{array}$ & $\begin{array}{l}\text { No. of } \\
\text { Animals } \\
\text { Exposed }\end{array}$ & $\begin{array}{l}\text { No. of } \\
\text { Animals } \\
\text { Dead }\end{array}$ & $\begin{array}{l}\text { Postexp. } \\
\text { Survival } \\
\text { (years) }\end{array}$ & $\begin{array}{l}\text { Lur } \\
\text { Tur }\end{array}$ & & & $\begin{array}{l}\text { Eobron- } \\
\text { ymph } \\
\text { Tumor }\end{array}$ & & ion \\
\hline $\begin{array}{l}\text { None } \\
\text { (Control) }\end{array}$ & 9 & 7 & 11.8 & 0 & (0) & 0 & (0) & 0 & (0) \\
\hline $2.5^{*}$ & 9 & 9 & 6.3 & 3 & (33) & 0 & (0) & 2 & (22) \\
\hline $4.5^{* *}$ & 9 & 9 & 6.2 & 1 & (11) & 2 & (22) & 1 & (11) \\
\hline $9.0^{* *}$ & 9 & 9 & 6.5 & 7 & (78) & 3 & (33) & 2 & (22) \\
\hline
\end{tabular}

All ${ }^{144} \mathrm{Ce}$-exposed dogs are dead, the last dying in 1985; 2 control dogs survive. Only a brief description of the experiment, with early results, has been published (Boecker, 1980). The 13 exposures at the $2.5-\mu \mathrm{Ci} / \mathrm{kg}$ level were designed to produce a 2-year cumulative dose to lung of $35,000 \mathrm{rad}$; the same 2-year cumulative dose was projected for the regimen re-establishing a lung burden of $9.0 \mu \mathrm{Ci} / \mathrm{kg}$. An approximately 3 -fold range of cumulative dose was observed in each exposure group, as evaluated by total-body counting. Only 1 hemangiosarcoma of the lung was observed, in sharp contrast to observations in single-exposure studies. The other 10 lung carcinomas appeared later than the carcinomas observed in single-exposure studies. Lymph node tumors were hemangiosarcomas.

Information relevant to this experiment will be found in the following Inhalation Toxicology Research Institute periodic reports:

\begin{tabular}{|c|c|c|c|c|c|}
\hline Issue Date & Doc. No. & Page Refs. & Issue Date & Doc. No. & Page Refs. \\
\hline 1974 (Dec) & $L F-49$ & $314-317$ & $1980(\mathrm{Dec})$ & LMF-84 & $99-103$ \\
\hline 1975 (Dec) & LF-52 & $226-229$ & 1981 (Dec) & LMF-91 & $134-137$ \\
\hline 1976 (Dec) & LF-56 & $278-282$ & $1983(\mathrm{Dec})$ & LMF-107 & $232-236$ \\
\hline 1977 (Dec) & LF-58 & $186-188$ & 1984 (Dec) & LMF-113 & 201-204 \\
\hline 1978 (Dec) & $L F-60$ & $180-183$ & 1985 (Dec) & LMF-114 & $212-215$ \\
\hline 1979 (Dec) & $L F-69$ & $182-186$ & 1986 (Dec) & LMF-115 & 204-207 \\
\hline
\end{tabular}




\subsubsection{2 $1973^{238} \mathrm{Pu}$}

Single inhalation of monodisperse plutonium oxide aerosol, AMAD $3.0 \mu \mathrm{m}$; dogs 12 to 14 months old, of both sexes, placed on experiment from 1973 to 1976. Results as of 30 September 1987 (Doc. LMF-120).

\begin{tabular}{|c|c|c|c|c|c|c|c|c|c|}
\hline EXPERIN & MENTAL & EXPERIN & NTAL RES & & & & & & \\
\hline DESIGN & & & Median & & & & & & \\
\hline Initial & No. of & No. of & Postexp. & No. & Incidence) & & & & \\
\hline $\begin{array}{l}\text { Burden } \\
(\mu \mathrm{Ci} / \mathrm{kg})\end{array}$ & $\begin{array}{l}\text { Animals } \\
\text { Exposed }\end{array}$ & $\begin{array}{l}\text { Animals } \\
\text { Dead }\end{array}$ & $\begin{array}{l}\text { Survival } \\
\text { (vears) }\end{array}$ & $\begin{array}{l}\text { Bo } \\
\text { Iur }\end{array}$ & & $\begin{array}{l}\text { Lu } \\
\text { Tur }\end{array}$ & & $\begin{array}{l}\text { Liv } \\
\text { IuI }\end{array}$ & \\
\hline $\begin{array}{l}\text { None } \\
\text { (Control) }\end{array}$ & 12 & 9 & 12.5 & 0 & (0) & 0 & (0) & 0 & $(0)$ \\
\hline 0.02 & 12 & 8 & 11.7 & 1 & (12) & 2 & (25) & 0 & $(0)$ \\
\hline 0.04 & 12 & 12 & 10.1 & 7 & $(58)$ & 4 & (33) & 0 & $(0)$ \\
\hline 0.07 & 12 & 12 & 9.9 & 7 & $(58)$ & 4 & (33) & 0 & $(0)$ \\
\hline 0.17 & 12 & 12 & 5.4 & 11 & (92) & 4 & (33) & 0 & $(0)$ \\
\hline 0.39 & 12 & 12 & 4.3 & 11 & (92) & 3 & $(25)$ & 0 & $(0)$ \\
\hline 0.77 & 12 & 12 & 3.5 & 7 & $(58)$ & 5 & (42) & 0 & $(0)$ \\
\hline
\end{tabular}

The 5 single-inhalation young-adult experiments with inhaled monodisperse plutonium oxide aerosols were designed, as a group, to explore the influence of alpha-particle dose distribution within the lung (McClellan, 1972, 1986). New techniques were developed to produce the monodisperse particles (Raabe, 1975; Talley, 1979). The 3.0- $\mathrm{m}$-AMAD particles had an actual diameter of about $1 \mu \mathrm{m}$ (Muggenburg, 1980).

Initial deposition was estimated from whole-body counting of gamma emissions from ${ }^{169} \mathrm{Yb}$ incorporated into the $\mathrm{PuO}_{2}$ particles as a short-lived tracer; retention and translocation were studied in supporting experiments involving the sacrifice of animals at intervals to 4 years postexposure (Hahn, 1981; Mewhinney, 1983). Translocation from the lung was much more rapid than that of ${ }^{239} \mathrm{PuO}_{2}$ and was a complex function of time, influenced by radiolytic fragmentation of the high-specific-activity ${ }^{238} \mathrm{PuO}_{2}$ particles (Diel, 1983). Estimated cumulative radiation doses from inhaled $3.0-\mu \mathrm{m}{ }^{238} \mathrm{PuO}_{2}$, at 4 years postexposure, to skeleton, liver, lung, and tracheobronchial lymph nodes, were in the ratio of 1:2:5:50 (Mewhinney, 1983). Estimated median-survival average radiation dose to bone ranged from about $100 \mathrm{rad}$ in the lowest exposure group to about $900 \mathrm{rad}$ in the highest (Doc. LMF-120). 
Particle size in the two ${ }^{238} \mathrm{PuO}_{2}$ experiments ( 3.0 or $1.5 \mu \mathrm{m}$ ) has not significantly influenced retention, translocation or biological effects (Hahn, 1981). The earliest observed biological effect was a lymphopenia, which occurred at the higher dose levels within 180 days; a $60 \%$ incidence of leukopenia was also noted at higher dose levels (Muggenburg, 1980). In addition to early deaths from radiation pneumonitis, other observed nonneoplastic effects included atrophy of the tracheobronchial lymph nodes and maxilloturbinates and hepatic degeneration (Hahn, 1981).

Lung and bone tumors have been observed and described in some detail (Hahn, 1981; Wolff, 1980), and risk factors have been estimated in several interpretations of partial results (Hahn, 1986; Mewhinney, 1986; Muggenburg, 1983; Raabe, 1984, 1986). Liver tumors have been described as incidental findings at death (Gillett, in press).

Information relevant to this experiment will be found in the following Inhalation Toxicology Research Institute periodic reports:

\begin{tabular}{|c|c|c|c|c|c|}
\hline Issue Date & Doc. No. & Page Refs. & Issue Date & Doc. No. & Page Refs. \\
\hline 1971 (Nov) & LF-44 & $7-19$ & 1979 (Dec) & $L F-69$ & $9-13$ \\
\hline \multirow[t]{2}{*}{$1973(\mathrm{Dec})$} & $L F-46$ & $1-30$ & & & $117-133$ \\
\hline & & $78-80$ & 1980 (Dec) & LMF-84 & $17-24$ \\
\hline \multirow[t]{2}{*}{$1974(\mathrm{Dec})$} & $L F-49$ & $37-42$ & & & $113-131$ \\
\hline & & $140-144$ & 1981 (Dec) & LMF-91 & $40-45$ \\
\hline \multirow[t]{2}{*}{1975 (Dec) } & $L F-52$ & $21-23$ & & & $145-158$ \\
\hline & & $193-203$ & 1982 (Dec) & LMF-102 & $322-335$ \\
\hline \multirow{2}{*}{1976 (Dec) } & $L F-56$ & $96-98$ & 1983 (Dec) & LMF-107 & $237-251$ \\
\hline & & $223-237$ & $1984(\mathrm{Dec})$ & $L M F-113$ & $210-224$ \\
\hline \multirow[t]{3}{*}{1977 (Dec) } & $L F-58$ & $40-43$ & 1985 (Dec) & $L M F-114$ & $220-235$ \\
\hline & & $117-134$ & 1986 (Dec) & LMF-115 & $208-225$ \\
\hline & & $139-143$ & 1987 (Dec) & LMF-120 & $228-247$ \\
\hline \multirow[t]{4}{*}{1978 (Dec) } & $L F-60$ & $16-20$ & & & $284-290$ \\
\hline & & $28-37$ & & & \\
\hline & & $127-144$ & & & \\
\hline & & $151-155$ & & & \\
\hline
\end{tabular}




\begin{abstract}
8.5.13 $1974^{238} \mathrm{Pu}$
Single inhalation of monodisperse plutonium oxide aerosol, AMAD $1.5 \mu \mathrm{m}$; dogs 12 to 15 months old, of both sexes, placed on experiment from 1974 to 1976. Results as of 30 September 1987 (Doc. LMF-120).
\end{abstract}

\begin{tabular}{|c|c|c|c|c|c|c|c|c|c|}
\hline \multirow{2}{*}{\multicolumn{2}{|c|}{$\begin{array}{l}\text { EXPERIMENTAL } \\
\text { DESIGN }\end{array}$}} & \multicolumn{8}{|c|}{ EXPERIMENTAL, RESULTS } \\
\hline & & \multirow{3}{*}{$\begin{array}{l}\text { No. of } \\
\text { Animals } \\
\text { Dead }\end{array}$} & \multirow{3}{*}{$\begin{array}{l}\text { Median } \\
\text { Postexp. } \\
\text { Survival } \\
\text { (years) }\end{array}$} & \multirow{2}{*}{\multicolumn{2}{|c|}{ No. (\% Incidence) }} & & & & \\
\hline Initial & No. of & & & & & & & & \\
\hline Burden & Animals & & & \multicolumn{2}{|c|}{$\begin{array}{l}\text { Bone } \\
\text { Tumor }\end{array}$} & \multicolumn{2}{|c|}{$\begin{array}{l}\text { Lung } \\
\text { Tumor }\end{array}$} & \multicolumn{2}{|c|}{$\begin{array}{l}\text { Liver } \\
\text { Tumor }\end{array}$} \\
\hline $\begin{array}{l}\text { None } \\
\text { (Control) }\end{array}$ & 12 & 7 & $>12.2$ & 0 & (0) & 2 & (29) & 0 & (0) \\
\hline 0.01 & 12 & 8 & $>11.8$ & 3 & $(38)$ & 1 & (12) & 0 & (0) \\
\hline 0.04 & 12 & 9 & 10.0 & 6 & $(67)$ & 1 & (11) & 3 & (33) \\
\hline 0.07 & 12 & 12 & 8.3 & 9 & $(75)$ & 2 & $(17)$ & 1 & (8) \\
\hline 0.16 & 12 & 12 & 5.7 & 10 & (83) & 2 & (17) & 1 & (8) \\
\hline 0.28 & 12 & 12 & 4.3 & 11 & (92) & 1 & (8) & 0 & (0) \\
\hline 0.68 & 12 & 12 & 3.6 & 6 & $(50)$ & 5 & $(42)$ & 0 & (0) \\
\hline
\end{tabular}

The 5 single-inhalation young-adult experiments with inhaled monodisperse plutonium oxide aerosols were designed, as a group, to explore the influence of alpha-particle dose distribution within the lung (McClellan, 1972, 1986). New techniques were developed to produce the monodisperse particles (Raabe, 1975; Talley, 1979). The 1.5- $\mu \mathrm{m}$-AMAD particles had an actual diameter of $0.44 \mu \mathrm{m}$ (Muggenburg, 1980).

Initial deposition was estimated from whole-body counting of gamma emissions from ${ }^{169} \mathrm{Yb}$ incorporated into the $\mathrm{PuO}_{2}$ particles as a short-lived tracer; retention and translocation were studied in supporting experiments involving the sacrifice of exposed animals at intervals to 4 years postexposure (Hahn, 1981; Mewhinney, 1983). Translocation from the lung was much more rapid than that of ${ }^{239} \mathrm{PuO}_{2}$, and was a complex function of time, influenced by radiolytic fragmentation of the high-specific-activity ${ }^{238} \mathrm{PuO}_{2}$ particles (Diel, 1983). Estimated cumulative radiation doses from inhaled $1.5-\mu \mathrm{m}{ }^{238} \mathrm{PuO}_{2}$, at 4 years postexposure, to skeleton, liver, lung, and tracheobronchial lymph nodes, were in the ratio of 1:2:6:200 (Mewhinney, 1983). Estimated median-survival average radiation dose to bone ranged from about $50 \mathrm{rad}$ in the lowest exposure group to nearly $600 \mathrm{rad}$ in the highest ( Doc. $L M F-120$ ). 
The microdistribution of inhaled $1.5-\mu \mathrm{m}{ }^{238} \mathrm{PuO}_{2}$ translocated to liver was studied in supporting experiments, with comparisons to intravenously injected ${ }^{238} \mathrm{Pu}$ and ${ }^{239} \mathrm{Pu}$ (Gearhart, 1980). This distribution was a function of exposure level and time postexposure; for high initial lung burdens there was evidence of particle transfer to liver. Particle size in the two ${ }^{238} \mathrm{PuO}_{2}$ experiments ( 3.0 or 1.5 $\mu \mathrm{m})$ has not appeared to significantly influence retention, translocation or biological effects (Hahn, 1981).

The earliest observed biological effect was a lymphopenia, which occurred at the higher dose levels within 180 days; a $60 \%$ incidence of leukopenia was also noted at higher dose levels (Muggenburg, 1980). Data on pulmonary function were obtained from dogs exposed to $1.5-\mu \mathrm{m}{ }^{238} \mathrm{PuO}_{2}$ who died with radiation pneumonitis. These data were compared with similar data from dogs exposed to ${ }^{144} \mathrm{Ce}$ (Mauderly, 1980). In addition to early deaths from radiation pneumonitis, other observed nonneoplastic effects included atrophy of the tracheobronchial lymph nodes and maxilloturbinates, and hepatic degeneration (Hahn, 1981).

Lung and bone tumors have been observed and described in some detail (Hahn, 1981; Wolff, 1980); risk factors have been estimated in several interpretations of partial results (Hahn, 1986; Mewhinney, 1986; Muggenburg, 1983; Raabe, 1984, 1986). Liver tumors have been described, usually as incidental findings at death (Gillett, in press).

Information relevant to this experiment will be found in the following Inhalation Toxicology Research Institute periodic reports:

\begin{tabular}{|c|c|c|c|c|c|}
\hline Issue Date & Doc. No. & Page Refs. & Issue Date & Doc. No. & Page Refs. \\
\hline 1971 (Nov) & LF-44 & $7-19$ & 1978 (Dec) & LF-60 & $16-20$ \\
\hline \multirow[t]{2}{*}{1973 (Dec) } & LF-46 & $1-30$ & & & $28-37$ \\
\hline & & $78-80$ & & & $127-144$ \\
\hline \multirow[t]{2}{*}{1974 (Dec) } & LF-49 & $37-42$ & & & $151-155$ \\
\hline & & $140-144$ & 1979 (Dec) & LF-69 & $9-13$ \\
\hline \multirow[t]{2}{*}{1975 (Dec) } & LF-52 & $21-23$ & & & $117-133$ \\
\hline & & $193-203$ & 1980 (Dec) & LMF-84 & $17-24$ \\
\hline \multirow[t]{2}{*}{1976 (Dec) } & $L F-56$ & $96-98$ & & & 113-131 \\
\hline & & $223-237$ & 1981 (Dec) & LMF-91 & $40-45$ \\
\hline \multirow[t]{3}{*}{1977 (Dec) } & LF-58 & $40-43$ & & & $145-158$ \\
\hline & & $117-134$ & 1982 (Dec) & LMF-102 & $322-335$ \\
\hline & & $139-143$ & $1983(\mathrm{Dec})$ & LMF-107 & $237-251$ \\
\hline
\end{tabular}




$\begin{array}{lllllll}\text { Issue Date } & \text { Doc. No. } & \text { Page Refs. } & \text { Issue Date } & & \text { Doc. No. } & \text { Page Refs. } \\ 1984 \text { (Dec) } & \text { LMF-113 } & 210-224 & 1987 \text { (Dec) } & \text { LMF-120 } & 228-247 \\ 1985 \text { (Dec) } & \text { LMF-114 } & 220-235 & & & 284-290 \\ 1986 \text { (Dec) } & \text { LMF-115 } & 208-225 & & & \end{array}$




\begin{abstract}
8.5.14 $1977^{239} \mathrm{Pu}$
Single inhalation of monodisperse plutonium oxide aerosol, AMAD $0.75 \mu \mathrm{m}$; dogs 12 to 15 months old, of both sexes, placed on experiment from 1977 to 1979. Results as of 30 September 1987 (Doc. LMF-120).
\end{abstract}

\begin{tabular}{|c|c|c|c|c|c|c|c|c|c|}
\hline \multirow{2}{*}{\multicolumn{2}{|c|}{$\begin{array}{l}\text { EXPERIMENTAL } \\
\text { DESIGN }\end{array}$}} & \multicolumn{8}{|c|}{ EXPERIMENTAL RESULTS } \\
\hline & & & Median & & & & & & \\
\hline Initial & No. of & No. of & Postexp. & \multicolumn{6}{|c|}{ No. $(\%$ Incidence) } \\
\hline Burden & Animals & Animals & Survival & \multicolumn{2}{|c|}{ Bone } & \multirow{2}{*}{\multicolumn{2}{|c|}{$\begin{array}{l}\text { Lung } \\
\text { Tumor }\end{array}$}} & \multirow{2}{*}{\multicolumn{2}{|c|}{$\begin{array}{l}\text { Liver } \\
\text { Iumor }\end{array}$}} \\
\hline$(\mu \mathrm{Ci} / \mathrm{kg})$ & Exposed & Dead & (vears) & Tun & & & & & \\
\hline $\begin{array}{l}\text { None } \\
\text { (Control) }\end{array}$ & 12 & 2 & $>9.3$ & 0 & (0) & 0 & $(0)$ & 0 & $(0)$ \\
\hline 0.014 & 12 & 3 & $>10.1$ & 0 & $(0)$ & 2 & $(67)$ & 0 & $(0)$ \\
\hline 0.041 & 12 & 9 & 8.8 & 0 & $(0)$ & 7 & (78) & 0 & $(0)$ \\
\hline 0.062 & 12 & 12 & 5.8 & 0 & $(0)$ & 11 & $(92)$ & 0 & $(0)$ \\
\hline 0.16 & 12 & 12 & 4.1 & 0 & (0) & 7 & $(58)$ & 0 & $(0)$ \\
\hline
\end{tabular}

The 5 single-inhalation young-adult experiments with inhaled monodisperse plutonium oxide aerosols were designed, as a group, to explore the influence of alpha-particle dose distribution within the lung (McClellan, 1972, 1986). New techniques were developed to produce the monodisperse particles (Raabe, 1975, Talley, 1979). The $0.75-\mu \mathrm{m}-\mathrm{AMAD}$ particles had an actual diameter of about 0.18 $\mu \mathrm{m}$ (Guilmette, 1984b).

Initial deposition was estimated from whole-body counting of gamma emissions from ${ }^{169} \mathrm{Yb}$ incorporated into the $\mathrm{PuO}_{2}$ particles as a short-lived tracer; retention and translocation were studied in supporting experiments involving the sacrifice of animals at intervals to 2 years postexposure (Guilmette, 1984). In these studies a half-time of 680 days was measured for retention of $90 \%$ of $0.72-\mu \mathrm{m}$ ${ }^{239} \mathrm{PuO}_{2}$ particles deposited in the lung; a value much longer than that observed for ${ }^{238} \mathrm{PuO}_{2}$, about one-half that observed for $1.4-\mu \mathrm{m}{ }^{239} \mathrm{PuO}_{2}$ particles, and about one-third that observed for $2.8-\mu \mathrm{m}{ }^{239} \mathrm{PuO}_{2}$ particles. For all particle sizes of ${ }^{239} \mathrm{PuO}_{2}$, the major route of elimination was via feces, with about $15 \%$ of the initial lung burden translocated to pulmonary lymph nodes, about $0.2 \%$ to liver, and about $0.1 \%$ to skeleton by 2 years postexposure.

Effects thus far observed show no clear dependence on particle size. The earliest observed biological effect was a lymphopenia, which occurred at the higher dose levels within 180 days; no depression of neutrophil counts was observed, in contrast to findings with $238 \mathrm{PuO}_{2}$ (Guilmette, 1984b). The primary cause of 
death has been radiation pneumonitis, still appearing at 8 years postexposure; the numerous lung tumors are usually incidental findings at autopsy (Diel, 1983b; Guilmette, 1984b; Hahn, 1986; Scott, 1986). Preliminary analyses of incomplete data on lung tumor incidence suggest risk factors for the $0.75-\mu \mathrm{m}$ particles that are not significantly different from those for $1.5-\mu \mathrm{m}$ or $3.0-\mu \mathrm{m}$ particles (Boecker, 1988; Muggenburg, in press).

Information relevant to this experiment will be found in the following Inhalation Toxicology Research Institute periodic reports:

\begin{tabular}{|c|c|c|c|c|c|}
\hline Issue Date & Doc. No. & Page Refs. & Issue Date & Doc. No & Page Refs. \\
\hline 1971 (Nov) & $L F-44$ & $7-19$ & 1982 (Dec) & LMF-102 & $336-343$ \\
\hline 1973 (Dec) & LF-46 & $1-30$ & $1983(\mathrm{Dec})$ & LMF-107 & $144-148$ \\
\hline 1975 (Dec) & LF-52 & $21-23$ & & & $252-259$ \\
\hline 1977 (Dec) & LF-58 & $135-138$ & & & $292-297$ \\
\hline \multirow[t]{2}{*}{$1978(\mathrm{Dec})$} & $L F-60$ & $34-37$ & 1984 (Dec) & LMF-113 & $89-101$ \\
\hline & & $145-150$ & & & $225-232$ \\
\hline \multirow[t]{2}{*}{1979 (Dec) } & LF-69 & $14-17$ & 1985 (Dec) & LMF-114 & $236-243$ \\
\hline & & $134-140$ & $1986(\mathrm{Dec})$ & LMF-115 & $226-232$ \\
\hline \multirow[t]{2}{*}{$1980(\mathrm{Dec})$} & LMF-84 & $25-28$ & 1987 (Dec) & LMF-120 & $248-259$ \\
\hline & & $132-140$ & & & \\
\hline \multirow[t]{2}{*}{1981 (Dec) } & LMF-91 & $46-52$ & & & \\
\hline & & $159-166$ & & & \\
\hline
\end{tabular}


8.5.15 $1977^{239} \mathrm{Pu}$

Single inhalation of monodisperse plutonium oxide aerosol, AMAD $1.5 \mu \mathrm{m}$; dogs 12 to 15 months old, of both sexes, placed on experiment from 1977 to 1979. Results as of 30 September 1987

(Doc. $L M F-120$ ).

\begin{tabular}{|c|c|c|c|c|c|c|c|c|c|}
\hline \multirow{2}{*}{\multicolumn{2}{|c|}{$\begin{array}{l}\text { EXPERIMENTAL } \\
\text { DESIGN }\end{array}$}} & \multicolumn{8}{|c|}{ EXPERIMENTAL RESULTS } \\
\hline & & \multirow{4}{*}{$\begin{array}{l}\text { No. of } \\
\text { Animals } \\
\text { Dead } \\
\end{array}$} & \multirow{3}{*}{$\begin{array}{l}\text { Median } \\
\text { Postexp. } \\
\text { Survival }\end{array}$} & & & & & & \\
\hline Initial & No. of & & & \multicolumn{2}{|c|}{ №. (\% Incidence) } & & & & \\
\hline Burden & Animals & & & \multicolumn{2}{|c|}{ Bone } & \multicolumn{2}{|c|}{ Lung } & \multicolumn{2}{|c|}{ Liver } \\
\hline$(\mu \mathrm{Ci} / \mathrm{kg})$ & Exposed & & (years) & Iun & & Tur & & Tum & \\
\hline None & 12 & 0 & $>9.9$ & 0 & (0) & 0 & (0) & 0 & (0) \\
\hline 0.0029 & 12 & 0 & $>9.9$ & 0 & (0) & 0 & (0) & 0 & (0) \\
\hline 0.0098 & 12 & 2 & $>9.9$ & 0 & (0) & 1 & $(50)$ & 0 & $(0)$ \\
\hline 0.021 & 12 & 4 & $>9.2$ & 0 & (0) & 2 & $(50)$ & 0 & (0) \\
\hline 0.05 & 12 & 9 & 7.5 & 0 & (0) & 6 & (67) & 0 & (0) \\
\hline 0.11 & 12 & 12 & 4.9 & 0 & (0) & 8 & (67) & 0 & (0) \\
\hline 0.18 & 12 & 12 & 4.8 & 0 & (0) & 7 & $(58)$ & 0 & (0) \\
\hline 0.32 & 12 & 12 & 2.1 & 0 & (0) & 1 & (8) & 0 & $(0)$ \\
\hline 0.79 & 12 & 12 & 0.9 & 0 & (0) & 0 & (0) & 0 & (0) \\
\hline
\end{tabular}

The 5 single-inhalation young-adult experiments with inhaled monodisperse plutonium oxide aerosols were designed, as a group, to explore the influence of alpha-particle dose distribution within the lung (McClellan, 1972, 1986). New techniques were developed to produce the monodisperse particles (Raabe, 1975, Talley, 1979). The 1.5- $\mu \mathrm{m}$-AMAD particles had an actual diameter of about 0.44 $\mu \mathrm{m}$ (Guilmette, 1984b).

Initial deposition was estimated from whole-body counting of gamma emissions from ${ }^{169} \mathrm{Yb}$ incorporated into the $\mathrm{PuO}_{2}$ particles as a short-lived tracer; retention and translocation were studied in supporting experiments involving the sacrifice of exposed animals at intervals to 2 years postexposure (Guilmette, 1984). Detailed retention and translocation data have also been reported for life-span animals dying within 1100 days postexposure (Guilmette, 1987). A half-time of 1400 days was measured for retention of $68 \%$ of $1.4-\mu \mathrm{m}{ }^{239} \mathrm{PuO}_{2}$ particles deposited in the lung; a value much longer than that observed for ${ }^{238} \mathrm{PuO}_{2}$, about twice that observed for $0.72 \cdot \mu \mathrm{m}{ }^{239} \mathrm{PuO}_{2}$ particles, and about three-fourths that observed for $2.8-\mu \mathrm{m}{ }^{239} \mathrm{PuO}_{2}$ particles. For all particle sizes of ${ }^{239} \mathrm{PuO}_{2}$, the major route 
of elimination was via feces, with about $15 \%$ of the initial lung burden translocated to pulmonary lymph nodes, about $0.2 \%$ to liver, and about $0.1 \%$ to skeleton by 2 years postexposure.

Early distribution data from both sacrifice and life-span animals have been compared with similar data from immature and aged dogs (Guilmette, 1987b). The major age-related effect is that seen in the skeleton, with significantly greater deposition in immature animals, and significantly smaller deposition in aged animals, than that seen in young adults.

Effects thus far observed show no clear dependence on particle size. The earliest observed biological effect was a lymphopenia, which occurred at the higher dose levels within 180 days; no depression of neutrophil counts was observed, in contrast to findings with ${ }^{238} \mathrm{PuO}_{2}$ (Guilmette, $1984 \mathrm{~b}$ ). The primary cause of death has been radiation pneumonitis, still appearing at 8 years postexposure; the numerous lung tumors are usually incidental findings at autopsy (Diel, 1983b; Guilmette, 1984b; Hahn, 1986, Scott, 1986). Preliminary analyses of incomplete data on lung tumor incidence suggest risk factors for the $1.5-\mu \mathrm{m}{ }^{239} \mathrm{PuO}_{2}$ particles that are not significantly different from those for $0.75-\mu \mathrm{m}$ or $3.0-\mu \mathrm{m}$ particles (Boecker, 1988; Muggenburg, in press). Data on early effects have been compared with similar data from immature and aged dogs; the incidence of fatal radiation pneumonitis is lower in the immature dogs and higher in the aged dogs (Guilmette, 1988b).

Information relevant to this experiment will be found in the following Inhalation Toxicology Research Institute periodic reports:

\begin{tabular}{|c|c|c|c|c|c|}
\hline Issue Date & Dec. Ne. & Page Refs. & Issue Date & Doc. No. & Page Refs. \\
\hline 1971 (Nov) & $L F-44$ & $7-19$ & $1982(\mathrm{Dec})$ & LMF-102 & $336-343$ \\
\hline 1973 (Dec) & $L F-46$ & $1-30$ & $1983(\mathrm{Dec})$ & LMF-107 & $144-148$ \\
\hline 1975 (Dec) & LF-52 & $21-23$ & & & $252-259$ \\
\hline 1977 (Dec) & $L F-58$ & $135-138$ & & & $292-297$ \\
\hline \multirow[t]{2}{*}{1978 (Dec) } & $L F-60$ & $34-37$ & 1984 (Dec) & LMF-113 & $89-101$ \\
\hline & & $145-150$ & & & $225-232$ \\
\hline \multirow[t]{2}{*}{1979 (Dec) } & LF-69 & $14-17$ & 1985 (Dec) & LMF-114 & $236-243$ \\
\hline & & $134-140$ & $1986(\mathrm{Dec})$ & LMF-115 & $226-232$ \\
\hline \multirow[t]{2}{*}{1980 (Dec) } & LMF-84 & $25-28$ & 1987 (Dec) & LMF-120 & $248-259$ \\
\hline & & $132-140$ & & & \\
\hline \multirow[t]{2}{*}{1981 (Dec) } & LMF-91 & $46-52$ & & & \\
\hline & & $159-166$ & & & \\
\hline
\end{tabular}


8.5.16 $1977^{239} \mathrm{Pu}$

Single inhalation of monodisperse plutonium oxide aerosol, AMAD $3.0 \mu \mathrm{m}$; dogs 12 to 15 months old, of both sexes, placed on experiment from 1977 to 1979. Results as of 30 September 1987

(Doc. LMF-120).

\begin{tabular}{|c|c|c|c|c|c|c|c|c|c|}
\hline \multirow{2}{*}{\multicolumn{2}{|c|}{$\begin{array}{l}\text { EXPERIMENTAL } \\
\text { DESIGN }\end{array}$}} & \multicolumn{8}{|c|}{ EXPERIMENTAL RESULTS } \\
\hline & & \multirow{4}{*}{$\begin{array}{l}\text { No. of } \\
\text { Animals } \\
\text { Dead } \\
\end{array}$} & \multirow{4}{*}{$\begin{array}{l}\text { Median } \\
\text { Postexp. } \\
\text { Survival } \\
\text { (years) }\end{array}$} & & & & & & \\
\hline Initial & No. of & & & \multicolumn{2}{|c|}{ №. (\% Incidence) } & & & & \\
\hline Burden & Animals & & & \multicolumn{2}{|c|}{ Bone } & \multicolumn{2}{|c|}{ Lung } & \multicolumn{2}{|c|}{ Liver } \\
\hline$(\mu \mathrm{Cl} / \mathrm{kg})$ & Exposed & & & Tun & & Iun & & Tum & \\
\hline $\begin{array}{l}\text { None } \\
\text { (Control) }\end{array}$ & 11 & 1 & $>9.7$ & 0 & (0) & 0 & (0) & 0 & $(0)$ \\
\hline 0.019 & 12 & 3 & $>10.2$ & 0 & (0) & 3 & $(100)$ & 0 & $(0)$ \\
\hline 0.039 & 12 & 8 & $>8.3$ & 0 & (0) & 7 & (88) & 0 & (0) \\
\hline 0.11 & 12 & 12 & 6.2 & 0 & (0) & 11 & (92) & 0 & $(0)$ \\
\hline 0.25 & 12 & 12 & 3.7 & 0 & (0) & 6 & (50) & 0 & (0) \\
\hline 0.49 & 12 & 12 & 2.0 & 0 & (0) & 2 & (17) & 0 & (0) \\
\hline 1.0 & 12 & 12 & 1.2 & 0 & (0) & 0 & (0) & 0 & (0) \\
\hline
\end{tabular}

The 5 single-inhalation young-adult experiments with inhaled monodisperse plutonium oxide aerosols were designed, as a group, to explore the influence of alpha-particle dose distribution within the lung (McClellan, 1972, 1986). New techniques were developed to produce the monodisperse particles (Raabe, 1975, Talley, 1979). The 3.0- $\mu \mathrm{m}-\mathrm{AMAD}$ particles had an actual diameter of about 0.96 $\mu \mathrm{m}$ (Guilmette, 1984b).

Initial deposition was estimated from whole-body counting of gamma emissions from ${ }^{169} \mathrm{Yb}$ incorporated into the $\mathrm{PuO}_{2}$ particles as a short-lived tracer; retention and translocation were studied in supporting experiments involving the sacrifice of exposed animals at intervals to 2 years postexposure (Guilmette, 1984). Detailed retention and translocation data have also been reported for life-span animals dying within 1100 days postexposure (Guilmette, 1987). A half-time of 1800 days was measured for retention of $82 \%$ of $2.8-\mu \mathrm{m}{ }^{239} \mathrm{PuO}_{2}$ particles deposited in the lung; a value much longer than that observed for ${ }^{238} \mathrm{PuO}_{2}$, about three times that observed for $0.72-\mu \mathrm{m}{ }^{239} \mathrm{PuO}_{2}$ particles, and somewhat greater than that observed for $1.4-\mu \mathrm{m}{ }^{239} \mathrm{PuO}_{2}$ particles.

For all particle sizes of ${ }^{239} \mathrm{PuO}_{2}$, the major route of elimination was via feces, with about $15 \%$ of the initial lung burden translocated to pulmonary lymph 
nodes, about $0.2 \%$ to liver, and about $0.1 \%$ to skeleton by 2 years postexposure. In another supporting dosimetry study, the microdistribution of dose in the lung was studied for $3.0-\mu \mathrm{m}{ }^{239} \mathrm{PuO}_{2}$ particles (Diel, 1984).

Effects thus far observed show no clear dependence on particle size. The earliest observed biological effect was a lymphopenia, which occurred at the higher dose levels within 180 days; no depression of neutrophil counts was observed, in contrast to findings with ${ }^{238} \mathrm{PuO}_{2}$ (Guilmette, 1984b). The primary cause of death has been radiation pneumonitis, still appearing at 8 years postexposure; the numerous lung tumors are usually incidental findings at autopsy (Diel, 1983b; Guilmette, 1984b; Hahn, 1986; Scott, 1986). Preliminary analyses of incomplete data on lung tumor incidence suggest risk factors for the $3.0-\mu \mathrm{m}{ }^{239} \mathrm{PuO}_{2}$ particles that are not significantly different from those for $0.75-\mu \mathrm{m}$ or $1.5-\mu \mathrm{m}$ particles (Boecker, 1988; Muggenburg, in press).

Information relevant to this experiment will be found in the following Inhalation Toxicology Research Institute periodic reports:

\begin{tabular}{|c|c|c|c|c|c|}
\hline Issue Date & Doc. No. & Page Refs. & Issue Date & Doc. Ne & Page Refs. \\
\hline 1971 (Nov) & LF-44 & $7-19$ & 1982 (Dec) & LMF-102 & $336-343$ \\
\hline 1973 (Dec) & $L F-46$ & $1-30$ & $1983(\mathrm{Dec})$ & LMF-107 & $144-148$ \\
\hline 1975 (Dec) & $L F-52$ & $21-23$ & & & $252-259$ \\
\hline 1977 (Dec) & LF-58 & $135-138$ & & & $292-297$ \\
\hline \multirow[t]{2}{*}{1978 (Dec) } & $L F-60$ & $34-37$ & $1984(\mathrm{Dec})$ & LMF-113 & $89-101$ \\
\hline & & $145-150$ & & & $225-232$ \\
\hline \multirow[t]{2}{*}{1979 (Dec) } & $L F \cdot 69$ & $14-17$ & 1985 (Dec) & LMF-114 & $236-243$ \\
\hline & & $134-140$ & $1986(\mathrm{Dec})$ & LMF-115 & $226-232$ \\
\hline \multirow[t]{2}{*}{1980 (Dec) } & LMF-84 & $25-28$ & $1987(\mathrm{Dec})$ & LMF-120 & $248-259$ \\
\hline & & $132-140$ & & & \\
\hline \multirow[t]{2}{*}{1981 (Dec) } & LMF-91 & $46-52$ & & & \\
\hline & & $159-166$ & & & \\
\hline
\end{tabular}


8.5.17 $1977{ }^{239} \mathrm{Pu}$

Twenty inhalations at 6-month intervals of monodisperse plutonium oxide aerosol, AMAD $0.75 \mu \mathrm{m}$; dogs 12 to 15 months old, of both sexes, given first exposure from 1977 to 1978. Results as of 30 September 1987 (Doc. LMF-120).

\begin{tabular}{|c|c|c|c|c|c|c|c|c|c|}
\hline \multirow{2}{*}{\multicolumn{2}{|c|}{$\begin{array}{l}\text { EXPERIMENTAL } \\
\text { DESIGN }\end{array}$}} & \multicolumn{8}{|c|}{ EXPERIMENTAL RESULTS } \\
\hline & & \multirow{4}{*}{$\begin{array}{l}\text { No. of } \\
\text { Animals } \\
\text { Dead } \\
\end{array}$} & \multirow{4}{*}{$\begin{array}{l}\text { Median } \\
\text { Postexp. } \\
\text { Survival } \\
\text { (years) } \\
\end{array}$} & & & & & & \\
\hline Initial & No. of & & & \multicolumn{2}{|c|}{ №. (\% Incidence) } & & & & \\
\hline Burden & Animals & & & \multicolumn{2}{|c|}{ Bone } & \multicolumn{2}{|c|}{ Lung } & \multirow{2}{*}{\multicolumn{2}{|c|}{$\begin{array}{l}\text { Liver } \\
\text { Tumor }\end{array}$}} \\
\hline$(\mu \mathrm{Ci} / \mathrm{kg})$ & Exposed & & & Tun & & Tur & & & \\
\hline $\begin{array}{l}\text { None } \\
\text { (Control) }\end{array}$ & 12 & 2 & $>9.9$ & 0 & (0) & 0 & (0) & 0 & (0) \\
\hline $0.0016^{\circ}$ & 24 & 13 & $>9.8$ & 0 & (0) & 8 & (62) & 0 & (0) \\
\hline $0.015^{\circ}$ & 12 & 12 & 5.2 & 1 & (8) & 4 & (33) & 0 & $(0)$ \\
\hline $0.019^{* *}$ & 24 & 11 & $>9.8$ & 0 & (0) & 8 & (73) & 0 & (0) \\
\hline
\end{tabular}

* Mean incremental deposition at each exposure

* Dogs received only one exposure

Initial deposition was estimated from whole-body counting of gamma emissions from ${ }^{169} \mathrm{Yb}$ incorporated into the $\mathrm{PuO}_{2}$ particles as a short-lived tracer. In addition to the animals detailed above, 12 dogs were exposed at 6 -month intervals for dosimetric studies, 3 to be sacrificed after 1, 3, 5, and 10 years. Results after 2 years indicate that retention following each exposure was independent of previous exposure history; from 2 to $3 \%$ of the alveolar deposit was translocated to tracheobronchial lymph nodes and less than $0.1 \%$ to other tissues (Diel, 1982). Biological effects have been described only in the report literature (Doc. LMF114). The preliminary conclusion is that effects correlate with total radiation dose without regard to the time course of exposure.

Information relevant to this experiment will be found in the following Inhalation Toxicology Research Institute periodic reports:

\begin{tabular}{|c|c|c|c|c|c|}
\hline Issue Date & Doc. No. & Page Refs. & Issue Date & Doc. No & Page Refs. \\
\hline 1977 (Dec) & $L F-58$ & $189-192$ & 1983 (Dec) & LMF-107 & $269-273$ \\
\hline $1978(\mathrm{Dec})$ & LF -60 & $167-170$ & 1984 (Dec) & LMF-113 & $242-246$ \\
\hline 1979 (Dec) & LF-69 & $179-181$ & 1985 (Dec) & LMF-114 & $254-258$ \\
\hline 1980 (Dec) & LMF-84 & $146-148$ & 1986 (Dec) & LMF-115 & $243-246$ \\
\hline 1981 (Dec) & LMF-91 & $174-177$ & 1987 (Dec) & LMF-120 & $271-283$ \\
\hline $1982(\mathrm{Dec})$ & LMF-102 & $364-370$ & & & \\
\hline
\end{tabular}


8.5.18 $1979{ }^{239} \mathrm{Pu}$

Single inhalation of monodisperse plutonium oxide aerosol, AMAD $1.5 \mu \mathrm{m}$; dogs 2.6 to 3.6 months old, of both sexes, exposed from 1979 to 1983. Results as of 30 September 1987 (Doc. LMF120).

\begin{tabular}{|c|c|c|c|c|c|c|c|c|c|}
\hline \multirow{2}{*}{\multicolumn{2}{|c|}{$\begin{array}{l}\text { EXPERIMENTAL } \\
\text { DESIGN }\end{array}$}} & \multicolumn{8}{|c|}{ EXPERIMENTAL RESULTS } \\
\hline & & & Median & & & & & & \\
\hline Initial & No. of & No. of & Postexp. & \multicolumn{6}{|c|}{ No. (\% Incidence) } \\
\hline Burden & Animals & Animals & Survival & \multicolumn{2}{|c|}{$\begin{array}{l}\text { Bone } \\
\text { Tumor }\end{array}$} & \multicolumn{2}{|c|}{$\begin{array}{l}\text { Lung } \\
\text { Tumor }\end{array}$} & \multicolumn{2}{|c|}{$\begin{array}{l}\text { Liver } \\
\text { Tumor }\end{array}$} \\
\hline $\begin{array}{l}\text { None } \\
\text { (Control) }\end{array}$ & 12 & 1 & $>5.8$ & 0 & (0) & 0 & (0) & 0 & (0) \\
\hline 0.0004 & 12 & 1 & $>5.8$ & 0 & (0) & 0 & (0) & 0 & $(0)$ \\
\hline 0.0025 & 12 & 1 & $>5.9$ & 0 & (0) & 0 & (0) & 0 & (0) \\
\hline 0.013 & 12 & 1 & $>5.5$ & 0 & (0) & 0 & (0) & 0 & $(0)$ \\
\hline 0.024 & 12 & 4 & $>5.8$ & 0 & (0) & 2 & $(50)$ & 0 & $(0)$ \\
\hline 0.053 & 12 & 0 & $>5.5$ & 0 & (0) & 0 & $(0)$ & 0 & $(0)$ \\
\hline 0.097 & 12 & 2 & $>5.3$ & 0 & $(0)$ & 2 & $(100)$ & 0 & $(0)$ \\
\hline 0.20 & 12 & 5 & $>5.5$ & 0 & (0) & 3 & $(60)$ & 0 & $(0)$ \\
\hline 0.55 & 12 & 10 & 4.4 & 0 & $(0)$ & 9 & $(90)$ & 0 & $(0)$ \\
\hline
\end{tabular}

Only brief descriptions of, and early results from, this experiment have appeared in the published literature (Guilmette, 1987b, 1988b). Initial deposition was estimated from whole-body counting of gamma emissions from ${ }^{169} \mathrm{Yb}$ incorporated into the $\mathrm{PuO}_{2}$ particles as a short-lived tracer.

In addition to those detailed above, other dogs were exposed for sacrifice at intervals to 2 years postexposure for dosimetric purposes. Distribution data from these sacrifice dogs have been compared with similar data from animals exposed as young adults or as aged dogs (Guilmette, 1987b). Immature dogs exhibited a greater fractional deposition of plutonium in the skeleton; radiation dose to lung and tracheobronchial lymph nodes per microcurie inhaled did not vary greatly among the three age groups (Guilmette, 1988b).

Radiation-induced effects observed during the first 5 years postexposure were limited to pneumonitis and fibrosis, pulmonary carcinoma, and lymph node atrophy; the immature dogs showed a lower incidence of pneumonitis and fibrosis than young-adult and aged dogs (Guilmette, 1988b). 
Information relevant to this experiment will be found in the following Inhalation Toxicology Research Institute periodic reports:

\begin{tabular}{|c|c|c|c|c|c|}
\hline Issue Date & Doc. No. & Page Refs. & Issue Date & Doc. No & Page Refs. \\
\hline 1979 (Dec) & LF-69 & $145-149$ & 1984 (Dec) & LMF-113 & $233-236$ \\
\hline 1980 (Dec) & LMF-84 & $141-142$ & 1985 (Dec) & LMF-114 & $244-248$ \\
\hline 1981 (Dec) & LMF-91 & $167-168$ & $1986(\mathrm{Dec})$ & LMF-115 & $105-108$ \\
\hline 1982 (Dec) & LMF-102 & $344-346$ & & & $233-238$ \\
\hline \multirow[t]{2}{*}{1983 (Dec) } & LMF-107 & $260-263$ & 1987 (Dec) & LMF-120 & $154-161$ \\
\hline & & $292-297$ & & & $260-265$ \\
\hline
\end{tabular}




\begin{abstract}
8.5.19 $1979{ }^{239} \mathrm{Pu}$
Single inhalation of monodisperse plutonium oxide aerosol, AMAD $1.5 \mu \mathrm{m}$; dogs 7 to 10 years old, of both sexes, exposed from 1979 to 1982. Results as of 30 September 1987 (Doc. LMF-120).
\end{abstract}

\begin{tabular}{|c|c|c|c|c|c|c|c|c|c|}
\hline \multirow{2}{*}{\multicolumn{2}{|c|}{$\begin{array}{l}\text { EXPERIMENTAL } \\
\text { DESIGN }\end{array}$}} & \multicolumn{8}{|c|}{ EXPERIMENTAL RESULTS } \\
\hline & & \multirow{4}{*}{$\begin{array}{l}\text { No. of } \\
\text { Animals } \\
\text { Dead } \\
\end{array}$} & \multirow{4}{*}{$\begin{array}{l}\text { Median } \\
\text { Postexp. } \\
\text { Survival } \\
\text { (years) }\end{array}$} & & & & & & \\
\hline Initial & No. of & & & \multicolumn{2}{|c|}{ No. (\% Incidence) } & & & & \\
\hline Burden & Animals & & & \multicolumn{2}{|c|}{ Bone } & \multicolumn{2}{|c|}{ Lung } & \multirow{2}{*}{\multicolumn{2}{|c|}{$\begin{array}{l}\text { Liver } \\
\text { Tumor }\end{array}$}} \\
\hline$(\mu \mathrm{Ci} / \mathrm{kg})$ & Exposed & & & Iun & & Tun & & & \\
\hline None & 12 & 11 & 5.0 & 0 & $(0)$ & 1 & (9) & 0 & (0) \\
\hline 0.03 & 12 & 10 & 5.0 & 0 & $(0)$ & 3 & (30) & 0 & (0) \\
\hline 0.09 & 12 & 12 & 2.9 & 0 & $(0)$ & 1 & (8) & 0 & (0) \\
\hline 0.16 & 12 & 12 & 1.4 & 0 & $(0)$ & 0 & $(0)$ & 0 & (0) \\
\hline 0.37 & 12 & 12 & 0.8 & 0 & (0) & 0 & (0) & 1 & (8) \\
\hline
\end{tabular}

Only brief descriptions of, and early results from, this experiment have appeared in the published literature (Guilmette, 1987b, 1988b). Initial deposition was estimated from whole-body counting of gamma emissions from ${ }^{169} \mathrm{Yb}$ incorporated into the $\mathrm{PuO}_{2}$ particles as a short-lived tracer. Only a few dogs remain alive in the control and lower-dose groups.

Distribution data have been compared with similar data from animals exposed as young adults and as immature dogs (Guilmette, 1987b). Fractional deposition of plutonium in the skeleton was lower in the aged dogs; radiation dose to lung and tracheobronchial lymph nodes per microcurie inhaled did not vary greatly among the three age groups (Guilmette, 1988b).

Radiation-induced effects observed during the first 5 years postexposure were limited to pneumonitis and fibrosis, pulmonary carcinoma and lymph node atrophy; the aged dogs showed a higher incidence of pneumonitis and fibrosis than young-adult and juvenile dogs, with early deaths at higher exposure levels attributable to this effect (Guilmette, 1988b).

Information relevant to this experiment will be found in the following Inhalation Toxicology Research Institute periodic reports: 


\begin{tabular}{|c|c|c|c|c|c|}
\hline Issue Date & Doc. No. & Page Refs. & Issue Date & Doc. No & Page Refs. \\
\hline 1979 (Dec) & LF-69 & $141-144$ & 1984 (Dec) & LMF-113 & $237-241$ \\
\hline 1980 (Dec) & LMF-84 & 143-145 & 1985 (Dec) & LMF-114 & $249-253$ \\
\hline 1981 (Dec) & LMF-91 & $169-173$ & & & $344-347$ \\
\hline 1982 (Dec) & LMF-102 & $347-351$ & $1986(\mathrm{Dec})$ & LMF-115 & $239-242$ \\
\hline \multirow[t]{2}{*}{1983 (Dec) } & LMF-107 & $264-268$ & 1987 (Dec) & LMF-120 & $154-161$ \\
\hline & & 292-297 & & & $266-270$ \\
\hline
\end{tabular}




\section{BIBLIOGRAPHY AND SENIOR-AUTHOR CITATION INDEX (with biographical sketches of principal contributors)}

This is a reasonably complete bibliography of publications in the scientific literature that pertain to the life-span beagle studies sponsored by the U.S. Department of Energy and its predecessor agencies. Most, but not all, of the publications have been referenced in the text of this document.

Two categories of references are distinguished in the listing. Those preceded by a bullet $(\bullet)$ are considered primary references. Primary references are those relating directly to the design, conduct, results, or interpretation of the life-span studies; they also include reports on supporting studies performed as a necessary adjunct to the design, conduct, or interpretation of the life-span studies.

Secondary references, which are not preceded by a bullet, include publications that report findings not related to the primary purpose of the life-span studies, but which are derived from the life-span studies; or reports of ancillary studies that would probably not have been conducted except for the existence of the lifespan studies. Also included in this category are publications in which the results of the life-span studies are applied in a context more general than the life-span studies themselves, and which add nothing in the way of interpretation or understanding of these studies. The distinction between a primary and a secondary reference is ultimately a matter of judgment and has sometimes been based on a less-than-thorough review of the publication in question.

Except in a few critical instances, the main text of this document does not make reference to the periodic progress reports that have been issued by the various laboratories, nor are these reports listed in this bibliography. A listing of these reports appears in Chapter 10, and page references to these reports appear at the conclusion of the individual experiment summaries of Chapter 8 .

Because of the large number of entries that appear for many senior authors, a scheme of alphabetical and chronological listing has been employed that differs from that commonly employed in bibliographies. Only the senior author's name is considered in determining the alphabetical positioning of an entry. Different papers by the same senior author are then arranged by date of publication, with no reference to other authors whose names may appear on the paper. Where more than one publication appeared in the same year, the publications are distinguished by letters appended to the year, i.e., 1988, 1988b, 1988c, etc. Thus, in looking for a publication by a given senior author, an unambiguous order can be followed, based only on the date of publication. With this system, callouts in the text are also simplified, consisting only of the last name of the senior author 
(initials only if necessary to distinguish between senior authors with the same last name), and the year of publication (with letters appended where necessary).

This bibliography also doubles as a senior-author citation index. Appended to each cited entry in the bibliography are references (in brackets) to the text pages on which the paper is cited.

Another unusual feature of this bibliography is the alphabetical interspersing, among the listed publications, of brief biographical sketches of the principal contributors to the life-span beagle studies.

Adelman R.D., Spangler W.L., Beasom F., Ishizaki G. and Conzelman G.M. 1981. Frusemide enhancement of netilmicin nephrotoxicity in dogs. J. Antimicrobial Chemotherapy 7:431-440.

Amtmann E., Oyama J. and Fisher G.L. 1976. Effect of chronic centrifugation on the musculoskeletal system of the dog. Anat. Embryol. 149:71-78.

"Bud" Andersen directed the beagle studies at the University of California, Davis from their inception in 1951 until Leo Bustad took over in that capacity in 1965. He was particularly involved in the study of $x$-ray effects in female beagles and in the housing and veterinary medical routines essential to the conduct of life-span beagle studies.

Andersen A.C. 1955. Debarking in a kennel: Technic and results. Vet. Med. 1:409-411.

Andersen A.C. and Hart G.H. 1955b. Kennel construction and management in relation to longevity studies in the dog. J. Am. Vet. Med. Assoc. 126:366-373.

- Andersen A.C. 1957. A substance observed within the vascular system of dogs receiving lethal exposures of whole-body x-irradiation. Radiat. Res. $6: 361-370$.

$[60,157]$

Andersen A.C. 1957b. Puppy production to the weaning age. J. Am. Vet. Med. Assoc. 130:151-158.

Andersen A.C. and Gee W. 1957c. Blood values in the beagle, M.S.U. Vet. 18:16-21.

Andersen A.C. 1958. A comparison of beagles released for private ownership with those maintained under kennel conditions. J. Am. Vet. Med. Assoc. 132:95-96.

Andersen A.C. and Gee W. 1958b. Normal blood values in the beagle. Vet. Med. 53:135-138. 
Andersen A.C. and Shultz F.T. 1958c. Inherited (congenital) cataract in the dog. Am. J. Pathol. 34:965-975.

Andersen A.C. and Wooten E. 1959. The estrous cycle of the dog. In Reproduction in Domestic Animals (H.H. Cole and P.T. Cupps, eds., Academic Press, New York) 359-397.

Andersen A.C. and Goldman M. 1960. An evaluation of an outdoor kennel for dogs. J. Am. Vet. Med. Assoc. 137:1 29-135.

Andersen A.C. and Parker H.R. 1960b. The production and use of beagles for radiobiological research. Proc. Anim. Care Panel 9:61-66.

- Andersen A.C., Schultz F.T. and Hage T.J. 1961. The effect of total-body $\mathrm{x}$-irradiation on reproduction of the female beagle to 4 years of age. Radiat. Res. 15:745-753.

$[83,157]$

- Andersen A.C. and Goldman M. 1962. Pathologic sequelae in beagles following continuous feeding of $\mathrm{Sr}^{90}$ at a toxic level. In Some Aspects of Internal Irradiation (T.F. Dougherty et al., eds., Pergamon Press, Oxford) 319-328.

$[68,72,74,159]$

- Andersen A.C. 1962b. Life-span studies on irradiated beagles. Small Anim. Clin. 1962:506-512.

- Andersen A.C. and Johnson R.M. 1962c. Erythroblastic malignancy in a beagle. J. Am. Vet. Med. Assoc. 141:944-946.

- Andersen A.C. and McKelvie D.H. 1962d. Long-term experiments in relation to treatment effects: Radiobiological and gerontological problems. Proc. Anim. Care Panel 12:169-182.

Andersen A.C., McKelvie D.H. and Phemister R. 1962e. Reproductive fitness of the female beagle. J. Am. Vet. Med. Assoc. 141:1451-1454.

Andersen A.C. 1962f. Practical information from research in veterinary radiobiology. Southwest. Vet. 16:1-8.

Andersen A.C. 1963. Carcinoma of the bladder in a beagle. J. Am. Vet. Med. Assoc. 143:30-33.

Andersen A.C. 1963b. Carcinoma of the uterus in a beagle. J. Am. Vet. Med. Assoc. 143:500-502.

Andersen A.C. 1963c. Granulosa-cell tumor in a beagle. J. Am. Vet. Med. Assoc. 143:384-386.

Andersen A.C. 1963d. Programming a radionuclide animal treatment facility. Lab. Anim. Care 13:299-309.

- Andersen A.C. 1963e. Reproductive ability of the aging x-irradiated and sham-treated female beagle. In Proceedings of an International Symposium on the Effects of Ionizing Radiation in the Reproductive System (Pergamon Press, New York) 323-331.

$[83,157]$ 
- Andersen A.C. 1963f. Syndromes affecting reproduction in normal and $\mathrm{x}$-irradiated female beagles. In Proceedings of an International Symposium on the Effects of Ionizing Radiation in the Reproductive System (Pergamon Press, New York) 377-392.

$[83,157]$

Andersen A.C. and Floyd M. 1963g. Growth and development of the femur in the beagle. Am. J. Vet. Res. 24:348-351.

- Andersen A.C., Goldman M., Della Rosa R. and McKelvie D. $\mathbf{1 9 6 3 h . ~}$ Fallout hazards to man studied through life-span tests with beagles. Calif. Agric. 1963 (Dec.):2-3.

[107]

Andersen A.C. 1964. Air conditioned cages designed to minimize kennel problems. Lab. Anim. Care 14:292-303.

Andersen A.C. and Johnson R.M. 1964b. Bile duct carcinoma in a beagle. Am. J. Vet. Res, 25:1562-1563.

Andersen A.C. and Johnson R.M. 1964c. Carcinoma of the thyroid in a beagle. Am. J. Vet. Res. 25:861-864.

Andersen A.C. and Potts C. 1964d. Odorless dog cage rooms. All-Pets Magazine 1964 (Jan.):39-42.

- Andersen A.C. 1965. Effects of ionizing radiation on blood. In Veterinary Hematology, 2nd Edition (O. Schalm, ed., Lea and Febiger, Philadelphia) 471-477.

Andersen A.C. 1965b. Reproductive ability of female beagles in relation to advancing age. Exp. Gerontol. 1:189-192.

Andersen A.C. and Rosenblatt L.S. 1965c. Survival of beagles under natural and laboratory conditions. Exp. Gerontol. 1:193-199.

- Andersen A.C. and Guttman P. 1966. Lung neoplasms in whole-body $\mathrm{X}$-irradiated beagles. In Lung Tumors in Animals (L. Severi, ed., Univ. of Perugia, Italy) 359-366.

- Andersen A.C. and Rosenblatt L.S. 1968. Effects of fractionated whole-body $x$-ray exposures on reproductive ability and median survival of female dogs (beagles). In The Proceedings of a Symposium on Dose Rate in Mammalian Radiation Biology (D.G. Brown et al., eds., CONF-680410, NTIS, Springfield, VA) 11.1-11.12.

- Andersen A.C. and Rosenblatt L.S. 1969. The effect of whole-body x-irradiation on the median lifespan of female dogs (beagles). Radiat. Res. 39:177-200.

$[6,17,21,32,81,156]$

- Andersen A.C. ed. 1970. The Beagle as an Experimental Dog, Iowa State Univ. Press, Ames, Iowa.

- Andersen A.C. and Goldman M. 1970b. Growth and development [of the beagle]. In The Beagle as an Experimental Dog (A.C. Andersen, ed., Iowa State Univ. Press, Ames, Iowa) 43-105.

Andersen A.C. 1970c. Kennel facilities. In The Beagle as an Experimental Dog (A.C. Andersen, ed., Iowa State Univ. Press, Ames, Iowa) 10-15. 
Andersen A.C. 1970d. Reproduction [of the beagle]. In The Beagle as an Experimental Dog (A.C. Andersen, ed., Iowa State Univ. Press, Ames, Iowa) 31-39.

- Andersen A.C. 1970e. Skeletal system. A. Osteogenesis [in the beagle]. In The Beagle as an Experimental Dog (A.C. Andersen, ed., Iowa Sate Univ. Press, Ames, Iowa) 149-158.

- Andersen A.C. 1970f. Digestive system [of the beagle]. In The Beagle as an Experimental Dog (A.C. Andersen, ed., Iowa State Univ. Press, Ames, Iowa) $226-231$.

- Andersen A.C. and Schalm O.W. 1970g. Cardiovascular system. C. Hematology [of the beagle]. In The Beagle as an Experimental Dog (A.C. Andersen, ed., Iowa State Univ. Press, Ames, Iowa) 261-281.

Andersen A.C. 1970h. Renal system [of the beagle]. In The Beagle as an Experimental Dog (A.C. Andersen, ed., Iowa State Univ. Press, Ames, Iowa. 294-295.

- Andersen A.C. 1970i. Reproductive system [of the beagle]. B. Female. In The Beagle as an Experimental Dog (A.C. Andersen, ed., Iowa State Univ. Press, Ames, Iowa) 312-326.

- Andersen A.C. 1970j. Eye [of the beagle]. A. Gross and subgross. In The Beagle as an Experimental Dog (A.C. Andersen, ed., Iowa State Univ. Press, Ames, Iowa) 374-385.

- Andersen A.C. 1970k. Pathology [in the beagle]. A. General pathology. In The Beagle as an Experimental Dog (A.C. Andersen ed., Iowa State Univ. Press, Ames, Iowa) 520-546.

- Andersen A.C. 1970l. Pathology [in the beagle]. D. Pathology of the eye. In The Beagle as an Experimental Dog (A.C. Andersen ed., Iowa State Univ. Press, Ames, Iowa) 574-580.

Andersen A.C. and Simpson M.E. 1973. The Ovary and Reproductive Cycle of the Dog (Beagle), Geron-X Inc., Los Altos, California.

- Andersen A.C. and Rosenblatt L.S. 1976. Reproductive ability of female dogs (beagles) surviving a single, midlethal, whole-body x-ray exposure. Radiat Res. 68:361-370.

$[83,157]$

- Angleton G.M. and Lo Presti C.A. 1977. A summary of the dosimetrics for exposures to ${ }^{60} \mathrm{Co}$ of the beagles entered into the CRHL long-term study. Health Phys. 33:100-103.

$[20,22]$

- Angleton G.M., Lee A.C. and Benjamin S.A. 1986. Use of linear model analysis techniques in the evaluation of radiation effects on the life span of the beagle. In Life-Span Radiation Effects Studies in Animals: What Can They Tell Us? (R.C. Thompson and J.A. Mahaffey, eds., CONF-830951, NTIS, Springfield, VA) $160-171$.

$[29,81]$

- Angleton G.M., Benjamin S.A. and Lee A.C. 1988. Health effects of low-level irradiation during development: Experimental design and prenatal and early neonatal mortality in beagles exposed to ${ }^{60} \mathrm{Co}$ gamma rays. Radiat. Res. 115:70-83. 
Jim Arnold, first at Argonne National Laboratory working mostly with rats, and from 1951 to 1956 at the University of Utah, working with beagles, pioneered in the development of autoradiographic tecniques for studying the microscopic distribution of radionuclides. He was particularly concerned with the distribution and biological effects of alpha emitters in bone. These studies were continued and extended by Webster S.S. Jee and his colleagues at the University of Utah.

- Arnold J.S. and Jee W.S.S. 1959. Autoradiography in the localization and radiation dosage of $\mathrm{Ra}^{226}$ and $\mathrm{Pu}^{239}$ in the bones of dogs. Lab. Invest. 8:194-204.

$[53,54,69,127,131]$

- Arnold J.S. and Jee W.S.S. 1962. Pattern of longterm skeletal remodeling revealed by radioautographic distribution of $\mathrm{Pu}^{239}$ in dogs. Health Phys. 8:705-707.

$[53,54,69,127]$

Arnold J.S., Bartley M.H., Tont S.A. and Jenkins D.P. 1966. Skeletal changes in aging and disease. Clin. Orthop. 49:17-39.

- Arnold J.S. 1976. The relation of trabecular bone morphology to plutonium and radium toxicity. In Health Effects of Plutonium and Radium (W.S.S. Jee, ed., JW Press, Salt Lake City) 573-586.

Dave Atherton, a radiochemist, was, from 1952 until his death in 1983, a critical contributor to the design, control and evaluation of nearly all of the University of Utah beagle studies. Although seldom a senior author, he was a prolific contributor to the Utah effort.

- Atherton D.R., Stover B.J. and Mays C.W. 1965. Soft tissue retention of ${ }^{226} \mathrm{Ra}$ in the beagle. Health Phys. 11:101-108.

$[42,130]$

- Atherton D.R. and Stover B.J. 1966. Soft tissue retention of strontium-90 in the beagle. Nature 21 2:703-704.

- Atherton D.R. and Lloyd R.D. 1972. The distribution and retention of ${ }^{249} \mathrm{Cf}$ in beagle soft tissue. Health Phys. 22:675-677.

$[49,143]$

- Atherton D.R., Stevens W., Bates D.S. and Bruenger F.W. 1976. Skeletal retention of ${ }^{239} \mathrm{Pu}(\mathrm{IV})$ in beagles injected at three months of age. In Health Effects of Plutonium and Radium (W.S.S. Jee, ed., JW Press, Salt Lake City) 71-80.

$[47,52]$

- Atherton D.R., Stevens W., Bruenger F.W., Woodbury L., Stover B.J., Smith J.M. and Wrenn M.E. 1986. Life Expectancy for the University of Utah beagle colony and selection of a control group. In Life-Span Radiation Effects Studies in Animals: What Can They Tell Us? (R.C. Thompson and J.A. Mahaffey, eds., CONF-830951, NTIS, Springfield, VA) 243-252.

$[17,18,82,126,130,133,136,139]$ 
Bill Bair developed inhalation exposure capabilities at Pacific Northwest Laboratory and intitiated the first studies with inhaled plutonium in beagles in the late 1950s. In 1968 he became manager of the entire PNL biology research program. He has since main. tained a close interest in the beagle inhalation studies and in the application of the beagle data to the estimation of human risks and to the establishment of exposure limits for workers.

- Bair W.J. 1960. Radioisotope toxicity: From pulmonary absorption. In Radioisotopes in the Biosphere (R.S. Caldecott and L.A. Snyder, eds., Univ. of Minnesota, Minneapolis) 431-448.

- Bair W.J. 1961. Deposition, retention, translocation and excretion of radioactive particles. In Inhaled Particles and Vapours (C.N. Davies, ed., Pergamon Press, New York) 192-208.

$[35,66]$

- Bair W.J. and MeClanahan B.J. 1961b. Plutonium inhalation studies. II. Excretion and translocation of inhaled $\mathrm{Pu}^{239} \mathrm{O}_{2}$ dust. Arch. Environ. Health $2: 648-655$

- Bair W.J. and Willard D.H. 1962. Plutonium inhalation studies. IV. Mortality in dogs after inhalation of $\mathrm{Pu}^{239} \mathrm{O}_{2}$. Radiat. Res. 16:811-821.

$[46,61,66,174]$

- Bair W.J., Willard D.H., Herring J.P. and George L.A.II. 1962b. Retention, translocation and excretion of inhaled $\mathrm{Pu}^{239} \mathrm{O}_{2}$. Health Phys. 8:639-649.

$[35,46,174]$

- Bair W.J. and Willard D.H. 1963. Plutonium inhalation studies. III. Effect of particle size and total dose on deposition, retention and translocation. Health Phys. 9:253-266.

$[25,35,47,174,176]$

- Bair W.J., Tombropoulos E.G.and Park J.F. 1963b. Distribution and removal of transuranic elements and cerium deposited by the inhalation route. In Diagnosis and Treatment of Radioactive Poisoning (International Atomic Energy Agency, Vienna) 319-344.

$[35,89.174]$

- Bair W.J., Stuart B.O., Park J.F. and Clarke W.J. 1964. Factors affecting retention, translocation and excretion of radioactive particles. In Radiological Health and Safety in Mining and Milling of Nuclear Materials. Vol.I (International Atomic Energy Agency, Vienna) 253-274.

$[35,174,182]$

Bair W.J. and Dilley J.V. 1967. Pulmonary clearance of ${ }^{59} \mathrm{Fe}_{2} \mathrm{O}_{3}$ and ${ }^{51} \mathrm{Cr}_{2} \mathrm{O}_{3}$ in rats and dogs exposed to cigarette smoke. In Inhaled Particles and Vapours. II. (C.N. Davies ed., Pergamon Press, Oxford) 251-271.

- Bair W.J. and Park J.F. 1968. Comparative disposition of four types of plutonium dioxides inhaled by dogs. In Proceedings of the First International Congress of Radiation Protection, Pt. 1 (W.S. Snyder et al., eds., Pergamon Press, Oxford) 181-197.

$[35,174]$

Bair W.J. and Smith V.H. 1969. Radionuclide contamination and removal. In Progress in Nuclear Energy. Series XII. Health Physics, Vol.II (A.M.F. Duhamel, ed., Pergamon Press, Oxford) 157-223.

Bair W.J., Porter N.S., Brown, D.P. and Wehner A.P. 1969b. Apparatus for direct inhalation of cigarette smoke by dogs. J. Appl. Physiol. 26:847-850. 
Bair W.J. 1970. Inhalation of radionuclides and carcinogenesis. In Inhalation Carcinogenesis (M.G. Hanna, Jr., et al., eds., CONF-691001, NTIS, Springfield, VA) 77-101.

- Bair W.J. 1970b. Toxicology of inhaled plutonium. In Proceedings of the Eleventh AEC Air Cleaning Conference (M.W. First and J.M. Morgan Jr., eds., CONF-700816, Vol. 2, NTIS, Springfield, VA) 697-720. [174, 175, 184]

- Bair W.J. 1970c. Toxicity of inhaled plutonium. [in Japanese] Hoshasen Kagaku 13:217-221.

- Bair W.J. 1971. Toxicology of inhaled plutonium: Experimental animal studies. In Radiation Protection Problems Relating to Transuranium Elements (EUR-4612, Commission of the European Communities) 145-185.

- Bair W.J., Ballou J.E., Park J.F. and Sanders C.L. 1973. Plutonium in soft tissues with emphasis on the respiratory tract. In Uranium-PlutoniumTransplutonic Elements (H.C. Hodge, et al., eds., Springer-Verlag, New York) 503-568. $[35,36,106,174,175,176]$

- Bair W.J. and Thompson R.C. 1974. Plutonium: Biomedical research. Science 183:715-722.

$[82,106,107,111,175]$

- Bair W.J. 1974b. Toxicology of plutonium. Adv. Radiat. Biol. 4:255-315.

$[106,107,175,184]$

- Bair W.J., Richmond C.R. and Wachholz B.W. 1974c. A Radiobiological Assessment of the Spatial Distribution of Radiation Dose from Inhaled Plutonium (WASH-1320, N'TIS, Springfield, VA).

$[107,111,175,176]$

- Bair W.J., Willard D.H., Nelson I.C. and Case A.C. 1974d. Comparative distribution and excretion of ${ }^{237} \mathrm{Pu}$ and ${ }^{239} \mathrm{Pu}$ nitrates in beagle dogs. Health Phys. 27:392-396.

- Bair W.J. 1974e. The biological effects of transuranium elements in experimental animals. In Plutonium and other Transuranium Elements: Sources, Environmental Distribution and Biomedical Effects (WASH-1359, NTIS, Springfield, VA) 171-241.

Bair W.J. 1974f. Considerations in assessing the potential harm to populations exposed to low levels of plutonium in air. In Population Dose Evaluation and Standards for Man and His Environment (International Atomic Energy Agency, Vienna) 435-449.

$[107,111,115,175]$

Bair W.J. 1974g. Consideration of reactor accident exposure guides for plutonium. In Proceedings of the Fast Reactor Safety Meeting (CONF740401-P1, NTIS, Springfield, VA).

Bair W.J. 1975. Biomedical aspects of plutonium. In Public Issues of Nuclear Power (H.S. Isbin ed., Univ. of Minnesota Press, Minneapolis) 686-729.

Bair W.J. and Thomas J.M. 1976. Prediction of the health effects of inhaled transuranium elements from experimental animal data. In Transuranium Nuclides in the Environment (International Atomic Energy Agency, Vienna) 569-585.

$[107,111]$ 
- Bair W.J. 1976b. Recent animal studies on the deposition, retention and translocation of plutonium and other transuranic compounds. In Diagnosis and Treatment of Incorporated Radionuclides (International Atomic Energy Agency, Vienna) 51-83.

- Bair W.J. 1977. Current status of the hot particle issue (a review of relevant experimental and theoretical approaches). In Proceedings of the IVth International Congress, Vol. 3 (International Radiation Protection Association, Paris) 703-710.

- Bair W.J. 1979. Metabolism and biological effects of alpha-emitting radionuclides. In Radiation Research. Proceedings. of the 6th International Congress of Radiation Research (S. Okada et al., eds., Japanese Association for Radiation Research, Tokyo) 903-912.

- Bair W.J., Metivier H., Park J.F., Masse R., Stevens D.L., Lafuma J., Watson C.R. and Nolibe D. 1980. Comparison of early mortality in baboons and dogs after inhalation of ${ }^{239} \mathrm{PuO}_{2}$. Radiat. Res. 82:588-610. [46,62, 174]

- Barnes J.E. 1971. Distribution of inhaled radionuclides in the respiratory tract. Health Phys. 21:227-232.

$[34,35,50,195]$

Barnes J.E. and Osborn L.A. 1972. Radiation dose distribution in the beagle during radiographic exposure. Am. J. Vet. Res. 33:131-136.

- Barnes J.E., McClellan R.O., Hobbs C.H. and Kanapilly G.M. 1972 b. Toxicity in the dog of inhaled ${ }^{90} \mathrm{Y}$ in fused clay particles: Distribution, retention kinetics, and dosimetry. Radiat. Res. 49:416-429.

$[40,195]$

Barnes J.E., Kanapilly G.M. and Newton G.J. 1976. Cobalt-60 oxide aerosols: Methods of production and short-term retention and distribution kinetics in the beagle dog. Health Phys. 30:391-398.

[96]

- Bartley M.H., Bromley R.G., Targonski S., Seamans S.W. and Jee W.S.S. 1967. Comparison of some quantitative studies of trabecular bone. Stereologia $6: 1$.

- Bartley M.H.Jr., Taylor G.N. and Jee W.S.S. 1970. Skeletal system. D. Teeth and Mandible [of the beagle]. In The Beagle as an Experimental Dog (A.C. Andersen, ed., Iowa State Univ. Press, Ames, Iowa) 189-21.5.

Baxter D.W., Rosenthal M.W., Russell J.J., Moretti E., Chladek D. and Lindenbaum A. 1973. Comparison of monomeric and polymeric plutonium in the dog and mouse. Radiat. Res. 54:556-565.

Baxter D.W., Rosenthal M.W. and Lindenbaum A. 1973b. Decorporation of monomeric plutonium from the dog by glucan and/or DTPA. Radiat. Res. $55: 144-152$.

Beddoe A.H. and Spiers F.W. 1979. A comparative study of the dosimetry of bone-seeking radionuclides in man, rhesus monkey, beagle and miniature pig. Radiat. Res. 80:423-439.

[117] 
Steve Benjamin, a veterinary pathologist at the Inhalation Toxicology Research Institute from 1970 until 1977, was involved in the initiation, and the description and interpretation of effects from, the early ITRI studies. In 1977 he moved to Colorado State University, where he has managed their life-span studies of prenatally and postnatally exposed beagles, sponsored by the Bureau of Radiological Health and the National Cancer Institute.

- Benjamin S.A., Boecker B.B., Chiffelle T.L., Hahn F.F., Hobbs C.H., Jones R.K., McClellan R.O., Pickrell J.A. and Redman H.C. 1973. Neoplasia in beagle dogs after inhalation of ${ }^{144} \mathrm{CeCl}_{3}$. In Radionuclide Carcinogenesis C.L. Sander's et al., eds., CONF-720505, NTIS, Springfield, VA) 181-200.

$[39,61,63,73,82,186,187]$

- Benjamin S.A., Brooks A.L. and Jones R.K. 1975. Effect of pulmonary irradiation from internal emitters on the transformation and kinetics of canine lymphocytes in vitro. In The Cell Cycle in Malignancy and Immunity (J.C. Hampton, ed., CONF-731005, NTIS, Springfield, VA) 224-238.

$[61,66,187,191]$

- Benjamin S.A., Hahn F.F., Chiffelle T.L., Boecker B.B., Hobbs C.H., Jones R.K., McClellan R.O. and Snipes M.B. 1975b. Occurrence of hemangiosarcomas in beagles with internally deposited radionuclides. Cancer Res. $35: 1745-1755$.

$[74,77,79,185,191,200]$

Benjamin S.A. and Ferris A.C. 1975c. Dextran potentiation of the canine lymphocyte response to plant mitogens. Proc. Soc. Exp. Biol. Med. 148:247250 .

- Benjamin S.A., Jones R.K., Snipes M.B. and Lustgarten C.S. 1976. Comparative effects of inhaled relatively insoluble forms of ${ }^{90} \mathrm{Y},{ }^{144} \mathrm{Ce}$, and ${ }^{90} \mathrm{Sr}$ on canine peripheral lymphocyte function. In Radiation and the Lymphatic System (J.E. Ballou, ed., CONF-740930, NTIS, Springfield, VA) 90-99.

$[61,66,191,196,199]$

- Benjamin S.A., Hahn F.F. and Boecker B.B. 1978. Effects of chronic pulmonary irradiation on peripheral lymphocytes and their function in the dog. Radiat. Res. 75:121-137.

$[61,66,191]$

- Benjamin S.A., Boecker B.B., Cuddihy R.G. and McClellan R.O. 1979. Nasal carcinomas in beagles after inhalation of relatively soluble forms of beta-emitting radionuclides. J. Natl. Cancer Inst. 63:133-139.

$[76,112,185,187,188]$

- Benjamin S.A., Lee A.C., Angleton G. M., Jaenke R.S., Saunders W.J., Miller G.K. and Brewster R.D. 1986. Life-span radiation effects studies in prenatally and postnatally exposed beagle dogs at Colorado State University. In Life-Span Radiation Effects Studies in Animals: What Can They Tell Us? (R.C. Thompson and J.A. Mahaffey, eds., CONF-830951, NTIS, Springfield, VA) $1-13$

$[4,20,22,81]$

- Benjamin S.A., Angleton G.M., Lee A.C., Saunders W.J., Miller G.K., Jaenke R.S., Brewster R.D. and Long R.I. 1986b. Neoplasia in beagles that received whole-body irradiation during prenatal or postnatal development. In Life-Span Radiation Effects Studies in Animals: What Can They Tell Us? (R.C. Thompson and J.A. Mahaffey, eds., CONF-830951, NTIS, Springfield, VA) $142-159$ 
- Berliner D.L., Berliner M.L. and Dougherty T.F. 1962. The effects of chronic irradiation by internally deposited radionuclides on corticosteroid biosynthesis. In Some Aspects of Internal Irradiation (T.F. Dougherty et al., eds., Pergamon Press, New York) 179-186.

- Berliner D.L., Stevens W., Nabors C.J. Jr. and Maxwell R.E. 1969. Biochemical changes induced by -internally deposited radionuclides in beagle dog blood: A statistical study. In Delayed Effects of Bone Seeking Radionuclides (C.W. Mays et al., eds., Univ. of Utah Press, Salt Lake City) 471-488.

$[67,127,131,134,137,140]$

Bice D.E., Harris D.L., Hill J.O., Muggenburg B.A. and Wolff R.K. 1980. Immune responses after localized lung immunization in the dog. Am. Rev. Respir. Dis. 122:755-760.

Bice D.E., Harris D.L. and Muggenburg B.A. 1980b. Regional immunologic responses following localized deposition of antigen in the lung. Exp. Lung Res. 1:33-41.

Bice D.E. and Muggenburg B.A. 1985. Effect of age on antibody responses after lung immunization. Am. Rev. Respir. Dis. 132:661-665.

Bice D.E. 1985b. Methods and approaches for assessing immunotoxicity of the lower respiratory tract. In Toxicology of the Immune System (J.H. Dean, ed., Raven Press, New York) 145-157.

- Bielfelt S.W., Wilson A.J., Redman H.C., McClellan R.O. and Rosenblatt L.S. 1969. A breeding program for the establishment and maintenance of a stable gene pool in a beagle dog colony to be utilized for long-term experiments. Am J. Vet. Res. 30:2221-2229.

Bielfelt S.W., Redman H.C. and McClellan R.O. 1971. Sire- and sexrelated differences in rates of epileptiform seizures in a purebred beagle dog colony. Am. J. Vet. Res. 32:2039-2048.

$[17,104]$

Binn L.N., Marchwicki R.H., Eckermann E.H. and Fritz T.E. 1981. Viral antibody studies of laboratory dogs with diarrheal disease. Am. J. Vet. Res. 42:1665-1667.

$[17,103]$

- Bistline R.W., Watters R.L. and Lebel J.L. 1972. A study of translocation dynamics of plutonium and americium from simulated puncture wounds in beagle dogs. Health Phys. 22:829-831.

$[20,88,93]$

Bistline R.W., Lebel J.L. and Dagle G.E. 1976. Translocation dynamies of $\mathrm{Pu}\left(\mathrm{NO}_{3}\right)_{4}$ and $\mathrm{PuO}_{2}$ from puncture wounds to lymph nodes and major organs of beagles. In Radiation and the Lymphatic System (J.E. Ballou, ed., CONF740930, NTIS, Springfield, VA) 10-18.

$[20,88,93]$

- Blair H.A. 1968. Radiation dose-time relations for induction of bone tumors in the dog and skin tumors in the rat. Radiat. Res. 34:501-522.

$[118,127,131,134,137]$

- Blair H.A. 1972. Radiation dose-time relations for induction of osteosarcoma in mice and dogs and their bearing on maximal permissible burden of ${ }^{90} \mathrm{Sr}$ in man. Health Phys. 23:759-765.

$[73,111,118,127,131,140]$ 
Bruce Boecker has been associated with the Inhalation Toxicology Research Institute's life-span dog program since its inception in 1961. He was responsible for the development of apparatus and protocols for the early ITRI inhalation exposures and was particularly involved with the studies of inhaled fission products and with studies involving repeated exposures. Since 1975 he has been an Assistant Director of ITRI with responsibility for the nuclear program and completion of the life-span dog studies.

- Boecker B.B., Aguilar F.L. and Mercer T.T. 1964. A canine inhalation exposure apparatus utilizing a whole body plethysmograph. Health Phys. 10:1077-1089.

- Boecker B.B. and McClellan R.O. 1968. The effects of solubility on the bioassay for inhaled radionuclides. In Diagnosis and Treatment of Deposited Radionuclides (H.A. Kornberg and W.D. Norwood, eds., Excerpta Medica Foundation, Amsterdam) 234-242.

$[39,41]$

- Boecker B.B. 1969. Comparison of ${ }^{137} \mathrm{Cs}$ metabolism in the beagle dog following inhalation and intravenous injection. Health Phys. 16:785-788.

$[33,34,39,41,193]$

- Boecker B.B. 1969b. The metabolism of ${ }^{137} \mathrm{Cs}$ inhaled as ${ }^{137} \mathrm{CsCl}$ by the beagle dog. Proc. Soc. Exp. Biol. Med. 130:966-971.

$[24,39,41]$

- Boecker B.B. 1972. Toxicity of ${ }^{137} \mathrm{CsCl}$ in the beagle: Metabolism and dosimetry. Radiat. Res. 50:556-573.

$[24,28,39,193]$

Boecker B.B., Muggenburg B.A., McClellan R.O., Clarkson S.P., Mares F.J. and Benjamin S.A. 1974. Removal of ${ }^{144}$ Ce in fused clay particles from the beagle dog lung by bronchopulmonary lavage. Health Phys. 26:505-517.

[89]

- Boecker B.B. and Cuddihy R.G. 1974b. Toxicity of ${ }^{144} \mathrm{Ce}$ inhaled as ${ }^{144} \mathrm{CeCl}_{3}$ by the beagle: Metabolism and dosimetry. Radiat. Res. 60:133-154.

$[39,111,186]$

- Boecker B.B., Thomas R.G. and McClellan R.O. 1977. Accumulation and retention of ${ }^{137} \mathrm{Cs}$-labelled fused aluminosilicate particles by beagle dogs after repeated inhalation exposures. In Inhaled Particles IV, Part 1 (W.H. Walton, ed., Pergamon, New York) 221-236.

$[10,41]$

- Boecker B.B., Hahn F.F., Mauderly J.L. and McClellan R.O. 1980. Tumorigenic responses from single or repeated inhalation exposures to relatively insoluble aerosols of ${ }^{144} \mathrm{Ce}$. In Proceedings of the 5th International Congress of the International Radiation Protection Association (Israel Health Physics Society, Jerusalem) 257-260.

$[40,61,77,205]$

- Boecker B.B., Muggenburg B.A., Hahn F.F., Jones R.K. and McClellan R.O. 1984. Inhalation toxicology of ${ }^{144} \mathrm{CeCl}_{3}$ in the beagle dog. In: Radiaion-Risk-Protection, Vol 1 (International Radiation Protection Association, Berlin) 355-358. $[39,61,63,73,74,76,79,112,186,187]$

- Boecker B.B., Hahn F.F., Cuddihy R.G., Snipes M.B. and McClellan R.O. 1986. Is the human nasal cavity at risk from inhaled radionuclides? In Life-Span Radiation Effects Studies in Animals: What Can They Tell Us? (R.C. Thompson and J.A. Mahaffey, eds., CONF-830951, NTIS, Springfield, VA) $564-577$. $[38,51,76,112,185,187,188,192,194]$ 
- Boecker B.B., Hahn F.F., Muggenburg B.A., Guilmette R.A., Griffith W.C. and McClellan R.O. 1988. The relative effectiveness of inhaled alpha- and beta-emitting radionuclides in producing lung cancer. In Radiation Protection Practice. Proceedings of the 7 th International Congress of the International Radiation Protection Association. Vol. 2 (Pergamon, Sydney, Australia) 1059-1062.

$[78,108,111,198,212,214,216]$

Steve Book, a physiologist at the University of California, Davis, from 1973 to 1985, was involved in the conduct and interpretation of results from their ${ }^{90} \mathrm{Sr}$ and $226 \mathrm{Ra}$ ingestion studies. He also conducted ancillary studies of radioiodine metabolism in beagles and rodents.

Book S.A. 1976. Influence of age on thyroidal ${ }^{131} \mathrm{I}$ uptake in beagle pups. Lab. Anim. Sci. 26:443-449.

Book S.A. 1977. Age related changes in serum thyroxine and ${ }^{125}$ I-triiodothyronine resin sponge uptake in the young dog. Lab. Anim. Sci. 27:646-650.

- Book S.A. 1980. The canine as a model in radiobiologic research. In The Canine as a Biomedical Research Model: Immunological,Hematological, and Oncological Aspects (M. Shifrine and F.D. Wilson, eds., DOE/TIC-10191, NTIS, Springfield, VA) 315-330.

$[4,7,15,22,24,72,74,82,158,162]$

- Book S.A., Spangler W.L. and Swartz L.A. 1982. Effects of lifetime ingestion of ${ }^{90} \mathrm{Sr}$ in beagle dogs. Radiat. Res. 90:244-251.

[22, 38, 82, 159]

- Book S.A., Spangler W.L., Parks N.J., Rosenblatt L.S. and Goldman M. 1983. Effects of long-term, low-level exposures from strontium-90 and radium-226 in beagle dogs. In Proceedings of the Seventh International Congress of Radiation Research, Vol. C (J.J. Broerse et al., eds., Martinus Nijhoff, Amsterdam) C7-01.

Book S.A. and Goldman M. 1983b. Radioactive Fallout: An Overview of Internal Emitter Research in the Era of Atmospheric Nuclear Weapons Testing (UCD 472-505, NTIS, Springfield, VA).

[107]

- Book S.A., Rosenblatt L.S. and Goldman M. 1986. Lifetime effects of longterm exposures to strontium-90 and radium-226 in beagle dogs. In Life-Span Radiation Effects Studies in Animals: What Can They Tell Us? (R.C. Thompson and J.A. Mahaffey, eds., CONF-830951, NTIS, Springfield, VA) 646-659.

$[72,74,82,158,162]$

Brennan P.C. and Simkins R.C. 1970. Throat flora of a closed colony of beagles. Proc. Soc. Exp. Biol. Med. 134:566-570.

Brooks A.L., Diel J.H. and McClellan R.O. 1979. The influence of testicular microanatomy on the potential genetic dose from internally deposited ${ }^{239} \mathrm{Pu}$ citrate in Chinese hamster, mouse, and man. Radiat. Res. 77:292-302.

Brown M.G. and Park J.F. 1968. Control of dental calculus in experimental beagles. Lab. Anim. Care 18:527-535. 
Brownstein D.G., Rebar A.H., Bice D.E., Muggenburg B.A. and Hill J.O. 1980. Immunology of the lower respiratory tract: Serial morphologic changes in the lungs and tracheobronchial lymph nodes of dogs after intrapulmonary immunization with sheep erythrocytes. Am. J. Pathol. 98:499-513.

Fred Bruenger, since 1958, has been involved in the chemical and biochemical aspects of all of the life-span beagle studies conducted at the University of Utah, with emphasis during recent years on studies of the effect of age at exposure. He was particularly concerned with the development and application of analytical procedures, including extensive exploration of cellular and subcellular distribution of internally deposited radionuclides.

Bruenger F.W., Atherton D.R. and Stover B.J. 1963. Determination of Sr 90 and $\mathrm{Th}^{228}$ in biological materials. Health Phys. 9:232-235.

Bruenger F.W., Stover B.J. and Atherton D.R. 1963b. Determination of plutonium in biological material by solvent extraction with primary amines. Anal. Chem. 35:1671-1673.

Bruenger F.W., Stevens W. and Stover B.J. 1967. Half-periods of serum proteins in the dog. Am. J. Vet. Res. 28:1699-1703.

Bruenger F.W., Stover B.J. and Atherton D.R. 1967b. The incorporation of various metal ions into in vivo- and in vitro-produced melanin. Radiat. Res. $32: 1-12$.

- Bruenger F.W., Stevens W. and Stover B.J. 1969. Americium-241 in the blood: In vivo and in vitro observations, Radiat. Res. 37:349-360. [49,141]

- Bruenger F.W., Stover B.J., Stevens W. and Atherton D.R. 1969 b. Exchange of ${ }^{239} \mathrm{PuIV}$ between transferrin and ferritin in vitro. Health Phys. $16: 339-340$.

- Bruenger F.W., Stover B.J. and Stevens W. 1971. ${ }^{239} \mathrm{Pu}(\mathrm{IV})$ : Its subcellular distribution and association with ferritin in the canine liver. Health Phys. 21:679-687.

$[55,127]$

- Bruenger F.W., Atherton D.R. and Stevens W. 1972. Intracellular distribution of ${ }^{249} \mathrm{Cf}$ in canine liver. Health Phys. 22:685-689.

Bruenger F.W. and Stevens W. 1973. The association of ${ }^{210} \mathrm{~Pb}$ with constituents of erythrocytes. Health Phys. 25:37-42.

[98]

- Bruenger F.W., Stevens W., Atherton D.R., Bates D.S. and Grube B.J. 1976. The subcellular distribution of ${ }^{239} \mathrm{PuIV}$ in beagle livers as determined by zonal centrifugation and isoelectric focusing. In Health Effects of Plutonium and Radium (W.S.S. Jee, ed., JW Press, Salt Lake City) 199-210. [55, 127]

- Bruenger F.W., Stevens W., Atherton D.R. and Grube B.J. 1976b. The subcellular distribution of some actinide elements in the beagle liver. In Health Effects of Plutonium and Radium (W.S.S. Jee, ed., JW Press, Salt Lake City) 211-221.

$[56,127,141,143,145,149]$

- Bruenger F.W., Grube B.J., Atherton D.R., Taylor G.N. and Stevens W. 1976c. Subcellular distribution of curium in beagle liver. Radiat. Res. 66:443-452. 
- Bruenger F.W., Stevens W., Atherton D.R., Smith J.M., Howerton G. and Buster D.S. 1978. Distribution of ${ }^{239} \mathrm{Pu}$ in neonatal beagles. In Developmental Toxicology of Energy-Related Pollutants (D.D. Mahlum et al., eds., CONF-771017, NTIS, Springfield, VA) 344-360.

$[47,52,147]$

- Bruenger F.W., Stevens W., Stover B.J., Taylor G.N., Smith J.M., Buster D.S. and Atherton D.R. 1980. The distribution and pathological effects of $\mathrm{Pu}$ in juvenile beagles. Radiat. Res. 84:325-342.

$[47,52,55,63,66,69,147]$

- Bruenger F.W., Stevens W., Atherton D.R., Roswell R.L. and Smith J.M. 1981. Biological mechanisms and translocation kinetics of particulate plutonium. In Actinides in Man and Animals (M.E. Wrenn, ed., RD Press, Salt Lake City) 413-426.

$[23,45,127]$

- Bruenger F.W., Smith J.M., Atherton D.R., Jee W.S.S., Lloyd R.D. and Stevens W. 1983. Skeletal retention and distribution of ${ }^{226} \mathrm{Ra}$ and ${ }^{239} \mathrm{Pu}$ in beagles injected at ages ranging from 2 days to 5 years. Health Phys. 44, Supp.l: 513-527.

$[43,47,54,147,150,151,153]$

- Bruenger F.W., Smith J.M., Atherton D.R., Miller S.C., Jee W.S.S. and Stevens W. 1984. Radiation dose rates to the proximal humerus of growing beagles injected with ${ }^{239} \mathrm{Pu}$. Radiat. Res. 97:302-317.

$[47,54,147]$

A veteran of the Manhattan Project at the University of Chicago, Austin Brues was director of biological and medical research at Argonne National Laboratory from the formation of the laboratory until 1962. He conducted some of the first experiments with radionuclides in dogs during the war years. He was one of the group of "Founding Fathers" that established the experimental protocols for the earliest beagle studies at the University of Utah, and served as a consultant to that laboratory for many years.

- Brues A.M. 1975. The use of animal data in derivation of standards. Health Phys. 29:521-524.

Dave Bruner filled a critical administrative role, with scientific understanding, during the years when the majority of the life-span beagle studies were initiated. He served as Chief of the Medical Branch of the Division of Biology and Medicine of the Atomic Energy Commission from 1956 to 1960, and as Assistant Director of the Division from 1960 to 1972 . He played a central role in the establishment of the laboratory that became the Inhalation Toxicology Research Institute.

- Bruner H.D. 1969. The objectives of long-term toxicity studies. In Delayed Effects of Bone-Seeking Radionuclides (C.W. Mays et al., eds., Univ. of Utah Press, Salt Lake City) 1-6.

$[4,13]$

Bryant B.J. and Shifrine M. 1972. Histogenesis of lymph nodes during development of the dog. J. Reticuloendothelial Soc. 12:96-107.

Bryant B.J., Shifrine M. and McNeill C. 1973. Cell-mediated immune response in the developing dog. Int. Arch. Allergy Appl. Immunol. 45:937942.

- Bulgin M.S., Munn S.L. and Gee W. 1970. Hematologic changes to 4 and one-half years of age in clinically normal beagles. J. Am. Vet. Med. Assoc. 157:1064- 1070. 
Bulgin M.S., Shifrine M. and Galligan S.J. 1971. Electrophoretic analyses of normal beagle serum proteins. Lab. Anim. Sci. 21:275-279.

Leo Bustad began his career at Pacific Northwest Laboratory, where, during the 1950 s and early 1960 s he directed experiments with radionuclides in sheep and miniature swine. His research association with the beagle began in 1965 when he assumed directorship of the radiobiology program at the University of California, Davis, a position he held until 1973. He has since continued to influence the field of large-animal research as Dean (now emeritus) of the College of Veterinary Medicine of Washington State University.

- Bustad L.K., Goldman M., Rosenblatt L.S., McKelvie D.H. and Hertzendorf I.I. 1969. Hematopoietic changes in beagles fed ${ }^{90} \mathrm{Sr}$. In Delayed Effects of Bone-Seeking Radionuclides (C.W. Mays et al., eds., Univ. of Utah Press, Salt Lake City) 279-291.

$[65,67,72,159]$

- Bustad L.K. 1969b. Effects of single or fractionated $x$ irradiation and of bone-seeking radionuclides on mammals: A review. In Biological Implications of the Nuclear Age (CONF-690303, NTIS, Springfield, VA) 231-253.

$[65,69,181,83]$

- Bustad L.K., Goldman M., Rosenblatt L.S., Mays C.W., Hetherington N.W., Bair W.J., McClellan R.O., Richmond C.R. and Rowland R.E. 1972. Evaluation of long-term effects of exposure to internally deposited radionuclides. In Peaceful Uses of Atomic Energy, Vol. 11 (United Nations, New York) $125-140$.

[106]

Bustad L.K., Stitzel K.A., Haro E.K. and Goldman M. 1972b. The choice of the beagle for radiobiologic studies. In Radiobiology of Plutonium (B.J. Stover and W.S.S. Jee, eds., JW Press, Salt Lake City) 203-211.

Bustad L.K., Andersen A.C., Chrisp C.E., Fisher G.L., Shifrine M., White R.G. and Wilson F.D. 1973. Clinical applications of research in a large beagle colony, with emphasis on aging. In Proceedings of the 23rd Gaines Veterinary Symposium, Pullman, Washington, 21-28

Bustad L.K., Goldman M. and Rosenblatt L. 1976. Inferences on radiation carcinogenesis revealed by selected studies in animals. In Biology of Radiation Carcinogenesis (J.M. Yuhas et al., eds., Raven Press, New York) $13-29$.

Cain G.R., Kawakami T.G. and Jain N.C. 1985. Radiation-induced megakaryoblastic leukemia in a dog. Vet. Pathol. 22:641-643.

Cain G.R., Cardinet G.H. III, Cuddon P.A., Gale R.P. and Champlin R. 1986. Myasthenia gravis and polymyositis in a dog following fetal hematopoietic cell transplantation. Transplantation 41:21-25.

Cain G.R., Feldman B.F., Kawakami T.G. and Jain N.C. 1986b. Platelet dysplasia associated with megakaryoblastic leukemia in a dog. J. Am. Vet. Med. Assoc. 188:529-530. 
Calder S.E., Taylor G.N., Lloyd R.D., Petersen R.V., Atherton D.R. and Mays C.W. 1978. Zn-DTPA administered by slow-release implant. Health Phys. 35:785-790.

Carrig C.B., Morgan J.P. and Pool R.R. 1974. A study of degenerative joint disease. In Proceedings of 24 th Gaines Veterinary Symposium, Ithaca, New York, 12-17.

- Carrig C.B., Morgan J.P. and Pool R.R. 1975. Effects of x-irradiation on the distal ulnar growth plate of the dog: Gross radiographic changes. J. Am. Vet. Radiol. Soc. 16:211-215.

Carrig C.B., Morgan J.P. and Pool R.R. 1975b. Effects of asynchronous growth of the radius and ulna on the canine elbow joint following experimental retardation of longitudinal growth of the ulna. J. Am. Anim. Hosp. Assoc. 11:560-567.

[103]

- Casarett G.W. 1970. Pathological changes after protracted exposure to low dose radiation. In Late Effects of Radiation (R.J.M. Fry et al., eds., Taylor and Francis, London) 85-100.

$[4,19,81,83]$

Chrisp C.E. and Spangler W.L. 1980. The malignant canine mammary tumor as a model for the study of human breast cancer. In The Canine as a Biomedical Research Model: Immunological, Hematological, and Oncological Aspects (M. Shifrine and F.D. Wilson, eds., DOE/TIC-10191, NTIS, Springfield, VA) 331-349.

$[16,80,120,159,163]$

Christensen W.R. and Sosman M.C. 1951. A preliminary report on telepaque, a new cholecystographic medium. Am. J. Roentgenol. 66:764-768.

- Christensen W.R., Smith C.C., Rehfeld C.E. and Taylor G.N. 1962. Radiographic changes in internally irradiated dogs. In Some Aspects of Internal Irradiation (T.F. Dougherty et al., eds., Pergamon Press, Oxford) 63-77.

$[28,69,70,127,131,134,137]$

- Christensen W.R., Jee W.S.S., Taylor G.N. and Nebeker N. 1972. Distribution of internal radiation-induced osteogenic sarcoma in various species. In Radiobiology of Plutonium (B.J. Stover and W.S.S. Jee, eds, JW Press, Salt Lake City) 195-202.

Clapper W.E. and Meade G.H. 1963. Normal flora of the nose, throat, and lower intestine of dogs. J. Bacteriol. 85:643-648.

- Clapper W.E., Boecker B.B., Sanchez A. and Levy J. 1970. Anamnestic response to Leptospira canicola and infectious canine hepatitis antigens in beagles exposed to ${ }^{90} \mathrm{Sr}$ by inhalation or intravenous injection. Radiat. Res. 43:112-130.

- Clapper W.E. 1970b. Microbiology [in the beagle]. A. Gastrointestinal tract. In The Beagle as an Experimental Dog (A.C. Andersen, ed., Iowa State Univ. Press, Ames, Iowa) 469-473.

- Clapper W.E. 1970c. Microbiology [in the beagle]. B. Respiratory tract. In The Beagle as an Experimental Dog (A.C. Andersen, ed., Iowa State Univ. Press, Ames, Iowa) 474-478.

Clapper W.E. 1970d. Comments on viruses recovered from dogs. J. Am. Vet. Med. Assoc. 156:1678-1680. 
Bill Clarke, a veterinary pathologist at Pacific Northwest Laboratory from the mid-1950s until 1972, was responsible for interpretation of pathologic responses to plutonium in the first studies with inhaled plutonium in the beagle; he also made the early observations of lung carcinomas in these studies.

- Clarke W.J. and Bair W.J. 1964. Plutonium inhalation studies. VI. Pathologic effects of inhaled plutonium particles in dogs. Health Phys. 10:391-398.

$[50,61,71,174,175]$

- Clarke W.J., Park J.F., Palotay J.L. and Bair W.J. 1964b. Bronchioloalveolar tumors of the canine lung following inhalation of plutonium particles. Am. Rev. Respir. Dis. 90:963-967.

$[46,78,175]$

- Clarke W.J., Park J.F. and Bair W.J. 1966. Plutonium particle-induced neoplasia of the canine lung. II. Histopathology and conclusions. In Lung Tumors in Animals (L. Severi, ed., Univ. of Perugia, Italy) 345-355.

$[50,71,78,119,175]$

- Clarke W.J., Park J.F., Palotay J.L. and Bair W.J. 1966b. Plutonium inhalation studies. VII. Bronchiolo-alveolar carcinomas of the canine lung following plutonium particle inhalation. Health Phys. 12:609-613.

$[46,78,119,175]$

- Cochran T.H., Jee W.S.S., Stover B.J. and Taylor G.N. 1962. Liver injury in beagles with $\mathrm{Pu}^{239}$ : Distribution, dosage and damage. Health Phys. 8:699-703.

$[55,63,127]$

Cohen B.L. 1975. Hazards in Plutonium Dispersal. Draft report, Institute for Energy Analysis, Oak Ridge Associated Universities, Oak Ridge.

Cohen B.L. 1977. Hazards from plutonium toxicity. Health Phys. 32:359-379.

Colgrove G.S. and Shifrine M. 1980. Canine immunology: Current status. In The Canine as a Biomedical Research Model: Immunological, Hematological, and Oncological Aspects (M. Shifrine and F.D. Wilson, eds., DOE/TIC-10191, NTIS, Springfield, VA) 43-66.

Colgrove G.S., Shifrine M. and Wilson F.D. 1980b. Canine tumor immunology. In The Canine as a Biomedical Research Model: Immunological, Hematological and Oncological Aspects (M. Shifrine and R.D. Wilson, eds., DOE/TIC-10191, NTIS, Springfield, VA) 83-98.

Cowgill L.D. and Spangler W.L. 1981. Renal insufficiency in geriatric dogs. In The Veterinary Clinics of North America: Small Animal Practice (J.J. Brace, ed., Saunders, Philadelphia) 727-748.

Doug Craig, an aerosol physicist at Pacific Northwest Laboratory from 1969 until 1978, developed the exposure techniques for the plutonium inhalation experiments initiated during the 1970s at PNL; he also described plutonium deposition in the respiratory tract of beagles.

Craig D.K. 1971. The interpretation of rotameter air flow readings. Health Phys. 21:328-332. 
- Craig D.K. Thomas J.M., Decker J.R. and Park J.F. 1972. Alveolar deposition of ${ }^{239} \mathrm{PuO}_{2}$ aerosols in beagle dogs as a function of respiration and aerosol parameters. Health Phys. 22:845-855.

$[25,180]$

- Craig D.K., Buschbom R.L. and Herring J.P. 1973. Relationships between nebulizer suspension concentration, concentration and size distribution of ${ }^{239} \mathrm{PuO}_{2}$ aerosols generated for animal inhalation experiments. Health Phys. 24:637-644.

$[25,26,180]$

- Craig D.K., Park J.F. and Ryan J.L. 1976. Effect of physicochemical properties on metabolism of transuranium oxide inhaled by beagle dogs. In Aerosole in Naturwissenschaft, Medizin und Technik Chemie der Umweltaerosole (V. Bohlau and H. Straubel, eds., Gesellschaft fur Aerosolforschung, e.V., Bad Soden, W. Germany) 429-445.

- Craig D.K., Ballou J.E., Dagle G.E., Mahlum D.D., Park J.F., Sanders C.L., Sikov M.R. and Stuart B.O. 1978. Deposition, translocation and effects of transuranic particles inhaled by experimental animals. In Airborne Radioactivity (American Nuclear Society, La Grange Park, IL) 191-221.

- Craig D.K., Park J.F., Powers G.J. and Catt D.L. 1979. The disposition of americium-241 oxide following inhalation by beagles. Radiat. Res. 78:455-473.

$[47,48,101]$

Fred Cross assumed direction of the Pacific Northwest Laboratory research program relating to uranium-miner exposures in 1978, by which time the studies with beagles had been largely completed. His subsequent rodent and hamster studies have contributed to a total body of data, including the beagle data, which he has applied to the evaluation of uranium-miner risks and to the evaluation of risks from radon in homes.

- Cross F.T., Filipy R.E., Loscutoff S.M., Mihalko P.J., Palmer R.F. and Busch R.H. 1981. Histopathologic, morphometric and physiologic investigation of lungs of dogs exposed to uranium ore dust. In Radiation Hazards in Mining: Control, Measurement, and Medical Aspects (M. Gomez, ed. American Institute of Mining, Metallurgical, and Petroleum Engineers, New York) 228-235.

$[9,98,99]$

- Cross F.T., Palmer R.F., Filipy R.E., Dagle G.E. and Stuart B.O. 1982. Carcinogenic effects of radon daughters, uranium ore dust and cigarette smoke in beagle dogs. Health Phys. 42:33-52.

$[4,9,19,26,76,78,92,98]$

- Cross F.T., Palmer R.F., Busch R.H., Dagle G.E., Filipy R.E. and Ragan H.A. 1986. An overview of PNL radon experiments with reference to epidemiological data. In Life-Span Radiation Effects Studies in Animals: What Can They Tell Us? (R.C. Thompson and J.A. Mahaffey, eds., CONF-830951, NTIS, Springfield, VA) 608-623.

$[62,76,78]$

- Crump K.S., Rosenblatt L.S. and Schneiderman M.A. 1986. Panel Discussions. In Life-Span Radiation Effects Studies in Animals: What Can They Tell Us? (RC. Thompson and J.A. Mahaffey, eds., CONF-830951, NTIS, Springfield, VA) 97-106, 237-242, 343-348, 450-454, 496-500, 624-627, 710-715.

$[16,18,29,81,98,113]$ 
Dick Cuddihy, at the Inhalation Toxicology Research Institute since 1967, has been principally concerned with modeling the distribution kinetics of inhaled materials, often involving special experiments ancillary to the life-span experiments, and with radionuclides not included in the life-span experiments. He has more recently concentrated on the application of the life-span beagle data in the analysis of rishs to man.

Cuddihy R.G. and Boecker B.B. 1970. Kinetics of lanthanum retention and tissue distribution in the beagle dog following administration of ${ }^{140} \mathrm{LaCl}_{3}$ by inhalation, gavage and injection. Health Phys. 19:419-426.

Cuddihy R.G. and Griffith W.C. 1972. A biological model describing tissue distribution and whole-body retention of barium and lanthanum in beagle dogs after inhalation and gavage. Health Phys. 23:621-633.

$[97,111,112]$

- Cuddihy R.G., Brownstein D.G., Raabe O.G. and Kanapilly G.M. 1973. Respiratory tract deposition of inhaled polydisperse aerosols in beagle dogs. Aerosol Sci. 4:35-45.

- Cuddihy R.G. and Boecker B.B. 1973b. Controlled administration of respiratory tract burdens of inhaled radioactive aerosols in beagle dogs. Toxicol. Appl. Pharmacol. 25:597-605.

[25, 190, 195, 197, 199]

Cuddihy R.G., Hall R.P. and Griffith W.C. 1974. Inhalation exposures to barium aerosols: Physical, chemical and mathematical analysis. Health Phys. 26:405-416.

- Cuddihy R.G., Griffith W.C. and Boecker B.B. 1974b. Biological modeling for predicting retention patterns of inhaled contaminants. In Proceedings of the Third International Congress of the International Radiation Protection Association (W.S.Snyder, ed., CONF-730907-P2, NTIS, Springfield, VA) 1243 1248 .

- Cuddihy R.G., Gomez S.R. and Pfleger R.C. 1975. Inhalation exposures of beagle dogs to cerium aerosols: Physical, chemical and mathematical analysis. Health Phys. 29:257-265.

$[39,186]$

- Cuddihy R.G., McClellan R.O., Mewhinney J.A. and Muggenburg B.A. 1976. Correlations between the metabolic behavior of inhaled and intravenously injected plutonium in beagle dogs. In Health Effects of Plutonium and Radium (W.S.S. Jee, ed., JW Press, Salt Lake City) 169-182.

$[46,174,175]$

- Cuddihy R.G., Boecker B.B., McClellan R.O. and Kanapilly G.M. 1976b. ${ }^{144} \mathrm{Ce}$ in tissues of beagle dogs after inhalation of $\mathrm{CeCl}_{3}$ with special emphasis on endocrine glands and reproductive organs. Health Phys. 30:53-59.

- Cuddihy R.G. 1978. Deposition and retention of inhaled niobium in beagle dogs. Health Phys. 34:167-176.

$[96,111]$

- Cuddihy R.G., Boecker B.B. and Griffith W.C. 1979. Modelling the deposition and clearance of inhaled radionuclides. In Biological Implications of Radionuclides Released from Nuclear Industries. Vol. II (International Atomic Energy Agency, Vienna) 77-90.

$[112,186]$

- Cuddihy R.G. 1981. Modeling the metabolism of actinide elements. In Actinides in Man and Animals (M.E. Wrenn ed., RD Press, Salt Lake City) 617-628.

[112] 
- Cuddihy R.G. 1982. Risks of radiation-induced lung cancer. In Critical Issues in Setting Radiation Dose Limits (National Council on Radiation Protection and Measurements, Washington, DC) 133-152.

$[107,115,175]$

Cuddihy R.G., Boecker B.B., Hahn F.F., Muggenburg B.A. and McClellan R.O. In Press. Radiation risk assessment: Current state and future directions. In Proceedings of the United Nations Conference on Peaceful Uses of Nuclear Energy, held in Geneva, Switzerland, March 26-27, 1987.

$[113,115]$

Roger Culbertson, veterinary pathologist at the University of California, Davis, since 1980, has primary responsibility for oncologic histopathology and its integration into the overall interpretation of the life-span beagle experiments.

Culbertson M.R. 1982. Hemangiosarcoma of the canine skin and tongue. Vet. Pathol. 19:556-558.

Culbertson M.R. 1983. Esophageal/gastric leiomyoma in the laboratory beagle. J. Am. Vet. Med. Assoc. 183:1168-1171.

Jerry Dagle, a veterinary pathologist, first worked with beagles on the Colorado State University project. At Pacific Northwest Laboratory since 1973, he has been responsible for histopathologic interpretation on all of the life-span studies with inhaled plutonium in beagles and has been principal investigator for the experiment with inhaled $\mathrm{Pu}\left(\mathrm{NO}_{3}\right)_{4}$.

Dagle G.E., Phemister R.D., Lebel J.L., Jaenke R. and Watters R.L. 1975. Plutonium-induced popliteal lymphadenitis in beagles. Radiat. Res. 61:239250.

$[20,71,93]$

Dagle G.E., Lebel J.L., Phemister R.D., Watters R.L. and Gomez L.S. $1975 \mathrm{~b}$. Translocation kinetics of plutonium oxide from the popliteal lymph nodes of beagles. Health Phys. 28:395-398.

$[20,93]$

- Dagle G.E., Lund J.E. and Park J.F. 1976. Pulmonary lesions induced by inhaled plutonium in beagles. In Health Effects of Plutonium and Radium (W.S.S. Jee, ed., JW Press, Salt Lake City) 161-167.

$[61,78,119,174,175]$

- Dagle G.E. and Park J.F. 1976b. Plutonium-induced lymphadenitis in beagles. In Radiation and the Lymphatic System (J.E. Ballou, ed., CONF740930, NTIS, Springfield, VA) 239-245.

$[50,71,79,93,175]$

- Dagle G.E., Park J.F., Ragan H.A. and Morris J.E. 1979. Toxicity of inhaled plutonium in dogs. In Biological Implications of Radionuclides Released from Nuclear Industries, Vol. I (International Atomic Energy Agency, Vienna) 105-119. [25, 35, 46, 61, 66, 68, 69, 71, 82, 94, 178, 180, 182]

Dagle G.E., Zwicker G.M., Adee R.R. and Park J.F. 1979b. Cytoplasmic inclusions in urinary bladder epithelium of dogs. Vet. Pathol. 16:258-259. 
- Dagle G.E., Sanders C.L., Park J.F. and Mahaffey J.A. 1980. Pulmonary carcinogenesis with inhaled plutonium in rats and dogs. In Pulmonary Toxicology of Respirable Particles (C.L. Sanders et al., eds., CONF-791002, NTIS, Springfield, VA) 601-615.

- Dagle G.E., Cannon W.C., Stevens D.L. and McShane J.F. 1983. Comparative disposition of inhaled ${ }^{238} \mathrm{Pu}$ and ${ }^{239} \mathrm{Pu}$ nitrates in beagles. Health Phys. 44:275-277.

$[36,47,182]$

- Dagle G.E. and Sanders C.L. 1984. Radionuclide injury to the lung. Environ. Health Perspect. 55:129-137.

$[107,115]$

Dagle G.E., Bistline R.W., Lebel J.L. and Watters R.L. 1984b. Plutonium-induced wounds in beagles. Health Phys. 47:73-84.

$[20,93]$

- Dagle G.E., Park J.F., Weller R.E., Ragan, H.A., McClanahan B.J. and Fisher D.R. 1985. Skeletal lesions from inhaled plutonium in beagles. In Metals in Bone (N.D. Priest, ed., MTP Press, Lancaster) 333-341.

$[46,52,69,70,74,75]$

- Dagle, G.E., Park J.F., Weller R.E., Ragan H.A. and Stevens D.L. 1986. Pathology associated with inhaled plutonium in beagles. In Life-Span Radiation Effects Studies in Animals: What Can They Tell Us? (R.C. Thompson and J.A. Mahaffey, eds., CONF-830951, NTIS, Springfield, VA) 471-476.

$[55,61,64,69,74,78,79,178,180,183]$

Decker C.F., Kaspar L.V. and Norris W.P. 1964. The variation of strontium metabolism with age in the dog. Radiat. Res. 23:475-490.

$[37,139]$

Deeg H.J., Storb R., Prentice R., Fritz T.E., Weiden P. L., Sale G.E., Graham T.C. and Thomas E.D. 1980. Increased cancer risk in canine radiation chimeras. Blood 55:233-239.

Deeg H.J., Prentice R., Fritz T.E., Sale G.E., Lombard L.S., Thomas E.D. and Storb R. 1983. Increased incidence of malignant tumors in dogs after total body irradiation and marrow transplantation. Int. J. Radiat. Oncol. Biol. Phys. 9:1505-1511.

Rocco Della Rosa, from 1960 until the early 1970s, was concerned with preparations for, and the conduct of, the ${ }^{90} \mathrm{Sr}$ ingestion experiment at the University of California, Davis. An understanding of the metabolic complexities of this experiment, involving changes in absorption, distribution and retention from conception to adulthood, was of critical importance to its design and conduct.

Della Rosa R.J., Smith F.A. and Stannard J.N. 1961. The renal excretion of strontium and calcium in dogs. Int. J. Radiat. Biol. 3:557-578.

- Della Rosa R.J., Goldman M., Andersen A.C., Mays C.W. and Stover B.J. 1965. Absorption and retention of ingested strontium and calcium in beagles as a function of age. Nature 205:197-198.

$[34,37,107,159]$

- Della Rosa R.J., Peterson G. and Gielow F. 1966. Strontium-90 in beagle hair. Nature 211:777-779.

[107, 159] 
- Della Rosa R.J., Goldman M. and Bustad L.K. 1968. Assessment of ${ }^{90} \mathrm{Sr}$ body burdens in vivo in relation to the metabolic time course following uniform skeletal contamination. In Diagnosis and Treatment of Deposited Radionuclides (H.A. Kornberg and W.D. Norwood, eds., Excerpta Medica Foundation, Amsterdam) 152-163.

- Della Rosa R.J., Wolf H.G. and Goldman M. 1968b. Translocation of ${ }^{90} \mathrm{Sr}$ from maternal skeleton to progeny during gestation and lactation. In Proceedings of the First International Congress on Radiation Protection (W.S. Snyder ed., Pergamon Press, Oxford) 201-204.

$[37,107,159]$

- Della Rosa R.J., McKelvie D.H., Goldman M. and Rosenblatt L.S. 1969. Metabolism and toxicity of ${ }^{90} \mathrm{Sr}$ in the immature beagle. In Radiation Biology of the Fetal and Juvenile Mammal (M.R. Sikov and D.D. Mahlum eds., CONF- 690501, NTIS, Springfield, VA) 529-541. [37, 65, 68, 107, 159]

- Della Rosa R.J., Goldman M., Wolf H.G. and Rosenblatt L.S. 1972. Application of canine metabolic data to man. In Biomedical Implications of Radiostrontium Exposure (M. Goldman and L.K. Bustad, eds., CONF-710201, NTIS, Springfield, VA) 52-67.

$[22,83,107,159,160]$

Detweiler D.K., Buchanan J.W., Fregin G.F. and Hill J.D. 1970. Cardiovascular system [of the beagle]. A. Heart. In The Beagle as an Experimental Dog (A.C. Andersen, ed., Iowa State Univ. Press, Ames, Iowa) 232-246.

Joe Diel, at the Inhalation Toxicology Research Institute since 1975, is a mathematical physicist who has been concerned with dosimetric interpretations of the life-span beagle data. He has been particularly involved in the modeling of pulmonary processes, in the study of the long-term effects of repeated inhalation exposure, and in the management of ITRI computer activities.

- Diel J.H. and Mewhinney J.A. 1980. Toxicity of inhaled ${ }^{238} \mathrm{PuO}_{2}$. I. Metabolism. In Book of Papers, 5th International Congress of the International Radiation Protection Association. Vol. II (Israel Health Physics Soc., Jerusalem) $107-110$.

$[35,46]$

- Diel J.H. and Lundgren D.L. 1982. Repeated inhalation exposure of beagle dogs to ${ }^{239} \mathrm{PuO}_{2}$ : Retention and translocation. Health Phys. 43:655-662.

$[10,25,28,35,45,217]$

- Diel J.H. and Mewhinney J.A. 1983. Fragmentation of inhaled ${ }^{238} \mathrm{PuO}_{2}$ particles in lung. Health Phys. 44:135-143.

$[36,50,206,208]$

- Diel J.H., Guilmette R.A., Hahn F.F., Lundgren D.L., Mewhinney J.A., Muggenburg B.A., Snipes M.B., Boecker B.B. and McClellan R.O. $1983 \mathbf{b .}$ Dosimetry of internally deposited radionuclides in lung and its usefulness in predicting biological effects. In Current Concepts in Lung Dosimetry (D.R. Fisher, ed., CONF-820492-Pt.1, NTIS, Springfield, VA) 18-28.

$[62,82,107,212,214,216]$

- Diel J.H., Mewhinney J.A. and Guilmette R.A. 1984. Microscopic dose distribution around plutonium dioxide particles in lungs of hamsters, rats, and dogs. Radiat. Environ. Biophys, 23:171-177.

$[50,216]$

- Dilley J.V. 1972. The origin of urinary taurine excretion during chronic radiation injury. Radiat. Res. 50:191-196.

$[68,175]$ 
Dolphin G.W. 1971. The biological problems in the radiological protection of workers exposed to ${ }^{239} \mathrm{Pu}$. Health Phys. 20:549-557.

$[106,111]$

Dolphin G.W. 1972. Problems associated with setting safe levels for working with plutonium. Health Phys. 22:937-942.

Dolphin G.W. 1974. Hot particles. Radiol. Protect. Bull. 1974 (8):8-10.

Dolphin G.W. 1978. Problems in radiological protection involving alphaemitters in bone. In Biological Effects of ${ }^{224} \mathrm{Ra}$ (W.A. Muller and H.G. Ebert, eds., Nijhoff, The Hague) 182-200.

[109]

Donovan E.F. and Wyman M. 1970. Clinical aspects of the eye [of the beagle]. B. The ocular fundus. In The Beagle as an Experimental Dog (A.C. Andersen ed., Iowa State Univ. Press, Ames, Iowa) 599-601.

Jean Dougherty, at the University of Utah from the beginning of the life-span studies until 1976, was responsible for the very detailed hematologic studies that were conducted in all of their early ex. periments. These were the first effects data obtainable from these experiments and, as such, were of particular importance in gauging the appropriateness of the chosen exposure levels.

- Dougherty J.H., Bowers J.Z., Bay R.C. and Keyanonda P. 1955. Comparison of hematologic effects of internally deposited radium and plutonium in dogs. Radiology 65:253-259.

$[64,65,127,131]$

- Dougherty J.H. 1962. Some hematological responses to internal irradiation in the beagle. In Some Aspects of Internal Irradiation (T.F. Dougherty et al., eds., Pergamon Press, Oxford) 79-93.

$[65,127,131,134,137,140]$

- Dougherty J.H. and Rosenblatt L.S. 1965. Changes in the hemogram of the beagle with age. J. Gerontol. 20:131-138.

- Dougherty J.H. and Rosenblatt L.S. 1969. Leukocyte depression in beagles injected with ${ }^{226} \mathrm{Ra}$ or ${ }^{239} \mathrm{Pu}$. In Delayed Effect of Bone-Seeking Radionuclides (C.W. Mays et al., eds., Univ. of Utah Press, Salt Lake City) 457-470.

[65. 127. 131]

- Dougherty J.H. and Rosenblatt L.S. 1970. The comparative toxicity of ${ }^{226} \mathrm{Ra},{ }^{239} \mathrm{Pu},{ }^{228} \mathrm{Th},{ }^{228} \mathrm{Ra}$, and ${ }^{90} \mathrm{Sr}$ to leukocytes of beagles. Radiat. Res. 43:56-70.

$[65,110,127,128,131,134,137,140]$

- Dougherty J.H. and Rosenblatt L.S. 1971. Long-term hematological effects of internal emitters in beagles. Radiat. Res. 48:319-331.

$[28,65,110,127,131,134,137,140]$

- Dougherty J.H. 1972. The hematologic changes induced by ${ }^{239} \mathrm{Pu}$ in beagles. In Radiobiology of Plutonium (B.J. Stover and W.S.S. Jee, eds., JW Press, Salt Lake City) 75-86.

$[65,127]$

- Dougherty J.H., Taylor G.N. and Mays C.W. 1972b. Strontium-90 toxicity in adult beagles after acute exposure. In: Biomedical Implications of Radiostrontium Exposure (M. Goldman and L.K. Bustad, eds., CONF-710201, NTIS, Springfield, VA) 259-276.

$[61,65,140]$ 
Tom Dougherty was Chairman of the Department of Anatomy and Director of the Radiobiology Division at the University of Utah from 1954 until his death in 1974. His directorship thus spanned the period during which all but a few of the life-span experiments were initiated. He had particular interests in the hematological and endocrine aspects of these studies.

- Dougherty T.F., Stover B.J., Dougherty J.H., Jee W.S.S., Mays C.W., Rehfeld C.E., Christensen W.R. and Goldthorpe H.C. 1962. Studies of the biological effects of $\mathrm{Ra}^{226}, \mathrm{Pu}^{239}, \mathrm{Ra}^{228}\left(\mathrm{MsTh}_{1}\right), \mathrm{Th}^{228}(\mathrm{RdTh})$, and $\mathrm{Sr}^{90}$ in adult beagles. Radiat Res. 17:625-681.

$[7,13,16,17,29,73,126,127,130,131,133,134,136,137]$

- Dougherty T.F. 1962b. Study of the long term biological effects of internal irradiation in adult beagles. In Some Aspects of Internal Irradiation (T.F. Dougherty et al., eds., Pergamon Press, Oxford) 3-6.

- Dougherty T.F. 1962c. Incidence of bone cancer in internally irradiated dogs. In Some Aspects of Internal Irradiation (T.F. Dougherty et al., eds., Pergamon Press, Oxford) 47-61.

$[73,127,131,134,137]$

- Dougherty T.F., Jee W.S.S., Mays C.W. and Stover B.J. (eds.). $1962 d$. Some Aspects of Internal Irradiation (Pergamon, Oxford).

- Dougherty T.F. and Mays C.W. 1969. Bone cancer induced by internally deposited emitters in beagles. In Radiation-Induced Cancer (International Atomic Energy Agency, Vienna) 361-367.

$[73,109,131,134,137,140]$

- Doyle D.E., Lombard L.S. and Fritz T.E. 1981. A direct data entry system for toxicologic pathology. Toxicol. Pathol. 9:22-29.

Dubin S. and Westcott R.J. 1969. Functional residual capacity of normal unanesthetized beagle dogs. Am. J. Vet. Res. 30:2027-2030.

Dubin S. 1970. Lung compliance of normal unanesthetized beagle dogs. Am J. Vet. Res. 31:895-902.

[26]

Dubin S., Wilson A.J. and Pfleger, R.C. 1971. Changes in respiratory function of beagle dogs given pulmonary lavage. Am. J. Vet. Res. 32:2033-2038.

$[26,90]$

Dudley R.E., Muggenburg B.A., Cuddihy R.G. and McClellan R. O. 1980. Absorption of diethylenetriaminepentaacetic acid (DTPA) from the respiratory tracts of beagle dogs. Am. Ind. Hyg. Assoc. J. 41:5-11.

[88]

- DuMouchel W.H., Dedrick R.L. and Raabe O.G. 1981. Dose-response analyses of bone cancers from radium. Science 214:206-208. $\quad[76,116,163]$

- Dungworth D.L., Goldman M. and McKelvie D.H. 1968. Development of a form of myelogenous leukemia in beagles continuously exposed to strontium-90. Exp. Hematol. (Oak Ridge, Tenn.) 16:54.

$[65,72,159]$

- Dungworth D.L., Goldman M., Switzer J.W. and McKelvie D.H. 1969. Development of a myeloproliferative disorder in beagles continuously exposed to ${ }^{90} \mathrm{Sr}$. Blood 34:610-632.

$[65,72,94,159]$ 
While not directly involved with life-span beagle studies, Pat Durbin has been one of the most active users of the data on radionuclide distribution and effects obtained from these experiments. She has been particularly concerned with the correlation of the beagle data with that from other experimental animals and from humans, and with the explanation of these data in terms of basic chemical and biological principles.

Durbin P.W. 1972. Plutonium in man: A new look at the old data. In Radiobiology of Plutonium (B.J. Stover and W.S.S. Jee, eds., JW Press, Salt Lake City) 469-530.

- Durbin P.W. 1973. Metabolism and biological effects of the transplutonium elements. In Uranium-Plutonium-Transplutonic Elements (H.C. Hodge et al., eds., Springer-Verlag, New York) 739-896.

[106]

- Durbin P. 1974. Behavior of plutonium in animals and man. In Plutonium Information Meeting for an Ad Hoc Subcommittee of the Advisory Committee on Reactor Safeguards, (CONF-740115, NTIS, Springfield, VA) 30-55.

- Durbin P.W. 1975. Plutonium in mammals: Influence of plutonium chemistry, route of administration, and physiological status of the animal on initial distribution and long-term metabolism. Health Phys. 29:495-510. [106]

Durbin P.W. and Jeung N. 1976. Reassessment of distribution of plutonium in the human body based on experiments with non-human primates. In Health Effects of Plutonium and Radium (W.S.S. Jee, ed., JW Press, Salt Lake City) 297-313.

$[111,117]$

- Durbin P.W. (ed) 1978. Workshop on Research Needs in Actinide Biology: Proceedings. CONF-77049l, NTIS, Springfield, VA.

Durbin P.R. and Schmidt C.T. 1985. The U. S. Transuranium Registry report on the ${ }^{241} \mathrm{Am}$ content of a whole body. V. Implications for metabolic modelling. Health Phys. 49:623-661.[

- Dyck J.A., Shifrine M., Klein A.K., Rosenblatt L.S. and Kawakami T. 1986. Spontaneous cell-mediated cytolysis by peripheral blood cells obtained from whole-body chronically irradiated beagle dogs. Radiat. Res. 106:31-40.

Edsall J.T. 1976. Toxicity of plutonium and some other actinides. Bull. Atomic Sci. 32(7):27-37.

Eidson A.F., Damon E.G., Hahn F.F. and Griffith W.C. In Press. Scaling results from extrapolating a model of uranium excretion rates in beagle dog studies to man. Health Phys.

[99]

Ekins M.B., Waring G.O. and Harris R.R. 1980. Oval lipid corneal opacities in beagles. Part II: Natural history over four years and study of tear function. J. Am. Anim. Hosp. Assoc. 16:601-605. 
Ellis L.C. and Berliner D.L. 1967. The effects of ionizing radiations on endocrine cells. VI. Alterations in androgen biosynthesis by canine testicular tissue after the internal deposition of some radionuclides. Radiat Res. $32: 520-537$.

Epstein R.J., Haumann J.R. and Keener R.B. 1968. An implantable telemetry unit for accurate body temperature measurements. J. Appl. Physiol. 24:439-445.

Robley Evans, a Massachusetts Institute of Technology physicist (now retired), is known in this field primarily for his extensive studies on humans exposed to internally deposited radium. He was influential in the initiation and early direction of the first lifespan beagle experiments at the University of Utah, where, along with Austin Brues and Wright Langham, he is known as one of the "Founding Fathers." He served as a consultant to the Utah program for many years.

Evans R.D., Keane A.T. and Shanahan M.M. 1972. Radiogenic effects in man of long-term skeletal alpha-irradiation. In Radiobiology of Plutonium (B.J. Stover and W.S.S. Jee, eds, JW Press, Salt Lake City) 431-468.

$[7,113,133,136]$

Felicetti S.A., Thomas R.G. and McClellan R.O. 1974. Retention of inhaled antimony-124 in the beagle dog as a function of temperature of aerosol formation. Health Phys. 26:525-531.

Felicetti S.A., Silbaugh S.A. and Muggenburg B.A. 1975. Effect of flow rate of lavage fluid on the removal of radioactive particles from the lung by bronchopulmonary lavage. Health Phys. 28:399-403.

Felicetti S.A., Silbaugh S.A., Muggenburg B.A. and Hahn F.F. 1975 b. Effect of time post-exposure on the effectiveness of bronchopulmonary lavage in removing inhaled ${ }^{144} \mathrm{Ce}$ in fused clay from beagle dogs. Health Phys. 29:8996

Felicetti S.A., Wolff R.K. and Muggenburg B.A. 1981. Comparison of tracheal mucous transport in rats, guinea pigs, rabbits, and dogs. J. Appl. Physiol. 51:1612-1617.

Fellows M.H., Clark L. Jr., O'Toole J.J., Kimmel D.B. and Jee W.S.S. 1975. An improved technique for neutron-induced autoradiography of bone containing plutonium. Health Phys. 29:97-101.

$[53,121]$

Filipy R.E., Stuart B.O., Palmer R.F., Ragan H.A. and Hackett P.L. 1974. The effects of inhaled uranium mine air contaminants in beagle dogs. In Experimental Lung Cancer: Carcinogenesis and Bioassays (E.Karbe and J.F Park, eds., Springer Verlag, New York) 403-410. 
Miriam Finkel is best known for her extensive and pioneering studies of radionuclide carcinogenesis in mice, which began dur. ing the war years at the University of Chicago and continued at Argonne National Laboratory until her retirement in 1980. She was involved to a lesser extent in early studies with dogs and with two life-span studies with ${ }^{90} \mathrm{Sr}$ in beagles. Her mouse data have often been employed in interspecies comparisons with life-span beagle data.

- Finkel M.P., Biskis B.O. and Bergstrand P.J. 1960. Radioisotope toxicity: Significance of chronic administration. In Radioisotopes in the Biosphere (R.S. Caldecott and L.A. Snyder, eds., Univ, of Minnesota, Minneapolis) 461-473.

$[8,22,61,82]$

- Finkel M.P., Biskis B.O., Greco I. and Camden R.W. 1972. Strontium-90 toxicity in dogs: Status of Argonne study on influence of age and dosage pattern. In Biomedical Implications of Radiostrontium Exposure (M. Goldman and L.K. Bustad, eds., CONF-710201, NTIS, Springfield, VA) 285-312.

$[8,22,61,72,82,165,166]$

Fish B.R., Keilholtz G.W., Snyder W.S. and Swisher C.D. 1972. Calculation of Doses Due to Accidentally Released Plutonium from an LMFBR. ORNL-NSIC-74, NTIS, Springfield, VA.

- Fisher D.R., Cannon W.C., Hadley R.T. and Park J.F. 1986. Preliminary evaluation of lung doses for dogs exposed to ${ }^{239} \mathrm{PuO}_{2}$. In Life-Span Radiation Effects Studies in Animals: What Can They Tell Us? (R.C. Thompson and J.A. Mahaffey, eds., CONF-830951, NTIS, Springfield, VA) 683-696.

$[35,61,78,82,178,179]$

Fisher G.L. 1974. Comment on in vivo measurement of canine bone mineral. In Proceedings of the International Conference on Bone Mineral Measurement (R.B. Maxess, ed., NIH 75-633, NTIS, Springfield, VA) 282-284.

- Fisher G.L., Nash C.P., Parks N.J. and Gee N.A. 1976. Chemical quantitation of the tapetum lucidum with application to ${ }^{226} \mathrm{Ra}$ dosimetry of the canine eye. In Health Effects of Plutonium and Radium (W.S.S. Jee, ed., JW Press, Salt Lake City) 373-387.

$[42,56,131,162]$

Fisher G.L. 1977. Effects of disease on serum copper and zinc values in the beagle. Am. J. Vet. Res. 38:935-940.

[104]

- Fisher G.L. and Shifrine M. 1977b. Serum-copper and serum-zinc levels in dogs and humans with neoplasia. In Biological Implications of Metals in the Environment, (H. Drucker and R.E. Wildung, eds., CONF-750929, NTIS, Springfield, VA) 507-522.

[104]

Foreman R.E. and Boecker B.B. 1969. Radioiodine metabolism in the beagle dog: The importance of age and mode of ${ }^{131} \mathrm{I}$ exposure. Proc. Soc. Exp. Biol. Med. 131:980-985.

Fox M.W. 1970. Central nervous system [of the beagle]. In:The Beagle as an Experimental Dog (A.C. Andersen, ed., Iowa State Univ. Press, Ames, Iowa) $350-373$.

- Frazier M.E., Park J.F., Jee W.S.S. and Taylor G. 1976. RNA-instructed DNA polymerase activity in radiation-induced osteosarcomas. In Health Effects of Plutonium and Radium (W.S.S. Jee, ed., JW Press, Salt Lake City) 707-716. 
- Frazier M.E., Lund J.E. and Busch R.H. 1976b. In vitro interactions of lymphocytes and cultured cells from beagles with plutonium-induced bone tumors. In Radiation and the Lymphatic System (J.E. Ballou ed., CONF740930, NTIS, Springfield, VA) 197-202.

- Frazier M.E., Seed T.M., Scott L.L. and Stiegler G.L. 1987. Radiationinduced carcinogenesis in dogs. In Radiation Research: Proceedings of the 8th International Congress of Radiation Research, Edinburgh, July 1987. Vol. 2 (E.M. Fielden, et al., eds., Taylor and Francis, London) 488-493. $[11,29,72,78,121,179,183]$

Tom Fritz has been at Argonne National Laboratory since 1963. He has continued and extended the chronic, external, ${ }^{60}$ Co-gammairradiation studies with beagles, begun by Bill Norris, developing in the process an elegant animal model for radiation leukemogenesis. Since 1979 he has been Associate Director of Argonne's Division of Biological and Medical Research.

- Fritz T.E., Norris W.P., Rehfeld C.E. and Poole C.M. 1970. Myeloproliferative disease in beagle dogs given protracted whole-body irradiation or single doses of ${ }^{144} \mathrm{Ce}$. In Myeloproliferative Disorders of Animals and Man (W.J. Clarke, et al., eds., CONF 680529, NTIS, Springfield, VA) 219-241.

$[8,24,60,61,63,64,65,72,73,74,167]$

Fritz T.E., Zeman R.C. and Zelle M.R. 1970b. Pathology and familial incidence of thyroiditis in a closed beagle colony. Exp. Mol. Pathol. 12:14-30.

$[17,102]$

Fritz T.E., Norris W.P. and Kretz N.D. 1970c. Influence of lymphocytic thyroiditis on iodine metabolism in the beagle. Proc. Soc. Exp. Biol. Med. $134: 450-458$.

[102]

Fritz T.E. 1971. Thyroiditis in the dog. In Proceedings of the 19th World Veterinary Congress, Mexico City, 1055-1058.

[102]

Fritz T.E., Norris W.P., Taylor J.A. and Kretz N.D. 1972. Effect of $\mathrm{x}$-irradiation on the thyroid gland of beagles fed a restricted iodide diet. Radiat. Res. 51:333-360.

[102]

- Fritz T.E., Norris W.P. and Tolle D.V. 1973. Myelogenous leukemia and related myeloproliferative disorders in beagles continuously exposed to ${ }^{60} \mathrm{Co}$ gamma-radiation. In Unifying Concepts of Leukemia, Bibl. haemat. Vol. 39 (R.M. Dutcher and L. Chieco-Bianchi, eds., Karger, Basel) 170-188.

$[60,64,72,170]$

Fritz T.E., Lombard L.S., Tyler S.A. and Norris W.P. 1976. Pathology and familial incidence of orchitis and its relation to thyroiditis in a closed beagle colony. Exp. Mol. Pathol. 24:142-158.

$[17,102]$

- Fritz T.E., Norris W.P., Tolle D.V., Seed T.M., Poole C.M., Lombard L.S. and Doyle D.E. 1978. Relationship of dose rate and total dose to responses of continuously irradiated beagles. In Late Biological Effects of Ionizing Radiation, Vol.II (International Atomic Energy Agency, Vienna) 71-82.

Fritz T.E. 1979. Canine enteritis caused by a parvovirus-Illinois. J. Am. Vet. Med. Assoc. 174:6. 
Fritz T.E. and Rose N.R. 1980. Autoimmune diseases of canines. In The Canine as a Biomedical Research Model: Immunological, Hematological, and Oncological Aspects (M. Shifrine and F.D. Wilson eds., DOE/TIC 10191, NTIS, Springfield, VA) 231-243.

- Fritz T.E., Tolle D.V., Doyle D.E., Seed T.M. and Cullen S.M. 1982. Hematologic responses of beagles exposed continuously to low doses of ${ }^{60} \mathrm{Co}$ gamma radiation. In Experimental Hematology Today 1982 (S.J. Baum, G.D. Ledney and S. Thierfelder, eds., S. Karger, New York) 229-240. [21, 64, 170]

- Fritz T.E., Tolle D.V. and Seed T.M. 1985. Chapter 7. The preleukemic syndrome in radiation-induced myelogenous leukemia and related myeloproliferative disorders. In The Preleukemic Syndrome (Hemopoietic Dysplasia) (G.C. Bagby, ed., CRC Press, Cleveland) 87-100.

$[72,118,119,170]$

- Fritz T.E., Seed T.M., Tolle D.V. and Lombard L.S. 1986. Late effects of protracted whole-body irradiation of beagles by cobalt-60 gamma rays. In Life-Span Radiation Effects Studies in Animals: What Can They Tell Us? (R.C. Thompson and J.A. Mahaffey, eds., CONF-830951, NTIS, Springfield, VA) $116-141$. $[9,17,20,21,60,64,72,80,118,119,170,172,173]$

Galvin J.B., Bice D.E. and Muggenburg B.A. 1986. Comparison of cell-mediated and humoral immunity in the dog lung after localized lung immunization. J. Leuk. Biol. 39:359-370.

Garsd A., Goldman M. and Rosenblatt L.S. 1981. The prediction of skeletal mass in growing and adult beagles. Growth 45:29-41.

Garsd A. and Shifrine M. 1982. Environmental factors affecting seasonal variation in immunity of clinically normal dogs. Int. J. Biometeorol. 26:121-128

Gavankar H.G. 1972. Risks and Emergency Radiation Doses from Plutonium Dioxide Exposures (Thesis), TID-26142, Univ, of California, Berkeley.

$[111,115]$

Gavankar R., Durbin P.W. and Pigford T.H. 1974. Basis for assessment of radiation risks for a hypothetical release of plutonium dioxide from a fast reactor accident. In Proceedings of the Fast Reactor Safety Meeting (CONF-740401-P1, NTIS, Springfield, VA) 498-513.

$[111,115]$

- Gearhart J.M., Diel J.H. and McClellan R.O. 1980. Intrahepatic distribution of plutonium in beagles. Radiat. Res. 84:343-352.

$[55,209]$

German Ministry of the Interior (Bonn) 1976. On the toxicity of inhaled hot particles with special reference to plutonium. Recommendations of the Strahlenschutz-kommission (in German). English translation in Radiat. Environ. Biophys. 15:1-11 (1978).

[107]

- Gies R.A., Cross F.T. and Dagle G.E. 1987. A histologic study of the influence of cigarette smoking in suppressing Rn daughter carcinogenesis in dogs. Health Phys. 53:527-529. 
Ethel Gilbert, a statistician at Pacific Northwest Laboratory, has been conducting statistical analyses on the data from PNL life-span beagle studies since 1985 .

- Gilbert E.S. In Press. Time-related factors in the study of risks in animals and man. Health Phys.

$[29,113]$

Nancy Gillett, a veterinary pathologist at the Inhalation Toxicology Research Institute since 1984, has assumed a central role in the conduct of the life-span beagle studies, including especially the preparation for publication of the biological effects data from these studies.

- Gillett N.A., Hahn F.F., Mewhinney J.A. and Muggenberg B.A. 1985. Osteosarcoma development following single inhalation exposure to americium-241 in beagle dogs. Radiat. Res. 104:83-93.

$[69,74,101,120]$

- Gillett N.A., Muggenburg B.A., Boecker B.B., Hahn F.F., Seiler F.A., Rebar A.H., Jones R.K. and McClellan R.O. 1987. Single inhalation exposure to ${ }^{90} \mathrm{SrCl}_{2}$ in the beagle dog: Hematological effects. Radiat. Res. 110:267-288. $[60,65,72,184,185]$

- Gillett N.A., Muggenburg B.A., Boecker B.B., Griffith W.C., Hahn F.F. and McClellan R.O. 1987b. Single inhalation exposure to ${ }^{90} \mathrm{SrCl}_{2}$ in the beagle dog: Late biological effects. J. Natl. Cancer Inst. 79:359-376.

$[69,70,72,74,75,76,120,184,185]$

- Gillett N.A., Muggenburg B.A., Mewhinney J.A., Hahn F.F., Seiler F.A., Boecker B.B. and McClellan R.O. In Press. Primary liver tumors in beagle dogs exposed by inhalation to aerosols of plutonium-238 dioxide. Am. J. Pathol.

$[16,68,79,121,207,209]$

Gillett N.A., Gerlach G., Cassidy J.J. and Brown S.A. In Press b. Mechanical and morphological evaluation of age-related changes in the beagle spine. Acta Orthopaed. Scand.

[103]

G[illette] R. 1974. Plutonium and the "hot particle problem": Environmental group proposes a draconian answer. Science 183:834-835.

[107]

Giri S.N., Parker H.R., Spangler W.L., Misra H.P., Ishizaki G., Schiedt M.J. and Chandler D.B. 1982. Pharmacokinetics of $\left[{ }^{14} \mathrm{C}\right]$-paraquat and associated biochemical and pathologic changes in beagle dogs following intravenous administration. Fundam. Appl. Toxicol. 2:261-269.

[103]

Giri S.N., Curry D.L., Stabenfeldt G., Spangler W.L., Chandler D.B. and Schiedt M.J. 1983. Effects of paraquat on plasma glucose, cortisol, catecholamines, and insulin in the beagle. Environ. Res. 30:80-88.

[103]

- Glad B.W., Mays C.W. and Fisher W. 1960. Strontium studies in beagles. Radiat. Res. 12:672-681. $[37,139]$

Gofman J.W. 1975. Cancer Hazard from Inhaled Plutonium, Committee for Nuclear Responsibility, Dublin, CA.

$[107,111]$

Gofman J.W. 1975b. Estimated Production of Human Lung Cancers by Plutonium from Worldwide Fallout, Committee for Nuclear Responsibility, Dublin, CA. 
Gofman J.W. 1976. The plutonium controversy. J. Am. Med. Assoc. 236:284-286.

Marvin Goldman became associated with the life-span beagle studies at the University of California, Davis, in 1958. He was associate director of the project from 1967 to 1973 and director from 1973 until 1985. He specialized in the metabolic, dosimetric and pathologic effects of radiostrontium and in the development of models for extrapolating radiation risks from beagles to humans.

Goldman M., Anderson R.P., Edgerley E.,Jr. and Ray A.D. 1963. The removal of strontium ${ }^{90}$ from organic waste. Health Phys. 9:847-855.

- Goldman M. and Della Rosa R.J. 1967. Studies on the dynamics of strontium metabolism under condition of continual ingestion to maturity. In Strontium Metabolism (J. Lenihan et al., eds., Academic Press, London) $181-194$

- Goldman M., Della Rosa R.J. and McKelvie D.H. 1969. Metabolic, dosimetric, and pathological consequences in the skeletons of beagles fed ${ }^{90} \mathrm{Sr}$. In Delayed Effects of Bone-Seeking Radionuclides (C.W. Mays et al., eds, Univ. of Utah Press, Salt Lake City) 61-77.

$[7,22,37,68,159,161]$

- Goldman M., Dungworth D.L., Bulgin M.S., Rosenblatt L.S. Richards W.P.C. and Bustad L.K. 1969b. Radiation-induced neoplasms in beagles after administrations of ${ }^{90} \mathrm{Sr}$ and ${ }^{226} \mathrm{Ra}$. In Radiation-Induced Cancer (International Atomic Energy Agency, Vienna) 345-360.

$[16,24,65,68,72,73,159,161,163]$

- Goldman M. 1970. Skeletal mineralization [of the beagle]. In The Beagle as an Experimental Dog (A.C. Andersen, ed., Iowa State Univ. Press, Ames, Iowa) $216-225$.

- Goldman M., Williams R.J.R. and Bulgin M.S. 1970b. Application of ${ }^{18} \mathrm{~F}$ to image ${ }^{226}$ Ra-induced bone lesions. J. Nucl. Med. 11:208-213.

Goldman M., Pool R.R., Wilson F.D. and West J.E. 1971. Nuclear medicine studies on the bone and marrow of beagles. In Proceedings of the Twenty-First Gaines Veterinary Symposium, Ames, Iowa, 29-34.

- Goldman M. and Bustad L.K. 1972. Proceedings synthesis. In Biomedical Implications of Radiostrontium Exposure (M. Goldman and L.K. Bustad, eds., CONF-710201, NTIS, Springfield, VA) 1-16.

[159]

- Goldman M., Della Rosa R.J. and Momeni M.H. 1972b. Radiation dose to beagles from continuous ${ }^{90} \mathrm{Sr}$ exposure. In Biomedical Implications of Radiostrontium Exposure (M. Goldman and L.K. Bustad, eds., CONF-710201, NTIS, Springfield, VA) 103-113.

$[22,38,53,159]$

- Goldman M., Pool R., Momeni M.H., Wilson F., Williams R.J.R., Chrisp C., Rosenblatt L.S. and Bustad L.K. $1972 \mathrm{c}$. Quantitation of ${ }^{90} \mathrm{Sr}$ toxicity in dogs. In Second International Conference on Strontium Metabolism, Glasgow (CONF-720818, NTIS, Springfield, VA) 17-29.

$[38,65,72,74,76,82,159]$

- Goldman M. and Bustad L.K. (eds.). 1972d. Biomedical Implications of Radiostrontium Exposure. CONF-710201, NTIS, Springfield, VA. 
- Goldman M., Rosenblatt L.S., Hetherington N.W. and Finkel M.P. 1973. Scaling dose, time, and incidence of radium-induced osteosarcomas of mice and dogs to man. In Radionuclide Carcinogenesis (C.L. Sanders et al., eds., CONF-720505, NTIS, Springfield, VA) 347-357.

$[76,113,115,131,162,163]$

- Goldman M., Wilson F.D., Rosenblatt L.S. and Book S.A. 1976. Early radiation effects on bone and blood as "predictors" of osteosarcoma, a late effect. In Biological and Environmental Effects of Low-Level Radiation, Vol. I (International Atomic Energy Agency, Vienna) 335-342.

$[117,127,131,140,160,163]$

Goldman M. 1982. Ionizing radiation and its risk. West. J. Med. 137:540547.

- Goldman M., Rosenblatt L.S. and Book S.A. 1986. Lifetime radiation effects research in animals: An overview of the status and philosophy of studies at University of California-Davis Laboratory for Energy-Related Health Research. In Life-Span Radiation Effects Studies in Animals: What Can They Tell Us? (R.C. Thompson and J.A. Mahaffey, eds., CONF-830951, NTIS, Springfield, VA) 53-65.

$[10,19,73,76,113]$

Goldman M. 1986b. Experimental carcinogenesis in the skeleton. In Radiation Carcinogenesis (A.C. Upton, ed., Elsevier) 215-231.

- Goldthorpe H.C. 1962. The effect of ageing and internal emitters on blood chemistry. In Some Aspects of Internal Irradiation (T.F. Dougherty et al., eds., Pergamon Press, Oxford) 117-129.

$[16,66,67]$

Gomez L.S., Lebel J.L. and Watters R.L. 1972. The effect of lymph node removal on $\mathrm{PuO}_{2}$ translocation. Health Phys. 22:833-836.

$[20,88,93]$

Gomez L.S., Bistline R.W., Dagle G.E. Lebel J.L. and Watters R.L. 1974. Effect of delayed lymphadenectomy on ${ }^{239} \mathrm{PuO}_{2}$ translocation in dogs. Health Phys. 27:213-215.

$[20,88,93]$

- Grahn D. and Fritz T.E. 1986. Chronic radiation injury with mice and dogs exposed to external whole-body irradiation at the Argonne National Laboratory. In Life-Span Radiation Effects Studies in Animals: What Can They Tell Us? (R.C. Thompson and J.A. Mahaffey, eds., CONF-830951, NTIS, Springfield, VA) 14-31.

$[32,72,170,172]$

Greene S.A., Lundgren D.L., Snipes M.B. and Wolff R.K. 1987. Deposition and clearance of particles inhaled by beagle dogs previously exposed to $\mathrm{SO}_{2}$. Exper. Lung Res. 13:41 7-425.

- Gridgeman N.T. 1971. Methods of assay of the relative toxicity of certain bone-seeking radionuclides. Radiat. Res. 48:291-302.

$[73,109,127,128,131,134,137,140]$

Although Bill Griffith's name does not often appear as senior author, he has been an important contributor to the modeling and statistical aspects of a variety of papers emanating from the Inhalation Toxicology Research Institute since the early 1970s. His current emphasis is on the analysis of the beagle data for extrapolation to human exposure situations. 
Griffith W.C., Mewhinney J.A., Muggenburg B.A., Boecker B.B. and Cuddihy R.G. 1983. Bioassay model for estimating body burdens of ${ }^{241} \mathrm{Am}$ from excretion analyses. Health Phys. 44, Supp.1:545-554.

$[108,118]$

- Griffith W.C., Lundgren D.L., Hahn F.F., Boecker B.B. and McClellan R.O. 1986. An interspecies comparison of the biological effects of an inhaled, relatively insoluble beta emitter. In Life-Span Radiation Effects Studies in Animals: What Can They Tell Us? (R.C. Thompson and J.A. Mahaffey, eds., CONF-830951, NTIS, Springfield, VA) 501-520.

$[115,191]$

Ray Guilmette, after several years of decorporation studies with actinides in rats at Argonne National Laboratory, moved to the Inhalation Toxicology Research Institute in 1977, where he has retained his interest in decorporation, as well as other aspects of the metabolism and effects of inhaled radionuclides. His current special interests involve the life-span beagle studies with inhaled ${ }^{2}{ }^{39} \mathrm{PuO}_{2}$

Guilmette R.A., Moretti E.S. and Lindenbaum A. 1979. Toward an optimal DTPA therapy for decorporation of actinides: Time-dose relationships for plutonium in the dog. I. Radiat. Res. 78:415-428.

- Guilmette R.A., Diel J.H., Muggenburg B.A., Mewhinney J.A., Boecker B.B. and McClellan R.O. 1984. Biokinetics of inhaled ${ }^{239} \mathrm{PuO}_{2}$ in the beagle dog: Effect of aerosol particle size. Int. J. Radiat. Biol. 45:563-581.

$[35,46,111,112,211,213,215]$

- Guilmette R.A., Muggenburg B.A., Hahn F.F., Diel J.H., Mewhinney J.A., Boecker B.B. and McClellan R.O. 1984b. Biological response of beagle dogs to inhaled monodisperse aerosols of ${ }^{239} \mathrm{PuO}_{2}$. In Radiation-Risk-Protection, Vol. I (A.Kaul et al., eds., International Radiation Protection Association, Berlin) 384-387.

$[66,211,212,213,214,215,216$

Guilmette R.A., Muggenburg B.A. and Cambron B.L. 1986. Bronchoalveolar lavage: A new bioassay tool for plutonium inhalation exposures. $J$. Occup. Med. 28:492-496.

$[90,101]$

- Guilmette R.A., Muggenburg B.A., Hahn F.F., Mewhinney J.A., Seiler F.A., Boecker B.B. and McClellan R.O. 1987. Dosimetry of ${ }^{239} \mathrm{Pu}$ in dogs that inhaled monodisperse aerosols of ${ }^{239} \mathrm{PuO}_{2}$. Radiat. Res. 110:199-218.

$[35,46,51,55,56,112,213,215]$

- Guilmette R.A., Boecker B.B., Muggenburg B.A., Hahn F.F. and McClellan R.O. 1987b. Age-related effects on the disposition and dosimetry of inhaled ${ }^{239} \mathrm{Pu}$ or ${ }^{144} \mathrm{Ce}$ in immature or aged beagle dogs. In Age-Related Factors in Radionuclide Metabolism and Dosimetry (G.B. Gerber, H. Metivier and H. Smith, eds., Martinus Nijhoff, Dordrecht) 109-120.

$[34,36,41,47,191,201,203,214,218,220]$

Guilmette R.A. and Muggenburg B.A. 1988. Reducing the radiation dose from inhaled americium-241 using continuously administered DTPA therapy. Int. J. Radiat. Biol. 53:261-271.

$[87,89]$

- Guilmette R.A., Muggenburg B.A., Hahn F.F., Boecker B.B. and McClellan R.O. $\mathbf{1 9 8 8 b}$. Age effects on the induction of radiation- induced lung disease in beagles that inhaled ${ }^{239} \mathrm{PuO}_{2}$ aerosols: Status report. In Radiation Protection Practice. Proceedings of the 7 th International Congress of the International Radiation Protection Association. Vol. 2 (Pergamon, Sydney, Australia) 1063-1066.

$[47,62,78,214,218,220]$ 
Guilmette R.A. and Kanapilly G.M. In Press. Biokinetics and dosimetry of inhaled curium aerosols in beagles: Effect of aerosol chemical form. Health Phys.

$[101,102,111]$

Guilmette R.A. and Mewhinney J.A. In Press b. A biokinetic model of inhaled curium compounds in dogs: Application to human exposure data. Health Phys.

$[102,108]$

Guttman P.H. and Andersen A.C. 1968. Progressive intercapillary glomerulosclerosis in aging and irradiated beagles. Radiat Res. 35:45-60.

- Guttman P.H. 1970. Pathology [in the beagle]. B. Renal pathology. In The Beagle as an Experimental Dog (A.C. Andersen, ed., Iowa State Univ. Press, Ames, Iowa) 546-558.

Fletcher Hahn, a veterinary pathologist at the Inhalation Toxicology Research Institute since 1971, has been associated with the design and conduct of the life-span beagle studies, and particularly with evaluations of radionuclide carcinogenicity. His research emphasis has been on dose-response relationships in the lung, tracheobronchial lymph nodes, and other segments of the respiratory tract.

- Hahn F.F., Benjamin S.A., Boecker B.B., Chiffelle T.L., Hobbs C.H., Jones R.K., McClellan R.O. and Redman H.C. 1973. Induction of pulmonary neoplasia in beagle dogs by inhaled ${ }^{144} \mathrm{Ce}$ fused-clay particles. In Radionuclide Carcinogenesis (C.L. Sanders et al., eds., CONF-720505, NT'TS, Springfield, VA) $201-214$

- Hahn F.F., Benjamin S.A., Boecker B.B., Chiffelle T.L., Hobbs C.H., Jones R.K., McClellan R.O., Pickrell J.A. and Redman H. C. 1973b. Primary pulmonary neoplasms in beagle dogs exposed to aerosols of ${ }^{144} \mathrm{Ce}$ in fused-clay particles. J. Natl. Cancer Inst. 50:675-698.

$[40,66,68,77,191]$

- Hahn F.F., Barnes J.E., Hobbs C.H. and Mauderly J.L. 1975. Effect of ${ }^{90} \mathrm{Y}$ inhaled in fused clay particles on the gastrointestinal tract of beagles. Radiat. Res. 61:444-456.

$[40,63,195]$

- Hahn F.F., Boecker B.B., Hobbs C.H., Jones R.K. and Muggenburg B.A. 1976. Effect of ${ }^{144} \mathrm{Ce}$ inhaled in fused-clay particles on the tracheobronchial lymph nodes. In Radiation and the Lymphatic System (J.E. Ballou, ed. CONF-740930, NTIS, Springfield, VA) 218-224.

$[40,50,61,70,191]$

- Hahn F.F., Benjamin S.A., Boecker B.B., Hobbs C.H., Jones R.K., McClellan R.O. and Snipes M.B. 1977. Comparative pulmonary carcinogenicity of inhaled beta-emitting radionuclides in beagle dogs. In Inhaled Particles IV, Part 2 (W.H. Walton, ed., Pergamon, New York) 625-635.

$[77,110,191,198,200]$

- Hahn F.F. and Boecker B.B. 1980. Tumors of the tracheobronchial lymph nodes in beagle dogs after inhalation of a relatively insoluble form of cerium-144. In Pulmonary Toxicology of Respirable Particles (C.L. Sanders et al., eds., CONF-791002, N'TIS, Springfield, VA) 591-600.

$[79,191]$ 
- Hahn F.F., Mewhinney J.A., Merickel B.S., Guilmette R.A., Boecker B.B. and McClellan R.O. 1981. Piimary bone neoplasms in beagle dogs exposed by inhalation to aerosols of plutonium-238 dioxide. J. Natl. Cancer Inst. 67:917-927.

$[35,45,61,69,70,71,74,75,76,78,206,207,208,209]$

- Hahn F.F., Boecker B.B., Cuddihy R.G., Hobbs C.H., McClellan R.O. and Snipes M.B. 1983. Influence of radiation dose patterns on lung tumor incidence in dogs that inhaled beta emitters. In Proceedings 7 th International Congress of Radiation Research, Vol.C (J.J. Broerse et al., eds., Martinus Nijhoff, Amsterdam) C7-03.

$[82,119]$

- Hahn F.F., Boecker B.B., Cuddihy R.G., Hobbs C.H., McClellan R.O. and Snipes M.B. 1983b. Influence of radiation dose patterns on lung tumor incidence in dogs that inhaled beta emitters: A preliminary report. Radiat. Res. 96:505-517.

$[63,77,78,82,118,119,196,198,200]$

- Hahn F.F., Muggenburg B.A., Boecker B.B., Cuddihy R.G., Griffith W.C., Guilmette R.A., McClellan R.O. and Mewhinney J.A. 1986. Insights into radionuclide-induced lung cancer in people from life-span studies in beagle dogs. In Life-Span Radiation Effects Studies in Animals: What Can They Tell Us? (R.C. Thompson and J.A. Mahaffey, eds., CONF-830951, NTIS, Springfield, VA) $521-534$

$[77,78,79,82,110,112,115,119,191,198,200,207,209,212,214,216]$

- Hahn F.F., McClellan R.O., Boecker B.B. and Muggenburg B.A. 1988. Future development of biological understanding of radiation protection: Implications on nonstochastic effects. Health Phys. 55:303-313.

$[61,63,70]$

Hahn F.F. 1989. Carcinogenic responses of the lung to inhaled materials. In Concepts in Inhalation Toxicology, Chapter 12 (R.O. McClellan and R.F. Henderson, eds., Hemisphere Publishing, New York, NY) 313-346.

- Hahn F.F., Boecker B.B., McClellan R.O., Cuddihy R.G., Snipes M.B., Muggenburg B.A. and Hobbs C.H. In Press. Organs and cells at risk after inhalation of insoluble beta-emitting radionuclides: Lessons from experimental studies. In Proceedings of the Second International Workshop on Lung Dosimetry, Cambridge, England.

$[77,79,82,191,196,198,200]$

- Hahn F.F., Boecker B.B., Muggenburg B.A., Scott B.R. and McClellan R.O. In Press b. Irradiation of lymph nodes after deposition of radioactive particles in the lung. In Proceedings of the L.H. Gray Conference on Low Dose Radiation Biological Bases of Risk Assessment. Symposium held in Oxford, September 11-15, 1988

$[40,78,79,80,191]$

- Hanika C. and Rebar A.H. 1980. Ureteral transitional cell carcinoma in the dog. Vet. Pathol. 17:643-646.

Hardt A.B. and Jee W.S.S. 1982. Trabecular bone structural variation in biopsy sites of the beagle ilium. Calcif. Tissue Int. 34:391-395.

Harkema J.R., Mason M.J., Kusewitt D.F. and Pickrell J.A. 1982. Cholecystotomy as treatment for obstructive jaundice in a dog. J. Am. Vet. Med. Assoc. 181:815-816.

Harley N.H. and Pasternack B.S. 1976. A comparison of the dose to cells on trabecular bone surfaces from plutonium-239 and radium-226 based on experimental alpha absorption measurements. Health Phys. 30:35-46. [111, 117] 
Harmsen A.G., Bice D.E. and Muggenburg B.A. 1985. The effect of local antibody responses on in vivo and in vitro phagocytosis by pulmonary alveolar macrophages. J. Leukocyte Biol. 37:483-492.

Harmsen A.G., Muggenburg B.A., Snipes M.B. and Bice D.E. 1985b. The role of macrophages in particle translocation from lungs to lymph nodes. Science 230:1277-1280.

$[92,94]$

Harmsen A.G., Mason M.J., Muggenburg B.A., Gillett N.A., Jarpe M.A. and Bice D.E. 1987. Migration of neutrophils from lung to tracheobronchial lymph node. J. Leukocyte Biol. 41:95-103.

$[92,94]$

Harris W.R., Friedman S.B. and Silberman D. 1984. Behavior of vanadate and vanadyl ion in canine blood. J. Inorg. Biochem. 20:157-169.

[104]

Hart B.L. 1970. Reproductive system [of the beagle]. A. Male. In The Beagle as an Experimental Dog (A.C. Andersen, ed., Iowa State Univ. Press, Ames, Iowa) 296-312.

Hashimoto E.G. and Jee W.S.S. 1976. Cells at risk from bone-seeking radionuclides-A Review. In The Health Effects of Plutonium and Radium (W.S.S. Jee, ed., JW Press, Salt Lake City) 643-656.

$[119,121]$

Healy J.W. 1974. A Proposed Interim Standard for Plutonium in Soils (LA-5483-MS, NTIS, Springfield, VA).

[107, 111]

Hems G. and Mole R.H. 1966. The relative toxicities of radium 226, plutonium 239 and strontium 90 for bone tumour induction. Br. J. Radiol. 39:719-726.

$[111,113]$

Henderson R.F. and Pfleger R.C. 1972. Surface tension studies of phosphatidyl glycerol isolated from the lungs of beagle dogs. Lipids 7:492-494. [91]

Henderson R.F., Waide J.J. and Pfleger R.C. 1974. Methods for determining the fraction of pulmonary surfactant lipid removed from the lung of beagle dogs by lavage. Arch. Int. Physiol. Biochim. 82:259-272.

Henderson R.F., Waide J.J. and Pfleger R.C. 1975. Replacement time for alveolar lipid removed by pulmonary lavage: Effects of multiple lavage on lung lipids. Arch. Int. Physiol. Biochim. 83:261-269.

- Hobbs C.H., Barnes J.E., McClellan R.O., Chiffelle T.L., Jones R.K., Lundgren D.L., Mauderly J.L., Pickrell J.A. and Rypka E.W. 1972. Toxicity in the dog of inhaled ${ }^{90} \mathrm{Y}$ in fused clay particles: Early biological effects. Radiat. Res. 49:430-460.

$[61,66,196]$

Holmberg C.A. and Wilson F.D. 1980. Lymphoid neoplasia in the canine. In The Canine as a Biomedical Research Model: Immunological, Hematological and Oncological Aspects (M. Shifrine and R.D. Wilson, eds., DOE/TIC10191, NTIS, Springfield, VA) 296-314.

$[93,118]$

Hoopes P.J., Gillette E.L. and Benjamin S.A. 1985. The pathogenesis of radiation nephropathy in the dog. Radiat. Res. 104:406-419.

[64]

Horn P.L. and Shifrine M. 1973. Proton irradiation of beagle eyes. II. Clinical and histopathologic observations for $35 \mathrm{MeV}$ protons. Radiat. Res. 53:255-266. 
Howard E.B. 1967. Immunologic defect in mastocytoma-bearing dogs. $J$. Am. Vet. Med. Assoc. 151:1308-1310.

- Howard E.B. 1970. The morphology of experimental lung tumors in beagle dogs. In Morphology of Experimental Respiratory Carcinogenesis (P. Nettesheim et al., eds., CONF-700501, NTIS, Springfield, VA) 147-160.

Hubbard L.B. and Williamson F.S. 1969. Gamma-ray doses for all points in spheres and cylinders with uniformly distributed sources. Phys. Med. Biol. 14:255-267.

Hubbard L.B. and Williamson F.S. 1971. Scatter corrections in bounded media for the doses from internally emitted gamma rays. Phys. Med. Biol. 16:35-46.

- Ingrao G. and Jee W.S.S. 1985. Pu microdistribution study in cortical bones of beagle dogs. In Metals in Bone (N.D. Priest, ed., MTP Press, Lancaster) $199-210$.

International Commission on Radiological Protection 1968. Review of the Radiosensitivity of the Tissues in Bone. ICRP Publication 11, Pergamon Press, New York.

International Commission on Radiological Protection 1972. Metabolism of Compounds of Plutonium and other Actinides. ICRP Publication 19, Pergamon Press, New York.

$[111,112]$

International Commission on Radiological Protection 1979. Limits for Intakes of Radionuclides by Workers. Part 1. ICRP Publication 30 (Part 1), Pergamon Press, New York.

$[111,112]$

- International Commission on Radiological Protection 1980. Biological Effects of Inhaled Radionuclides. ICRP Publication 31, Pergamon Press, New York. $[112,174,175,176]$

International Commission on Radiological Protection 1980b. Limits for Intakes of Radionuclides by Workers. Part 2. ICRP Publication 30 (Part 2), Pergamon Press, New York.

International Commission on Radiological Protection 1981. Limits for Intakes of Radionuclides by Workers. Part 3. ICRP Publication 30 (Part 3), Pergamon Press, New York.

$[111,112]$

International Commission on Radiological Protection 1986. The Metabolism of Plutonium and Related Elements. ICRP Publication 48, Pergamon Press, New York.

- James A.C. and Kember N.F. 1972. Dose measurements from skeletal plutonium compared in mammalian species. In Radiobiology of Plutonium (B.J. Stover and W.S.S. Jee, eds., JW Press, Salt Lake City) 281-291.

[117] 
Jaroslow B.N., Suhrbier K.M. and Fritz T.E. 1974. Decline and restoration of antibody-forming capacity in aging beagle dogs. J. Immunol. 112:14671476.

"Web" Jee, working first as a graduate student with Jim Arnold, has been concerned with radionuclides in bone, at the University of Utah, since 1952. He has been particulary interested in radionuclide and radiation-dose distribution on a cellular scale, and on the physiogical explanations of both carcinogenic and noncarcinogenic effects of radiation in bones and teeth. He was acting director of the Utah project from 1974 until 1979.

- Jee W.S.S. and Arnold J.S. 1960. Radioisotopes in the teeth of dogs-I. The distribution of plutonium, radium, radiothorium, mesothorium and strontium and the sequence of histopathologic changes in teeth containing plutonium. Arch. Oral Biol. 2:215-238. [37, 42, 44, 45, 53, 70, 127, 131, 137, 139]

- Jee W.S.S. and Arnold J.S. 1960b. Effect of internally deposited radioisotopes upon blood vessels of cortical bones. Proc. Soc. Exp. Biol. Med. 105:351-356.

$[53,69,121,127,131,137]$

- Jee W.S.S. and Arnold J.S. 1960c. India ink-gelatin vascular injection of skeletal tissues. Stain Tech. 35:59-65. $[69,121]$

- Jee W.S.S. and Arnold J.S. 1961. The toxicity of plutonium deposited in skeletal tissues of beagles. I. The relation of the distribution of plutonium to the sequence of histopathologic bone changes. Lab. Invest. 10:797-825.

$[28,69,121,127]$

- Jee W.S.S., Stover B.J., Taylor G.N. and Christensen W.R. 1962. The skeletal toxicity of $\mathrm{Pu}^{239}$ in adult beagles. Health Phys. 8:599-607. [74, 127]

- Jee W.S.S. 1962b. Histopathological endpoints in compact bones receiving alpha irradiation. In Some Aspects of Internal Irradiation (T.F. Dougherty et al., eds., Pergamon Press, Oxford) 95-116.

$[69,121,127,131,134,137]$

- Jee W.S.S., Arnold J.S., Cochran T.H., Twente J.A. and Mical R.S. 1962c. Relationship of microdistribution of alpha particles to damage. In Some Aspects of Internal Irradiation (T.F. Dougherty et al., eds., Pergamon Press, Oxford) 27-45.

$[53,69,127,131]$

Jee W.S.S. and Nolan P.D. 1963. Origin of osteoclasts from the fusion of phagocytes. Nature 200:225-226.

[121]

- Jee W.S.S. 1964. A critical survey of the analysis of microscopic distribution of some bone-seeking radionuclides and assessment of absorbed dose. In Assessment of Radioactivity in Man, Vol. I (International Atomic Energy Agency, Vienna) 369-393.

$[53,54,127,131,137]$

Jee W.S.S. and Mical R.S. 1964b. Quantitative studies of trabecular bone remodeling. Anat. Rec. 148:294.

[121]

Jee W.S.S. 1964c. The influence of reduced local vascularity on the rate of internal reconstruction of adult long bone cortex. In Bone Biodynamics (H.M. Frost, ed., Little Brown, Boston) 259-277.

[121] 
- Jee W.S.S., Dockum N.L., Mical R.S., Arnold J.S. and Looney W.B. 1967. Distribution of thorium daughters in bone. Ann. NY Acad. Sci. 145:660-673.

- Jee W.S.S., Bartley M.H., Dockum N.L., Yee J. and Kenner G. H. 1969. Vascular changes in bones following bone-seeking radionuclides. In Delayed Effects of Bone-Seeking Radionuclides (C.W. Mays et al., eds., Univ. of Utah Press, Salt Lake City) 437-455.

$[69,121,127,131,134,137]$

- Jee W.S.S., Bartley M.H. Jr., Cooper R.R. and Dockum N.L. 1970. Skeletal system. C. Bone structure [in the beagle]. In The Beagle as an Experimental Dog (A.C. Andersen, ed., Iowa State Univ. Press, Ames, Iowa) 162-188.

- Jee W.S.S. 1971. Bone-seeking radionuclides and bone. In Pathology of Irradiation (C.C. Berdjis, ed., Williams and Wilkins, Baltimore) 186-212.

$[53,54,69,119,121,127,131,134,137,139]$

- Jee W.S.S. 1972. Distribution and toxicity of ${ }^{239} \mathrm{Pu}$ in bones. Health Phys. 22:583-595.

$[54,69,75,119]$

Jee W.S.S., Dell R.B. and Miller L.G. 1972b. High resolution neutron-induced autoradiography of bone containing ${ }^{239} \mathrm{Pu}$. Health Phys. 22:761-763.

- Jee W.S.S. 1972c. ${ }^{239} \mathrm{Pu}$ in bones as visualized by photographic and neutron-induced autoradiography. In Radiobiology of Plutonium (B.J. Stover and W.S.S. Jee, eds., JW Press, Salt Lake City) 171-193.

$[28,53,54,127]$

- Jee W.S.S. (ed.). 1976. The Health Effects of Plutonium and Radium, JW Press, Salt Lake City.

- Jee W.S.S., Atherton D.R., Bruenger F., Dougherty J.H., Lloyd R.D., Mays C..W., Nabors C.J. Jr., Stevens W., Stover B.J., Taylor G.N. and Woodbury L.A. 1976 b. Current status of Utah long-term $239 \mathrm{Pu}$ studies. In Biological and Environmental Effects of Low-Level Radiation. Vol. II (International Atomic Energy Agency, Vienna) 79-94.

$[43,46,55,63,74,75,77,114,126,147,150]$

Jee W.S.S. and Kimmel D.B. 1977. Bone cell origin at the endosteal surface. In Bone Histomorphometry, Second International Workshop (P. Meunier ed., Armour Montagu, Paris) 113-132.

[121]

Jee W.S.S. and Kimmel D.B. 1980. Evaluation of permissible exposure to plutonium in occupational workers. In Health Implications of New Energy Technologies (W.N. Rom and V.E. Archer, eds., Ann Arbor Science, Ann Arbor) 445-58.

$[111,112,117]$

- Jee W.S.S., Wronski T.J., Smith J.M., Kimmel D.B., Miller S.C. and Stover B.J. 1981. Selection of bone samples for ${ }^{239} \mathrm{Pu}$ analyses in man. In Actinides in Man and Animals (M.E. Wrenn, ed., RD Press, Salt Lake City) 407-412.

[108]

Jee W.S.S. 1981b. Research use of dogs and cats. Skeletal system (pp. 190-196). Joints (p. 197). Comparative models of selected problems of the human elderly; gingivitis and periodontitis, degenerative joint disease (pp. 352-358); osteoporosis (pp. 358-365). In Mammalian Models for Research in Aging (National Academy Press, Washington, DC). 
- Jee W.S.S., Dell R.B., Parks N.J., Miller S.C. and Wrenn M.E. 1985. Toxicity of plutonium and americium: Relationship of bone composition to location of ${ }^{226} \mathrm{Ra},{ }^{239} \mathrm{Pu}$ and ${ }^{241} \mathrm{Am}$-induced bone sarcomas. In Metals in Bone (N.D. Priest, ed., MTP Press, Lancaster) 155-174.

$[76,120]$

- Jee W.S.S., Parks N.J., Miller S.C. and Dell R.B. 1986. Relationship of bone composition to the location of radium-induced bone cancer. In The Radiobiology of Radium and Thorotrast (W. Gossner et al., eds., Urban and Schwarzenberg, Munich) 75-78.

$[76,120]$

Jochle W., Tomlinson R.V. and Andersen A.C. 1973. Prostaglandin effects on plasma progesterone levels in the pregnant and cycling dog (beagle). Prostaglandins 3:209-217.

Jochle W., Lamond D.R. and Andersen A.C. 1975. Mestranol as an abortifacient in the bitch. Theriogenology 4:1-9.

Jochle W. and Andersen A.C. 1977. The estrous cycle in the dog: A review. Theriogenology 7:113-140.

Johnson L.J., Watters R.L., Lagerquist C.R. and Hammond S.E. 1970. Relative distribution of plutonium and americium following experimental $\mathrm{PuO}_{2}$ implants. Health Phys. 19:743-749.

Johnson R.M. and Andersen A.C. 1961. A self-waterer for dogs. J. Am. Vet. Med. Assoc. 138:639-640.

Johnson R.M., Andersen A.C. and Gee W. 1963. Parasitism in an established dog kennel. Lab. Anim. Care 13:731-736.

Jones C.W., Mays C.W., Taylor G.N., Lloyd R.D. and Packer S.M. 1986. Reducing the cancer risk of ${ }^{239} \mathrm{Pu}$ by chelation therapy. Radiat. Res. 107:296-306.

- Jones R.K., Hahn F.F., Hobbs C.H., Benjamin S.A., Boecker B.B., McClellan R.O. and Slauson D.O. 1974. Pulmonary carcinogenesis and chronic beta irradiation of lung. In Experimental Lung Cancer: Carcinogenesis and Bioassays (E.Karbe and J.F. Park, eds. Springer-Verlag, New York) 454-467.

$[40,41,77,191,200]$

- Jones R.K., Boecker B.B., Pickrell J.A., Hobbs C.H. and McClellan R.O. 1976. Influence of radiation-dose pattern from inhaled beta-gamma-emitting radionuclides on canine peripheral lymphocytes. In Radiation and the Lymphatic System (J.E. Ballou, ed., CONF-740930, NTIS, Springfield, VA) 83-89.

$[61,66,191,196,198,199]$

Kanapilly G.M., Raabe O.G., Goh C.H.T. and Chimenti R.A. 1973. Measurement of in vitro dissolution of aerosol particles for comparison to in vivo dissolution in the lower respiratory tract after inhalation. Health Phys. 24:497-507.

Kaspar L.V. and Norris W.P. 1969. Fixation of cesium-137 by urinary calculi. Health Phys. 16:798-800. 
Kaspar L.V. and Norris W.P. 1977. Serum chemistry values of normal dogs (beagles): Associations with age, sex, and family line. Lab. Anim. Sci. 27:980- 985 .

$[16,66]$

Kaspar L.V., Poole C.M. and Norris W.P. 1978. Incidence of struvite urinary calculi in two ancestral lines of beagles. Lab. Anim. Sci. 28:545-550.

Kaspar L.V. and Seed T.M. 1984. CFU-GM colony-enhancing activity in sera of dogs under acute and chronic gamma-irradiation regimens. Acta Haematol. 71:189-197.

$[95,121]$

Keen C.L., Lonnerdal B. and Fisher G.L. 1981. Age-related variations in hepatic iron, copper, zinc and selenium concentrations in beagles. Am. J. Vet. Res. 42:1884-1887.

- Kelly G., Kerkof P.R., Brooks A.L. and Haley P.J. In Press. Oncogene activation in radiation-induced lung tumours. In Proceedings of the L.H. Gray Conference on Low Dose Radiation Biological Bases of Risk Assessment. Symposium held in Oxford, September 11-15, 1988.

$[11,29,78,121]$

- Kimmel D.B., Jee W.S.S., Wronski T.J., Atherton D.R., Schlenker R.A. and Stover B.J. 1976. A morphometric and autoradiographic study of the ulna and second lumbar vertebral body of young adult beagles. In Health Effects of Plutonium and Radium (W.S.S. Jee, ed., JW Press, Salt Lake City) 105-126.

$[54,76,119,121,127]$

Kimmel D.B. and Jee W.S.S. 1977. Morphometric measurements in tissue bands isometric to an irregular reference line. In Bone Histomorphometry, Second International Workshop (P. Meunier, ed., Armour Montagu, Paris) 97-102.

[121]

- Kimmel D.B. and Jee W.S.S. 1982. A quantitative histologic study of bone turnover in young adult beagles. Anat. Rec. 203:31-45.

$[76,103,121,127]$

King R.R., Mauderly J.L., Hahn F.F., Wolff R.K., Muggenburg B.A. and Pickrell J.A. 1985. Pulmonary function studies in a dog with pulmonary thromboembolism associated with Cushing's disease. J. Am. Anim. Hosp. Assoc. 21:555-562.

[104]

Klein A.K., Dyck J.A., Stitzel K.A., Shimizu J., Fox L.A. and Taylor N. 1983. Characterization of canine fetal lymphohematopoiesis: Studies of CFU-GM, CFU-L and CFU-F. Exp. Hematol. 11:263-274.

Klein A.K., Lynch J.A., Dyck J.A., Shimizu J.A., Fox L.A. and Stitzel K.A. 1984. The influence of fibroblast-like cells derived from canine fetal hematopoietic tissues on the regulation of lymphohematopoiesis. Int. J. Cell Cloning $2: 20-33$

$[10,95]$

- Klein A.K., Rosenblatt L.S., Stitzel K.A., Greenberg B. and Woo L. 1984b. In vitro radiation response studies on bone marrow fibroblasts (CFU-F) obtained from normal and chronically irradiated dogs. Leuk. Res. 8:473- 481.

- Klein A.K., Dyck J.A., Shimizu J.A., Stitzel K.A., Wilson F.D. and Cain G.R. 1985. Effect of continuous, whole-body gamma irradiation upon canine lymphohematopoietic (CFU-GM, CFU-L) progenitors and a possible hematopoietic regulatory population. Radiat Res. 10l:332-350. 
Knox S. and Shifrine M. 1979. Cell-mediated immunity in the dog in relation to disease: A review. Comp. Immunol. Microbiol. Infect. Dis. 2:405-415.

Knox S.J., Wilson F.D., Miller C.H., Rosenblatt L.S. and Shifrine M. 1981. Growth of macrophage colonies from normal canine peripheral blood: Morphological, cytochemical and functional parameters. Stem Cells 1:325-344.

- Kotrappa P. and Moss O.R. 1971. Production of relatively monodisperse aerosols for inhalation experiments by aerosol centrifugation. Health Phys. 21:531-535.

- Kotrappa P., Wilkinson C.J. and Boyd H.A. 1972. Technology for the production of monodisperse aerosols of oxides of transuranic elements for inhalation experiments. Health Phys. 22:837-843.

Wright Langham, at Los Alamos during the war years, was responsible for some of the first biological experiments with plutonium in rats and in humans. He, more than any other person, was insistent that life-span studies were required in large animals; an insistence that led to the original beagle studies at the University of Utah. Wright, along with Austin Brues and Robley Evans, were known as the "Founding Fathers" of these experiments, in which they played an important planning role. He served for many years as a consultant to the Utah program.

Langham W.H. 1959. Physiology and toxicology of plutonium-239 and its industrial medical control. Health Phys. 2:172-185.

Langham W.H., Lawrence J.N.P., McClelland J. and Hempelmann L.H. 1962. The Los Alamos scientific laboratory's experience with plutonium in man. Health Phys. 8:753-760.

Langham W.H. 1964. Physiological properties of plutonium and assessment of body burden in man. In Assessment of Radioactivity in Man. Vol. 2 (International Atomic Energy Agency, Vienna) 565-581.

Langham W.H. 1969. Biological Considerations of Nonnuclear Incidents Involving Nuclear Warheads. UCRL-50639, NTIS, Springfield, VA.

Leach L.J., Spiegl C.J., Wilson R.H., Sylvester G.E. and Lauterbach K.E. 1959. A multiple chamber exposure unit designed for chronic inhalation studies. Am. Ind. Hyg. Assoc. J. 20:13-22.

Leach L.J., Maynard E.A., Hodge H.C., Scott J.K., Yuile C.L., Sylvester G.E. and Wilson H.B. 1970. A five-year inhalation study with natural uranium dioxide ( $\mathrm{UO}_{2}$ ) dust- $\mathrm{I}$. Retention and biologic effect in the monkey, dog and rat. Health Phys. 18:599-612.

$[24,93,99]$

Lebel J.L., Bull E.H., Johnson J.L. and Watters R.L. 1970. Lymphosarcoma associated with nodal concentration of plutonium in dogs: A preliminary report. Am. J. Vet. Res. 31:1513-1516. 
Lebel J.L., Bistline R.W., Schallberger J.A., Dagle G.E. and Gomez L.S. 1976. Studies of plutonium and the lymphatic system: Six years of progress at Colorado State University. In Radiation and the Lymphatic System (J.E. Ballou, ed., CONF-740930, NTIS, Springfield, VA) 2-9.

$[4,20,93]$

Leeds S.E. 1976. The pulmonary lymphatics and radiation. In Radiation and the Lymphatic System (J.E. Ballou, ed., CONF-740930, NTIS, Springfield, VA) $36-45$.

Leggett R.W. and Eckerman K.F. 1984. Model for the age-dependent skeletal retention of plutonium. In Radiation-Risk-Protection, Vol. I (A. Kaul et al., eds., Int. Radiat. Protect. Assoc., Berlin) 454-457.

[111]

Leggett R.W., Eckerman K.F., Dunning D.E.Jr., Cristy M., CrawfordBrown D.J. and Williams L.R. 1984b. On Estimating Dose Rates to Organs as a Function of Age Following Internal Exposure to Radionuclides. ORNL/TM-8265, NTIS, Springfield, VA.

[111]

Leggett R.W. 1984c. Bioassay Data and a Retention-Excretion Model for Systemic Plutonium. NUREG/CR-3346 (ORNL/TM-8795), NTIS, Springfield, VA.

[108]

Leggett R.W. 1985. A model of the retention, translocation and excretion of systemic Pu. Health Phys. 49:1115-1137.

$[108,111]$

Leggett R.W. and Eckerman K.F. 1987. A method for estimating the systemic burden of Pu from urinalyses. Health Phys. 52:337-346.

[108]

Leonard A., Decat G. and Fritz T.E. 1982. Radiosensitivity and life span of dog peripheral blood lymphocytes. Mutat. Res. 92:257-263.

Levy J., Meade G.H. and Clapper W.E. 1969. Antigenicity of canine tissue cultures. Proc. Soc. Exp. Biol. Med. 132:478-483.

Lindenbaum A. and Rosenthal M.W. 1972. Deposition patterns and toxicity of plutonium and americium in liver. Health Phys. 22:597-605.

- Lloyd E. and Hodges D. 1971. Quantitative characterization of bone: A computer analysis of microradiographs. Clin. Orthop. 78:230-250.

$[54,117]$

- Lloyd E. and Marshall J.H. 1972. Toxicity of ${ }^{239} \mathrm{Pu}$ relative to ${ }^{226} \mathrm{Ra}$ in man and dog. In Radiobiology of Plutonium (B.J. Stover and W.S.S. Jee, eds., JW Press, Salt Lake City) 377-383.

Ray Lloyd has been involved, since 1961 , in the radionuclide retention and dosimetry aspects of all of the University of Utah life-span beagle studies. He has also been involved in a variety of ancillary beagle studies, designed to improve dosimetric interpretations, and to develop practical procedures for the therapeutic removal of inter. nally deposited radionuclides.

- Lloyd R.D., Mays C.W., Fisher W. and Hintze R. 1962. Total body gammaray counting of $\mathrm{Pu}^{239}$ in beagles. Health Phys. 8:777-780.

- Lloyd R.D., Mays C.W. and Atherton D.R. 1963. ${ }^{90} \mathrm{Sr}+{ }^{90} \mathrm{Y}$ bremsstrahlung efficiency in bone. Int. J. Radiat. Biol. 7:339-351.

$[37,139]$

- Lloyd R.D., Mays C.W., Taylor G.N. and Atherton D.R. 1970. Americium-241 studies in beagles. Health Phys. 18:149-156. 
- Lloyd R.D., Mays C.W., Taylor G.N. and Williams J.L. 1972. Californium excretion and retention by beagles injected with ${ }^{249} \mathrm{Cf}$ or ${ }^{252} \mathrm{Cf}$. Health Phys. $22: 667-673$.

$[48,49,52,143,145]$

- Lloyd R.D., Jee W.S.S., Atherton D.R., Taylor G.N. and Mays C.W. $1972 b$. Americium-241 in beagles: Biological effects and skeletal distribution. In Radiobiology of Plutonium (B.J. Stover and W.S.S. Jee, eds., JW Press, Salt Lake City) 141-148. $[48,52,55,63,64,71,141,142]$

Lloyd R.D., Atherton D.R., Mays C.W., McFarland S.S. and Williams J.L. 1974. The early excretion, retention and distribution of injected curium citrate in beagles. Health Phys. 27:61-67.

$[101,102]$

- Lloyd R.D., Dockum J.G., Atherton D.R., Mays C.W. and Williams J.L. 1975. The early retention, excretion and distribution of injected einsteinium citrate in beagles. Health Phys. 28:585-589.

$[10,49,149]$

Lloyd R.D., McFarland S.S., Taylor G.N., Williams J.L. and Mays C.W 1975b. Decorporation of ${ }^{241} \mathrm{Am}$ in beagles by DTPA. Radiat. Res. 62:97-106.

$[87,113]$

Lloyd R.D., Taylor G.N., Mays C.W., McFarland S.S. and Atherton D.R. 1975c. DTPA therapy of ${ }^{241} \mathrm{Am}$ from a simulated wound site. Health Phys. 29:808-811.

$[87,113]$

- Lloyd R.D. and Mays C.W. 1975d. Determining liver retention of transuranium elements in living beagles. Radiat. Environ. Biophys. 12:139-145.

$[47,48,102,141,143,145]$

- Lloyd R.D., Mays C.W., Atherton D.R. and Bruenger F.W. 1975 e. ${ }^{210} \mathrm{~Pb}$ studies in beagles. Health Phys. 28:575-583.

- Lloyd R.D., Mays C.W., McFarland S.S., Atherton D.R. and Williams J.L. 1976. Californium studies in beagles. Radiat. Res. 65:462-473.

$[10,48,49,74,110,111,143,145]$

- Lloyd R.D., Atherton D.R., McFarland S.S., Mays C.W., Stevens W., Williams J.L. and Taylor G.N. 1976b. Studies of injected ${ }^{237} \mathrm{Pu}(\mathrm{IV})$ citrate in beagles. Health Phys. 30:47-52.

$[44,127]$

Lloyd R.D., Mays C.W., McFarland S.S., Taylor G.N. and Atherton D.R. $1976 \mathrm{c}$. A comparison of Ca-DTPA and Zn-DTPA for chelating ${ }^{241} \mathrm{Am}$ in beagles. Health Phys. 31:281-284.

Lloyd R.D. 1976d. $^{237} \mathrm{Pu}$ studies in beagles. Applicability to environmental ${ }^{239} \mathrm{Pu}$ problems. Health Phys. 31:186-187.

$[23,44]$

- Lloyd R.D., Mays C.W. and Atherton D.R. 1976e. Distribution of injected ${ }^{226} \mathrm{Ra}$ and ${ }^{90} \mathrm{Sr}$ in the beagle skeleton. Health Phys. 30:183-189.

$[51,130,131,139]$

Lloyd R.D., Mays C.W., Atherton D.R. and Taylor G.N. 1976f. ${ }^{90} \mathrm{Sr}+{ }^{90} \mathrm{Y}$ bremsstrahlung efficiency predicted for humans. Radiat. Environ. Biophys. 13:229-237.

[113]

- Lloyd R.D., Taysum D.H. and Mays C.W. 1976g. Design and calibration of a total-body counting system for measuring radioactivity in beagles. Int. J. Appl. Radiat. Isotopes 27:103-111.

$[28,37,42,47,130,133,137,139,141]$ 
- Lloyd R.D., Mays C.W., Atherton D.R., Taylor G.N. and Van Dilla M.A. 1976h. Retention and skeletal dosimetry of injected ${ }^{226} \mathrm{Ra},{ }^{228} \mathrm{Ra}$, and ${ }^{90} \mathrm{Sr}$ in beagles. Radiat. Res. 66:274-287. $[37,42,130,133,139]$

Lloyd R.D., Taylor G.N., Boseman J.J., Mays C.W. and Atherton D.R. 1977. Further comparison of Ca-DTPA and Zn-DTPA for removal of ${ }^{241} \mathrm{Am}$ from beagles. Health Phys. 33:92-94.

- Lloyd R.D., McFarland S.S., Atherton D.R., Bruenger F.W., Taylor G.N. and Mays C.W. 1978. Early retention of ${ }^{237} \mathrm{Pu}+{ }^{239} \mathrm{Pu}$ in mature beagles. Health Phys. 35:211-215.

$[47,150]$

Lloyd R.D., Boseman J.J., Taylor G.N., Bruenger F.W., Atherton D.R., Stevens W. and Mays C.W. 1978b. Decorporation from beagles of a mixture of monomeric and particulate plutonium using Ca-DTPA and Zn-DTPA: Dependence upon frequency of administration. Health Phys. 35:217-227. [87]

- Lloyd R.D., McFarland S.S., Atherton D.R. and Mays C.W. 1978c. Plutonium retention, excretion, and distribution in juvenile beagles soon after injection. Radiat. Res. 75:633-641.

$[47,52,147]$

Lloyd R.D., Taylor G.N., Mays C.W., Jones C.W., Bruenger F.W and Atherton D.R. 1979. Dependency of chelation efficacy upon time after first DTPA injection. Radiat. Res. 78:448-454.

Lloyd R.D., Jones C.W., Taylor G.N., Mays C.W. and Atherton D.R. 1979b. $\mathrm{Pu}$ and Am decorportation in beagles: Effects of magnitude of initial Ca-DTPA injection upon chelation efficacy. Radiat. Res. 79:630-634.

- Lloyd R.D., Mays C.W., Taylor G.N., Atherton D.R., Bruenger F.W. and Jones C.W. 1982. Radium-224 retention, distribution, and dosimetry in beagles. Radiat Res. 92:280-295.

$[10,42,43,51,154]$

- Lloyd R.D., Jones C.W., Bruenger F.W., Atherton D.R. and Mays C.W. 1983. Radium retention and dosimetry in juvenile beagles. Radiat. Res. 94:295-304. Erratum 96:218.

$[43,51,151]$

- Lloyd R.D., Taylor G.N., Jones C.W. and Mays C.W. 1983b. Radium retention and dosimetry in the St. Bernard. Radiat. Res. 95:150-157.

$[43,51,131]$

- Lloyd R.D., Bruenger F.W., Jones C.W., Taylor G.N. and Mays C.W. 1983c. Radium retention in mature beagles injected at 5 years of age. Radiat. Res. 94:210-216.

$[43,51,153]$

- Lloyd R.D., Bruenger F.W., Mays C.W. and Jones C.W. 1983d. Skeletal radon-to-radium ratios in neonatal, juvenile and mature beagles and in adult St. Bernards. Health Phys. 44:61-63. $[42,43,130,131,151,153]$

Lloyd R.D., Bruenger F.W., Mays C.W., Atherton D.R., Jones C.W., Taylor G.N., Stevens W., Durbin P.W., Jeung N., Jones E.S., Kappel M.J., Raymond K.N. and Weitl F.L. 1984. Removal of Pu and Am from beagles and mice by 3,4,3-LICAM(C) or 3,4,3-LICAM(S). Radiat. Res. 99:106-128.

[88]

- Lloyd R.D., Jones C.W., Taylor G.N., Bruenger F.W., Mays C.W. and Wrenn M.E. 1984 b. Plutonium-237 and ${ }^{239} \mathrm{Pu}$ retention in a St. Bernard. Health Phys. 47:629-631.

$[46,128]$ 
- Lloyd R.D., Mays C.W., Jones C.W., Atherton D.R., Bruenger F.W., Shabestari L.R. and Wrenn M.E. 1984c. Retention and dosimetry of injected ${ }^{241} \mathrm{Am}$ in beagles. Radiat. Res. 100:564-575.

$[47,48,52,141]$

- Lloyd R.D., Jones C.W., Mays C.W., Atherton D.R., Bruenger F.W. and Taylor G.N. 1984 d. ${ }^{228}$ Th retention and dosimetry in beagles. Radiat. Res. 98:614-628.

$[43,44,49,52,136,137]$

- Lloyd R.D., Wrenn M.E., Taylor G.N., Mays C.W., Jee W.S.S., Bruenger R.W., Miller S.C. and Paschoa A.S. 1986. Toxicity of ${ }^{228}$ Ra and ${ }^{228}$ Th relative to ${ }^{226} \mathrm{Ra}$ for bone sarcoma induction in beagles. In The Radiobiology of Radium and Thorotrast (W. Gossner et al., eds., Urban and Schwarzenberg, Munich) 65-69.

$[74,109,133,134,136,137]$

- Lloyd R.D., Mays C.W. and Atherton D.R. 1986b. Skeletal dosimetry of injected ${ }^{226} \mathrm{Ra}$ in young adult beagles. In The Radiobiology of Radium and Thorotrast (W. Gossner et al., eds., Urban and Schwarzenberg, Munich) 70-74.

$[42,130,131]$

Long A.B. 1971. Plutonium inhalation: The burden of negligible consequence. Nucl. News 14 (6):69-73.

[111]

Lovins A.B. and Patterson W.C. 1975. Plutonium particles: Some like them hot. Nature 254:278-280.

[107]

Luick J.R., Parker H.R. and Andersen A.C. 1960. Composition of beagle dog milk. Am. J. Physiol. 199:731-732.

Lund J.E. and Park J.F. 1978. Focal mastocytosis in lymph nodes from a beagle dog. Vet. Pathol. 15:64-67.

Lundgren D.L., Clapper W.E. and Sanchez A. 1968. Isolation of human enteroviruses from beagle dogs. Proc. Soc. Exp. Biol. Med. 128:463-467.

Lundgren D.L. and Thorpe B.D. 1968b. A serological survey of dogs, native rodents and humans in New Mexico. Bull. Wildlife Dis. Assoc. 4:140-141.

Lundgren D.L. and Clapper W.E. 1969. Neutralization of canine herpesvirus by dog and human serums: A survey. Am. J. Vet. Res. 30:479-482. [16]

Lundgren D.L., Magnuson M.G. and Clapper W.E. 1969b. A serological survey in dogs for antibody to human respiratory viruses. Lab. Anim. Care 19:352-359.

Lundgren D.L., Sanchez A., Magnuson M.G. and Clapper W.E. 1970. A survey for human enteroviruses in dogs and man. Arch. Gesam. Virusforsch. 32:229-235.

Lundgren D.L., Hobbs C.H. and Clapper W.E. 1971. Experimental infection of beagle dogs with Coxsackievirus Type B1. Am. J. Vet. Res. 32:609-613. 
- Madewell B.R., Pool R.R. and Leighton R.L. 1977. Osteogenic sarcoma at the site of a chronic nonunion fracture and internal fixation device in a dog. J. Am. Vet. Med. Assoc. 171:187-189.

Magrane W.G. 1970. Clinical aspects of the eye [of the beagle]. A. The adnexa and anterior portion of the eye. In The Beagle as an Experimental Dog (A.C. Andersen, ed., Iowa State Univ. Press, Ames, Iowa) 597-599.

Mahaffey J.A., Sanders C.L., Dagle G.E. and Park J.F. 1981. A comparison of $\mathrm{Pu}$ metabolism and pulmonary tumors in dogs and rats exposed to ${ }^{239} \mathrm{PuO}_{2}$. In Actinides in Man and Animals (M.E. Wrenn, ed., RD Press, Salt Lake City) 541-555.

$[35,46,82,115,174,175]$

Marshall J.H. 1969. The retention of radionuclides in bone. In Delayed Effects of Bone-Seeking Radionuclides (C.W. Mays et al., eds., Univ. of Utah Press, Salt Lake City) 7-27.

$[51,109,119]$

Marshall J.H. and Lloyd E. 1973. The effect of the remodelling of bone upon the relative toxicities of radium and plutonium in man and dog. In Radionuclide Carcinogenesis (C.L. Sanders et al., eds., CONF-720505, NTIS, Springfield, VA) 421-436.

$[109,127,128,131]$

Martin R.K., Albright J.P., Jee W.S.S., Taylor G.N. and Clarke W.R. 1981. Bone loss in the beagle tibia: Influence of age, weight, and sex. Calcif. Tissue Int. 33:233-238.

Mason M.J., Gillett N.A. and Muggenburg B.A. 1987. Clinical, pathological, and epidemiological aspects of canine parvoviral enteritis in an unvaccinated closed beagle colony: 1978-1985. J. Am. Anim. Hosp. Assoc. 23:183-192.

Joe Mauderly, a veterinarian at Inhalation Toxicology Research Institute since 1969, has pioneered in the development of procedures for study of pulmonary function. These procedures allow noninvasive assessment of functional changes related to radiation doses delivered to the respiratory tract and contiguous tissues.

Mauderly J.L. 1972. Steady-state carbon monoxide-diffusing capacity of unanesthetized beagle dogs. Am J. Vet. Res. 33:1485-1491.

- Mauderly J.L., Pickrell J.A., Hobbs C.H., Benjamin S.A., Hahn F.F., Jones R.K. and Barnes J.E. 1973. The effects of inhaled ${ }^{90} \mathrm{Y}$ fused clay aerosol on pulmonary function and related parameters of the beagle dog. Radiat. Res. 56:83-96.

Mauderly J.L. and Pickrell J.A. 1973b. Pulmonary function testing of unanesthetized beagle dogs. In Research Animals in Medicine (L.T. Harrison ed., NIH 72-333, NTIS, Springfield, VA) 665-669.

Mauderly J.L. 1974. Influence of sex and age on the pulmonary function of the unanesthetized beagle dog. J. Gerontol. 29:282-289.

- Mauderly J.L., Mewhinney J.A. and Boecker B.B. 1980. A comparison of the effects of inhaled alpha- and beta-emitting radionuclides on pulmonary function in the dog. In Pulmonary Toxicology of Respirable Particles (C.L. Sanders et al., eds., CONF-791002, NTIS, Springfield, VA) 404-419.

$[61,62,191,209]$ 
- Mauderly J.L., Muggenburg B.A., Hahn F.F. and Boecker B.B. 1980 b. The effects of inhaled ${ }^{144} \mathrm{Ce}$ on cardiopulmonary function and histopathology of the dog. Radiat. Res. 84:307-324.

$[16,61,191]$

Mauderly J.L., Silbaugh S.A., Likens S.A., Harkema J.R. and Johnson W.K. 1983. Comparisons of the respiratory functional responses of animals and man. In Proceedings, 13 th Conference on Environmental Toxicology (AFAMRL-TR-82-101, NTIS, Springfield, VA) 147-170.

"Chuck" Mays was the principal radiological physicist for the University of Utah life-span beagle studies, from 1957 until 1988. As such, he was involved with radiation measurement problems and with the dosimetric interpretation of these measurements. More recently he has become involved with the interpretation of dose-response relationships and with the employment of chelating agents for therapeutic removal of internally deposited radionuclides.

- Mays C.W., Van Dilla M.A., Floyd R.L. and Arnold J.S. 1958. Radon retention in radium-injected beagles. Radiat. Res. 8:480-489.

$[42,130]$

- Mays C.W., Haldin R. and Van Dilla M.A. 1958b. Thoron exhalation in radiothorium-burdened beagles. Radiat. Res. 9:438-444. [42, 43, 133, 137]

- Mays C.W., Taysum D.H., Fisher W. and Glad B.W. 1958c. Bremsstrahlung counting of $\mathrm{Sr}^{90}$ injected dogs. Health Phys. 1:282-287. [37, 139]

Mays C.W. 1959. Radionuclides and bone cancer. Science 130:1600. [107]

- Mays C.W., Dougherty T.F., Taylor G.N., Lloyd R.D., Stover B.J., Jee W.S.S., Christensen W.R., Dougherty J.H. and Atherton D.R. 1969. Radiation-induced bone cancer in beagles. In Delayed Effects of Bone-Seeking Radionuclides (C.W. Mays et al., eds., Univ. of Utah Press, Salt Lake City) 387-408.

$[65,73,82,109,126,127,128,131,134,137,140]$

- Mays C.W., Jee W.S.S., Lloyd R.D., Stover B.J., Dougherty J.H. and Taylor G.N. (eds.). 1969b. Delayed Effects of Bone-Seeking Radionuclides (Univ. of Utah Press, Salt Lake City).

[13]

- Mays C.W., Taylor G.N., Jee W.S.S. and Dougherty T.F. 1970. Speculated risk to bone and liver from ${ }^{239} \mathrm{Pu}$. Health Phys. 19:601-610.

$[79,113,128]$

- Mays C.W. and Lloyd R.D. 1972. Bone sarcoma incidence vs. alpha particle dose. In Radiobiology of Plutonium (B.J. Stover and W.S.S. Jee, eds., JW Press, Salt Lake City) 409-430. $[73,120,127,131,134,137]$

- Mays C.W. and Dougherty T.F. 1972b. Progress in the beagle studies at the University of Utah. Health Phys. 22:793-801.

$[143,145]$

- Mays C.W. and Lloyd R.D. 1972c. Bone sarcoma risk from ${ }^{90} \mathrm{Sr}$. In Biomedical Implications of Radiostrontium Exposure (M. Goldman and L.K. Bustad eds., CONF-710201, NTIS, Springfield, VA) 352-375.

$[73,107,113,120,140]$

- Mays C.W. and Lloyd R.D. 1972d. Predicted toxicity of ${ }^{90} \mathrm{Sr}$ in humans. In Second International Conference on Strontium Metabolism (CONF-720818, NTIS, Springfield, VA) 181-205.

$[107,113]$ 
- Mays C.W., Lloyd R.D. and Van Dilla M.A. 1975. Fractional radon retention in bone. Health Phys. 29:761-765.

$[42,130]$

- Mays C.W., Spiess H., Taylor G.N., Lloyd R.D., Jee W.S.S., McFarland S.S., Taysum D.H., Brammer T.W., Brammer D. and Pollard T.A. 1976. Estimated risk to human bone from ${ }^{239} \mathrm{Pu}$. In Health Effects of Plutonium and Radium (W.S.S. Jee, ed., JW Press, Salt Lake City) 343-362.

$[114,128,131]$

- Mays C.W. 1976b. Estimated risk from ${ }^{239} \mathrm{Pu}$ to human bone, liver and lung. In Biological and Environmental Effects of Low-Level Radiation, Vol. II (International Atomic Energy Agency, Vienna) 373-384. [111, 114, 128, 131]

Mays C.W., Taylor G.N., Lloyd R.D., McFarland S.S., Fisher D.R., Calder S.E. and Boseman J. 1977. DTPA research at the University of Utah. In Proceedings of the IVth International Congress, Vol. 4 (International Radiation Protection Association, Paris) 12ll-1214.

Mays C.W., Spiess H. and Gerspach A. 1978. Skeletal effects following ${ }^{224} \mathrm{Ra}$ injections into humans. Health Phys. 35:83-90.

- Mays C.W. 1980. Discussion of plutonium toxicity. In National Energy Issues: How Do We Decide? Plutonium as a Test Case (R.G. Sachs, ed., Bellinger, Cambridge, MA) 127-142.

[120]

- Mays C.W. and Finkel M.P. 1980b. RBE of alpha particles vs beta particles in bone sarcoma induction. In Book of Papers, Fifth International Congress of the International Radiation Protection Association, Vol. 2 (Israel Health Physics Society, Jerusalem) 401-405.

$[73,109,140]$

Mays C.W., Taylor G.N., Lloyd R.D. and Wrenn M.E. 1981. Status of chelation research: A review. In Actinides in Man and Animals (M.E. Wrenn, ed., RD Press, Salt Lake City) 351-368.

[86]

Mays C.W. 1982. Risk estimates for liver. In Critical Issues in Setting Radiation Dose Limits, (National Council on Radiation Protection and Measurements, Washington, DC) $182-200$.

$[79,114]$

Mays C.W., Lloyd R.D., Jones C.W., Bruenger F.W., Taylor G.N., Durbin P.W., White D. and Raymond K.N. 1986. Decorporation of Pu and Am from beagles with delayed daily injections of 3,4,3-LICAM(C) or Zn-DTPA. Health Phys. 50:530-534.

[88]

- Mays C.W., Taylor G.N. and Lloyd R.D. 1986b. Toxicity ratios: Their use and abuse in predicting the risk from induced cancer. In Life-Span Radiation Effects Studies in Animals: What Can They Tell Us? (R.C. Thompson and J.A. Mahaffey, eds., CONF-830951, NTIS, Springfield, VA) 299-310.

$[7,74,75,113,126,128,131]$

Mays C.W., Spiess H., Chmelevsky D. and Kellerer A. 1986c. Bone sarcoma cumulative tumor rates in patients injected with ${ }^{224} \mathrm{Ra}$. In The Radiobiology of Radium and Thorotrast (W. Gossner et al., eds., Urban and Schwarzenberg, Munich) [Strahlentherapie Suppl. 80:27-31].

[10]

- Mays C.W., Lloyd R.D., Taylor G.N. and Wrenn M.E. 1987. Cancer incidence and lifespan vs. alpha-particle dose in beagles. Health Phys. 52:617-624.

$[73,74,82,109,133,134,137,142]$ 
Mays C.W. 1988. Alpha-particle-induced cancer in humans. Health Phys. 55:637-652.

Roger McClellan spent 5 years working with ${ }^{90} \mathrm{Sr}$ in miniature swine at Pacific Northwest Laboratory and a year in AEC's Division of Biology and Medicine before being chosen in 1966 to direct the laboratory that would become the Inhalation Toxicology Research Institute. He served as Director of ITRI until his departure in 1988. He was a major contributor to both the fission product and transuranic studies in beagles and in other laboratory animals.

- McClellan R.O. and Jones R.K. 1969. ${ }^{90}$ Sr-induced neoplasia: A selective review. In Delayed Effects of Bone-Seeking Radionuclides (C.W. Mays et al., eds., Univ. of Utah Press, Salt Lake City) 293-322.

[119]

- McClellan R.O., Barnes J.E., Boecker B.B., Chiffelle T.L., Hobbs C.H., Jones R.K., Mauderly J.L., Pickrell J.A. and Redman H.C. 1970. Toxicity of beta-emitting radionuclides inhaled in fused clay particles-An experimental approach. In Morphology of Experimental Respiratory Carcinogenesis ( $\mathrm{P}$. Nettesheim, et al., eds., CONF-700501, NTIS, Springfield, VA) 395-415.

$[9,25,40,61,191]$

- McClellan R.O. 1970b. [Untitled comments on the toxicity of inhaled radionuclides] In Late Effects of Radiation (R.M. Fry et al., eds., Taylor and Francis, London) 225-231.

- McClellan R.O. 1972. Progress in studies with transuranic elements at the Lovelace Foundation. Health Phys. 22:815-822.

$[10,89,113,206,208,211,213,215]$

- McClellan R.O., Boyd H.A., Gallegos A.F. and Thomas R.G. 1972 b. Retention and distribution of ${ }^{244} \mathrm{Cm}$ following inhalation of ${ }^{244} \mathrm{CmCl}_{3}$ and ${ }^{244} \mathrm{CmO}_{1.73}$ by beagle dogs. Health Phys. 22:877-885.

$[101,102,111]$

- McClellan R.O., Boecker B.B., Jones R.K., Barnes J.E., Chiffelle T.L., Hobbs C.H. and Redman H.C. 1972c. Toxicity of inhaled radiostrontium in experimental animals. In Biomedical Implications of Radiostrontium Exposure (M. Goldman and L.K. Bustad, eds., CONF-710201, NTIS, Springfield, VA) $149-167$ $[33,34,38,60,61,107,139,184,185]$

- McClellan R.O., Benjamin S.A., Boecker B.B., Chiffelle T.L., Hobbs C.H., Jones R.K., Pickrell J.A. and Redman H.C. 1973. Neoplasms in dogs that inhaled ${ }^{90} \mathrm{SrCl}_{2}$. In Radionuclide Carcinogenesis (C.L. Sanders et al., eds., CONF-720505, NTIS, Springfield, VA) 215-232.

$[74,82,185]$

- McClellan R.O., Boecker B.B., Hahn F.F., Hobbs C.H., Jones R.K. and Snipes M.B. 1974. Comparative toxicity of inhaled beta-emitting radionuclides in beagle dogs. In Proceedings of the Third International Congress of the International Radiation Protection Association (W. S. Snyder, ed., CONF-730907-P1, N'TIS, Springfield, VA) 208-213.

$[40,41,61,197,199]$

- McClellan R.O., Benjamin S.A., Boecker B.B., Hahn F.F., Hobbs C.H., Jones R.K. and Lundgren D.L. 1976. Influence of variations in dose and dose rates on biological effects of inhaled beta-emitting radionuclides. In Biological and Environmental Effects of Low-Level Radiation, Vol. II. (International Atomic Energy Agency, Vienna) 3-19.

$[38,77,185,186,187,188,192,196,198,200]$ 
- McClellan R.O., Hahn F.F., Snipes M.B., Cuddihy R.G. and Boecker B.B. 1979. Metabolism and somatic effect of beta-emitting radionuclides. In Proceedings of the 6th International Congress of Radiation Research (S. Okada et al., eds., Toppan Printing, Tokyo) 894-902.

$[38,77,80,112,185,187,188,192,196,198,200]$

- McClellan R.O., Boecker B.B., Cuddihy R.G., Griffith W.C., Hahn F.F., Muggenburg B.A., Scott B.R. and Seiler F.A. 1982. Health effects from internally deposited radionuclides released in nuclear disasters. In The Control of Exposure of the Public to Ionizing Radiation in the Event of Accident or Attack (National Council on Radiation Protection and Measurements, Bethesda) 28-39.

$[62,106,118,185,186,187,188,191,192,194,196,198,200]$

- McClellan R.O., Boecker B.B., Hahn F.F., Jones R.K., Muggenburg B.A. Redman H.C. and Snipes B. 1983. Toxicity of inhaled ${ }^{90} \mathrm{SrCl}_{2}$. In Proceedings of the 7 th International Congress of Radiation Research. Vol. C. (J.J. Broerse et al., eds., Martinus Nijhoff, Amsterdam) C7-05. $\quad[38,82,184,185]$

- McClellan R.O., Boecker B.B., Hahn F.F. and Muggenburg B.A. 1986. Lovelace ITRI studies on the toxicity of inhaled radionuclides in beagle dogs. In Life-Span Radiation Effects Studies in Animals: What Can They Tell Us? (R.C. Thompson and J.A. Mahaffey, eds., CONF-830951, NTIS, Springfield, VA) $74-96$ $[25,36,77,82,107,185,187,192,196,206,208,211,213,215]$

- McClellan R.O., Boecker B.B., Hahn F.F., Muggenburg B.A, and Cuddihy R.G. 1986b. Carcinogenic effects of inhaled radionuclides. In Radiation Carcinogenesis and DNA Alterations (F.J. Burns, A.C. Upton and G. Sillini, eds., Plenum) 147-154.

[115]

McClellan R.O. 1988. Prospects for new information relevant to radiation protection from studies of experimental animals. Health Phys. 55:279-293.

McDonald K.E., Park J.F., Dagle G.E., Sanders C.L. and Olson R.J. 1975. Removal of inhaled ${ }^{239} \mathrm{PuO}_{2}$ from beagle dogs by pulmonary lavage. Health Phys. 29:804-805.

McDonald K.E., Dilley J.V., Sanders C.L. and Mahaffey J.A. 1979. The influence of DTPA on the metabolism of inhaled ${ }^{239} \mathrm{PuF}_{4}$ in beagles. Health Phys. 36:632-635. $[89,100]$

McFarland L.Z. 1970. Topographical anatomy [of the beagle]. In The Beagle as an Experimental Dog (A.C. Andersen, ed., Iowa State Univ. Press, Ames, Iowa) 106-125.

Douglas McKelvie was a veterinary pathologist with the life-span beagle studies at the University of California, Davis, from 1960 to 1968. From 1968 until 1974 he filled a similar role for the program of life-span beagle studies at Colorado State University.

McKelvie D.H. and Andersen A.C. 1963. Neonatal deaths in relation to the total production of experimental beagles to the weaning age. Lab. Anim. Care 13:725-730.

McKelvie D.H., Andersen A.C. and Parker H. 1963b. A new dog cage for hospitals and research. Petfood Industry, Jan-Feb.

McKelvie D.H. and Shultz F.T. 1964. Methods of observing and recording data in long-term studies on beagles. Lab. Anim. Care 14:118-124. $\quad[19,26]$ 
- McKelvie D.H., Shultz F.T., Parcher J.W. and Rosenblatt L.S. 1966. Random selection of beagles to maintain heterogeneity and minimize bias in a lifespan experiment. Lab. Anim. Care 16:337-344.

- McKelvie D.H., Powers S. and McKim F. 1966b. Microanalytical procedures for blood chemistry long-term study on beagles. Am J. Vet. Res. 27:1405-1412.

McKelvie D.H. and Andersen A.C. 1966c. Production and care of laboratory beagles. J. Inst. Anim. Tech. 17:25-33.

- McKelvie D.H., Powell T.J. and Lee S. 1967. Programming for computer storage, retrieval, and analyses of clinical chemistry data in a life-span study on beagles. Lab. Anim. Care 17:494-500.

- McKelvie D.H., Munn S. and Bentley S. 1968. Serum chemistry changes during early growth in beagles maintained on a diet containing ${ }^{90} \mathrm{Sr} . A m$. J. Vet. Clin. Pathol. 2:153-160.

$[67,159]$

McKelvie D.H. 1970. Care and management [of the beagle]. In The Beagle as an Experimental Dog (A.C. Andersen, ed., Iowa State Univ. Press, Ames, Iowa) 16-21.

- McKelvie D.H. 1970b. Cardiovascular system [of the beagle]. D. Blood serum chemistry. In The Beagle as an Experimental Dog (A.C. Andersen, ed., Iowa State Univ. Press, Ames, Iowa) 281-284.

McKelvie D.H., Andersen A.C., Rosenblatt L.S. and Bustad L. K. 1971. The standardized dog as a laboratory animal. In Defining the Laboratory Animal (National Academy of Sciences, Washington, DC) 263-281.

- McShane J.F., Dagle G.E. and Park J.F. 1980. Pulmonary distribution of inhaled ${ }^{239} \mathrm{PuO}_{2}$ in dogs. In Pulmonary Toxicology of Respirable Particles (C.L. Sanders et al., eds., CONF-791002, NTIS, Springfield, VA) 248-255.

Medical Research Council (London). 1975. Toxicity of Plutonium. Medical Research Council, London.

$[106,107,109,111]$

Meikle A.W., Collier E.S., Stringham J.D., Fang S-M and Taylor G.N. 1981. Elevated intranuclear dihydrotestosterone in prostatic hyperplasia of aging dogs. J. Steroid Biochem. 14:331-335.

- Mercer T.T., Tillery M.I. and Chow H.Y. 1968. Operating characteristics of some compressed-air nebulizers. Am. Ind. Hyg. Assoc. J. 29:66-78. [24]

- Mercer T.T., Tillery M.L. and Newton G.J. 1970. A multi-stage, low flow rate cascade impactor. Aerosol. Sci. 1:9-15.

Merickel B.S., Hahn F.F., Rebar C.H., Muggenburg B.A., Brownstein D.G., Rebar A.H and DeNicola D.B. 1980. Acute parvoviral enteritis in a closed beagle dog colony. Lab. Anim. Sci. 30:874-878.

$[17,103]$

Jim Mewhinney, a radiobiologist at the Inhalation Toxicology Research Institute since 1964, has been involved with modeling the distribution and retention of inhaled plutonium, americium and other radionuclides, employing data from the life-span beagle studies and ancillary short-term studies, and with the derivation of dose-response relationships from the life-span beagle data. 
- Mewhinney J.A., Muggenburg B.A., McClellan R.O. and Miglio J.J. 1976. The effect of varying physical and chemical characteristics of inhaled ptonium aerosols on metabolism and excretion. In Diagnosis and Treatment of Incorporated Radionuclides (International Atomic Energy Agency, Vienna) 87-97.

- Mewhinney J.A., Griffith W.C. and Muggenburg B.A. 1980. Proposed retention model for human inhalation exposure to ${ }^{24} \mathrm{AmO}_{2}$ In Book of Papers, 5th International Congress of the International Radiation Protection Association, Vol. II. (Israel Health Physics Soc., Jerusalem) 111-114.

$[48,111,112,118]$

- Mewhinney J.A. and Craig D.K. 1981. Studies of americium in laboratory animals. In Actinides in Man and Animals (M.E. Wrenn ed., RD Press, Salt Lake City) 427-441.

$[47,48,101,111,112]$

- Mewhinney J.A., Griffith W.C. and Muggenburg B.A. 1982. The influence of aerosol size on retention and translocation of ${ }^{241} \mathrm{Am}$ following inhalation of ${ }^{241} \mathrm{AmO}_{2}$ by beagles. Health Phys. 42:611-627. $[47,48,101,111,112]$

- Mewhinney J.A. and Griffith W.C. 1982b. Models of Am metabolism in beagles and humans. Health Phys. 42:629-644.

$[48,111,112,118]$

- Mewhinney J.A. and Diel J.H. 1983. Retention of inhaled ${ }^{238} \mathrm{PuO}_{2}$ in beagles: A mechanistic approach to description. Health Phys. 45:39-60.

$[35,36,46,111,112,206,208]$

- Mewhinney J.A. and Griffith W.C. 1983b. A tissue distribution model for assessment of human inhalation exposures to ${ }^{241} \mathrm{AmO}_{2}$. Health Phys. 44, Supp.1:537-544.

$[48,111,112,118]$

Mewhinney J.A., Eidson A.F. and Boecker B.B. 1984. Interspecies comparison of the metabolism and dosimetry of inhaled mixed oxides of plutonium and uranium. In Radiation-Risk-Protection, Vol.I (A. Kaul et al., eds., Int. Radiat. Protect. Assoc., Berlin) 490-493.

[100]

- Mewhinney J.A., Griffith W.C., Hahn F.F., Snipes M.B., Boecker B.B. and McClellan R.O. 1986. Incidence of bone cancer in beagles after inhalation of ${ }^{90} \mathrm{SrCl}_{2}$ or ${ }^{238} \mathrm{PuO}_{2}$ : Implications for estimation of risk to humans. In Life-Span Radiation Effects Studies in Animals: What Can They Tell Us? (R.C. Thompson and J.A. Mahaffey, eds., CONF-830951, NTIS, Springfield, VA) 535-555. $[29,76,82,110,116,131,184,185,207,209]$

- Mewhinney J.A., Muggenburg B.A. and Griffith W.C. In Press. Modeling the influence of age at exposure and species on the fate of inhaled ${ }^{241} \mathrm{AmO}_{2}$. Health Phys.

[101]

Michaelson S.M. 1970. Endocrine system [of the beagle]. In The Beagle as an Experimental Dog (A.C. Andersen ed., Iowa State Univ. Press, Ames Iowa) $412-449$.

Miglio J.J., Muggenburg B.A. and Brooks A.L. 1977. A rapid method for determining the relative solubility of plutonium aerosols. Health Phys. 33:449-457.

[35]

Miller C.H., Carbonell A.R., Peng R., MacKenzie M.R. and Shifrine M. 1978. Cell surface markers on canine lymphocytes. Am. J. Vet. Res. 39:1191-1194. 
Miller C.H. and MacKenzie M.R. 1980. Cell-surface markers of canine lymphocytes. In The Canine as a Biomedical Research Model: Immunological, Hematological, and Oncological Aspects_(M. Shifrine and F.D. Wilson, eds., DOE/TIC-10191, NTIS, Springfield, VA) 67-82.

Scott Miller is a relative newcomer to the life-span beagle studies at the University of Utah. Since 1977, he has been principally involved in cellular-level studies in bone and bone marrow. With cur. tailment of the Utah beagle program in 1987, he was placed in administrative charge of the effort that remained.

Miller S.C., Bowman B.M., Smith J.M. and Jee W.S.S. 1980. Characterization of endosteal bone-lining cells from fatty marrow bone sites in adult beagles. Anat. Rec. 198:163-173.

$[16,119,121]$

Miller S.C. and Jee W.S.S. 1980b. The microvascular bed of fatty bone marrow in the adult beagle. Metab. Bone Dis. and Rel. Res. 2:239-246.

$[16,76,120,121]$

- Miller S.C. 1982. Localization of plutonium-24l in the testis. An interspecies comparison using light and electron microscope autoradiography. Int. J. Radiat. Biol. 41:633-643.

- Miller S.C., Rowland H.G. and Bowman B.M. 1985. Distributions of cell populations within alpha-particle range of plutonium deposits in the rat and beagle testis. Radiat. Res. 101:102-110.

- Miller S.C., Jee W.S.S., Smith J.M. and Wronski T.J. 1986. Tissue characteristics of high- and low-incidence plutonium-induced osteogenic sarcoma sites in life-span beagles. In Life-Span Radiation Effects Studies in Animals: What Can They Tell Us? (R.C. Thompson and J.A. Mahaffey, eds., CONF830951, NTIS, Springfield, VA) 286-298.

$[75,76,119,120,121,127,128]$

Martin Minthorn retired in 1988 from his position as Director of the Health Effects Research Division of the Office of Health and Environmental Research of the Department of Energy. In that capacity, Martin was always supportive of the life-span beagle studies in spite of frequent budgetary discouragement.

- Mitchell C.E. and Longwell B.B. 1970. The effects of adrenocorticotrophin hormone and metyrapone on the excretion of urinary steroids by beagle dogs burdened with strontium-90. Radiat. Res. 41:78-88. $[67,185]$

Miyabayashi T. and Morgan J.P. 1984. Gastric empyting in the normal dog: A contrast radiographic technique. Vet. Radiol. 25(4):187-191. [16, 103]

Miyabayashi T., Morgan J.P., Atilola M.A.O. and Muhumuza L. 1986. Small intestinal empyting time in normal beagle dogs. A contrast radiographic study. Vet. Radiol. 27(5):164- 168.

$[16,103]$

Mole R.H. 1969. Endosteal sensitivity to tumor induction by radiation in different species: A partial answer to an unsolved question? In Delayed Effects of Bone-Seeking Radionuclides (C.W. Mays et al., eds., Univ. of Utah Press, Salt Lake City) 249-261.

Mike Momeni, during the early 1970s at the University of California, Davis, contributed extensively to the dosimetric interpretation of the ${ }^{90} \mathrm{Sr},{ }^{226} \mathrm{Ra}$ and ${ }^{60} \mathrm{Co}$ gamma-irradiation studies. 
Momeni M.H., Cahill T.A. and Horn P.L. 1973. Proton irradiation of beagle eyes. I. $\mathrm{Si}(\mathrm{Li})$ and thermoluminescent dosimetry of 20,35 , and $45 \mathrm{MeV}$ protons. Radiat. Res. 53:15-23.

Momeni M.H., Worden L. and Goldman M. 1974. Dosimetry and facilities of UCD outdoor-indoor ${ }^{60}$ Co irradiator. Health Phys. 26:469-472.

- Momeni M.H. 1975. Comments on the concepts of biophysical dose and dose rate in continuous irradiation. Health Phys. 28:307-308.

- Momeni M.H., Jow N. and Bradley E. 1975b. Temporal and spatial marrow dose from ${ }^{90} \mathrm{Sr}-{ }^{90} \mathrm{Y}$ in beagles: A measurement of geometrydistribution factor. Health Phys. 28:285-294.

$[53,159]$

Momeni M.H. and Wilson F.D. 1975c. Studies on the bone marrow cellularity and marrow progenitor populations in beagles including age-related changes using two techniques for correction of variable hemodilution. In Proceedings of the Fourth Annual Meeting of the International Society for Experimental Hematology, Trogir, Yugoslavia.

[121]

- Momeni M.H. and Pool R.R. 1975d. Bone and bone marrow spaces in dosimetry of beagle skeletons. Health Phys. 29:877-881.

- Momeni M.H., Jow N. and Bradley E. 1976. ${ }^{90} \mathrm{Sr}-{ }^{90} \mathrm{Y}$ dose distribution in beagles: Injection relative to ingestion. Health Phys. 30:3-19.

$[23,33,34,38,53,139,159,161]$

- Momeni M.H., Williams R.J.R., Fisher G.L. and Rosenblatt L.S. $1976 \mathbf{b}$. Local dosimetry and qualitative changes in ${ }^{226} \mathrm{Ra}-$ and ${ }^{90} \mathrm{Sr}^{90}{ }^{90} \mathrm{Y}$-labeled beagle humeri. Health Phys. 30:21-34.

$[53,68,110,159,162,163]$

- Momeni M.H., Rosenblatt L.S. and Jow N. 1976c. Retention and distribution of ${ }^{226} \mathrm{Ra}$ in beagles. Health Phys. 30:369-380.

$[28,43,162]$

- Momeni M.H., Williams J.R., Jow N. and Rosenblatt L.S. 1976d. Dose rates, dose and time effects of ${ }^{90} \mathrm{Sr}+{ }^{90} \mathrm{Y}$ and ${ }^{226} \mathrm{Ra}$ on beagle skeleton. Health Physics 30:381-390. $[43,53,68,110,159,162]$

- Momeni M.H., Williams J.R. and Rosenblatt L.S. 1976e. Radiation induced skeletal changes in beagle: Dose rates, dose, and age effect analysis from ${ }^{226} \mathrm{Ra}$. In Health Effects of Plutonium and Radium (W.S.S. Jee, ed., JW Press, Salt Lake City) 389-408.

$[68,69,163]$

Moore W. Jr. 1970. Cytogenetics [of the beagle]. In The Beagle as an Experimental Dog (A.C. Andersen ed., Iowa State Univ. Press, Ames, Iowa) 510-519.

Joe Morgan, a veterinary radiologist with the University of California, Davis life-span dog studies since 1974, has been principally concerned with clinical, histologic, and radiologic effects in bone.

Morgan J.P., Fisher G.L., McNeill K.L. and Oyama J. 1979. Abnormal canine bone development associated with hypergravity exposure. Am. J. Vet. Res. 40:346-350. 
- Morgan J.P. and Pool R.R. 1982. Radium-226-induced bone lesions in beagles: Clinical, radiologic, and histologic observations. Vet. Radiol. 23:261271.

$[27,68,69,163]$

- Morgan J.P., Pool R.R. and Kirsh I.E. 1983. Comparison of radiological changes in humans and beagles with skeletal deposits of radium. Health Phys. 44, Supp.l:353-363.

$[68,69,163]$

Morgan J.P., Miyabayashi T. and Choy S. 1986. Cervical spine motion: Radiographic study. Am. J. Vet. Res. 47:2165-2169.

[103]

Morgan J.P. and Rosenblatt L.S. 1986b. Canine hip dysplasia: The pelvic inlet parameter in diagnosis. Calif. Vet. 40(3):15-18.

[103]

Morgan K.Z. 1975. Suggested reduction of permissible exposure to plutonium and other transuranium elements. Am. Ind. Hyg. Assoc. J. 36:567-575.

$[107,111]$

Morrin L.A., Waring G.O. and Spangler W. 1982. Oval lipid corneal opacities in beagles: Ultrastructure of normal beagle cornea. Am J. Vet. Res. 43:443-453.

Moss W.D. and Tietjen G.L. In Press. Reassessment of human and beagle dog plutonium excretion data based on reevaluation of human plutonium excretion data. Health Phys.

[108]

Moulton J.E., Taylor D.O., Dorn C.R. and Andersen A.C. 1970. Canine mammary tumors. Pathol. Vet. 78:289-320.

Moulton J.E., Rosenblatt L.S. and Goldman M. 1986. Mammary tumors in a colony of beagle dogs. Vet. Pathol. 23:741-749.

$[16,80]$

Bruce Muggenburg, a veterinary physiologist at the Inhalation Toxicology Research Institute since 1969, was involved during the 1970 s in the development and evaluation of techniques for the removal of inhaled, insoluble radionuclides from the lung using pulmonary lavage. He has primary responsibility for management of the ITRI beagle colony and is a key contributor to the completion of the life-span studies, especially from the chemical point of view.

Muggenburg B.A., Pfleger R.C., Cuddihy R.G. and McClellan R.O. 1972. The removal of inhaled ${ }^{144} \mathrm{CeCl}_{3}$ from beagle dogs. III. Bilateral bronchopulmonary lavage with a DTPA solution. Health Phys, 23:611-619.

[89]

Muggenburg B.A., Mauderly J.L., Pickrell J.A., Chiffelle T. L., Jones R.K., Luft U.C., McClellan R.O. and Pfleger R.C. 1972b. Pathophysiologic sequelae of bronchopulmonary lavage in the dog. Am. Rev. Respir. Dis. 106:219-232.

[90]

Muggenburg B.A. and Mauderly J.L. 1974. Cardioplulmonary function of awake, sedated, and anesthetized beagle dogs. J. Appl. Physiol. 37:152-157.

Muggenburg B.A., Mauderly J.L., Boecker B.B., Hahn F.F. and McClellan R.O. 1975. Prevention of radiation pneumonitis from inhaled cerium-144 by lung lavage in beagle dogs. Am. Rev. Respir. Dis. 111:795-802.

[90]

Muggenburg B.A. and Mauderly J.L. 1975b. Lung lavage using a singlelumen endotracheal tube. J. Appl. Physiol. 38:922-926. 
Muggenburg B.A., Mewhinney J.A., Slauson D.O., Miglio J.J. Ruoff L., Mersch S. and McClellan R.O. 1976. The removal of inhaled $239 \mathrm{Pu}$ from beagle dogs by bronchopulmonary lavage and chelation therapy. Health Phys. 31:315-321.

Muggenburg B.A., Mewhinney J.A., Miglio J.J., Slauson D.O. and McClellan R.O. 1976b. The removal of inhaled ${ }^{239} \mathrm{Pu}$ and ${ }^{238} \mathrm{Pu}$ from beagle dogs by lung lavage and chelation treatment. In Diagnosis and Treatment of Incorporated Radionuclides (International Atomic Energy Agency, Vienna) 341-355.

Muggenburg B.A., Felicetti S.A. and Silbaugh S.A. 1977. Removal of inhaled radioactive particles by lung lavage-A review. Health Phys. 33:213220.

- Muggenburg B.A., Mewhinney J.A., Merickel B.S., Boecker B.B., Hahn F.F., Guilmette R.A., Mauderly J.L. and McClellan R.O. 1980. Toxicity of inhaled ${ }^{238} \mathrm{PuO}_{2}$. II. Biological effects in beagle dogs. In Book of Papers, 5 th International Congress of the International Radiation Protection Association, Vol II. (Israel Health Physics Soc., Jerusalem) 115-118.

$[61,66,74,78,206,207,208,209]$

Muggenburg B.A., Mauderly J.L., Halliwell W.H. and Slauson D.O. 1980b. Cardiopulmonary function and morphologic changes in beagle dogs after multiple lung lavages. Arch. Environ. Health 35:85-91.

Muggenburg B.A. and Mewhinney J.A. 1981. Removal of inhaled ${ }^{241} \mathrm{Am}$ oxide particles of various sizes from beagle dogs using lung lavage and chelation treatment. Health Phys. 4l:123-133.

Muggenburg B.A., Mewhinney J.A., Guilmette R.A. and McClellan R.O. 1981b. Removal of inhaled plutonium and americium from dogs using lung lavage and DTPA. In Actinides in Man and Animals (M.E.Wrenn, ed., RD Press, Salt Lake City) 387-393.

[90]

- Muggenburg B.A., McClellan R.O., Boecker B.B., Mauderly J.L. and Hahn F.F. 1981c. Long-term biologic effects in dogs treated with lung lavage after inhalation of ${ }^{144} \mathrm{Ce}$ in fused aluminosilicate particles. In Actinides in Man and Animals (M.E. Wrenn, ed., RD Press, Salt Lake City) 395-400. [90, 191]

Muggenburg B.A., Boecker B.B., Diel J.H and Snipes M.B. 1981 d. Observations on the lung retention of inhaled, relatively insoluble, environmentally-related particles. Chest $80 \mathrm{~S}: 19 \mathrm{~S}-21 \mathrm{~S}$.

- Muggenburg B.A., Mewhinney J.A., Griffith W.C., Hahn F.F., McClellan R.O., Boecker B.B. and Scott B.R. 1983. Dose-response relationships for bone cancers from plutonium in dogs and people. Health Phys. 44, Supp.1:529-535.

$[114,128,131,207,209]$

- Muggenburg B.A., Boecker B.B., Hahn F.F., Griffith W.G. and McClellan R.O. 1986. The risk of liver tumors in dogs and man from radioactive aerosols. In Life-Span Radiation Effects Studies in Animals: What Can They Tell US? (R.C. Thompson and J.A. Mahaffey, eds., CONF-830951, NTIS, Springfield, VA) 556-563.

$[63,79,114,187,194]$

- Muggenburg B.A., Wolff R.K., Mauderly J.L., Plaggmier M.M., Hahn F.F., Guilmette R.A. and Gerlach R.F. 1988. Cardiopulmonary function of dogs with plutonium-induced chronic lung injury. Radiat. Res. 115:314-324. 
- Muggenburg B.A., Guilmette R.A., Hahn F.F., Griffith W.C., Boecker B.B. and McClellan R.O. In Press. The effect of nonuniformity of alpha irradiation of the lung on biological effects. In Proceedings of the L.H. Gray Conference on Low Dose Radiation Biological Bases of Risk Assessment. Symposium held in Oxford, September 11-15, 1988.

$[78,108,212,214,216]$

- Nabors C.J.Jr., Stevens W. and Maxwell R.E. 1972. Comparative effects of ${ }^{239} \mathrm{Pu}$ and ${ }^{241} \mathrm{Am}$ on biochemical parameters: Effect of dose and radionuclide burden time. In Radiobiology of Plutonium (B.J. Stover and W.S.S. Jee, eds., JW Press, Salt Lake City) 87-103.

$[67,127,142]$

- Nabors C.J., Jr. and Hinckley J.S. 1975. Cortisol metabolism in skin of beagles bearing ${ }^{241} \mathrm{Am}$. Radiat. Res. 61:513-518.

$[67,142]$

National Academy of Sciences - National Research Council - Subcommittee on Inhalation Hazards of the Committee on Pathologic Effects of Atomic Radiation. 1961. Effects of Inhaled Radioactive Particles, Publication 848. National Academy of Sciences - National Research Council, Washington, DC.

[105]

National Academy of Sciences - National Research Council - Ad Hoc Committee on "Hot Particles" of the Advisory Committee on the Biological Effects of Ionizing Radiations. 1976. Health Effects of Alpha-Emitting Particles in the Respiratory Tract. National Academy of Sciences - National Research Council, Washington, DC [EPA 520/4-76-013].

[107]

National Academy of Sciences - National Research Council - Institute of Laboratory Animal Resources. 1981. Animal Models for Research on Aging. National Academy Press, Washington, DC.

National Academy of Sciences - National Research Council - Committee on the Biological Effects of Ionizing Radiations. 1988. Health Risks of Radon and other Internally Deposited Alpha-Emitters (BEIR IV). National Academy Press, Washington, DC.

$[106,114]$

National Council on Radiation Protection and Measurements. 1975. Alpha-Emitting Particles in Lungs. NCRP Report No. 46. National Council on Radiation Protection and Measurements, Washington DC. [107]

- National Council on Radiation Protection and Measurements. 1977. Cesium-137 From The Environment To Man: Metabolism And Dose. NCRP Report No. 52. National Council on Radiation Protection and Measurements, Washington DC.

$[8,24,61,63,80,107,111,168]$

Nenot, J.C. and Stather J.W. 1979. Toxicity of Plutonium, Americium and Curium, Pergamon, Oxford.

[106]

- Nilsson A., Morgan J.P. and Book S.A. 1985. Investigations of ${ }^{90} \mathrm{Sr}$ in dogs. I. Pathogenesis of radiation-induced bone tumors. Acta Radiol. Oncol. 24:95-111.

$[69,74,75,119,120,159]$

- Nilsson A. and Book S.A. 1987. Occurrence and distribution of bone tumors in beagle dogs exposed to ${ }^{90}$ Sr. Acta Oncol. 26:133-138. [74, 75, 82, 159] 
Bill Norris was a veteran of the Manhattan Project, at the University of Chicago, and after the war remained at Argonne National Laboratory until his retirement in 1979. He conducted early nonlife-span studies with ${ }^{90} \mathrm{Sr}$ in beagles, and later life-span studies with ${ }^{144} \mathrm{Ce}$ and ${ }^{137} \mathrm{Cs}$, and with continuous whole-body ${ }^{60} \mathrm{Co}$ gamma-ray exposure.

- Norris W.P., Fritz T.E., Rehfeld C.E. and Poole C.M. 1968. The response of the beagle dog to cobalt- 60 gamma radiation: Determination of the $\mathrm{LD}$ and description of associated changes. Radiat. Res. 35:681-708. $[9,17,27,81]$

Norris W.P., Fritz T.E. and Tayler J.A. 1970. Cycle of accomodation to restricted dietary iodide in the thyroid gland of the beagle dog. Am. J. Vet. Res. 31:21-33.

- Norris W.P. and Fritz T.E. 1972. Interactions of total dose and dose rate in determining tissue responses to ionizing radiations. In Radiobiology of Plutonium (B.J. Stover and W.S.S. Jee, eds., JW Press, Salt Lake City) 243-260.

$[9,21,60,64,72,170]$

- Norris W.P., Tyler S.A. and Sacher G.A. 1976. An interspecies comparison of responses of mice and dogs to continuous ${ }^{60} \mathrm{Co}$ gamma-irradiation. In Biological and Environmental Effects of Low-Level Radiation. Vol. 1 (International Atomic Energy Agency, Vienna) 147-156.

$[60,64,72,81,170,172]$

Norwood W.D. 1959. The determination of injury from the internally deposited radioisotope plutonium. J. Occup. Med. 1:269-276.

Norwood W.D. 1962b. Therapeutic removal of plutonium in humans. Health Phys. 8:747-750.

Norwood W.D. 1972. Plutonium $\left({ }^{239} \mathrm{Pu}\right)$ toxicity diagnosis and therapy. $J$. Occup. Med. 14:37-44.

Palmer R.F., Stuart B.O. and Filipy R.E. 1975. Biological effects of daily inhalation of radon and its short-lived daughters in experimental animals. In Noble Gases (R.E. Stanley and A.A. Moghissi, eds., CONF-730915, NTIS, Springfield, VA) 507-519.

Parcher J.W. and Williams J.R. 1970. Skeletal system. B. Ossification [in the beagle]. In The Beagle as an Experimental Dog (A.C. Andersen, ed., Iowa State University Press, Ames, Iowa) 158-161.

- Park H.Z., Whitson S.W. and Jee W.S.S. 1972. Vascular theory of radiation injury to bone. In Radiobiology of Plutonium (B.L. Stover and W.S.S. Jee, eds., JW Press, Salt Lake City) 305-322.

$[69,127,131,134,137,140,142]$

Jim Park, at Pacific Northwest Laboratory since 1961, was involved with the early plutonium inhalation studies with beagles and has been principal investigator for the ${ }^{239} \mathrm{PuO}_{2}$ and ${ }^{238} \mathrm{PuO}_{2}$ life-span studies initiated in the early 1970 s.

- Park J.F., Willard D.H., Marks S., West J.E., Vogt G.S. and Bair W.J. 1962. Acute and chronic toxicity of inhaled plutonium in dogs. Health Phys. $8: 651-657$

$[50,61,66,68,71,79,174]$ 
- Park J.F., Clarke W.J. and Bair W.J. 1964. Chronic effects of inhaled plutonium in dogs. Health Phys. 10:1211-1217.

- Park J.F., Clarke W.J. and Bair W.J. 1966. Plutonium particle-induced neoplasia of the canine lung. I. Clinical and gross pathology. In Lung Tumours in Animals (L.Severi, ed., Univ. of Perugia, Italy) 33l-344.

$[46,66,78,175]$

- Park J.F., Howard E.B. and Bair W.J. 1969. Acute toxicity of inhaled $238 \mathrm{PuO}_{2}$ in beagle dogs. AFWL-TR-69-75, Air Force Systems Command, Kirtland Air Force Base, NM.

$[36,46,61,63,66,71,100,176]$

Park J.F., Clarke W.J. and Bair W.J. 1970. Respiratory system [of the beagle]. In The Beagle as an Experimental Dog (A.C.Andersen, ed., Iowa State Univ. Press, Ames, Iowa) 285-293.

- Park J.F., Howard E.B., Stuart B.O., Wehner A.P. and Dilley J.V. 1971. Cocarcinogenic studies in pulmonary carcinogenesis. In Morphology of Experimental Respiratory Carcinogenesis (P. Nettesheim et al., eds., CONF-700501, NTIS, Springfield, VA) 417-436.

$[26,46,66,71,78,94,99]$

- Park J.F., Bair W.J. and Busch R.H. 1972. Progress in beagle dog studies with transuranium elements at Battelle-Northwest. Health Phys. 22:803-810.

$[10,35,46,61,63,66,78,82,174,175,176,182]$

- Park J.F., Catt D.L., Craig D.K., Olson R.J. and Smith V. H. 1974. Solubility changes of ${ }^{238} \mathrm{Pu}$ oxide in water suspension and effect on biological behavior after inhalation by beagle dogs. In Proceedings of the Third International Congress of the International Radiation Protection Association (W. S. Snyder, ed., CONF-730907-Pl, NTIS, Springfield, VA) 719-724. [36, 176]

- Park J.F. 1975. Late effects of inhaled plutonium in dogs. In Radiation Research - Biomedical, Chemical, and Physical Perspectives (O.F. Nygaard et al., eds., Academic Press, New York) 1233-1247.

$[66,82,176,178,180]$

- Park J.F., Lund J.E., Ragan H.A., Hackett P.L. and Frazier M.E. 1976. Bone tumors induced by inhalation of ${ }^{238} \mathrm{PuO}_{2}$ in dogs. In Recent Results in Cancer Research, Vol. 54 (E. Grundmann, ed., Springer-Verlag, Berlin) 17-35. $[8,17,25,36,46,52,66,68,74,75,78,120,176]$

- Park J.F. 1981. Metabolic model of inhaled plutonium for radiation protection purposes: Experimental animal studies. In Actinides in Man and Animals (M.E. Wrenn, ed., RD Press, Salt Lake City) 283-300.

$[46,47,111,112,178,180,182]$

- Park J.F., Dagle G.E., Ragan H.A., Weller R.E. and Stevens D.L. 1986. Current status of life-span studies with inhaled plutonium in beagles at Pacific Northwest Laboratory. In Life-Span Radiation Effects Studies in Animals: What Can they Tell Us? (R.C. Thompson and J.A. Mahaffey, eds., CONF-830951, NTIS, Springfield, VA) 455-470.

$[10,16,25,33,36,46,61,64,66,68,69,71,74,78,178,180,182,183]$

- Park J.F., Sanders C.L., Weller R.E., Gilbert E.S., Buschbom R.L., Lauhala K.E. and McDonald K.E. In Press. Comparative toxicology of inhaled ${ }^{239} \mathrm{PuO}_{2}$ in dogs and rats. Health Phys.

$[35,46,78,178]$

- Parkinson J.E. 1956. Plasma iron studies in normal beagle dogs. Proc. Soc. Exp. Biol. Med. 92:266-268. 
- Parkinson J.E. and Dougherty, J.H. 1958. Effect of internal emitters on red cell and plasma volumes of beagle dogs. Proc. Soc. Exp. Biol. Med. 97:722725 .

- Parkinson J.E. 1959. The effect of internal emitters on red cell survival in beagle dogs. Radiat. Res. 10:63-66.

Jim Parks, at the University of California, Davis since 1972, has been involved primarily in the radionuclide distribution and dosimetry aspects of the Davis ${ }^{90} \mathrm{Sr}$ and ${ }^{226} \mathrm{Ra}$ chronic administration studies.

- Parks N.J., Pool R.R. and Williams J.R. 1978. Variation of radon retention parameters for radium-burdened dog skeletons as a function of exposure age and dosage level. Radiat. Res. 73:274-287.

$[42,162]$

- Parks N.J., Pool R.R., Williams J.R. and Wolf H.G. 1978b. Age and dosage-level dependence of radium retention in beagles. Radiat Res. 75:617-632.

$[43,162]$

Parks N.J. and Tsuboi K.K. 1978c. Emulsion scintillation counting of radium and radon. Int. J. Appl. Radiat. Isotopes 29:77-80.

- Parks N.J. 1980. Life span dynamics of intra-skeletal radionuclide distribution in radium-injected beagles. Health Phys. 38:11-20. $\quad[51,75,112,163]$

- Parks N.J. and Keane A.T. 1983. Consideration of age-dependent radium retention in people on the basis of the beagle model. Health Phys. 44, Supp. l:103-112.

- Parks N.J., Book S.A. and Pool R.R. 1984. Squamous cell carcinoma in the jaws of beagles exposed to ${ }^{90} \mathrm{Sr}$ throughout life: Beta flux measurements at the mandible and tooth surfaces and a hypothesis for tumorigenesis. Radiat. Res. 100:139-156.

$[53,77,118,159]$

- Parks N.J. 1985. Dynamics of lifespan strontium-90 distribution in beagles with uniformly labelled skeletons acquired by radionuclide ingestion from in utero to adulthood. In Metals in Bone (N.D. Priest, ed., MTP Press, Lancaster) 107-116.

$[51,112,159]$

Parks N.J., Jee W.S.S., Dell R.B. and Miller G.E. 1986. Assessment of cortical and trabecular bone distribution in the beagle skeleton by neutron activation analysis. Anat. Rec. 215:230-250.

[103]

Peneyra R.S. and Jaenke R.S. 1985. Functional and morphologic damage in the neonatally irradiated canine kidney. Radiat. Res. 104:166-177.

[64]

- Peterson A.V., Prentice R.L. and Marek P. 1982. Relationship between dose of injected ${ }^{239} \mathrm{Pu}$ and bone sarcoma mortality in young adult beagles. Radiat. Res. 90:77-89.

$[29,76,127]$

Peto R., et al. 1980. Guidelines for simple sensitive significance tests for carcinogenic effects in long-term animal experiments. In Long-Term and Short-Term Screening Assays for Chemical Carcinogens: A Critical Appraisal (IARC Monographs, Supp. 2, International Agency for Research on Cancer, France) 311-426. 
Pfleger R.C., Wilson A.J. and McClellan R.O. 1969. Pulmonary lavage as a therapeutic measure for removing inhaled "insoluble" materials from the lung. Health Phys. 16:758-763.

Pfleger R.C., Wilson A.J., Cuddihy R.G. and McClellan R.O. 1969 b. Bronchopulmonary lavage for removal of inhaled insoluble materials from the lung. Dis. Chest 56:524-530.

Pfleger R.C. and Johnson G.E. 1971. Acetate incorporation into lipids of canine lung cells. Proc. Soc. Exp. Biol. Med. 138:811-813.

Pfleger R.C. and Thomas H.G. 1971b. Beagle dog pulmonary surfactant lipids. Arch. Internal Med. 127:863-872.

Pfleger R.C., Muggenburg B A., Sesline D.H., Harvey J.W., Cuddihy R.G. and McClellan R.O. 1972. The removal of inhaled ${ }^{144} \mathrm{CeCl}_{3}$ from beagle dogs. I. Unilateral bronchopulmonary lavage with a DTPA solution. Health Phys. 23:595-603.

Pfleger R.C., Muggenburg B.A., Cuddihy R.G. and McClellan R.O. 1972b. Removal of inhaled ${ }^{144} \mathrm{CeCl}_{3}$ from beagle dogs. II. Intravenous administration of a DTPA solution. Health Phys. 23:605-609.

- Pfleger R.C., Boecker B.B., Redman H.C., Pickrell J.A., Mauderly J.L., Jones R.K., Benjamin S.A. and McClellan R.O. 1975. Biological alterations resulting from chronic lung irradiation. I. The pulmonary lipid composition, physiology and pathology after inhalation by beagle dogs of ${ }^{144}$ Ce-labeled fused clay aerosols. Radiat. Res. 63:275-298.

$[61,68,191]$

Phalen R.F., Yeh H.C., Schum G.M. and Raabe O.G. 1978. Application of an idealized model to morphometry of the mammalian tracheobronchial tree. Anat. Rec. 190:167-176.

John Pickrell, a veterinary physiologist at the Inhalation Toxicology Research Institute from 1968 to 1988 , had responsibility during most of this time for clinical pathology in the beagle studies. He was particularly interested in noncarcinogenic responses of the lung to chronic irradiation, such as pneumonitis and fibrosis.

- Pickrell J.A., Wilson A.J., McClellan R.O., Stewart E.V. and Redman H.C. 1970. Evaluation of hypothyroidism in a beagle dog colony. In Advances in Automated Analysis/Biomedical Profiling, Vol. III. (Mediad Inc., White Plains) 143-147.

Pickrell J.A., Dubin S.E. and Elliott J.C. 1971. Normal respiratory parameters of unanesthetized beagle dogs. Lab. Anim. Sci. 21:677-679.

Pickrell J.A., Mauderly J.L., Muggenburg B.A. and Luft U.C. 1973. Influence of fasting on blood gas tension, $\mathrm{pH}$, and related values in dogs. Am. J. Vet. Res. 34:805-808.

Pickrell J.A., Schluter S.J., Belasich J.J., Stewart E.V., Meyer J., Hobbs C.H. and Jones R.K. 1974. Relationship of age of normal dogs to blood serum constituents and reliability of measured single values. Am. J. Vet. Res. 35:897-903. 
- Pickrell J.A., Harris D.V., Pfleger R.C., Benjamin S.A., Belasich J.J., Jones R.K. and McClellan R.O. 1975. Biological alterations resulting from chronic lung irradiation. II. Connective tissue alterations following inhalation of ${ }^{144} \mathrm{Ce}$ fused clay aerosol in beagle dogs. Radiat. Res. 63:299-309.

$[61,68,191]$

- Pickrell J.A., Schnizlein C.T., Hahn F.F., Snipes M.B. and Jones R.K. 1978. Radiation-induced pulmonary fibrosis: Study of changes in collagen constituents in different lung regions of beagle dogs after inhalation of betaemitting radionuclides. Radiat. Res. 74:363-377. [61, 68, 191, 196, 198, 199, 203]

Pindak F.F. and Clapper W.E. 1964. Isolation of enteric cytopathogenic human orphan virus type 6 from dogs. Am. J. Vet. Res. 25:52-54.

Pindak F.F., Clapper W.E. and Sherrod J.H. 1965. Incidence and distribution of spirochetes in the digestive tract of dogs. Am. J. Vet. Res. 26:1391-1402.

Pindak F.F. and Clapper W.E. 1966. Experimental infection of beagles with ECHO virus type 6. Tex. Rep. Biol. Med. 24:466-472.

Erich Polig of the Kernforschungszentrum, Karlsruhe, West Germany, has developed highly sophisticated computerized techniques for studying the microdistribution and localized dosimetry of alpha emitters in bone, which he has applied in recent years to the life-span beagle material from the University of Utah studies.

- Polig E., Smith J.M. and Jee W.S.S. 1984. 241-Am in the beagle skeleton: Microdistribution and local dosimetry. Radiat. Environ. Biophys. 23:121-125.

$[55,141]$

- Polig E., Smith J.M. and Jee W.S.S. 1984b. Microdistribution and localized dosimetry of ${ }^{241} \mathrm{Am}$ in bones of beagle dogs. Int. J. Radiat. Biol. 46:143-160.

$[55,141]$

Polig E. 1985. Localized alpha-dosimetry for cancer induction studies. In Metals in Bone (N.D. Priest, ed., MTP Press, Lancaster) 269-284.

Polig E., Bruenger F.W. and Jee W.S.S. 1986. Quantitative autoradiography of radium-226 in bone: 1. The measurement technique. Radiat. Protect. Dosim. 16:205-211.

Polig E. and Jee W.S.S. 1986b. Cell-specific radiation dosimetry in the skeleton. Calcif. Tissue Int. 39:119-122.

Polig E. and Jee W.S.S. 1986C. Computerized analysis of ${ }^{226} \mathrm{Ra}$ autoradiographs and its application. Strahlentherapie 80 (Suppl.):104-110.

Polig E. and Jee W.S.S. 1987. Bone age and remodeling: A mathematical treatise. Calcif. Tissue Int. 41:130-136.

- Polig E. 1987b. Quantitative autoradiography of 226-radium in bone: 2 . Data analysis. Radiat. Protect. Dosim. 19:139-149.

- Polig E. In Press. Biomathematical modeling of plutonium-239 retention, skeletal label kinetics and local dosimetry in dogs. Health Phys.

$[45,52,55,127]$ 
Roy Pool, a veterinary pathologist at the University of California, Davis since 1969, has studied the pathogenesis of carcinogenic and noncarcinogenic effects in bones of the life-span beagles from the Davis ${ }^{90} \mathrm{Sr}$ and ${ }^{226}$ Ra studies, particularly in comparison to similar lesions in human bone.

Pool R.R., Williams R.J.R. and Bulgin M. 1972. Disseminated calcinosis cutis in a dog. J. Am. Vet. Med. Assoc. 161:291-293.

- Pool R.R., Williams R.J.R. and Goldman M. 1972b. Strontium-90 toxicity in adult beagles after continuous ingestion. In Biomedical Implications of Radiostrontium Exposure (M. Goldman and L.K. Bustad, eds., CONF-710201, NTIS, Springfield, VA) 277-284.

$[74,75,159]$

- Pool R.R., Williams R.J.R., Goldman M. and Rosenblatt L. 1973. Comparison of bone-tumor sites in beagles continually fed ${ }^{90} \mathrm{Sr}$ or injected with ${ }^{226} \mathrm{Ra}$ as a means of scaling risk to humans. In Radionuclide Carcinogenesis (C.L. Sanders et al., eds., CONF-720505, NTIS, Springfield, VA) $475-486$.

$[69,74,75,114,159,160,162,163]$

- Pool R.R. Williams R.J.R. and Goldman M. 1973b. Induction of tumors involving bone in beagles fed toxic levels of strontium 90. Am. J. Roentgenol. Radium Therap. Nucl. Med. 118:900-908.

$[74,75,120,159]$

Pool R.R. and Wolf H.G. 1974. An unusual case of canine osteosarcoma. Cancer 34:771-779.

Pool R.R. 1978. Tumors of bone and cartilage. In Tumors in Domestic Animals, 2nd Ed. (J.E. Moulton, ed., Univ. of California Press, Berkeley) 89-149.

- Pool R.R., Morgan J.P., Parks N.J., Farnham J.E. and Littman M.S. 1983. Comparative pathogenesis of radium-induced intracortical bone lesions in humans and beagles. Health Phys. 44 Supp.1:155-177. $\quad$ [68, 69, 114, 120, 163]

- Priest N.D. (ed.) 1985. Metals in Bone. MTP Press, Lancaster.

Otto Raabe, an aerosol physicist at the Inhalation Toxicology Research Institute from 1966 to 1976, was particularly concerned with the development of techniques for production and characterization of various kinds of aerosols. He then moved to the University of California, Davis, where he continued his aerosol research and has worked on dose-response-time relationships in the life-span beagle data.

- Raabe O.G., Kanapilly G.M. and Newton G.J. 1971. New methods for the generation of aerosols of insoluble particles for use in inhalation studies. In Inhaled Particles III, Vol. I (W.H. Walton, ed., Unwin Brothers, Old Woking, Surrey, England) 3-17.

$[25,34,39,190,195,197,199]$

- Raabe O.G., Boyd H.A., Kanapilly G.M., Wilkinson C.J. and Newton G.J. 1975. Development and use of a system for routine production of monodisperse particles of ${ }^{238} \mathrm{PuO}_{2}$ and evaluation of gamma emitting labels. Health Phys. 28:655-667.

$[10,25,26,28,206,208,211,213,215]$ 
- Raabe O.G. and Goldman M. 1979. A predictive model of early mortality following acute inhalation of $\mathrm{PuO}_{2}$ aerosols. Radiat. Res. 78:264-277.

$[62,76,110,116,118,174,191,199]$

- Raabe O.G., Book S.A. and Parks N.J. 1980. Bone cancer from radium: Canine dose response explains data for mice and humans. Science 208:61-64.

$[76,116,163]$

- Raabe O.G., Parks N.J. and Book S.A. 1981. Dose-response relationships for bone tumors in beagles exposed to ${ }^{226} \mathrm{Ra}$ and ${ }^{90} \mathrm{Sr}$. Health Phys. 40:863-880.

$[38,43,74,76,109,116,158,159,160,162]$

- Raabe O.G., Book S.A., Parks N.J., Chrisp C.E. and Goldman M. 1981b. Lifetime studies of ${ }^{226} \mathrm{Ra}$ and ${ }^{90} \mathrm{Sr}$ toxicity in beagles--A status report. Radiat. Res. 86:515-528.

$[38,43,76,82,116,158,162]$

- Raabe O.G., Book S.A. and Parks N.J. 1983. Lifetime bone cancer doseresponse relationships in beagles and people from skeletal burdens of ${ }^{226} \mathrm{Ra}$ and ${ }^{90}$ Sr. Health Phys. 44 Supp.1:33-48. $\quad[76,109,116,159,160,163]$

- Raabe O.G. 1984. Comparison of the carcinogenicity of radium and boneseeking actinides. Health Phys. 46:1241-1258.

$[76,109,116,127,131,134,137,142,143,145,163,207,209]$

- Raabe O.G. 1986. Use of three-dimensional lognormal dose-response surfaces in lifetime studies of radiation-induced cancer. In Life-Span Radiation Effects Studies in Animals: What Can They Tell Us? (R.C. Thompson and J.A. Mahaffey, eds., CONF-830951, NTIS, Springfield, VA) 320-342.

$[76,109,116,127,131,134,137,142,143,145,163,107,209]$

- Raabe O.G. 1986b. Reply to Wrenn, Lloyd, Mays and Taylor concerning the carcinogenecity of $\mathrm{Ra}$ and bone-seeking actinides. Health Phys. 51:679682 .

$[76,109,116,127,131,134,137,142,143,145,163]$

- Raabe O.G. 1987. Three-dimensional dose-response models of competing risks and natural life span. Fundam. Appl. Toxicol. 8:465-473. [116, 175, 179]

Raabe O.G. In Press. Scaling of fatal cancer risks from laboratory animals to man. Health Phys.

[116]

Harvey Ragan, a veterinary clinical pathologist, has, since 1962 , been involved in the hematological and clinical chemical studies for all of the Pacific Northwest Laboratory life-span beagle studies.

- Ragan H.A., Park J.F., Olson R.J. and Buschbom R.L. 1976. Lymphocytopenia induced in beagle dogs by inhalation of ${ }^{239} \mathrm{PuO}_{2}$. In Radiation and the Lymphatic System (J.E. Ballou, ed., CONF-740930, NTIS, Springfield, VA) 100-105.

$[66,178]$

- Ragan H.A., Buschbom R.L., Park J.F., Dagle G.E. and Weller R.E. 1986. Hematologic effects of inhaled plutonium in beagles. In Life-Span Radiation Effects Studies in Animals: What Can They Tell Us? (R.C. Thompson and J.A. Mahaffey, eds., CONF-830951, NTIS, Springfield, VA) 477-487.

$[66,178,180,183]$

- Ratzlaff M.H. 1970. Cardiovascular system [of the beagle]. B. Lymphatics. In The Beagle as an Experimental Dog (A.C. Andersen, ed., Iowa State Univ. Press, Ames, Iowa) 246-260 
Rebar A.H., DeNicola D.B. and Muggenburg B.A. 1980. Bronchopulmonary lavage cytology in the dog: Normal findings. Vet. Pathol. 17:294-304.

"Ham" Redman, a veterinarian at the Inhalation Toxicology Research Institute from 1961 to 1984, was responsible for establishing the ITRI closed beagle colony and for implementing the breeding program used to maintain a stable gene pool. He was particulary involved in the conduct of the fission product life-span studies.

Redman H.C. and Weir J.E. 1969. Detection of naturally occurring neurologic disorders of beagle dogs by electroencephalography. Am. J. Vet. Res. 30:2075-2082.

$[17,104]$

Redman H.C., Wilson A.J., Bielfelt S.W. and McClellan R.O. 1970. Beagle dog production experience at the Fission Product Inhalation Program (19611968). Lab. Anim. Care 20:61-68.

$[16,17]$

- Redman H.C., McClellan R.O., Jones R.K., Boecker B.B., Chiffelle T.L., Pickrell J.A. and Rypka E.W. 1972. Toxicity of ${ }^{137} \mathrm{CsCl}$ in the beagle. Early biological effects. Radiat. Res. 50:629-648.

$[24,29,60,61,65,67,193]$

Redman H.C., Hogan J.E. and Wilson G.L. 1972b. Effect of intermittent light stimulation singly and combined with pentylenetetrazol on the electroencephalogram and clinical response of the beagle dog. Am. J. Vet. Res. 33:677-685.

$[17,104]$

- Redman H.C., Hahn F.F. and Muggenburg B.A. 1973. Electroencephalographic correlates of a metastatic brain tumor in a beagle dog. Am. J. Vet. Res. 34:681-684.

Reece W.O. 1970. The treadmill and evaluation of stress [in the beagle]. In The Beagle as an Experimental Dog (A.C. Andersen ed., Iowa State Univ. Press, Ames, Iowa) 594-596.

Carl Rehfeld was a veterinarian at the University of Utah project, from 1955 to 1961. From 1961 until 1971 he filled a similar role at Argonne National Laboratory. A unique contribution to the research with beagles was his study of the genetic relationships of the several beagle colonies.

- Rehfeld C.E., Stover B.J., Taylor G.N., Atherton D.R. and Schneebeli G. 1960. Eye changes in beagles following intravenous injectrion of radium 226. J. Am. Vet. Med. Assoc. 136:562-564.

- Rehfeld C.E., Stover B.J., Taylor G.N. and Mays C.W. 1962. Fracture incidence in beagles receiving single injections of radium or plutonium. In Some Aspects of Internal Irradiation (T.F. Dougherty et al., eds., Pergamon, New York) 131-143. $[69,127,131]$

Rehfeld C.E., Bacus J.W., Pagels J.A. and Dipert M.H. 1967. Computer calculation of Wright's inbreeding coefficient. J. Heredity 58:81-84.

Rehfeld C.E. 1970. Definition of relationships in a closed beagle colony. Am. J. Vet. Res. 31:723-732.

Rehfeld C.E., Dammin G.J. and Hester W.J. 1970b. Skin graft survival in partially inbred beagles. Am. J. Vet. Res. 31:733-745. 
Rehfeld C.E., Blomquist J.A. and Taylor G.N. 1972. The beagles. In Radiobiology of Plutonium (B.J. Stover and W.S.S. Jee, eds., JW Press, Salt Lake City) 47-57.

"Chet" Richmond, although never directly involved in the life-span studies with beagles, conducted many shorter-term radionuclide studies in dogs while at the Los Alamos National Laboratory in the 1960 s and was an influential advocate for beagle studies while in the Division of Biology and Medicine of the Atomic Energy Commission from 1968 to 1971.

- Richmond C.R. and Furchner J.E. 1970. Whole-body counting [of the beagle]. In The Beagle as an Experimental Dog (A.C. Andersen, ed., Iowa State Univ. Press, Ames, Iowa) 588-593.

- Richmond C.R. 1970b. Program planning in inhalation carcinogenesis: USAEC plans. In Inhalation Carcinogenesis (M.G. Hanna et al., eds., CONF-691001, NTIS, Springfield, VA) 483-497.

$[4,13]$

Richmond C.R. and Furchner J.E. 1970c. Radionuclide metabolism [in the beagle]. In The Beagle as an Experimental Dog (A.C. Andersen ed., Iowa State Univ. Press, Ames, Iowa) 479-488.

- Richmond C.R. and Thomas R.L. 1975. Plutonium and other actinide elements in gonadal tissue of man and animals. Health Phys. 29:241-250.

Richmond C.R., Thompson R.C., Stather J.W., Coulon R.B. and Healy J.W. 1976. Plutonium - Implications for man and his environment (panel discussion). In Transuranium Nuclides in the Environment (International Atomic Energy Agency, Vienna) 627-646.

$[107,111]$

Robertson J.S. and Cohn S.H. 1964. Evaluation of plutonium exposures in man. Health Phys. 10:373-389.

$[108,111]$

Rosenberg H.M., Rehfeld C.E. and Emmering T.E. 1966. A method for the epidemiologic assessment of periodontal health-disease state in a beagle hound colony. J. Periodontol. 37:208-213.

Leon Rosenblatt has been associated, as a statistical consultant, with the University of Utah life-span beagle studies and with the Inhalation Toxicology Research Institute beagle studies, but most extensively and continuously with the program at the University of California, Davis. He is now heading the effort to complete the Davis studies.

- Rosenblatt L.S. and Goldman M. 1967. The use of probit analysis to estimate dose effects on postirradiation leukocyte depressions. Health Phys. 13:795- 798

$[29,65,163]$

- Rosenblatt L.S. 1970. Long-term experiments. In The Beagle as an Experimental Dog (A.C. Andersen, ed., Iowa State Univ. Press, Ames, Iowa) 583-587.

$[13,14,19,26]$

- Rosenblatt L.S., Hetherington N.H., Goldman M. and Bustad L.K. 1971. Evaluation of tumor incidence following exposure to internal emitters by application of the logistic dose-response surface. Health Phys. 21:869-875.

$[76,113,115,163]$ 
- Rosenblatt L.S. 1972. Determination of cumulative tumor incidences and their use in quantitative evaluation of radionuclide carcinogenesis. In Biomedical Implications of Radiostrontium Exposure (M. Goldman and L.K. Bustad, eds., CONF-710201, NTIS, Springfield, VA) 345-351. [115, 163]

- Rosenblatt L.S., Bielfelt S.W. and Della Rosa R.J. 1972b. Effects of radiostrontium on puppy mortality and litter size in dogs. In Biomedical Implications of Radiostrontium Exposure (M. Goldman and L.K. Bustad, eds., CONF-710201, NTIS, Springfield, VA) 334-344.

- Rosenblatt L.S., Goldman M., Book S.A. and Momeni M.H. 1976. Extrapolation of radiation-induced tumour incidence from animals to man. In Biological and Environmental Effects of Low-Level Radiation. Vol.1 (International Atomic Energy Agency, Vienna) 237-242.

$[76,113,115,128,131,163]$

- Rosenblatt L.S., Book S.A. and Goldman M. 1986. Effects of x-irradiation of young female beagles on life span and tumor incidence. In Life-Span Radiation Effects Studies in Animals: What Can They Tell Us? (R.C. Thompson and J.A. Mahaffey, eds., CONF-830951, N'TIS, Springfield, VA) 628-645.

$[17,27,72,77,80,81,156]$

Rosenthal M.W., Lindenbaum A., Baxter D.W., Kalesperis G.S., Moretti E.S. and Russell J.J. 1975. Removal of polymeric plutonium from dogs with DTPA and glucan. Radiat. Res. 63:253-261.

Rosenthal M.W., Rahman Y.E., Moretti E.S. and Cerny E.A. 1975b. Removal of polymeric plutonium by DTPA directed into cells by liposome encapsulation. Radiat. Res. 63:262-274.

Rowland R.E. and Lloyd E. 1969. Deposition patterns of bone-seeking isotopes in man. In Environmental Contamination by Radioactive Materials (International Atomic Energy Agency, Vienna) 63-72.

- Ruhmann A.G. and Berliner D.L. 1965. Serum lactic dehydrogenase levels in adult beagle dogs with internally deposited radionuclides. Radiat. Res. 26:287-294.

$[67,127,131,134,137,140]$

Ryan M.T. and Poston J.W. 1987. The impact of an isotopic effect on the interpretation of bioassay data for Pu. Health Phys. 53:255-259.

[108]

George Sacher, a radiation biologist at Argonne National Laboratory since Manhattan Project days, is better known for his studies of late effects of ionizing radiation in mice, but he was also importantly involved, until his death in 1980, in the design and interpretation of such studies in dogs, which he saw as necessary for extrapolation of late-effects data from animals to humans.

- Sanders C.L. and Park J.F. 1971. Pulmonary distribution of alpha dose from ${ }^{239} \mathrm{PuO}_{2}$ and induction of neoplasia in rats and dogs. In Inhaled Particles III, Vol. I (W.H. Walton, ed., Unwin, Old Woking, England) 489497.

Sanders C.L. 1972. Deposition patterns and the toxicity of transuranium elements in lung. Health Phys. 22:607-615. 
- Sanderson M.G. and Seed T.M. 1978. The effect of daily prenatal gamma irradiation on ovarian cortex of beagle pups. In Ninth International Congress on Electron Microscopy, Vol. II (Microscopical Society of Canada, Toronto) $488-489$.

Schallberger J.A., Dewhirst M.W. and Lebel J.L. 1976. Lymph transport of soluble and insoluble plutonium. In Radiation and the Lymphatic System (J.E. Ballou, ed., CONF-740930, NTIS, Springfield, VA) 19-27.

- Schlenker R.A. and Marshall J.H. 1975. Thicknesses of the deposits of plutonium and radium at bone surfaces in the beagle. Health Phys. 29:649654.

- Schlenker R.A. and Smith J.M. 1986. Argonne-Utah studies of ${ }^{224} \mathrm{Ra}$ endosteal surface dosimetry. Strahlentherapie 80 (Suppl.):93-98. [42, 54, 154]

Schlenker R.A. 1988. Skeletal ${ }^{212} \mathrm{~Pb}$ retention following ${ }^{224} \mathrm{Ra}$ injection: Extrapolation of animal data to adult humans. Health Phys. 54:383-396. [117]

Schnizlein C.T. and Bice D.E. 1980. Production and partial characterization of dog macrophage migration inhibitory factor. J. Reticuloendothel. Soc. 27:393-400.

Schofield G.B. and Dolphin G.W. 1974. U.K. experience on the medical aspects of radiological protection of workers handling plutonium. Ann. Occup. Hyg. 17:73-83.

Schreider J.P. and Raabe O.G. 1980. Replica casts of the entire respiratory airways of experimental animals. J. Environ. Pathol. Toxicol. $4: 427-435$.

Schreider J.P. and Raabe O.G. 1981. Anatomy of the nasal-pharyngeal airway of experimental animals. Anat Rec. 200:195-205.

Schreider J.P. 1983. Nasal airway anatomy and inhalation deposition in experimental animals and people. In Nasal Tumors in Animals and Man (G. Reznik and S.F. Stinson, eds., CRC Press, Boca Raton, Florida) 1-26.

Bobby Scott, a biophysicist and mathematician at the Inhalation Toxicology Research Institute since 1977, is involved in efforts to model dose-response-time data from the life-span beagle experiments in a manner that will prove most useful for extrapolation to humans.

- Scott B.R. 1979. Hazard-function method of resolving radiation doseresponse curves. Health Phys. 36:323-332. $[62,110,118,191,196,197,199]$

- Scott B.R. and Hahn F.F. 1980. A model that leads to the Weibull distribution function to characterize early radiation response probabilities. Health Phys. 39:521-530. [61, 62, 116, 118, 174, 185, 186, 188, 191, 193, 196, 197, 199]

- Scott B.R. 1980b. Model for early death caused by radiation pneumonitis and pulmonary fibrosis after inhaling insoluble radiocative particles. Bull. Math. Biol. 42:447-459.

$[62,118,174,191,196,197,199]$ 
- Scott B.R. 1980c. Proposed estimates of the probability of inducing pulmonary injury sufficient to cause death from radiation pneumonitis and pulmonary fibrosis after briefly inhaling a mixture of insoluble beta-emitting particles. Health Phys. 38:635-642.

$[62,118,191,196,197,200]$

- Scott B.R. 1982. Method of analysis of monotone dose-response probabilities after long-term exposure to a toxicant. Health Phys. 42:305-315.

[118]

- Scott B.R., Hahn F.F., Guilmette R.A., Muggenburg B.A., Snipes M.B., Boecker B.B. and McClellan R.O. 1986. Use of studies with laboratory animals to assess the potential early health effects of combined internal alpha and beta irradiation. In Life-Span Radiation Effects Studies in Animals. What Can They Tell Us? (R.C. Thompson and J.A. Mahaffey, eds., CONF830951, NTIS, Springfield, VA) 578-591.

$[118,200,212,214,216]$

Scott B.R., Hahn F.F., McClellan R.O. and Seiler F.A. In Press. Risk estimators for radiation-induced bone marrow syndrome lethality in humans. Risk Analysis.

[118]

Sedlak, A. 1986. Logistic estimate of the final incidence of late radiation effects. Health Phys. 51:357-364.

Tom Seed, an experimental hematologist and microbiologist at Argonne National Laboratory since 1975, has employed data from the life-span, continuous gamma-ray exposure studies, as well as from short-term "mechanistic" studies, to develop a highly detailed model of radiation leukemogenesis.

- Seed T.M., Tolle D.V., Fritz T.E., Devine R.L., Poole C.M. and Norris W.P. 1977. Irradiation-induced erythroleukemia and myelogenous leukemia in the beagle dog: Hematology and ultrastructure. Blood 50:1061-1079.

$[64,72,118,119,170]$

- Seed T.M., Sanderson M.H., Gutzeit D.L., Fritz T.E., Tolle D.V., Poole C.M. and Norris W.P. $1977 \mathrm{~b}$. Ovarian dysfunction in fetally irradiated beagles. In 35th Annual Proceedings of the Electron Microscopy Society of America (G.W. Bailey, ed., Claitor's Division, Baton Rouge, LA) 658-659. [65, 71, 83]

- Seed T.M., Tolle D.V., Fritz T.E., Cullen S.M., Kaspar L.V. and Poole C.M. 1978. Haemopathological consequences of protracted gamma irradiation in the beagle: Pre-clinical phases of leukaemia induction. In Late Biological Effects of Ionizing Radiation, Vol. I. (International Atomic Energy Agency, Vienna) 531-545.

$[64,72,118,119,170]$

Seed T.M., Chubb G.T. and Kaspar L.V. 1979. Processing and viewing of blood forming cells cloned in soft agar cultures. Micron 9:17-18.

[119]

- Seed T.M., Chubb G.T., Ulrich R.G., Wright B.J. and Kaspar L.V. 1979 b. Preparation and observation by SEM of hemopoietic cells cloned in soft agar. In Scanning Electron Microscopy/1979/III. (R.P. Becker and O. Johari, ed., SEM Inc., AMF O'Hare, Chicago) 397-410.

- Seed T.M., Cullen S.M., Kaspar L.V., Tolle D.V. and Fritz T.E. 1980. Hemopathologic consequences of protracted gamma irradiation: Alterations in granulocyte reserves and granulocyte mobilization. Blood 56:42-51.

$[64,119,170]$ 
- Seed T.M., Chubb G.T. and Tolle D.V. 1982. Sequential changes in bone marrow architecture during continuous low dose gamma irradiation. In Scanning Electron Microscopy/1981/IV. (O. Johari, ed., SEM Inc., AMF O'Hare, Chicago) 61-72.

$[64,119,170]$

- Seed T.M., Kaspar L.V., Tolle D.V. and Fritz T.E. 1982b. Hemopathologic predisposition and survival time under continuous gamma irradiation: Responses mediated by altered radiosensitivity of hemopoietic progenitors. Exp. Hematol. 10, Supp.12:232-248.

$[64,119,170]$

- Seed T.M., Chubb G.T., Tolle D.V., Fritz T.E., Poole C.M., Doyle D.E., Lombard L.S. and Kaspar L.V. 1983. The ultrastructure of radiation-induced endosteal myelofibrosis in the dog. In Scanning Electron Microscopy/ $1982 / I$. (O.Johari, et al., eds., SEM Inc., AMF O'Hare, Chicago) 377-391.

$[64,119,170]$

- Seed T.M., Fritz T.E., Tolle D.V., Poole C.M., Lombard L.S., Doyle D.E., Kaspar L.V., Cullen S.M. and Carnes B.A. 1984. Survival patterns and hemopathological responses of dogs under continuous gamma irradiation. In Responses of Different Species to Total Body Irradiation (J.J. Broerse and T.J. MacVittie, eds., Martinus Nijhoff, Boston) 137-159.

$[72,118,119,170]$

- Seed T.M., Kaspar L.V., Fritz T.E. and Tolle D.V. 1985. Cellular responses in chronic radiation leukemogenesis. In Carcinogenesis, Vol. $10(\mathrm{E}$. Huberman and S.H. Barr, eds., Raven Press, New York) 363-379.

- Seed T.M., Kaspar L.V., Tolle D.V. and Fritz T.E. 1987. Chronic radiation leukemogenesis: Postnatal hematopathologic effects resulting from in-utero irradiation. Leukemia Res. 11:171-179. $[72,95,119,170,172]$

- Seed T.M. 1987b. Structure-function relationships in radiation-induced cell and tissue lesions: Special references to the contributions of scanning electron microscopy and hematopoietic tissue responses. Scanning Microsc. 1:255-272.

$[119,170,172]$

Sekhri K.K. and Faulkin L.J., Jr. 1970. Reproductive system [of the beagle]. C. Mammary gland. In The Beagle as an Experimental Dog (A.C. Andersen, ed., Iowa State Univ. Press, Ames, Iowa) 327-349.

Shabistari L., Taylor G.N. and Angus W. 1967. Dental eruption pattern of the beagle. J. Dent. Res. 46:276-278.

Moshe Shifrine, at the University of California, Davis since 1968 , has made extensive use of the beagle, in life-span and shorter studies, to investigate leukemogenesis and the immune system.

- Shifrine M., Bulgin M.S., Dollarhide N.E., Wolf H.G., Taylor N.J., Wilson F.D., Dungworth D.L. and Zee Y-C. 1971. Transplantation of radiationinduced canine myelomonocytic leukaemia. Nature 232:405-406.

Shifrine M., Smith J.B., Bulgin M.S., Bryant B.J., Zee Y-C. and Osburn B.I. 1971b. Response of canine fetuses and neonates to antigenic stimulation. $J$. Immunol. 107:965-970.

- Shifrine M., Munn S.L., Rosenblatt L.S., Bulgin M.S. and Wilson F.D. 1973. Hematologic changes to 60 days of age in clinically normal beagles. Lab. Anim. Sci. 23:894-898. 
Shifrine M. and Stormont C. 1973b. Hemoglobins, haptoglobins, and transferrins in beagles. Lab. Anim. Sci. 23:704-706.

- Shifrine M., Wolf H.G., Taylor N.J., Galligan S.J., Wilson F.D., Colgrove G.S. and Bustad L.K. 1973c. Transplantation of radiation-induced canine myelomonocytic leukemia. In Unifying Concepts of Leukemia. Bibliography of Haematology No. 39 (R.M. Dutcher and L. Chieco-Bianchi, eds., Karger, Basel) 158-169.

- Shifrine M., Chrisp C.E., Wilson F.D. and Heffernon U. 1973d. Lysozyme (muramidase) activity in canine myelogenous leukemia. Am. J. Vet. Res. 34:695-696.

Shifrine M., Pappagianis D. and Neves J. 1975. Double electroimmunodiffusion: A rapid diagnostic test for canine coccidioidomycosis. Am. J. Vet. Res. 36:819-820.

Shifrine M. and Bryant B.J. 1976. Leukemia allotransplants in canine fetuses: Influence of host age and immune responsiveness. Proc. Soc. Exp. Biol. Med. 151:307-309.

Shifrine M., Thilsted J. and Pappagianis D. 1976b. Canine transfer factor. In Transfer Factor - Basic Properties and Clinical Applications (M.S. Ascher et al., eds., Academic Press, New York) 349-357.

Shifrine M., Taylor N.J., Rosenblatt L.S. and Wilson F.D. 1978. Comparison of whole blood and purified canine lymphocytes in a lymphocytestimulation microassay. Am. J. Vet. Res. 39:687-690.

- Shifrine M. and Wilson F.D. (eds.). 1980. The Canine as a Biomedical Research Model: Immunological, Hematological, and Oncological Aspects. DOE/TIC-10191, NTIS, Springfield, VA.

$[13,16,93]$

Shifrine M., Rosenblatt L.S., Taylor N., Hetherington N.W., Matthews V.J. and Wilson F.D. 1980b. Seasonal variations in lectin-induced lymphocyte transformation in the beagle dog. J. Interdisciplinary Cycle Res. 11:219-231.

Shifrine M., Taylor N., Rosenblatt L.S. and Wilson F.D. 1980c. Seasonal variation in cell mediated immunity of clinically normal dogs. Exp. Hematol. 8:318-326.

Shifrine M., Garsd A., Christiansen J.A. and Rosenblatt L.S. 1982. Photoperiod and cell mediated immunity of clinically normal dogs. $J$. Interdisciplinary Cycle Res. 13:177-186.

Shifrine M. and Cain G.R. 1984. Canine immunology: In search of new frontiers. In The Canine as a Biomedical Model (M.R. Gilman, ed.) 33-40.

Shively J.N. and Epling G.P. 1970. Eye [of the beagle]. B. Fine structure. In The Beagle as an Experimental Dog (A.C. Andersen, ed., Iowa State Univ. Press, Ames, Iowa) 386-411.

Shultz F.T. 1970. Genetics [of the beagle]. In The Beagle as an Experimental Dog (A.C. Andersen, ed., Iowa State Univ. Press, Ames, Iowa) 489509. 
Silbaugh S.A., Felicetti S.A., Muggenburg B.A. and Boecker B.B. 1975. Multiple bronchopulmonary lavages for the removal of ${ }^{144} \mathrm{Ce}$ in fused clay particles from beagle dog lungs. Health Phys. 29:81-88.

Silbaugh S.A., Muggenburg B.A., Mauderly J.L. and Crain C.R. 1977. Cardiopulmonary function in dogs during lung lavage and unilateral hypoxia. J. Appl. Physiol. 43:778-783.

Sinclair W.K. 1963. Absorbed dose in biological specimens irradiated externally with cobalt-60 gamma radiation. Radiat. Res. 20:288-297.

Singh N.P., Wrenn M.E., McInroy J.F., Gonzales E., Cross F.T. and Jackson P.O. 1986. Ratios of ${ }^{234} \mathrm{U},{ }^{238} \mathrm{U}$ and ${ }^{230} \mathrm{Th}$ in dog lungs exposed to $\mathrm{U}$ ore dust: An interlaboratory comparison. Health Phys. 50:292-297.

- Singh N.P., Zimmerman C.J., Taylor G.N. and Wrenn M.E. 1987. Distribution of low-level concentrations of ${ }^{239,240} \mathrm{Pu}$ in tissues of a beagle. Health Phys. 52:373-377.

Singh N.P., Zimmerman C.J., Taylor G.N. and Wrenn M.E. 1988. The beagle: An appropriate experimental animal for extrapolating the organ distribution pattern of Th in humans. Health Phys. 54:293-299.

Slauson D.O., Osburn B.I., Shifrine M. and Dungworth D.L. 1975. Regression of feline sarcoma virus-induced sarcomas in dogs. I. Morphologic investigations. J. Natl. Cancer Inst. 54:361-370.

Slauson D.O., Osburn B.I., Shifrine M. and Dungworth D.L. 1975b. Regression of feline sarcoma virus-induced sarcomas in dogs. II. Immunololgic investigations. J. Natl. Cancer Inst. 54:371-377.

- Slauson D.O., Hahn F.F., Benjamin S.A., Chiffelle T.L. and Jones R.K 1976. Inflammatory sequences in acute pulmonary radiation injury. $A m$. $J$. Pathol. 82:549-566.

$[61,196]$

- Slauson D.O., Hahn F.F. and Chiffelle T.L. 1977. The pulmonary vascular pathology of experimental radiation pneumonitis. Am. J. Pathol. 88:635-648.

$[61,196]$

Smith J.M., Taylor G.N. and Jee W.S.S. 1980. A technique of neutroninduced (fission-track) autoradiography with histological detail. Radiat. Res. $84: 579-585$.

[53]

- Smith J.M., Miller S.C. and Jee W.S.S. 1984. The relationship of bone marrow type and microvasculature to the microdistribution and local dosimetry of plutonium in the adult skeleton. Radiat. Res. 99:324-335.

$[52,127]$

Smith V.H., Ballou J.E., Lund, J.E., Dagle G.E., Ragan H.A., Busch R.H., Hackett P.L. and Willard D.W. 1976. Aspects of inhaled DTPA toxicity in the rat, hamster and beagle dog and treatment effectiveness for excorporation of plutonium from the rat. In Diagnosis and Treatment of Incorporated Radionuclides (International Atomic Energy Agency, Vienna) 517.530.

"Burt" Snipes, at the Inhalation Toxicology Research Institute since 1971, has contributed to the radiobiologic aspects of life-span studies of inhaled, long-lived, beta-emitting radionuclides, being particularly concerned with the hinetic behavior of insoluble particles in the lung and pulmonary lymph nodes. 
- Snipes M.B., Hulbert A.J. and Runkle G.E. 1975. Radiation dose patterns for inhaled radionuclides. In Biomedical Dosimetry (International Atomic Energy Agency, Vienna) 683-694.

$[50,199]$

Snipes M.B., Runkle G.E. and Muggenburg B.A. 1979. Influence of lavage treatment on the distribution patterns of inhaled, relatively insoluble particles in the lung. Health Phys. 37:201-206.

[90]

Snipes M.B. 1981. Metabolism and dosimetry of $106 \mathrm{Ru}$ inhaled as ${ }^{106} \mathrm{RuO}_{4}$ by beagle dogs. Health Phys. 41:303-317. [97, 111]

- Snipes M.B., Boecker B.B. and McClellan R.O. 1983. Retention of monodisperse or polydisperse aluminosilicate particles inhaled by dogs, rats, and mice. Toxicol. Appl. Pharmacol. 69:345-362.

$[34,41,112]$

- Snipes M.B., Muggenburg B.A. and Bice D.E. 1983b. Translocation of particles from lung lobes or the peritoneal cavity to regional lymph nodes in beagle dogs. J. Toxicol. Environ. Health 11:703-712.

Snipes M.B. 1983c. Retention of relatively insoluble particles inhaled by dogs, rats and mice. In Current Concepts in Lung Dosimetry (D.R. Fisher, ed., CONF-820492-Pt 1, NTIS, Springfield, VA) 73-79.

$[34,41]$

Snipes M.B., Chavez G.T. and Muggenburg B.A. 1984. Disposition of 3-, 7-, and $13-\mu \mathrm{m}$ microspheres instilled into lungs of dogs. Environ. Res. 33:333-342.

Snipes M.B., Boecker B.B. and McClellan R.O. 1984b. Respiratory tract clearance of inhaled particles in laboratory animals. In Lung Modelling for Inhalation of Radioactive Materials (H. Smith and G. Gerber, eds., Commission of the European Communities, Report EUR-9384 EN) 63-76. [41, 112]

Snipes M.B., McClellan R.O., Mauderly J.L. and Wolff R.K. In Press. Retention patterns for inhaled particles in lung: Comparisons between laboratory animals and humans for chronic exposures. Health Phys. [92]

Snyder W.S. 1964b. On the estimation of a systemic body burden of plutonium. In Assessment of Radioactivity in Man, Vol. II (International Atomic Energy Agency, Vienna) 583-588.

[108]

- Solarz A.K. 1964. Effects of early adult x-irradiation upon emotional display in the aged female beagle: A negative finding. Psychol. Rep. 15:927-930.

- Solarz A.K. 1964b. The effects of $x$-irradiation on the dominance behavior of the adult female beagle: A methodological problem. Radiat Res. 23:611-619.

$[83,157]$

Solarz A.K. 1970. Behavior [of the beagle]. In The Beagle as an Experimental Dog (A.C. Andersen, ed., Iowa State Univ.Press, Ames, Iowa) 453-468.

Bill Spangler, a veterinary pathologist at the University of California, Davis since 1978, has had primary responsibility for gerontologic pathology, especially renal changes, in the life-span beagles. 
Spangler W.L., Gribble D.H. and Weiser M.G. 1977. Canine hypertension: A review. J. Am. Vet. Med. Assoc. 170:995-998.

Spangler W.L. and Muggli F.M. 1978, Seizure-induced rhabdomyolysis accompanied by acute renal failure in a dog. J. Am. Vet. Med. Assoc. 172:1190-1194.

Spangler W.L. 1979. Ultrastructure of the juxtaglomerular apparatus in the dog in a sodium-balanced state. Am. J. Vet. Res. 40:802-808.

[103]

Spangler W.L. 1979b. Pathophysiologic response of the juxtaglomerular apparatus to dietary sodium restriction in the dog. Am. J. Vet. Res. 40:809819.

Spangler W.L., Adelman R.D., Conzelman G.M. Jr. and Ishizaki G. 1980. Gentamicin nephrotoxicity in the dog: Sequential light and electron microscopy. Vet. Pathol. 17:206-217.

[103]

Spangler W.L., Waring G.O. and Morrin L.A. 1982. Oval lipid corneal opacities in beagles. V. Ultrastructure. Vet. Pathol. 19:150-159.

Bill Spiers, an expert in radiation dosimetry at Leeds University, in England, has made extensive comparisons of the microscopic dose patterns of bone-seeking radionuclides in beagle and human bone.

- Spiers F.W. 1969. Beta particle dosimetry in trabecular bone. In Delayed Effects of Bone-Seeking Radionuclides (C.W. Mays et al., eds., Univ.of Utah Press, Salt Lake City) 95-108.

$[53,54,117,139]$

- Spiers F.W., Zanelli G.D., Darley P.J., Whitwell J.R. and Goldman M. 1972. Beta-particle dose rates in human and animal bone. In Biomedical Implications of Radiostrontium Exposure (M. Goldman and L.K. Bustad, eds., CONF-710201, NTIS, Springfield, VA) 130-148. $[53,54,117,159]$

- Spiers F.W. and Whitwell J.R. 1976. Dosimetry of ${ }^{239} \mathrm{Pu}$ and ${ }^{226} \mathrm{Ra}$ in man and animals. In Health Effects of Plutonium and Radium (W.S.S. Jee, ed., JW Press, Salt Lake City) 537-552.

$[54,117,127,128,131]$

- Spiers F.W. and Vaughan J. 1976b. Hazards of plutonium with special reference to the skeleton. Nature 259:531-534.

111,117

Spiers F.W., Whitwell J.R. and Beddoe A.H. 1978. Calculated dose factors for the radiosensitive tissues in bone irradiated by surface-deposited radionuclides. Phys. Med. Biol. 23:481-494.

- Spiers F.W. and Beddoe A.H. 1983. Sites of incidence of osteosarcoma in the long bones of man and the beagle. Health Phys. 44,Supp.1:49-64.

$[75,128,131,134,137,140,159,160,163]$

Spiers F.W. 1988. Particle dosimetry in bone and the toxicity of boneseeking radionuclides. Phys. Med. Biol. 33:395-411.

$[54,75,117,128,131,139,140,159,160,163]$

- Spiess H., Gerspach A. and Mays C.W. 1978. Soft-tissue effects following ${ }^{224} \mathrm{Ra}$ injections into humans. Health Phys. 35:61-81.

$[10,64]$ 
Spinelli J.S. and Williams J.R. 1968. Barium enemas in normal beagle dogs. Lab. Anim. Care 18:387-390.

Stanley J.A., Eidson A.F. and Mewhinney J.A. 1982. Distribution, retention and dosimetry of plutonium and americium in the rat, dog and monkey after inhalation of an industrial-mixed uranium and plutonium oxide aerosol. Health Phys. 43:521-530.

[100]

- Stannard J.N. 1988. Radioactivity and Health: A History. DOE/RL/ 01830 -T59 (DE-88013791). Pacific Northwest Laboratory, Richland, WA.

$[4,5,6,8,13,19,24]$

- Stara J.F., Nelson N.S., Della Rosa R.J. and Bustad L.K. 1971. Comparative metabolism of radionuclides in mammals: A review. Health Phys. 20:113-137.

[106]

- Stevens D.L. and Dagle G.E. 1986. Nonparametric statistical techniques used in dose estimation for beagles exposed to inhaled plutonium nitrate. In Life-Span Radiation Effects Studies in Animals: What Can They Tell Us? (R.C. Thompson and J.A. Mahaffey, eds., CONF-830951, N'TIS, Springfield, VA) $660-674$

$[36,46,182]$

- Stevens D.L. and Park J.F. 1986b. Estimation of initial lung deposition of inhaled ${ }^{238} \mathrm{PuO}_{2}$ in beagles. In Life-Span Radiation Effects Studies in Animals: What Can They Tell Us? (R.C. Thompson and J.A. Mahaffey, eds. CONF-830951, NTIS, Springfield, VA) 675-682.

Walter Stevens, associated with the life-span beagle studies at the University of Utah since 1962, has been particularly interested in molecular-level associations and effects of internally deposited radionuclides.

- Stevens W. and Berliner D.L. 1964. Serum transaminase levels in beagle dogs burdened with plutonium-239. Radiat. Res. 23:420-429.

$[67,127]$

Stevens W., Bruenger F.W. and Stover B.J. 1965. In vitro studies of the interactions of PuIV with blood proteins. Radiat. Res. 26:114-123.

[49]

- Stevens W., Nabors C.J.Jr and Berliner D.L. 1967. A comparison of serum transaminase levels and other serum constituents in dogs burdened with ${ }^{239} \mathrm{Pu},{ }^{228} \mathrm{Th},{ }^{228} \mathrm{Ra}$, and ${ }^{226} \mathrm{Ra}$. Ann. NY Acad. Sci. 145:817-829.

$[28,67,127,131,134,137]$

- Stevens W., Bruenger F.W. and Stover B.J. 1968. In vivo studies on the interactions of PuIV with blood constituents. Radiat. Res. 33:490-500.

$[49,127]$

- Stevens W., Stover B.J., Bruenger F.W. and Taylor G.N. 1969. Some observations on the deposition of americium-241 in the thyroid gland of the beagle Radiat. Res. 39:201-206.

$[48,57,141,142]$

- Stevens W. and Bruenger F.W. 1972. Interaction of ${ }^{249} \mathrm{Cf}$ and ${ }^{252} \mathrm{Cf}$ with constituents of dog and human blood. Health Phys. 22:679-683.

$[48,49,143,145]$

- Stevens W., Stover B.J., Atherton D.R. and Bruenger F.W. 1975. Distribution and excretion of three chemical species of ${ }^{239} \mathrm{Pu}(\mathrm{IV})$ in the beagle. Health Phys. 28:387-394.

$[23,45,55]$ 
- Stevens W., Atherton D.R., Jee W.S.S., Buster D.S., Grube B.J., Bruenger F.W. and Lindenbaum A. 1976. Induction of osteogenic sarcoma by polymeric plutonium $\left({ }^{239} \mathrm{PuIV}\right)$ in beagles. In Health Effects of Plutonium and Radium (W.S.S. Jee, ed., JW Press, Salt Lake City) 81-95.

- Stevens W., Atherton D.R., Bates D., Lloyd R.D., Buster D.S. and Bruenger F.W. 1977. Retention and distribution of ${ }^{241}$ AmIII in neonatal beagles. Health Phys. 33:553-559.

$[48,53,56,142]$

Stevens W., Bruenger F.W., Atherton D.R., Buster D.S. and Howerton G. 1978. Retention and distribution of ${ }^{241} \mathrm{Am}$ and ${ }^{65} \mathrm{Zn}$, given as DTPA chelates in rats and of $\left[{ }^{14} \mathrm{C}\right] \mathrm{DTPA}$ in rats and beagles. Radiat. Res. 75:397-409.

Stevens W., Bruenger F.W., Atherton D.R., Smith J.M. and Taylor G.N. 1980. The distribution and retention of hexavalent ${ }^{233} \mathrm{U}$ in the beagle. Radiat. Res. 83:109-126.

[100]

- Stitzel K.A., Wilson F.D. and Shifrine M. 1982. Survival of dogs exposed to continuous whole-body gamma irradiation postpartum or beginning in utero and continuing after birth. In Experimental Hematology Today (S.J. Baum, G.D. Ledney and S. Thierfelder, eds., S. Karger, Basel) 261-266.

$[95,119]$

Stone J.M. and Shifrine M. 1970. Radionuclide marking of canine fetuses for studying ontogeny of the immune response. J. Immunol. 104:1562-1564.

Betsy Stover has been a major player in the University of Utah lifespan beagle program from its inception in 1950. She continues to maintain an interest in the program since her departure from Salt Lake City in 1970. Her concern has centered on metabolic and dosimetric aspects of the studies, and, more recently, on the modeling of life-shortening effects.

Stover B.J., Atherton D.R. and Arnold J.S. 1957. Comparative metabolism of $\mathrm{Ca}^{45}$ and $\mathrm{Ra}^{226}$. Proc. Soc. Exp. Biol. Med. 94:269-272.

- Stover B.J. and Atherton D.R. 1958. Metabolism of $\mathrm{Sr}^{90}$ in adult beagle dogs. Proc. Soc. Exp. Biol. Med. 99:201-205.

$[37,130,139]$

- Stover B.J. 1959. Metabolism of radioactive isotopes which deposit mainly in the skeleton. Health Phys. 1:373-378.

$[45,106,127,130]$

- Stover B.J., Atherton D.R. and Keller N. 1959b. Metabolism of $\mathrm{Pu}^{239}$ in adult beagle dogs. Radiat. Res. 10:130-147.

$[23,45,127]$

- Stover B.J. 1959c. $\mathrm{Pb}^{212}$ (ThB) tracer studies in adult beagle dogs. Proc. Soc. Exp. Biol. Med. 100:269-272.

$[42,43,133,137]$

- Stover B.J., Atherton D.R., Keller N. and Buster D.S. 1960. Metabolism of the $\mathrm{Th}^{228}$ decay series in adult beagle dogs. I. Th228 (RdTh). Radiat. Res. $12: 657-671$.

$[44,137]$

Stover B.J., Goldman M. and Andersen A.C. 1961. Failure of a dog to discriminate between strontium-90 and calcium given orally. Nature 191:713-714.

$[34,159]$

- Stover B.J., Atherton D.R. and Mays C.W. 1962. Studies of the retention and distribution of $\mathrm{Ra}^{226}, \mathrm{Pu}^{239}, \mathrm{Ra}^{228}\left(\mathrm{MsTh}_{1}\right), \mathrm{Th}^{228}(\mathrm{RdTh})$, and $\mathrm{Sr}^{90}$ in adult beagles. In Some Aspects of Internal Irradiation (T.F. Dougherty et al., eds., Pergamon, New York) 7-25.

$[23,37,42,44,45,56,127,130,131,133,137,139]$ 
- Stover B.J., Atherton D.R., Bruenger F.W. and Buster D.S. 1962 b. Further studies of the metabolism of $\mathrm{Pu}^{239}$ in adult beagles. Health Phys. 8:589-597.

$[33,44,45,127]$

- Stover B.J. and Jee W.S.S. 1963. Some effects of long-term alpha irradiation on the composition and structure of bone. Health Phys. 9:267-275.

$[69,127]$

- Stover, B.J., Atherton D.R., Bruenger F.W. and Mays C.W. 1965. Distribution of ${ }^{226} \mathrm{Ra}$ between blood cells and plasma. Health Phys. 11:617-622.

$[49,131]$

- Stover B.J., Atherton D.R., Buster D.S. and Keller N. 1965b. The Th228 decay series in adult beagles: $\mathrm{Ra}^{224}, \mathrm{~Pb}^{212}$, and $\mathrm{Bi}^{212}$ in blood and excreta. Radiat Res. 26:226-243.

$[43,137]$

- Stover B.J., Atherton D.R., Buster D.S. and Bruenger F.W. 1965c. The $\mathrm{Th}^{228}$ decay series in adult beagles: $\mathrm{Ra}^{224}, \mathrm{~Pb}^{212}$, and $\mathrm{Bi}^{212}$ in selected bones and soft tissues. Radiat. Res. 26:132-145.

$[43,137]$

Stover B.J., Ruhmann A.G. and Atherton D.R. 1966. The action of chelating agents on ${ }^{212} \mathrm{~Pb}$ in the blood: The particular value of the steady state after ${ }^{228} \mathrm{Th}$ administration to the beagle. Int. J. Radiat. Biol. 11:27-34.

[86]

- Stover B.J., Atherton D.R., Bruenger F.W. and Buster D.S. $1968 .{ }^{239} \mathrm{Pu}$ in liver, spleen, and kidneys of the beagle. Health Phys. 14:193-197.

$[45,127]$

Stover B.J., Bruenger F.W. and Stevens W. 1968b. The reaction of PuIV with the iron transport system in human blood serum. Radiat. Res. 33:381394.

- Stover B.J. and Buster D.S. 1968c. Metabolism and localization of thorium series nuclides after entry into blood: Observations in animals. In The Dosimetry and Toxicity of Thorotrast (International Atomic Energy Agency, Vienna) 53-64.

- Stover B.J., Atherton D.R., Bruenger F.W. and Buster D.S. 1969. Pu-239(IV): Its distribution in the beagle. In Delayed Effects of Bone-Seeking Radionuclides (C.W. Mays et al., eds., Univ. of Utah Press, Salt Lake City) 109-123.

$[45,52,127]$

- Stover B.J., Bruenger F.W. and Stevens W. 1970. Association of americium with ferritin in the canine liver. Radiat. Res. 43:173-186.

[56]

- Stover B.J. and Eyring H. 1970b. The dynamics of life. I. Death from internal irradiation by ${ }^{239} \mathrm{Pu}$ and ${ }^{226} \mathrm{Ra}$, aging, cancer, and other diseases. Proc. Natl. Acad. Sci. USA 66:132-139.

$[76,81,115,127,131]$

- Stover B.J., Atherton D.R. and Buster D.S. 1971. Protracted hepatic, splenic and renal retention of ${ }^{239} \mathrm{Pu}$ in the beagle. Health Phys. 20:369-374. [45, 127]

- Stover B.J. and Jee W.S.S. (eds.). 1972. Radiobiology of Plutonium. JW Press, Univ. of Utah, Salt Lake City.

- Stover B.J., Stevens W. and Bruenger F.W. 1972b. Chemical associations of ${ }^{239} \mathrm{Pu}(\mathrm{IV})$ and ${ }^{241} \mathrm{Am}$ (III) in blood, liver and thyroid. In Radiobiology of Plutonium (B.J. Stover and W.S.S. Jee, eds., JW Press, Salt Lake City) 129-140.

$[49,55,56]$ 
- Stover B.J., Atherton D.R. and Buster D.S. 1972c. Retention of ${ }^{239} \mathrm{Pu}(\mathrm{IV})$ in the beagle. In Radiobiology of Plutonium (B.J. Stover and W.S.S. Jee, eds, JW Press, Salt Lake City) 149-169.

- Stover B.J., Eyring H. and Atherton D.R. 1972d. Effects of ${ }^{239} \mathrm{Pu}$ and related radionuclides on survival of the adult beagles. In Radiobiology of Plutonium (B.J. Stover and W.S.S. Jee, eds., JW Press, Salt Lake City) 385-407.

$[81,82,130]$

- Stover B.J. 1972e. Life shortening consequent to internal irradiation from ${ }_{239}$ Pu. Health Phys. 22:823-827.

$[81,82]$

- Stover B.J. and Stover C.N.Jr. 1972f. The Laboratory for Radiobiology at the University of Utah. In Radiobiology of Plutonium (B.J. Stover and W.S.S. Jee, eds., JW Press, Salt Lake City) 29-46.

$[7,9,10,13,15,126]$

- Stover B.J. and Atherton D.R. 1974. Kinetics of the skeletal retention of ${ }^{239} \mathrm{Pu}(\mathrm{IV})$. Radiat. Res. 60:525-535.

$[45,127]$

- Stover B.J. 1975. Dose-response relationships for beagles injected with ${ }^{239} \mathrm{Pu}$ (IV) or ${ }^{241} \mathrm{Am}$ (III). In Radiation Research - Biomedical, Chemical, and Physical Perspectives (O.R. Nygaard et al., eds., Academic Press, New York) 1266-1277.

$[45,81,126]$

- Stover B.J. 1976. ${ }^{239} \mathrm{Pu}(\mathrm{IV})$ : Skeletal dosimetry in the beagle. In Health Effects of Plutonium and Radium (W.S.S. Jee, ed., JW Press, Salt Lake City) 97-103.

- Stover B.J., Atherton D.R., Stevens W., Buster D.S. and Bruenger F.W. 1977. Effect of dose level on skeletal retention of ${ }^{239} \mathrm{Pu}(\mathrm{IV})$ in the beagle. Radiat. Res. 69:442-458.

$[45,127]$

- Stover B.J. 1981. Toxicology of ${ }^{228} \mathrm{Th}$ in young adult beagles: Potential relationship to the thorium fuel cycle. In Actinides in Man and Animals (M.E. Wrenn, ed., RD Press, Salt Lake City) 483-492.

$[43,136,137]$

- Stover B.J., Wrenn M.E., Jee W.S.S. and Atherton D.R. 1986. The Eyring-Stover theory of survival applied to life-span radiation effects studies in animals. In Life-Span Radiation Effects Studies in Animals: What Can They Tell Us? (R.C. Thompson and J.A. Mahaffey, eds., CONF-830951, NTIS, Springfield, VA) $311-319$.

$[81,127,131]$

Straus R., Wurm M., Kositchek R.J. and Weiner J.M. 1970. Pathology [in the beagle]. C. Spontaneous atherosclerotic lesions of the aorta and coronary vessels. In The Beagle as an Experimental Dog (A.C. Andersen, ed., Iowa State Univ. Press, Ames, Iowa) 558-573.

Bruce Stuart was associated from 1962 until 1974 with some of the earliest Pacific Northwest Laboratory radionuclide inhalation studies, including, in particular, the uranium mine atmosphere inhalation studies in beagles.

Stuart B.O., Casey H.W. and Bair W.J. 1964. Acute and chronic effects of inhaled ${ }^{144} \mathrm{CeO}_{2}$ in dogs. Health Phys. 10:1203-1209. 
- Stuart B.O., Bair W.J. and Park J.F. 1968. Interpretation of excretion data from beagle dogs after ${ }^{239} \mathrm{Pu}$ inhalation. In Diagnosis and Treatment of Deposited Radionuclides (H.A. Kornberg and W.D. Norwood, eds., Excerpta Medica Foundation, Amsterdam) 243-255.

- Stuart B.O., Dionne P.J. and Bair W.J. 1970. A dynamic simulation of the retention and translocation of inhaled plutonium oxide in beagle dogs. In Proceedings of the 11th AEC Air Cleaning Conference, Vol.2 (M.W. First and J.M. Morgan, Jr., eds., CONF-700816, NTIS, Springfield, VA) 721-737.

$[35,46,174]$

- Stuart B.O., Willard D.H. and Howard E.B. 1970b. Uranium mine air contaminants in dogs and hamsters. In Inhalation Carcinogenesis (M.G. Hanna, Jr. et al., eds., CONF-691001, NTIS, Springfield, VA) 413-427.

$[9,26,92,99]$

- Stuart B.o., Willard D.H. and Howard E.B. 1971. Studies of inhaled radon daughters, uranium ore dust, diesel exhaust, and cigarette smoke in dogs and hamsters. In Inhaled Particles. III. Vol. 1. (W.H. Walton, ed., Unwin Bros., Old Woking, Surrey, England) 543-560. [4, 9, 19, 26, 92, 99]

Stuart B.O. (Panel Chairman) 1973. Inhalation Risks from Radioactive Contaminants. International Atomic Energy Agency, Vienna.

Stuart B.O. 1973b. Deposition of inhaled aerosols. Arch. Internal Med. 131:60-73.

Stuart B.O. 1976. Selection of animal models for evaluation of inhalation hazards in man. In Air Pollution and the Lung: Proceedings of the Twentieth Annual "OHOLO" Biological Conference (E.F. Aharonson, et al., eds., John Wiley \& Sons, New York) 268-288.

Stuart B.O. 1976b. Deposition and clearance of inhaled particles. Environ. Health Perspect. 16:41-53.

- Stuart B.O., Palmer R.F., Filipy R.E., Dagle G.E. and McDonald K.E. 1977. Respiratory tract carcinogenesis in large and small experimental animals following daily inhalation of radon daughters and uranium ore dust. In: Proceedings IVth International Congress, Vol. 4. (International Radiation Protection Association, Paris) 1337-1340.

Sullivan M.F. and Willard D.H. 1978. The beagle dog as an animal model for marihuana smoking studies. Toxicol. Appl. Pharmacol. 45:445-462.

Sullivan M.F. 1980. Absorption of actinide elements from the gastrointestinal tract of rats, guinea pigs and dogs. Health Phys. 38:159-171.

[100]

Swinth K.L., Griffin B.I. and Park J.F. 1967. Whole body counting of inhaled plutonium in dogs. Health Phys. 13:1223-1231.

Swinth K.L. 1968. Interpreting counting data for internally deposited plutonium. In: Diagnosis and Treatment of Deposited Radionuclides (H.A. Kornberg and W.D. Norwood, eds., Excerpta Medica Foundation, Amsterdam) 208-221.

[101]

Swinth K.L., Park J.F. and Moldofsky P.J. 1972b. Counting plutonium in the tracheobronchial lymph nodes. Health Phys. 22:899-904.

[101] 
Swinth K.L., Park J.F., Voelz G.L. and Ewins J.H. 1976. In vivo detection of plutonium in the tracheobronchial lymph nodes with a fiber-optic coupled scintillator. In Radiation and the Lymphatic System (J.E. Ballou, ed., CONF-740930, NTIS, Springfield, VA) 59-66.

- Talley D., Raabe O.G. and Mewhinney J.A. 1979. Lovelace aerosol particle separator design modifications. In Aerosol Measurement (D.A. Lundgren et al., eds., Univ. of Florida Press, Gainesville, FL). $\quad$ [26, 206, 208, 211, 213, 215]

Tamplin A.R. and Cochran T.B. 1974. Radiation Standards for Hot Particles. Natural Resources Defense Council, Washington, DC.

Tamplin A.R. and Cochran T.B. 1974b. NRDC Comments on WASH 1535, Draft Environmental Statement, Liquid Metal Fast Breeder Reactor Program. Re: Vol. II, Part 2, Section 4.6.5, Particle Lung Dose Effects, pp. 4.6-89 to 4.6-105. Natural Resources Defense Council, Washington, DC.

[107]

Tamplin A.R. and Cochran T.B. 1974c. The Hot Particle Issue: A Critique of WASH 1320 as it Relates to the Hot Particle Hypothesis. Natural Resources Defense Council, Washington, DC.

[107]

Taylor D.M. 1964. IV. The metabolism of the transuranic elements. $B r$. J. Radiol. 37:95-100.

[106]

Taylor D.M. 1972. Interactions between transuranium elements and the components of cells and tissues. Health Phys. 22:575-581.

[106]

Taylor D.M. 1972b. Cellular deposition and retention of ${ }^{239} \mathrm{Pu}$ in relation to the induction of neoplasms. In Radiobiology of Plutonium (B.J. Stover and W.S.S. Jee, eds., JW Press, Salt Lake City) 273-279.

[106]

Taylor D.M. 1985. The retention of plutonium in human bone: A reconnaissance. In Metals in Bone (N.D. Priest, ed., MTP Press, Lancaster) 221-228.

Glenn Taylor has been the principal veterinary pathologist with the University of Utah life-span beagle studies since 1961. He has been especially concerned with the production of liver tumors and with a wide variety of nonosteosarcoma effects, such as effects in the thyroid, the eye, and the liver.

- Taylor G.N., Christensen W.R., Jee W.S.S., Rehfeld C.E. and Fisher W. 1962. Anatomical distribution of fractures in beagles injected with $\mathrm{Pu}^{239}$ Health Phys. 8:609-613.

$[69,127]$

- Taylor G.N., Rehfeld C.E., Schneebeli G. and Johnson H.A. 1962b. Eye changes induced by internal irradiation. In Some Aspects of Internal Ir radiation (T.F. Dougherty et al., eds., Pergamon, New York) 163-178.

$[42,44,56,71,127,131,134,137]$

- Taylor G.N., Christensen W.R., Jee W.S.S. and Rehfeld C.E. 1964. Gingival ulceration in beagles induced by Ra-226, Ra-228 or Th-228. J. Dent. Res. 43:35-43.

$[70,77]$ 
- Taylor G.N., Stover B.J., Jee W.S.S. and Mays C.W. 1964b. Selective deposition of radium in normal and neoplastic melanocytes. Radiat. Res. 21:285-298.

$[42,56,71,131]$

- Taylor G.N., Christensen W.R., Jee W.S.S., Rehfeld C.E. and Petermann P. 1966. Intercomparison of pathological fractures in beagles injected with ${ }^{226} \mathrm{Ra},{ }^{228} \mathrm{Ra},{ }^{239} \mathrm{Pu}$ or ${ }^{90} \mathrm{Sr}$. Health Phys. 12:361-367.

$[69,127,131,134]$

- Taylor G.N., Jee W.S.S., Christensen W.R., Rehfeld C.E. and Nebeker N. 1966b. Thorium-228 induced fractures in beagles. Health Phys. 12:889-893.

$[69,137]$

- Taylor G.N., Dougherty T.F., Shabestari L. and Dougherty J. H. 1969. Soft-tissue tumors in internally irradiated beagles. In Delayed Effects of Bone-Seeeking Radionuclides (C.W. Mays et al., eds., Univ. of Utah Press, Salt Lake City) 323-336.

$[79,80,128,131,134]$

- Taylor G.N., Rehfeld C.E., Christensen W.R. and Jee W.S.S. 1969 b. Influence of ${ }^{226} \mathrm{Ra}$ and ${ }^{239} \mathrm{Pu}$ on the dental root canal of the dog. J. Dent. Res. 48:924-927.

$[70,127]$

- Taylor G.N., Jee W.S.S., Dockum N. and Hromyk E. 1969c. Microscopic distribution of americium-241 in the beagle thyroid gland. Health Phys. $17: 723-725$.

$[57,142]$

- Taylor G.N., Dougherty T.F. and Christensen W.R. 1971. Some toxicity aspects of internally deposited ${ }^{239} \mathrm{Pu}$. In Pathology of Irradiation (C.C. Berdjis, ed., Williams and Wilkins, Baltimore) 110-119. $[55,63,67,69,70,74,127]$

- Taylor G.N., Jee W.S.S., Mays C.W., Dell R.B., Williams J.L. and Shabestari L. 1972. Microscopic distribution of californium-249 and berkelium-249 in the soft tissues of beagles. Health Phys. 22:691-693.

$[48,55,56,57,143]$

- Taylor G.N., Jee W.S.S., Williams J.L. and Shabestari L. 1972b. Hepatic changes induced by ${ }^{239} \mathrm{Pu}$. In Radiobiology of Plutonium (B.J. Stover and W.S.S. Jee, eds., JW Press, Salt Lake City) 105-127.

$[55,63,79,127,128,141,142]$

- Taylor G.N., Christensen W.R., Shabestari, L. and Jee W.S.S. 1972c. The general syndrome induced by ${ }^{239} \mathrm{Pu}$ in the beagle. In Radiobiology of Plutonium (B.J. Stover and W.S.S. Jee, eds., JW Press, Salt Lake City) 59-74. [126]

- Taylor G.N., Dougherty T.F., Mays C.W., Lloyd R.D., Atherton D.R. and Jee W.S.S. 1972d. Radium-induced eye melanomas in dogs. Radiat. Res. 51:361-373.

$[56,80,131,134,137]$

Taylor G.N., Williams J.L., Roberts L., Atherton D.R. and Shabestari L. 1974. Increased toxicity of $\mathrm{Na}_{3} \mathrm{CaDTPA}$ when given by protracted administration. Health Phys. 27:285-288.

- Taylor G.N., Jee W.S.S. and Mays C.W. 1976. Some similarities of radium and plutonium toxicity in the beagle and man. In Health Effects of Plutonium and Radium (W.S.S. Jee, ed., JW Press, Salt Lake City) 523-536.

$[69,70,72,74,79,80,114,127,128,131,134,137]$

- Taylor G.N., Shabestari L., Williams J., Mays C.W., Angus W. and McFarland S. 1976b. Mammary neoplasia in a closed beagle colony. Cancer Res. 36:2740-2743.

$[16,80,120,128,131,134,137,140]$ 
Taylor G.N., Lloyd R.D., Boseman J.J., Atherton D.R. and Mays C.W. 1978. Removal of plutonium from beagles using Ca-DTPA and Zn-DTPA: Effects of initial DTPA injection. Health Phys. 35:201-210.

Taylor G.N. and Mays C.W. 1978b. Fetal injury induced by Ca-DTPA in dogs. Health Phys. 35:858-860.

- Taylor G.N., Shabestari L., Angus W., Lloyd R.D. and Mays C.W. 1979. Primary pulmonic tumors in beagles. Am. J. Vet. Res. 40:1316-1318. [16, 77]

- Taylor G.N., Thurman G.B., Mays C.W., Shabestari L., Angus W. and Atherton D.R. 1981. Plutonium-induced osteosarcomas in the St. Bernard. Radiat. Res. 88:180-186.

$[74,75,128]$

- Taylor G.N., Mays C.W., Wrenn M.E. and Shabestari L. 1983. Incidence of liver tumors in beagles with body burdens of Pu-239 or Am-241. In Proceedings 7 th International Congress of Radiation Research (J.J. Broerse et al., eds., Martinus Nijhoff, Amsterdam) C7-08.

- Taylor G.N., Mays C.W., Lloyd R.D., Gardner P.A., Talbot L.R., McFarland S.S., Pollard T.A., Atherton D.R., VanMoorhem D., Brammer D., Brammer T.W., Ayoroa G. and Taysum D.H. 1983b. Comparative toxicity of ${ }^{226} \mathrm{Ra}$, ${ }^{239} \mathrm{Pu},{ }^{241} \mathrm{Am},{ }^{249} \mathrm{Cf}$, and ${ }^{252} \mathrm{Cf}$ in $\mathrm{C} 57 \mathrm{BL} / \mathrm{Do}$ black and albino mice. Radiat. Res. 95:584-601.

$[74,110,145]$

- Taylor G.N., Mays C.W., Wrenn M.E., Shabestari L. and Lloyd R.D. 1986. Incidence of liver tumors in beagles with body burdens of ${ }^{239} \mathrm{Pu}$ or ${ }^{241} \mathrm{Am}$. In Life-Span Radiation Effects Studies in Animals: What Can They Tell Us? (R.C. Thompson and J.A. Mahaffey, eds., CONF-830951, NTIS, Springfield, VA) $268-285$.

$[55,63,79,127,128,142]$

Taylor N., Shifrine M., Wolf H.G. and Trommerhausen-Smith A. 1975. Canine osteosarcoma karyotypes from an original tumor, its metastasis, and tumor cells in tissue culture. Transplant. Proc. 7:485-493.

- Taysum, D.H., Evans F.G., Hammer W.M., Jee W.S.S., Rehfeld C.E. and Blake L.W. 1962. Radionuclides and bone strength. In Some Aspects of Internal Irradiation (T.F. Dougherty et al., eds., Pergamon, New York) 145-162.

Theilen G.H., Leighton R., Pool R. and Park R.D. 1977. Treatment of canine osteosarcoma for limb preservation using osteotomy, adjuvant radiotherapy and chemotherapy (a case report). Pet Practice-Vet. Med./Small Anim. Clin. 1977:179-183.

Thilsted J.P. and Shifrine M. 1977. Lymphocyte transformation in the dog: Response of lymphocytes from normal and immune dogs to phytohemaglutinin, coccidioidin, and purified-protein derivative. Am. J. Vet. Res. 38:81-87.

Thilsted J.P. and Shifrine M. 1978. Delayed cutaneous hypersensitivity in the dog: Reaction to tuberculin Purified Protein Derivative and coccidioidin. Am. J. Vet. Res. 39:1702-1705.

Thilsted J.P. and Shifrine M. 1979. Studies on the transfer of tuberculin specific CMI in the dog. In Immune Regulators in Transfer Factor (A. Khan, C. Kirkpatrick and N.O. Hill, eds., Academic Press, New York) 673-678. 
Thilsted J.P., Shifrine M. and Wiger N. 1979b. Correlation of in vitro and in vivo tests for cell-mediated immunity in the dog. Am. J. Vet. Res. 40:13131315.

Thilsted J.P., Shifrine M., Wilson F.D., Stitzel K. and Indrieri C. $\mathbf{1 9 8 0}$. Graft-versus-host disease in two immunocompetent dogs. Exp. Hematol. $8: 351-360$.

Bob Thomas was associated from its inception in 1961 with the program of life-span studies in beagles that became the Inhalation Toxicology Research Institute. After a sojurn at the Los Alamos Scientific Laboratory, from 1974 to 1984, he moved to the Office of Health and Environmental Research of the Department of Energy, where he has oversight responsibility for all of DOE's radiation biology programs, including the life-span beagle projects.

- Thomas R.G. 1964. Influence of aerosol properties upon gross distribution and excretion. Health Phys. 10:1013-1028.

[106]

Thomas R.G. 1968. Transport of relatively insoluble materials from lung to lymph nodes. Health Phys. 14:111-117.

[106]

Thomas R.G. 1970. Estimation of ${ }^{241}$ Am body burdens by analysis of whole-body scanning, excreta, and body weight data. Health Phys. 19:751-755.

$[47,101,108]$

- Thomas R.G. 1971. Retention kinetics of inhaled fused aluminosilicate particles. In Inhaled Particles III. Vol. 1. (W.H. Walton, ed., Unwin Bros., Surrey, England) 193-200.

Thomas R.G. 1972. An interspecies model for retention of inhaled particles. In Assessment of Airborne Particles. Fundamentals, Applications and Implications to Inhalation Toxicity (T.T. Mercer, et al., eds., Charles C. Thomas, Springfield, IL) 405-420.

[112]

Thomas R.G., McClellan R.O., Thomas R.L., Chiffelle T.L., Hobbs C.H., Jones R.K., Mauderly J.L. and Pickrell J.A. 1972b. Metabolism, dosimetry and biological effects of inhaled ${ }^{241} \mathrm{Am}$ in beagle dogs. Health Phys, 22:863871 .

$[47,48,101]$

- Thomas R.G. 1972c. Tracheobronchial lymph node involvement following inhalation of alpha emitters. In Radiobiology of Plutonium (B.J. Stover and W.S.S. Jee, eds., JW Press, Salt Lake City) 231-241.

$[111,112]$

Thomas R.G., Healy J.W. and McInroy J.F. 1984. Plutonium partitioning among internal organs. Health Phys. 46:839-844.

Roy Thompson has had no direct involvement in the conduct of experiments with beagles but has been closely associated with those who did, since he came to the Pacific Northwest Laboratory in 1950. He has made many applications of the beagle data, and since his retirement in 1985 has been preparing a review of the total research effort on life-span radiation effect studies in beagles.

Thompson R.C. 1960. Vertebrate radiobiology: Metabolism of internal emitters. Ann. Rev. Nucl. Sci. 10:531-560 
Thompson R.C. 1967. Biological factors. In Plutonium Handbook: A Guide to the Technology, Vol. 2 (O.J. Wick, ed., Gordon and Breach, New York) 785-829.

Thompson R.C. 1968. Properties of radionuclides in tissues: A new look at some old problems. In Diagnosis and Treatment of Deposited Radionuclides (H.A. Kornberg and W.D. Norwood eds., Excerpta Medica Foundation, Amsterdam) 39-44.

Thompson R.C., Park J.F. and Bair W.J. 1972. Some speculative extensions to man of animal risk data on plutonium. In Radiobiology of Plutonium (B.J. Stover and W.S.S. Jee, eds., JW Press, Salt Lake City) 221 230.

$[82,111,115]$

Thompson R.C. 1974. Effects of plutonium in animals. In Plutonium Information Meeting for an Ad Hoc Subcommittee of the Advisory Committee on Reactor Safeguards (CONF-740115, NTIS, Springfield, VA) 56-63.

[111]

Thompson R.C. $\mathbf{1 9 7 4 b . ~ I m p l i c a t i o n s ~ w i t h ~ r e s p e c t ~ t o ~ p r o t e c t i o n ~ c r i t e r i a . ~}$ In Plutonium and other Transuranium Elements: Sources, Environmental Distribution and Biomedical Effects (WASH-1359, NTIS, Springfield, VA) 271-289.

Thompson R.C. 1975. Animal data on plutonium toxicity. Health Phys. 29:511-519.

Thompson R.C. 1975b. Transuranium element toxicity-Dose-response relationships at low exposure levels. Summary and speculative interpretation relative to exposure limits. In Radiation Research-Biomedical, Chemical, and Physical Perspectives (O.F. Nygaard et al., eds., Academic Press, New York) 1278-1284.

$[107,111]$

Thompson R.C. 1977. Role of animal toxicity studies in the evaluation of human health risks from internally deposited transuranics. In Proceedings IVth International Congress, Vol. 4 (International Radiation Protection Association, Paris) 1333-1336.

$[106,107]$

Thompson R.C. 1983. 1976 Hanford americium exposure incident: Overview and perspective. Health Phys. 45:837-845. [87, 113]

- Thompson R.C. 1984. Life-span radiation effects studies in animals: What can they tell us? In Radiation-Risk-Protection, Vol I (A. Kaul et al., eds., International Radiation Protection Association, Berlin) 347-350.

- Thompson R.C. and Mahaffey J.A. (eds.) 1986. Life-Span Radiation Effects Studies in Animals: What Can They Tell Us? CONF-830951, NTIS, Springfield, VA.

- Thompson R.C., Cross F.T., Dagle G.E., Park J.F. and Sanders C.L. 1986b. DOE life-span radiation effects studies at Pacific Northwest Laboratory. In Life-Span Radiation Effects Studies in Animals: What Can They Tell Us? (R.C. Thompson and J.A. Mahaffey, eds., CONF-830951, NTIS, Springfield, VA) 66-73.

- Thompson R.C. 1988. The legacy of life-span dog studies. Health Phys. $55: 483-485$ 
- Thurman G.B., Mays C.W., Taylor G.N., Christensen W.R., Rehfeld C.E. and Dougherty T.F. 1971. Growth dymanics of beagle osteosarcomas. Growth 35:119-125.

$[75,128,131,134,137,140]$

- Thurman, G.B., Mays C.W., Taylor G.N., Keane A.T. and Sissons H.A 1973. Skeletal location of radiation-induced and naturally occurring osteosarcomas in man and dog. Cancer Res. 33:1604-1607.

Tjalma R.A. 1966. Canine bone sarcoma: Estimation of relative risk as a function of body size. J. Natl. Cancer Inst. 36:1137-1150.

David Tolle, a clinical hematologist at Argonne National Laboratory since 1963, has played a principal role in diagnostic hematology and differential cytopathology in the leukemogenesis and radiation toxicology programs that have developed from the chronic gamma-ray exposure experiments with beagles.

- Tolle D.V., Fritz T.E. and Norris W.P. 1977. Radiation-induced erythroleukemia in the beagle dog. A hematologic summary of five cases. Am. J. Pathol. 87:499-510. $[64,72,170]$

- Tolle D.V., Seed T.M., Fritz T.E., Lombard L.S., Poole C.M. and Norris W.P. 1979. Acute monocytic leukemia in an irradiated beagle. Vet. Pathol. 16:243-254. $[64,72,172]$

- Tolle D.V., Seed T.M., Fritz T.E. and Norris W.P. 1979b. Irradiation-induced canine leukemia: A proposed new model. Incidence and Hematopathology. In Experimental Hematology Today 1979. (S.J. Baum and G.D. Ledney, eds., Springer-Verlag, New York) 247-256.

$[64,72,118,119,170,172]$

- Tolle D.V., Fritz T.E., Seed T.M., Cullen S.M., Lombard L.S. and Poole C.M. 1982. Leukemia induction in beagles exposed continuously to ${ }^{60} \mathrm{Co}$ gamma irradiation: Hematopathology. In Experimental Hematology Today 1982 (S.J. Baum, G.D. Ledney and S. Thierfelder, eds., S. Karger, Basel) 241-249.

$[64,72,118,119,170]$

- Tolle D.V., Cullen S.M., Seed T.M. and Fritz T.E. 1983. Circulating micromegakaryocytes preceding leukemia in three dogs exposed to $2.5 \mathrm{R} /$ day gamma radiation.. Vet. Pathol. 20:111-114.

- Tolle D.V., Seed T.M., Cullen S.M., Poole C.M. and Fritz T.E. 1983b. Aberrant megakaryocytopoiesis preceding radiation-induced leukemia in the dog. In Scanning Electron Microscopy/1982/I (O. Johari, R.M. Albrecht and T.,M. Seed, eds., SEM Inc., AMF O'Hare, Chicago IL) 367-376. [64, 119, 170]

Tombropoulos E.G., Bair W.J. and Park J.F. 1963. Effect of diethylenetriamine-pentaacetic acid and polypropylenoglycolethylene oxide polymer on excretion of inhaled ${ }^{239} \mathrm{Pu}_{2}$ in dogs. Nature 198:703-704.

[89]

Tombropoulos E.G. 1964. Review of therapeutic procedures for removal of inhaled radioactive materials. Health Phys. 10:1251-1257.

[89]

- Totter J.R. 1972. Biological research with plutonium 1944-1984. In Radiobiology of Plutonium (B.J. Stover and W.S.S. Jee, eds., JW Press, Salt Lake City) 23-27.

$[4,10,13]$ 
- Tsai H-C.C., Della Rosa R.J., Rosenblatt L.S. and Nix N. 1970. Radium-226 effects on the mucopolysaccharides of cartilage. Health Phys. 18:71-73.

$[68,163]$

Tuttle W.C. and Westerberg S.C. 1974. Alpha-1 globulin trypsin inhibitor in canine surfactant protein. Proc. Soc. Exp. Biol. Med. 146:232-235.

- Twente J.A. and Jee W.S.S. 1961. The determination of localized concentration of $\mathrm{Pu}^{239}$ in bone. Health Phys. 5:142-148.

Tyler S.A. and Norris W.P. 1968. An algorithm for selecting acceptable animals from a colony of beagles. Growth 32:235-253.

- Tyler S.A., Kretz N.D. and Norris W.P. 1969. Dependence of ${ }^{137} \mathrm{Cs}$ retention on age in the fetal and juvenile beagle dog. In Radiation Biology of the Fetal and Juvenile Mammal (M.R. Sikov and D.D. Mahlum, eds., CONF69050l, NTIS, Springfield, VA) 191-205.

United Nations Scientific Committee on the Effects of Atomic Radiation. 1972. Ionizing Radiation: Levels and Effects. Vol. II: Effects. United Nations, New York.

United Nations Scientific Committee on the Effects of Atomic Radiation. 1977. Sources and Effects of Ionizing Radiation. United Nations, New York.

[106]

United Nations Scientific Committee on the Effects of Atomic Radiation. 1982. Ionizing Radiation: Sources and Biological Effects. United Nations, New York.

[106]

United States Atomic Energy Commission. 1974. Liquid Metal Fast Breeder Reactor Program. Environmental Statement. Vol. II. WASH-1535, NTIS, Springfield, VA.

United States Energy Research and Development Administration. 1975. Final Environmental Statement, Liquid Metal Fast Breeder Reactor Program. Vols. 1, 2, and 3. ERDA-1535, NTIS, Springfield, VA.

United States Nuclear Regulatory Commission. 1976. Natural Resources Defense Council. Denial of Petition for Rule Making. Fed. Reg. 41(71):15371-15379.

Valberg P.A., Wolff R.K. and Mauderly J.L. 1985. Redistribution of retained particles: Effect of hyperpnea. Am. Rev. Respir. Dis. 131:273-280.

Marvin Van Dilla was the original radiological physicist on the University of Utah beagle project, from 1951 to 1957, and had a major hand in the design and initiation of the early Utah studies.

- Van Dilla M.A. and Stover B.J. 1956. On the role of radiothorium ( $\mathrm{Th}^{228}$ ) in radium poisoning. Radiology 66:400-402.

$[7,43,137]$ 
- Van Dilla M.A., Stover B.J. and Arnold J.S. 1957. On the retention and translocation of $\mathrm{Ra}^{224}$ (Th X) in dogs. Am. J. Roentgenol. Radium Therap. Nucl. Med. 77:503-510.

$[43,137]$

- Van Dilla M.A., Stover B.J., Floyd R.L., Atherton D.R. and Taysum D.H. 1958. Radium $\left(\mathrm{Ra}^{226}\right)$ and radon $\left(\mathrm{Em}^{222}\right)$ metabolism in dogs. Radiat. Res. $8: 417-437$

$[42,43,130]$

Vaughan J. 1965. Non-uniformity of radiation dose in space with special reference to radiological protection. Int. J. Radiat. Biol. 9:51 3-543.

[105]

Vaughan J. 1971. Haematological consequences of radioisotope incorporation with particular reference to plutonium, thorium, radium, iron, and gold. In Manual on Radiation Hematology (International Atomic Energy Agency, Vienna) 215-242

Vaughan J., Bleaney B. and Taylor D.M. 1973. Distribution, excretion and effects of plutonium as a bone-seeker. In Uranium-Plutonium-Transplutonic Elements (H.C. Hodge, et al., eds., Springer-Verlag, New York) 349-502.

$[105,176]$

Vaughan J.M. 1973b. Effects of Irradiation on the Skeleton. Oxford Univ. Press, New York.

[105]

Vaughan J. 1976. Plutonium-A possible leukaemic risk. In Health Effects of Plutonium and Radium (W.S.S. Jee, ed., JW Press, Salt Lake City) 691-705.

[73]

Waligora S.J.,Jr. 1971. Pulmonary retention of zirconium oxide $\left({ }^{95} \mathrm{Nb}\right)$ in man and beagle dogs. Health Phys. 20:89-91.

$[96,111]$

Waring G.O., Muggli F.M. and MacMillan A. 1977. Oval corneal opacities in beagles. J. Am. Anim. Hosp. Assoc. 13:204-208.

Waring G.O., Spangler W.L., Roth A.M. and MacMillan A.D. 1978. Oval corneal opacities in beagles: Clinical, histochemical and ultrastructural description. Metabol. Ophthalmol. 2:75.

Waring G.O., Ekins M.B., Spangler W.L., Roth A.M. and MacMillan A.D. 1979. Oval lipid corneal opacities in beagles and crystalline lipid corneal opacities in Siberian Huskies. Metabol. Pediatr. Ophthalmol. 3:203-213. [103]

Waring G.O., MacMillan A.D., Roth A.M., Spangler W.L. and Ekins M.B. 1979b. Lipid corneal opacities in beagles and Siberian huskies. Proc. Am. Coll. Vet. Ophthalmol. 10:1-27.

[103]

Warner M.R. 1977. Response of beagle mammary dysplasias to various hormone supplements in vitro. Cancer Res. 37:2062-2067.

Warner R.L. and McFarland L.Z. 1970. Integument [of the beagle]. In The Beagle as an Experimental Dog (A.C. Andersen, ed., Iowa State Univ. Press, Ames, Iowa) 126-148.

- Watters R.L. and Lebel J.L. 1972. Progress in the beagle studies at Colorado State University. Health Phys. 22:811-814.

$[4,93]$ 
Watts L. 1975. Clearance rates of insoluble plutonium-239 compounds from the lung. Health Phys. 29:53-59.

Weiser M.G., Spangler W.L. and Gribble D.H. 1977. Blood pressure measurement in the dog. J. Am. Vet. Med. Assoc. 171:364-368.

Dick Weller, a veterinary oncologist at Pacific Northwest Laboratory since 1980, is responsible for clinical pathology and oncology studies in beagles exposed to inhaled plutonium.

- Weller R.E., Park J.F., Stevens D.L. and Park C.L. 1986. Application of a systematized nomenclature of veterinary medicine (SNOVET) to diagnostic coding and retrieval of clinical life-span data in beagle dogs exposed to plutonium. In Life-Span Radiation Effects Studies in Animals: What Can They Tell Us? (R.C. Thompson and J.A. Mahaffey, eds., CONF-830951, NTIS, Springfield, VA) 488-495.

Weller R.E., Dagle G.E. and Perry R.L. 1986b. A comparison of thyroid carcinoma in two dogs. Mod. Vet. Pract. 2:116-119.

- West J.E. and Bair W.J. 1964. Plutonium inhalation studies. V. Radiation syndrome in beagles after inhalation of plutonium dioxide. Radiat. Res. 22:489-506.

$[35,61,62,66,174]$

- Whaley S.L., Wolff R.K., Muggenburg B.A. and Snipes M.B. 1986. Mucociliary clearance and particle retention in the maxillary and ethmoid turbinate regions of beagle dogs. J. Toxicol. Environ. Health 19:569-580.

- Whaley S.L., Muggenburg B.A., Seiler F.A. and Wolff R.K. 1987. Effeect of aging on tracheal mucociliary clearance in beagle dogs. J. Appl. Physiol. 62:1331-1334.

Whaley S.L., Renken S., Muggenburg B.A. and Wolff R.K. 1988. Technique for aerosol deposition restricted to the nose in beagle dogs. J. Toxicol. Environ. Health 23:519-525.

- Whittemore A.S. and McMillan A. 1982. Osteosarcomas among beagles exposed to ${ }^{239} \mathrm{Pu}$. Radiat. Res. 90:41-56.

$[76,116,127]$

Floyd Wilson's contributions at the University of California, Davis during the decade of the 1970s centered around studies of leukemogenesis. These studies derived from the life-span experiments with ${ }^{90} \mathrm{Sr}$ and ${ }^{226} \mathrm{Ra}$, and from non-life-span gamma-irradiation experiments.

- Wilson F.D. and Shifrine M. 1972. Radiation-induced transplantable myelomonocytic leukemia in beagles: Hematologic parameters. Exp. Hematol. (Oak Ridge Tenn.) 22:41-44. $[65,72,94,95,159]$

- Wilson F.D., Pool R.R., Stitzel K, and Momeni M.H. 1976. The bone-bone marrow interface (endosteum) potential relationship of microenvironments in the regulation of response to internal emitters. In Health Effects of Plutonium and Radium (W.S.S.Jee, ed., JW Press, Salt Lake City) 617-642.

$[121,163]$ 
Wilson F.D. and Shifrine M. 1977. In vitro growth of canine and murine granulocyte-monocyte, fibroblast and lymphocyte colonies: Preliminary results. In Regulatory Mechanisms in Lymphocyte Activation (D.O. Lucas, ed., Academic Press, New York) 572-574.

Wilson F.D. and Shifrine M. 1978. The canine as a model for immunohematological studies of leukemia. In Animal Models of Comparative and Developmental Aspects of Immunity and Disease (M.E. Gershwin and E.L. Cooper, eds., Pergamon, New York) 346-358.

$[94,118]$

Wilson F.D., Shifrine M., Gershwin M.E., Spangler W. and Dyck J. $1978 b$. Growth of canine T-lymphocyte colonies in vitro. Exp. Hematol. 6:539-548.

Wilson F.D., Stitzel K.A., Klein A.K., Shifrine M., Graham R., Jones M., Bradley E. and Rosenblatt L.S. 1978c. Quantitative response of bone marrow colony-forming units (CFU-C and PFU-C) in weanling beagles exposed to acute whole-body gamma irradiation. Radiat. Res. 74:289-297.

$[95,121]$

- Wilson F.D. and Shifrine M. 1980. The canine as a model for studies on stem and progenitor cells of lymphohematopoiesis. In The Canine as a Biomedical Research Model: Immunological, Hematological, and Oncological Aspects (M. Shifrine and F.D. Wilson, eds., DOE/TIC-10191, NTIS, Springfield, Va) 3-42.

$[94,118]$

Wilson F.D., Dyck J.A., Knox S.J. and Shifrine M. 1980b. A "whole-blood" technique for the quantitation of canine "T-lymphocyte" progenitors using a semisolid culture system. Exp. Hematol. 8:1031-1039.

Wilson F.D., Tavassoli M., Greenberg B.R., Hinds D. and Klein A.K. 1981. Morphological studies on "adherent cells" in bone marrow cultures from humans, dogs, and mice. Stem Cells 1:15-29.

Winchell H.S., Pollycove M., Andersen A.C. and Lawrence J.H. 1964. Relatively selective beta irradiation of lymphatic structures in the dog using Y 90 -DTPA. Blood 23:321-336.

Wittmier J.R. and James C.K. 1983. A computerized system for veterinary medical records. Lab. Anim. Sci. 33:101-105.

- Wolf H.G., Della Rosa R.J. and Andersen A.C. 1966. Nutritional management of a large experimental beagle colony. Lab. Anim. Care 16:309-315. [16]

Wolf H.G., Della Rosa R.J. and Corbin J.E. 1970. Nutriton [of the beagle]. In The Beagle as an Experimental Dog (A.C. Andersen, ed., Iowa State Univ. Press, Ames, Iowa) 22-30.

Wolf H.G. 1973. The use and misuse of supplementation. In Proceedings of 23 rd Gaines Veterinary Symposium, Pullman, WA, 8-10.

Wolff R.K. and Muggenburg B.A. 1979. Comparison of two methods of measuring tracheal mucous velocity in anesthetized beagle dogs. Am. Rev. Respir. Dis. 120:137-142.

- Wolff R.K., Merickel B.S., Rebar A.H. and Mewhinney J.A. 1980. Comparison of bone scans and radiography for detecting bone neoplasms in dogs exposed to ${ }^{238} \mathrm{PuO}_{2}$. Am J. Vet. Res. 41:1804-1807.

$[28,207,209]$ 
Wolff R.K., Kanapilly G.M., DeNee P.B. and McClellan R.O. 1981. Deposition of $0.1 \mu \mathrm{m}$ chain aggregate aerosols in beagle dogs. J. Aerosol Sci. 12:119-129.

Wolff R.K., Muggenburg B.A. and Silbaugh S.A. 1981b. Effect of 0.3 and $0.9 \mu \mathrm{m}$ sulfuric acid aerosols on tracheal mucous clearance in beagle dogs. Am. Rev. Respir. Dis. 123:291-294.

Wolff R.K., Hardy S.C. and Muggenburg B.A. 1982. Effect of radiolabeled materials on tracheal mucous clearance in beagle dogs. Am. Rev. Respir. Dis. 126:505-508.

Wolff R.K., Kanapilly G.M., Gray R.H. and McClellan R.O. 1984. Deposition and retention of inhaled aggregate ${ }^{67} \mathrm{Ga}_{2} 0_{3}$ particles in beagle dogs, Fischer-344 rats, and CD-1 mice. Am. Ind. Hyg. Assoc. J. 45:377-381. [91]

Wolff R.K., Beierman W.F., Eng P., Mason M.J., Harkema J.R. and Muggenburg B.A. 1988. Detection of a liver tumor in a beagle dog using single photon emission computed tomography. Vet. Radiol. 29:179-183. [28]

Wolff R.K., Kitzman J.V., Muggenburg B.A. and Mauderly J.L. In Press. Clearance of $99 \mathrm{~m}$ Tc-DTPA from four sites in the respiratory tract of dogs. $J$. Aerosol Med.

[91]

"Ed" Wrenn, after an eventful career in environmental radiobiology at New York University, assumed directorship of the University of Utah Radiobiology Laboratory in 1979, a position he held until 1986.

- Wrenn M.E., ed. 1981. Actinides in Man and Animals. RD Press, Univ. of Utah, Salt Lake City.

Wrenn M.E. and Roswell R.L. 1981b. A review of ${ }^{241} \mathrm{Am}$ accumulation by man and an estimation of the carcinogenic risks. In Actinides in Man and Animals (M.E. Wrenn, ed., RD Press, Salt Lake City) 443-453.

Wrenn M.E., Durbin P.W., Howard B., Lipsztein J., Rundo J., Still E.T. and Willis D.L. 1985. Metabolism of ingested U and Ra. Health Phys. 48:601-633.

- Wrenn M.E., Taylor G.N., Stevens W., Mays C.W., Jee W.S.S., Lloyd R.D., Atherton D.R., Bruenger F.W., Miller S.C., Smith J.M., Shabestari L.R., Woodbury L.A. and Stover B.J. 1986. DOE life-span radiation effects studies in experimental animals at University of Utah Division of Radiobiology. In Life-Span Radiation Effects Studies in Animals: What Can They Tell Us? (R.C. Thompson and J.A. Mahaffey, eds., CONF-830951, NTIS, Springfield, VA) $32-52$. $[10,74,75,126,131,142,143,145,147,150,151,153,154]$

- Wrenn M.E., Taylor G.N., Stevens W., Mays C.W., Jee W.S.S., Lloyd R.D., Atherton D.R., Bruenger F.W., Kimmel D.B., Miller S.C., Shabestari L., Smith J.M., Woodbury L. and Stover B.J. 1986b. Summary of dosimetry, pathology, and dose response for bone sarcomas in beagles injected with radium-226. In Life Span Radiation Effects Studies in Animals: What Can They Tell Us? (R.C. Thompson and J.A. Mahaffey, eds., CONF-830951, NTIS, Springfield, VA) 253-267.

$[42,73,75,130,137]$

- Wrenn M.E., Lloyd R.D., Mays C.W. and Taylor G.N. 1986c. Relative effectiveness of alpha emitters for bone sarcoma induction. Health Phys. $51: 677-679$.

$[109,116,127,131,134,142,143,145,163]$ 
- Wronski T.J., Smith J.M. and Jee W.S.S. 1980. The microdistribution and retention of injected ${ }^{239} \mathrm{Pu}$ on trabecular bone surfaces of the beagle: Implications for the induction of osteosarcoma. Radiat. Res. 83:74-89.

$[53,76,119,121,127,128]$

Wronski T.J., Smith J.M. and Jee W.S.S. 1981. Variations in mineral apposition rate of trabecular bone within the beagle skeleton. Calcif. Tissue Int. 33:583-586.

- Zanelli G.D., Darley P.J. and Goldman M. 1971. Marrow absorbed dose-rates in bones of beagle dogs raised on diets containing strontium-90. In Proceedings 5th International Meeting (French Society for Radiation Protection, Grenoble, France) 381-400.

$[53,159]$ 



\section{REPORT LITERATURE}

Although this document has been based very largely upon publications in the open scientific literature, some descriptions of, and observations from the lifespan beagle experiments, and the detailed tabulations of individual animal data, will be found only in U.S. DOE/ERDA/AEC reports published from the various laboratories. A chronological listing of these reports, from each laboratory, is provided in this chapter. The individual "Experiment Summaries" of the Appendix (Chapter 8 ) include page-number references to these reports.

\begin{tabular}{|c|c|c|c|}
\hline \multicolumn{2}{|c|}{ Report Date } & $\begin{array}{l}\text { Document } \\
\text { Number }\end{array}$ & $\begin{array}{l}\text { Document } \\
\text { Iite }\end{array}$ \\
\hline Unive & rsity 0 & Itah Radiobio & Laboratory Reports \\
\hline 1954 & Jun & TID-7639 & Consultants Meeting \\
\hline 1955 & Mar & AECU-3418 & Annual Report \\
\hline 1955 & Sep & AECU-3109 & Semi-Annual Report \\
\hline 1956 & Mar & TID-16458 & Annual Report \\
\hline 1956 & Sep & TID-16459 & Semi-Annual Report \\
\hline 1957 & Mar & AECU-3522 & Annual Report \\
\hline 1957 & Sep & AECU-3583 & Semi-Annual Report \\
\hline 1958 & Mar & $\mathrm{COO}-215$ & Annual Report \\
\hline 1958 & Mar & $\mathrm{COO}-216$ & Escape of Radon and Thoron \\
\hline 1958 & Sep & $\mathrm{COO}-217$ & Semi-Annual Report \\
\hline 1959 & Feb & AECU-4112 & Radioactive Fallout \\
\hline 1959 & Mar & $\mathrm{COO}-218$ & Annual Report \\
\hline 1959 & Sep & COO-219 & Semi-Annual Report \\
\hline 1960 & Mar & $\mathrm{COO}-220$ & Research in Radiobiology \\
\hline 1960 & Aug & $\mathrm{COO}-221$ & Interim Report on ${ }^{90} \mathrm{Sr}$ \\
\hline 1960 & Sep & $\mathrm{COO}-222$ & Research in Radiobiology \\
\hline 1961 & Mar & $\mathrm{COO}-223$ & Research in Radiobiology \\
\hline 1961 & Sep & $\mathrm{COO}-224$ & Research in Radiobiology \\
\hline 1962 & Mar & $\mathrm{COO}-225$ & Research in Radiobiology \\
\hline 1962 & Sep & $\mathrm{COO}-226$ & Research in Radiobiology \\
\hline 1963 & Mar & $\mathrm{COO}-227$ & Research in Radiobiology \\
\hline 1963 & Sep & $\mathrm{COO}-228$ & Research in Radiobiology \\
\hline 1964 & Mar & $C 00-119-229$ & Research in Radiobiology \\
\hline 1964 & Jul & COO-119-230 & Radiobiology Safety Manual \\
\hline 1964 & Sep & COO-119-231 & Research in Radiobiology \\
\hline
\end{tabular}




\begin{tabular}{|c|c|c|c|}
\hline \multicolumn{2}{|c|}{ Report Date } & \multirow{2}{*}{$\begin{array}{l}\text { Document } \\
\text { Number }\end{array}$} & \multirow{2}{*}{$\begin{array}{l}\text { Document } \\
\text { Title }\end{array}$} \\
\hline Year & Month & & \\
\hline \multicolumn{4}{|c|}{ University of Utah Radiobiology Laboratory Reports (Continued) } \\
\hline 1965 & Mar & $\mathrm{COO}-119-232$ & Research in Radiobiology \\
\hline 1965 & Sep & COO-119-233 & Research in Radiobiology \\
\hline 1966 & Mar & $\mathrm{COO}-119-234$ & Research in Radiobiology \\
\hline 1966 & Sep & COO-119-235 & Research in Radiobiology \\
\hline 1967 & Mar & COO-119-236 & Research in Radiobiology \\
\hline 1968 & Mar & COO-119-237 & Research in Radiobiology \\
\hline 1968 & Aug & COO-119-238 & $\mathrm{Rb}$ in $\mathrm{RBC}$, Plasma, and Urine \\
\hline 1968 & Dec & COO-119-239 & $\mathrm{Cs}, \mathrm{Rb}$, and $\mathrm{K}$ Metabolism \\
\hline 1969 & Mar & COO-119-240 & Research in Radiobiology \\
\hline 1970 & Mar & COO-119-241 & Retention and Dosimetry \\
\hline 1971 & Jan & COO-119-242 & Research in Radiobiology \\
\hline 1971 & $\operatorname{Jan}$ & COO-119-243 & Osteosarcoma Growth Dynamics \\
\hline 1971 & Mar & $\mathrm{COO}-119-244$ & Research in Radiobiology \\
\hline 1971 & May & COO-119-245 & Radiobiology Safety Manual \\
\hline 1972 & Mar & COO-119-246 & Research in Radiobiology \\
\hline 1972 & Oct & $\mathrm{COO}-119-247$ & Rb and Cs Metabolism \\
\hline 1973 & Mar & $\mathrm{COO}-119-248$ & Research in Radiobiology \\
\hline 1974 & Mar & COO-119-249 & Research in Radiobiology \\
\hline 1975 & Mar & COO-119-250 & Research in Radiobiology \\
\hline 1976 & Mar & COO-119-251 & Research in Radiobiology \\
\hline 1977 & Mar & $\mathrm{COO}-119-252$ & Research in Radiobiology \\
\hline 1978 & Mar & $\mathrm{COO}-119-253$ & Research in Radiobiology \\
\hline 1979 & Mar & COO-119-254 & Research in Radiobiology \\
\hline 1980 & Jan & COO-119-255 & Radiobiology Safety Manual \\
\hline 1980 & Mar & COO-119-256 & Research in Radiobiology \\
\hline 1982 & Mar & $\mathrm{COO}-119-257$ & Research in Radiobiology \\
\hline 1983 & Mar & COO-119-258 & Research in Radiobiology \\
\hline 1984 & Dec & COO-119-259 & Research in Radiobiology \\
\hline 1985 & Dec & $\mathrm{COO}-119-261$ & Research in Radiobiology \\
\hline 1986 & Dec & COO-119-262 & Research in Radiobiology \\
\hline 1987 & Dec & COO-119-263 & Research in Radiobiology \\
\hline
\end{tabular}

University of California, Davis, Laboratory for Energy-Related Health Research (Radiobiology Laboratory) Reports

1956

1957 Jun
Effect of X-Radiation on the Work Capacity and Longevity of the

Dog, Fifth Annual Progress Report

Effect of X-Radiation on Work Capacity and Longevity of the Dog 


\begin{tabular}{|c|c|c|c|}
\hline \multicolumn{2}{|c|}{ Repert Date } & \multirow{2}{*}{$\begin{array}{l}\text { Document } \\
\text { Number }\end{array}$} & \multirow{2}{*}{$\begin{array}{l}\text { Document } \\
\text { Tite }\end{array}$} \\
\hline Year & Month & & \\
\hline \multicolumn{4}{|c|}{$\begin{array}{l}\text { University of California, Davis, Laboratory for Energy-Related Health Research } \\
\text { (Radiobiology Laboratory) Reports (Continued) }\end{array}$} \\
\hline 1958 & Jun & & Effect of X-Radiation on Work Capacity and Longevity of the Dog \\
\hline 1958 & & & $\begin{array}{l}\text { Effects of Sr-90 Administered During the Growth Period of the } \\
\text { Dog }\end{array}$ \\
\hline 1959 & Jun & & Effect of X-Radiation on Work Capacity and Longevity of the Dog \\
\hline $1959-6$ & & & $\begin{array}{l}\text { Effects of Continual } \mathrm{Sr}^{90} \text { Ingestion During the Growth Period of } \\
\text { the Beagle and Its Relation to } \mathrm{Ra}^{226} \text { Toxicity }\end{array}$ \\
\hline 1960 & Jun & & Effects of X-Radiation on Work Capacity and Longevity of the Dog \\
\hline 1961 & Jul & UCD 101 & Effects of X-Radiation on Work Capacity and Longevity of the Dog \\
\hline 1961 & & UCD 102 & Effects of X-Irradiation on Reproduction in Female Beagles \\
\hline 1961 & & UCD 103 & Development of the Ovary in the Beagle \\
\hline 1961 & Sep & UCD 104 & $\begin{array}{l}\text { Effects of Continual } \mathrm{Sr}^{90} \text { Ingestion During the Growth Period of } \\
\text { the Beagle and Its Relation to } \mathrm{Ra}^{226} \text { Toxicity }\end{array}$ \\
\hline 1962 & Jun & UCD 105 & Effects of X-Radiation on Work Capacity and Longevity of the Dog \\
\hline 1962 & Jun & UCD 106 & $\begin{array}{l}\text { Efffects of Continual } \mathrm{Sr}^{90} \text { Ingestion During the Growth Period of } \\
\text { the Beagle and Its Relation to } \mathrm{Ra}^{226} \text { Toxicity }\end{array}$ \\
\hline 1963 & Jun & UCD 107 & Effects of X-Radiation on Work Capacity and Longevity of the Dog \\
\hline 1963 & Aug & UCD 108 & $\begin{array}{l}\text { Effects of Continual } \mathrm{Sr}^{90} \text { Ingestion During the Growth Period of } \\
\text { the Beagle and Its Relation to } \mathrm{Ra}^{226} \text { Toxicity }\end{array}$ \\
\hline 1964 & Jun & UCD 472-109 & Effects of X-Radiation on Work Capacity and Longevity of the Dog \\
\hline 1964 & Jul & UCD $472-110$ & $\begin{array}{l}\text { Effects of Continued } \mathrm{Sr}^{90} \text { Ingestion During the Growth Period of } \\
\text { the Beagle and Its Relation to } \mathrm{Ra}^{226} \text { Toxicity }\end{array}$ \\
\hline 1965 & Apr & UCD $472-111$ & $\begin{array}{l}\text { Effects of X-Irradiation on Work Capacity and Longevity of the } \\
\text { Dog }\end{array}$ \\
\hline 1965 & Jun & UCD $472-112$ & $\begin{array}{l}\text { Effects of Continued Sr-90 Ingestion During the Growth Period of } \\
\text { the Beagle and Its Relation to Ra-226 Toxicity }\end{array}$ \\
\hline 1967 & Jun & UCD $472-113$ & 1966 Annual Report \\
\hline 1968 & Jun & UCD $472-114$ & 1967 Annual Report \\
\hline 1969 & Jun & UCD $472-115$ & 1968 Annual Report \\
\hline 1970 & Jun & UCD $472-116$ & 1969 Annual Report \\
\hline 1971 & Jun & UCD $472-117$ & 1970 Annual Report \\
\hline 1972 & Jun & UCD $472-118$ & 1971 Annual Report \\
\hline 1973 & Jun & UCD $472-119$ & 1972 Annual Report \\
\hline 1974 & Jun & UCD $472-120$ & 1973 Annual Repont \\
\hline 1975 & Jun & UCD $472-121$ & 1974 Annual Report \\
\hline 1976 & Jun & UCD $472-122$ & 1975 Annual Report \\
\hline 1977 & Oct & UCD $472-123$ & 1976 Annual Report \\
\hline 1978 & Oct & UCD $472-124$ & 1977 Annual Report \\
\hline
\end{tabular}




\begin{tabular}{|c|c|c|c|}
\hline \multicolumn{2}{|c|}{ Repent Date } & $\begin{array}{l}\text { Document } \\
\text { Number }\end{array}$ & $\begin{array}{l}\text { Document } \\
\text { Tithe }\end{array}$ \\
\hline \multicolumn{4}{|c|}{$\begin{array}{l}\text { University of California, Davis, Laboratory for Energy-Related Health Research } \\
\text { (Radiobiology Laboratory) Reports (Continued) }\end{array}$} \\
\hline 1979 & Oct & UCD 472-125 & 1978-79 Annual Report \\
\hline 1981 & & UCD $472-126$ & 1980 Annual Report \\
\hline 1982 & Sep & UCD $472-127$ & 1981 Annual Report \\
\hline 1983 & Aug & UCD $472-128$ & 1982 Annual Report \\
\hline 1984 & Jul & UCD 472-129 & 1983 Annual Report \\
\hline 1985 & Nov & UCD $472-130$ & 1984 Annual Report \\
\hline 1987 & Jun & UCD 472-131 & 1985 Annual Report \\
\hline \multicolumn{4}{|c|}{ Argonne National Laboratory, Division of Biological and Medical Research Reports } \\
\hline 1955 & Apr & ANL-5426 & Quarterly Report \\
\hline 1956 & Jan & ANL-5518 & Quarterly Report \\
\hline 1957 & Mar & ANL-5696 & Quarterly Report, October, November, December, 1956 \\
\hline 1959 & Dec & ANL-6093 & Semiannual Report, July through December, 1958 \\
\hline 1961 & Nov & ANL-6464 & Semiannual Report, January through June, 1961 \\
\hline 1962 & Apr & ANL-6535 & Semiannual Report, July through December, 1961 \\
\hline 1963 & May & ANL-6723 & Semiannual Report, January through June, 1962 \\
\hline 1963 & Oct & ANL-6790 & Semiannual Report, July through December, 1962 \\
\hline 1964 & Jan & ANL-6823 & Semiannual Report, January through June, 1963 \\
\hline 1964 & Jun & ANL-6906 & Semiannual Report, July through December, 1963 \\
\hline 1964 & Dec & ANL-6971 & Annual Report 1964 \\
\hline 1965 & Dec & ANL-7136 & Annual Report 1965 \\
\hline 1966 & Dec & ANL-7278 & Annual Report 1966 \\
\hline 1967 & Dec & ANL-7409 & Annual Report 1967 \\
\hline 1968 & Dec & ANL-7535 & Annual Report 1968 \\
\hline 1969 & Dec & ANL-7635 & Annual Report 1969 \\
\hline 1970 & Dec & ANL -7770 & Annual Report 1970 \\
\hline 1971 & Dec & ANL-7870 & Annual Report 1971 \\
\hline 1972 & Dec & ANL-7970 & Annual Report 1972 \\
\hline 1973 & Dec & ANL-8070 & Annual Report 1973 \\
\hline 1974 & Dec & ANL-75-30 & Annual Report 1974 \\
\hline 1975 & Dec & ANL-76-99 & Annual Report 1975 \\
\hline 1976 & Dec & ANL- $-77-55$ & Annual Report 1976 \\
\hline 1977 & Dec & ANL-78-90 & Annual Report 1977 \\
\hline 1978 & Dec & ANL-79-90 & Annual Report 1978 \\
\hline 1980 & Dec & ANL-80-90 & Annual Report 1979 \\
\hline 1981 & Aug & ANL-81-50 & Annual Report 1980 \\
\hline
\end{tabular}




\begin{tabular}{lll} 
Report Date & $\begin{array}{l}\text { Document } \\
\text { Year Month }\end{array}$ & $\begin{array}{l}\text { Document } \\
\text { Tithe }\end{array}$ \\
\hline
\end{tabular}

Argonne National Laboratory, Division of Biological and Medical Research Reports (Continued)

$\begin{array}{llll}1982 & \text { Jun } & \text { ANL-82-35 } & \text { Annual Technical Report 1981 } \\ 1983 & \text { May } & \text { ANL-83-40 } & \text { Annual Technical Report } 1982 \\ 1984 & \text { Aug } & \text { ANL-84-30 } & \text { Annual Research Summary 1983 } \\ 1985 & \text { Aug } & \text { ANL-85-30 } & \text { Research Summary 1984-1985 }\end{array}$

\section{Pacific Northwest Laboratory (Hanford Laboratory) Reports}

\begin{tabular}{|c|c|c|c|}
\hline 1960 & Jan & $H W-65500$ & Hanford Biology Research Annual Report for 1959 \\
\hline 1961 & Jan & $H W-69500$ & Hanford Biology Research Annual Report for 1960 \\
\hline 1962 & Jan & $\mathrm{HW}-72500$ & Hanford Biology Research Annual Report for 1961 \\
\hline 1963 & Jan & $\mathrm{HW}-76000$ & Hanford Biology Research Annual Report for 1962 \\
\hline 1964 & Jan & HW-80500 & Hanford Biology Research Annual Report for 1962 \\
\hline 1965 & Jan & BNWL-122 & Hanford Biology Research Annual Report for 1964 \\
\hline 1966 & Jan & BNWL-280 & Annual Report for 1965 in the Biological Sciences \\
\hline 1967 & Jul & BNWL-480 & Annual Report for 1966, Vol. 1, Biological Sciences \\
\hline 1968 & May & BNWL-714 & Annual Report for 1967, Vol. 1, Biological Sciences \\
\hline 1970 & Jan & BNWL-1050 & Annual Report for 1968, Vol. 1, Part 1, Biological Sciences \\
\hline 1970 & Aug & BNWL-1306 & Annual Report for 1969, Vol. 1, Part 1, Biological Sciences \\
\hline 1971 & Oct & BNWL-1550 & Annual Report for 1970, Vol. 1, Part 1, Biological Sciences \\
\hline 1972 & Sep & BNWL-1650 & Annual Report for 1971, Vol. 1, Part 1, Biological Sciences \\
\hline 1973 & Apr & BNWL-1750 & Annual Report for 1972, Vol. 1, Part 1, Biological Sciences \\
\hline 1974 & Aug & BNWL-1850 & Annual Report for 1973, Part 1, Biological Sciences \\
\hline 1975 & Mar & BNWL-1950 & Annual Report for 1974, Part 1, Biomedical Sciences \\
\hline 1976 & Jan & BNWL-2000 & Annual Report for 1975, Part 1, Biomedical Sciences \\
\hline 1977 & May & BNWL-2100 & Annual Report for 1976, Part 1, Biomedical Sciences \\
\hline 1978 & Feb & PNL-2500 & Annual Report for 1977, Part 1, Biomedical Sciences \\
\hline 1979 & Feb & PNL-2850 & Annual Report for 1978, Part 1, Biomedical Sciences \\
\hline 1980 & Feb & PNL-3300 & Annual Report for 1979, Part 1, Biomedical Sciences \\
\hline 1981 & Feb & PNL-3700 & Annual Report for 1980, Part 1, Biomedical Sciences \\
\hline 1982 & Feb & PNL-4100 & Annual Report for 1981, Part 1, Biomedical Sciences \\
\hline 1983 & Feb & PNL-4600 & Annual Report for 1982, Part 1, Biomedical Sciences \\
\hline 1984 & Feb & PNL-5000 & Annual Report for 1983, Part 1, Biomedical Sciences \\
\hline 1985 & Feb & PNL-5500 & Annual Report for 1984, Part 1, Biomedical Sciences \\
\hline 1986 & Feb & PNL-5750 & Annual Report for 1985, Part 1, Biomedical Sciences \\
\hline 1987 & Feb & PNL-6100 & Annual Report for 1986, Part 1, Biomedical Sciences \\
\hline 1988 & Feb & PNL-6500 & Annual Report for 1987, Part 1, Biomedical Sciences \\
\hline
\end{tabular}




\begin{tabular}{|c|c|c|c|}
\hline \multicolumn{2}{|c|}{ Report Date } & $\begin{array}{l}\text { Document } \\
\text { Number }\end{array}$ & $\begin{array}{l}\text { Document } \\
\text { Tite }\end{array}$ \\
\hline \multicolumn{4}{|c|}{ Inhalation Toxicology Research Institute Reports } \\
\hline 1962 & Sep & LF-2 & $\begin{array}{l}\text { The Flora of Healthy Dogs I. Bacteria and Fungi of the Nose, } \\
\text { Throat, and Lower Intestine }\end{array}$ \\
\hline 1963 & Jun & LF-8 & $\begin{array}{l}\text { Flora of Healthy Dogs II. Isolation of Enteroviruses from Lower } \\
\text { Intestine }\end{array}$ \\
\hline 1963 & Aug & $L F-10$ & $\begin{array}{l}\text { The Development of a Continuous Cell Line from Normal Dog } \\
\text { Liver and Its Susceptibility to Viruses }\end{array}$ \\
\hline 1964 & Jun & LF-15 & The Bactericidal Activity of the Serum of Healthy Beagles \\
\hline 1964 & Sep & $L F-17$ & $\begin{array}{l}\text { Response to Secondary Antigenic Stimulus after Whole Body } \\
\text { X-irradiation in the Beagle }\end{array}$ \\
\hline 1964 & Oct & LF-16 & $\begin{array}{l}\text { The Design of a Canine Inhalation Exposure Apparatus } \\
\text { Incorporating a Whole Body Plethysmograph }\end{array}$ \\
\hline 1964 & Nov & LF-19 & $\begin{array}{l}\text { Flora of Healthy Dogs III. Incidence and Distribution of } \\
\text { Spirochetes in the Digestive Tract of Dogs }\end{array}$ \\
\hline 1965 & May & $L F-25$ & Experimental Infection of Beagles with Echo Virus Type 6 \\
\hline 1965 & Aug & $L F-24$ & $\begin{array}{l}\text { Management Procedures for Animal Colonies Serving the Fission } \\
\text { Product Inhalation Program }\end{array}$ \\
\hline 1965 & Aug & $L F-26$ & Susceptibility of Tissue Cultures of Canine Origin to Viruses \\
\hline 1965 & Sep & $L F-27$ & Intestinal Spirochetes and Disease in Dogs \\
\hline 1965 & Sep & LF-28 & $\begin{array}{l}\text { Selective Summary of Studies on the Fission Product Inhalation } \\
\text { Program from July } 1964 \text { through June } 1965\end{array}$ \\
\hline 1966 & Jun & LF-29 & $\begin{array}{l}\text { Immune Response to a Secondary Stimulus with Leptospira } \\
\text { Canicola and Infectious Canine Hepatitis in Beagles Exposed } \\
\text { to } \mathrm{Sr}^{90}\end{array}$ \\
\hline 1966 & Nov & LF-33 & $\begin{array}{l}\text { Selective Summary of Studies on the Fission Product Inhalation } \\
\text { Program from July } 1965 \text { through June } 1966\end{array}$ \\
\hline 1967 & Oct & LF-36 & $\begin{array}{l}\text { Viruses Isolated from Beagle Dogs Exposed to Aerosols of }{ }^{90} \mathrm{Sr} \\
\text { and }{ }^{144} \mathrm{Ce}\end{array}$ \\
\hline 1967 & Nov & LF-38 & Fission Product Inhalation Program Annual Report 1966-1967 \\
\hline 1968 & Nov & LF-39 & Fission Product Inhalation Program Annual Report 1967-1968 \\
\hline 1969 & Nov & $L F-41$ & Fission Product Inhalation Program Annual Report 1968-1969 \\
\hline 1970 & Nov & $L F-43$ & Fission Product Inhalation Program Annual Report 1969-1970 \\
\hline 1971 & Nov & LF-44 & Fission Product Inhalation Program Annual Report 1970-1971 \\
\hline 1972 & Nov & LF-45 & Fission Product Inhalation Program Annual Report 1971-1972 \\
\hline 1973 & Dec & LF-46 & Annual Report 1972-1973 \\
\hline 1974 & Dec & $L F-49$ & Annual Report 1973-1974 \\
\hline 1975 & Dec & $L F-52$ & Annual Report 1974-1975 \\
\hline 1976 & Dec & LF-56 & Annual Report 1975-1976 \\
\hline 1977 & Dec & LF-58 & Annual Report 1976-1977 \\
\hline
\end{tabular}




\begin{tabular}{|c|c|c|c|}
\hline \multicolumn{2}{|c|}{ Report Date } & \multirow{2}{*}{$\begin{array}{l}\text { Document } \\
\text { Number }\end{array}$} & \multirow{2}{*}{$\begin{array}{l}\text { Document } \\
\text { Title }\end{array}$} \\
\hline Year & Month & & \\
\hline \multicolumn{4}{|c|}{ Inhalation Toxicology Research Institute Reports (Con } \\
\hline 1978 & Dec & LF-60 & Annual Report 1977-1978 \\
\hline 1979 & Dec & LF-69 & Annual Report 1978-1979 \\
\hline 1980 & Dec & LMF-84 & Annual Report 1979-1980 \\
\hline 1981 & Dec & LMF-91 & Annual Report 1980-1981 \\
\hline 1982 & Dec & LMF-102 & Annual Report 1981-1982 \\
\hline 1983 & Dec & LMF-107 & Annual Report 1982-1983 \\
\hline 1984 & Dec & LMF-113 & Annual Report 1983-1984 \\
\hline 1985 & Dec & LMF-114 & Annual Report 1984-1985 \\
\hline 1986 & Dec & LMF-115 & Annual Report 1985-1986 \\
\hline 1987 & Dec & LMF-120 & Annual Report 1986-1987 \\
\hline
\end{tabular}


